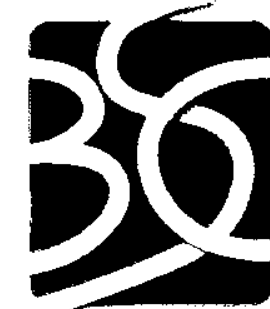

BECHTEL SAIC comâü
QA:QA

MDL-NBS-HS-000010 REV 02

November 2004

\title{
Site-Scale Saturated Zone Transport
}

Prepared for:

U.S. Department of Energy

Office of Civilian Radioactive Waste Management

Office of Repository Development

1551 Hillshire Drive

Las Vegas, Nevada 89134-6321

Prepared by:

Bechtel SAIC Company, LLC

1180 Town Center Drive

Las Vegas, Nevada 89144

Under Contract Number

DE-AC28-01RW12101 


\section{DISCLAIMER}

This report was prepared as an account of work sponsored by an agency of the United States Government. Neither the United States Government nor any agency thereof, nor any of their employees, nor any of their contractors, subcontractors or their employees, makes any warranty, express or implied, or assumes any legal liability or responsibility for the accuracy, completeness, or any third party's use or the results of such use of any information, apparatus, product, or process disclosed, or represents that its use would not infringe privately owned rights. Reference herein to any specific commercial product, process, or service by trade name, trademark, manufacturer, or otherwise, does not necessarily constitute or imply its endorsement, recommendation, or favoring by the United States Government or any agency thereof or its contractors or subcontractors. The views and opinions of authors expressed herein do not necessarily state or reflect those of the United States Government or any agency thereof. 
QA: QA

Site-Scale Saturated Zone Transport

MDL-NBS-HS-000010 REV 02

November 2004 
2. Type of Methematical Modd

8 Procass Moded

Abtracion moded

Sysian Model

Dowotibe intonded Lee of Modat

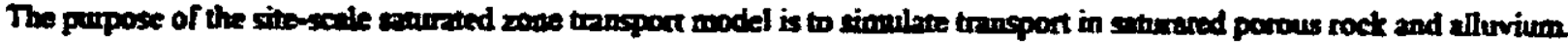

3. The

Sitorade Sanowed Zone Troupoirt

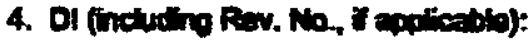

MDLANESFS-OOOOLIO REV O2

\section{Toted Appondices}

Nine
6. No of Pages in Eaph Appondix

A-64, B-6, C-24, D-6, E-10, F-16, G-38, H-8, $1-8$

\begin{tabular}{|c|c|c|c|}
\hline & Pinted Name & Signemine & Dete \\
\hline 7. Origintor & S. Kellorir & & \\
\hline $\begin{array}{l}\text { 8. Independont Technicat } \\
\text { Revinumer }\end{array}$ & L. Chenurvedi & & \\
\hline 9. Chector & E. Kalievina & & \\
\hline 10. QER & P. Buentiaje & & $11 / 2$ \\
\hline 11. Respontiols Monapallood & B.w. Aroold & $T$ & $11 / 02$ \\
\hline 12. Rosponsible Mentager & M. Zmi & & (I) \\
\hline
\end{tabular}

Change Hitary

\begin{tabular}{|c|c|}
\hline 14. Ravison Na & 15. Deseription of Change \\
\hline REV OD & Initial inese \\
\hline REV OI & 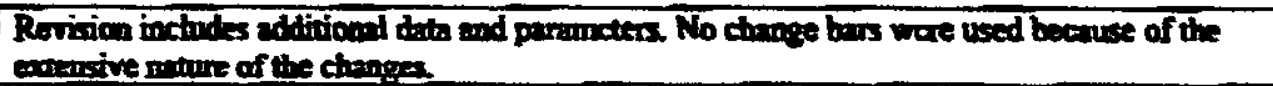 \\
\hline BEV ol Entw OOL & Rerpoure to CR 1162 \\
\hline REV 01 Exin 002 & Responses to CR 221 and CR 2019 \\
\hline REV O2 & 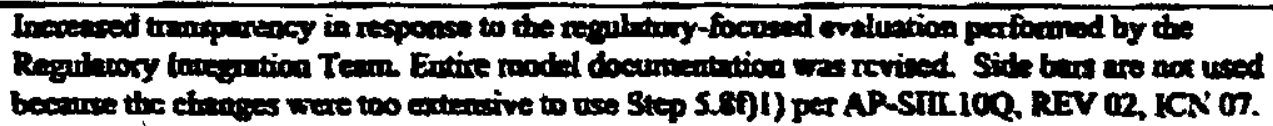 \\
\hline
\end{tabular}


INTENTIONALLY LEFT BLANK 


\section{CONTENTS}

Page

ACRONYMS AND ABBREVIATIONS xix

1. PURPOSE $1-1$

2. QUALITY ASSURANCE ...................................................................................... 2-1

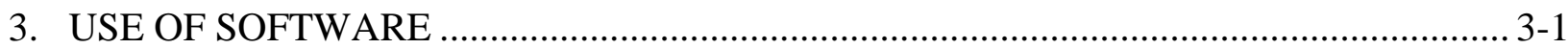

3.1 SOFTWARE TRACKED BY CONFIGURATION MANAGEMENT ..................... 3-1

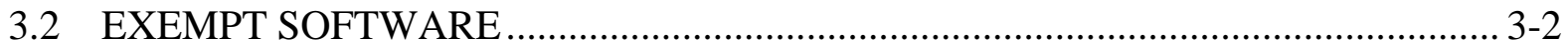

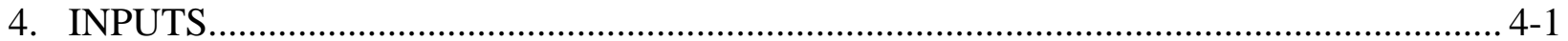

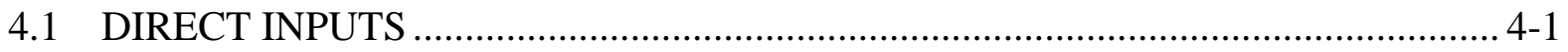

4.1.1 Data and Technical Information ......................................................... 4-1

4.1.2 Parameters and Parameter Uncertainty ........................................................... 4-6

4.1.2.1 Specific Discharge Multiplier …………………............................. 4-8

4.1.2.2 Horizontal Anisotropy in Permeability …………………………..... 4-8

4.1.2.3 Bulk Density in Alluvium ............................................................ 4-8

4.1.2.4 Sorption Coefficient in Alluvium................................................... 4-9

4.1.2.5 Effective Porosity in Alluvium............................................................ 4-9

4.1.2.6 Retardation Factor in Alluvium for Irreversible Colloids ............... 4-10

4.1.2.7 Flowing Interval Porosity ............................................................. 4-10

4.1.2.8 Flowing Interval Spacing ……..................................................... 4-10

4.1.2.9 Matrix Porosity in Volcanics......................................................... 4-11

4.1.2.10 Effective Diffusion Coefficient in Volcanics ................................... 4-11

4.1.2.11 Matrix Sorption Coefficient in Volcanics ....................................... 4-12

4.1.2.12 Retardation Factor in Volcanics for Irreversible Colloids .............. 4-12

4.1.2.13 Groundwater Concentrations of Colloids........................................ 4-12

4.1.2.14 Sorption Coefficient onto Colloids................................................... 4-12

4.1.2.15 Fraction of Colloids Transported Unretarded ................................. 4-13

4.1.2.16 Dispersivity, Longitudinal............................................................. 4-13

4.1.2.17 Dispersivity, Transverse, Horizontal.............................................. 4-13

4.1.2.18 Dispersivity, Transverse, Vertical ................................................ 4-14

4.1.3 Accuracy, Precision, And Representativeness............................................... 4-14

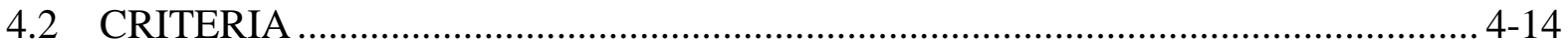

4.3 CODES, STANDARDS, AND REGULATIONS ................................................. 4-17

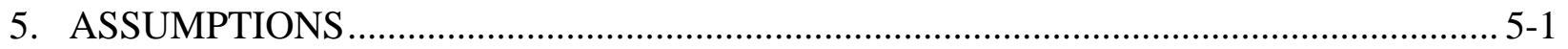

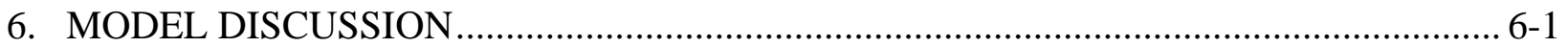

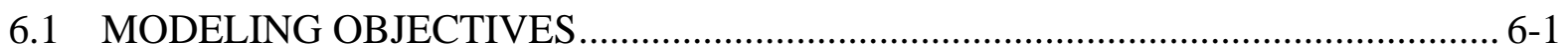

6.2 FEATURES, EVENTS, AND PROCESSES FOR THIS MODEL REPORT .............. 6-2

6.3 BASE-CASE CONCEPTUAL MODEL .............................................................. 6-4

6.4 MATHEMATICAL AND COMPUTATIONAL FORMULATION OF

BASE-CASE CONCEPTUAL MODEL ........................................................... 6-12 


\section{CONTENTS (Continued)}

Page

6.4.1 Overview of Model Formulation

6.4.2 Mathematical Model Description

6.4.2.1 Advective Dispersive Transport 6-12

6.4.2.2 General Form of the Dispersion Tensor for Axisymmetric Media 6-13

6.4.2.2.1 Special Cases of the Dispersion Tensor 6-16

6.4.2.2.2 New Form of the Dispersion Tensor for Axisymmetric Media 6-18

6.4.2.2.3 Dispersion Tensor Proposed by Burnett and Frind..... 6-22

6.4.2.3 Random-Walk Particle-Tracking Method ....................................... 6-24

6.4.2.4 Matrix Diffusion in Fractured Geological Media............................. 6-29

6.4.2.4.1 Mathematical Description of Matrix Diffusion and Sorption...................................................................... 6-30

6.4.2.5 Mathematical Description of Sorptive Transport in Alluvium ......6-34

6.4.2.6 Colloid-Facilitated Transport .......................................................6-34

6.4.2.6.1 Radionuclides Attached Irreversibly to the Colloids ...................................................................... 6-35

6.4.2.6.2 Radionuclides Attached Reversibly to the Colloids .................................................................... 6-36

6.4.3 Base-Case Model Inputs ........................................................................... 6-37

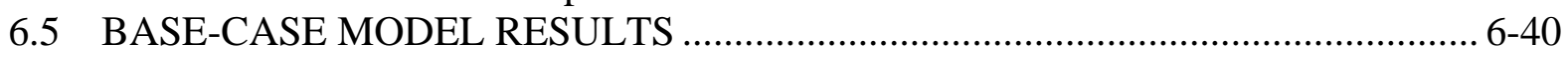

6.6 ALTERNATIVE CONCEPTUAL MODELS ..................................................... 6-44

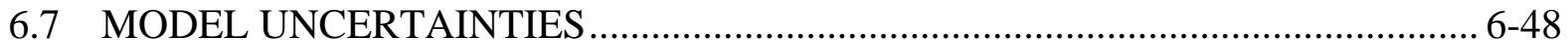

6.7.1 Propagation of Uncertainty in the Input Parameter Values to the Output ...... 6-50

6.7.1.1 Specific Discharge Multiplier ........................................................ 6-51

6.7.1.2 Horizontal Permeability Anisotropy …………….............................. 6-51

6.7.1.3 Bulk Density in Alluvium ............................................................. 6-53

6.7.1.4 Sorption Coefficient in Alluvium..................................................6-54

6.7.1.5 Effective Porosity in Alluvium........................................................6-55

6.7.1.6 Retardation Factor in Alluvium for Radionuclides Attached Irreversibly to Colloids .................................................................. 6-56

6.7.1.7 Reversible Sorption onto Colloids in the Alluvium ......................... 6-57

6.7.1.8 Flowing Interval Aperture in Volcanics..........................................6-58

6.7.1.9 Effective Diffusion Coefficient in Volcanics .................................. 6-59

6.7.1.10 Matrix Sorption Coefficient in Volcanics ....................................... 6-60

6.7.1.11 Retardation Factor in Volcanics for Radionuclides Attached Irreversibly to Colloids .............................................................. 6-61

6.7.1.12 Reversible Sorption onto Colloids in the Volcanics ........................ 6-62

6.7.1.13 Longitudinal Dispersivity .................................................................6-63

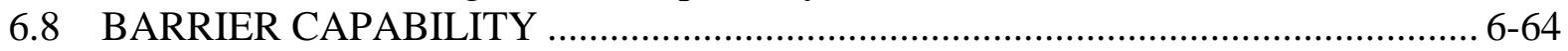

6.8.1 Introduction............................................................................................ 6-64

6.8.2 Saturated Zone Subsystem Performance: Sorption in Volcanics and Alluvium ..... 6-65 


\section{CONTENTS (Continued)}

Page

6.8.3 Saturated-Zone Subsystem Performance: Fractured Volcanic Tuffs and Alluvium

6.8.4 Saturated-Zone Sensitivity Analyses: Advection, Diffusion, and Dispersion 6-66

6.8.5 Colloid-Facilitated Transport in the Saturated Zone $6-73$

6.8.6 Discussion of Saturated-Zone Barrier Performance 6-75

7. VALIDATION. $7-1$

7.1 CONFIDENCE BUILDING DURING MODEL DEVELOPMENT TO ESTABLISH SCIENTIFIC BASIS AND ACCURACY FOR INTENDED USE....... 7-2

7.1.1 Analogue Studies to Support Transport Parameters .......................................... 7-4

7.1.1.1 Studies Performed at the Nevada Test Site ........................................ 7-4

7.1.1.1.1 General Conclusions from NTS Investigations ............ 7-5

7.1.1.1.2 Radionuclide Transport in Alluvium (CAMBRIC Test)........................................................ 7-7

7.1.1.1.3 Radionuclide Transport in the Volcanics (TYBO-BENHAM Study) ............................................ 7-7

7.1.1.2 Transport of Uranium at Natural Analogue Sites.............................. 7-9

7.1.2 Submodel Components ................................................................................ 7-10

7.1.2.1 Advection Through Fractures in the Volcanics............................... 7-10

7.1.2.2 Dispersion in the Volcanics....................................................... 7-12

7.1.2.3 Matrix Diffusion in the Volcanics............................................... 7-14

7.1.2.4 Sorption in the Volcanics ........................................................... 7-14

7.1.2.5 Colloid-Facilitated Transport in the Volcanics ............................... 7-15

7.1.2.5.1 Radionuclides Attached Irreversibly to the Colloids ................................................................... 7-15

7.1.2.5.2 Radionuclides Attached Reversibly to the Colloids ................................................................... 7-16

7.1.2.6 Advection in the Alluvium .......................................................... 7-17

7.1.2.6.1 Alluvial Tests at NC-EWDP-19D1........................... 7-17

7.1.2.6.2 CAMBRIC (NTS) ................................................ 7-17

7.1.2.7 Diffusion and Dispersion in the Alluvium ................................... 7-18

7.1.2.8 Sorption in the Alluvium.......................................................... 7-19

7.1.2.9 Colloid-Facilitated Transport in the Alluvium................................. 7-19

7.1.2.9.1 Radionuclides Attached Irreversibly to the Colloids ...................................................................... 7-21

7.1.2.9.2 Radionuclides Attached Reversibly to the Colloids .

7.1.3 Comparison of Flow Paths and Transit Times Against Those Computed by an Independent Site-Scale Model

7.2 POST-DEVELOPMENT VALIDATION TO SUPPORT THE SCIENTIFIC BASIS OF THE MODEL $7-22$

7.2.1 Comparison of Flow Paths Against Those Deduced from Hydrochemistry Data 


\section{CONTENTS (Continued)}

Page

7.2.2 Comparison of Transit Times Against Those Deduced from ${ }^{14} \mathrm{C}$ Data ........... 7-25

7.3 VALIDATION SUMMARY …………………..................................................... 7-28

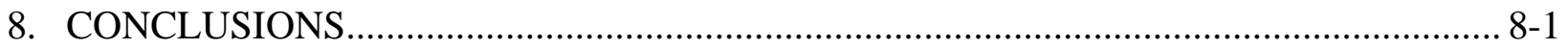

8.1 SUMMARY OF MODELING ACTIVITIES …………………………………... 8-2

8.1.1 Hydrogeologic Setting and Conceptual Flow Model........................................ 8-2

8.1.2 Conceptual Model of Transport .......................................................................... 8-3

8.1.3 Model Validation and Confidence Building................................................ 8-3

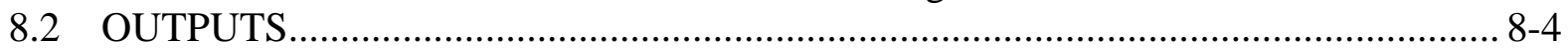

8.2.1 Technical Output........................................................................................... 8-4

8.2.2 Developed Output Listed by Data Tracking Number .......................................... 8-4

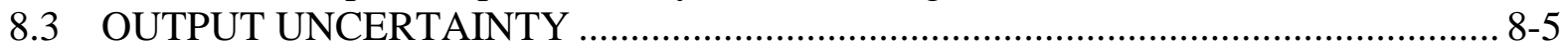

8.4 HOW THE ACCEPTANCE CRITERIA ARE ADDRESSED ................................... 8-5

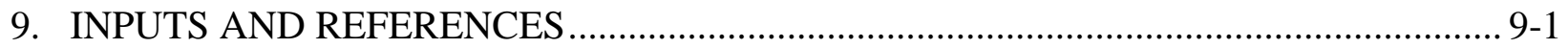

9.1 DOCUMENTS CITED ...................................................................................... 9-1

9.2 CODES, STANDARDS, REGULATIONS, AND PROCEDURES ........................... 9-14

9.3 SOURCE DATA, LISTED BY DATA TRACKING NUMBER …........................... 9-16

9.4 OUTPUT DATA, LISTED BY DATA TRACKING NUMBER ………................. 9-19

9.5 SOFTWARE CODES ..................................................................................... 9-20

APPENDIX A - TECHNICAL BASIS FOR SORPTION-COEFFICIENT PROBABILITY DISTRIBUTION FUNCTIONS........................................ A-1

APPENDIX B - THE COLLOID-FACILITATED TRANSPORT OF REVERSIBLY ATTACHED RADIONUCLIDES.............................................................

APPENDIX C - DERIVATION OF COMPOSITE UPSCALED $K_{D}$ DISTRIBUTIONS........C-1

APPENDIX D - JUSTIFICATION OF LOCAL EQUILIBRIUM APPROACH FOR MODELING RADIONUCLIDE TRANSPORT IN THE SZ ........................ D-1

APPENDIX E - TRANSPORT SIMULATIONS WITH ALTERNATE FLOW

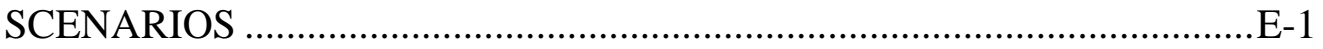

APPENDIX F - REDOX MEASUREMENTS IN SATURATED ZONE WATERS ...............F-1

APPENDIX G - RADIONUCLIDE SORPTION PARAMETERS FROM BATCH AND COLUMN TRANSPORT EXPERIMENTS IN SATURATED ALLUVIUM AND DISCUSSION OF EARLY BREAKTHROUGH BEHAVIOR IN COLUMN EXPERIMENTS............................................... G-1

APPENDIX H - QUALIFICATION OF SORPTION DATA DTNS:

LA0305AM831341.001 AND LA0310AM831341.001 ................................ H-1

APPENDIX I - QUALIFICATION OF COMPOSITIONS OF WATERS FROM

UE-25 J-13 AND UE-25 P\#1(CARBONATE) 


\section{FIGURES}

1-1. A Schematic of the Flow of Information Among SZ Reports

6.3-1. Schematic Illustration of the Conceptual Model of Transport Processes in the Volcanic Tuffs and the Alluvium .

6.4-1. Schematic of the Matrix Diffusion Submodel ......................................................... 6-31

6.5-1. Breakthrough Curve at the 18-km Boundary for the Transport Base Case (for a Conservative, Nonsorbing Radionuclide)........................................................... 6-42

6.5-2. Particle Tracks Resulting from the Site-Scale Saturated Zone Base-Case Transport Model

6.7-1. Propagation of Input Uncertainty in the Specific Discharge to the Output Breakthrough Curves at the 18-km Boundary $6-52$

6.7-2. Propagation of Input Uncertainty in the Horizontal Permeability Anisotropy Ratio to the Output Breakthrough Curves at the 18-km Boundary 6-53

6.7-3. Propagation of Input Uncertainty in the Bulk Density of Alluvium to the Output Breakthrough Curves at the 18-km Boundary

6.7-4. Propagation of Input Uncertainty in the Sorption Coefficient in Alluvium to the Output Breakthrough Curves at the 18-km Boundary .... $6-55$

6.7-5. Propagation of Input Uncertainty in the Effective Porosity of Alluvium to the Breakthrough Curves at the 18-km Boundary ..... 6-56

6.7-6. Propagation of Input Uncertainty in the Colloid Retardation Factor in Alluvium for Irreversible Colloids to the Breakthrough Curves at the 18-km Boundary... $6-57$

6.7-7. Propagation of Input Uncertainty in the Colloid Retardation Factor in Alluvium for Reversible Colloids to the Output Breakthrough Curves at the 18-km Boundary $6-58$

6.7-8. Propagation of Input Uncertainty in the Flowing Interval Aperture in Volcanics to the Breakthrough Curves at the 18-km Boundary .....

6.7-9. Propagation of Input Uncertainty in the Effective Diffusion Coefficient in Volcanics to the Output Breakthrough Curves at the 18-km Boundary

6.7-10. Propagation of Input Uncertainty in the Matrix Sorption Coefficient in Volcanics to the Output Breakthrough Curves at the 18-km Boundary ..... $6-61$

6.7-11. Propagation of Input Uncertainty in the Colloid Retardation Factor in Volcanics for Irreversible Colloids to the Output Breakthrough Curves at the 18-km Boundary.....

6.7-12. Propagation of Input Uncertainty in the Distribution Parameter Kc in Volcanics for Reversible Colloids to the Output Breakthrough Curves at the 18-km Boundary

6.7-13. Propagation of Input Uncertainty in the Longitudinal Dispersivity to the Output Breakthrough Curves at the 18-km Boundary

6.8-1a. Breakthrough Curves for the Base Case, Conservative Radionuclide, and Sorbing Radionuclides: 18-km Boundary. 6-66

6.8-1b. Breakthrough Curves for the Base Case, Conservative Radionuclides, and Sorbing Radionuclides: Volcanic/Alluvium Boundary . 


\section{FIGURES (Continued)}

6.8-2a. Breakthrough Curves Comparing the Base Case, Nondispersive, and Nondiffusive Cases: 18-km Boundary....

6.8-2b. Breakthrough Curves Comparing the Base Case, Nondispersive, and Nondiffusive Cases: Volcanic/Alluvium Boundary ...

6.8-3a. Breakthrough Curves for the Base-Case Parameters with Point Source and Distributed Source for Input to the Saturated Zone: 18-km Boundary....

6.8-3b. Comparison of Breakthrough Curves for the Point Source and Distributed Source, Base Case, and Nondiffusive Case: Volcanic/Alluvium Boundary

6.8-4a. Breakthrough Curves for the Base Case and Cases with Lower and Higher Specific Discharge: 18-km Boundary....

6.8-4b. Breakthrough Curves for the Base Case and Cases with Lower and Higher Specific Discharge: Volcanic/Alluvium Boundary .....

6.8-5a. Comparison of Breakthrough Curves for the Base Case and Radionuclides Irreversibly Attached to Colloids: 18-km Boundary

6.8-5b. Comparison of Breakthrough Curves for the Base Case and Radionuclides Irreversibly Attached to Colloids: Volcanic/Alluvium Boundary

7-1. Locations of Underground Nuclear Tests and the Specific Locations of the TYBO and BENHAM Tests at the Nevada Test Site

7-2. Schematic of Possible Pathways from the BENHAM Test to the ER-20-5 Observation Wells

7-3. Normalized Tracer Concentrations Versus Time in the Bullfrog Tuff Tracer Test Conducted from October 1996 to September 1997

7-4. Longitudinal Dispersivity as a Function of Test Scale in Several Tracer Tests Conducted in the Vicinity of Yucca Mountain.....

7-5. Plot of Longitudinal Dispersivity Versus Length Scale Showing the Range of C-Wells Values Derived from Interpretations of the Prow Pass and Bullfrog Multiple-Tracer Tests.

7-6. $\quad$ Normalized Concentrations of PFBA and 360-nm-Diameter Carboxylate-Modified Polystyrene Latex Microspheres in the Bullfrog Tuff Tracer Test.....

7-7. $\quad$ Normalized Concentrations of Tracers in Production Water from NC-EWDP-19D1 as a Function of Gallons Pumped After a Rest Period of Approximately 0.5 Hour

7-8. Column Data (Concentration in the units of milli-equivalent/liter) and MULTRAN Fits for Experiments with a LiBr Injection Concentration of $0.0275 \mathrm{M}$

7-9a. Transport Pathways Deduced from Hydrochemistry. $7-23$

7-9b. Transport Pathways Deduced from Hydrochemistry Data (in red, enlarged from 7-9a) Overlaying Flow Paths Calculated from the Site-Scale SZ Transport Model (in black) for Tracer Particles Starting at the Repository Footprint.

A-1. Americium Sorption Coefficients on Devitrified Tuff Versus Calculated Final Americium Concentration in Solution..... 


\section{FIGURES (Continued)}

Page

A-2. Americium Sorption Coefficients for Devitrified Tuff as a Function of Experiment Duration........................................................................................ A-11

A-3. Americium Sorption Coefficients on Devitrified Tuff Versus pH ............................ A-12

A-4. Americium Sorption Coefficients on Quartz from Beall et al. and Model Fit ........... A-13

A-5. Americium Sorption Coefficients on Zeolitic Tuff Versus Calculated Final Americium Concentration in Solution .................................................................... A-14

A-6. Americium Sorption Coefficients for Zeolitic Tuff as a Function of Duration of Sorption and Desorption Experiments ............................................................... A-15

A-7. Americium Sorption Coefficient Data and Modeling Results for Zeolitic Tuff as a Function of $\mathrm{pH}$

A-8. Cesium Sorption Coefficients on Devitrified Tuff Versus Calculated Final Cesium Concentration in Solution.......................................................................... A-17

A-9. Freundlich Isotherm Fit to Sorption-Coefficient Data for Cesium on Devitrified Tuff Sample G1-2840 in J-13 Water ................................................................. A-18

A-11. Cesium Sorption Coefficients on Zeolitic Tuff Versus Calculated Final Cesium Concentration in Solution ........................................................................... A-20

A-12. Freundlich Isotherm Fit to Sorption-Coefficient Data for Sample YM-38 in J-13 Water ................................................................................................... A-20

A-13. Cesium Sorption Coefficients on Zeolitic Tuff Versus Experiment Duration for Sorption (Forward) and Desorption (Backward) Experiments .................................... A-21

A-14. Neptunium Sorption Coefficients on Devitrified Tuff Versus Calculated Final Neptunium Concentration in Solution ............................................................... A-23

A-15. Neptunium Sorption Coefficients on Devitrified Tuff Versus Calculated Final Neptunium Concentration in Solution ....

A-16. Neptunium Sorption Coefficients on Devitrified Tuff Versus Experiment Duration for Sorption (Forward) and Desorption (Backward) Experiments

A-17. Neptunium Sorption Coefficients on Devitrified Tuff in J-13 and Synthetic p\#1 Waters Versus Solution pH in Sorption (Forward) and Desorption (Backward) Experiments

A-18. Neptunium Sorption Coefficients on Zeolitic Tuff Versus Calculated Final Neptunium Concentration in Solution

A-19. Neptunium Sorption Coefficients on Zeolitic Tuff Versus Calculated Final Neptunium Concentration in Solution with Data from Oversaturated Experiments Removed...

A-20. Neptunium Sorption Coefficients on Zeolitic Tuff Versus Experiment Duration for Sorption (Forward) and Desorption (Backward) Experiments

A-21. Neptunium Sorption Coefficients on Devitrified Tuff in J-13 Water and Synthetic p\#1 Water Versus Solution pH in Sorption (Forward) and Desorption (Backward) Experiments ...

A-22. Plutonium Sorption Coefficients on Devitrified Tuff Versus Calculated Final Plutonium Concentration in Solution... A-30

A-23. Plutonium Sorption Coefficients Versus Calculated Final Plutonium Solution Concentration (M/L) for Experiments with Samples YM-22 and G4-272. 


\section{FIGURES (Continued)}

Page

A-24. Plutonium Sorption Coefficients on Devitrified Tuff Versus Experiment Duration for Sorption (Forward) and Desorption (Backward) Experiments

A-25. Plutonium Sorption Coefficients on Devitrified Tuff in J-13 Water and Synthetic p\#1 Water Versus Solution pH in Sorption (Forward) and Desorption (Backward) Experiments

A-26. Plutonium Sorption Coefficients on Devitrified Tuff in J-13 Water and Synthetic p\#1 Water Versus Solution pH in Sorption (Forward) and Desorption (Backward) Experiments with Durations Greater than 40 Days ............................ A-33

A-27. Plutonium Sorption Coefficients Versus Eh as Predicted by PHREEQC Model...... A-34

A-28. Plutonium Sorption Coefficients on Zeolitic Tuff Versus Calculated Final Plutonium Concentration in Solution...

A-29. Plutonium Sorption Coefficients on Zeolitic Tuff Versus Experiment Duration for Sorption (Forward) and Desorption (Backward) Experiments

A-30. Plutonium Sorption Coefficients on Zeolitic Tuff in J-13 Water and Synthetic p\#1 Water Versus Solution pH in Sorption (Forward) and Desorption (Backward) Experiments with Durations Greater than 40 Days ............................. A-37

A-31. Protactinium Sorption Coefficients Versus pH................................................... A-38

A-32. Barium and Radium Sorption Coefficients on Devitrified Tuff Versus

Calculated Final Barium or Radium Concentrations in Solution A-41

A-33. Barium and Radium Sorption Coefficients on Devitrified Tuff Versus Experiment Duration for Sorption (Forward) and Desorption (Backward)

Experiments A-42

A-34. Barium and Radium Sorption Coefficients on Devitrified Tuff Versus

Experiment Duration for Sorption (Forward) and Desorption (Backward) Experiments

A-35. Barium and Radium Sorption Coefficients on Zeolitic Tuff Versus Calculated

Final Barium or Radium Concentrations in Solution A-43

A-36. Isotherm Diagram for Barium Sorption on Zeolitic Tuff Sample YM-38 in J-13 Water. A-44

A-37. Barium and Radium Sorption Coefficients on Zeolitic Tuff in J-13 Water Versus Experiment Duration for Sorption (Forward) and Desorption

(Backward) Experiments

A-38. Strontium Sorption Coefficients on Devitrified Tuff Versus Calculated Final

Strontium Concentration in Solution A-46

A-39. Strontium Sorption Coefficients on Devitrified Tuff Versus Experiment Duration for Sorption (Forward) and Desorption (Backward) Experiments . A-47

A-40. Strontium Sorption Coefficients on Devitrified Tuff Versus Experiment Duration for Sorption (Forward) and Desorption (Backward) Experiments with Reduced Range

A-41. Strontium Sorption Coefficients on Zeolitic Tuff Versus Calculated Final Strontium Concentration in Solution .... A-48

A-42. Strontium Sorption Coefficients Versus Calculated Final Solution Concentration (M/L) for Sample YM-38 in J-13 Water. 


\section{FIGURES (Continued)}

Page

A-43. Strontium Sorption Coefficients on Zeolitic Tuff Versus Experiment Duration for Sorption (Forward) and Desorption (Backward) Experiments

A-44. Strontium Sorption Coefficients on Zeolitic Tuff With Fine Fraction Removed Versus Experiment Duration for Sorption (Forward) and Desorption (Backward) Experiments

A-45. Thorium Sorption Coefficients on Tuff Versus Calculated Final Thorium

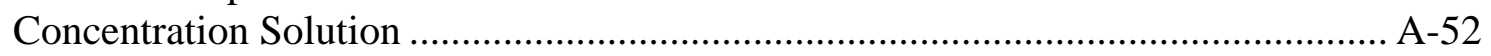

A-46. Thorium Sorption Coefficients on Tuff Versus pH ..................................................... A-52

A-47. Uranium Sorption Coefficients on Devitrified Tuff Versus Calculated Final Uranium Concentration in Solution....

A-48. Uranium Sorption Coefficients on Devitrified Tuff Versus Experiment Duration for Sorption (Forward) and Desorption (Backward) Experiments .............. A-55

A-49. Uranium Sorption Coefficients on Devitrified Tuff Versus pH ................................ A-55

A-50. Uranium Sorption Coefficients on Devitrified Tuff in p\#1 (Volcanics) Water Versus $\mathrm{pH}$. A-56

A-51. Uranium Sorption Coefficients on Zeolitic Tuff Versus Calculated Final Uranium Concentration in Solution

A-52. Uranium Sorption Coefficients on Zeolitic Tuff as a Function of Experiment Duration .

A-53. Uranium Sorption Coefficients for Zeolitic Tuff in J-13 Water Plotted as a Function of $\mathrm{pH}$..... A-58

A-54. Uranium Sorption Coefficients for Zeolitic Tuff in Synthetic p\#1 Water as a Function of $\mathrm{pH}$.

C-1. $\quad$ A Schematic Representation of the Definition of Effective $K_{d}$......................................

C-2. The Processes During Transport of a Radionuclide in a Fractured Media ......................-3

C-3. Representation of the Breakthrough Curves Used to Calculate Effective Matrix

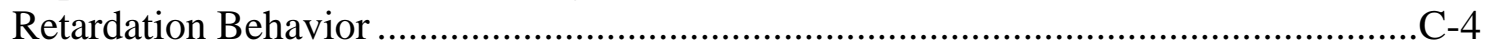

C-4. Calculated Semivariogram and Model Fit in the Horizontal Direction ...........................-8

C-5. Calculated Semivariogram and Model Fit in the Vertical Direction ................................-8

C-6. $\quad$ Comparison of Breakthrough Behavior Predicted by the Calculated Effective $K_{d}$.....

C-7. Plot of Composite Cumulative Probability Distribution for Americium, Thorium, and Protactinium in Volcanics and Alluvium.

C-8. Plot of Composite Cumulative Probability Distribution for Strontium in Volcanics and Alluvium

C-9. Plot of Composite Cumulative Probability Distribution for Uranium in Volcanics.

C-10. Plot of Composite Cumulative Probability Distribution for Uranium in

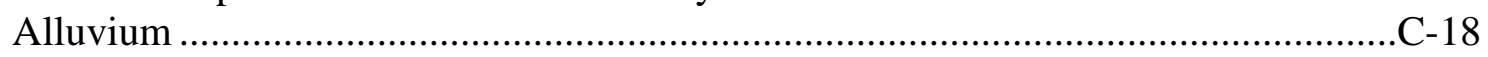

C-11. Plot of Composite Cumulative Probability Distribution for Neptunium in

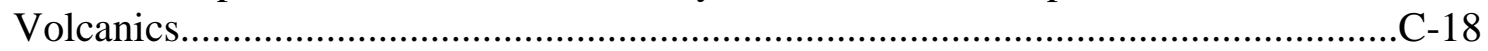

C-12. Plot of Composite Cumulative Probability Distribution for Neptunium in Alluvium ..C-19 


\section{FIGURES (Continued)}

C-13. Plot of Composite Cumulative Probability Distribution for Plutonium in Volcanics.

C-14. Plot of Composite Cumulative Probability Distribution for Plutonium in Alluvium

C-15. Plot of Composite Cumulative Probability Distribution for Cesium in Volcanics.

C-16. Plot of Composite Cumulative Probability Distribution for Cesium in

Alluvium

E-1. Breakthrough Curves for Nonsorbing Radionuclides for Future Glacial-transition Climatic Conditions Using Two Alternative Approaches

E-2. Breakthrough Curves for Neptunium for Future Glacial-transition Climatic Conditions Using Two Alternative Approaches

E-3 Breakthrough Curves at the Boundary to the Accessible Environment at $18 \mathrm{~km}$ for the alternate flow models and the Base-Case Flow Model (Using Base-Case Transport Properties)

E-4. Comparison of the Breakthrough Curves for the Alternate Flow Model AM2

With and Without Matrix Diffusion ..... E-6

E-5. Breakthrough Curves for the Case of a Moderately Sorbing Radionuclide Such As Np Using the AM2 Flow Model and Base-Case Flow Model

F-1. Locations of the Wells Sampled for Redox Analysis

F-2. Measured Eh Values Relative to the Standard Hydrogen Electrode Versus Measured Values of Dissolved Oxygen

F-3. Plot of Duration of Field Measurement (in minutes) Versus Measured Eh Value in mv Relative to the Standard Hydrogen Electrode for Oxidizing Waters

F-4. $\quad$ Measured pH Values Versus Measured Eh Values and Eh Values Calculated From the $\mathrm{DO} / \mathrm{H}_{2} \mathrm{O}, \mathrm{Mn}^{2+} / \mathrm{MnO}_{2}, \mathrm{NO}_{2}^{-} / \mathrm{NO}_{3}^{-}$, and $\mathrm{Fe}^{2+} / \mathrm{Fe}(\mathrm{OH})_{3}$ Couples in Oxidizing Groundwaters ( $\mathrm{DO}>1.0 \mathrm{mg} / \mathrm{L}$ )

F-5. $\quad$ Measured Eh Values Versus pH Values and Measured pH Values Versus Eh Values Calculated on the Basis of the $\mathrm{Fe}^{2+} / \mathrm{FeS}_{2}$ (pyrite) Couple

G-1. Sorption Kinetics of ${ }^{237} \mathrm{~Np}$ to Yucca Mountain Alluvium .......................................... G-8

G-2. Batch $K_{d}$ Values for $\mathrm{Np}(\mathrm{V})$ in Different Intervals and Size Fractions......................... G-9

G-3. Batch $K_{d}$ Values for Np(V) in Waters from Boreholes NC-EWDP-3S and

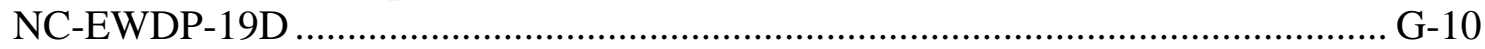

G-4. Batch $K_{d}$ Values for ${ }^{237} \mathrm{~Np}$ in Solutions with Different Ionic Strengths ..................... G-11

G-5. Surface Area, Combined Smectite and Clinoptilolite, and $K_{d}$ Values for $\mathrm{Np}(\mathrm{V})$....... G-12

G-6. Batch $K_{d}$ Values for ${ }^{129} \mathrm{I}$ and ${ }^{99} \mathrm{Tc}$ in Alluvium ....................................................... G-14

G-7. $\quad K_{d}$ Values for ${ }^{237} \mathrm{~Np}$ and ${ }^{233} \mathrm{U}$ in Alluvium...............................................................

G-8. $\quad$ Batch $K_{d}$ Values for ${ }^{233} \mathrm{U}$ in NC-EWDP-19D Zone 1 and Zone 4 Waters .................. G-15

G-9. Batch $K_{d}$ Values for ${ }^{233} \mathrm{U}$ onto Alluvium as a Function of Time ................................ G-17

G-10. Cumulative Release of Sorbed ${ }^{233} \mathrm{U}$ from NC-EWDP-19IM1A and NC-EWDP-10SA Alluvium G-17 


\section{FIGURES (Continued)}

G-11. Release of Sorbed ${ }^{233} \mathrm{U}$ as a Function of Eluted Volume of Groundwater ................. G-18

G-12. Tritiated Water and Neptunium Breakthrough Curves in Columns 1 and 2.............. G-20

G-13. Neptunium Breakthrough Curves in Columns 3, 4, and 5....................................... G-21

G-14. $\quad{ }^{233}$ U Breakthrough Curves in the Three Alluvium Column Experiments................... G-22

G-15. Predicted Breakthrough Curves as a Function of the Product of Adsorption Rate Constant $(\mathrm{k})$ and Nonsorbed Residence Time in the Column ( $\mathrm{t})$................................. G-24

G-16. A Schematic of Adsorption Residence Time Distribution in which there is a Probability of 0.5 of Having No Sorption, and a Probability of 0.5 of Having an Adsorption Residence Time of 1 (arbitrary time units)....

G-17. A Schematic of Adsorption Residence Time Distribution in which there is a Probability of 0.25 of Having No Sorption, and a Probability of 0.5 of Having an Adsorption Residence Time of 1, and a Probability of 0.25 of Having an Adsorption Residence Time of 2 (arbitrary time units)....

G-18. Adsorption Residence Time Distribution Corresponding to First-order Desorption with a Rate Constant of $0.2 \mathrm{hr}^{-1}$, and Three Distributions Defined by Equation G-1 that are Discussed in the Text. Distribution 1 is an Approximation of the First-Order Desorption Distribution, Distribution 2 is an Approximation to a First-Order Desorption Distribution with a Rate Constant of $1 \mathrm{hr}^{-1}$, and Distribution 3 Has the Same Area Under the Curve as Distribution 1, but with an Effective Adsorption Rate about 4 Times Lower

G-19. Breakthrough Curves Computed by Spreadsheet Analysis When Residence Time Distribution 1 From G-18 is Sampled Four Times per Hour of Nonsorbed Residence Time in the Column. The Products of the Adsorption Rate Constant and the Nonsorbed Residence Time Associated with Each Breakthrough Curve are Listed in the Legend. These Products are the Same as Those Associated With the Breakthrough Curves of G-15.

G-20. Breakthrough Curves Obtained by Sampling Distribution 2 Five Times More Frequently Than Distribution 1 But With the Nonsorbed Residence Time in the Column Being Five Times Longer for Distribution 1

G-21. Breakthrough Curves Obtained by Sampling Distribution 1 and Distribution 3 at the Same Frequency (four times per hour) With the Nonsorbed Residence Time in the Column Being Four Times Longer for Distribution 3

G-22. Adsorption Residence Time Distribution that has a Low Probability of Very Long Residence Times (i.e., a Small Fraction of Very Strong Adsorption Sites)

G-23. Percent of Solute Sorbed as a Function of Time in a Simulated Batch Sorption Experiment Using the Adsorption Residence Time Distribution of G-22 at a Sampling Frequency of Once Every 2 Hours

G-24. Fraction of Solute Desorbed as a Function of Time in Simulated Desorption Experiments Initiated After 10 Hours and 200 Hours of Sorption, Respectively, Using the Adsorption Residence Time Distribution of Figure G-22 G-35 


\section{FIGURES (Continued)}

Page

G-25. Simulated Breakthrough Curves as a Function of Relative Flow Rate for a Solute That Has an Adsorption Residence Time Distribution Given by Figure G-22. These Breakthrough Curves are Qualitatively Comparable to Those for Neptunium in G-13

H-1. Barium Sorption Coefficient on Zeolitic Tuff in J-13 Water Versus Calculated Final Barium-133 Solution Concentration................................................................ H-2

H-2. Uranium Sorption Coefficient on Devitrified Tuff in J-13 Water Versus pH .............. H-3 


\section{TABLES}

Page

3-1. Computer Software and Routines ........................................................................ 3-1

3-2. Exempt Software............................................................................................ 3-2

4-1. Input Data and Technical Information ................................................................ 4-2

4-2. Input Parameters and Range of Values for the Site-Scale SZ Transport Model .......... 4-6

4-3. Project Requirements and Acceptance Criteria Applicable to This Model Report .... 4-14

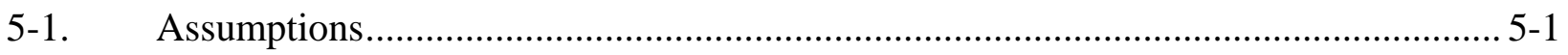

6.2-1. Features, Events, and Processes Included in TSPA-LA and Relevant to This Model Report ...................................................................................................... 6-3

6.4-1. Colloid-Facilitated Transport of Radionuclides............................................................ 6-35

6.4-2. Base-Case Model Inputs ...................................................................................... 6-38

6.4-3. Additional Parameters Needed for Abstraction Analysis ............................................. 6-40

6.6-1a. Key Components and Basis for the Alternative Conceptual Models Excluded from Further Consideration Because They Lead to Transit Times Greater than the Base-Case Results ............................................................................................... 6-44

6.6-1b. Key Components, Disposition, and Basis for Alternative Conceptual Models that are Implicitly Included in the Site-Scale SZ Transport Model ............................. 6-47

6.7-1. Effect of Parameter Ranges on 50 Percent Breakthrough Times ................................. 6-50

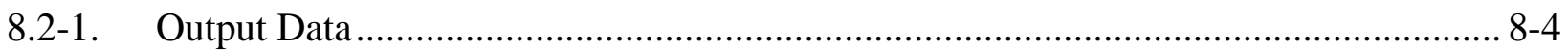

A-1. Surface Areas $\left(\mathrm{m}^{2} / \mathrm{g}\right)$ for Yucca Mountain Tuffs......................................................... A-3

A-2a. Compositions of Waters from UE-25 J-13 and UE-25 p\#1 ……................................ A-6

A-2b. Comparative Information on the Range of Concentrations From Wells in the SZ Transport Model Area..................................................................................... A-7

A-3. Surface Complexation Reactions for Americium ........................................................ A-13

A-4. Small-Scale Probability Distribution Functions for $K_{d}$ s in the Saturated Zone Developed on the Basis of Laboratory Data from Core Samples ................................ A-60

A-5. Recommended SZ $K_{d}$ Correlations ............................................................................ A-62

C-1. $\quad$ List of Wells for Which Mineral Abundance Data Were Available................................-5

C-2. Spatial Correlation Parameters for Mineralogic Rock Type Data ...................................-9

C-3. Proportions of Zeolitic and Devitrified Rocks in Output Realizations............................-9

C-4. Statistical Distributions of Experimentally Observed $K_{d}$ Values .....................................9

C-5. Comparison of Input and Mean Output CDFs for Uranium ............................................

C-6. Comparison of Input and Mean Output CDFs for Cesium ............................................-10

C-7. Comparison of Input and Mean Output CDFs for Neptunium .....................................-10

C-8. Comparison of Input and Mean Output CDFs for Plutonium.......................................-10

C-9. Values of Properties Used in Flow and Transport Calculations ......................................-11

C-10. Values of Diffusion Coefficients Used for the Particle-Tracking Calculations ...........-11

C-11. Statistics of Calculated Effective $K_{d}$ Values ............................................................... 


\section{TABLES (Continued)}

Page

C-12. Effect of Changes in Correlation Length on Effective $K_{d}$ Distributions for

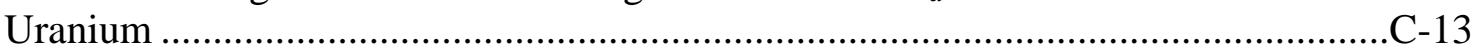

C-13. Statistics of Calculated Effective $K_{d}$ Values for Uranium for Different Hydraulic Gradients .................................................................................................................. -13

C-14. Recommended Composite Distribution for $K_{d}$ s In Volcanics and Alluvium ...............-17

F-1. Water Quality Data for Samples from Borehole NC-EWDP-22S ...............................F-10

G-1. Redox Measurements in Groundwater in Nye County Boreholes................................ G-3

G-2. Boreholes and Sample Preparation Methods ……………………………............ G-5

G-3. Borehole NC-EWDP-03S and NC-EWDP-19D Water Composition ........................... G-6

G-4. Mineral Abundance and Surface Areas for Selected Alluvium Samples used in Np Sorption Tests .................................................................................................. G-7

G-5. Experimental Conditions for Testing Kinetics of $\mathrm{Np}(\mathrm{V})$ Sorption to Alluvium ......... G-8

G-6. Chemical Composition of NC-EWDP-19D Waters ………………………….......... G-13

G-7. Quantitative X-ray Diffraction Results of Alluvium Used in Second Set of Experiments ...................................................................................................... G-13

G-8. $\quad \mathrm{Np}(\mathrm{V})$ Column Study (I) .................................................................................. G-19

G-9. $\quad \mathrm{Np}(\mathrm{V})$ Column Study (II)................................................................................. G-19

G-10. Uranium Column Experiments .......................................................................... G-22

I-1 Compositions of Waters from UE-25 J-13 and UE-25 p\#1 ……...................................

I-2. Comparison of Composition of Water from UE-25 J-13..............................................

I-3. Comparison of Compositions of Water from UE-25 p\#1(Carbonate)............................. I-4 


\section{ACRONYMS AND ABBREVIATIONS}

ATC (Nye Country) Alluvial Testing Complex

CDF cumulative distribution function

DIRS Document Input Reference System

DO dissolved oxygen

EWDP Early Warning Drilling Program

FEPs features, events, and processes

LA license application

LANL Los Alamos National Laboratory

NC-EWDP Nye County Early Warning Drilling Program

NNWSI Nevada Nuclear Waste Storage Investigations

NRC U.S. Nuclear Regulatory Commission

NTS Nevada Test Site

ORP oxidation/reduction potential

PFBA difluorobenzoic acid

QA quality assurance

QAM Quality Assurance Manual

SHE standard hydrogen electrode

SME subject matter expert

STN software tracking number

SZ saturated zone

TSPA total system performance assessment

UZ unsaturated zone

XRD X-ray diffraction

YMP Yucca Mountain Project

YMRP Yucca Mountain Review Plan, Final Report 


\section{INTENTIONALLY LEFT BLANK}




\section{PURPOSE}

This work provides a site-scale transport model for calculating radionuclide transport in the saturated zone (SZ) at Yucca Mountain, for use in the abstractions model in support of Total System Performance Assessment for License Application (TSPA-LA). The purpose of this model report is to provide documentation for the components of the site-scale SZ transport model in accordance with administrative procedure AP-SIII.10Q, Models.

The initial documentation of this model report was conducted under the Technical Work Plan For: Saturated Zone Flow and Transport Modeling and Testing (BSC 2003 [DIRS 163965]). The model report has been revised in accordance with the Technical Work Plan For: Natural System - Saturated Zone Analysis and Model Report Integration, Section 2.1.1.4 (BSC 2004 [DIRS 171421]) to incorporate Regulatory Integration Team comments. All activities listed in the technical work plan that are appropriate to the transport model are documented in this report and are described in Section 2.1.1.4 (BSC 2004 [DIRS 171421]). This report documents: (1) the advection-dispersion transport model including matrix diffusion (Sections 6.3 and 6.4); (2) a description and validation of the transport model (Sections 6.3 and 7); (3) the numerical methods for simulating radionuclide transport (Section 6.4); (4) the parameters (sorption coefficient, $K_{d}$ ) and their uncertainty distributions used for modeling radionuclide sorption (Appendices $A$ and C); (5) the parameters used for modeling colloid-facilitated radionuclide transport (Table 4-1, Section 6.4.2.6, and Appendix B); and (6) alternative conceptual models and their dispositions (Section 6.6).

The intended use of this model is to simulate transport in saturated fractured porous rock (double porosity) and alluvium. The particle-tracking method of simulating radionuclide transport is incorporated in the finite-volume heat and mass transfer numerical analysis (FEHM) computer code, (FEHM V2.20, STN: 10086-2.20-00) (LANL 2003 [DIRS 161725]) and is described in Section 6.4 of this report. FEHM is a three-dimensional (3-D), finite-volume, finite-element, heat and mass flow-and-transport code.

This report documents the features and capabilities of the site-scale transport model for calculating radionuclide transport in the SZ at Yucca Mountain in support of the TSPA-LA. Correlative flow-model calculations using FEHM are carried out and documented in the model report Saturated Zone Site-Scale Flow Model (BSC 2004 [DIRS 170037]). The velocity fields are calculated by the flow model independent of the transport processes and supplied as a part of the output package from the flow model, which is then used as inputs to the transport model. Several SZ analysis model reports provide information and data needed as feed-ins for this report, and this report in turn provides technical product outputs that feed into other SZ reports. The details of inputs to the site-scale transport model are provided in Section 4.

Figure 1-1 shows the relationship of this report to other model reports that also pertain to flow and transport in the SZ. Figure 1-1 also shows the flow of key information among the SZ reports. It should be noted that Figure 1-1 does not contain a complete representation of the data and parameter inputs and outputs of all SZ reports, nor does it show inputs external to this suite of SZ reports. The primary input model to this report is the SZ site-scale flow model. In addition, several other reports provide the technical basis for the conceptual models of radionuclide transport in fractured and porous media and in association with ground water 
colloids. A complete listing and justification of the input data are given in Table 4-1. The output model from this report is the SZ site-scale transport model, which forms the basis of the abstraction models for use in the TSPA-LA. The outputs from this report are listed in Table 8.2-1.

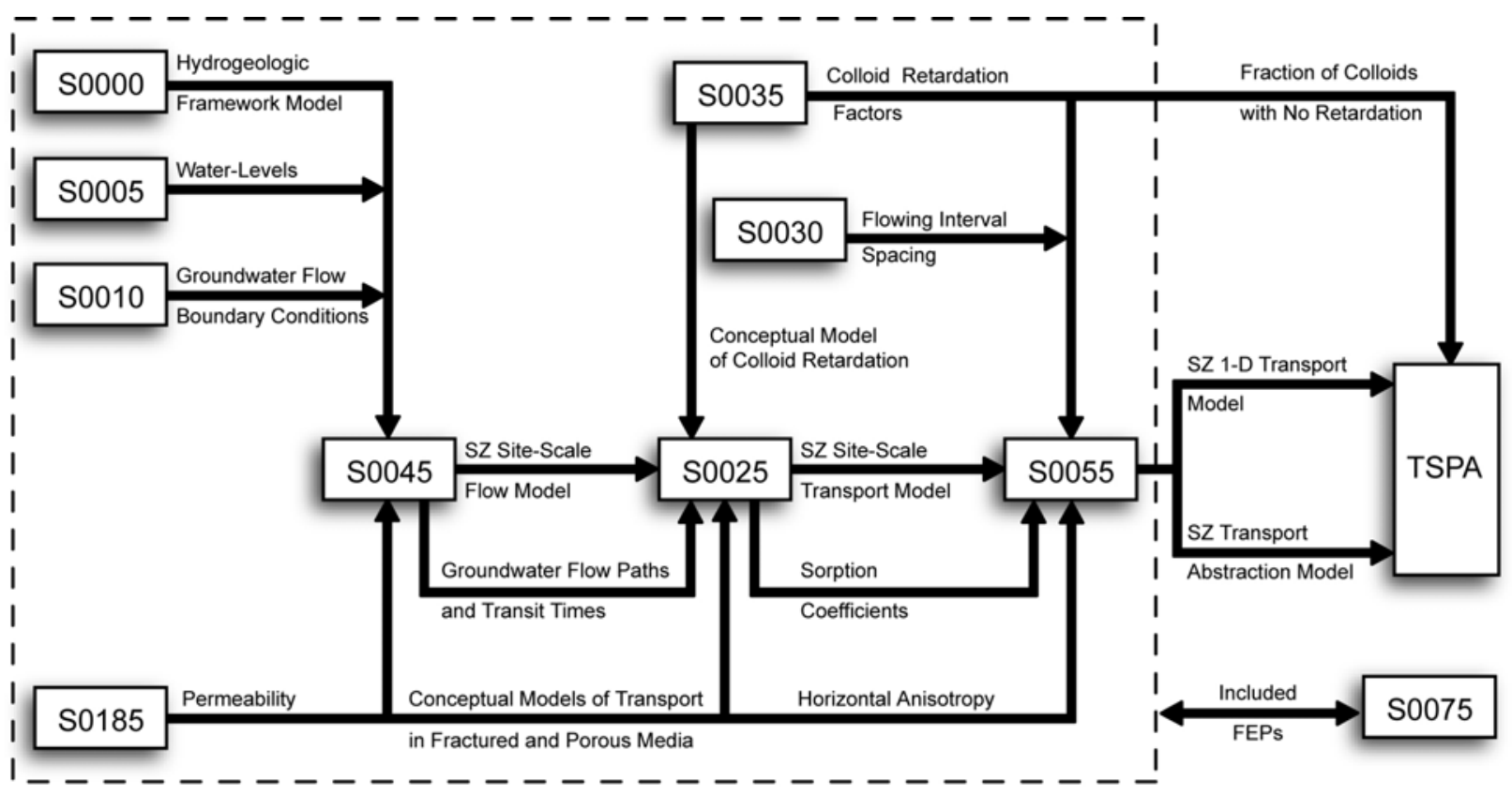

\section{Legend}

S0000 - Hydrogeologic Framework Model

S0005 - Water-Level Data Analysis

S0010 - Recharge and Lateral Groundwater Flow Boundary Conditions

S0025 - Site-Scale Saturated Zone Transport

S0030 - Probability Distribution for Flowing Interval Spacing

S0035 - Saturated Zone Colloid Transport

S0045 - Site-Scale Saturated Zone Flow Model

S0055 - Saturated Zone Flow and Transport Model Abstraction

S0075 - Features, Events, and Processes in SZ Flow and Transport

S0185 - Saturated Zone In-Situ Testing
MDL-NBS-HS-000024

ANL-NBS-HS-000034

ANL-NBS-MD-000010

MDL-NBS-HS-000010

ANL-NBS-MD-000003

ANL-NBS-HS-000031

MDL-NBS-HS-000011

MDL-NBS-HS-000021

ANL-NBS-MD-000002

ANL-NBS-HS-000039

Figure 1-1. A Schematic of the Flow of Information Among SZ Reports

The geohydrologic setting to be modeled is complex, with multifaceted and diverse geochemical interactions possible between the groundwater, solutes, and geological materials. Also, the intended use by the TSPA-LA requires a computationally efficient model that is amenable to repeated runs for stochastic simulations. The approach taken in this report is to construct a plausible conceptual model of transport that represents the important SZ transport processes and also supports the TSPA-LA. Alternate conceptual models and the implications of these models for transport predictions are evaluated relative to the base-case model. A number of relevant features, events, and processes (FEPs) are included in this report (Section 6.2). The manner of their inclusion is described in various sections of this report. The excluded FEPs are discussed in Features, Events, and Processes in SZ Flow and Transport (BSC 2004 [DIRS 170013]). The 
transport of chemical species in groundwater generally leads to retardation of the transported species with respect to the bulk movement of the groundwater. The radionuclide transport times can be several orders of magnitude longer than those for the bulk water. The processes of importance to site-scale SZ transport model are radionuclide dispersion, diffusion into the rock matrix and subsequent radionuclide sorption onto matrix surfaces, and colloid-facilitated radionuclide transport. These processes are included in the site-scale SZ transport model (Sections 6.3 and 6.4). The sorption of radionuclides onto fracture surfaces is not included in this model as a conservative approach.

The process of radionuclide adsorption within the rock matrix is represented using a sorption coefficient $\left(K_{d}\right)$ approach, which is presented in this report with justification for its use (Sections 6.3, 6.4, 6.6, and Appendix A). Probability distributions for the $K_{d}$ values for radionuclides of interest are derived based on data from the field, laboratory, literature, and models of sorption reactions. The probability distributions are designed to include expected variations in environmental parameters that can influence the sorption behavior of the radionuclides of interest. To make the transport calculations more efficient computationally, the approach taken is to use two separate single-valued sorption coefficients to calculate transport rates in the volcanic and alluvial portions of the flow path in the SZ. The justification for this approach is provided in this report (Appendices A and C). Also, the process of colloid-facilitated radionuclide transport is represented using the colloid partition coefficient $\left(K_{c}\right)$ approach. This modeling approach and its justification are presented in this report (Sections 6.3, 6.4, 6.6, and Appendix B).

The methodology for computing the transport of radionuclides within the SZ has been revised to capture a variety of different processes. This document presents the conceptual, mathematical, and computational aspects of a radionuclide transport model for the SZ and satisfies a requirement of the Yucca Mountain Project (YMP) to produce scientifically defensible transport predictions. The numerical techniques required to implement the method are described in Section 6.4.

Model validation activities presented in this report provide increased confidence that the model is a reasonable representation of the transport at Yucca Mountain near the repository site (Section 7). Because of the time and spatial scales involved and the fact that radionuclides cannot be used as tracers in field experiments, confidence-building activities during model development as well as postdevelopment validation are documented in this report. Recognizing that the model is being used to perform probabilistic calculations in which parameter uncertainties are propagated through the model, the intent of this validation is to confirm that radionuclide parameters and processes included in the site-scale SZ transport model are adequately represented with sufficient accuracy. Confidence building during model development (Section 7.1) is carried out by a series of different approaches that include: (1) comparison to analogue sites, (2) submodel-data comparisons, (3) model-data comparisons, and (4) comparison with data published in referenced journals. The data used in confidence building for the relevant transport parameters (e.g., sorption coefficients), submodel processes (e.g., advection, sorption), and site-scale model processes (e.g., flow pathways, transit times) are based on laboratory testing, field tests, natural analogue sites, and expert elicitations. Postdevelopment validation (Section 7.2) is carried out by comparison of model predictions with inferences based on geochemical data. By demonstrating that the parameters and processes 
selected have an experimental or observational basis, the model is validated for use in a stochastic analysis that establishes ranges of potential behavior of the SZ transport system.

The technical output of this report is composed of the site-scale SZ transport model and associated input and output files (base-case transport files); and SZ distribution coefficients $\left(K_{d} \mathrm{~S}\right)$ data for uranium, neptunium, plutonium, cesium, americium, protactinium, strontium, thorium, radium, carbon, technetium, and iodine (Table C-14). The output breakthrough curves and transport times will be integrated into the SZ flow and transport abstractions model for use in the TSPA-LA calculations.

When using the site-scale SZ transport model for calculations, limitations that must be noted are:

- Input parameter values/ranges. The transport model is intended for use with stochastic simulations using large uncertainty ranges for particular parameters such as specific discharge, fracture spacing and aperture, diffusion coefficient, and sorption coefficients. Care should be exercised in interpreting individual simulations for single sets of parameter values. Also, care should be exercised if the parameters that are used fall outside the range of parameter values (Table 4-2) or outside the range established by model validation (Section 7.3).

- Useable path-line distances. The flow field underlying the site-scale SZ transport model is based on the dual-porosity, effective-continuum approach requiring large grid blocks that effectively average fracture, rock matrix, and alluvium properties. Also, the parallel fracture model used to model advection/diffusion in the volcanics is valid only for grid-block sizes much larger than the mean flowing-interval spacing of $20 \mathrm{~m}$. It is recommended in the Saturated Zone Site-Scale Flow Model that to produce meaningful results, the flow path should be long compared to the grid-block size (BSC 2004 [DIRS 170037], Section 8) for modeling the fractured system using the effectivecontinuum approach. Because the grid-block size is $500 \mathrm{~m}$, a minimum distance of $2 \mathrm{~km}$ is recommended for path lines used in performance assessment calculations.

- Sufficient number of input particles for particle tracking. Radionuclide transport is implemented in the Site-scale SZ transport model using particle tracking with a random walk method (Sections 6.4.2.3 and 6.4.2.4). In order to obtain reproducible results, a sufficient number of particles must be input into the model. The base-case transport model utilizes 1,000 input particles (output DTN: LA0306SK831231.001), considered on the basis of expert judgment and previous experience to be sufficient for obtaining a smooth breakthrough curve at the boundary of the accessible environment at $18 \mathrm{~km}$ (Figure 6.5-1). However, a larger number of input particles may be required, depending on the purpose of the model use.

Note that the six-digit numerical identifier following each reference callout is the YMP Document Input Reference System (DIRS) number, the purpose of which is to assist in locating a specific reference in the reference list in Section 9 and in the DIRS database. 


\section{QUALITY ASSURANCE}

Development of this model report and the supporting modeling activities has been determined to be subject to the YMP quality assurance (QA) program ) and Technical Work Plan For: Natural System - Saturated Zone Flow and Transport Modeling and Testing Analysis and Model Report Integration (BSC 2004 [DIRS 171421]). Approved QA procedures identified in the technical work plan have been used to conduct and document the activities described in this model report. The technical work plan also identifies the methods used to control the electronic management of data included in Section 8 of the technical work plan. The site-scale SZ transport model development and model validation was conducted under the Technical Work Plan For: Saturated Zone Flow and Transport Modeling and Testing (BSC 2003 [DIRS 163965].

This model report provides a validated site-scale SZ transport model which is important in demonstrating compliance with the postclosure performance objectives prescribed in 10 CFR 63.113 (DIRS 156605). Therefore, the SZ is classified on the Q-List (BSC 2004 [DIRS 168361], Table A-2) as "SC" (safety category), reflecting its importance to waste isolation, as defined in AP-2.22Q, Classification Analyses and Maintenance of the Q-List. This report contributes to the analysis and modeling data used to support postclosure performance assessment; the conclusions do not directly impact preclosure engineered features important to safety, as defined in AP-2.22Q. 


\section{INTENTIONALLY LEFT BLANK}




\section{USE OF SOFTWARE}

\subsection{SOFTWARE TRACKED BY CONFIGURATION MANAGEMENT}

The computer software code used as the basis to model SZ transport in this report is FEHM. This version of the code includes the particle-tracking algorithm described in this report and was obtained from Software Configuration Management. The other codes listed in Table 3-1 were used in the analysis described in the appendices. All were obtained from Software Configuration Management, used only within the range of validation as required by LP-SI.11Q-BSC REV 0 ICN 1, Software Management, and are appropriate for the application in this report. Input and output files for this report are listed in Section 8.2.2 and identified in the respective discussions in Sections 6, 7, and 8.

Table 3-1. Computer Software and Routines

\begin{tabular}{|c|c|c|c|c|}
\hline $\begin{array}{c}\text { Software } \\
\text { Title/Version } \\
\text { Number }\end{array}$ & $\begin{array}{l}\text { Software } \\
\text { Tracking } \\
\text { Number }\end{array}$ & Code Usage & $\begin{array}{l}\text { Computer: Type, } \\
\text { Platform, and } \\
\text { Location }\end{array}$ & References \\
\hline FEHM V 2.20 & $10086-2.20-00$ & $\begin{array}{l}\text { Used for calculations } \\
\text { throughout this model report. } \\
\text { The FEHM } \vee 2.20 \text { application is } \\
\text { based on a finite-volume/ } \\
\text { finite-element heat- and } \\
\text { mass-transfer code that } \\
\text { simulates nonisothermal, } \\
\text { multiphase, multicomponent } \\
\text { flow and solute transport in } \\
\text { porous media. }\end{array}$ & $\begin{array}{l}\text { Sun, PC } \\
\text { Sun OS } 5.7 \text { and } \\
5.8, \text { Microsoft } \\
\text { Windows 2000, } \\
\text { Linux } 7.1 \text {, } \\
\text { Location: LANL. }\end{array}$ & $\begin{array}{l}\text { LANL } 2003 \\
\text { (DIRS 161725) }\end{array}$ \\
\hline cr8sptr.c V 2.0 & $10927-2.0-00$ & $\begin{array}{l}\text { Used to create an input file for } \\
\text { sptr macro in FEHM. }\end{array}$ & $\begin{array}{l}\text { Sun, Sun OS 5.7, } \\
\text { Location: LANL }\end{array}$ & $\begin{array}{l}\text { SNL } 2002 \\
\text { (DIRS 163836) }\end{array}$ \\
\hline calc_cdf.c V 1.0 & $10924-1.0-00$ & $\begin{array}{l}\text { Used to calculate the } \\
\text { cumulative distribution function } \\
\text { of the stochastic distributions of } \\
K_{d} \text {. }\end{array}$ & $\begin{array}{l}\text { Sun, Sun OS } 5.7 \text {, } \\
\text { Location: LANL }\end{array}$ & $\begin{array}{l}\text { SNL } 2000 \\
\text { (DIRS 149117) }\end{array}$ \\
\hline GS2FEHM.C V 1.0 & $10923-1.0-00$ & $\begin{array}{l}\text { Used to create an input file for } \\
\text { the perm macro in FEHM. }\end{array}$ & $\begin{array}{l}\text { Sun, Sun OS } 5.7 \text {, } \\
\text { Location: LANL }\end{array}$ & $\begin{array}{l}\text { SNL } 2002 \\
\text { (DIRS 163837) }\end{array}$ \\
\hline $\begin{array}{l}\text { GSLIB } \\
\text { V1.0GAMV3V1.201 }\end{array}$ & $\begin{array}{l}\text { 10398-1.0GAM } \\
\text { V3V1.201-00 }\end{array}$ & $\begin{array}{l}\text { Used to calculate a 3-D } \\
\text { variogram of input data. }\end{array}$ & $\begin{array}{l}\text { Sun, Sun OS 5.5.1, } \\
\text { Location: LANL }\end{array}$ & $\begin{array}{l}\text { LBNL } 2000 \\
\text { (DIRS 153099) }\end{array}$ \\
\hline $\begin{array}{l}\text { GSLIB } \\
\text { V2.0MSISIMV2.0 }\end{array}$ & $\begin{array}{l}\text { 10098-2.0MSI } \\
\text { SIMV2.0-00 }\end{array}$ & $\begin{array}{l}\text { Used to generate a stochastic } \\
\text { distribution of parameters, such } \\
\text { as } K_{d} \text { and permeability. }\end{array}$ & $\begin{array}{l}\text { Sun, UNIX, } \\
\text { Location: LANL }\end{array}$ & $\begin{array}{l}\text { SNL } 2000 \\
\text { (DIRS 149114) }\end{array}$ \\
\hline PHREEQC V 2.3 & $10068-2.3-00$ & $\begin{array}{l}\text { Calculates surface } \\
\text { complexation reactions for } \\
\text { radionuclides. }\end{array}$ & $\begin{array}{l}\text { PC, Location: } \\
\text { LANL }\end{array}$ & $\begin{array}{l}\text { BSC } 2001 \\
\text { (DIRS 155323) }\end{array}$ \\
\hline RELAP V2.0 & $10551-2.0-00$ & Generate breakthrough curves & $\begin{array}{l}\text { PC, Location : } \\
\text { LANL }\end{array}$ & $\begin{array}{l}\text { LANL } 2002 \\
\text { (DIRS 159065) }\end{array}$ \\
\hline FRACT_p V1.0 & $11009-1.0-00$ & $\begin{array}{l}\text { Calculates data that correlate } \\
\text { concentrations with time for } \\
\text { transport in the fractured media. }\end{array}$ & $\begin{array}{l}\text { PC, LINUX } 2.4 .18, \\
\text { Location: LANL }\end{array}$ & $\begin{array}{l}\text { LANL } 2003 \\
\text { (DIRS 164509) }\end{array}$ \\
\hline
\end{tabular}

LANL=Los Alamos National Laboratory; OS=operating system; PC=personal computer; 


\subsection{EXEMPT SOFTWARE}

Commercial, off-the-shelf software used in support of this model report is listed in Table 3-2. This software is exempt from the requirements of LP-SI.11Q-BSC, REV 0 ICN 1.

\section{Table 3-2. Exempt Software}

\begin{tabular}{|c|c|c|}
\hline $\begin{array}{c}\text { Software Name and } \\
\text { Version }\end{array}$ & Description & $\begin{array}{c}\text { Computer and } \\
\text { Platform Identification }\end{array}$ \\
\hline Microsoft Excel 2000 & $\begin{array}{l}\text { Used for preparing spreadsheets of data and plotting } \\
\text { graphs. No data analysis was done with this software. Only } \\
\text { built-in standard functions in this software were used. No } \\
\text { software routines or macros were used with this software to } \\
\text { prepare this report. The output was visually checked for } \\
\text { correctness. }\end{array}$ & $\begin{array}{l}\text { PC, Microsoft Windows } \\
\text { 2000/NT }\end{array}$ \\
\hline $\begin{array}{l}\text { FORTNER SUN } \\
\text { PLOT }\end{array}$ & $\begin{array}{l}\text { Used for plotting graphs. No data analysis was done with } \\
\text { this software. Only built-in standard functions in this } \\
\text { software were used. No software routines or macros were } \\
\text { used with this software to prepare this report. The output } \\
\text { was visually checked for correctness. }\end{array}$ & $\begin{array}{l}\text { Sun with UNIX OS, } \\
\text { FORTRAN }\end{array}$ \\
\hline SURFER V6.03 & $\begin{array}{l}\text { Used for plotting and visualization of analysis results in } \\
\text { figures shown in this report. No data analysis was done with } \\
\text { this software. Only built-in standard functions in this } \\
\text { software were used. No software routines or macros were } \\
\text { used with this software to prepare this report. The output } \\
\text { was visually checked for correctness. }\end{array}$ & $\begin{array}{l}\text { PC, Microsoft Windows } \\
\text { 2000/NT }\end{array}$ \\
\hline
\end{tabular}

$\mathrm{PC}=$ personal computer 


\section{INPUTS}

\subsection{DIRECT INPUTS}

This section identifies all input data and parameters that are used in this modeling activity.

\subsubsection{Data and Technical Information}

The data providing input for the development of parameters used in the modeling activities documented in this report are listed in Table 4-1. Several SZ reports provide information and data needed as feed-ins for this report, and this report in turn provides technical product outputs that feed into other SZ reports. This flow of information is shown schematically in Figure 1-1. The base-case SZ site-scale flow model (DTN: LA0304TM831231.002 [DIRS 163788]) forms the starting point data for this transport model. The development of this flow model, including the conceptual model, various alternate conceptual models, the choice of parameter values for base-case flow model, and the appropriateness of data and technical information used in this model are discussed in detail in Saturated Zone Site-Scale Flow Model (BSC 2004 [DIRS 170037], Sections 4, 5 and 6). For a number of parameters that are needed in the transport model, the selection of ranges of values and uncertainty distributions are presented in Saturated Zone Flow and Transport Model Abstraction (BSC 2004 [DIRS 170042], Section 6.5.2; DTN: SN0310T0502103.009 [DIRS 168763]). Where available, sorption coefficient data for radionuclides of interest on rock and water samples from Yucca Mountain were used for developing the $K_{d}$ distributions that are used in this model report, as described in detail in Appendix A. These sorption coefficient data were augmented by technical information available in the literature on systems with similar geochemical characteristics. Mineralogic composition data are available on core samples taken from boreholes in the Yucca Mountain area. Since these data are site specific, they were considered the most appropriate data for use in stochastic analysis of $K_{d}$ distributions described in Appendix C. These input data and technical information and their sources are provided in Table 4-1. Parameters needed for modeling colloid-facilitated transport (Table 4-2) were obtained from Saturated Zone Colloid Transport (BSC 2004 [DIRS 170006], Sections 6.4, 6.5, and 6.6) and Waste Form and In-Drift ColloidsAssociated Radionuclide Concentrations: Abstraction and Summary (BSC 2004 [DIRS 170025], Section 6.3). Justification for the choice of data and technical information for selecting the range of values (presented in Table 4-2) of all other parameters needed for the site-scale SZ transport model is given in detail in Saturated Zone Flow and Transport Model Abstraction (BSC 2004 [DIRS 170042], Section 6.5.2). The qualification of LA0310AM831341.001 (DIRS 165865) and LA0305AM831341.001 (DIRS 163789) is presented in Appendix H and the qualification of water composition as documented in Ogard and Kerrisk (1984 [DIRS 100783]) is presented in Appendix I. The qualification status of the remaining input sources is provided in the Technical Data Management System. A discussion of the selection of the range of values for each model parameter using the available data is presented in Section 4.1.2. 
Table 4-1. Input Data and Technical Information

\begin{tabular}{|c|c|c|}
\hline Data Description & Source & Data Tracking Number \\
\hline \multicolumn{3}{|c|}{ Data and Technical Information for Uncertainty Distribution of Parameters } \\
\hline $\begin{array}{l}\text { Uncertainty distribution for } \\
\text { parameters used in the SZ } \\
\text { transport abstractions model }\end{array}$ & $\begin{array}{l}\text { BSC } 2004 \text { (DIRS 170042), } \\
\text { Section } 6.5 .2\end{array}$ & SN0310T0502103.009 (DIRS 168763) \\
\hline \multicolumn{3}{|c|}{ Data and Technical Information for Base-Case Flow Model } \\
\hline $\begin{array}{l}\text { FEHM V2.20 files for base-case } \\
\text { flow model }\end{array}$ & $\begin{array}{l}\text { BSC } 2004 \text { (DIRS 170037), entire } \\
\text { document }\end{array}$ & LA0304TM831231.002 (DIRS 163788) \\
\hline \multicolumn{3}{|c|}{ Data and Technical Information for Sorption Coefficient Data } \\
\hline $\begin{array}{l}\text { Input data file (YMP_R3.DAT) for } \\
\text { thermo-dynamic data software code } \\
\text { PHREEQC, Version } 2.3\end{array}$ & $\begin{array}{l}\text { BSC } 2004 \text { ([DIRS 167621], } \\
\text { Section 6.3.2.2) }\end{array}$ & $\begin{array}{l}\text { MO0403SPAYMPR3.000 } \\
\text { (DIRS 170937) }\end{array}$ \\
\hline $\begin{array}{l}\text { Americium, protactinium, plutonium, } \\
\text { and thorium sorption coefficients on } \\
\text { silica and surface area for silica } \\
\text { sample }\end{array}$ & $\begin{array}{l}\text { Allard et al. } 1983 \\
\text { (DIRS 162982), pp. 6, 9, 10, 12; } \\
\text { Allard et al. 1980 } \\
\text { (DIRS 104410), p. 478; Beall et } \\
\text { al. } 1986 \text { (DIRS 162983), entire } \\
\text { document. }\end{array}$ & $\begin{array}{l}\text { See Notes } 1 \text { and } 2 \text { below for } \\
\text { justification of the use of the data }\end{array}$ \\
\hline $\begin{array}{l}\text { Density of sorption sites, on the } \\
\text { solid surface, uranium and } \\
\text { neptunium surface complexation } \\
\text { binding constants on silica }\end{array}$ & $\begin{array}{l}\text { Pabalan et al. } 1998 \\
\text { (DIRS 162987), p. } 124\end{array}$ & $\begin{array}{l}\text { See Note } 3 \text { below for the justification of } \\
\text { the use of the data }\end{array}$ \\
\hline $\begin{array}{l}\text { Batch sorption coefficient data for } \\
\text { barium on Yucca Mountain tuffs in } \\
\text { representative water compositions }\end{array}$ & N/A & LA0407AM831341.001 (DIRS 170623) \\
\hline $\begin{array}{l}\text { Batch sorption coefficient data for } \\
\text { cesium on Yucca Mountain tuffs in } \\
\text { representative water compositions }\end{array}$ & N/A & LA0407AM831341.002 (DIRS 170621) \\
\hline $\begin{array}{l}\text { Batch sorption coefficient data for } \\
\text { strontium on Yucca Mountain tuffs } \\
\text { in representative water } \\
\text { compositions }\end{array}$ & $\mathrm{N} / \mathrm{A}$ & LA0407AM831341.003 (DIRS 170626) \\
\hline $\begin{array}{l}\text { Batch sorption coefficient data for } \\
\text { neptunium on Yucca Mountain tuffs } \\
\text { in representative water } \\
\text { compositions }\end{array}$ & $\mathrm{N} / \mathrm{A}$ & LA0407AM831341.004 (DIRS 170622) \\
\hline $\begin{array}{l}\text { Batch sorption coefficient data for } \\
\text { plutonium on Yucca Mountain tuffs } \\
\text { in representative water } \\
\text { compositions }\end{array}$ & N/A & LA0407AM831341.005 (DIRS 170625) \\
\hline $\begin{array}{l}\text { Batch sorption coefficient data for } \\
\text { uranium on Yucca Mountain tuffs in } \\
\text { representative water compositions }\end{array}$ & N/A & LA0407AM831341.006 (DIRS 170628) \\
\hline $\begin{array}{l}\text { Neptunium, uranium, plutonium, } \\
\text { barium, strontium, cesium sorption } \\
\text { coefficients on Yucca Mountain } \\
\text { tuffs }\end{array}$ & $\mathrm{N} / \mathrm{A}$ & $\begin{array}{l}\text { LA0305AM831341.001 (DIRS 163789) } \\
\text { (Data are qualified in Appendix H), } \\
\text { LA0302MD831341.003 (DIRS 163784), } \\
\text { LA0302MD831341.004 (DIRS 163785) }\end{array}$ \\
\hline
\end{tabular}


Table 4-1. Input Data and Technical Information (Continued)

\begin{tabular}{|c|c|c|}
\hline Data Description & Source & Data Tracking Number \\
\hline \multicolumn{3}{|c|}{ Data and Technical Information for Sorption Coefficient Data (Continued) } \\
\hline $\begin{array}{l}\text { Static measurements for UE-25 } \\
\text { WT\#17, 01-JUL-98 }\end{array}$ & N/A & LAAM831311AQ98.010 (DIRS 149522) \\
\hline $\begin{array}{l}\text { Eh-pH field measurements on Nye } \\
\text { County EWDP wells }\end{array}$ & N/A & $\begin{array}{l}\text { LA0206AM831234.001 (DIRS 160051) } \\
\text { LA9907AM831234.009 (DIRS 149209) } \\
\text { LA9907AM831234.011 (DIRS 149212) }\end{array}$ \\
\hline $\begin{array}{l}\text { Flow-through cell and static } \\
\text { measurements at UE-25 WT\#3 }\end{array}$ & N/A & $\begin{array}{l}\text { LAAM831311AQ98.007 } \\
\text { (DIRS 149520) }\end{array}$ \\
\hline $\begin{array}{l}\text { Geochemical field measurements } \\
\text { on Nye County EWDP wells. }\end{array}$ & N/A & LA0206AM831234.002 (DIRS 163852) \\
\hline $\begin{array}{l}\text { Adsorption of } \mathrm{Np}-237 \text { in three types } \\
\text { of alluvium as a function of time and } \\
\text { stratigraphic position }\end{array}$ & N/A & LA0109MD831341.001 (DIRS 156870) \\
\hline $\begin{array}{l}\text { Adsorption of Tc-99 in three types } \\
\text { of alluvium as a function of time and } \\
\text { stratigraphic position }\end{array}$ & N/A & LA0109MD831341.002 (DIRS 156871) \\
\hline $\begin{array}{l}\text { Sorption/desorption measurements } \\
\text { of cesium on Yucca Mountain tuff }\end{array}$ & N/A & $\begin{array}{l}\text { LA0310AM831341.001 (DIRS 165865) } \\
\text { (Data are qualified in Appendix H) }\end{array}$ \\
\hline $\begin{array}{l}\text { Water chemistry for } \mathrm{J}-13 \text { water and } \\
\mathrm{pH} \text { in } \mathrm{p} \# 1 \text { water }\end{array}$ & N/A & $\begin{array}{l}\text { MO0007MAJIONPH.011 } \\
\text { (DIRS 151524). }\end{array}$ \\
\hline $\begin{array}{l}\text { Deprotonation constants and } \\
\text { binding constants for aluminum } \\
\text { and silica }\end{array}$ & $\begin{array}{l}\text { Dixit and Van Cappellen } 2002 \\
\text { (DIRS 162985), p. } 2565\end{array}$ & $\begin{array}{l}\text { See Note } 5 \text { below for the justification of } \\
\text { the use of the data }\end{array}$ \\
\hline $\begin{array}{l}\text { Binding constants for sodium on } \\
\text { silica }\end{array}$ & $\begin{array}{l}\text { Marmier et al. } 1999 \\
\text { (DIRS 162986), p. } 228\end{array}$ & $\begin{array}{l}\text { See Note } 6 \text { below for the justification of } \\
\text { the use of the data }\end{array}$ \\
\hline $\begin{array}{l}\text { Binding constants for neptunium on } \\
\text { silica }\end{array}$ & $\begin{array}{l}\text { Turner et al. } 1998 \\
\text { (DIRS 162989) p. } 264\end{array}$ & $\begin{array}{l}\text { See Note } 4 \text { below for the justification of } \\
\text { the use of the data }\end{array}$ \\
\hline $\begin{array}{l}\text { Water compositions for samples } \\
\text { from wells J-13 and } p \# 1\end{array}$ & $\begin{array}{l}\text { Ogard and Kerrisk } 1984 \\
\text { (DIRS 100783) }\end{array}$ & Data are qualified in Appendix I \\
\hline $\begin{array}{l}\text { Colloid retardation factors for the } \\
\text { SZ fractured volcanics }\end{array}$ & N/A & LA0303HV831352.004 (DIRS 163559) \\
\hline \multicolumn{3}{|c|}{ Boundary of the Accessible Environment at $18 \mathrm{~km}$} \\
\hline $\begin{array}{l}\text { Boundary of the accessible } \\
\text { environment at } 18 \mathrm{~km}\end{array}$ & 10 CFR 63.302 (DIRS 156605) & $\begin{array}{l}\text { See Note } 7 \text { below for the justification of } \\
\text { the use of the data }\end{array}$ \\
\hline \multicolumn{3}{|c|}{ Data and Information for Stochastic Modeling of $K_{d}$} \\
\hline $\begin{array}{l}\text { Mineralogic data for Yucca } \\
\text { Mountain. }\end{array}$ & N/A & LASC831321AQ98.001 (DIRS 109047) \\
\hline $\begin{array}{l}\text { XRD data describing mineralogic } \\
\text { composition of core samples from } \\
\text { wells }\end{array}$ & $\begin{array}{l}\text { Chipera et al. } 1995 \\
\text { (DIRS 111081), entire document }\end{array}$ & LA000000000086.002 (DIRS 107144) \\
\hline $\begin{array}{l}\text { XRD data describing mineralogic } \\
\text { composition of core samples from } \\
\text { wells }\end{array}$ & N/A & $\begin{array}{l}\text { LAJC831321AQ98.005 (DIRS 109004), } \\
\text { LASC831321AQ98.001 (DIRS 109047), } \\
\text { LADV831321AQ99.001 (DIRS 109044) } \\
\text { MO0408K8313211.000 (DIRS 171437) }\end{array}$ \\
\hline $\begin{array}{l}\text { XRD data describing mineralogic } \\
\text { composition of core samples from } \\
\text { wells }\end{array}$ & $\begin{array}{l}\text { Steinborn } 2002 \text { (DIRS 160702), } \\
\text { entire document }\end{array}$ & $\begin{array}{l}\text { MO0101XRDMINAB.001 } \\
\text { (DIRS 163796), } \\
\text { MO0106XRDDRILC.003 } \\
\text { (DIRS 163797), } \\
\text { MO0101XRDDRILC.002 } \\
\text { (DIRS 163795) }\end{array}$ \\
\hline
\end{tabular}


Table 4-1. Input Data and Technical Information (Continued)

\begin{tabular}{|l|l|l|}
\hline \multicolumn{1}{|c|}{ Data Description } & \multicolumn{1}{|c|}{ Source } & \multicolumn{1}{c|}{ Data Tracking Number } \\
\hline \multicolumn{2}{|c|}{ Data and Information for Stochastic Modeling of $\mathbf{K}_{\mathbf{d}}$ (Continued) } \\
\hline $\begin{array}{l}\text { Values of diffusion coefficients used } \\
\text { for stochastic analysis }\end{array}$ & N/A & LA0003JC831362.001 (DIRS 149557) \\
\hline $\begin{array}{l}\text { Hydraulic gradient used in } \\
\text { stochastic modeling on a 550-m } \\
\text { block }\end{array}$ & $\begin{array}{l}\text { CRWMS M\&O 2000 } \\
\text { (DIRS 152259), p. 14, Sec. 5.2. }\end{array}$ & Product output \\
\hline
\end{tabular}

Notes on data obtained from outside sources

1) Data from Allard et al. (1980 [DIRS 104410]) and Beall et al. (1986 [DIRS 162983]) were used for calculating americium sorption coefficient over the range of geochemical conditions expected to occur at the Yucca Mountain repository. The calculations were conducted using the geochemical code PHREEQC (PHREEQC V2.3, STN: 10068-2.3-00) (BSC 2001 [DIRS 155323]). Site-specific americium sorption coefficient data are available only for J-13 water. To evaluate the impact of variations in groundwater compositions on americium sorption coefficients, a surface complexation modeling exercise was carried out. In order to model americium surface complexation reactions with PHREEQC, one or more surface species must be selected along with appropriate binding constants for these species. The surface species and binding constants were obtained through an inverse approach where experimental data for the binding of americium on silica presented by Beall et al. (1986 [DIRS 162983]) were fit with a surface complexation model. In order to do this inverse analysis, the surface area reported by Allard et al. (1980 [DIRS 104410]) was used. Two americium surface species and their binding constants were derived in fitting the data. These species and binding constants were then used to evaluate the water compositional dependence of americium sorption coefficients on silica. The americium sorption coefficient data presented by Beall et al. were published in a paper titled "Americium Speciation and Distribution Coefficients in a Granitic Water," published in Scientific Basis for Nuclear Waste Management IX, a symposium held September 11, 1985, in Stockholm, Sweden. The surface area measurement on silica was published in a paper titled "The sorption of Actinides in Igneous Rocks," published in the journal Nuclear Technology (Volume 49, page 474480). Articles submitted to this journal are peer reviewed. Dr. Allard and Dr. Beall worked extensively together as part of the Swedish Nuclear Waste Program. Drs. Allard and Beall have published a comprehensive set of sorption coefficients for radionuclides-of-interest on rocks similar in composition to the tuffs at Yucca Mountain. Drs. Allard and Beall both have extensive backgrounds in radiochemistry and in the measurement of sorption coefficients on silicate rocks. Thus, the americium sorption coefficient data is deemed reliable and appropriate for use in modeling americium sorption behavior in Yucca Mountain samples.

2) The available data on site-specific sorption coefficients for protactinium are very limited. Further, the available data were not obtained under conditions (i.e., pH values) expected in the SZ. Sorption coefficient data for protactinium on silica in a simple electrolyte were obtained by Allard et al. (1983 [DIRS 162982]). These data were obtained over a range of $\mathrm{pH}$ values that is directly applicable to the $\mathrm{SZ}$ at Yucca Mountain. The protactinium sorption coefficient data presented by Allard et al. were published in a paper titled "Sorption Behavior of Actinides in Well-defined Oxidation States," published by the Swedish Nuclear Energy Agency. The work of the Swedish Nuclear Energy Agency is well respected in the international nuclear waste community. Dr. Allard has published a comprehensive set of sorption coefficients for radionuclides-of-interest on rocks similar in composition to the tuffs at Yucca Mountain. Dr. Allard has an extensive background in radiochemistry and in the measurement of sorption coefficients on silicate rocks. Thus, the protactinium sorption coefficient data he has published is deemed reliable and appropriate for use in modeling americium sorption behavior in Yucca Mountain samples. 
Table 4-1. Input Data and Technical Information (Continued)

Notes on data obtained from outside sources (continued)

3) Site-specific sorption coefficient data for uranium have been obtained for the major rock types and a range of water compositions. To obtain a more complete understanding of the relationship between uranium sorption and variations in water composition, modeling calculations were carried out using the code PHREEQC. As part of the input to the PHREEQC surface complexation model, surface species must be selected along with appropriate binding constants for these species. The surface species and binding constants for uranium sorption on silica were obtained from a journal article by Pabalan et al. (1998 [DIRS 162987]) titled "Uranium" Sorption onto Selected Mineral Surfaces, Key Geochemical Parameters." The paper was published in a volume titled "Adsorption of Metals by Geomedia" published by the Academic Press. This volume presented the state-of-the-art in understanding of the sorption behavior of metals in geomedia at the time it was published. The work described in the paper was carried out by the authors while at the Southwest Research Center under contract with the U.S. Nuclear Regulatory Commission (NRC). The work at the Southwest Research Center is well respected in the nuclear waste community. Thus, the uranium surface species and binding constants reported by Pabalan et al. (1998 [DIRS 162987]) are judged to be reliable and appropriate for use in modeling uranium sorption behavior on silica.

4) Site-specific sorption coefficient data for neptunium have been obtained for the major rock types and a range of water compositions. To obtain a more complete understanding of the relationship between neptunium sorption and variations in water composition, modeling calculations were carried out using the code PHREEQC. As part of the input to the PHREEQC surface complexation model, surface species must be selected along with appropriate binding constants for these species and site densities. The surface species, binding constants, and site density for neptunium sorption on silica were obtained from a journal article by Turner et al. (1998 [DIRS 162989]) titled "Neptunium (V) Sorption on Montmorillonite: An Experimental and Surface Complexation Modeling Study," published in the journal Clays and Clay Minerals. Articles submitted to this journal are peer reviewed. The work described in the paper was carried out by the authors while at the Southwest Research Center under contract with the NRC. The work at the Southwest Research Center is well respected in the nuclear waste community. Thus, the neptunium surface species and binding constants reported by Turner et al. is judged to be reliable and appropriate for use in modeling neptunium sorption behavior in the Yucca Mountain flow system.

5) As part of the input to the PHREEQC surface complexation model, acidity constants are required for the chosen solid surface (silica). These acidity constants quantify the binding of hydrogen ions to the solid surface. Dixit and Van Cappellen (2002 [DIRS 162985]) have derived acidity constants for silica surfaces in a paper titled "Surface Chemistry and Reactivity of Biogenic Silica," published in the journal Geochimica et Cosmochimica Acta, Volume 66, pp. 2559 to 2568. Articles submitted to this journal are peer reviewed. The journal is published by The Geochemical Society, which is a premier source of information to the international geochemical community. Based on this information, the surface acidity constants reported by Dixit and Van Cappellen are judged to be reliable and appropriate for use in PHREEQC surface complexation modeling on silica.

6) In modeling surface complexation reactions, competition among species in solution for surface sites, and in particular, competition from the major cations in solution, must be considered. In order to include such competitive effects, binding constants for the major cations on the chosen surface (silica) are required. Marmier et al. (1999 [DIRS 162986]) derived a binding constant for sodium on silica surfaces in a paper titled "Surface Complexation Modeling of $\mathrm{Yb}(\mathrm{III})$ and $\mathrm{Cs}(\mathrm{I})$ Sorption on Silica," published in the Journal of Colloid and Interface Science, Volume 212, pp. 228 to 233. Articles submitted to this journal are peer reviewed. This journal is a premier source of information to the international surface science community. Based on this information, the binding constant for sodium on silica reported by Marmier et al. is judged to be reliable and appropriate for use in PHREEQC surface complexation modeling on silica.

7) The boundary of the accessible environment at $18 \mathrm{~km}$ is used as an input to the site-scale SZ transport model for calculating breakthrough curves. Any radionuclides crossing the vertical east-west plane across the entire site-scale SZ flow model, $18 \mathrm{~km}$ downstream from the repository footprint, are counted as breakthrough particles. This boundary is established by 10 CFR 63.302 (DIRS 156605), which, as a code of federal regulations, is judged to be an established fact.

EWDP=Early Warning Drilling Program; SZ=saturated zone; XRD=X-ray diffraction; 


\subsubsection{Parameters and Parameter Uncertainty}

The range of values for each input parameter is presented in Table 4-2 and discussed in Sections 4.1.2.1 through 4.1.2.18 and in Appendix A. The ranges of sorption coefficient values presented in Table 4-2 encompass all the radionuclides of interest since the intent of this report is to present a site-scale SZ transport model that can be used by the TSPA-LA with any of these radionuclides. The distributions for individual radionuclides are presented in Table C-14. Basecase values for most parameters were chosen to be the median values for the distributions except for sorption coefficients, which were taken to be 0 to represent a nonsorbing radionuclide such as ${ }^{14} \mathrm{C}$.

Table 4-2. Input Parameters and Range of Values for the Site-Scale SZ Transport Model

\begin{tabular}{|c|c|c|c|c|c|}
\hline Parameter & $\begin{array}{c}\text { Base- } \\
\text { Case } \\
\text { Value(s) }\end{array}$ & $\begin{array}{l}\text { Uncertainty } \\
\text { Range }\end{array}$ & Units & $\begin{array}{l}\text { Variable } \\
\text { Type }\end{array}$ & Source/DTN \\
\hline $\begin{array}{l}\text { Specific discharge } \\
\text { multiplier }^{\mathrm{a}}\end{array}$ & $1^{b}$ & $1 / 30-10$ & - & Stochastic & $\begin{array}{l}\text { BSC } 2004 \text { (DIRS 170042) } \\
\text { SN0306T0502103.009 } \\
\text { (DIRS 168763) }\end{array}$ \\
\hline $\begin{array}{l}\text { Permeability horizontal } \\
\text { anisotropy ratio }\end{array}$ & $4.2^{\mathrm{C}}$ & $0.05-20$ & - & Stochastic & $\begin{array}{l}\text { SN0310T0502103.009 } \\
\text { (DIRS 168763) }\end{array}$ \\
\hline Bulk density in alluvium & $1,910^{c}$ & $\begin{array}{l}1,669- \\
2,151^{d}\end{array}$ & $\mathrm{~kg} / \mathrm{m}^{3}$ & Stochastic & $\begin{array}{l}\text { BSC } 2004 \text { (DIRS 170042) } \\
\text { SN0310T0502103.009 } \\
\text { (DIRS 168763) }\end{array}$ \\
\hline $\begin{array}{l}\text { Sorption coefficient in } \\
\text { alluvium }\end{array}$ & $0.0^{\mathrm{e}}$ & $0-10,000$ & $\mathrm{~mL} / \mathrm{g}$ & Stochastic & Appendices $\mathrm{A}$ and $\mathrm{C}$ \\
\hline $\begin{array}{l}\text { Effective porosity in the } \\
\text { alluvium, fraction }\end{array}$ & $0.18^{b}$ & $0.02^{k}-0.3$ & - & Stochastic & $\begin{array}{l}\text { BSC } 2004 \text { (DIRS 170042) } \\
\text { SN0310T0502103.009 } \\
\text { (DIRS 168763) }\end{array}$ \\
\hline $\begin{array}{l}\text { Colloid retardation factor in } \\
\text { alluvium for irreversible } \\
\text { colloids }\end{array}$ & $N / A^{j}$ & $8-5,188$ & - & Stochastic & $\begin{array}{l}\text { BSC } 2004 \text { (DIRS 170006) } \\
\text { LA0303HV831352.004 } \\
\text { (DIRS 163559) }\end{array}$ \\
\hline $\begin{array}{l}\text { Colloid retardation factor in } \\
\text { alluvium for nonsorbing } \\
\text { radionuclides }\end{array}$ & $1^{g}$ & - & - & - & - \\
\hline $\begin{array}{l}\text { Flowing interval porosity, } \\
\text { fraction }^{\prime}\end{array}$ & $0.01^{f}$ & $0.00001-0.1$ & - & Stochastic & $\begin{array}{l}\text { BSC } 2004 \text { (DIRS 170042) } \\
\text { SN0310T0502103.009 } \\
\text { (DIRS 168763) }\end{array}$ \\
\hline Flowing interval spacing & $20^{b}$ & $1.22-417$ & $\mathrm{~m}$ & Stochastic & $\begin{array}{l}\text { SN0310T0502103.009 } \\
\text { (DIRS 168763) }\end{array}$ \\
\hline $\begin{array}{l}\text { Matrix porosity in volcanics, } \\
\text { fraction }\end{array}$ & $0.15-0.25^{h}$ & $N / A$ & - & $\begin{array}{l}\text { Assigned } \\
\text { value for each } \\
\text { unit; not a } \\
\text { stochastic } \\
\text { parameter }\end{array}$ & $\begin{array}{l}\text { BSC } 2004 \text { (DIRS 170042) } \\
\text { SNO310T0502103.009 } \\
\text { (DIRS 168763) }\end{array}$ \\
\hline $\begin{array}{l}\text { Effective diffusion } \\
\text { coefficient in volcanics }\end{array}$ & $5.0 \times \underset{b}{10^{-11}}$ & $\begin{array}{l}5.0 \times 10^{-12}- \\
5.0 \times 10^{-10}\end{array}$ & $\mathrm{~m}^{2} / \mathrm{s}$ & Stochastic & $\begin{array}{l}\text { BSC } 2004 \text { (DIRS 170042) } \\
\text { SN0310T0502103.009 } \\
\text { (DIRS 168763) }\end{array}$ \\
\hline $\begin{array}{l}\text { Matrix sorption coefficient in } \\
\text { volcanics }\end{array}$ & $0.0^{\mathrm{e}}$ & $0-10,000$ & $\mathrm{~mL} / \mathrm{g}$ & Stochastic & Appendices $\mathrm{A}$ and $\mathrm{C}$ \\
\hline $\begin{array}{l}\text { Colloid retardation factor in } \\
\text { volcanics for irreversible } \\
\text { colloids }\end{array}$ & $N / A^{j}$ & $6.0-794^{\prime}$ & - & Stochastic & $\begin{array}{l}\text { BSC } 2004 \text { (DIRS 170006) } \\
\text { LA0303HV831352.002 } \\
\text { (DIRS 163558) }\end{array}$ \\
\hline
\end{tabular}


Table 4-2. Input Parameters and Range of Values for the Site-Scale SZ Transport Model (Continued)

\begin{tabular}{|c|c|c|c|c|c|}
\hline Parameter & $\begin{array}{c}\text { Base- } \\
\text { Case } \\
\text { Value(s) }\end{array}$ & $\begin{array}{l}\text { Uncertainty } \\
\text { Range }\end{array}$ & Units & $\begin{array}{c}\text { Variable } \\
\text { Type }\end{array}$ & Source/DTN \\
\hline $\begin{array}{l}\text { Colloid retardation factor in } \\
\text { volcanics related to } \\
\text { nonsorbing radionuclides }\end{array}$ & $1^{g}$ & - & - & - & - \\
\hline $\begin{array}{l}\text { Groundwater concentration } \\
\text { of colloids }\end{array}$ & $N / A^{j}$ & $\begin{array}{c}10^{-9}- \\
2.5 \times 10^{-4}\end{array}$ & $\mathrm{~g} / \mathrm{mL}$ & Stochastic & $\begin{array}{l}\text { BSC } 2004 \text { (DIRS 170025) } \\
\text { SN0306T0504103.005 } \\
\text { (DIRS 164132) }\end{array}$ \\
\hline $\begin{array}{l}\text { Groundwater concentration } \\
\text { of colloids related to } \\
\text { nonsorbing radionuclides }\end{array}$ & $0^{g}$ & - & $\mathrm{g} / \mathrm{mL}$ & - & - \\
\hline $\begin{array}{l}\text { Sorption coefficient onto } \\
\text { colloids }\end{array}$ & $N / A^{j}$ & $10^{1}-10^{7}$ & $\mathrm{~mL} / \mathrm{g}$ & Stochastic & $\begin{array}{l}\text { BSC } 2004 \text { (DIRS 170025) } \\
\text { SN0306T0504103.006 } \\
\text { (DIRS 164131) }\end{array}$ \\
\hline $\begin{array}{l}\text { Sorption coefficient onto } \\
\text { colloids for nonsorbing } \\
\text { radionuclides }\end{array}$ & $0^{g}$ & - & $\mathrm{mL} / \mathrm{g}$ & - & - \\
\hline $\begin{array}{l}\text { Fraction of colloids } \\
\text { transported unretarded }\end{array}$ & $N / A^{j}$ & $\begin{array}{c}0.00034^{\mathrm{n}}- \\
0.0017^{\mathrm{n}}\end{array}$ & - & Stochastic & BSC 2004 (DIRS 170006) \\
\hline $\begin{array}{l}\text { Fraction of colloids } \\
\text { transported unretarded } \\
\text { related to nonsorbing } \\
\text { radionuclides }\end{array}$ & $0^{9}$ & - & - & - & - \\
\hline Dispersivity, longitudinal & 10.0 & $0.10-2,000$ & $\mathrm{~m}$ & Stochastic & Section 4.1.2.16 \\
\hline $\begin{array}{l}\text { Dispersivity, transverse, } \\
\text { horizontal }\end{array}$ & 0.05 & $0.0005-10$ & $\mathrm{~m}$ & Stochastic & Section 4.1.2.17 \\
\hline $\begin{array}{l}\text { Dispersivity, transverse, } \\
\text { vertical }\end{array}$ & 0.0005 & $\begin{array}{l}0.000005- \\
0.1\end{array}$ & $\mathrm{~m}$ & Stochastic & Section 4.1.2.18 \\
\hline
\end{tabular}

${ }^{a}$ The boundary fluxes, recharge rates and permeabilities were multiplied by this factor to vary the specific discharge, which has a base-case value of $0.67 \mathrm{~m} / \mathrm{year}$ from the repository to a distance of $5 \mathrm{~km}$ as shown in the Saturated Zone Site-Scale Flow Model. (BSC 2004 [DIRS 170037], Section 6.6.2.4).

${ }^{\mathrm{b}}$ Median value, given as the 0.5 probability value in the source data tracking number (DTN), see Section 4.1.2.5.

${ }^{c}$ Median value, interpolated to the 0.5 probability from the data given in the source DTN.

${ }^{\mathrm{d}}$ Range derived from the normal distribution given in Saturated Zone Flow and Transport Model Abstraction (BSC 2004 [DIRS 170042], Figure 6-16).

e Base case is taken to be the value for nonsorbing radionuclides.

${ }^{f}$ Base-case value taken at 0.8 probability, see Section 4.1.2.7.

${ }^{g}$ Base-case colloid sorption parameters are set to 0 , and retardation factors set to 1 , to simulate the base case of nonsorbing radionuclides.

h Each hydrostratigraphic unit was assigned a fixed value within this range.

' Flowing interval porosity is referred to as the "Fracture porosity in volcanic units" in DTN: SN0310T0502103.009 (DIRS 168763).

j These do not apply to the site-scale SZ transport model base case because the base case considers only the nonsorbing radionuclides, and hence also excludes colloid facilitated transport.

${ }^{k}$ As discussed in Section 4.1.2.5, this value differs from that given in the reference DTN, but this is a conservative choice that avoids potential numerical problems.

1 The value given here is lower than the value of 800 given in the source/DTN. This is a conservative choice.

${ }^{m}$ Note that the numbers in the source/DTN are in units of $\mathrm{mg} / \mathrm{l}$, which are converted to the units of this table, $\mathrm{g} / \mathrm{mL}$ by multiplying by $10^{-6}$. Also the value in this table of $2.5 \times 10^{-4}$ is somewhat different from the value of $2 \times 10^{-4}$ $\mathrm{g} / \mathrm{mL}$ given in the source/DTN. This is a conservative choice.

${ }^{n}$ Note that the lower limit given here is somewhat higher than the one given in the source/DTN and the upper limit given here is somewhat higher than that given in the source/DTN. This is a conservative choice. 


\subsubsection{Specific Discharge Multiplier}

Field values of groundwater-specific discharge in the SZ have been estimated from the tracer testing at the Alluvial Testing Complex as shown in Saturated Zone In-Situ Testing (BSC 2004 [DIRS 170010], Section 6.5) to be in the range of $1.2 \mathrm{~m}$ to $9.4 \mathrm{~m}$ per year. This information is combined with the recommendations from the SZ expert elicitation project presented in Saturated Zone Flow and Transport Expert Elicitation Project (CRWMS M\&O 1998 [DIRS 100353], p. 3-43) to create a distribution of the specific discharge multiplier in the range of 1/30 to 10 (DTN: SN0310T0502103.009 [DIRS 168763]). More details of this analysis are presented in Saturated Zone Flow and Transport Model Abstraction (BSC 2004 [DIRS 170042], Section 6.5.2.1). All the permeabilities in the base-case flow model are multiplied by this factor, and all the recharge values and boundary fluxes input to the model are also multiplied by this factor to preserve the calibration of the base-case SZ flow model as discussed in Saturated Zone Site-Scale Flow Model (BSC 2004 [DIRS 170037], Section 6.6; DTN: LA0304TM831231.002 [DIRS 163788]). The effect of the range of uncertainty in this parameter on the output breakthrough curves is presented in Section 6.7.1. The base-case multiplier for the site-scale SZ transport model is chosen to be 1 , corresponding to the median value used in the base-case SZ flow model.

\subsubsection{Horizontal Anisotropy in Permeability}

Field estimates of the horizontal permeability anisotropy ratio in the north-south/east-west direction were obtained from the long-term pumping test conducted at the C-wells complex presented in Saturated Zone In-Situ Testing (BSC 2004 [DIRS 170010], Section 6.2.6). These data were used to obtain a distribution of the anisotropy ratio in the range of 0.05 to 20 with a median value of 4.2 (DTN: SN0310T0502103.009 [DIRS 168763]). More details of this analysis are presented in Saturated Zone Flow and Transport Model Abstraction (BSC 2004 [DIRS 170042], Section 6.5.2.10). The effect of the range of uncertainty in this parameter on the output breakthrough curves is presented in Section 6.7.2. The base-case ratio is chosen to be the median value of 4.2 .

\subsubsection{Bulk Density in Alluvium}

Borehole gravimeter data (DTN: MO0105GPLOG19D.000 [DIRS 163480]) from the well NC-EWDP-19D1 was used in conjunction with laboratory grain-density measurements (USGS n.d. [DIRS 154495]) in estimating the uncertainty distribution of the bulk density. These data yielded a normal distribution with a mean of $1,910 \mathrm{~kg} / \mathrm{m}^{3}$ and standard deviation of $78 \mathrm{~kg} / \mathrm{m}^{3}$ (DTN: SN0310T0502103.009 [DIRS 168763]). More details of this analysis, including a graphical representation, are presented in Saturated Zone Flow and Transport Model Abstraction (BSC 2004 [DIRS 170042], Section 6.5.2.7 and Figure 6-16). From this graph, a lower bound of $1,669 \mathrm{~kg} / \mathrm{m}^{3}$ and an upper bound of $2,151 \mathrm{~kg} / \mathrm{m}^{3}$ are estimated. The effect of the range of uncertainty in this parameter on the output breakthrough curves is presented in Section 6.7.3. The base-case value is chosen to be the median value of $1,910 \mathrm{~kg} / \mathrm{m}^{3}$. 


\subsubsection{Sorption Coefficient in Alluvium}

Sorption coefficient measurements for neptunium and uranium on core samples from the alluvium to the south of Yucca Mountain are available (Section A.7.3.3 and Section A.7.9.3 of Appendix A). For the radionuclides of americium, cesium, plutonium, protactinium, radium, strontium and thorium, the data on devitrified tuff samples from the Yucca Mountain area are used, since devitrified tuff makes up a major portion of the alluvium (Sections A.7.1.3, A.7.2.3, A.7.4.3, A.7.5.3, A.7.6.3, A.7.7.3, and A.7.8.3 of Appendix A). These data and the analysis to obtain stochastic uncertainty distributions for the sorption coefficient are presented in detail in Appendix A. Uncertainty distributions with wide ranges were selected to account for the uncertainty in the sorption coefficient rising from the uncertainties associated with the conceptual model (Section 6), geochemical conditions, and the in situ rock mineralogy (output DTN: LA0310AM831341.002). The sensitivity of the breakthrough curves for each radionuclide to the uncertainty in its $K_{d}$ values is presented in Saturated Zone Flow and Transport Model Abstraction (BSC 2004 [DIRS 170042], Section 6.6). In this report only the overall uncertainty range that encompasses all the radionuclides, 0 to $10,000 \mathrm{~mL} / \mathrm{g}$ (Table $4-2$ ), is considered. The effect of the range of uncertainty in this parameter on the output breakthrough curves is presented in Section 6.7.4. A base-case value of 0 is chosen to represent the case of nonsorbing radionuclides.

\subsubsection{Effective Porosity in Alluvium}

The study of Bedinger et al. (1989 [DIRS 129676], p. A18, Table 1) on the hydraulic characteristics of alluvium within the Southwest Basin and Range Province is relevant to the local basin fill conditions and provides an uncertainty distribution for effective porosity. Further information is available from the following sources:

- Single-point, site-specific, effective porosity data from the well NC-EWDP-19D1 presented in Saturated Zone In-Situ Testing (BSC 2004 [DIRS 170010], Section G.4.2.4) with a value of 0.1

- Total porosity data from the CAMBRIC study (Burbey and Wheatcraft 1986 [DIRS 129679], pp. 23 to 24) with an average value of 0.34

- Total porosity data presented in Regional Groundwater Flow and Tritium Transport Modeling and Risk Assessment of the Underground Test Area, Nevada Test Site, Nevada (DOE 1997 [DIRS 103021], Tables 8-1 and 8-2) with values of 0.36 and 0.35 .

All these data are used as supporting information to develop an uncertainty distribution with an upper bound of 0.3, median value of 0.18 and lower limit of 0 (DTN: SN0310T0502103.009 [DIRS 168763]) for the effective porosity. More details of this analysis are presented in Saturated Zone Flow and Transport Model Abstraction (BSC 2004 [DIRS 170042], Section 6.5.2.3). The lower limit given in this report (Tables 4-2 and 6.4-2) is 0.02, a conservative choice that avoids potential numerical problems with the value 0 . The effect of the range of uncertainty in this parameter on the output breakthrough curves is presented in Section 6.7.5. The base-case value is chosen to be the median value of 0.18 . 


\subsubsection{Retardation Factor in Alluvium for Irreversible Colloids}

The development of colloid retardation factors based on experimental data specific to Yucca Mountain as well as field studies of bacteriophage transport in alluvial material is presented in Saturated Zone Colloid Transport (BSC 2004 [DIRS 170006], Section 6.5). An uncertainty distribution with a range of 8 to 5,188 and a median value of 33.9 is presented. More details of this analysis are presented in Saturated Zone Flow and Transport Model Abstraction (BSC 2004 [DIRS 170042], Section 6.5.2.11). The effect of the range of uncertainty in this parameter on the output breakthrough curves is presented in Section 6.7.6. The base-case value is set to 1 to simulate the transport of nonsorbing radionuclides.

\subsubsection{Flowing Interval Porosity}

At Yucca Mountain, a flowing interval is defined as the region in which significant groundwater flow occurs at a well. The fracture porosity characterizes these flowing intervals rather than individual fractures. Data from tests in unsaturated tuff in the Exploratory Studies Facility were used to estimate the uncertainty distribution with a range of 0.00001 to 0.1 and a median value of 0.001, using gas flow as presented in Analysis of Hydrologic Properties Data (BSC 2004 [DIRS 170038], Section 6.1.3.2); water flow (BSC 2004 [DIRS 170038], Section 6.1.3.4); cross-hole tracer tests at the C-Wells complex given in Report of Results of Hydraulic and Tracer Tests at the C-Holes Complex (CRWMS M\&O 1997 [DIRS 100328], pp. 2 to 4 and 28); Nevada Environmental Restoration Project tests presented in Regional Groundwater Flow and Tritium Transport Modeling and Risk Assessment of the Underground Test Area, Nevada Test Site, Nevada (DOE 1997 [DIRS 103021], p. 5-14); and laboratory measurements on core from the wells USW G-1, USW GU-3, USW G-4, and UE-25 a\#1, where parallel-plate fracture geometry model was used (Wilson et al. 1994 [DIRS 100191], Volume 1, Chapter 7, Table 7-19, p. 7-30). More details of this analysis are presented in Saturated Zone Flow and Transport Model Abstraction (BSC 2004 [DIRS 170042], Section 6.5.2.5). The flowing interval porosity enters the transport model indirectly through the flowing interval aperture parameter, which is computed as a product of the porosity and the spacing. The effect of the range of uncertainty in the flowing interval aperture on the output breakthrough curves is presented in Section 6.7.8. The base-case value of the flowing interval porosity is taken to be .01 (corresponding to 80 percent probability), which is greater than the median value of the distribution. This is because, as discussed in Saturated Zone Flow and Transport Model Abstraction, the median value of .001 is at the lower limit of the values obtained from field data acquired after TSPA site recommendation calculations were done- 0.01 is more representative of the average of this data (BSC 2004 [DIRS 170042], Section 6.5.2.5).

\subsubsection{Flowing Interval Spacing}

An uncertainty distribution with a range of $1.22 \mathrm{~m}$ to $417 \mathrm{~m}$ and a median value of $20 \mathrm{~m}$ was developed in Saturated Zone Flow and Transport Model Abstraction (BSC 2004 [DIRS 170042], Section 6.5.2.4). The borehole flow meter survey data and analysis presented in Probability Distribution for Flowing Interval Spacing (BSC 2004 [DIRS 170014], Section 6.5) were used in the above-referenced analysis. The flowing interval spacing enters the transport model indirectly through the flowing interval aperture parameter, which is computed as a product of the porosity and the spacing. The effect of the range of uncertainty in the flowing interval aperture on the 
output breakthrough curves is presented in Section 6.7.8. The base-case value of the flowing interval spacing was taken to be the median of the distribution, $20 \mathrm{~m}$.

\subsubsection{Matrix Porosity in Volcanics}

The matrix porosity in volcanic units is treated as a nonstochastic parameter, although it is allowed to vary from unit to unit (DTN: SN0310T0502103.009 [DIRS 168763]). It is acceptable to treat this parameter as a nonstochastic parameter because it enters the site-scale SZ transport model through a combination with the distribution coefficient (Equation 57, Section 6.4.2.4.1) or with the diffusion coefficient (Equations 64 and 74, Section 6.4.2.4.1 ), and both these coefficients are being treated as stochastic variables with wide ranges (Sections 4.1.2.10 and 4.1.2.11). Values in the range of 0.15 to 0.25 are assigned on a unit-by-unit basis. Values were chosen based on the Rock Properties Model (BSC 2004 [DIRS 170032], Table 6.4-2) and porosity data from Boreholes UE-25 p\#1, USW H-3, USW SD-7, USW G-3, USW H-1, USW G-4, USW H-5, and USW H-6 (DTN: SN0004T0501399.003 [DIRS 155045], MO0109HYMXPROP.001 [DIRS 155989], and MO0010CPORGLOG.002 [DIRS 155229]). More details of this analysis are presented in SZ Flow and Transport Model Abstraction (BSC 2004 [DIRS 170042], Section 6.5.2.18).

\subsubsection{Effective Diffusion Coefficient in Volcanics}

Matrix diffusion is a process in which diffusing particles move, via Brownian motion, through both mobile and immobile fluids. Diffusion is a Fickian process. Diffusing species move from high to low concentrations. It depends on the free water molecular diffusion coefficient for individual constituents and the characteristics of the flow path in which the diffusing species passes. Because diffusion through porous media is less than free water molecular diffusion, it is quantitatively defined as the effective diffusion coefficient, $D_{e}$. The variability in $D_{e}$ in saturated media is caused by the variability in: (1) the individual constituents' size (atom, ion, or molecule) and charge; (2) fluid temperature; and (3) the unique properties of a porous media's lithology at a microscopic scale, including the tortuosity of the media.

Reimus et al. reported diffusion cell measurements on numerous rock samples from the vicinity of Yucca Mountain (2002 [DIRS 162956], Tables 3-2 through 3-8; 2002 [DIRS 163008], Tables 2-4 and 2-5). These measurements give a correlation between the measured effective diffusion coefficient, sample porosity, and sample permeability. The range of values is corroborated by the site-specific values reported by Triay et al. (1993 [DIRS 145123], Tables 1 and 2) and Rundberg et al. (1987 [DIRS 106481]). The correlation given by Reimus et al. (2002 [DIRS 163008]) Equation 2.5, p. 2.25) was used to develop a range of values appropriate for the porosities and permeabilities of various units as reported in Flint (1998 [DIRS 100033], p. 89). These were scaled to account for the uncertainty and variation in the effective diffusion coefficient based on the species size and charge, leading to the final uncertainty range of $5 \times 10^{-12}$ to $5 \times 10^{-10} \mathrm{~m}^{2} / \mathrm{s}$, with a median value of $5.0 \times 10^{-11} \mathrm{~m}^{2} / \mathrm{sec}$ (DTN: SN0310T0502103.009 [DIRS 168763]). Details of this analysis are presented in Saturated Zone Flow and Transport Model Abstraction (BSC 2004 [DIRS 170042], Section 6.5.2.6). The effect of the range of uncertainty in this parameter on the output breakthrough curves is presented in Section 6.7.9. The median value of $5.0 \times 10^{-11} \mathrm{~m}^{2} / \mathrm{sec}$ was taken to be the base-case value. 


\subsubsection{Matrix Sorption Coefficient in Volcanics}

Sorption coefficients were measured on devitrified and zeolitic tuff samples from the Yucca Mountain area for the radionuclides americium, barium, cesium, neptunium, plutonium, protactinium, radium, strontium, thorium, and uranium. These data and analyses were used to obtain stochastic uncertainty distributions for the sorption coefficients presented in detail in Appendix A. These measurements represent a spatial scale on the order of centimeters. A stochastic scaling procedure was used to obtain from these distributions the uncertainty distributions on the scale of $500 \mathrm{~m}$, which is the scale of the grid blocks used in the site-scale SZ transport model. This stochastic analysis is presented in detail in Appendix C of this report. Stochastic analysis was combined with expert judgment (Appendix C, Section C-2) to develop uncertainty distributions with wide ranges. These were selected to account for the uncertainty in the sorption coefficient rising from the uncertainties associated with the conceptual model (Section 6), geochemical conditions, and the in situ rock mineralogy (output DTN: LA0310AM831341.002). The resulting distributions for individual radionuclides are given in Table C-14 and output DTN: LA0310AM831341.002. The overall uncertainty range that encompasses all the radionuclides is 0 to $10,000 \mathrm{~mL} / \mathrm{g}$ (Table C-14). The effect of the range of uncertainty in this parameter on the output breakthrough curves is presented in Section 6.7.10. A base-case value of 0 was chosen to represent the case of nonsorbing radionuclides such as $\mathrm{C}^{14}$.

\subsubsection{Retardation Factor in Volcanics for Irreversible Colloids}

Saturated Zone Colloid Transport describes the development of colloid retardation factors for fractured tuff from field and experimental data (BSC 2004 [DIRS 170006], Section 6.4). More details of this analysis are presented in Saturated Zone Flow and Transport Model Abstraction (BSC 2004 [DIRS 170042], Section 6.5.2.11) leading to an uncertainty distribution with a range of 6 to 800 and a median value of 26 (DTN: LA0303HV831352.002 [DIRS 163558]). The effect of the range of uncertainty in this parameter on the output breakthrough curves is presented in Section 6.7.11. Base-case value for the site-scale SZ transport model is set to 1 to simulate the transport of nonsorbing radionuclides.

\subsubsection{Groundwater Concentrations of Colloids}

The uncertainty distribution was developed in Waste Form and In-Drift Colloids-Associated Radionuclide Concentrations: Abstraction and Summary (BSC 2004 [DIRS 170025], Section 6.3) and DTN:SN0306T0504103.005 (DIRS 164132). A range of $10^{-9}$ to $2.5 \times 10^{-4}$ $\mathrm{g} / \mathrm{mL}$ with a median value of $10^{-7} \mathrm{~g} / \mathrm{mL}$ is given. This parameter enters the site-scale SZ transport model indirectly through the coefficient for reversible sorption onto colloids shown in Saturated Zone Flow and Transport Model Abstraction (BSC 2004 [DIRS 170042], Section 6.5.2.12). The effect of the range of uncertainty in this parameter on the output breakthrough curves is presented in Section 6.7.12. The value of this parameter in the base-case transport model is set to 0 to simulate the transport of nonsorbing radionuclides.

\subsubsection{Sorption Coefficient onto Colloids}

The uncertainty distributions for the coefficient of sorption of plutonium, americium, thorium, protactinium, and cesium onto colloids were developed in Waste Form and In-Drift 
Colloids-Associated Radionuclide Concentrations: Abstraction and Summary (BSC 2004 [DIRS 170025], Section 6.3) and DTN: SN0306T0504103.006 (DIRS 164131). A range of $10^{1}$ to $10^{7} \mathrm{~mL} / \mathrm{g}$ was used with a median value of $0.5 \times 10^{7} \mathrm{~mL} / \mathrm{g}$. More details are presented in Saturated Zone Flow and Transport Model Abstraction (BSC 2004 [DIRS 170042], Section 6.5.2.12). This parameter enters the site-scale SZ transport model indirectly through the coefficient for reversible sorption onto colloids. The effect of the range of uncertainty in this parameter on the output breakthrough curves is presented in Section 6.7.12. The value of this parameter in the base-case transport model is set to 0 to simulate the transport of nonsorbing radionuclides.

\subsubsection{Fraction of Colloids Transported Unretarded}

A discussion of the fraction of colloids transported with no retardation is in Saturated Zone Colloid Transport (BSC 2004 [DIRS 170006], Section 6.6). The range of uncertainty distribution of this fraction is taken to be 0.00034 to 0.0017 with a median value of 0.0005 . This parameter is applied in the TSPA-LA calculations after the breakthrough curves are calculated from the site-scale SZ transport model; hence, the influence of the uncertainty in this parameter on the breakthrough curves is not discussed in this report. The base-case value is set to 0 to simulate the transport of nonsorbing radionuclides.

\subsubsection{Dispersivity, Longitudinal}

As explained in Saturated Zone Flow and Transport Model Abstraction (BSC 2004 [DIRS 170042], Section 6.5.2.9), the uncertainty distribution for longitudinal dispersivity was taken to be truncated lognormal with the mean of 2 and the standard deviation of 0.75 (in the log space) (DTN: SN0310T0502103.009 [DIRS 168763]). The basis used in SZ Flow and Transport Model Abstraction for this distribution was expert elicitation (CRWMS M\&O 1998 [DIRS 100353], pp. 3-10, 3-11, and LG-12). Graphically, the estimated range of this distribution is $1 \mathrm{~m}$ to $20,000 \mathrm{~m}$ with a median value of $100 \mathrm{~m}$ as discussed in Saturated Zone Flow and Transport Model Abstraction (BSC 2004 [DIRS 170042]), Figure 6-18). As explained in SZ Flow and Transport Model Abstraction, these dispersivity values are on the scale of the site-scale SZ transport model and correspond to dispersivity values smaller by a factor of 10 when represented on the scale of $500 \mathrm{~m}$, the scale of computational grid blocks shown in Saturated Zone Flow and Transport Model Abstraction (BSC 2004 [DIRS 170042], Section 6.5.2.9 and Figure 6-19). Hence, the range of uncertainty for this parameter given in Table $4-2$ is $0.1 \mathrm{~m}$ to $2,000 \mathrm{~m}$. The effect of the range of uncertainty in this parameter on the output breakthrough curves is presented in Section 6.7.13. The base-case value is taken to be the median value on the $500-\mathrm{m}$ scale, $10 \mathrm{~m}$.

\subsubsection{Dispersivity, Transverse, Horizontal}

As explained in Saturated Zone Flow and Transport Model Abstraction the transverse horizontal dispersivity values were determined by dividing the longitudinal dispersivity by a factor of 200 (BSC 2004 [DIRS 170042], Section 6.5.2.9). The basis used in Saturated Zone Flow and Transport Model Abstraction for the use of this factor was expert elicitation given in Saturated Zone Flow and Transport Expert Elicitation Project (CRWMS M\&O 1998 [DIRS 100353], pp. 3-11, LG-11, and LG-14). The range of values is $0.0005 \mathrm{~m}$ to $10 \mathrm{~m}$. The base-case value is 
taken to be $0.05 \mathrm{~m}$, calculated as the median value of the longitudinal dispersivity (Section 4.1.2.16) of $10 \mathrm{~m}$ divided by 200.

\subsubsection{Dispersivity, Transverse, Vertical}

As explained in Saturated Zone Flow and Transport Model Abstraction, the transverse vertical dispersivity values were determined by dividing the transverse horizontal dispersivity by a factor of 100 (BSC 2004 [DIRS 170042], Section 6.5.2.9). The basis used in Saturated Zone Flow and Transport Model Abstraction for the use of this factor was expert elicitation discussed in Saturated Zone Flow and Transport Expert Elicitation Project (CRWMS M\&O 1998 [DIRS 100353], pp. 3-11, LG-11, and LG-14). The range of values is $0.000005 \mathrm{~m}$ to $0.1 \mathrm{~m}$. The base-case value is taken to be $0.0005 \mathrm{~m}$, calculated as the base value of the transverse horizontal dispersivity (Section 4.1.2.17) of $0.05 \mathrm{~m}$ divided by 100 .

\subsubsection{Accuracy, Precision, And Representativeness}

The site-scale SZ transport model is a theoretical framework involving a number of transport parameters that reflect the properties of the SZ, incorporated into a computer code using numerical methods. This model is intended for making TSPA-LA predictions using stochastic methods with a wide range of values that reflect uncertainty in the input parameters. The accuracy and precision of the output results depend upon the accuracy and precision of the input parameter values, the theoretical model and the numerical model. As seen in Table 4-2, the uncertainty ranges for all input parameters (except the matrix porosity in volcanics, which is a deterministic value per each lithologic unit) are at least 25 percent or more of the base-case value, and in most cases they are several orders of magnitude larger than the base-case value. The theoretical and mathematical methods selected for the computational transport model, as described in detail in Section 6, are well established in the literature and are sufficiently accurate to deal with these wide parameter ranges. The approach taken in this report is to select a range of values for each input parameter as described in Section 4.1.2 and evaluate the propagation of this uncertainty range to the output breakthrough curves as described in Section 8. Confidence in the representativeness of the model output is developed through the validation activities of comparison against field data and independent models, and other confidence-building activities described in detail in Section 7.

\subsection{CRITERIA}

General requirements to be satisfied by the TSPA-LA are stated in 10 CFR 63.114 (DIRS 156605). Technical requirements to be satisfied by the TSPA-LA are identified in the Yucca Mountain Project Requirements Document (Canori and Leitner 2003 [DIRS 166275]). The acceptance criteria that will be used by the NRC to determine whether the technical requirements have been met are identified in the Yucca Mountain Review Plan, Final Report (YMRP) (NRC 2003 [DIRS 163274]). The pertinent requirements and criteria for this report are summarized in Table 4-3.

Table 4-3. Project Requirements and Acceptance Criteria Applicable to This Model Report

\begin{tabular}{|c|c|c|c|}
\hline $\begin{array}{c}\text { Requirement } \\
\text { Number }^{\mathrm{a}}\end{array}$ & Requirement Title $^{\mathrm{a}}$ & $10{\text { CFR 63 } \text { Link }^{\mathrm{b}}}^{\mathrm{a}}$ & YMRP Acceptance Criteria $^{\mathrm{c}}$ \\
\hline
\end{tabular}




\begin{tabular}{|l|l|l|l|}
\hline PRD-002/T-015 & $\begin{array}{l}\text { Requirements for performance } \\
\text { assessment }\end{array}$ & $\begin{array}{l}\text { 10 CFR 63.114 (a)- } \\
\text { (c) and (e)-(g) }\end{array}$ & $\begin{array}{l}\text { Criteria 1(1), 1(2),1(3), 1(5), 1(6), 2 } \\
\text { (1), 2(3), 3(1), 3(2), 3(4), 3(5), 3(6), } \\
\text { (1), 4(2),4(3), and 4(4) for } \\
\text { radionuclide transport in the SZ }\end{array}$ \\
\hline
\end{tabular}

${ }^{\mathrm{a}}$ From Canori and Leitner 2003 (DIRS 166275).

${ }^{b}$ From 10 CFR 63 (DIRS 156605).

${ }^{c}$ From YMRP (NRC 2003 [DIRS 163274]).

The acceptance criteria identified in Section 2.2.1.3.9.3 of the YMRP (NRC 2003 [DIRS 163274]) are included below. In cases where subsidiary criteria are listed in the YMRP for a given criterion, only the subsidiary criteria addressed by this scientific analysis are listed below. How the acceptance criteria and the subsidiary criteria are addressed in this report is presented in Section 8.4.

\section{Acceptance Criteria from Section 2.2.1.3.9.3, Radionuclide Transport in the Saturated Zone}

\section{Acceptance Criterion 1: System Description and Model Integration Are Adequate}

(1) Total system performance assessment adequately incorporates important design features, physical phenomena, and couplings, and uses consistent and appropriate assumptions throughout the radionuclide transport in the saturated zone abstraction process.

(2) The description of the aspects of hydrology, geology, geochemistry, design features, physical phenomena, and couplings, that may affect radionuclide transport in the saturated zone, is adequate. For example, the description includes changes in transport properties in the saturated zone, from water-rock interaction. Conditions and assumptions in the abstraction of radionuclide transport in the saturated zone are readily identified, and consistent with the body of data presented in the description.

(3) The abstraction of radionuclide transport in the saturated zone uses assumptions, technical bases, data, and models that are appropriate and consistent with other related U.S. Department of Energy abstractions. For example, assumptions used for radionuclide transport in the saturated zone are consistent with the total system performance assessment abstractions of radionuclide release rates and solubility limits, and flow paths in the saturated zone (Sections 2.2.1.3.4 and 2.2.1.3.8 of the Yucca Mountain Review Plan, respectively). The descriptions and technical bases provide transparent and traceable support for the abstraction of radionuclide transport in the saturated zone.

(5) Sufficient data and technical bases for the inclusion of features, events, and processes related to radionuclide transport in the saturated zone in the total system performance assessment abstraction are provided; and

(6) Guidance in NUREG-1297 and NUREG-1298 (Altman et al. 1988 [DIRS 103597; DIRS 103750]), or other acceptable approaches for peer review and data qualification is followed. 


\section{Acceptance Criterion 2: Data Are Sufficient for Model Justification}

(1) Geological, hydrological, and geochemical values used in the license application are adequately justified (e.g., flow path lengths, sorption coefficients, retardation factors, colloid concentrations, etc.). Adequate descriptions of how the data were used, interpreted, and appropriately synthesized into the parameters are provided;

(3) Data on the geology, hydrology, and geochemistry of the saturated zone, including the influence of structural features, fracture distributions, fracture properties, and stratigraphy, used in the total system performance assessment abstraction, are based on appropriate techniques. These techniques may include laboratory experiments, sitespecific field measurements, natural analog research, and process-level modeling studies. As appropriate, sensitivity or uncertainty analyses used to support the U.S. Department of Energy total system performance assessment abstraction are adequate to determine the possible need for additional data.

\section{Acceptance Criterion 3: Data Uncertainty Is Characterized and Propagated Through the Model Abstraction}

(1) Models use parameter values, assumed ranges, probability distributions, and bounding assumptions that are technically defensible, reasonably account for uncertainties and variabilities, and do not result in an under-representation of the risk estimate.

(2) For those radionuclides where the total system performance assessment abstraction indicates that transport in fractures and matrix in the saturated zone is important to waste isolation: (i) estimated flow and transport parameters are appropriate and valid, based on techniques that may include laboratory experiments, field measurements, natural analog research, and process-level modeling studies conducted under conditions relevant to the saturated zone at Yucca Mountain; and (ii) models are demonstrated to adequately predict field transport test results. For example, if a sorption coefficient approach is used, the assumptions implicit in that approach are validated.

(4) Parameter values for processes, such as matrix diffusion, dispersion, and groundwater mixing, are based on reasonable assumptions about climate, aquifer properties, and ground-water volumetric fluxes (Section 2.2.1.3.8 of the Yucca Mountain Review Plan);

(5) Uncertainty is adequately represented in parameter development for conceptual models, process-level models, and alternative conceptual models considered in developing the abstraction of radionuclide transport in the saturated zone. This may be done either through sensitivity analyses or use of conservative limits.

(6) Where sufficient data do not exist, the definition of parameter values and conceptual models is based on appropriate use of other sources, such as expert elicitation conducted in accordance with NUREG-1563 (Kotra et at. 1996 [DIRS 100909]). If other approaches are used, the U.S. Department of Energy adequately justifies their use. 


\section{Acceptance Criterion 4: Model Uncertainty Is Characterized and Propagated Through the Model Abstraction}

(1) Alternative modeling approaches of features, events, and processes are considered and are consistent with available data and current scientific understanding, and the results and limitations are appropriately considered in the abstraction.

(2) Conceptual model uncertainties are adequately defined and documented, and effects on conclusions regarding performance are properly assessed.

(3) Consideration of conceptual model uncertainty is consistent with available site characterization data, laboratory experiments, field measurements, natural analogue information, and process-level modeling studies; and the treatment of conceptual model uncertainty does not result in an under-representation of the risk estimate.

(4) Appropriate alternative modeling approaches are consistent with available data and current scientific knowledge, and appropriately consider their results and limitations using tests and analyses that are sensitive to the processes modeled. For example, for radionuclide transport through fractures, the U.S. Department of Energy adequately considers alternative modeling approaches to develop its understanding of fracture distributions and ranges of fracture flow and transport properties in the saturated zone.

\subsection{CODES, STANDARDS, AND REGULATIONS}

No codes, standards, or regulations other than those identified in the Project Requirements Document (Canori and Leitner 2003 [DIRS 166275], Table 2-3) and determined to be applicable (Table 4-3) were used in this analysis. 
INTENTIONALLY LEFT BLANK 


\section{ASSUMPTIONS}

A list of the assumptions used in this model report is provided in Table 5-1. Subsections where assumptions are used are identified in the table. The conceptual model of transport representing the important transport processes in the SZ is presented in Section 6.3 of this report. Alternative conceptual models and the implications of these models are discussed in Section 6.6. The theoretical framework of this model and its computational implementation are presented in Section 6.4. The simplifications necessary to develop a model amenable to efficient computations for stochastic simulations are also discussed in Sections 6.3 and 6.6.

Table 5-1. Assumptions

\begin{tabular}{|c|c|c|c|}
\hline Number & Assumption & Rationale & $\begin{array}{l}\text { Location in } \\
\text { this Report }\end{array}$ \\
\hline 1 & $\begin{array}{l}\text { It is assumed that } \\
\text { sorption on individual } \\
\text { fracture surfaces need } \\
\text { not be included in the } \\
\text { site-scale SZ transport } \\
\text { model. }\end{array}$ & $\begin{array}{l}\text { Sorption on individual fracture surfaces can result in } \\
\text { significant retardation of radionuclide transport even for small } \\
\text { values of fracture sorption coefficients (Robinson } 1994 \\
\text { [DIRS 101154], Figure 7). However, the sorption coefficient } \\
\text { onto fracture surfaces is a strong function of the minerals } \\
\text { coating the fracture surfaces. Because of the lack of } \\
\text { sufficient data, there is uncertainty about the nature of the } \\
\text { fracture coatings in the volcanics along the potential } \\
\text { transport pathways in the SZ. Hence, as a conservative } \\
\text { approach, no credit is taken for sorption on individual fracture } \\
\text { surfaces in the SZ. This assumption requires no further } \\
\text { justification. }\end{array}$ & Section 6.3 \\
\hline 2 & $\begin{array}{l}\text { The derivation of the } \\
\text { probability distribution } \\
\text { for the sorption } \\
\text { coefficients assumes } \\
\text { that conditions are } \\
\text { oxidizing in the } \\
\text { groundwater in the SZ. }\end{array}$ & $\begin{array}{l}\text { Available measurements show that the waters in the SZ at } \\
\text { Yucca Mountain along the potential transport pathways } \\
\text { downstream of the repository footprint (Appendix F, } \\
\text { Table F-1;) are mostly in oxidizing conditions or in transition } \\
\text { between oxidizing-reducing conditions, with some of the } \\
\text { deeper waters being in reducing condition. There is } \\
\text { insufficient data to completely characterize in detail the } \\
\text { oxidation state of the water along the expected transport } \\
\text { pathways (Figures 7-9a and 7-9b) in the SZ. Further, the } \\
\text { assumption of oxidizing conditions leads to predictions of } \\
\text { lower values of sorption coefficients. Hence, as a } \\
\text { conservative approach, it is assumed that conditions are } \\
\text { oxidizing in the groundwater in the SZ. This assumption } \\
\text { requires no further justification. }\end{array}$ & $\begin{array}{l}\text { Appendix A } \\
\text { Section A7 }\end{array}$ \\
\hline
\end{tabular}


Table 5-1. Assumptions (Continued)

\begin{tabular}{|c|c|c|c|}
\hline Number & Assumption & Rationale & $\begin{array}{l}\text { Location in } \\
\text { this Report }\end{array}$ \\
\hline 3 & $\begin{array}{l}\text { For the radionuclides } \\
\text { americium, cesium, } \\
\text { plutonium, protactinium, } \\
\text { strontium, and thorium, } \\
\text { sorption coefficients in } \\
\text { alluvium are assumed } \\
\text { to be those } \\
\text { corresponding to the } \\
\text { values measured on } \\
\text { samples of devitrified } \\
\text { crushed tuff. }\end{array}$ & $\begin{array}{l}\text { There is insufficient data on the sorption coefficients of the } \\
\text { radionuclides americium, cesium, plutonium, protactinium, } \\
\text { strontium, and thorium in alluvium. Alluvium along the } \\
\text { potential transport pathways is composed largely of } \\
\text { disaggregated tuffaceous materials. Sources of data on } \\
\text { aquifer matrix compositions in the SZ are provided in } \\
\text { Table C-1. These data have been incorporated into a site } \\
\text { mineralogic model, Mineralogic Model (MM3.0) Report, } \\
\text { (BSC } 2004 \text { [DIRS 170031]) There are two dominant rock } \\
\text { types in the SZ along potential flow paths in volcanics to the } \\
\text { 18-km boundary: devitrified tuff and zeolitic tuff. Because } \\
\text { devitrified tuff makes up a major portion of the volcanic units } \\
\text { exposed at the surface, it should be a major component in } \\
\text { alluvium. In addition, clays and other secondary minerals are } \\
\text { enriched in alluvial materials. These characteristics would } \\
\text { result in higher sorption coefficients for alluvial materials } \\
\text { compared to intact devitrified tuff. Also, the sorption } \\
\text { coefficients onto zeolitic tuffs are higher than those on } \\
\text { devitrified tuffs. Hence, as a conservative approach, it is } \\
\text { assumed that, for the radionuclides americium, cesium, } \\
\text { plutonium, protactinium, strontium, and thorium, sorption } \\
\text { coefficients in alluvium are given by the corresponding } \\
\text { values measured on samples of devitrified crushed tuff. This } \\
\text { assumption needs no further justification. }\end{array}$ & $\begin{array}{l}\text { Appendix A, } \\
\text { Section A7 }\end{array}$ \\
\hline
\end{tabular}




\section{MODEL DISCUSSION}

This section presents a discussion of the site-scale SZ transport model. Section 6.1 summarizes the objectives of this modeling activity, a description of the problem, and model inputs and outputs. Section 6.2 lists the FEPs included in TSPA-LA and relevant to this model report. The excluded FEPs are discussed in Features, Events, and Processes in SZ Flow and Transport (BSC 2004 [DIRS 170013]). The base-case conceptual model is presented in Section 6.3. The mathematical formulation of the base-case conceptual model is presented in Section 6.4, and the base-case model results are given in Section 6.5. Alternative conceptual models are discussed and evaluated along with their dispositions in Section 6.6. Model uncertainties and propagation of input uncertainties to output results are presented in Section 6.7. In Section 6.8, a description of the $\mathrm{SZ}$ as a barrier to transport of radionuclides is given.

\subsection{MODELING OBJECTIVES}

As summarized in Section 1 of this report, the objective of the site-scale SZ transport model is to simulate the transport of radionuclides in the saturated, fractured volcanic rock and alluvium near Yucca Mountain. The geohydrologic setting to be modeled is multifaceted with complex and diverse geochemical interactions possible between the groundwater, solutes, and the geological materials. Also, the intended use requires a computationally efficient model that is amenable to repeated runs for stochastic simulations. The approach taken here is to construct a plausible conceptual model of transport that incorporates the main SZ transport processes and is amenable to efficient computation (Sections 6.3 and 6.4). Alternate conceptual models and the implications of these models for transport predictions are evaluated relative to this base-case model (Section 6.6).

This transport model takes the calibrated flow model (DTN: LA0304TM831231.002 [DIRS 163788]) described in detail in Saturated Zone Site-Scale Flow Model (BSC 2004 [DIRS 170037]) as the starting point. The dependence of the flow field on various material and fluid properties, such as permeability and viscosity, is discussed in the SZ site-scale flow model report. Using the steady-state flow-velocity field supplied by the flow model, this transport model incorporates the transport processes of advection, dispersion, diffusion, retardation, and colloid-facilitated transport to compute the downstream radionuclide concentrations. Input parameters to the transport model are dispersivities in the volcanics, matrix porosity, matrix diffusion coefficient, the sorption distribution coefficient $K_{d}$ in the matrix, flowing interval porosity, flowing interval spacing, flowing interval aperture, retardation factor in the flowing interval, bulk density of the alluvium, effective porosity of the alluvium, dispersivities in the alluvium, and the $K_{d}$ in the alluvium. These are listed along with the base case and range of values in Table 6.4-2 in Section 6.4.3 and in Table 4-2 in Section 4. The site-scale SZ transport model generates breakthrough curves for a unit point source, hence radionuclide release locations are not given as specific values. The radionuclide release locations for various scenarios are discussed in Saturated Zone Flow and Transport Model Abstraction (BSC 2004 [DIRS 170042], Sections 6.3.3 and 6.4.2.13). The output from the transport model consists of radionuclide breakthrough curves at the boundary of the accessible environment at $18 \mathrm{~km}$ for nonsorbing transport as well as radionuclides subject to reactive transport. The reactive transport includes dissolved species and colloid-facilitated transport. Breakthrough curves for aqueous species are calculated including the retardation due to sorption onto rock surfaces 
(Section 6.4.2.5). Breakthrough curves are generated for transport of radionuclides attached reversibly to the colloids using modified transport parameters (Section 6.4.2.6.2). Breakthrough curves for the radionuclides attached irreversibly to the colloids are generated using retardation factors for colloids (Section 6.4.2.6.1). A small fraction of colloids travels with the groundwater unretarded and is handled using the same breakthrough curves as those for nonsorbing radionuclides.

A variety of laboratory and field data supports the understanding of the transport processes of importance included in the transport model. The cross-hole tracer tests conducted at the C-wells complex support the use of a dual-porosity fracture flow and transport model of advection and dispersion coupled with a matrix-diffusion and matrix-sorption model Saturated Zone In-Situ Testing (BSC 2004 [DIRS 170010], Sections 6.3.3 and 6.3.4). Additionally, several laboratory-scale colloid-facilitated $\mathrm{Pu}$ transport experiments conducted in fractured volcanic rocks support the use of a colloid-facilitated transport model in the volcanics (Kersting and Reimus 2003 [DIRS 162421], Chapter 7; DTN: LA0301PR831361.003 [DIRS 162435]; LA0301PR831361.004 [DIRS 162436]). The model of transport in the alluvium with advection, dispersion, sorption, and colloid-facilitated transport is supported by the single-well tracer tests at the Nye County ATC wells as presented in Saturated Zone In-Situ Testing (BSC 2004 [DIRS 170010], Section 6.5). Laboratory column transport experiments in Yucca Mountain alluvium have indicated that sorption should be included in the alluvium transport model Saturated Zone In-Situ Testing (BSC 2004 [DIRS 170010], Section 6.5.6). Likewise, colloid-facilitated $\mathrm{Pu}$ transport experiments in laboratory-scale columns packed with Yucca Mountain alluvium (DTN: LA0301AA831352.001 [DIRS 162433]) have indicated that colloid-facilitated transport is a valid process to include in the alluvium transport model. These models are supported also by information available from analogue studies at the Nevada Test Site (NTS) (see Sections 7.1.1 and 7.1.2.6). The overall site-scale transport model is validated by comparison against transit times and flow paths deduced from hydrochemistry data (Section 7.2).

The site-scale SZ transport model is used directly in the model report Saturated Zone Flow and Transport Model Abstraction (BSC 2004 [DIRS 170042]) for generating a set of radionuclide breakthrough curves at the accessible environment for use in the TSPA-LA simulations of radionuclide release to the biosphere. The outputs from the transport model are transit times, flow paths, and breakthrough curves at the boundary of the accessible environment at $18 \mathrm{~km}$ for various radionuclides of concern. The results for the base case are given in Section 6.5.

\subsection{FEATURES, EVENTS, AND PROCESSES FOR THIS MODEL REPORT}

As stipulated in Technical Work Plan For: Natural System - Saturated Zone Analysis and Model Report Integration (BSC 2004 [DIRS 171421]) this model report addresses the SZ FEPs pertaining to the site-scale SZ transport that are included for TSPA-LA (Table 6.2-1). SZ FEPs that were excluded for TSPA-LA are described in Features, Events, and Processes in SZ Flow and Transport (BSC 2004 [DIRS 170013]. Table 6.2-1 provides a list of FEPs that are relevant to this model analysis in accordance with their assignment in the LA FEP list (DTN: MO0407SEPFEPLA.000 ([DIRS 170760]). Specific reference to the various sections within this document where issues related to each FEP are addressed is provided in the table. 
Table 6.2-1. Features, Events, and Processes Included in TSPA-LA and Relevant to This Model Report

\begin{tabular}{|c|c|c|c|}
\hline FEP No. & FEP Name & $\begin{array}{l}\text { Sections That } \\
\text { Support Disposition }\end{array}$ & $\begin{array}{c}\text { FEP Topic Addressed in Other SZ Analysis } \\
\text { or Model Reports }\end{array}$ \\
\hline 1.2.02.01.0A & Fractures & $6.3,6.4 .2 .4$ & $\begin{array}{l}\text { Upstream Feeds }^{\mathrm{a}}-\mathrm{BSC} 2004 \text { (DIRS 170037) } \\
\text { Corroborating }^{\mathrm{b}}-\text { BSC } 2004 \text { (DIRS 170014), } \\
\text { BSC } 2004 \text { (DIRS 170010), BSC } 2004 \\
\text { (DIRS 170008) }\end{array}$ \\
\hline 1.2.02.02.0A & Faults & $6.3,6.4 .2 .1,6.4 .2 .4$ & $\begin{array}{l}\text { Upstream Feeds }^{\mathrm{a}}-\text { BSC } 2004 \text { (DIRS 170037) } \\
\text { Corroborating }^{\mathrm{b}}-\mathrm{BSC} 2004 \text { (DIRS 170010), } \\
\text { BSC } 2004 \text { (DIRS 170008) }\end{array}$ \\
\hline 1.3.07.02.0A & $\begin{array}{l}\text { Water table rise } \\
\text { affects } \mathrm{SZ}\end{array}$ & 6.6, Appendix E & $\begin{array}{l}\text { Upstream Feeds }{ }^{\text {a }} \text {-BSC } 2004 \text { (DIRS 170037) } \\
\text { Corroborating }^{\mathrm{b}} \text {-BSC } 2004 \text { (DIRS 170009) }\end{array}$ \\
\hline 2.2.03.01.0A & Stratigraphy & 6.3 & $\begin{array}{l}\text { Upstream Feeds }^{a}-\text { BSC } 2004 \text { (DIRS 170037) } \\
\text { Corroborating }^{\mathrm{b}}-\text { BSC } 2004 \text { (DIRS 170008), } \\
\text { BSC } 2004 \text { (DIRS 170014), BSC } 2004 \\
\text { (DIRS 170010) }\end{array}$ \\
\hline $2.2 .03 .02 .0 \mathrm{~A}$ & $\begin{array}{l}\text { Rock properties of } \\
\text { host rock and other } \\
\text { units }\end{array}$ & $4.1 .2,6.3,6.6,7.1$ & $\begin{array}{l}\text { Upstream Feeds }^{a}-\text { BSC } 2004 \text { (DIRS 170037) } \\
\text { Corroborating }^{\mathrm{b}}-\text { BSC } 2004 \text { (DIRS 170010), } \\
\text { BSC } 2004 \text { (DIRS 170008), BSC } 2004 \\
\text { (DIRS 170014) }\end{array}$ \\
\hline 2.2.07.12.0A & $\begin{array}{l}\text { Saturated } \\
\text { groundwater flow in } \\
\text { the geosphere }\end{array}$ & $4.1 .2,6.3,6.4,6.6,7$ & $\begin{array}{l}\text { Upstream Feeds }^{a}-\text { BSC } 2004 \text { (DIRS 170037) } \\
\text { Corroborating }- \text { BSC } 2004 \text { (DIRS 170037), BSC } \\
2004 \text { (DIRS 170010), BSC } 2004 \\
\text { (DIRS 170014), BSC } 2004 \text { (DIRS 170015) }\end{array}$ \\
\hline $992.2 .07 .13 .0 \mathrm{~A}$ & $\begin{array}{l}\text { Water-conducting } \\
\text { features in the SZ }\end{array}$ & $4.1 .2,6.3,6.6$ & $\begin{array}{l}\text { Upstream Feeds }{ }^{a}-B S C 2004 \text { (DIRS 170037) } \\
\text { Corroborating }- \text { BSC } 2004 \text { (DIRS 170014), BSC } \\
2004 \text { (DIRS 170010), BSC } 2004 \text { (DIRS 170008) }\end{array}$ \\
\hline 2.2.07.15.0A & $\begin{array}{l}\text { Advection and } \\
\text { dispersion in the SZ }\end{array}$ & $\begin{array}{l}\text { 6.3 (Items } 2 \text { and } 6) \\
\text { 6.4.2.2, 6.4.2.3, } \\
4.1 .2 .1,4.1 .2 .16,7.1 \\
\end{array}$ & $\begin{array}{l}\text { Upstream Feeds }{ }^{\mathrm{a}}-\mathrm{BSC} 2004 \text { (DIRS 170037) } \\
\text { Corroborating }{ }^{\mathrm{b}} \text { - BSC 2004 (DIRS 170010), } \\
\text { BSC } 2004 \text { (DIRS 170015) }\end{array}$ \\
\hline 2.2.07.16.0A & $\begin{array}{l}\text { Dilution of } \\
\text { radionuclides in } \\
\text { groundwater }\end{array}$ & $6.3,6.4$ & $\begin{array}{l}\text { Upstream Feeds }{ }^{a}-N / A \\
\text { Corroborating }{ }^{\mathrm{b}}-\mathrm{N} / \mathrm{A}\end{array}$ \\
\hline $2 \cdot 2 \cdot 07.17 .0 \mathrm{~A}$ & Diffusion in the SZ & $\begin{array}{l}6.3 \text { (Item 3), 6.4.2.4 } \\
4.1 .2 .10,7.1\end{array}$ & $\begin{array}{l}\text { Upstream Feeds }{ }^{a}-N / A \\
\text { Corroborating }- \text { BSC 2004 (DIRS 170006), } \\
\text { BSC } 2004 \text { (DIRS 170010), BSC } 2004 \\
\text { (DIRS 170014) }\end{array}$ \\
\hline 2.2.08.01.0A & $\begin{array}{l}\text { Chemical } \\
\text { characteristics of } \\
\text { groundwater in the SZ }\end{array}$ & $\begin{array}{c}6.3 \text { (Items } 4 \text { and } 7) \\
\text { Appendix A (A.3 and } \\
\text { A.7) } \\
\end{array}$ & $\begin{array}{l}\text { Upstream Feeds }{ }^{\mathrm{a}}-\mathrm{N} / \mathrm{A} \\
\text { Corroborating }^{\mathrm{b}}-\mathrm{BSC} 2004 \text { (DIRS 170037) }\end{array}$ \\
\hline $2.2 .08 .06 .0 \mathrm{~A}$ & $\begin{array}{l}\text { Complexation in the } \\
\text { SZ }\end{array}$ & $\begin{array}{l}\text { 6.4.2.4.1, 6.4.2.5, } \\
\text { 6.4.2.6, Appendices A } \\
\text { (A.7 and A.8) and B }\end{array}$ & $\begin{array}{l}\text { Upstream Feeds }{ }^{a}-N / A \\
\text { Corroborating }{ }^{b}-N / A\end{array}$ \\
\hline 2.2.08.08.0A & $\begin{array}{l}\text { Matrix diffusion in the } \\
\text { SZ }\end{array}$ & $\begin{array}{l}6.3 \text { (Item 3), 6.4.2.4 } \\
\text { 4.1.2.10, } 7.1\end{array}$ & $\begin{array}{l}\text { Upstream Feeds }^{\mathrm{a}-\mathrm{N} / \mathrm{A}} \\
\text { Corroborating } \\
\text { BSC } 2004 \text { (DSC 2004 (DIRS 170010), BSC } 2004 \\
\text { (DIRS 170006) }\end{array}$ \\
\hline 2.2.08.09.0A & Sorption in the SZ & $\begin{array}{c}6.3 \text { (Items } 4 \text { and } 7) \\
6.4 .2 .4,6.4 .2 .5,7.1 \\
\text { Appendix A (A.7, A.8) }\end{array}$ & $\begin{array}{l}\text { Upstream Feeds }^{\mathrm{a}-N / A} \\
\text { Corroborating }^{\mathrm{b}}-\mathrm{BSC} 2004 \text { (DIRS 170006) }\end{array}$ \\
\hline 2.2.08.10.0A & $\begin{array}{l}\text { Colloidal transport in } \\
\text { the SZ }\end{array}$ & $\begin{array}{l}\text { 6.4.2.6, 7.1, } \\
\text { Appendix B }\end{array}$ & $\begin{array}{l}\text { Upstream Feeds }{ }^{\mathrm{a}-B S C} 2004 \text { (DIRS 170006) } \\
\text { Corroborating }^{\mathrm{b}}-\text { BSC } 2004 \text { (DIRS 170010) }\end{array}$ \\
\hline
\end{tabular}


Table 6.2-1. Features, Events, and Processes Included in TSPA-LA and Relevant to This Model Report (Continued)

\begin{tabular}{|c|c|c|c|}
\hline FEP No. & FEP Name & $\begin{array}{l}\text { Sections That } \\
\text { Support Disposition }\end{array}$ & $\begin{array}{c}\text { FEP Topic Addressed in Other SZ Analysis } \\
\text { or Model Reports }\end{array}$ \\
\hline $2.2 .10 .03 .0 \mathrm{~A}$ & $\begin{array}{l}\text { Natural Geothermal } \\
\text { Effects on Flow in the } \\
\text { SZ }\end{array}$ & 6.1 & $\begin{array}{l}\text { Upstream Feeds }{ }^{\text {a }}-\text { BSC } 2004 \text { (DIRS 170037) } \\
\text { Corroborating }^{b 9}-\text { N/A }\end{array}$ \\
\hline 2.2.12.00.0B & $\begin{array}{l}\text { Undetected features in } \\
\text { the SZ }\end{array}$ & $\begin{array}{l}\text { Appendix A, } \\
\text { Appendix C }\end{array}$ & $\begin{array}{l}\text { Upstream Feeds }{ }^{\mathrm{a}-B S C} 2004 \text { (DIRS 170037) } \\
\text { Corroborating }{ }^{\mathrm{b}}-\mathrm{BSC} 2004 \text { (DIRS 170008), } \\
\text { BSC } 2004 \text { (DIRS 170014), BSC } 2004 \\
\text { (DIRS 170010) }\end{array}$ \\
\hline
\end{tabular}

${ }^{a}$ Upstream Feeds-Aspects of the SZ FEP dispositions adopted in this report are a result of SZ analyses performed in a directly upstream SZ model or analyses.

${ }^{b}$ Corroborating-Corroborative aspect(s) of the FEP topic is (are) discussed in a SZ analysis or model report.

\subsection{BASE-CASE CONCEPTUAL MODEL}

The transport of chemical species in the groundwater involves a variety of processes such as advection, dispersion, diffusion, sorption, and colloid-facilitated transport. These processes, in general, lead to retardation of the migration of transported species with respect to the bulk movement of the groundwater. Hence, the radionuclide transport times can be several orders of magnitude longer than the transport times of ground water. Given the complex geohydrologic setting and the computational constraints imposed by the TSPA-LA methodology, the approach taken here is to construct a plausible conceptual transport model that incorporates the main SZ transport processes and is amenable to efficient computation. Alternate conceptual models and the implications of these models for transport predictions are evaluated relative to this base-case model.

The base-case conceptual model of SZ transport begins in the neighborhood of the repository footprint at the water table, where the radionuclides enter the water table, and ends at the boundary of the accessible environment at $18 \mathrm{~km}$ downstream from this footprint, where the radionuclides reaching the boundary are removed from the model. The flow path from the repository to the proposed boundary of the accessible environment at $18 \mathrm{~km}$ begins in the volcanic tuffs but ends in the alluvium, and different transport processes operate in the volcanic tuffs and the alluvium.

The components of the conceptual model follow: (1) Radionuclides enter the SZ via fluids percolating through the unsaturated zone (UZ) below the repository site. Within the SZ, they are transported with the groundwater that flows subhorizontally in a southerly or southeasterly direction. (2) The radionuclides advect and disperse with the groundwater through the fractured portions of the tuffs near the water table. (3) Fluid flow occurs preferentially within the flowing intervals, whereas stagnant fluid resides in the rock matrix. Solutes diffuse in and out of fluid within the rock matrix that is essentially stagnant. (4) Sorption reactions occur in volcanics between the rock matrix and some of the radionuclides, tending to retard the transport of these radionuclides. (5) Radionuclides can undergo colloid-facilitated transport in the volcanics. (6) The radionuclides advect and disperse with the groundwater through the alluvium. (7) Sorption reactions occur in alluvium between the rock and some of the radionuclides, tending to retard the transport of these radionuclides. (8) Radionuclides can undergo colloid-facilitated transport in 
alluvium. (9) The radionuclides reaching the model boundary, which is downstream from the boundary to the accessible environment at $18 \mathrm{~km}$ downstream of the repository footprint are removed from the model. These are schematically illustrated in Figure 6.3-1:

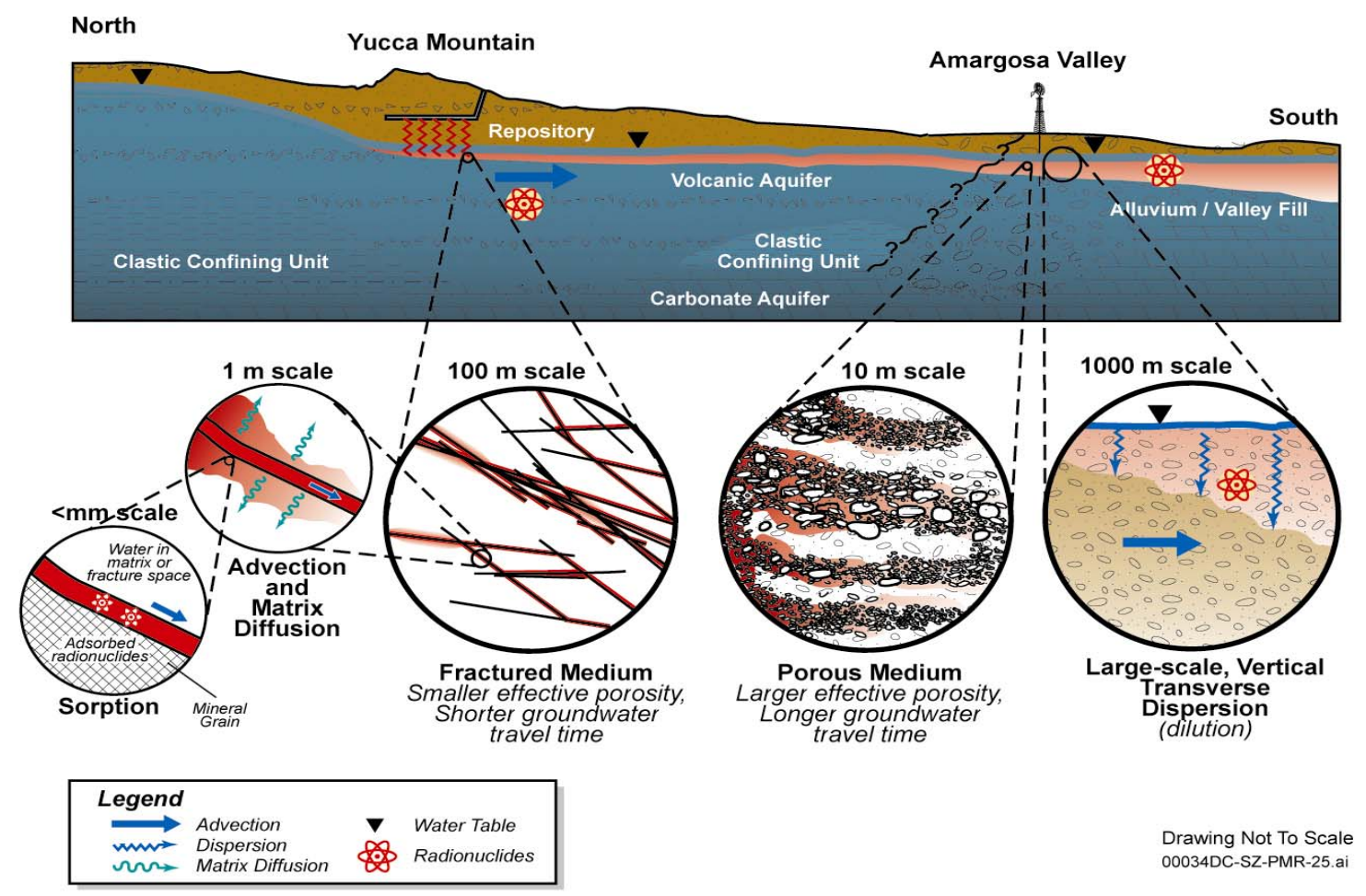

NOTE: For the sake of clarity, the following processes are not explicitly indicated in this figure: dispersion and colloid-facilitated transport in the volcanics; and advection, longitudinal dispersion, sorption, and colloidfacilitated transport in the alluvium.

Figure 6.3-1. Schematic Illustration of the Conceptual Model of Transport Processes in the Volcanic Tuffs and the Alluvium

A discussion of each model component follows:

1. Radionuclides enter the SZ via fluids percolating through the UZ below the repository site. The source of radionuclides in the site-scale SZ transport model is provided by the UZ transport model. The nature of this source term and its relationship to various intrusion scenarios is discussed in Saturated Zone Flow and Transport Model Abstraction (BSC 2004 [DIRS 170042], Sections 6.3.3 and 6.5.2.13). The site-scale SZ transport model generates breakthrough curves for a unit point source. The conceptual model of site-scale SZ transport is suitable for a range of locations of the source term, and the mathematical and computational model is designed to handle such variations. Thus, the SZ transport component of the site-scale SZ model is developed independently from a transport model for the UZ. As discussed in the Saturated Zone Flow and Transport Model Abstraction (BSC 2004 [DIRS 170042], 
Section 6.3.3), this approach leads to results that are generally conservative with respect to radionuclide concentrations and transport times.

As shown in Section 6.6.2.3 of the Saturated Zone Site-Scale Flow Model (BSC 2004 [DIRS 170037]), the general flow direction starting at the repository footprint is to the south. The flow lines remain shallow and subhorizontal within the SZ. Thus, fluid flow occurs within the fractured volcanics immediately below and downstream of the repository footprint, entering the alluvium further downstream, and continuing through the alluvium to the boundary of the accessible environment at $18 \mathrm{~km}$.

2. The radionuclides advect and disperse with the groundwater through the fractured portions of the tuffs near the water table. Flow occurs within the fractured portions of the tuffs near the water table. Flow intervals identified in well tests correlate with fracture locations (Erickson and Waddell 1985 [DIRS 105279], p. 18), the extent of fracturing correlates reasonably well with the degree of welding (Waddell et al. 1984 [DIRS 101064], p. 26), and the degree of welding is one of the criteria used to define the submembers within a lithologic unit. In the SZ flow model, such lithologic members are represented as single zones, each with its equivalent permeability (BSC 2004 [DIRS 170037], Table 6-22). This representation is also used in the transport model; however, the influence of heterogeneities within a permeability zone is incorporated into the model via dispersion and matrix diffusion as discussed below.

A distinction must be made between fracture zones and individual fractures. Fracture zones are typically spaced tens of meters apart, with thicknesses on the order of meters, and contain broken-up matrix blocks and many intersecting fractures that are conduits to flow and to diffusion and retardation. Individual fractures, on the other hand, tend to be spaced as close as a meter or less, have thicknesses on the order of fractions of millimeters, and may or may not contribute to the flow of groundwater.

The conceptual model is that high-permeability regions are offset by low-permeability regions due to the extensive faulting and fracturing observed in the volcanics (Luckey et al. 1996 [DIRS 100465], pp. 8 to 12) in the model domain. These low-permeability regions effectively will act as large-scale heterogeneities that give rise to large-scale macroscopic dispersion due to the tortuous nature of flow over the scale of hundreds of meters to kilometers. Field studies of transport and dispersion at a variety of length scales (from meters to kilometers) presented in Saturated Zone In-Situ Testing (BSC 2004 [DIRS 170010], Figures E-39 and E-40) and Neuman (1990 [DIRS 101464], Figure 1) show a trend toward larger apparent dispersion coefficients for transport over longer distances. The estimates of dispersivities from the C-wells tracer tests presented in Saturated Zone In-Situ Testing (BSC 2004 [DIRS 170010], Section E.4.1) fall within the range of values from other sites, suggesting that transport in the fractured tuffs exhibits similar dispersive characteristics.

In an equivalent-continuum dispersion model, hydrogeologic features that may be present at scales smaller than the size of a typical grid cell are simulated as averages. The equivalent-continuum model averages the concentration variations within a grid block into a single value for the block. Concentration differences at scales smaller 
than the grid cell, if present, are not resolved. Whether this distinction is important depends on specific performance criteria and scenarios for exposure to humans or plants. Because the site-scale SZ flow and transport model is used to calculate radionuclide mass reaching the accessible environment at $18 \mathrm{~km}$, which is then used to calculate ground water concentrations in a representative volume of 3000 acre-feet in accordance with 10 CFR 63.312 (DIRS 156605), the above approach is considered adequate.

3. Fluid flow occurs preferentially within the flowing intervals, whereas stagnant fluid resides in the rock matrix. Solutes diffuse in and out of fluid within the rock matrix that is essentially stagnant. Current hydrologic evidence supports the model of fluid flow within fracture zones in the moderately to densely welded tuffs of the SZ (e.g., Waddell et al. 1984 [DIRS 101064], Table 2, pp. 17 to 22). Hydraulic conductivities measured for core samples in the laboratory are orders of magnitude higher when the sample is fractured (Peters et al. 1984 [DIRS 121957], p. 60). Also, there generally is a positive correlation between fractures, identified using acoustic televiewer or borehole television tools, and zones of high transmissivity (Erickson and Waddell 1985 [DIRS 105279], Figure 3; Karasaki et al. 1990 [DIRS 148309], p. 811). The fluid travels preferentially within regions of large apertures with large sections of the fracture surface containing stagnant fluid or no fluid where the faces are in contact.

Matrix materials conduct no fluid under natural groundwater flow conditions but are physically connected to the fracture fluid through the pore network. When a dissolved species travels with the fluid within a fracture, it may migrate by molecular diffusion into the stagnant fluid in the rock matrix. Within the matrix, due to low permeability, the velocity for the bulk movement of the ground water is effectively zero; hence, the advection of the solute along the fracture is effectively zero. The Brownian motion of the solute molecules becomes dominant, and the effect of advection resumes when the solute re-enters the fracture. The result is a delay of the delivery of the solute to a downgradient location from what would be predicted if the solute had remained in the fracture.

Several theoretical, laboratory, and field studies have demonstrated the validity of the matrix-diffusion model. Sudicky and Frind (1981 [DIRS 148342], pp. 161 to 163) developed a model of flow in an aquifer with diffusion into a surrounding aquitard to show that the movement of ${ }^{14} \mathrm{C}$ can be much slower than predicted if only movement with the flowing water is considered. Maloszewski and Zuber (1985 [DIRS 148312], pp. 353 to 354) reached a similar conclusion with a model for ${ }^{14} \mathrm{C}$ transport that consists of uniform flow through a network of equally spaced fractures with diffusion into the surrounding rock matrix between the joints. Maloszewski and Zuber present analyses of several interwell tracer experiments that show that their matrix diffusion model can be used to provide simulations of these tests that are consistent with the values of matrix porosity obtained in the laboratory and aperture values estimated from hydraulic tests. In all cases, the results are superior when compared to previous analyses that did not include matrix diffusion effects. Finally, a data set of great relevance to the SZ beneath Yucca Mountain is the C-wells reactive tracer test presented in Saturated Zone In-Situ Testing (BSC 2004 [DIRS 170010], Section 6.3.), 
which demonstrated that models incorporating matrix diffusion provide more reasonable fits to the tracer experiment data than those that use a single continuum. The calculated transport times of SZ fluids determined from ${ }^{14} \mathrm{C}$ data (BSC 2004 [DIRS 170037], Table A6-11) are on the order of hundreds to thousands of years. As demonstrated in Figures 6.8-2a, 6.8-2b, 6.8-3a, and 6.8-3b of this report, model transit times without matrix diffusion are only on the order of several hundred years but become comparable with transit times estimated from the geochemical data when retardation due to matrix diffusion is included in the model. Thus, the ${ }^{14} \mathrm{C}$ ages are consistent with the conceptual model of interchange of solutes between fractures and matrix found in the matrix diffusion model.

Matrix diffusion is characterized using an abstracted model of uniform flow and transport in equally spaced, parallel fractures. This treatment represents a considerable simplification of the complex fracture network observed in volcanic rocks. It is acceptable because the particle-tracking model is intended to be used in large-scale simulations. The size of a typical computational grid block in the SZ flow and transport model is $500 \mathrm{~m}$ by $500 \mathrm{~m}$. Average flowing intervals are subvertical with an average spacing of $20 \mathrm{~m}$, orders of magnitude smaller than the grid block size. In such circumstances, the use of an abstracted dual-porosity model with equally spaced, parallel fractures to capture the transport behavior is reasonable. In numerical modeling studies, such uncertainties are commonly addressed through the use of simpler conceptual models, such as this one, combined with sensitivity analyses to assess the importance of the uncertain parameters to the final model result. Therefore, a broad range of flowing interval spacings is used (Table 4-2), and the influence on the final results is quantified (Section 6.7).

4. Sorption reactions occur in volcanics between the rock matrix and some of the radionuclides, tending to retard the transport of these radionuclides. Radionuclide-rock interactions potentially can occur on the surfaces of fractures and within the rock matrix. Sorption reactions are chemical reactions that involve the distribution of chemical constituents between water and solid surfaces. Measurements show that the majority of water samples from the SZ at the Yucca Mountain (Table A-2b) are in oxidizing conditions or in transition between oxidizing-reducing conditions. Further, other factors being equal, oxidizing conditions lead to lower values of sorption coefficients (Appendix A). Hence, this conceptual model takes the geochemical conditions along the entire flow path to be oxidizing.

Although the radionuclide-rock reactions can be complex in detail, they are represented in the transport model by a constant called the sorption coefficient $K_{d}$ (Freeze and Cherry 1979 [DIRS 101173], p. 403, Equation 9.13). The use of the $K_{d}$ model requires that the reactions must be in equilibrium, instantaneous (kinetics), linear, and reversible. The validity of these requirements at Yucca Mountain and their implications for the transport model are discussed in Section 6.6 on alternate conceptual models. The surface-area to fluid-volume ratio and the mineral distributions probably are different in the fractures as compared to the matrix. As a conservative approach, sorption on individual fracture surfaces is not included in the transport model. 
Maloszewski and Zuber (1985 [DIRS 148312], pp. 353 to 354) show that, at several sites, models that include the effect of chemical exchange reactions in the matrix lead to greater agreement with the field data than the models that do not include these effects. Of particular relevance to Yucca Mountain, the lithium tracer in the C-wells reactive tracer experiment shown in Saturated Zone In-Situ Testing (BSC 2004 [DIRS 170010], Section 6.3.; Robinson 1994 [DIRS 101154], pp. 86 to 93, Figures 5 and 8) was modeled using a matrix diffusion model with the sorption coefficient as an additional adjustable parameter. The fact that the early lithium response had the same timing as that of the nonsorbing tracers, but with a lower normalized peak concentration, is consistent with matrix diffusion coupled with sorption in the matrix (Robinson 1994 [DIRS 101154], Figure 7).

5. Radionuclides can undergo colloid-facilitated transport in the volcanics. Colloids are microscopic particles that are much larger than solute molecules. Solute molecules can absorb onto the colloid particles and be transported along with them. Because the colloidal particles are much larger in size and mass than the radionuclides that sorb onto them, the radionuclides do not affect the transport of the colloid particles. A discussion of colloid-facilitated transport is found in Appendix B of this report and presented in Saturated Zone Colloid Transport (BSC 2004 [DIRS 170006]), Section 6.4). The colloids in the SZ can be of several types including natural colloids (typically clay or silica), waste-form colloids resulting from degradation of spent nuclear fuel or glass, and iron-oxyhydroxide colloids resulting from degradation of the waste container presented in Waste Form and In-Drift Colloids-Associated Radionuclide Concentrations: Abstraction and Summary (BSC (2004 [DIRS 170025], Section 6.3.1). The transport of colloids themselves in groundwater, as well as the mechanisms of sorption of radionuclides onto these colloid particles, needs to be included in the transport model for the SZ. The irreversibly sorbed radionuclides are taken to transport identically to the colloids to which they are sorbed.

Mechanisms for transport of colloid particles in groundwater are distinct from those appropriate for the solute molecules and are considered in detail in Saturated Zone Colloid Transport BSC (2004 [DIRS 170006], Section 6.4). In the transport, movement of colloids is taken to occur within fractures only. Because of the relatively large size of the colloids, matrix diffusion of these particles is taken to be negligible. Several field observations have suggested that a small percentage of colloids transport with essentially no retardation in groundwater (Kersting et al. 1999 [DIRS 103282], p. 56, 58; Penrose et al. 1990 [DIRS 100811], p. 228), whereas the majority undergo either reversible or irreversible filtration, which can be described by a retardation factor. In this analysis, filtration is defined as the net effect of chemical sorption of the colloid onto the rock surface and the physical removal of colloids from the advective flow due to sieving and settling. The retardation factor is dependent on several factors such as colloid size, colloid type, and geochemical conditions (e.g., $\mathrm{pH}$, Eh, and ionic strength). Details are presented in Saturated Zone Colloid Transport (BSC 2004 [DIRS 170006], Section 6).

The radionuclides that are reversibly absorbed onto colloids are modeled using the $K_{\mathrm{c}}$ model. This model represents the equilibrium partitioning of radionuclides between 
the aqueous phase and the colloidal phase with the distribution coefficient (Appendix B, Equations B-2 through B-7). Based on the estimated rate constants and the range of transport times being considered for transport through saturated fractured tuffs, it is shown in Saturated Zone Colloid Transport (BSC 2004 [DIRS 170006], Section 6.7) that the equilibrium partitioning is valid for all but the shortest transport times and overestimates transport mobility of radionuclides for the shortest transport times. The distribution coefficient $K_{\mathrm{c}}$ is modeled as a function of radionuclide sorption properties, colloid substrate properties, aqueous chemistry, and colloid concentration, but independent of the properties of the immobile media through which transport occurs (Appendix B). The radionuclides that are irreversibly absorbed onto the colloids are modeled to transport in a manner identical to the colloids onto which they are sorbed. The transport of the colloids is simulated using the advection-dispersion equation.

Colloid-facilitated transport is not included in the base-case site-scale SZ transport model because it represents the extreme case of nonsorbing radionuclides.

6. The radionuclides advect and disperse with the groundwater through the alluvium. Alluvium is valley-fill material consisting of heterogeneous deposits of sand and gravel interbedded with mud and clay-sized materials (Waddell et al. 1984 [DIRS 101064], p. 27). This material is not well consolidated and tends to exhibit a more porous, less-fractured nature. Because of the heterogeneous nature of the medium, flow occurs through the more permeable regions within the alluvium, and the lower-permeability regions act as flow barriers. This characteristic tends to reduce the amount of porosity actually available to flow and transport as compared to the total large-scale porosity of the alluvium. To account for this, the effective flow porosity of the alluvium is considered to be a stochastic variable with a range of input values presented in Saturated Zone Flow and Transport Model Abstraction (BSC 2004 [DIRS 170042], Section 6.5.2.3). Dispersion is caused by heterogeneities at all scales, from the scale of individual pore spaces to the scale of the thickness of individual strata and the length of structural features such as faults. The spreading and dilution of radionuclides that result from these heterogeneities could be important to the performance of the repository. The largest heterogeneities are represented explicitly in Saturated Zone Site-Scale Flow Model (BSC 2004 [DIRS 170037], Section 6). For dispersion at smaller scales, the advective-dispersion model is used with dispersion characterized using a dispersion coefficient tensor.

7. Sorption reactions occur in alluvium between the solid surfaces and some of the radionuclides, tending to retard the transport of these radionuclides. In contrast to the fractured tuffs, there are no cross-hole, field-scale tracer transport tests in the alluvium south of Yucca Mountain to confirm the in situ sorption characteristics. However, the transport of sorbing solutes in porous media that is not controlled by fractures has been well studied (e.g., Freeze and Cherry 1979 [DIRS 101173], Chapter 9, pp. 385 to 457). Sorption coefficients onto alluvium from the Nye County wells have been measured for a few key radionuclides. For the remaining radionuclides, sorption coefficients have been estimated based on the corresponding values measured for crushed tuff (Appendix A). Radionuclides could also precipitate in the SZ, forming solid phases on 
the rock surfaces. The most credible mechanism for this effect to occur is through a different redox condition in the SZ. If the conditions were significantly more reducing, the valence states of actinides, such as $\mathrm{Np}, \mathrm{U}$, and $\mathrm{Pu}$, and fission products, such as Tc and I, could be lowered. Typically, in these groundwaters, this situation results in much lower solubilities of the radionuclides and hence, precipitation. This effect is an extreme form of retardation that renders the bulk of the radionuclide plume virtually immobile. The base-case model takes the conditions to be oxidizing, which results in higher solubilities and lower sorption coefficients than would be the case if reducing conditions were selected. Therefore, the base-case model produces more rapid transport of some radionuclides in that the use of lower valence states for these radionuclides would yield a less mobile species.

8. Radionuclides can undergo colloid-facilitated transport in alluvium. The conceptual model for colloid-facilitated transport in the alluvium is essentially the same as in fractured tuffs in that colloids are modeled as transported only by advective water (no diffusion into stagnant water or into grains), and colloid attachment and detachment onto alluvial surfaces are described by first-order rate expressions. Because the colloidal particles are much larger in size and mass than the radionuclides that sorb onto them, the radionuclides do not affect the transport of the colloid particles. The irreversibly sorbed radionuclides are taken to transport identically to the colloids to which they are sorbed. A discussion of colloid-facilitated transport is found in Appendix B of this report and in Saturated Zone Colloid Transport (BSC 2004 [DIRS 170006], Section 6.5). Laboratory site-specific data for colloid transport in the alluvium along with literature data are used to obtain distributions and bounds for attachment and detachment rate constants as given in Saturated Zone Colloid Transport (BSC 2004 [DIRS 170006], Section 6.5). Based on the estimated rate constants and the range of transport times being considered for transport through saturated fractured tuffs, it was shown in Saturated Zone Colloid Transport (BSC 2004 [DIRS 170006], Section 6.7) that the equilibrium partitioning is valid for all but the shortest transport times and overestimates transport mobility of radionuclides for the shortest transport times.

9. The radionuclides reaching the model boundary are removed from the model The boundary to the accessible environment is taken at $18 \mathrm{~km}$ downstream (10 CFR 63.302 [DIRS 156605]) of the repository footprint. As discussed above, the flow direction is generally southwards downstream from the repository. The southern boundary of the site-scale SZ flow model, which is also taken to be the southern boundary of the transport model, is more than $18 \mathrm{~km}$ downstream of the repository footprint (BSC 2004 [DIRS 170037]). Thus the site-scale SZ transport model encompasses the boundary to the accessible environment. The radionuclides reaching the model boundary are removed from the model, providing the boundary condition necessary for modeling the transport. 


\subsection{MATHEMATICAL AND COMPUTATIONAL FORMULATION OF BASE-CASE CONCEPTUAL MODEL}

\subsubsection{Overview of Model Formulation}

The mathematical model formulation of the conceptual model presented in Section 6.3 is described below. The advection-dispersion equation is stated in Section 6.4.2.1, and the development of a general form of the dispersion tensor for axisymmetric media is given in Section 6.4.2.2. A random-walk particle-tracking method for implementing advection-dispersion in a numerical code is presented in Section 6.4.2.3. A mathematical formulation to treat matrix diffusion in volcanics is presented next in Section 6.4.2.4, followed by a mathematical description of sorptive transport in alluvium in Section 6.4.2.5 and colloid-facilitated transport in Section 6.4.2.6. The computer implementation of the mathematical models in the code FEHM is verified with a suite of example problems using published examples, analytical and semianalytical solutions and examples run with different codes. These are documented in the verification report for FEHM.

\subsubsection{Mathematical Model Description}

\subsubsection{Advective Dispersive Transport}

The fundamental mass transport equation for transport of a nonreactive, dilute species in a saturated porous medium (with no sources or sinks) has the form (e.g., Bear 1972 [DIRS 156269], p. 617, Equation 10.5.2):

$$
\frac{\partial C}{\partial t}+\nabla \cdot(\vec{v} C)-\nabla \cdot\left(D^{\prime} \nabla C\right)=0
$$

where

$C$ denotes the solute concentration in units of moles per liter

$t$ is time

$\bar{v} \quad$ designates the pore-water velocity vector

$D^{\prime}$ denotes the dispersion tensor.

This equation serves as the starting point for all subsequent development below, and the sorbing and colloid-facillitated transport described later on. The pore water velocity is obtained from the flow field presented by the flow model (BSC 2004 [DIRS 170037]), taken to be independent of the transport model. The dispersion tensor $D^{\prime}$ appearing in Equation 1 is the sum of the mechanical dispersion tensor $(D)$ for the flow system and the coefficient of molecular diffusion $\left(D_{0}\right)$ in porous media. The effects of molecular diffusion are significant only at low flow velocities (Bear 1972 [DIRS 156269], p. 581). The following discussion deals with $D$ for the sake of simplicity. The effects of molecular diffusion can be accounted for by adding in the term $D_{0}$ to the diagonal components of the displacement matrix as in Equation 55. 
Generally, the pore-water velocity and the dispersion tensor vary spatially and temporally. Experimental studies of transport in groundwater have determined the nature of the dispersion tensor and the appropriate values of the dispersivity parameter. Gelhar (1997 [DIRS 145122], p. 164, Figure 8) showed that distinct values of the longitudinal dispersivity, the transverse dispersivity in the horizontal direction, and the transverse dispersivity in the vertical direction can be identified based on available field transport studies. The general conceptual model underlying the use of these three terms is one of horizontal flow with tortuous fine-scale flow through heterogeneous media. The details of transport through the heterogeneous media give rise to the spreading of solute in the direction of flow and, to a lesser extent, transverse to the direction of flow. Of course, groundwater flow, though generally horizontal, exhibits vertical velocities locally in regions of upward or downward gradients, such as in areas of recharge or discharge or when the flow is subject to variability in hydraulic conductivity that diverts water vertically.

In practice, it is difficult, from available data, to propose more complex forms of the dispersion process and to determine the alternate dispersivity values from field observations. Nevertheless, it is quite possible that more complex forms are more representative, given the complexity and variety of different heterogeneities present in nature. An important conclusion from the available field data is that longitudinal dispersion is a strong function of scale, that is, the travel length of a solute plume in the medium (e.g., Neuman 1990 [DIRS 101464], Figure 1). In a typical groundwater flow model at the scale of a flow basin, characteristic flow distances of tens to hundreds of meters vertically may be present, compared to hundreds to thousands of meters horizontally. Given the difference in scale, it is not clear that the longitudinal dispersivity in the vertical direction should be set equal to that in the horizontal direction. In addition, in stratified porous media containing heterogeneities such as irregularly shaped beds or clay lenses, the characteristic scale of the heterogeneity encountered by a solute will be different in the horizontal and vertical directions, yielding potentially different values for longitudinal dispersion. Therefore, one motivation for the development of the theory in this section is to propose a dispersion tensor that can be used to handle these more general scenarios.

\subsubsection{General Form of the Dispersion Tensor for Axisymmetric Media}

An axisymmetric medium is one that displays rotational symmetry about an axis. For example, a medium composed of horizontal layers of different geological materials, each of which is uniform within the layer, is an axisymmetric medium with the axis of symmetry being vertical. Consider a porous medium that, at the macroscale, exhibits an axis of symmetry $\bar{\lambda}$; that is, physical processes are invariant under rotations about $\bar{\lambda}$. To be considered a tensor, the collection of coefficients making up the dispersion tensor must satisfy certain transformation rules upon changing from one coordinate system to another. It can be shown that the most general, symmetric, second-order tensor that can be constructed from the vectors $\bar{\lambda}$ and the average macroscale pore velocity $\bar{v}$ is given by the dyadic (Poreh 1965 [DIRS 163847], p. 3911, Equation 8):

$$
\bar{D}=\alpha_{1} v \bar{I}+\alpha_{2} \frac{\bar{v} \bar{v}}{v}+\alpha_{3} v \bar{\lambda} \bar{\lambda}+\frac{1}{2} \alpha_{4}(\bar{\lambda} \bar{v}+\bar{v} \bar{\lambda})
$$

where 
$\bar{I} \quad$ represents the unit tensor

$v \quad$ denotes the magnitude of the solute velocity $\left(v^{2}=\sum v_{i}^{2}\right)$

the coefficients $\alpha_{i}$ are scalar quantities with the dimension of length.

Symmetry is imposed on the dispersion tensor. While traditionally this is taken to be the case for the dispersion tensor (Bear 1972 [DIRS 156269], p. 611), it is not a fundamental requirement. In this expression for the dispersion tensor, and in what follows, the contribution of molecular diffusion is not explicitly indicated for simplicity. It may be easily added to the diagonal elements.

The four coefficients $\alpha_{i}$ that appear in Equation 2 are, in general, functions of the scalar quantities $v$ and $\bar{\lambda} \cdot \bar{v}$, which may be functions of time and space for a variable velocity field, such as encountered in heterogeneous media, for example. $\bar{v}$ is an eigenvector of $\bar{D}$ provided that the coefficients $\alpha_{3}$ and $\alpha_{4}$ are related to each other through the relation:

$$
\alpha_{4}=-2 \cos \theta \alpha_{3}=-2 \frac{\bar{\lambda} \cdot \bar{v}}{v} \alpha_{3}
$$

where $\theta$ is the angle between the symmetry axis and the velocity vector.

Using Equation 3a, Equation 2 becomes

$$
\bar{D}=\alpha_{1} v \bar{I}+\alpha_{2} \frac{\bar{v} \bar{v}}{v}+\alpha_{3} v\left(\bar{\lambda} \bar{\lambda}-\frac{\cos \theta}{v}(\bar{\lambda} \bar{v}+\bar{v} \bar{\lambda})\right)
$$

When Equations 3a and 3b hold, the coefficients $\alpha_{i}$ may be related to their more conventional designations in terms of longitudinal and transverse dispersivity corresponding to eigenvalues of $\bar{D}$ associated with principal axes parallel and perpendicular to the direction of flow, respectively. In this case, two distinct longitudinal and transverse dispersivities can be defined. These correspond to flow parallel and perpendicular to the axis of symmetry and may be assigned as $\alpha_{L}^{H}, \alpha_{L}^{V}$ and $\alpha_{T}^{H}, \alpha_{T}^{V}$, respectively. The superscripts $V$ and $H$, designating "vertical" and "horizontal," are defined in relation to the axis of symmetry with $V$ parallel and $H$ perpendicular to the symmetry axis $\bar{\lambda}$.

For a general axisymmetric medium, it is possible that the principal axes are not aligned with the direction of flow. Let $\Phi$ denote the angle between the major principal axis of the dispersion tensor and the flow velocity. When $\Phi \neq 0$, the terms longitudinal and transverse are not meaningful. There is some precedent for $\Phi \neq 0$ in heterogeneous porous media (de Marsily 1986 [DIRS 100439], p. 250). Gelhar and Axness (1983 [DIRS 107296], pp. 166 to 170) and Neuman et al. (1987 [DIRS 147577], pp. 460 to 462) have demonstrated theoretically that, in general, $\Phi \neq 0$ is based on a stochastic analysis of transport in heterogeneous media. However, there is disagreement among the authors as to the sign of the angle $\Phi$. At present, there does not exist experimental or field confirmation of the situation when $\Phi \neq 0$, which appears difficult at best to establish. 
If Equation 3a holds so that one of the principal axes is aligned with the direction of flow, then (Lichtner et al. 2002 [DIRS 163821], Equation 19a and 19b):

$$
\alpha_{L}=\alpha_{1}+\alpha_{2}+\cos ^{2} \theta \alpha_{3}
$$

and

$$
\alpha_{T}=\alpha_{1}+\left(1-\cos ^{2} \theta\right) \alpha_{3}
$$

where, $\alpha_{L}$ is the longitudinal dispersivity and $\alpha_{T}$ is the transverse dispersivity, respectively.

Thus, when $\theta=\pi / 2$,

$$
\alpha_{L}=\alpha_{L}^{H}=\alpha_{1}+\alpha_{2}
$$

and

$$
\alpha_{T}=\alpha_{T}^{V}=\alpha_{1}+\alpha_{3}
$$

When $\theta=0$,

$$
\alpha_{L}=\alpha_{L}^{V}=\alpha_{1}+\alpha_{2}+\alpha_{3}
$$

and

$$
\alpha_{T}=\alpha_{T}^{H}=\alpha_{1}
$$

Solving 4a, 4c, and $4 \mathrm{f}$ for the $\alpha_{i}$ yields the inverse relations:

$$
\begin{gathered}
\alpha_{1}=\alpha_{T}^{H}, \\
\alpha_{2}=\alpha_{L}-\alpha_{T}^{H}-\frac{\cos ^{2} \theta}{1-\cos ^{2} \theta}\left(\alpha_{T}-\alpha_{T}^{H}\right) \\
\alpha_{3}=\frac{\alpha_{T}-\alpha_{T}^{H}}{1-\cos ^{2} \theta}
\end{gathered}
$$

With these results the dispersion tensor can be written in dyadic form as:

$$
\begin{gathered}
\bar{D}=\alpha_{T}^{H} v \bar{I}+\left[\alpha_{L}-\alpha_{T}^{H}-\frac{\cos ^{2} \theta}{1-\cos ^{2} \theta}\left(\alpha_{T}-\alpha_{T}^{H}\right)\right] \frac{\bar{v} \bar{v}}{v} \\
+\frac{\alpha_{T}-\alpha_{T}^{H}}{1-\cos ^{2} \theta} v\left[\bar{\lambda} \bar{\lambda}-\frac{\cos \theta}{v}(\bar{\lambda} \bar{v}+\bar{v} \bar{\lambda})\right]
\end{gathered}
$$


The longitudinal and transverse dispersivities, $\alpha_{L}$ and $\alpha_{T}$, can be arbitrary functions of the scalar quantities $v$ and $\theta$. Whether or not this four-parameter representation of the dispersion tensor provides an adequate description of flow at an angle to the symmetry axis, it needs to be verified by direct comparison with field observations.

\subsection{Special Cases of the Dispersion Tensor}

In this section, special cases corresponding to an isotropic medium and an axisymmetric medium with flow parallel and perpendicular to the symmetry axis are considered in more detail. These cases are implicit in the site-scale SZ transport model and arise for special values of the dispersion coefficients as discussed below for each case. It is useful to consider them for comparative purposes.

Isotropic Media-For the special case of an isotropic medium, $\bar{\lambda}=0$ since there is no preferred axis of symmetry, and it follows that the general form of the dispersion tensor reduces to the well-known form (Poreh 1965 [DIRS 163847], Equation 15):

$$
\bar{D}=\alpha_{1} v \bar{I}+\alpha_{2} \frac{\bar{v} \bar{v}}{v}
$$

To relate the coefficients $\alpha_{L}$ and $\alpha_{T}$ to the usual longitudinal and transverse dispersivity coefficients $\alpha_{L}$ and $\alpha_{T}$, note that $\bar{v}$ is an eigenvector of $\bar{D}$ with eigenvalue $\alpha_{1}+\alpha_{2}$, and

$$
\bar{D} \cdot \bar{v}=\left(\alpha_{1}+\alpha_{2}\right) \bar{v}
$$

Thus, it follows that $\alpha_{L}=\alpha_{1}+\alpha_{2}$. The remaining two eigenvectors represented by $\bar{\zeta}_{i}$, orthogonal to $\bar{v}$, belong to the degenerate eigenvalue $\alpha_{1} v$. Hence, it follows that

$$
\bar{D} \cdot \bar{\zeta}_{i}=\alpha_{1} v \bar{\zeta}_{i}
$$

Thus, $\alpha_{T}=\alpha_{1}$, and $\alpha_{2}=\alpha_{L}-\alpha_{T}$.

The resulting dispersion tensor for an isotropic porous medium has the form (Bear 1972 [DIRS 156269], p. 613, Equation 10.4.16):

$$
\begin{gathered}
\bar{D}=\alpha_{T} v I+\left(\alpha_{L}-\alpha_{T}\right) \frac{\bar{v} \bar{v}}{v}= \\
\left(\begin{array}{lll}
\alpha_{T} v+\left(\alpha_{L}-\alpha_{T}\right) \frac{v_{1}^{2}}{v} & \left(\alpha_{L}-\alpha_{T}\right) \frac{v_{1} v_{2}}{v} & \left(\alpha_{L}-\alpha_{T}\right) \frac{v_{1} v_{3}}{v} \\
\left(\alpha_{L}-\alpha_{T}\right) \frac{v_{2} v_{1}}{v} & \alpha_{T} v+\left(\alpha_{L}-\alpha_{T}\right) \frac{v_{2}^{2}}{v} & \left(\alpha_{L}-\alpha_{L}\right) \frac{v_{2} v_{3}}{v} \\
\left(\alpha_{L}-\alpha_{T}\right) \frac{v_{3} v_{1}}{v} & \left(\alpha_{L}-\alpha_{T}\right) \frac{v_{3} v_{2}}{v} & \alpha_{T} v+\left(\alpha_{L}-\alpha_{T}\right) \frac{v_{3}^{2}}{v}
\end{array}\right)
\end{gathered}
$$


Axisymmetric Medium: Flow Parallel to Symmetry Axis-For an axisymmetric medium with flow along the axis of symmetry, it follows that

$$
\bar{v}=v \bar{\lambda}
$$

and $\bar{D}$ becomes

$$
\bar{D}=\alpha_{1} v \bar{I}+\left(\alpha_{2}+\alpha_{3}+\alpha_{4}\right) v \bar{\lambda} \bar{\lambda}
$$

It is apparent that $\bar{v}$ (and $\bar{\lambda}$ ) is an eigenvector belonging to the eigenvalue $\left(\alpha_{1}+\alpha_{2}+\alpha_{3}+\alpha_{4}\right) v$, and the eigenvalue $\alpha_{1}$ is degenerate with eigenvectors in the plane normal to $\bar{\lambda}$.

Introducing the notation $\alpha_{L}^{V}$ for longitudinal dispersivity along the axis of symmetry and $\alpha_{T}^{H}$ for horizontal transverse dispersivity, the dispersion tensor can be written as:

$$
\bar{D}=\alpha_{T}^{H} v \bar{I}+\left(\alpha_{L}^{V}-\alpha_{T}^{H}\right) \frac{\bar{v} \bar{V}}{v}
$$

with $\alpha_{1}=\alpha_{T}^{H}$ and $\alpha_{2}+\alpha_{3}+\alpha_{4}=\alpha_{L}^{V}-\alpha_{T}^{H}$. This form of the dispersion tensor has the same form as that for an isotropic medium. Thus, $\bar{v}$ is a principal direction of the dispersion tensor satisfying

$$
\bar{D} \cdot \bar{v}=\alpha_{L}^{V} v \bar{v}
$$

with

$$
\alpha_{L}^{V}=\alpha_{1}+\alpha_{2}+\alpha_{3}+\alpha_{4}
$$

The remaining two eigenvectors are orthogonal to $\bar{v}$ but otherwise arbitrary, belonging to the degenerate eigenvalue $\alpha_{T}^{H} v$.

Axisymmetric Medium: Flow Perpendicular to Symmetry Axis-For the special case when $\bar{v}$ and $\bar{\lambda}$ are orthogonal and $\cos \theta=0$, it follows that

$$
\bar{D} \cdot \bar{v}=\left(\alpha_{1}+\alpha_{2}\right) \bar{v}+\frac{1}{2} \alpha_{4} v^{2} \bar{\lambda}
$$

and

$$
\bar{D} \cdot \bar{\lambda}=\frac{1}{2} \alpha_{4} \bar{v}+\left(\alpha_{1}+\alpha_{3}\right) v \bar{\lambda}
$$


From these relations it is apparent that, in general, neither $\bar{v}$ nor $\bar{\lambda}$ are eigenvectors of $\bar{D}$ unless $\alpha_{4}=0$, in which case both vectors $\bar{v}$ and $\bar{\lambda}$ are eigenvectors satisfying the eigenvalue equations

$$
\begin{aligned}
\bar{D} \cdot \bar{v} & =\left(\alpha_{1}+\alpha_{2}\right) \bar{v} \\
& =\alpha_{L}^{H} v \bar{v}
\end{aligned}
$$

and

$$
\begin{aligned}
\bar{D} \cdot \bar{\lambda} & =\left(\alpha_{1}+\alpha_{3}\right) v \bar{\lambda} \\
& =\alpha_{T}^{V} v \bar{\lambda}
\end{aligned}
$$

In this case there are two transverse dispersivities, horizontal and vertical, denoted by $\alpha_{T}^{H}$ and $\alpha_{T}^{V}$. Longitudinal dispersivity is denoted by $\alpha_{L}^{H}$ because, in general, it may be different from $\alpha_{L}^{T}$ for flow in the direction parallel to $\bar{\lambda}$.

Noting that $\bar{v} \times \bar{\lambda}$ is an eigenvector belonging to the eigenvalue $\alpha_{1}$, which may be identified with $\alpha_{T}^{H}$, it follows that

$$
\begin{gathered}
\alpha_{1}=\alpha_{T}^{H} \\
\alpha_{2}=\alpha_{L}^{H}-\alpha_{T}^{H}
\end{gathered}
$$

and

$$
\alpha_{3}=\alpha_{T}^{V}-\alpha_{T}^{H}
$$

Generally, $\alpha_{2}>\alpha_{3}$ since $\alpha_{2}$ involves the difference between longitudinal and transverse dispersivities, whereas $\alpha_{3}$ is proportional to the difference in transverse dispersivities.

\subsection{New Form of the Dispersion Tensor for Axisymmetric Media}

To construct a form of the dispersion tensor that could apply to flow at any arbitrary angle to the axis of symmetry and still honor tensorial transformation properties, the appropriate functional form of the coefficients $\alpha_{L}$ and $\alpha_{T}$ on the direction of flow must be determined. Two limiting cases must be met for flow parallel and perpendicular to the axis of symmetry. For flow parallel to the symmetry axis, longitudinal dispersion should reduce to the vertical longitudinal dispersivity $\alpha_{L}^{V}$, and transverse dispersion should be isotropic with dispersivity described by $\alpha_{T}^{V}$. For flow perpendicular to the axis of symmetry, longitudinal dispersion should reduce to the horizontal longitudinal dispersivity $\alpha_{L}^{H}$, with transverse dispersion described by the two coefficients, $\alpha_{T}^{V}$ and $\alpha_{T}^{H}$. Clearly, it is not possible to deduce a priori the form of the dispersion tensor for flow at an angle to the symmetry axis without additional information. It would be expected that the dispersion tensor would depend on the specific properties of the porous 
medium and even head differences. Without additional information, it is taken that the longitudinal and transverse dispersivities have the forms

$$
\alpha_{L}=G_{L}\left(\cos \theta ; \alpha_{L}^{V}, \alpha_{L}^{H}\right)
$$

and

$$
\alpha_{T}=G_{T}\left(\cos \theta ; \alpha_{T}^{V}, \alpha_{T}^{H}\right)
$$

where the only dependence is on the scalar $\cos \theta$ in addition to the dispersivity parameters. The functions $G_{L, T}$ satisfy the end member conditions

$$
\begin{gathered}
G_{L}\left(0 ; \alpha_{L}^{V}, \alpha_{L}^{H}\right)=\alpha_{L}^{V} \text { (horizontal flow) } \\
G_{T}\left(0 ; \alpha_{T}^{V}, \alpha_{T}^{H}\right)=\alpha_{T}^{H} \text { (horizontal flow) } \\
G_{L}\left(1 ; \alpha_{L}^{V}, \alpha_{L}^{H}\right)=\alpha_{L}^{H} \quad \text { (vertical flow) } \\
G_{T}\left(1 ; \alpha_{T}^{V}, \alpha_{T}^{H}\right)=\alpha_{T}^{V} \quad \text { (vertical flow) }
\end{gathered}
$$

but are otherwise arbitrary. To determine the functional form of $G_{L, T}$, it would be necessary to compare predictions based on a specific form of the dispersion tensor with actual field observations or to carry out numerical experiments involving heterogeneous media.

For the new form of the dispersion tensor proposed here, the following dependency on $\cos \theta$ is chosen:

$$
G_{L}=\alpha_{L}^{H}+\cos ^{2} \theta\left(\alpha_{L}^{V}-\alpha_{L}^{H}\right)
$$

and

$$
G_{T}=\alpha_{T}^{V}+\cos ^{2} \theta\left(\alpha_{T}^{H}-\alpha_{T}^{V}\right)
$$

With this choice of $G_{L, T}$, the desired behavior is obtained that is at least correct for the end member cases of flow parallel and perpendicular to the axis of symmetry. However, clearly an infinite number of functional forms are possible that satisfy the conditions of Equations 20a to 20d. In what follows, it is taken that the transverse horizontal dispersivity $\alpha_{T}^{H}$ has the same value for flow parallel and perpendicular to the axis of symmetry. However, it is not apparent that this necessarily must be the case, which would complicate the formulation. 
To obtain expressions for the coefficients $\alpha_{i}$ in terms of the set $\alpha_{L}^{V}, \alpha_{L}^{H}, \alpha_{T}^{V}$, and $\alpha_{T}^{H}$, the expressions for longitudinal and transverse dispersion given by Equations $4 \mathrm{a}$ and $4 \mathrm{~b}$ are equated to the desired forms given by

$$
\begin{aligned}
& \alpha_{L}=\alpha_{1}+\alpha_{2}+\cos ^{2} \theta \alpha_{3} \\
& =\alpha_{L}^{H}+\cos ^{2} \theta\left(\alpha_{L}^{V}-\alpha_{L}^{H}\right)
\end{aligned}
$$

and

$$
\begin{aligned}
& \alpha_{T}=\alpha_{1}+\left(1-\cos ^{2} \theta\right) \alpha_{3} \\
& =\alpha_{T}^{V}+\cos ^{2} \theta\left(\alpha_{T}^{H}-\alpha_{T}^{V}\right)
\end{aligned}
$$

As can be seen from these relations, the eigenvalue for longitudinal dispersion varies between the horizontal and vertical longitudinal dispersivities as the flow direction varies from perpendicular to parallel to the symmetry axis of the medium. Similarly, the eigenvalue for transverse dispersivity varies between the vertical and horizontal transverse dispersivities.

The coefficient $\alpha_{1}$ is taken to be given by

$$
\alpha_{1}=\alpha_{T}^{H}
$$

independent of the direction of the flow velocity relative to the symmetry axis. To see that this is reasonable, define the vector $\bar{\omega}$ as

$$
\bar{\omega}=\bar{\lambda}-\frac{\bar{\lambda} \cdot \bar{v}}{v^{2}} \bar{v}
$$

Then it is seen that the vector $\bar{\xi}_{3}=\bar{v} \times \bar{\omega}$ is an eigenvector of the dispersion tensor $\bar{D}$, and is perpendicular to the symmetry axis $\bar{\lambda}$ and the flow velocity $\bar{v}$ with eigenvalue $\alpha_{1} v$, and, hence, it should reflect only horizontal transverse dispersion. Equations 22a and 22b provide two equations for the two coefficients $\alpha_{2}$ and $\alpha_{3}$. It follows that

$$
\begin{gathered}
\alpha_{2}=\alpha_{L}^{H}-\alpha_{T}^{H}+\cos ^{2} \theta\left(\alpha_{L}^{V}-\alpha_{L}^{H}+\alpha_{T}^{V}-\alpha_{T}^{H}\right) \\
=\alpha_{L}-\alpha_{T}+\alpha_{T}^{V}-\alpha_{T}^{H}
\end{gathered}
$$

and

$$
\alpha_{3}=\alpha_{T}^{V}-\alpha_{T}^{H}
$$

with $\alpha_{4}$ given by Equation 3a. 
In these relations, $\alpha_{1}$ is seen to be independent of the angle $\theta$. This behavior is intuitively correct because the vector $\bar{\zeta}_{3}$ always remains perpendicular to the symmetry axis and the direction of flow, and, hence, the eigenvalue should always be equal to the horizontal transverse dispersivity. In addition, as expected, the eigenvalue corresponding to the eigenvector $\bar{\omega}$ varies from transverse vertical to transverse horizontal as the vectors $\bar{v}$ and $\bar{\omega}$ range from perpendicular to parallel.

In terms of individual matrix elements with $\lambda=(0,0,1)$, the dispersion tensor becomes (Lichtner et al. 2002 [DIRS 163821], Equations 47a to 47f):

$$
\begin{gathered}
D_{11}=\alpha_{L} \frac{v_{1}^{2}}{v}+\alpha_{T}^{H} \frac{v_{2}^{2}}{v}\left(1+\frac{v_{3}^{2}}{v_{1}^{2}+v_{2}^{2}}\right)+\alpha_{T} \frac{v_{3}^{2}}{v} \frac{v_{1}^{2}}{v_{1}^{2}+v_{2}^{2}} \\
D_{22}=\alpha_{T}^{H} \frac{v_{1}^{2}}{v}\left(1+\frac{v_{3}^{2}}{v_{1}^{2}+v_{2}^{2}}\right)+\alpha_{L} \frac{v_{2}^{2}}{v}+\alpha_{T} \frac{v_{3}^{2}}{v} \frac{v_{2}^{2}}{v_{1}^{2}+v_{2}^{2}} \\
D_{12}=\left[\begin{array}{c}
\alpha_{L}-\alpha_{T}^{H}\left(1+\frac{v_{3}^{2}}{v_{1}^{2}+v_{2}^{2}}\right)+\alpha_{T} \frac{\left.v_{1}^{2}+v_{2}^{2}\right)}{v_{1}^{2}+v_{2}^{2}}+\alpha_{L} \frac{v_{3}^{2}}{v} \\
\frac{v_{1} v_{2}}{v} \\
D_{13}=\left(\alpha_{L}-\alpha_{T}\right) \frac{v_{1} v_{3}}{v} \\
D_{23}=\left(\alpha_{L}-\alpha_{T}\right) \frac{v_{2} v_{3}}{v}
\end{array}\right.
\end{gathered}
$$

In these relations $\alpha_{T}$ and $\alpha_{L}$ are functions of $\cos \theta$ as given by Equations 22a and 22b.

According to this formulation, the dispersion tensor can be expressed in terms of the four dispersivity coefficients $\alpha_{L}^{V, H}$ and $\alpha_{T}^{V, H}$. By appropriately choosing $\alpha_{4}$, one of the principal axes can always be lined up with the direction of flow. Only through comparison with field data will it be possible to determine the correct form of the coefficients $\alpha_{i}$ in terms of the invariants $\theta$ and $v$.

In principle, at the laboratory scale the coefficients $\alpha_{L}^{H}, \alpha_{L}^{V}, \alpha_{T}^{H}$, and $\alpha_{T}^{V}$ could be estimated by conducting experiments on suitably oriented cores. One approach would be to estimate $\alpha_{L}^{H}$ and $\alpha_{L}^{V}$ by measuring tracer breakthrough curves on a core sample oriented normal and parallel, respectively, to the axis of symmetry. Transverse dispersivities $\alpha_{T}^{H}, \alpha_{T}^{V}$ could then be estimated by performing two-dimensional flow experiments on rock slabs, for example. Such results would be of significant theoretical interest. However, it is well known that dispersivity values 
are a strong function of scale; hence, it is necessary to estimate the dispersivity coefficients at a larger scale using field experiments. In the most general flow situation, the coefficients $\alpha_{1}, \alpha_{2}$, $\alpha_{3}$, and $\alpha_{4}$ could be estimated from field data using a numerical model in conjunction with nonlinear parameter estimation by fitting an observed 3-D plume. If a tracer test can be conducted in a portion of the aquifer where the fluid velocity is unidirectional and horizontal coinciding with the principal axis of the dispersion tensor (as often is the case), the parameter estimation procedure can be simplified considerably. In this case, the dispersion tensor simplifies to

$$
D_{11}=\alpha_{L}^{H} v, D_{22}=\alpha_{T}^{H} v, D_{33}=\alpha_{T}^{V} v
$$

with zero off-diagonal terms.

With appropriately spaced observation wells, the coefficients $\alpha_{L}^{H}, \alpha_{T}^{H}$ and, if enough 3-D information is available, $\alpha_{T}^{V}$ as well, can be estimated. Note that in this particular case, no information is available about $\alpha_{L}^{V}$. To obtain this coefficient, another test would have to be conducted in a portion of the field where the fluid velocity has a significant vertical component. If the spread of a plume can be measured in such a case, then Equations 26a to $26 \mathrm{f}$ can be used to estimate the value of the coefficient $\alpha_{L}^{V}$ and, perhaps, $\alpha_{T}^{V}$. Once the dispersivities are estimated for horizontal and vertical flow, the $\theta$ dependence of the dispersivity coefficients for flow at an angle to the symmetry axis could be tested.

\subsection{Dispersion Tensor Proposed by Burnett and Frind}

Burnett and Frind (1987 [DIRS 130526], Equations 6a to 6f) proposed a dispersion tensor for axisymmetric media, hereafter designated as $D_{B F}$ and referred to as the BF-dispersion tensor. The BF-dispersion tensor involves only three independent parameters. These refer to longitudinal dispersion $\alpha_{L}$, and transverse horizontal $\alpha_{T}^{H}$ and vertical $\alpha_{T}^{V}$ dispersion. Burnett and Frind (1987 [DIRS 130526], Equations 6a to 6f) derived the form of the dispersion tensor from the form for isotropic media to account for different transverse dispersivities in the horizontal and vertical directions as observed in natural stratified media with flow along the bedding plane (Anderson 1979 [DIRS 104397]). For example, Zheng and Bennett (1995 [DIRS 154702], pp. 45 to 46) have used the Burnett-Frind tensor to model dispersion in axisymmetric media. 
Burnett and Frind (1987 [DIRS 130526], Equations 6a to 6f) write the dispersion tensor as a matrix of coefficients in the form:

$$
\bar{D}_{B F}=\left(\begin{array}{ccc}
\alpha_{L} \frac{v_{1}^{2}}{v}+\alpha_{T}^{H} \frac{v_{2}^{2}}{v}+\alpha_{T}^{V} \frac{v_{3}^{2}}{v} & \left(\alpha_{L}-\alpha_{T}^{H}\right) \frac{v_{1} v_{2}}{v} & \left(\alpha_{L}-\alpha_{T}^{V}\right) \frac{v_{1} v_{3}}{v} \\
\left(\alpha_{L}-\alpha_{T}^{H}\right) \frac{v_{2} v_{1}}{v} & \alpha_{T}^{H} \frac{v_{1}^{2}}{v}+\alpha_{L} \frac{v_{2}^{2}}{v}+\alpha_{T}^{V} \frac{v_{3}^{2}}{v} & \left(\alpha_{L}-\alpha_{T}^{V}\right) \frac{v_{2} v_{3}}{v} \\
\left(\alpha_{L}-\alpha_{T}^{V}\right) \frac{v_{3} v_{1}}{v} & \left(\alpha_{L}-\alpha_{T}^{V}\right) \frac{v_{3} v_{2}}{v} & \alpha_{\mathrm{T}}^{\mathrm{V}} \frac{v_{1}^{2}+v_{2}^{2}}{v}+\alpha_{L} \frac{v_{3}^{2}}{v}
\end{array}\right)
$$

The diagonal elements may be written in a form similar to the isotropic case given in Equation 10 as

$$
\begin{gathered}
\left(\bar{D}_{B F}\right)_{11}=\alpha_{T}^{H} v+\left(\alpha_{L}-\alpha_{T}^{H}\right) \frac{v_{1}^{2}}{v}+\left(\alpha_{T}^{V}-\alpha_{T}^{H}\right) \frac{v_{3}^{2}}{v} \\
\left(\bar{D}_{B F}\right)_{22}=\alpha_{T}^{H} v+\left(\alpha_{L}-\alpha_{T}^{H}\right) \frac{v_{2}^{2}}{v}+\left(\alpha_{T}^{V}-\alpha_{T}^{H}\right) \frac{v_{3}^{2}}{v} \\
\left(\bar{D}_{B F}\right)_{33}=\alpha_{T}^{V} v+\left(\alpha_{L}-\alpha_{T}^{V}\right) \frac{v_{3}^{2}}{v}
\end{gathered}
$$

To determine the tensorial properties of the BF-dispersion tensor, it is first noted that $\bar{D}_{B F}$ be interpreted as referring to a particular coordinate system in which the symmetry axis of the medium lies along the z-axis: $\bar{\lambda}=(0,0,1)$. Then $v_{3}$ can be written as the scalar product $v_{3}=v \cos \theta=\bar{\lambda} \cdot \bar{v}$. With this in mind, the matrix $\bar{D}_{B F}$ can be expressed as

$$
\bar{D}_{B F}=\left[\alpha_{T}^{H}+\cos ^{2} \theta\left(\alpha_{T}^{V}-\alpha_{T}^{H}\right)\right] v \bar{I}+\left(\alpha_{L}-\alpha_{T}^{H}\right) \frac{\bar{v} \bar{v}}{v}+\left(\alpha_{T}^{V}-\alpha_{T}^{H}\right) \quad\left\lceil\bar{\lambda} \bar{\lambda}-\frac{\cos \theta}{v}(\bar{\lambda} \bar{v}+\bar{v} \bar{\lambda})\right]
$$

valid for any orientation of the coordinate system relative to the symmetry axis. Accordingly, $\bar{D}_{B F}$ is a tensor by construction. Comparing Equation 30 with Equations 3a, 3b, 5a, 5b, and 5c leads to the following identification of the coefficients $\alpha_{i}$ :

$$
\begin{gathered}
\alpha_{1}=\alpha_{T}^{H}+\cos ^{2} \theta\left(\alpha_{T}^{V}-\alpha_{T}^{H}\right) \\
\alpha_{2}=\alpha_{L}-\alpha_{T}^{H} \\
\alpha_{3}=\alpha_{T}^{V}-\alpha_{T}^{H}
\end{gathered}
$$


and

$$
\alpha_{4}=-2 \cos \theta\left(\alpha_{T}^{V}-\alpha_{T}^{H}\right)=-2 \cos \theta \alpha_{3}
$$

It follows that Equation 3a is satisfied for the BF-dispersion tensor and, thus, both $v$ and $\omega$ are eigenvectors belonging to eigenvalues $\alpha_{L} v$ and $\alpha_{T}^{V} v$, respectively, according to Equations 17a and 17b:

$$
\bar{D}_{B F} \cdot \bar{v}=\alpha_{L} v \bar{v}
$$

and

$$
\bar{D}_{B F} \cdot \bar{\omega}=\alpha_{T}^{V} v \bar{\omega}
$$

The eigenvector $\bar{\zeta}_{3}$, orthogonal to $\bar{v}$ and $\bar{\omega}$, belongs to the eigenvalue $\alpha_{1}$

$$
\bar{D}_{B F} \cdot \bar{\zeta}_{3}=\left[\alpha_{T}^{H}+\cos \theta\left(\alpha_{T}^{V}-\alpha_{T}^{H}\right)\right] v \bar{\zeta}_{3}
$$

There are certain limitations with the form of the BF-dispersion tensor. The relation for the generalized dispersion tensor given in Equation 23, in which $\alpha_{1}$ is independent of the angle $\theta$, differs from that of $\bar{D}_{B F}$ given in Equation 31a, in which $\alpha_{1}$ is a function of $\cos \theta$. Similarly, the generalized relation for $\alpha_{2}$ in Equation 25a is different from that obtained for the BF-dispersion tensor given in Equation 31b. In addition, for the BF-dispersion tensor, there is only one coefficient for longitudinal dispersion, which is, therefore, the same for flow in both the vertical and horizontal directions, i.e., parallel and perpendicular to the axis of symmetry, contrary to what one would expect for nonisotropic porous media.

However, for flow perpendicular to the axis of symmetry, the BF-tensor agrees with the more general form. And for small values of vertical velocity $v_{3}$, the generalized form of the dispersion tensor reduces to the form given by Burnett and Frind (1987 [DIRS 130526], Equations 6a to 6f). Hence, in situations where the axis of symmetry is vertical and the flow fields are horizontal to subhorizontal, as is the case at Yucca Mountain presented in Saturated Zone Site-Scale Flow Model (BSC 2004 [DIRS 170037], Section 6.6), this form of the dispersion tensor is adequate. This is the form that is used in the calculations presented in this report.

\subsubsection{Random-Walk Particle-Tracking Method}

Given a steady-state velocity field generated, for example, for an arbitrary permeability field, a random walk is superimposed on the flow field to describe dispersion and molecular diffusion. The general approach used in particle tracking is to replace the partial differential equation for the solute concentration $C$, generally expressed by Equation 1, with random-walk displacements defined in differential form by the Langevin equation (Gardiner 1997 [DIRS 145116], p. 80):

$$
d x=A(x, t) d t+B(x, t) d W(t)
$$


for position vector $x(t)$. The matrix $A$ represents the deterministic background displacement determined by $\bar{v}$ and, in addition, contains contributions from the dispersion tensor. The displacement matrix $B$ refers to a stochastic random-walk process that incorporates molecular diffusion and dispersion. The differential $d W(t)$ represents a Wiener process describing Brownian motion with the properties:

$$
<d W>=0
$$

and

$$
<d W(t) d W(t)>=I d t
$$

where the angular brackets represent the ensemble mean.

The equivalent Fokker-Plank equation corresponding to the Langevin equation (Equation 33) for the conditional probability $P\left(x, t \mid x_{0}, t_{0}\right)$ is given by (Gardiner 1997 [DIRS 145116], p. 97):

$$
\frac{\partial P}{\partial t}=-\nabla \cdot[A(x, t) P]+\nabla: \nabla\left[\frac{1}{2} B \tilde{B} P\right]
$$

where $\tilde{B}$ represents the transpose matrix. The Fokker-Plank equation may be written in the form of the transport equation by rearranging Equation 36 to obtain:

$$
\frac{\partial P}{\partial t}=-\nabla \cdot\left[\left(A(x, t)-\frac{1}{2} \nabla \cdot B \tilde{B}\right) P\right]+\nabla \cdot\left[\frac{1}{2} B \tilde{B} \nabla P\right]
$$

Comparing this modified Fokker-Plank equation with the continuum-based transport equation given in Equation 1 yields the identifications:

$$
P\left(x, t \mid x_{0}, t_{0}\right)=\frac{N_{A}}{N} C(x, t),
$$

where $N$ represents the number of particles and $N_{A}$ denotes Avogadro's number,

$$
A(x, t)=\vec{v}+\nabla \cdot D
$$

and

$$
\frac{1}{2} B \tilde{B}=D
$$

Therefore, it is necessary to obtain the displacement matrix $B$ based on the dispersion tensor $D$. To do this, the approach used by Tompson et al. (1987 [DIRS 145195], Appendix A) is followed in which a transformation that diagonalizes the dispersion tensor is carried out. By construction, the eigenvectors of the dispersion tensor depend only on the components of the flow velocity but not on the dispersivity values themselves. One eigenvector always points in the direction of the 
flow velocity. The other two eigenvectors are perpendicular to the direction of flow. The eigenvalue problem for $D$ reads:

$$
D e_{\lambda}=\lambda \bar{e}_{\lambda}
$$

with eigenvalue $\lambda$ and eigenvector $\bar{e}_{\lambda}$. Because the dispersion tensor is symmetric (Bear 1972 [DIRS 156269], p. 611), there exists an orthogonal transformation $U$ that diagonalizes $D$ (Tompson et al. 1987 [DIRS 145195], p. 106, Equation A-3):

$$
\tilde{U} \bar{D} U \tilde{U} e_{\lambda}=\lambda \tilde{U} \bar{e}_{\lambda}
$$

where $\tilde{U}$ is the transpose of $\mathrm{U}$, with

$$
\tilde{U} \hat{D} U=D
$$

where $D$ is a diagonal matrix, and $U$ satisfies the relations:

$$
U \tilde{U}=\tilde{U} U=I
$$

Expressing $\hat{D}$ in the form

$$
\hat{D}=Q \tilde{Q}
$$

with $Q$ diagonal, then gives

$$
2 D=2 U \hat{D} \tilde{U}=2 U Q \tilde{Q} \tilde{U}=2 U Q \tilde{U} \tilde{Q}=B \tilde{B}
$$

From this relation it follows that the displacement matrix $B$ is given by Tompson et al. (1987 [DIRS 145195], p. 107, Equation A-10):

$$
B=\sqrt{2} U Q
$$

The implementation of the particle-tracking model requires a finite difference form of Equation 5 at time step $n$, which in this model is given by:

$$
X_{i}^{n}=X_{i}^{n-1}+A_{i} \Delta t+\sqrt{\Delta t} \sum_{j} B_{i j} Z_{j}
$$

with

$$
d W_{j}=Z_{j} \sqrt{\Delta t}
$$


for a time step $\Delta t$, where $Z_{j}$ represents a random number. In matrix notation,

$$
X^{n}=X^{n-1}+A \Delta t+\sqrt{\Delta t} B Z
$$

Sampling $Z$ from a uniform distribution (Tompson et al. 1987 [DIRS 145195], p. 40) leads to the expression:

$$
Z=2 \sqrt{3} Z^{\prime}
$$

with $Z$ occurring with unit probability over the interval $-\frac{1}{2}$ to $\frac{1}{2}$. Then,

$$
<Z>=12<Z^{\prime} Z^{\prime}>=1
$$

since

$$
\left.<Z^{\prime} Z^{\prime}\right\rangle=\int_{-1 / 2}^{1 / 2} Z^{\prime 2} d Z^{\prime}=\frac{1}{12}
$$

The final step in the derivation is to determine the form of the displacement matrix $B$. Tompson et al. (1987 [DIRS 145195]) derived the expression for an isotropic system, but the equivalent derivation for an anisotropic dispersion model was not available and, hence, is given below for an axisymmetric medium (Lichtner et al. 2002 [DIRS 163821], Equation 62). The eigenvalues are distinct, and there exist three unique normalized eigenvectors. The matrix $U$ has the form:

$$
U=\left(\begin{array}{ccc}
\frac{v_{1}}{v} & -\frac{v_{1} v_{3}}{v \sqrt{v_{1}^{2}+v_{2}^{2}}} & -\frac{v_{2}}{\sqrt{v_{1}^{2}+v_{2}^{2}}} \\
\frac{v_{2}}{v} & -\frac{v_{2} v_{3}}{\sqrt{v_{1}^{2}+v_{2}^{2}}} & -\frac{v_{1}}{\sqrt{v_{1}^{2}+v_{2}^{2}}} \\
\frac{v_{3}}{v} & \frac{1}{v} \sqrt{v_{1}^{2}+v_{2}^{2}} & \mathrm{O}
\end{array}\right)
$$

In this case the displacement matrix $B$ is given by

$$
B=\left(\begin{array}{ccc}
\frac{v_{1}}{v} \sqrt{2\left(\alpha_{L} v+D_{0}\right)} & -\frac{v_{1} v_{3} \sqrt{2\left(\alpha_{T} v+D_{0}\right)}}{v \sqrt{v_{1}^{2}+v_{2}^{2}}} & -\frac{v_{2} \sqrt{2\left(\alpha_{T}^{H} v+D_{0}\right)}}{\sqrt{v_{1}^{2}+v_{2}^{2}}} \\
\frac{v_{2}}{v} \sqrt{2\left(\alpha_{L} v+D_{0}\right)} & -\frac{v_{2} v_{3} \sqrt{2\left(\alpha_{T} v+D_{0}\right)}}{v \sqrt{v_{1}^{2}+v_{2}^{2}}} & \frac{v_{1} \sqrt{2\left(\alpha_{T}^{H} v+D_{0}\right)}}{\sqrt{v_{1}^{2}+v_{2}^{2}}} \\
\frac{v_{3}}{v} \sqrt{2\left(\alpha_{L} v+D_{0}\right)} & \sqrt{2 \frac{v_{1}^{2}+v_{2}^{2}}{v^{2}}\left(\alpha_{T} v+D_{0}\right)} & 0
\end{array}\right)
$$


In summary, the particle trajectory is computed by a finite difference technique expressed in Equation 33. The first displacement term of this equation $(A \Delta t)$ is deterministic, with A defined in Equation 39. This expression captures the movement of particles in the streamlines defined by the flow field. The term $\nabla \cdot D \Delta t$ is required to reproduce the transport equation correctly for cases in which there are gradients in velocity or dispersion coefficient. It reduces to zero for uniform flow fields and constant dispersivity. What is retained in this case is transport along the flow streamline governed by the flow field. The second term in Equation 33 is a stochastic random-walk term to simulate dispersion, with the form of the matrix $B$ derived for an anisotropic dispersion coefficient tensor in Equation 55.

Determination of the advection portion of the deterministic term $A \Delta t$ requires that the velocity at the particle location be determined. In this version of the code FEHM, the method is restricted to orthogonal finite-element grids. This simplification means that the control volume associated with each grid point is a brick-shaped element. Velocity interpolation within a cell is then determined quickly and easily using the velocity interpolation scheme first derived by Pollock (1988 [DIRS 101466], Equations 4a to 5c). Using that scheme, the code determines, for a given particle at a given location within the cell, the time required to exit the cell and the location where it leaves. If this time is greater than the time step $\Delta t$, the particle location within the cell is computed. If the time is less than the time step $\Delta t$, the particle is forced to stop at this location and then proceed in another step within the adjoining cell. This process is repeated until the ending time $\Delta t$ is reached. At the end of this time step, the term $\nabla \cdot D \Delta t$ is used to move the particle deterministically to correct for gradients in the dispersion coefficient. A differencing scheme on the finite-element grid using a trilinear interpolation analogous to the method described by LaBolle et al. (1996 [DIRS 105039], pp. 587 to 588) is used to compute these terms, with the modification that the interpolated quantity is the local Darcy flux rather than the fluid velocity. This modification yields smoother results in situations such as those encountered at volcanic rock-alluvium interfaces, where local porosity can change by several orders of magnitude from a node to its neighbor. Finally, the random-walk term is applied (the final term in Equation 33) using the $B$ matrix derived above (Equation 55).

For this method to work properly, the time step must be selected such that, on average, a particle takes several time steps within each cell. In a system with large variations in pore-water velocity due to permeability and porosity differences from cell to cell, the appropriate time step can vary greatly throughout the domain. In FEHM, this factor is accounted for by dynamically determining the characteristic time step in an approach similar to that developed by Wen and Gomez-Hernandez (1996 [DIRS 130510], p. 137). In a given cell, the magnitude of the velocity in the cell is used to scale the time step. The time required to traverse the cell completely in each of the three coordinate directions is computed, and the minimum is determined. Then a user-defined parameter called the Courant factor is multiplied by this minimum time to obtain the time step for the particle within the cell. This approach ensures that several steps are taken by a particle within a cell but minimizes computational time by tailoring each time step to the characteristic velocities within the cells.

Applying the random-walk method on grids and flow fields, it was found that the theoretically simple inclusion of the $\nabla \cdot D$ term to correct for velocity gradients may not be sufficient to account for regions with highly variable velocity fields. In short, computation of $\nabla \cdot D$ on the scale of the finite-element grid may not be sufficient to capture the magnitude of this term 
adequately. For example, in high-permeability zones immediately adjacent to confining units of low permeability, the gradient is not captured sufficiently accurately to prevent the artificial meandering of a small number of particles into the low-permeability region. As a result, some particles are held up for an unrealistically long time in these zones, resulting in a nonconservative tailing of the solute breakthrough curve at a downstream location. To correct this problem, a user-defined velocity-scaling parameter can be defined to prohibit particles from entering the low-velocity domain by random-walk processes. If the ratio of the velocity before and after the random-walk jump is less than this parameter, the code prohibits the jump, and the particle is returned to the original position where another jump is taken with a different set of random numbers. This simple correction serves the same purpose as the $\nabla \cdot D$ term but is more foolproof in maintaining a physically meaningful set of random-walk jumps.

The particle tracking method presented here, as discussed in Lichtner et al. (2002 [DIRS 163821]), has the advantage that solute mass is automatically conserved as long the number of particles is tracked in the numerical implementation, and the method is free from numerical convergence and numerical dispersion problems encountered by the methods that use the approach of solving discretized forms of the advection dispersion equation (Equation 1). Further, The boundary conditions described in Section 6.3, item 9 are easily implemented by removing from further computations any particles that reach a model boundary.

To report the results of a particle-tracking simulation, two options are available. The first requires the definition of a zone consisting of a set of finite-element grid points representing a portion of the model domain where transport results are desired. For example, a "boundary of the accessible environment" compliance boundary, which is a given distance from the repository, can be defined by listing all of the nodes in the boundary. Then the code determines the first arrival time of each particle at any node in this fence and reports the cumulative arrival time distribution for all particles. This arrival-time distribution can then be converted to a ground water concentration using a representative volume of 3000 acre-feet in accordance with 10 CFR 63.312 (DIRS 156605), and the resulting curve can be used as the input to the performance assessment analysis. Alternatively, the concentration of particles at any cell in the finite-element domain can be reported as the number of particles residing in the cell divided by the fluid mass in the cell. Concentrations computed in this way represent the in situ concentration in response to the injection of a pulse of solute at time zero. To obtain the cumulative breakthrough curve, we may perform a time integration of these results, yielding the in situ concentration breakthrough curve at the node in response to a step change in concentration. Breakthrough curves for both pulse and step response curves can be obtained in the FEHM particle-tracking code.

\subsubsection{Matrix Diffusion in Fractured Geological Media}

To incorporate the influence of sorption and matrix diffusion, the residence time transfer function particle-tracking method outlined in the FEHM models and methods document (Zyvoloski et al. 1997 [DIRS 110491], pp. 41 to 42) has been adapted to the particle-tracking algorithm. In this method, adjustments to the transport time of a particle are made to account for the influence of physicochemical processes such as sorption and matrix diffusion. During its path along a streamline, the particle transport time is governed by a transfer function describing the probability of the particle spending a given length of time on that portion of its path. For a 
cumulative probability distribution function of particle residence times, the transport time of a particle along this portion of its path is computed by generating a random number between 0 and 1 and determining the corresponding residence time. On average, if a large number of particles travel through this portion of the model domain, the cumulative residence time distribution of particles will reproduce the shape of the transfer function. The form of the transfer function is derived from an analytical or numerical solution to capture the appropriate processes being considered. A suite of type curves were generated for numerically implementing the transfer function.

\subsection{Mathematical Description of Matrix Diffusion and Sorption}

In this particle-tracking algorithm, the schematic model depicted in Figure 6.4-1 is used to provide a transfer function for the case of fracture flow and diffusion between equally spaced fractures. In this model:

$\begin{array}{ll}z & \text { is the spatial coordinate along the fracture } \\ t & \text { is the time } \\ 2 b & \text { is the fracture aperture } \\ 2 B & \text { is the mean fracture spacing } \\ v & \text { is the linear groundwater velocity in the fracture } \\ \theta & \text { is the porosity of the matrix } \\ \mathrm{q} & \text { is the diffusive flux from fracture to matrix } \\ K_{d} & \text { is the distribution coefficient in the fracture } \\ k_{d}^{\prime} & \text { is the distribution coefficient in the matrix } \\ R^{\prime} & \text { is the retardation factor in the matrix } \\ R & \text { is the retardation factor in the fracture } \\ \rho_{b} & \text { is the bulk density of the matrix } \\ \rho_{w} & \text { is the density of water } \\ D^{\prime} & \text { is the matrix effective diffusion coefficient } \\ D & \text { is the dispersion coefficient in the fracture } \\ c & \text { is the concentration at } z \text { along the fracture } \\ c_{0} & \text { is the source concentration at } z=z_{0} .\end{array}$

The equations describing the radionuclide transport in the fractures and matrix are (Robinson 1994 [DIRS 101154], p. 81, Equations 1 and 2):

$$
\frac{\partial c}{\partial t}+\frac{v}{R} \frac{\partial c}{\partial z}-\frac{D}{R} \frac{\partial^{2} c}{\partial z^{2}}+\frac{q}{b R}=0
$$

and in the matrix:

$$
\frac{\partial c}{\partial t}-\frac{D^{\prime}}{R^{\prime}} \frac{\partial^{2} c}{\partial x^{2}}=0
$$




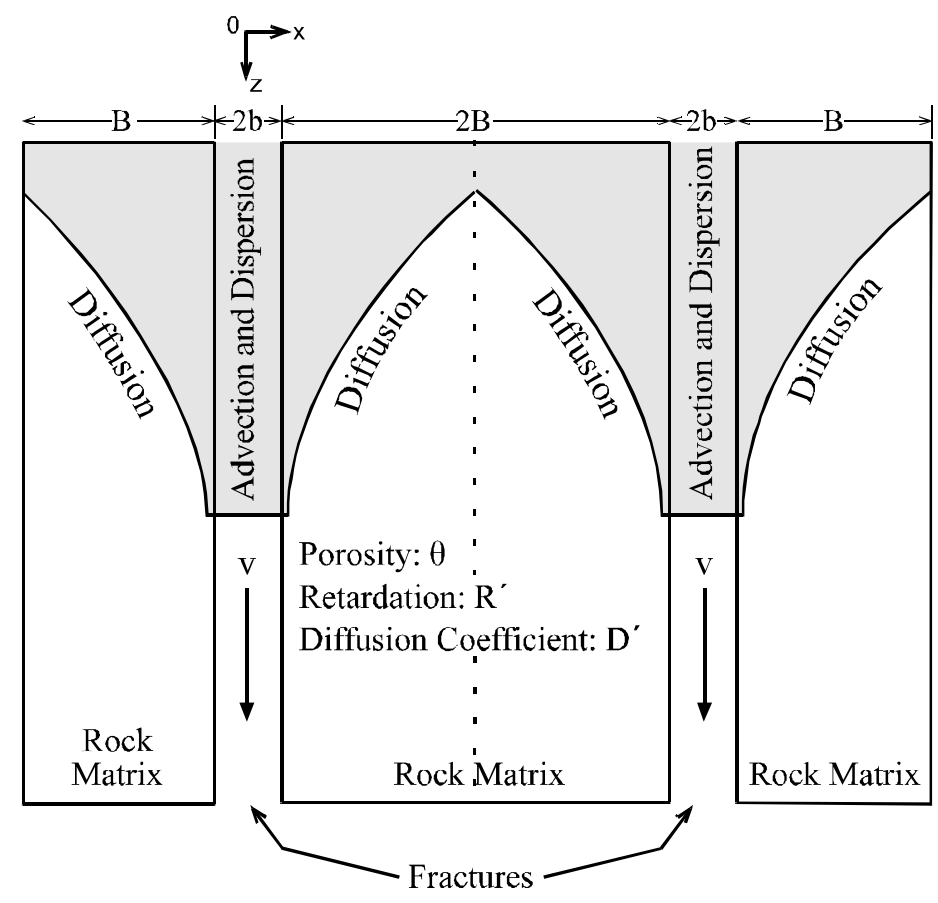

Figure 6.4-1. Schematic of the Matrix Diffusion Submodel

The distribution coefficient and the retardation factor are related by Freeze and Cherry (1979 [DIRS 101173], p. 404, Equation 9.14):

$$
R^{\prime}=1+\frac{\rho_{b}}{\theta} k_{d}^{\prime}
$$

The transient solution for contaminant transport with $D=0$ in parallel fractures, for $c=c_{0}$ at the inlet end with $z=0$, and $\partial c / \partial x=0$ at the center line between the fractures at $x=B$ (Figure 6.4-1) is given by Sudicky and Frind (1982 [DIRS 105043], p. 1637, Equation 28):

$$
\begin{gathered}
\frac{c}{c_{0}}=0, T^{0} \leq 0 \\
\frac{c}{c_{0}}=\frac{1}{\pi} \exp \left(\frac{R \lambda z}{v}\right) \int_{0}^{\infty} \frac{\varepsilon}{\lambda^{2}+\varepsilon^{4} / 4} \exp \left(\varepsilon_{R}{ }^{0}\right)\left[\exp \left(-\lambda T^{0}\right)\left\{\frac{\varepsilon^{2}}{2} \sin \left(\varepsilon_{I}{ }^{0}\right)-\lambda \cos \left(\varepsilon_{I}{ }^{0}\right)\right\}\right. \\
\left.+\frac{\varepsilon^{2}}{2} \sin \left(\Omega_{I}{ }^{0}\right)+\lambda \cos \left(\Omega_{I}{ }^{0}\right)\right] d \varepsilon, \quad T^{0}>0
\end{gathered}
$$

where $\lambda$ is a first-order decay constant, $\varepsilon$ is an integration variable, and

$$
T^{0}=t-\frac{R z}{v}
$$




$$
\begin{gathered}
\varepsilon_{R}{ }^{0}=-\frac{\omega \varepsilon}{2}\left(\frac{\sinh (\sigma \varepsilon)-\sin (\sigma \varepsilon)}{\cosh (\sigma \varepsilon)+\cos (\sigma \varepsilon)}\right) \\
\varepsilon_{I}{ }^{0}=\frac{\varepsilon^{2} T^{0}}{2}-\frac{\omega \varepsilon}{2}\left(\frac{\sinh (\sigma \varepsilon)+\sin (\sigma \varepsilon)}{\cosh (\sigma \varepsilon)+\cos (\sigma \varepsilon)}\right) \\
\Omega_{I}{ }^{0}=\frac{\omega \varepsilon}{2}\left(\frac{\sinh (\sigma \varepsilon)+\sin (\sigma \varepsilon)}{\cosh (\sigma \varepsilon)+\cos (\sigma \varepsilon)}\right)
\end{gathered}
$$

with

$$
\begin{gathered}
\omega=\frac{\theta\left(R^{\prime} D^{\prime}\right)^{1 / 2} z}{b v} \\
\sigma=\left(R^{\prime} / D^{\prime}\right)^{1 / 2}(B-b)
\end{gathered}
$$

Using the transformation variables:

$$
\begin{gathered}
\tau_{0}=\frac{Z}{v} \\
\varepsilon_{1}=\varepsilon \tau_{0}^{1 / 2}
\end{gathered}
$$

Equations 58 through 65 can be rewritten as:

$$
\begin{gathered}
\frac{c}{c_{0}}=0, T_{1}^{0} \leq 0 \\
\frac{c}{c_{0}}=\frac{1}{\pi} \exp \left(R \lambda \tau_{0}\right) \int_{0}^{\infty} \frac{\varepsilon_{1}}{\lambda^{2} \tau_{0}^{2}+\varepsilon_{1}{ }^{4} / 4} \exp \left(\varepsilon_{R}{ }^{0}\right)\left[\exp \left(-\lambda \tau_{0} T_{1}^{0}\right)\left\{\frac{\varepsilon_{1}^{2}}{2} \sin \left(\varepsilon_{I}{ }^{0}\right)-\lambda \tau_{0} \cos \left(\varepsilon_{I}{ }^{0}\right)\right\}\right. \\
\left.+\frac{\varepsilon_{1}^{2}}{2} \sin \left(\Omega_{I}{ }^{0}\right)+\lambda \tau_{0} \cos \left(\Omega_{I}{ }^{0}\right)\right] d \varepsilon_{1}, \quad T_{1}^{0}>0
\end{gathered}
$$

where

$$
\begin{gathered}
T_{1}^{0}=\frac{t}{\tau_{0}}-R \\
\varepsilon_{R}^{0}=-\frac{\omega_{1} \varepsilon_{1}}{2}\left(\frac{\sinh \left(\sigma_{1} \varepsilon_{1}\right)-\sin \left(\sigma_{1} \varepsilon_{1}\right)}{\cosh \left(\sigma_{1} \varepsilon_{1}\right)+\cos \left(\sigma_{1} \varepsilon_{1}\right)}\right)
\end{gathered}
$$




$$
\begin{gathered}
\varepsilon_{I}^{0}=\frac{\varepsilon_{1}^{2} T_{1}^{0}}{2}-\frac{\omega_{1} \varepsilon_{1}}{2}\left(\frac{\sinh \left(\sigma_{1} \varepsilon_{1}\right)+\sin \left(\sigma_{1} \varepsilon_{1}\right)}{\cosh \left(\sigma_{1} \varepsilon_{1}\right)+\cos \left(\sigma_{1} \varepsilon_{1}\right)}\right) \\
\Omega_{I}{ }^{0}=\frac{\omega_{1} \varepsilon_{1}}{2}\left(\frac{\sinh \left(\sigma_{1} \varepsilon_{1}\right)+\sin \left(\sigma_{1} \varepsilon_{1}\right)}{\cosh \left(\sigma_{1} \varepsilon_{1}\right)+\cos \left(\sigma_{1} \varepsilon_{1}\right)}\right)
\end{gathered}
$$

with

$$
\begin{gathered}
\omega_{1}=\frac{\theta\left(R^{\prime} D^{\prime} \tau_{0}\right)^{1 / 2}}{b} \\
\sigma_{1}=\left(\frac{R^{\prime}}{D^{\prime} \tau_{0}}\right)^{1 / 2}(B-b)
\end{gathered}
$$

For the case of no radioactive decay $(\lambda=0)$ :

$$
\frac{c}{c_{0}}=\frac{1}{\pi} \int_{0}^{\infty} \frac{2}{\varepsilon_{1}} \exp \left(\varepsilon_{R}{ }^{0}\right)\left[\sin \left(\varepsilon_{I}{ }^{0}\right)+\sin \left(\Omega_{I}{ }^{0}\right)\right] d \varepsilon_{1}
$$

Therefore, to implement this model, the algorithm requires the input of transport parameters defined in Equations 74 and 75. With the transport parameters and the unretarded transport time $\tau_{0}$ within a given portion of the path known from the advection part of the particle, values of $\omega_{1}$ and $\sigma_{1}$ are computed, thereby fully defining the transfer function for this portion of the particle's travel path. Given these parameters, the model returns a value of the delayed transport time of the particle from Equation 70 that is consistent with the matrix diffusion model. To implement this model in FEHM, a series of type curves were generated (Output DTN: LA0302RP831228.001) at specified values of $\omega_{1}$ and $\sigma_{1}$ using a code FRACT_p V1.0 (FRACT_p V1.0, STN: 11009-1.0-00) (LANL 2003 [DIRS 164509]). For given values of the parameters, the code performs a linear interpolation between the nearest type curves to obtain the result. This approach of tabulating the results of the analytical solution is much more computationally efficient than computing the values through integration at run time. Under limiting conditions of low diffusion and/or large fracture spacing, the infinite spacing solution of Tang et al. (1981 [DIRS 101160], p. 559, Eq. 35) implemented in the cell-based particle-tracking algorithm of FEHM can be used instead of the finite spacing model. A provision in the code allows the Tang solution to be invoked in this particle-tracking model as well, but it should be used only when the characteristic diffusion time to the centerline between the fractures (of order $\left.B^{2} / D^{\prime}\right)$ is much greater than the time of the simulation.

The final step of the model development is to integrate the matrix diffusion model with the random-walk transport model developed in Section 6.4.2.3. Specifically, the time intervals over which the time delays are applied must be set in a manner that allows for computationally efficient and accurate solutions to be obtained. In this model, the time delay is applied to a particle at the time at which it exits a cell, after having determined the cumulative time the particle spent in advective transport through the cell. Within a cell, the transport properties of 
diffusion and sorption are, by definition, uniform, so that a unique set of transport dimensionless parameters can be defined. Alternatively, the time delay could be applied at each segment of the particle path, resulting in potentially many time delays for a particle within each cell as it is transported by advection and random-walk dispersion. However, in initial prototype testing using this approach, it was determined that the technique, although theoretically equivalent to the application of time delay once per cell, showed that practical limitations of reduced accuracy and reduced computational efficiency resulted. Therefore, the code was developed with the time delay applied only at the time the particle exits the cell, which can occur either by advection or by random-walk dispersion.

To apply the time delay, the particle is held at that location until the time the simulation run catches up to the time of that particle, after which the particle is allowed to resume its transport. Finally, it is noted that for sorption without matrix diffusion, the time delay is computed deterministically by computing a retardation factor based on the sorption coefficient $K_{d}$, but otherwise, the method is identical to the matrix-diffusion method. Alternatively, this particular case could have been handled through a simple adjustment of the transport velocity, but the implementation using the time-delay method was simpler because it is consistent with the matrix-diffusion method just described.

\subsubsection{Mathematical Description of Sorptive Transport in Alluvium}

Equation 1 for advective-dispersive transport can be generalized to include sorption onto the rock surfaces as follows (Freeze and Cherry 1979 [DIRS 101173], Equations 9.9 and A10.14):

$$
\frac{\partial C}{\partial t}+\nabla \cdot(\vec{v} C)-\nabla \cdot(D \nabla C)=\frac{\rho_{b}}{\theta} \frac{\partial S}{\partial t}
$$

where $S$ is the mass of the transported species adsorbed on the solid per unit bulk dry mass of the porous medium. For linear, reversible, equilibrium sorption, the $K_{d}$ model can be used, and $S$ and $C$ are related as follows (Freeze and Cherry 1979 [DIRS 101173], Equation 9.12):

$$
S=K_{\mathrm{d}} C
$$

\subsubsection{Colloid-Facilitated Transport}

Radionuclides can attach to the colloids either reversibly or irreversibly. The radionuclides that are attached to the colloids reversibly are partitioned between the colloids and the aqueous phase. On the other hand, the radionuclides that are irreversibly attached to the colloids stay attached to the particles for the entire duration of the transit through the SZ to the boundary of the accessible environment at $18 \mathrm{~km}$. The colloid-facilitated transport of the radionuclides follows different mechanisms for the two different types of attachments, which are summarized in Table 6.4-1 and described below. 
Table 6.4-1. Colloid-Facilitated Transport of Radionuclides

\begin{tabular}{|c|c|c|}
\hline $\begin{array}{l}\text { Radionuclides Attached Reversibly to } \\
\text { Colloids }\end{array}$ & \multicolumn{2}{|c|}{$\begin{array}{l}\text { Radionuclides Attached Irreversibly to Colloids } \\
\text { (Transport in a Manner Identical to Colloids) }\end{array}$} \\
\hline \multirow[t]{2}{*}{$\begin{array}{l}\text { Radionuclides spend part of the time } \\
\text { attached to the colloids and part of the } \\
\text { time in the aqueous phase. }\end{array}$} & \multicolumn{2}{|c|}{$\begin{array}{l}\text { Radionuclides are embedded in the colloids, mostly those derived from } \\
\text { the waste form degradation. This is discussed in Saturated Zone Colloid } \\
\text { Transport (BSC } 2004 \text { [DIRS 170006], Sections } 6.3 \text { and 6.6). The } \\
\text { radionuclides travel in a manner identical to the colloids. }\end{array}$} \\
\hline & $\begin{array}{l}\text { Normal (Fraction that Undergoes } \\
\text { Reversible Filtration) }\end{array}$ & $\begin{array}{l}\text { Fast (Fraction That Does Not } \\
\text { Undergo Reversible Filtration) }\end{array}$ \\
\hline $\begin{array}{l}\text { Radionuclides are treated in the site- } \\
\text { scale SZ transport model using } \\
\text { equations similar to those for aqueous } \\
\text { phase transport (Appendix B) but with } \\
\text { modified parameters. } \\
\text { The diffusion coefficient in volcanics is } \\
\text { reduced with respect to that for aqueous } \\
\text { transport. This is given by Equation } 80 a \\
\text { in Section } 6.4 .2 .6 \text {. The equation is } \\
\text { derived in Saturated Zone Flow and } \\
\text { Transport Model Abstraction (BSC } 2004 \\
\text { [DIRS 170042], Section 6.5.1.1). The } \\
\text { sorption coefficient is modified and given } \\
\text { in Equation } 81, \text { Section } 6.4 .2 .6 \text { and } \\
\text { derived in Saturated Zone Flow and } \\
\text { Transport Model Abstraction (BSC } \\
\text { (2004 [DIRS } 170042 \text { ] Section } 6.5 .1 .1 \text { ). } \\
K_{c} \text { needed in Equation } 80 \text { a and } 81 \text { is } \\
\text { given in Equation } 80 \text { b. } \\
\text { The groundwater concentration of } \\
\text { colloids and the sorption coefficient onto } \\
\text { colloids are summarized in Table } 4-2 \\
\text { and taken from Waste Form and In-Drift } \\
\text { Colloids-Associated Radionuclide } \\
\text { Concentrations: Abstraction and } \\
\text { Summary (BSC } 2004 \text { [DIRS } 170025 \text { ], } \\
\text { Section 6.3.1). } \\
\text { Section } 7.1 .2 .5 .2 \text { gives } \\
\text { confidence-building arguments for this } \\
\text { process based on laboratory data and } \\
\text { theoretical considerations from } \\
\text { literature. } \\
\text { The transport of these radionuclides is } \\
\text { simulated in the site-scale SZ transport } \\
\text { model using the same approach as the } \\
\text { aqueous species but with modified } \\
\text { diffusion coefficient and } K_{d} \text { as described } \\
\text { above. }\end{array}$ & $\begin{array}{l}\text { The colloids undergo "reversible } \\
\text { filtration." This is discussed in } \\
\text { Saturated Zone Colloid Transport } \\
\text { (BSC } 2004 \text { [DIRS 170006], Section } \\
\text { 6.4) where field and laboratory data } \\
\text { are analyzed to get "attachment } \\
\text { rate constants" and "detachment } \\
\text { rate constants." These lead to a } \\
\text { retardation factor for the colloids } \\
\text { (Equation 79). } \\
\text { The same retardation factor applies } \\
\text { to the fraction of radionuclides } \\
\text { absorbed irreversibly onto colloids. } \\
\text { The range of values for this fraction } \\
\text { is given in Waste Form and In-Drift } \\
\text { Colloids-Associated Radionuclide } \\
\text { Concentrations: Abstraction and } \\
\text { Summary BSC } 2004 \\
\text { [DIRS } 170025 \text { ], Section 6.3). } \\
\text { Section 7.1.2.5.1 gives } \\
\text { confidence-building arguments for } \\
\text { "colloid filtration" based on C-wells } \\
\text { data. } \\
\text { The transport of these } \\
\text { radionuclides is simulated in the } \\
\text { site-scale SZ transport model using } \\
\text { the same approach as the aqueous } \\
\text { species but with zero diffusion } \\
\text { coefficient and colloid retardation } \\
\text { factor as explained above. }\end{array}$ & $\begin{array}{l}\text { A small fraction of colloids travels } \\
\text { with the groundwater without any } \\
\text { retardation. The radionuclides } \\
\text { sorbed onto this fraction also travel } \\
\text { without any retardation. The } \\
\text { transport times for this fraction are } \\
\text { the same as those for nonsorbing } \\
\text { radionuclides. } \\
\text { The range of values of this fraction } \\
\text { is given in Table } 4-2 \text { and discussed } \\
\text { in Waste Form and In-Drift } \\
\text { Colloids-Associated Radionuclide } \\
\text { Concentrations: Abstraction and } \\
\text { Summary (BSC } 2004 \\
\text { [DIRS } 170025], \text { Section } 6.3 \text { ). } \\
\text { Section 7.1.1.1.3 gives } \\
\text { confidence-building arguments for } \\
\text { the occurrence of this process } \\
\text { based on NTS data. } \\
\text { The breakthrough curves for these } \\
\text { radionuclides are identical to those } \\
\text { without sorption. }\end{array}$ \\
\hline
\end{tabular}

\subsection{Radionuclides Attached Irreversibly to the Colloids}

These radionuclides are embedded in the colloids and travel in a manner identical to the colloids themselves. The majority of the colloid particles undergo filtration during transport through the SZ; however, a small fraction travels with the movement of the bulk water without any retardation. The transport of colloid particles is included in the site-scale SZ transport model using the process of reversible filtration shown in Saturated Zone Colloid Transport (BSC 2004 
[DIRS 170006] Sections 6.4 and 6.5). The transport of the colloids is simulated using the advection-dispersion equation, and colloids are taken not to diffuse. Filtration of the colloids can be described by a retardation factor, $R_{c o l}$. In this analysis, filtration is defined as the net effect of chemical sorption of the colloid onto the rock surface and the physical removal of colloids from the advective flow due to sieving and settling. The value of $R_{\text {col }}$ is dependent on several factors such as colloid size, colloid type, and geochemical conditions (e.g., pH, Eh, and ionic strength) as shown in Saturated Zone Colloid Transport (BSC 2004 [DIRS 170006], Section 6.3). These factors are folded into the distribution of $R_{\text {col }}$ that has been developed from field and experimental data collected under varying geochemical conditions with different colloid types and sizes as discussed in Saturated Zone Colloid Transport (BSC 2004 [DIRS 170006], Tables 6-2 and 6-3). Attachment rate constants, $K_{\text {att }}$, and detachment rate constants, $K_{\text {det }}$, of colloids to the rock matrix have been measured, and $R_{\text {col }}$ distributions have been developed for the fractured volcanics and alluvium. The relationship between $R_{\text {col }}, K_{\text {att }}$,and $K_{\text {det }}$ is given by (BSC 2004 [DIRS 170006] Equation 6-3):

$$
R_{\text {col }}=1+\frac{K_{\mathrm{att}}}{K_{\mathrm{det}}}
$$

The attachment rate constant is also used to determine the fraction of the colloids that transport with no retardation. Specifically, colloids for which one over the attachment rate constant is smaller than the transport time through the system will transport with no retardation. The fraction of colloids that transport unretarded is documented in Saturated Zone Colloid Transport (BSC 2004 [DIRS 170006], Table 6-4).

\subsection{Radionuclides Attached Reversibly to the Colloids}

Radionuclides cannot diffuse into the matrix while attached to colloids because the colloid particles themselves cannot diffuse into the matrix (Section 6.3). Hence, the fraction of radionuclides that are attached irreversibly onto colloids does not experience any matrix diffusion and is transported at the same rate as the colloid particles. The fraction of radionuclides that are reversibly attached onto the colloids experiences a reduction in the diffusion process because this fraction can diffuse into the matrix only while unattached to the colloids. In the volcanics, this is implemented through a reduction in the effective diffusion coefficient for the radionuclides attached reversibly to colloids, given by the following equation given in Saturated Zone Flow and Transport Model Abstraction (BSC 2004 [DIRS 170042], Equation 6-9):

$$
D_{e}^{\text {adjusted }}=\frac{D_{e}}{\left(1+K_{c}\right)^{2}}
$$


where

$D_{e}^{\text {adjusted }}$ is the effective diffusion coefficient of the radionuclide adjusted for the effect of reversible attachment to colloids,

$D_{e}$ is the effective diffusion coefficient of the radionuclide in the matrix, and

$K_{\mathrm{c}}$ is the distribution parameter expressing the relative amount of radionuclide residing on the colloids with respect to that in the aqueous phase, given by (Appendix B of this report):

$$
K_{c}=C_{c} * K_{d-c}
$$

where

$C_{c}$ is the colloid concentration in the groundwater, and

$K_{d-c}$ is the sorption coefficient for the radionuclide onto the colloids.

In the alluvium, diffusion is not an issue; however, the sorption coefficient for the radionuclide onto the rock surface is modified due to the competition with the colloids as follows, shown in Saturated Zone Flow and Transport Model Abstraction (BSC 2004 [DIRS 170042], Section 6.5.1.1, Equation 6-5):

$$
K_{d}^{\text {new }}=\frac{K_{d}^{\text {original }}}{\left(1+K_{c}\right)}
$$

where

$K_{d}^{\text {original }}$ is the sorption coefficient for the radionuclide in the alluvium in the absence of colloids, and

$K_{d}^{\text {new }}$ is the sorption coefficient for the radionuclide in the alluvium in the presence of reversible attachment to colloids.

\subsubsection{Base-Case Model Inputs}

The base-case flow model, Saturated Zone Site-Scale Flow Model, (BSC 2004 [DIRS 170037], Section 6.6; DTN: LA0304TM831231.002 [DIRS 163788]) was used directly as an input to the base-case transport model. The flow model provides the numerical grid with geometric coefficients and the groundwater flow velocity field, which are used by the transport model. Modifications were made to the input file to include base-case transport parameter values. These modifications do not impact the results of the flow calculations. The parameters modified are rock bulk density, rock bulk porosity, effective porosity in alluvium, flowing-interval porosity, matrix porosity in volcanics, effective diffusion coefficient in the volcanic matrix, and flowing-interval spacing in volcanics. Table 6.4-2 provides a list of input 
parameters for the base-case transport model. This table gives the parameter name, its description and intended use, base-case value, type of uncertainty and the source DTN. Additional parameters that are not included in the base-case model but are needed for the flow and transport abstractions analysis (BSC 2004 [DIRS 170042]) and subsequent feed to the TSPA-LA are listed in Table 6.4-3. The values of all of these parameters involve uncertainties. For each parameter, the rationale for the selection of the range of value, probability distribution, and expected value is summarized in Section 4.1.2 and details are given in Appendix A and in Saturated Zone Flow and Transport Model Abstraction (BSC 2004 [DIRS 170042] Section 6.5.2). The transport base case reported in this section focuses on providing, as output, a single case of the transport model calculations. The ranges of input uncertainties are listed in Table 4-2. For most parameters, the base-case value was chosen to be the median of the uncertainty distribution, except for the sorption coefficient, which was assigned a value of 0 for the base case, which leads to faster transit times for the base case than the stochastic simulations. The propagation of the uncertainties to the output breakthrough curves (Output DTN: LA0309SK831231.001) is documented in Section 6.7.

Table 6.4-2. Base-Case Model Inputs

\begin{tabular}{|c|c|c|c|c|c|}
\hline $\begin{array}{c}\text { Input Name } \\
\text { (Name of the Variable } \\
\text { in the FEHM V } 2.20 \\
\text { Code) }\end{array}$ & $\begin{array}{l}\text { Input Description and } \\
\text { Intended Use }\end{array}$ & $\begin{array}{c}\text { Base-Case } \\
\text { Value }\end{array}$ & Units & $\begin{array}{c}\text { Type of } \\
\text { Uncertainty }\end{array}$ & Source/DTN \\
\hline $\begin{array}{l}\text { DENRD in the control } \\
\text { statement "rock" }\end{array}$ & $\begin{array}{l}\text { Bulk density in alluvium } \\
\text { needed for retardation } \\
\text { calculations in Equations } \\
76 \text { and } 77 .\end{array}$ & 1,910 & $\mathrm{~kg} / \mathrm{m}^{3}$ & Epistemic $^{a}$ & $\begin{array}{l}\text { Saturated Zone Flow } \\
\text { and Transport Model } \\
\text { Abstraction (BSC } 2004 \\
\text { [DIRS 170042) } \\
\text { SN0310T0502103.009 } \\
\text { [DIRS 168763] }\end{array}$ \\
\hline $\begin{array}{l}K_{d} \text { in the control } \\
\text { statement "sptr" }\end{array}$ & $\begin{array}{l}\text { Sorption coefficient in } \\
\text { alluvium needed for } \\
\text { retardation calculations } \\
\text { in Equations } 77 \text { and } 78 .\end{array}$ & 0.0 & $\mathrm{~mL} / \mathrm{g}$ & Epistemic & $\begin{array}{l}\text { Appendix A. } \\
\text { Output DTN: } \\
\text { LA0310AM831341.002 }\end{array}$ \\
\hline $\begin{array}{l}\text { PSD in the control } \\
\text { statement "rock" }\end{array}$ & $\begin{array}{l}\text { Effective porosity in the } \\
\text { alluvium needed for } \\
\text { converting Darcy flux to } \\
\text { fluid velocity and } \\
\text { retardation calculations } \\
\text { in Equations } 77 \text { and } 78 \text {. }\end{array}$ & 0.18 & - & Epistemic & $\begin{array}{l}\text { Saturated Zone Flow } \\
\text { and Transport Model } \\
\text { Abstraction BSC } 2004 \\
\text { [DIRS 170042]) } \\
\text { SN0310T0502103.009 } \\
\text { [DIRS 168763] }\end{array}$ \\
\hline $\begin{array}{l}\text { Enters indirectly via } \\
\text { the Flowing Interval } \\
\text { Aperture through the } \\
\text { relation }^{b} \\
\text { Porosity = } \\
\text { (Aperture/Spacing) }\end{array}$ & $\begin{array}{l}\text { Flowing interval porosity } \\
\text { needed for converting } \\
\text { Darcy flux to fluid velocity } \\
\text { and retardation } \\
\text { calculations in Equations } \\
56 \mathrm{a} \text { and 56b. }\end{array}$ & 0.01 & - & Epistemic & $\begin{array}{l}\text { Saturated Zone Flow } \\
\text { and Transport Model } \\
\text { Abstraction (BSC } 2004 \\
\text { [DIRS 170042]) }\end{array}$ \\
\hline $\begin{array}{l}\text { Enters indirectly via } \\
\text { the Flowing Interval } \\
\text { Aperture through the } \\
\text { relation }^{\text {b }} \\
\text { Porosity = } \\
\text { (Aperture/Spacing) }\end{array}$ & $\begin{array}{l}\text { Flowing interval spacing } \\
\text { needed for converting } \\
\text { Darcy flux to fluid velocity } \\
\text { and retardation } \\
\text { calculations in Equations } \\
56 \mathrm{a} \text { and 56b. }\end{array}$ & 20 & $\mathrm{~m}$ & Epistemic & $\begin{array}{l}\text { SN0310T0502103.009 } \\
\text { [DIRS 168763] }\end{array}$ \\
\hline
\end{tabular}


Table 6.4-2. Base-Case Model Inputs (Continued)

\begin{tabular}{|c|c|c|c|c|c|}
\hline $\begin{array}{c}\text { Input Name } \\
\text { (Name of the Variable } \\
\text { in the FEHM V } 2.20 \\
\text { Code) }\end{array}$ & $\begin{array}{l}\text { Input Description and } \\
\text { Intended Use }\end{array}$ & $\begin{array}{c}\text { Base-Case } \\
\text { Value }\end{array}$ & Units & $\begin{array}{c}\text { Type of } \\
\text { Uncertainty }\end{array}$ & Source/DTN \\
\hline $\begin{array}{l}\text { APERTURE in the } \\
\text { control statement } \\
\text { "sptr"b }\end{array}$ & $\begin{array}{l}\text { Flowing interval aperture } \\
\text { needed for converting } \\
\text { Darcy flux to fluid velocity } \\
\text { and retardation } \\
\text { calculations in Equations } \\
56 \text { a and 56b. }\end{array}$ & 0.2 & $\mathrm{~m}$ & Epistemic & $\begin{array}{l}\text { Obtained as the } \\
\text { product of flowing } \\
\text { interval porosity and } \\
\text { flowing interval } \\
\text { spacing given this } \\
\text { table. }\end{array}$ \\
\hline $\begin{array}{l}\text { POR_MATRIX in the } \\
\text { control statement } \\
\text { "sptr," fraction }\end{array}$ & $\begin{array}{l}\text { Matrix porosity in } \\
\text { volcanics, needed for } \\
\text { diffusion and retardation } \\
\text { calculations in Equations } \\
56 \mathrm{~b} \text { and } 57 .\end{array}$ & $0.15-0.25$ & - & Epistemic & $\begin{array}{l}\text { Saturated Zone Flow } \\
\text { and Transport Model } \\
\text { Abstraction (BSC } \\
\text { 2004 [DIRS 170042]) } \\
\text { SN0306T0502103.007 } \\
\text { [DIRS 168763] }\end{array}$ \\
\hline $\begin{array}{l}\text { DIFM in the control } \\
\text { statement "sptr" }\end{array}$ & $\begin{array}{l}\text { Effective diffusion } \\
\text { coefficient in volcanics, } \\
\text { needed for diffusion } \\
\text { calculations in Equation } \\
57 \mathrm{~b} \text {. }\end{array}$ & $5.0 \times 10^{-11}$ & $\mathrm{~m}^{2} / \mathrm{s}$ & Epistemic & $\begin{array}{l}\text { Saturated Zone Flow } \\
\text { and Transport Model } \\
\text { Abstraction (BSC 2004 } \\
\text { [DIRS 170042]) } \\
\text { SNO310T0502103.009 } \\
\text { [DIRS 168763] }\end{array}$ \\
\hline $\begin{array}{l}K_{d} \text { in the control } \\
\text { statement "sptr" }\end{array}$ & $\begin{array}{l}\text { Matrix sorption } \\
\text { coefficient in volcanics, } \\
\text { needed for retardation } \\
\text { calculations in Equations } \\
56 \text { a and } 57 .\end{array}$ & 0.0 & $\mathrm{~mL} / \mathrm{g}$ & Epistemic & $\begin{array}{l}\text { Output DTN: } \\
\text { LA0310AM831341.002 }\end{array}$ \\
\hline $\begin{array}{l}\text { AL in the control } \\
\text { statement "sptr" }\end{array}$ & $\begin{array}{l}\text { Dispersivity, longitudinal, } \\
\text { needed for dispersion } \\
\text { calculations in Equations } \\
1 \text { and } 56 a \text {. }\end{array}$ & 10.0 & $\mathrm{~m}$ & Epistemic & Section 4.1.2.16 \\
\hline $\begin{array}{l}\text { ATH in the control } \\
\text { statement "sptr" }\end{array}$ & $\begin{array}{l}\text { Dispersivity, transverse, } \\
\text { horizontal, needed for } \\
\text { dispersion calculations in } \\
\text { Equation } 1 .\end{array}$ & 0.05 & $\mathrm{~m}$ & Epistemic & Section 4.1.2.17 \\
\hline $\begin{array}{l}\text { ATV in the control } \\
\text { statement "sptr" }\end{array}$ & $\begin{array}{l}\text { Dispersivity, transverse, } \\
\text { vertical, needed for } \\
\text { dispersion calculations in } \\
\text { Equation } 1 .\end{array}$ & 0.0005 & $\mathrm{~m}$ & Epistemic & Section 4.1.2.18 \\
\hline
\end{tabular}

Source: Output DTN: LA0306SK831231.001.

${ }^{a}$ Epistemic uncertainty is defined as uncertainty in the parameter space of a conceptual model for which some knowledge is obtainable as given in Guidelines for Developing and Documenting Alternative Conceptual Models, Model Abstractions, and Parameter Uncertainty in the Total System Performance Assessment for the License Application. (BSC 2002 [DIRS 158794], Section 4.1.1).

${ }^{\mathrm{b}}$ Input listed in Table 4-2 for flowing-interval porosity was multiplied by flowing interval spacing to obtain flowing interval aperture. 
Table 6.4-3. Additional Parameters Needed for Abstraction Analysis

\begin{tabular}{|c|c|c|c|c|c|}
\hline $\begin{array}{c}\text { Input Name } \\
\text { (Variable Name and the } \\
\text { Control Statement where it } \\
\text { Appears in the } \\
\text { FEHM V } 2.20 \text { Code) }\end{array}$ & Input Description & $\begin{array}{c}\text { Base- } \\
\text { Case } \\
\text { Value(s) }\end{array}$ & Units & $\begin{array}{c}\text { Type of } \\
\text { Uncertainty }\end{array}$ & Source/DTN \\
\hline $\begin{array}{l}\text { Multiplying factor }{ }^{a} \text { for SKD in } \\
\text { control statement "flow" }\end{array}$ & $\begin{array}{l}\text { Specific discharge } \\
\text { multiplication factor }\end{array}$ & 1 & - & Stochastic & $\begin{array}{l}\text { Saturated Zone Flow } \\
\text { and Transport Model } \\
\text { Abstraction (BSC } 2004 \\
\text { [DIRS 170042]) } \\
\text { SN0310T0502103.009 } \\
\text { [DIRS 168763] }\end{array}$ \\
\hline $\begin{array}{l}\text { SCALEX, SCALEY in control } \\
\text { statement "fper," ratio }\end{array}$ & $\begin{array}{l}\text { Permeability } \\
\text { horizontal anisotropy }\end{array}$ & 4.2 & - & Stochastic & $\begin{array}{l}\text { SN0310T0502103.009 } \\
\text { [DIRS 168763] }\end{array}$ \\
\hline $\begin{array}{l}\text { RD_FRAC in the control } \\
\text { statement "sptr," ratio }\end{array}$ & $\begin{array}{l}\text { Colloid retardation } \\
\text { factor in volcanics for } \\
\text { irreversible colloids }\end{array}$ & 1 & - & Epistemic & Table 4-2 \\
\hline $\begin{array}{l}\text { Needed in Equations } 79 \text { and } \\
80 \text { for calculating the relative } \\
\text { concentration of radionuclide } \\
\text { on colloids, needed for } \\
\text { colloid- facilitated reversible } \\
\text { transport }\end{array}$ & $\begin{array}{l}\text { Groundwater } \\
\text { concentration of } \\
\text { colloids }\end{array}$ & 0 & $\mathrm{~g} / \mathrm{mL}$ & Epistemic & Table 4-2 \\
\hline $\begin{array}{l}\text { Needed in Equations } 79 \text { and } \\
80 \text { for calculating the relative } \\
\text { concentration of radionuclide } \\
\text { on colloids, needed for } \\
\text { colloid- facilitated reversible } \\
\text { transport }\end{array}$ & $\begin{array}{l}\text { Sorption coefficient } \\
\text { onto colloids }\end{array}$ & 0 & $\mathrm{~mL} / \mathrm{g}$ & Epistemic & Table 4-2 \\
\hline $\begin{array}{l}\text { In TSPA calculations, used } \\
\text { for post processing the } \\
\text { breakthrough curves } \\
\text { generated by the transport } \\
\text { model, fraction }\end{array}$ & $\begin{array}{l}\text { Fraction of colloids } \\
\text { transported } \\
\text { unretarded }\end{array}$ & $0.0005^{b}$ & - & Epistemic & $\begin{array}{l}\text { Saturated Zone Colloid } \\
\text { Transport (BSC } 2004 \\
\text { DIRS 170006], Section } \\
6.6 \text { ) }\end{array}$ \\
\hline $\begin{array}{l}\text { Used for calculating colloid } \\
\text { sorption coefficient using } \\
\text { Equation } 57, \text { which is in turn } \\
\text { input as the variable } K_{d} \text { in the } \\
\text { control statement "sptr," ratio }\end{array}$ & $\begin{array}{l}\text { Colloid retardation } \\
\text { factor in alluvium for } \\
\text { irreversible colloids }\end{array}$ & 1 & - & Epistemic & Table 4-2 \\
\hline
\end{tabular}

${ }^{a}$ Base-case permeabilities and recharge and boundary fluxes are multiplied by this factor to vary specific discharge without affecting the flow calibration.

$\mathrm{b}$ This value is different from the base-case value given in Table 4-2. This parameter does not enter the base-case transport model directly. It is used in TSPA calculations for postprocessing the breakthrough curves output from this report.

\subsection{BASE-CASE MODEL RESULTS}

The base-case model results (output DTN: LA0306SK831231.001) are discussed in this section.

The conceptual and mathematical model described in Sections 6.3 and 6.4 was implemented in the numerical code FEHM V 2.20, a finite-volume/finite-element heat- and mass-transfer code that simulates nonisothermal, multiphase, multicomponent flow and solute transport in porous media. The details of this code, its usage and verification example are given in the Validation Test Plan (VTP) for the FEHM Application Version 2.20 (LANL 2003 [DIRS 164150]). The 
calibrated base-case site-scale SZ flow model (DTN: LA0304TM831231.002 [DIRS 163788]), which is described in detail in the SZ flow model report, Site-Scale Saturated Zone Flow Model, (BSC 2004 [DIRS 170037]), was used as the starting input.

The purpose of this model report is to provide a site-scale SZ transport model to be used as the starting point for the SZ abstractions model presented in Saturated Zone Flow and Transport Model Abstraction (BSC 2004 [DIRS 170042], Section 6.3) for use in the TSPA-LA calculations. For this purpose, a single base-case transport model is presented here along with its outputs. The propagations of uncertainties in the input parameters to the output breakthrough curves are presented in Section 6.7 by documenting the breakthrough curves at the boundary of the accessible environment at $18 \mathrm{~km}$ (10 CFR 63.302 [DIRS 156605]) for minimum and maximum values of the various parameters. The barrier capabilities of the SZ transport are presented in Section 6.8 of this report, where the influence of key parameters on the radionuclide breakthrough is discussed.

The input transport parameter values and the sources for these values for the base-case model are given in Table 6.4-2. Particle source locations were distributed over the anticipated repository footprint at the water table (BSC 2004 [DIRS 170042], Section 6.5.2.13). Calculations were performed for an instantaneous release of particles at the source location. The breakthrough curve at the boundary of the accessible environment at $18 \mathrm{~km}$ was calculated by starting 1,000 particles distributed over the repository footprint and by outputting the cumulative number of particles crossing an east-west vertical plane across the entire width and depth of the model. Output of this model is shown in Figure 6.5-1 where normalized cumulative mass is plotted on the $y$-axis and the time in years on the log scale on the $x$-axis.

The base case serves as a reference point for exploring the role of processes and features of the system in subsequent simulations. The solid black curve in Figure 6.5-1a shows the breakthrough curve at the $18 \mathrm{~km}$ boundary, which can also be thought of as an arrival time distribution for transport through the SZ. This is the breakthrough curve for a conservative, nonsorbing radionuclide in the absence of radioactive decay. Breakthrough times on the order of hundreds of years are predicted for the bulk of the mass arriving at the water table, with transport times extending into the thousands of years for the slowest moving 20 percent of the mass. This breakthrough curve in Figure 6.5-1 corresponds to a breakthrough time at 50 percent concentration of 705 years.

A similar model calculation was performed where the number of input tracer particles was changed (from 1,000 in the base case) to nine particles spread over the repository footprint, and the output option was changed to produce detailed particle tracks as they moved from the source location to the boundary of the accessible environment at $18 \mathrm{~km}$. These are plotted against a shaded relief map of the SZ site scale model area in Figure 6.5-2. Note that there is very minimal transverse spreading of the flow paths due to the small value of transverse dispersivities (Table 6.4-2). The flow paths in Figure 6.5-2 appear to converge toward the southern portion of the model due to the large-scale heterogeneities that are explicitly included in the hydrologic framework model shown in Saturated Zone Site-Scale Flow Model (BSC 2004 [DIRS 170037], Table 6-17). 


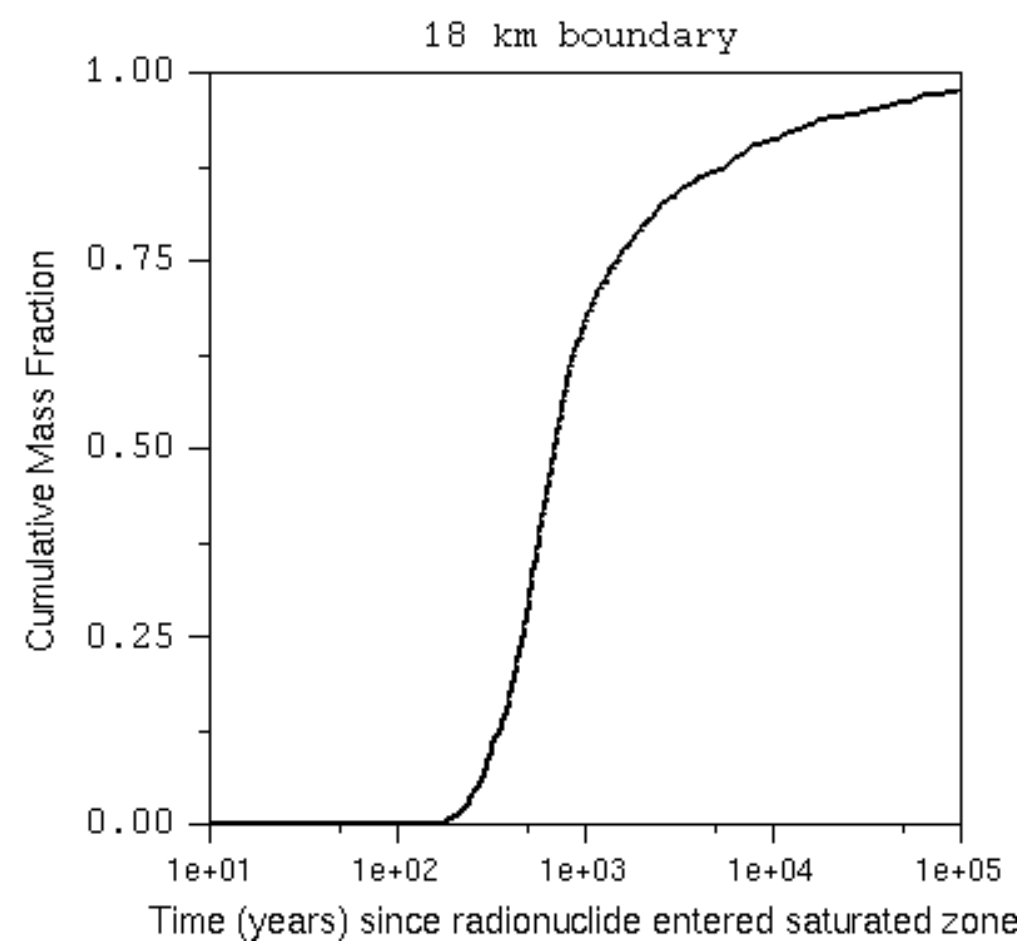

Output DTN: LA0306SK831231.001.

NOTE: Mass breakthrough curves and median transport times are for an instantaneous source, present-day climate, and do not include radionuclide decay. Particle source locations were distributed over the anticipated repository footprint.

Figure 6.5-1. Breakthrough Curve at the 18-km Boundary for the Transport Base Case (for a Conservative, Nonsorbing Radionuclide) 


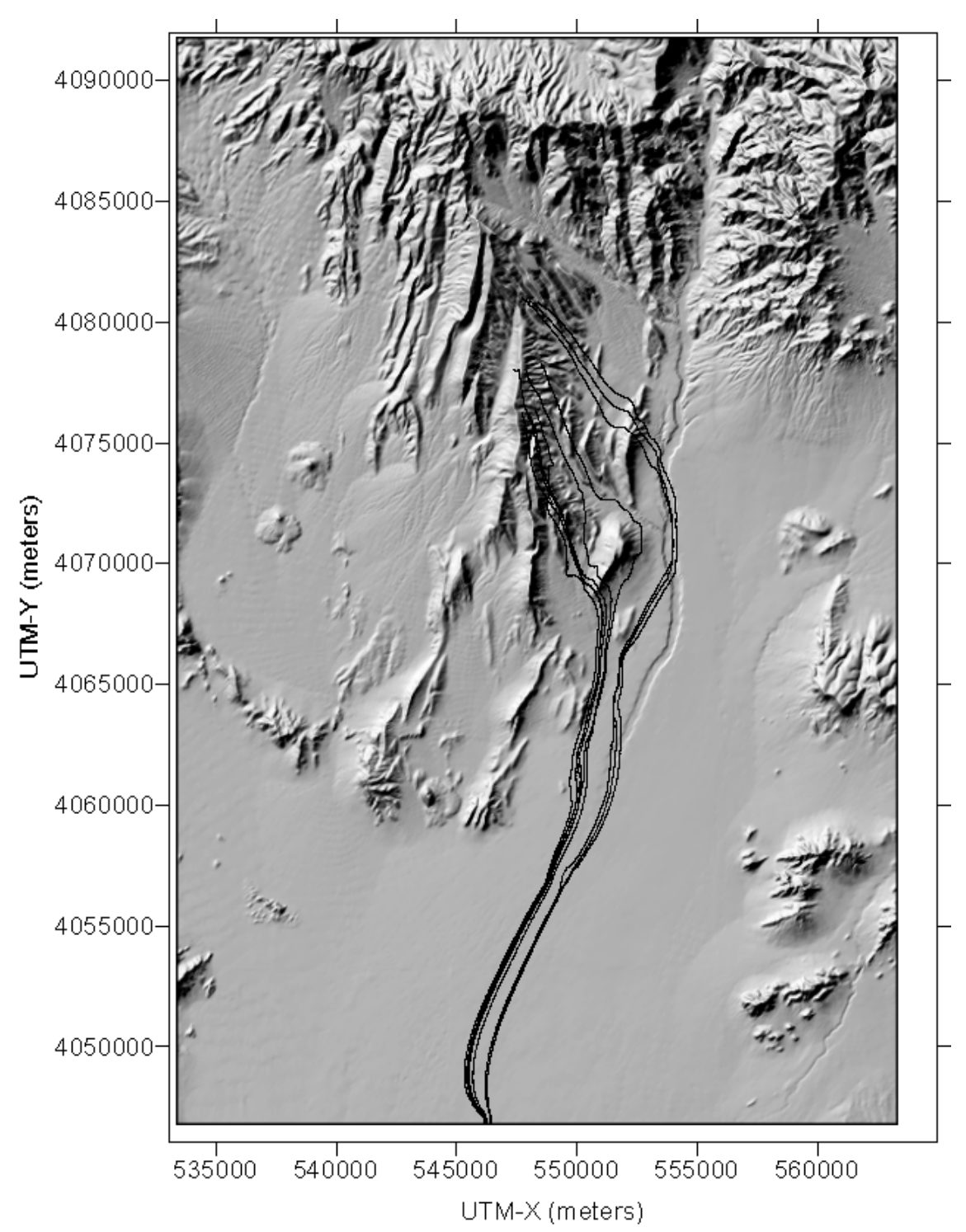

Output DTN: LA0307SK831231.001.

NOTE: Mass breakthrough curves and median transport times are for an instantaneous source, present-day climate, and do not include radionuclide decay. Particle source locations were distributed over the anticipated repository footprint.

Figure 6.5-2. Particle Tracks Resulting from the Site-Scale Saturated Zone Base-Case Transport Model 


\subsection{ALTERNATIVE CONCEPTUAL MODELS}

Credible alternatives to the conceptual model of SZ transport presented in Section 6.3 were evaluated in regards to their impact on the radionuclide transit times from the repository footprint to the boundary of the accessible environment at $18 \mathrm{~km}$. The alternative conceptual models fall into two classes. One class consists of the alternative conceptual models that are excluded from further consideration because they lead to transit times greater than those calculated by the sitescale SZ transport model. The key components of each of these alternative conceptual models, and the basis for the disposition are presented in Table 6.6-1a. The other class consists of alternative conceptual models that are implicitly included in the site-scale SZ transport model through the range of uncertainty in parameter values. The key components of each of these alternative conceptual models, the disposition of each alternative conceptual model and the basis for the disposition are presented in Table 6.6-1b.

Table 6.6-1a. Key Components and Basis for the Alternative Conceptual Models Excluded from Further Consideration Because They Lead to Transit Times Greater than the Base-Case Results

\begin{tabular}{|c|c|c|}
\hline $\begin{array}{c}\text { Alternative } \\
\text { Conceptual } \\
\text { Model }\end{array}$ & Key Components & Basis \\
\hline $\begin{array}{l}\text { Fluid flow in } \\
\text { matrix blocks }\end{array}$ & $\begin{array}{l}\text { The matrix material in } \\
\text { the intervening space } \\
\text { between the flowing } \\
\text { intervals in the } \\
\text { volcanics has } \\
\text { significant } \\
\text { permeability and } \\
\text { transmits significant } \\
\text { amounts of fluid by } \\
\text { advection for the flow } \\
\text { conditions in the SZ. }\end{array}$ & $\begin{array}{l}\text { There are three situations possible: (1) flow occurs from the fractures } \\
\text { into the matrix, (2) flow occurs from the matrix into the fractures, and } \\
\text { (3) flow occurs independently within matrix blocks. In the first case, } \\
\text { flow out of the fractures into the matrix would enhance the effects of } \\
\text { the matrix diffusion, leading to transit times greater than those } \\
\text { calculated by this model. In the second case, considering the } \\
\text { steady-state nature of the flow system as presented in Saturated Zone } \\
\text { Site-Scale Flow Model (BSC } 2004 \text { [DIRS } 170037 \text { ], Sections } 5 \text { and } 6 \text { ), } \\
\text { if some flow is occurring out of the matrix into the fractures, then } \\
\text { equivalent flow must also occur from fractures into the matrix blocks. } \\
\text { Thus, the matrix diffusion effects will be negated in some areas and } \\
\text { enhanced in others, and the overall effect on the effective diffusion } \\
\text { coefficient will be small. In the third case, an effective porosity would } \\
\text { have to be used that is larger than that being used for the fractured } \\
\text { flowing intervals, thus leading to transit times greater than those } \\
\text { calculated by this model. }\end{array}$ \\
\hline $\begin{array}{l}\text { Irreversible } \\
\text { sorption }\end{array}$ & $\begin{array}{l}\text { Rate of desorption of } \\
\text { radionuclides from the } \\
\text { rock surfaces is } \\
\text { slower than the rate of } \\
\text { absorption. }\end{array}$ & $\begin{array}{l}\text { Sorption reactions that are not fully reversible result in rates of } \\
\text { transport that are slower than would be the case for fully reversible } \\
\text { reactions. Therefore, for radionuclides that sorb irreversibly, using a } \\
\text { sorption coefficient would result in diminished total radionuclide mass } \\
\text { breakthrough from the SZ. A potential scenario for which this } \\
\text { simplification could lead to longer transit times is when deposition of } \\
\text { radionuclides takes place under the ambient geochemical conditions, } \\
\text { and later, because of geochemical changes, the deposited } \\
\text { radionuclides re-enter the aqueous phase and are transported } \\
\text { downstream as a pulse. On the basis of available geochemical and } \\
\text { mineralogical data, this situation is not considered likely. It is being } \\
\text { addressed as a FEP in Features, Events, and Processes in SZ Flow } \\
\text { and Transport (BSC } 2004 \text { [DIRS 170013]). }\end{array}$ \\
\hline
\end{tabular}


Table 6.6-1a. Key Components and Basis for the Alternative Conceptual Models Excluded from Further Consideration Because They Lead to Transit Times Greater than the Base-Case Results (Continued)

\begin{tabular}{|c|c|c|}
\hline $\begin{array}{c}\text { Alternative } \\
\text { Conceptual } \\
\text { Model }\end{array}$ & Key Components & Basis \\
\hline \multirow[t]{2}{*}{$\begin{array}{l}\text { Radionuclide } \\
\text { precipitation }\end{array}$} & \multirow[t]{2}{*}{$\begin{array}{l}\text { Radionuclides could } \\
\text { precipitate in the SZ, } \\
\text { forming solid phases } \\
\text { on the rock surfaces. }\end{array}$} & $\begin{array}{l}\text { Thermodynamically, chemical species in supersaturation possess a } \\
\text { driving force that favors the formation of solid phases. The result for a } \\
\text { radionuclide would be an extreme form of retardation that renders the } \\
\text { bulk of the radionuclide plume virtually immobile. Given that most } \\
\text { radionuclides reaching the } \mathrm{SZ} \text { would need to transport there in } \\
\text { aqueous solution, thermodynamic conditions along the flow path would } \\
\text { have to change (relative to the UZ) for species to precipitate. The } \\
\text { most credible mechanism for this to occur would be through a different } \\
\text { redox condition in the SZ. If the conditions were significantly more } \\
\text { reducing, the valence state of actinides, such as Np, U, and Pu, and } \\
\text { fission products, such as Tc and I, could be lowered. Typically, in } \\
\text { these groundwaters, this condition would result in much lower } \\
\text { solubilities of the radionuclides and hence, precipitation. There are } \\
\text { insufficient measurements and data concerning the redox behavior of } \\
\text { these radionuclides to warrant including redox behavior in model } \\
\text { calculations. In all cases, the radionuclides in question have been } \\
\text { assigned to their highest valence state, which results in higher } \\
\text { solubilities and lower sorption coefficients than would be the case if the } \\
\text { lower valence state were selected. }\end{array}$ \\
\hline & & $\begin{array}{l}\text { A potential scenario for which this simplification could be less } \\
\text { straightforward is when deposition of radionuclides takes place under } \\
\text { the ambient geochemical conditions, and later, because of } \\
\text { geochemical changes, the deposited radionuclides re-enter the } \\
\text { aqueous phase and are transported downstream as a pulse. On the } \\
\text { basis of available geochemical and mineralogical data, this situation is } \\
\text { not considered likely. It is being addressed as a FEP in Features, } \\
\text { Events, and Processes in SZ Flow and Transport (BSC } 2004 \\
\text { [DIRS 170013]. }\end{array}$ \\
\hline Water table rise & $\begin{array}{l}\text { Future water table } \\
\text { rise could elevate the } \\
\text { water table, leading to } \\
\text { radionuclide transport } \\
\text { through geological } \\
\text { horizons currently } \\
\text { considered outside of } \\
\text { the SZ. }\end{array}$ & $\begin{array}{l}\text { Wetter, glacial climatic conditions could occur in the future at the } \\
\text { Yucca Mountain site within the } 10,000 \text {-year period of regulatory } \\
\text { concern presented in Total System Performance Assessment for the } \\
\text { Site Recommendation (CRWMS M\&O } 2000 \text { [DIRS 153246], } \\
\text { Section 1.8.4.3). These changes in the climate relative to present } \\
\text { conditions would affect groundwater flow in the SZ by increasing the } \\
\text { amount of recharge to the regional groundwater flow system. These } \\
\text { regional and local increases in recharge will tend to increase the } \\
\text { groundwater flux through the SZ system and lead to a rise in the water } \\
\text { table beneath Yucca Mountain. In previous analyses, the effect of this } \\
\text { on the SZ breakthrough curves was modeled using a scaling factor } \\
\text { representing the alternative climate state as given in Saturated Zone } \\
\text { Flow and Transport Model Abstraction (BSC } 2004 \text { [DIRS } 170042 \text { ], } \\
\text { Section } 6.5 \text { ). The scaling factor used in this approach is the ratio of } \\
\text { average SZ groundwater flux under the future climatic conditions to the } \\
\text { flux under present conditions. However, this approach uses the same } \\
\text { flow path for radionuclide transport through the SZ under wetter } \\
\text { climatic conditions of the future. Alternatively, the rise in the water } \\
\text { table due to climatic changes could be included in the model with } \\
\text { potentially different flow paths through different hydrogeologic units in } \\
\text { the SZ. }\end{array}$ \\
\hline
\end{tabular}


Table 6.6-1a. Key Components and Basis for the Alternative Conceptual Models Excluded from Further Consideration Because They Lead to Transit Times Greater than the Base-Case Results (Continued)

\begin{tabular}{|c|c|c|}
\hline $\begin{array}{c}\text { Alternative } \\
\text { Conceptual } \\
\text { Model }\end{array}$ & Key Components & Basis \\
\hline $\begin{array}{l}\text { Water table rise } \\
\text { (continued) }\end{array}$ & $\begin{array}{l}\text { Future water table } \\
\text { rise could elevate the } \\
\text { water table, leading to } \\
\text { radionuclide transport } \\
\text { through geological } \\
\text { horizons currently } \\
\text { considered outside of } \\
\text { the SZ (continued) }\end{array}$ & $\begin{array}{l}\text { Saturated Zone Site-Scale Flow Model (BSC } 2004 \text { ([DIRS 170037], } \\
\text { Section 6.4.5) presents a modeling exercise that adapts the site-scale } \\
\text { SZ flow model to include the effects of estimated water table rise. The } \\
\text { results of particle-tracking simulations using this adapted model to the } \\
\text { simple flux-scaling approach are presented in Appendix E. The results } \\
\text { presented in Figures E-1 and E-2 indicate that the model with the } \\
\text { higher water table results in longer simulated transport times for both } \\
\text { the nonsorbing species and for neptunium. The simplified approach of } \\
\text { scaling the breakthrough curves from the SZ site-scale flow model } \\
\text { with the present water-table elevations is, thus, an acceptable } \\
\text { representation of transport in the SZ under wetter, glacial climatic } \\
\text { conditions relative to the adapted model that incorporates water-table } \\
\text { rise associated with future conditions. The reason for the longer } \\
\text { transport times using the water-table-rise model relates to the } \\
\text { hydrogeologic units encountered by a radionuclide plume arriving at } \\
\text { the water table. Transport must occur through lower-permeability } \\
\text { confining units, and the flow-path distance through the alluvium is } \\
\text { predicted to be longer for the water-table-rise model. }\end{array}$ \\
\hline $\begin{array}{l}\text { Diffusion into } \\
\text { low- permeability } \\
\text { zones }\end{array}$ & $\begin{array}{l}\text { Within alluvium, } \\
\text { solutes can diffuse } \\
\text { into low- permeability } \\
\text { zones, later diffusing } \\
\text { out into the flow, } \\
\text { which leads to a long } \\
\text { tail for the } \\
\text { radionuclide } \\
\text { breakthrough curve. }\end{array}$ & $\begin{array}{l}\text { This phenomenon was not observed to occur in the single-hole tracer } \\
\text { tests conducted at the ATC presented in Saturated Zone In-Situ } \\
\text { Testing (BSC } 2004 \text { [DIRS } 170010 \text { ], Section } 6.5 ., \text { Figures G-18 and } \\
\text { G-19). On a larger scale, this scenario will lead to lower } \\
\text { concentrations at breakthrough and longer times for reaching } \\
50 \text { percent breakthrough concentrations. Thus, the conceptual model } \\
\text { presented in this model report is acceptable. }\end{array}$ \\
\hline $\begin{array}{l}\text { Alternate flow } \\
\text { paths resulting } \\
\text { from alternate } \\
\text { flow models. }\end{array}$ & $\begin{array}{l}\text { Alternate flow models } \\
\text { may lead to flow } \\
\text { paths that are } \\
\text { different from the } \\
\text { base case, which may } \\
\text { lead to different } \\
\text { breakthrough times. }\end{array}$ & $\begin{array}{l}\text { Alternate flow models have been developed resulting from revised } \\
\text { hydrologic framework model, additional water level data, revised } \\
\text { recharge distribution, updated boundary fluxes, additional permeability } \\
\text { data, and further evaluation of the flow models shown in Saturated } \\
\text { Zone Site-Scale Flow Model (BSC } 2004 \text { [DIRS } 170037] \\
\text { Section } 6.4 .6 \text { ). As discussed in Sections } 6.4 .6 .3 .5 \text { and } 6.4 .6 .3 .7 \text { of } \\
\text { Saturated Zone Site-Scale Flow Model (BSC } 2004 \text { [DIRS } 170037] \text { ), } \\
\text { the spatial distribution and magnitude of the flow velocities differs } \\
\text { significantly from the base-case results, with the specific discharge for } \\
\text { the alternate flow models being in the range of } 0.459 \text { to } 0.653 \text { m/year } \\
\text { as compared to } 1.3 \text { m/year for the base case. This leads to } \\
\text { significantly different flow paths for the radionuclides, as shown in } \\
\text { Figures } 6-19,6-20,6-21 \text { of Saturated Zone Site-Scale Flow Model } \\
\text { (BSC } 2004 \text { [DIRS } 170037] \text {. The resulting breakthrough curves using } \\
\text { the same transport parameter values as those used in the base-case } \\
\text { transport model (Table } 6.4-2 \text { ) are shown in Figure E-3. The transit } \\
\text { times are significantly longer, in excess of } 6500 \text { years for } 50 \text { percent } \\
\text { breakthrough for nonsorbing radionuclides, as compared to } 705 \text { years } \\
\text { for the base case (Section } 6.5 \text { ). The transit time for the case of even a } \\
\text { moderately sorbing radionuclide such Np (with } K_{d} \text {-volcanics }=1.3 \mathrm{~mL} / \mathrm{g} \text {, } \\
K_{d} \text {-alluvium=6.3 mL/g) becomes in excess of } 75,000 \text { years. Thus, } \\
\text { these alternative flow models are excluded from further consideration } \\
\text { because they lead to transit times greater than those calculated by the } \\
\text { site-scale SZ transport model. }\end{array}$ \\
\hline
\end{tabular}


Table 6.6-1b. Key Components, Disposition, and Basis for Alternative Conceptual Models that are Implicitly Included in the Site-Scale SZ Transport Model

\begin{tabular}{|c|c|c|c|}
\hline $\begin{array}{c}\text { Alternative } \\
\text { Conceptual } \\
\text { Model }\end{array}$ & $\begin{array}{c}\text { Key } \\
\text { Components }\end{array}$ & Disposition & Basis \\
\hline $\begin{array}{l}\text { Sorption } \\
\text { reactions are } \\
\text { not } \\
\text { instantaneous }\end{array}$ & $\begin{array}{l}\text { Reaction rates } \\
\text { for sorption } \\
\text { kinetics are } \\
\text { slow compared } \\
\text { to the rates of } \\
\text { solute } \\
\text { transport. }\end{array}$ & $\begin{array}{l}\text { This alternative } \\
\text { conceptual model is } \\
\text { implicitly included in } \\
\text { the site-scale SZ } \\
\text { transport model } \\
\text { through the range } \\
\text { of uncertainty in the } \\
\text { sorption- coefficient } \\
\text { values. }\end{array}$ & $\begin{array}{l}\text { Among the radionuclides of concern, Pu has the slowest } \\
\text { reaction kinetics (Appendix A). Calculations of the } \\
\text { Damköhler number (Da) (Appendix D) for reaction rates, } \\
\text { and analysis of laboratory data (presented in Appendix } G \text { ) } \\
\text { for plutonium indicate that, for typical transport times } \\
\text { through the SZ, the local equilibrium approach is valid). } \\
\text { However, in localized areas, flow rates may be fast } \\
\text { enough for kinetic limitations to be a factor for Pu } \\
\text { (Appendix } A \text { ). The possibility of the sorption reaction rate } \\
\text { being slow relative to the flow rate would lead to in situ } \\
\text { sorption experienced by the radionuclides being less than } \\
\text { that estimated on the basis of equilibrium } K_{d} \text { values, i.e., } \\
\text { the in situ } K_{d} \text { would be lower than the equilibrium values } \\
\text { measured in the laboratory. Accommodation is made for } \\
\text { this by biasing the sorption-coefficient distributions } \\
\text { downward for the radionuclides of interest that appear to } \\
\text { have slow sorption kinetics (Appendices A and C). }\end{array}$ \\
\hline $\begin{array}{l}\text { Nonlinear } \\
\text { sorption }\end{array}$ & $\begin{array}{l}\text { Sorption } \\
\text { reactions have } \\
\text { to be modeled } \\
\text { using nonlinear } \\
\text { isotherms. }\end{array}$ & $\begin{array}{l}\text { This alternative } \\
\text { conceptual model is } \\
\text { implicitly included in } \\
\text { the site-scale SZ } \\
\text { transport model } \\
\text { through the range } \\
\text { of uncertainty in the } \\
\text { sorption- coefficient } \\
\text { values. }\end{array}$ & $\begin{array}{l}\text { The } K_{d} \text { model is based on treating the concentration of } \\
\text { the radionuclides absorbed onto the rock surface as a } \\
\text { linear function of the concentration of that species in the } \\
\text { aqueous solution. This treatment is valid at low } \\
\text { concentrations, but at higher concentrations, as } \\
\text { absorption sites start getting saturated, the absorbed } \\
\text { concentration starts falling below the value predicted by } \\
\text { this linear relationship. This effect results in a lower } \\
\text { apparent value of } K_{d} \text { at higher concentrations, which is } \\
\text { accounted for in the model by biasing the } K_{d} \text { distributions } \\
\text { at low values (Appendix A). The use of low } K_{d} \text { values } \\
\text { could lead to underestimating the long-term tail of the } \\
\text { breakthrough curve; however, since the long-term tailing } \\
\text { generally does not occur during the } 10,000 \text {-year } \\
\text { regulatory period, such biasing is acceptable. }\end{array}$ \\
\hline $\begin{array}{l}\text { Locally } \\
\text { varying } \\
\text { sorption } \\
\text { parameters }\end{array}$ & $\begin{array}{l}\text { Sorption } \\
\text { parameters are } \\
\text { strong } \\
\text { functions of } \\
\text { local water } \\
\text { chemistry, rock } \\
\text { mineralogy, } \\
\text { and solute } \\
\text { concentrations. } \\
\text { The properties } \\
\text { have to be } \\
\text { calculated } \\
\text { locally at each } \\
\text { node along the } \\
\text { travel path. }\end{array}$ & $\begin{array}{l}\text { This alternative } \\
\text { conceptual model is } \\
\text { implicitly included in } \\
\text { the site-scale SZ } \\
\text { transport model } \\
\text { through the range } \\
\text { of uncertainty in the } \\
\text { coefficient values } \\
\text { for sorption onto the } \\
\text { rocks and colloids } \\
\text { and for colloid } \\
\text { retardation factors. }\end{array}$ & $\begin{array}{l}\text { The approach taken in this model report is to use linear } \\
\text { transport equations with transport parameters appearing } \\
\text { in the equations being treated as "effective" stochastic } \\
\text { variables appropriate for the model scale. This approach } \\
\text { is consistent with the current level of available data. } \\
\text { Transport parameters such as the } K_{d} \text { coefficients depend } \\
\text { on type and concentration of the species, rock } \\
\text { mineralogy, and groundwater pH and Eh. In the present } \\
\text { model, broad distributions have been developed for } \\
\text { effective } K_{d} S \text { (Appendices A, and C). No credit is being } \\
\text { taken for sorption onto zeolites, and the } K_{d} \text { distributions } \\
\text { presented in Appendix A are based on silica surfaces. } \\
\text { Given the current level of data available, water pH data } \\
\text { are being treated as spatially random along the transport } \\
\text { path, and oxidizing conditions are taken as a conservative } \\
\text { approach, as justified in Section } 6.3 \text { of this report. }\end{array}$ \\
\hline
\end{tabular}


Table 6.6-1b. Key Components, Disposition, and Basis for Alternative Conceptual Models that are Implicitly Included in the Site-Scale SZ Transport Model (Continued)

\begin{tabular}{|c|c|c|c|}
\hline $\begin{array}{c}\text { Alternative } \\
\text { Conceptual } \\
\text { Model }\end{array}$ & $\begin{array}{c}\text { Key } \\
\text { Components }\end{array}$ & Disposition & Basis \\
\hline $\begin{array}{l}\text { Locally } \\
\text { varying } \\
\text { sorption } \\
\text { parameters } \\
\text { (continued) }\end{array}$ & $\begin{array}{l}\text { Sorption } \\
\text { parameters are } \\
\text { strong } \\
\text { functions of } \\
\text { local water } \\
\text { chemistry, rock } \\
\text { mineralogy, } \\
\text { and solute } \\
\text { concentrations. } \\
\text { The properties } \\
\text { have to be } \\
\text { calculated } \\
\text { locally at each } \\
\text { node along the } \\
\text { travel path. } \\
\text { (continued) }\end{array}$ & $\begin{array}{l}\text { This alternative } \\
\text { conceptual model is } \\
\text { implicitly included in } \\
\text { the site-scale SZ } \\
\text { transport model } \\
\text { through the range } \\
\text { of uncertainty in the } \\
\text { coefficient values } \\
\text { for sorption onto the } \\
\text { rocks and colloids } \\
\text { and for colloid } \\
\text { retardation factors. } \\
\text { (continued) }\end{array}$ & $\begin{array}{l}\text { To evaluate the effect of scale on the } K_{d} \text { distributions, } \\
\text { calculations were performed to capture the effect of } \\
\text { spatial variability of rock types and variability in water } \\
\text { chemistries on effective } K_{d} \text { (Appendix C). The effect of } \\
\text { variability in water chemistry was captured in the input } K_{d} \\
\text { distributions that were used for effective } K_{d} \text { calculations. } \\
\text { It was observed that the effective } K_{d} \text { distributions } \\
\text { calculated for a single } 500-m \times 500-m \text { grid block were } \\
\text { narrower than the input } K_{d} \text { distributions. During } \\
\text { performance assessment modeling studies, calculations } \\
\text { will be made through multiple runs, each with a distinct } K_{d} \\
\text { value sampled from the above-mentioned effective } K_{d} \\
\text { distribution. This approach will be more approximate than } \\
\text { assigning } K_{d} \text { values on a node basis, as the latter } \\
\text { approach will lead to breakthrough behavior that can be } \\
\text { described by an even narrower distribution than the } \\
\text { effective } K_{d} \text { distribution. }\end{array}$ \\
\hline $\begin{array}{l}\text { Channeling in } \\
\text { alluvium }\end{array}$ & $\begin{array}{l}\text { In alluvium, } \\
\text { high- } \\
\text { permeability } \\
\text { channels exist } \\
\text { that can } \\
\text { provide } \\
\text { preferential } \\
\text { pathways for } \\
\text { flow and } \\
\text { transport. }\end{array}$ & $\begin{array}{l}\text { This alternative } \\
\text { conceptual model is } \\
\text { implicitly included in } \\
\text { the site-scale SZ } \\
\text { transport model } \\
\text { through the range } \\
\text { of uncertainty in the } \\
\text { effective porosity } \\
\text { values. }\end{array}$ & $\begin{array}{l}\text { The conceptual model presented in this model report } \\
\text { uses the effective-continuum approach, using effective } \\
\text { values averaged over the grid block sizes on the order of } \\
500 \mathrm{~m} \times 500 \mathrm{~m} \times 50 \mathrm{~m} \text { for the parameters of interest, } \\
\text { such as porosity. The effective porosity being used in the } \\
\text { TSPA-LA calculations is represented by a normal } \\
\text { distribution with the expected value of } 0.18 \text { and a value of } \\
0.027 \text { at } 3 \text { standard deviations below the mean shown in } \\
\text { Saturated Zone Flow and Transport Model Abstraction } \\
\text { (BSC } 2004 \text { [DIRS } 170042 \text { ], Section } 6.5 .2 .3 \text { ). Thus, the } \\
\text { PA calculations allow for the eventuality that all the flow is } \\
\text { concentrated within a small fraction of the alluvium, } \\
\text { corresponding to the low effective porosity of } 0.027 \text {. The } \\
\text { conceptual model presented in this model report is } \\
\text { appropriate for these ranges of values. }\end{array}$ \\
\hline
\end{tabular}

\subsection{MODEL UNCERTAINTIES}

Model-form uncertainty as presented in Guidelines for Developing and Documenting Alternative Conceptual Models, Model Abstractions, and Parameter Uncertainty in the Total System Performance Assessment for the License Application (BSC 2002 [DIRS 158794], Section 4.1.1) in regards to the transport model is unavoidable given the sparseness of observed data and the limited amount of information available to corroborate or refute alternative models. This form of uncertainty is explicitly addressed by discussing alternative conceptual models in Section 6.4 of this report. The discussion and screening of the alternative conceptual models is based on available understanding and data. As discussed in Section 6.6, several of the alternative conceptual models are implicitly included in the model through the use of uncertainty distributions for parameter values. Although the model is meant to represent the SZ transport accurately, for the case of those alternative conceptual models that could not be included in the model, the transport model was selected such that it resulted in transit times faster than those expected for the alternative conceptual model. 
There are uncertainties associated with scaling parameter values from the scale of measurements to the scale of interest. Much of the data used for deriving parameter values in this report are from laboratory or field experiments conducted on spatial and temporal scales much smaller than those in the site-scale SZ model. Most of the measurements are done on sample sizes less than $1 \mathrm{~m}$, with the exception of the C-wells and ATC field tests, which were conducted on the scale of tens of meters. This scale is still several orders of magnitude smaller than the site scale. Nevertheless, these parameter values reflect the rock properties on the scale of measurement. Because the objective here is to calculate cumulative breakthrough curves at a boundary of the accessible environment, large grid spacing $(500 \mathrm{~m} \times 500 \mathrm{~m})$ is used in the numerical model. This leads to significant averaging of properties in the model calculations, thereby reducing the sensitivity of the output results to the stochastic variations in the parameter values. This is well demonstrated by the analysis presented in Appendix $\mathrm{C}$ for upscaling the distribution of the $K_{\mathrm{d}}$ values from a $4-\mathrm{m}$ to a $500-\mathrm{m}$ scale in the absence of spatial correlations. These calculations demonstrate that the resulting 500-m-scale $K_{d}$ distribution is actually narrower, with a standard deviation of $0.7 \mathrm{~mL} / \mathrm{g}$ (and a mean of $6.99 \mathrm{~mL} / \mathrm{g}$ ) than the two starting 4-m-scale $K_{d}$ distributions with standard deviations of $2 \mathrm{~mL} / \mathrm{g}$ and $3.6 \mathrm{~mL} / \mathrm{g}$. However, geological formations are inherently inhomogeneous, and they incorporate fractures, faults, and other heterogeneities on a variety of scales. Spatial correlations over some length scales often exist. Thus, it is difficult to extrapolate the measurements on a small scale to the SZ site scale.

There is epistemic uncertainty as presented in Guidelines for Developing and Documenting Alternative Conceptual Models, Model Abstractions, and Parameter Uncertainty in the Total System Performance Assessment for the License Application (BSC 2002 [DIRS 158794], Section 4.1.1) in the parameter space of the base-case conceptual model. This form of uncertainty is explicitly described by using probability distributions for appropriate model parameters. Expected uncertainty ranges for the various transport parameters are discussed in Section 4.1.2 and summarized in Table 4-2 of this report. The development and discussion of the $K_{\mathrm{d}}$ parameters needed for modeling reactive transport and the $K_{\mathrm{c}}$ parameters needed for modeling colloid-facilitated transport are presented in Appendices A, B, and C of this report. The probability distributions for all other model parameters are presented and discussed in the model report Saturated Zone Flow and Transport Model Abstraction (BSC 2004 [DIRS 170042], Section 6.5.2). These uncertainties are propagated through the model to the output breakthrough curves, presented in Section 6.7.1. Radionuclide transit times are most sensitive to groundwater-specific discharge. This is because increasing the specific discharge not only increases the advective velocity but also reduces the time available for matrix diffusion to be effective. In assessing the sensitivity of breakthrough times to the specific discharge through the model, permeabilities of the various units are scaled along with the specific discharge to preserve the model calibration. Other parameters of importance to the breakthrough times are matrix diffusion, the sorption coefficient in the volcanics as well as the alluvium, the effective fracture porosity in the volcanics (as reflected in the flowing interval aperture and spacing), the effective porosity in the alluvium, and the sorption coefficients for reversible colloids and retardation factor for irreversible colloids in the volcanics and alluvium. The volcanic retardation factors are important since the distribution contains large values that result in significant retardation even though transport times in the volcanics are shorter than the transport times in the alluvium. The alluvium retardation factors are lower than the volcanics but the contaminants spend the majority of the time in the alluvium. Therefore, any retardation in the alluvium results in large effects in the overall transport time. It should be also noted that although the time for 50 percent 
breakthrough shows only a moderate sensitivity to the value of longitudinal dispersivity (Figure 6.7-13), the leading and tailing edge of the breakthrough curve are significantly affected for dispersivity values near the upper limit. Thus, dispersivity could be an important parameter to consider in situations where the first and last arrival times for radionuclides play an important role. Quantification of the sensitivity of the model output breakthrough curves for various radionuclides of concern to parameter uncertainties are further evaluated in the model report Saturated Zone Flow and Transport Model Abstraction (BSC 2004 [DIRS 170042], Sections 6.7 and 8).

\subsubsection{Propagation of Uncertainty in the Input Parameter Values to the Output}

Starting with the SZ site-scale base-case transport model, sensitivity of the output breakthrough curves to each of the uncertain input parameters was investigated by considering the upper and lower levels of each parameter individually (Table 6.7-1).

Table 6.7-1. Effect of Parameter Ranges on 50 Percent Breakthrough Times

\begin{tabular}{|c|c|c|c|c|}
\hline & Parameter & $\begin{array}{c}\text { Base-case } \\
\text { Value(s) }\end{array}$ & $\begin{array}{l}\text { Uncertainty Range } \\
\text { (Units) }\end{array}$ & $\begin{array}{l}\text { Output Range, Time } \\
\text { (Years) for } 50 \% \\
\text { Breakthrough }\end{array}$ \\
\hline 1 & $\begin{array}{l}\text { Specific discharge multiplication } \\
\text { factor }^{\mathrm{a}}\end{array}$ & 1 & $1 / 30-10$ & 50 to $>10,000$ \\
\hline 2 & Permeability horizontal anisotropy & 4.2 & $\begin{array}{l}0.05-20 \\
\text { (ratio) }\end{array}$ & 352 to 922 \\
\hline 3 & Bulk density in alluvium & 1,910 & $\begin{array}{c}1,669-2,151 \\
\mathrm{~kg} / \mathrm{m}^{3}\end{array}$ & 705 \\
\hline 4 & Sorption coefficient in alluvium & 0.0 & $\begin{array}{c}0-10,000 \\
(\mathrm{~mL} / \mathrm{g})\end{array}$ & 705 to $>10,000$ \\
\hline 5 & Effective porosity in the alluvium & 0.18 & $\begin{array}{l}0.02-0.3 \\
\text { (fraction) }\end{array}$ & 349 to 913 \\
\hline 6 & $\begin{array}{l}\text { Colloid retardation factor in } \\
\text { alluvium for irreversible colloids }\end{array}$ & 1 & $\begin{array}{l}8-5,188 \\
\text { (ratio) }\end{array}$ & 705 to $>10,000$ \\
\hline 7 & $\begin{array}{l}\text { Effective sorption coefficient for } \\
\text { radionuclides with reversible } \\
\text { sorption onto colloids in alluvium }\end{array}$ & 0 & $\begin{array}{l}0-10,000 \\
(\mathrm{~mL} / \mathrm{g})\end{array}$ & 705 to $>10,000$ \\
\hline 8 & Flowing interval aperture ${ }^{c}$ & 0.2 & $\begin{array}{c}1.2 \times 10^{-5}-41.7^{d} \\
(\mathrm{~m})\end{array}$ & 443 to $>10,000$ \\
\hline 9 & $\begin{array}{l}\text { Effective diffusion coefficient in } \\
\text { volcanics }\end{array}$ & $5.0 \times 10^{-11}$ & $\begin{array}{c}5.0 \times 10^{-12}-5.0 \mathrm{x} \\
10^{-10} \\
\left(\mathrm{~m}^{2} / \mathrm{s}\right)\end{array}$ & 520 to 2,100 \\
\hline 10 & $\begin{array}{l}\text { Matrix sorption coefficient in } \\
\text { volcanics }\end{array}$ & 0.0 & $\begin{array}{c}0-10,000 \\
(\mathrm{~mL} / \mathrm{g})\end{array}$ & 705 to $>10,000$ \\
\hline 11 & $\begin{array}{l}\text { Colloid retardation factor in } \\
\text { volcanics for irreversible colloids }\end{array}$ & 1 & $\begin{array}{l}6-794 \\
\text { (ratio) }\end{array}$ & 705 to $>10,000$ \\
\hline
\end{tabular}


Table 6.7-1.Effect of Parameter Ranges on 50 Percent Breakthrough Times (Continued)

\begin{tabular}{|l|l|c|c|c|}
\hline & \multicolumn{1}{|c|}{ Parameter } & $\begin{array}{c}\text { Base-case } \\
\text { Value(s) }\end{array}$ & $\begin{array}{c}\text { Uncertainty Range } \\
\text { (Units) }\end{array}$ & $\begin{array}{c}\text { Output Range, Time } \\
\text { (Years) for 50\% } \\
\text { Breakthrough }\end{array}$ \\
\hline 12 & $\begin{array}{l}\text { Effective sorption coefficient for } \\
\text { radionuclides with reversible } \\
\text { sorption onto colloids in volcanic }\end{array}$ & 0 & $\begin{array}{c}0-10,000 \\
\text { (mL/g) }\end{array}$ & 705 to $>10,000$ \\
\hline 13 & Dispersivity, longitudinal & 10.0 & $\begin{array}{c}0.10-2,000 \\
(\mathrm{~m})\end{array}$ & 664 to 1,486 \\
\hline
\end{tabular}

${ }^{a}$ Base-case permeabilities and the recharge and boundary fluxes are multiplied by this factor to vary specific discharge without affecting the flow calibration.

b See Equations 79 to 81 in Section 6.4.2.6.

c Flowing interval aperture is the product of the flowing interval porosity and flowing interval spacing.

${ }^{d}$ This value is made to address the case when flowing interval spacing is very large.

\subsubsection{Specific Discharge Multiplier}

The results from simulations that evaluate the effect of changes in the specific discharge are shown in Figure 6.7-1. In these simulations, the base-case flow model was modified to scale the input recharge fluxes by the same factor as the rock permeabilities to preserve the model calibration. The results show that output is very sensitive to the level of uncertainty in this parameter. For the case of upper limit of specific discharge multiplier where the base-case fluxes are multiplied by a factor of 10, time to 50 percent breakthrough is 50 years (with extremely fast fluid flow such as would be expected for the very unlikely case of a high-permeability channel going continuously over the distance of $18 \mathrm{~km}$ in a highly faulted region). The lower limit of specific discharge multiplier, a factor of $1 / 30$, leads to breakthrough times at greater than 10,000 years. This indicates that information that helps reduce the uncertainty of this parameter will greatly improve the ability of the model to predict the results.

\subsubsection{Horizontal Permeability Anisotropy}

The results from simulations that evaluate the effect of changes in the ratio of horizontal permeability anisotropy are shown in Figure 6.7-2. The base-case flow model was modified to include the permeability ratios; however, note that the flow model was not recalibrated for the different values of permeability. The output shows a moderate level of variation resulting from the uncertainty limits of this parameter $(0.05$ to 20 , Table 6.7-1) with the time for 50 percent breakthrough varying between 352 to 922 years. This variation results mainly from the variation in the flow paths due to the changed velocity fields corresponding to the different ratios of the east-west versus the north-south permeabilities.

\subsubsection{Bulk Density in Alluvium}

The results from simulations that evaluate the effect of changes in the bulk density in the alluvium are shown in Figure 6.7-3. Note that these simulations are for the base case without sorption. There is no significant difference between the breakthrough curves because the bulk density enters the transport calculations only through the retardation factor as given in Equation 57. 


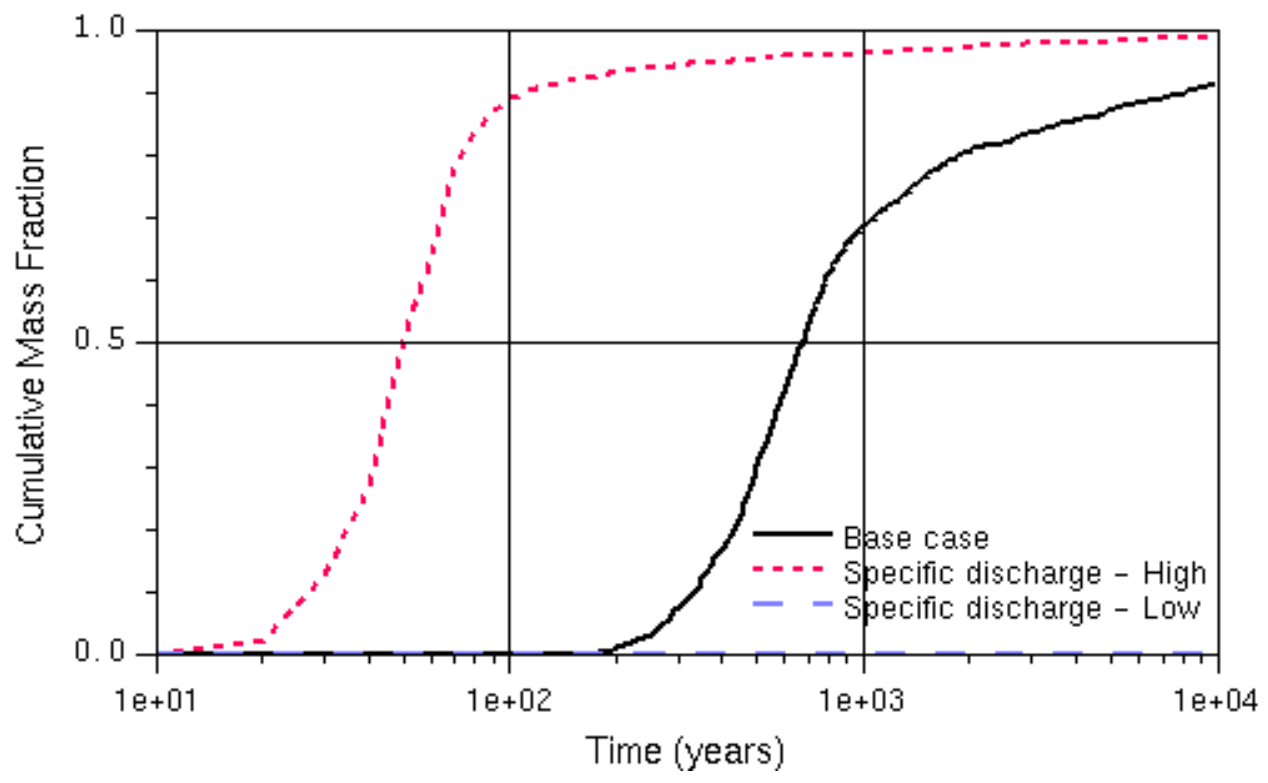

Output DTN: LA0307SK831231.002.

NOTES: Mass breakthrough curves and median transport times are for an instantaneous source, present-day climate, and do not include radionuclide decay. Particle source locations were distributed over the anticipated repository footprint.

The output results are stored in folder sp_discharge1_30 in file 02-calib-expected.sptr3 and in folder sp_discharge10 in file 02-calib-expected.sptr3. Column 1 is the elapsed time in days. Plotted on the $Y$ axis is the third column (the total number of particles crossing the boundary by this time) divided by 1,000.

Figure 6.7-1. Propagation of Input Uncertainty in the Specific Discharge to the Output Breakthrough Curves at the 18-km Boundary 


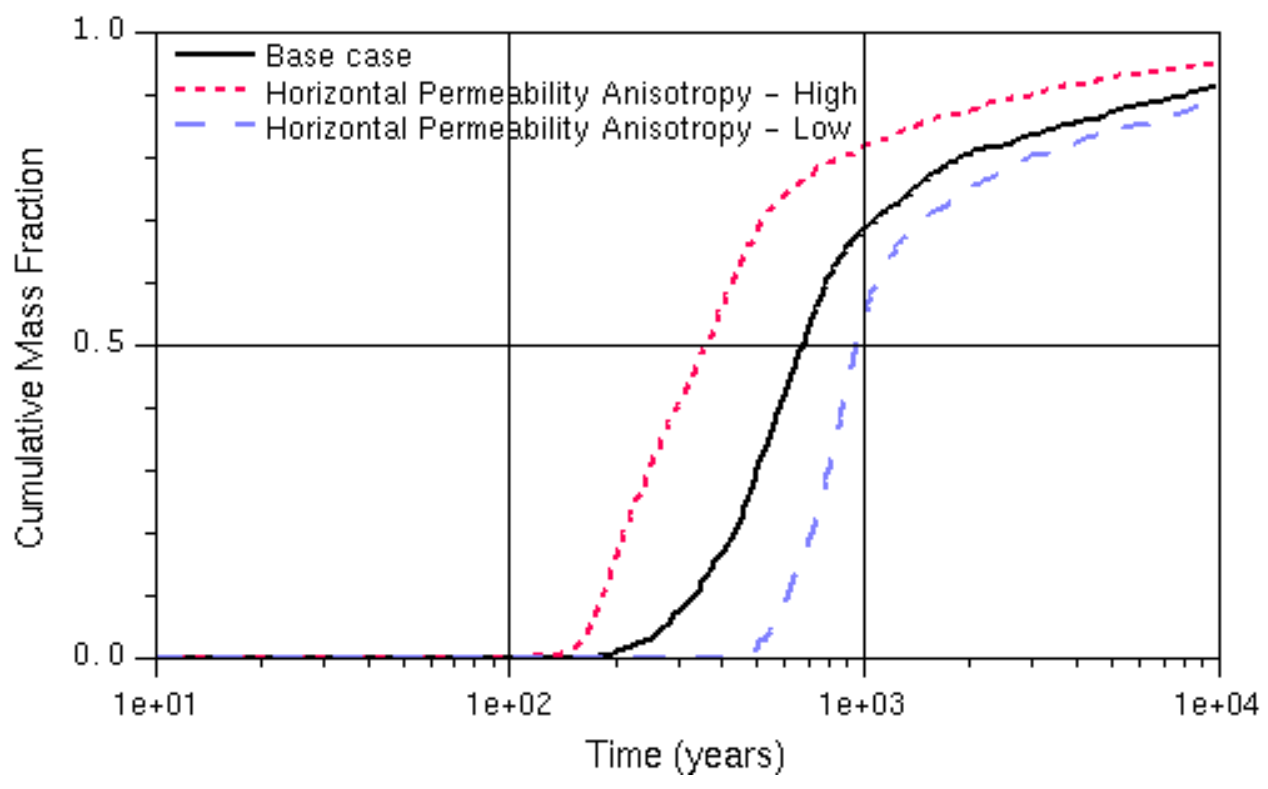

Output DTN: LA0309SK831231.001.

NOTES: Mass breakthrough curves and median transport times are for an instantaneous source, present-day climate, and do not include radionuclide decay. Particle source locations were distributed over the anticipated repository footprint.

The output results are stored in folder permhorzani-h.d in file sens_permhorzani-h.sptr3 and in folder permhorzani-I.d in file sens_permhorzani-I.sptr3. Column 1 is the elapsed time in days. Plotted on the $Y$ axis is the third column (the total number of particles crossing the boundary by this time) divided by 1,000.

Figure 6.7-2. Propagation of Input Uncertainty in the Horizontal Permeability Anisotropy Ratio to the Output Breakthrough Curves at the 18-km Boundary 


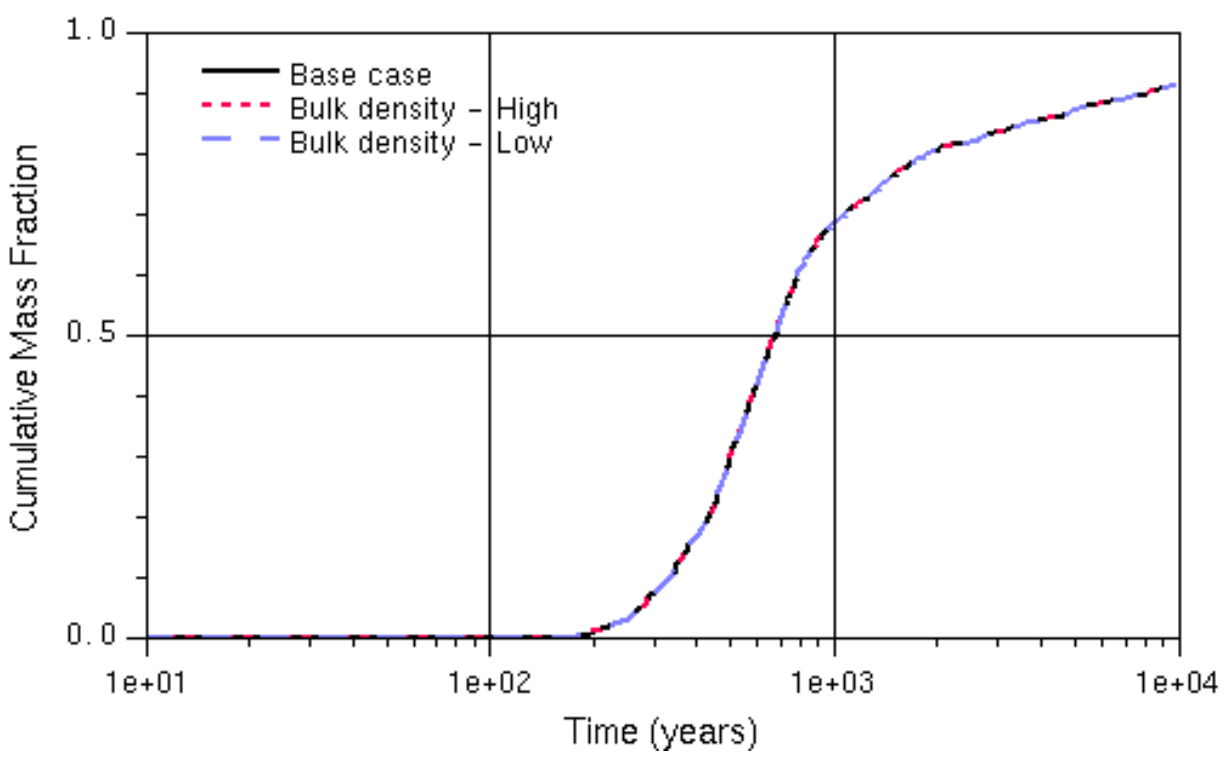

Output DTN: LA0309SK831231.001.

NOTES: Mass breakthrough curves and median transport times are for an instantaneous source, present-day climate, and do not include radionuclide decay. Particle source locations were distributed over the anticipated repository footprint.

The output results are stored in folder bulkdens-h.d in file bulkdens-h.sptr3 and in folder bulkdens-l.d in file bulkdens-I.sptr3. Column 1 is the elapsed time in days. Plotted on the $Y$ axis is the third column (the total number of particles crossing the boundary by this time) divided by 1,000 .

Figure 6.7-3. Propagation of Input Uncertainty in the Bulk Density of Alluvium to the Output Breakthrough Curves at the 18-km Boundary

\subsubsection{Sorption Coefficient in Alluvium}

The results from simulations that evaluate the effect of changes in sorption coefficient in alluvium on the output breakthrough curves are shown in Figure 6.7-4. The results indicate that when the sorption coefficient is high, no breakthrough of the tracers is observed in 10,000 years. Thus, the alluvium could form a very effective barrier for sorbing radionuclides. The base-case simulation is the same as for the low-sorption case because the base-case value of the sorption coefficient is 0 , meant to capture the behavior of a nonsorbing radionuclide. 


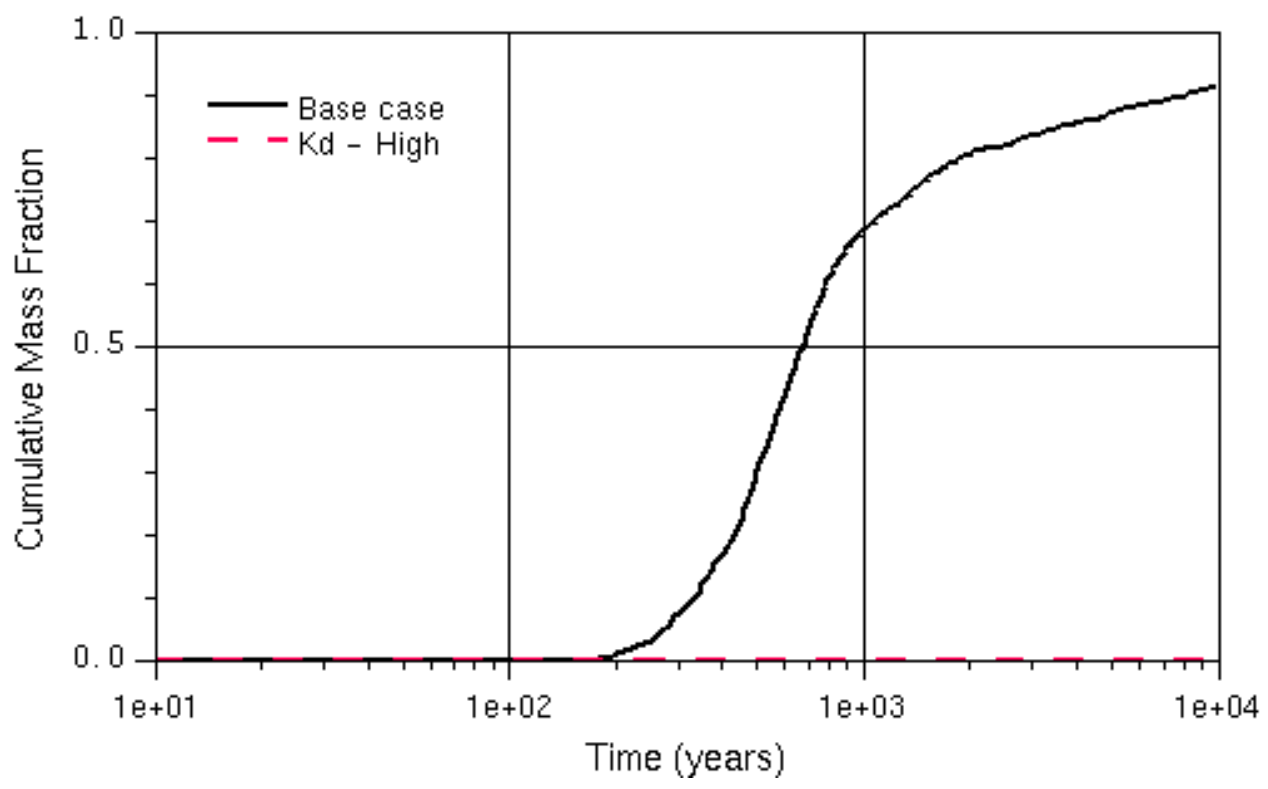

Output DTN: LA0309SK831231.001.

NOTES: Mass breakthrough curves and median transport times are for an instantaneous source, present-day climate, and do not include radionuclide decay. Particle source locations were distributed over the anticipated repository footprint.

The output results are stored in folder kd-alvm-h.d in file sens_kd-alvm-h.sptr3. Column 1 is the elapsed time in days. Plotted on the $Y$ axis is the third column (the total number of particles crossing the boundary by this time) divided by 1,000 .

Figure 6.7-4. Propagation of Input Uncertainty in the Sorption Coefficient in Alluvium to the Output Breakthrough Curves at the 18-km Boundary

\subsubsection{Effective Porosity in Alluvium}

The results from simulations that evaluate the effect of changes in the effective porosity in the alluvium are shown in Figure 6.7-5. Note that in these simulations, the influence of the alluvium porosity on the retardation factor (Equation 57) does not show up because the base case does not include sorption. Thus, the effect shown in Figure 6.7-5 is that resulting from the effect of porosity on the fluid velocity through the relation:

$$
\bar{v}=\frac{\bar{u}}{\theta}
$$

where

$\bar{u}$ is the Darcy velocity obtained from the base-case flow model

$\bar{v}$ is the fluid velocity

$\theta$ is the porosity. 


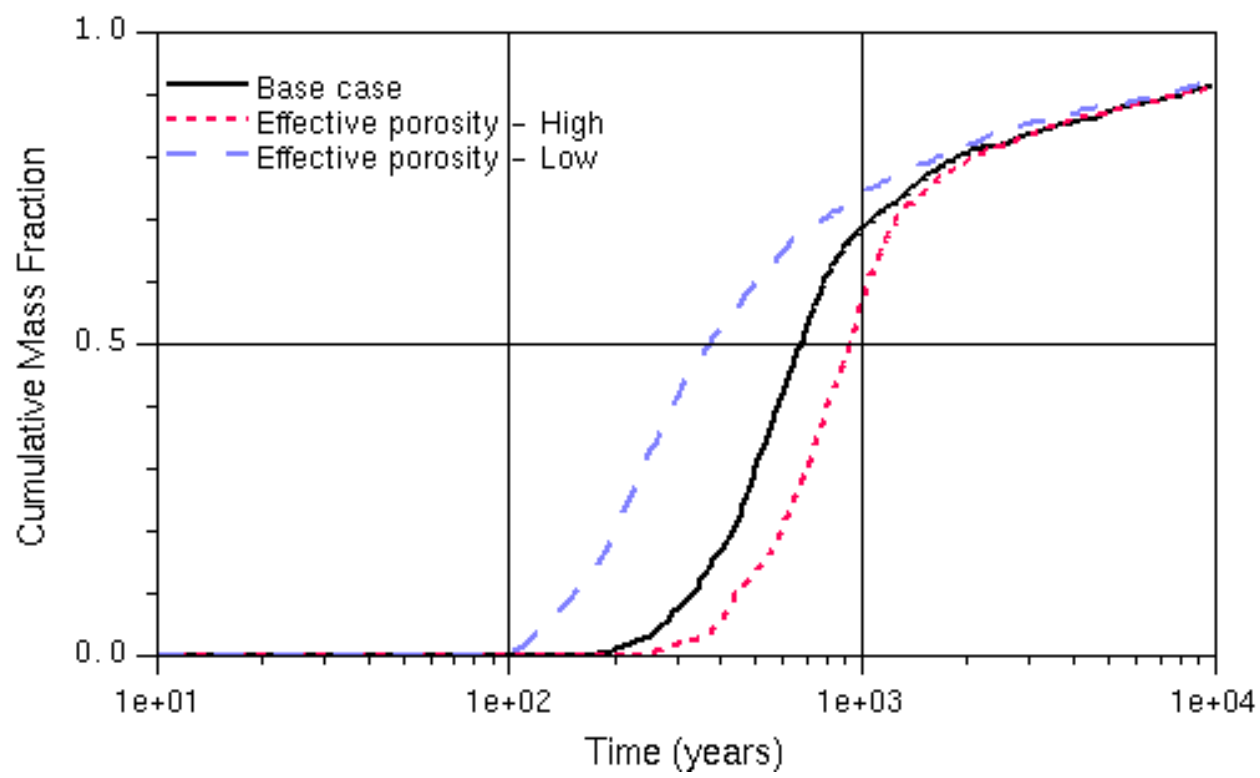

Output DTN: LA0309SK831231.001.

NOTES: Mass breakthrough curves and median transport times are for an instantaneous source, present-day climate, and do not include radionuclide decay. Particle source locations were distributed over the anticipated repository footprint.

The output results are stored in folder effporalvm-h.d in file sens_effporalvm-h.sptr3 and in folder effporalvm-I.d in file sens_effporalvm-I.sptr3. Column 1 is the elapsed time in days. Plotted on the $Y$ axis is the third column (the total number of particles crossing the boundary by this time) divided by 1,000.

Figure 6.7-5. Propagation of Input Uncertainty in the Effective Porosity of Alluvium to the Breakthrough Curves at the 18-km Boundary

A moderate sensitivity is seen in Table 6.7-1 with the 50 percent breakthrough time spanning 349 to 913 years for the input range of values from 0.02 to 0.3 .

\subsubsection{Retardation Factor in Alluvium for Radionuclides Attached Irreversibly to Colloids}

The results from simulations that evaluate the effect of changes in the colloid retardation factor in the alluvium are shown in Figure 6.7-6. As discussed in Section 6.4.2.6, these results include the retardation of the colloid particles due to reversible filtration in the porous media. The effect of colloids transported unretarded is applied in the TSPA-LA calculations after the breakthrough curves are calculated from the site-scale SZ transport model, and hence it is not considered here. For comparison, the base case, which considers nonsorbing radionuclides without any retardation, but includes matrix diffusion in the volcanic portion of the flow path, is also shown. Note that the retardation arises from the reversible filtration of the colloids in the alluvium. The range of uncertainty in the retardation factor of 8 to 5,188 translates into an output uncertainty range of 2,472 to greater than 10,000 years for the 50 percent breakthrough time. Recall that breakthrough time for the base case is 705 years. 


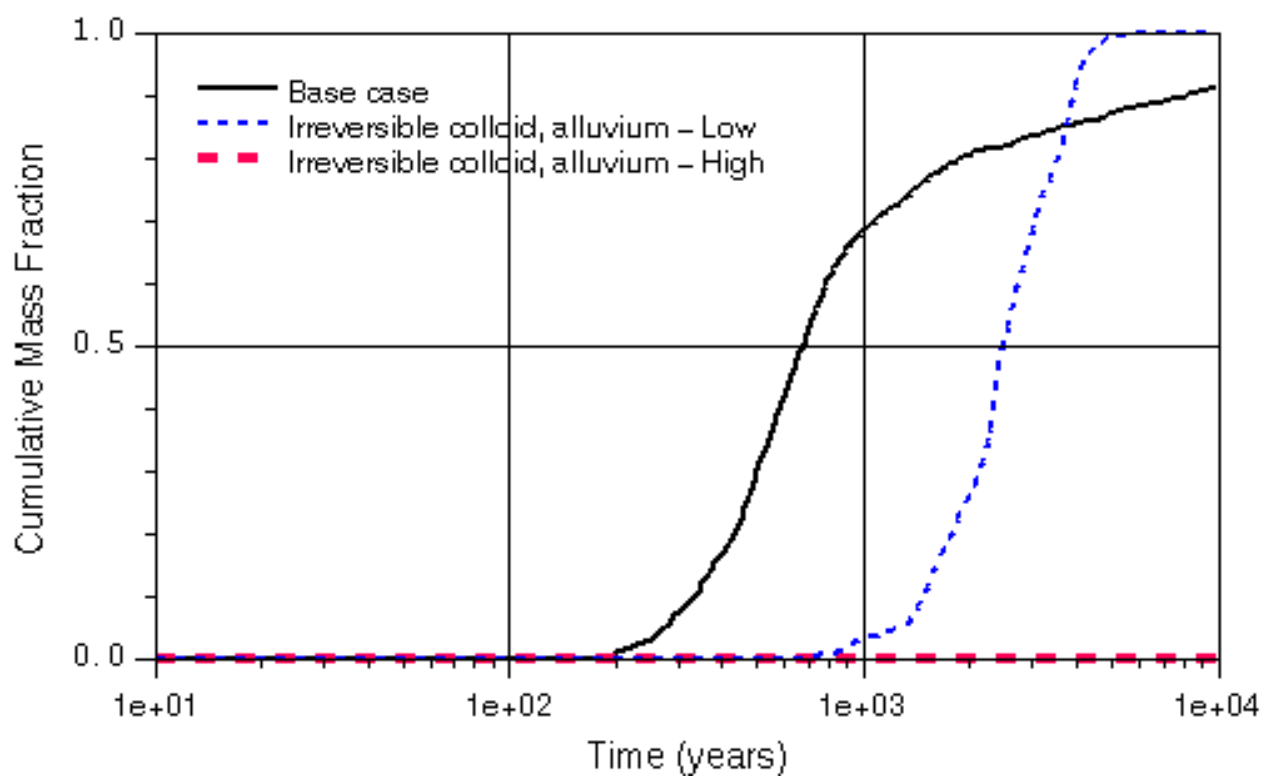

Output DTN: LA0309SK831231.001.

NOTES: Mass breakthrough curves and median transport times are for an instantaneous source, present-day climate, and do not include radionuclide decay. Particle source locations were distributed over the anticipated repository footprint.

Effect of colloids transported unretarded is applied in the TSPA-LA calculations after the breakthrough curves are calculated from the site-scale SZ transport model, and hence it is not considered here.

The output results are stored in folder coll-a-ir-h.d in file sens_coll-a-ir-h.sptr3 and in folder coll-a-ir-l.d in file sens_coll-a-ir-I.sptr3. Column 1 is the elapsed time in days. Plotted on the $Y$ axis is the third column (the total number of particles crossing the boundary by this time) divided by 1,000.

Figure 6.7-6. Propagation of Input Uncertainty in the Colloid Retardation Factor in Alluvium for Irreversible Colloids to the Breakthrough Curves at the 18-km Boundary

\subsubsection{Reversible Sorption onto Colloids in the Alluvium}

The results from simulations that evaluate the effect of changes in distribution parameters for reversible sorption onto colloids in alluvium are shown in Figure 6.7-7. Note that by using Equations 80a, 80b, and 81 of this report, the modified sorption coefficient for this case has the same range of values as the original sorption coefficient in alluvium; that is, 0 to $10,000 \mathrm{~mL} / \mathrm{g}$ (Table 6.7-1). Thus, the results for the lower limiting case are identical to the base case, and those for the upper limiting case show no breakthrough at all within 10,000 years. 


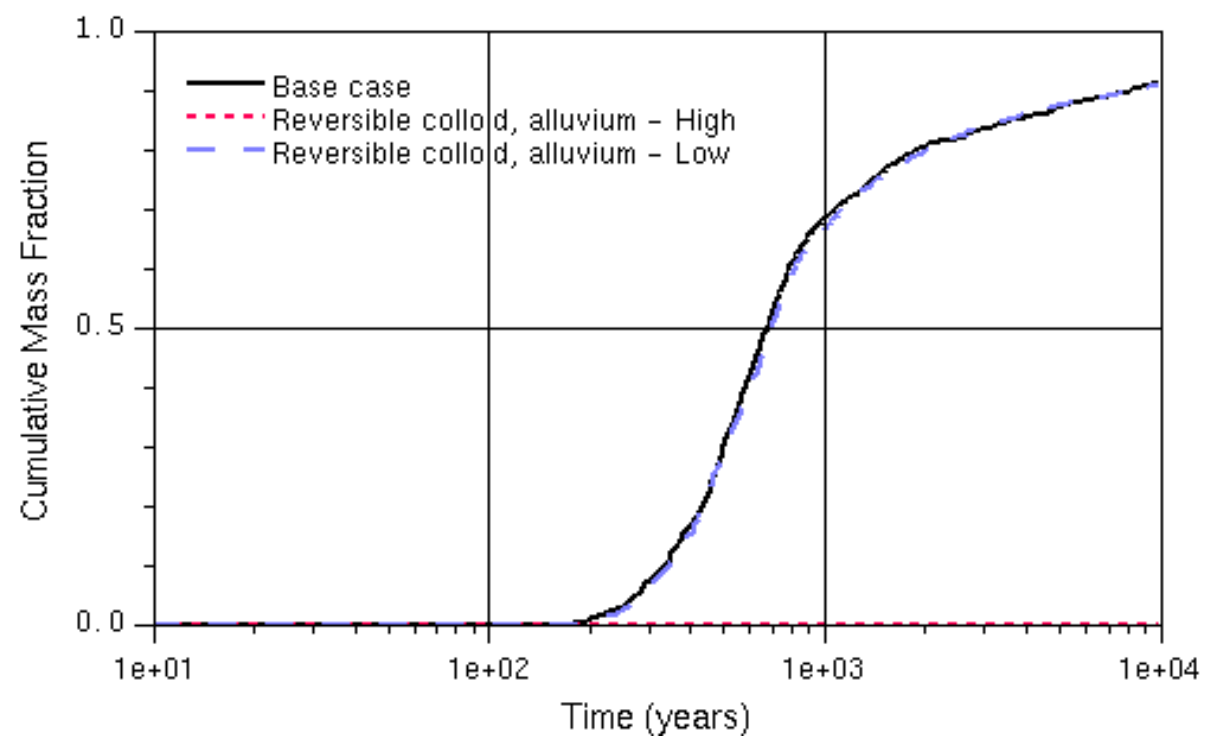

Output DTN: LA0309SK831231.001.

NOTES: Mass breakthrough curves and median transport times are for an instantaneous source, present-day climate, and do not include radionuclide decay. Particle source locations were distributed over the anticipated repository footprint.

The output results are stored in folder coll-a-rev-h.d in file sens_coll-a-rev-h.sptr3 and in folder coll-a-rev-l.d in file sens coll-a-rev-l.sptr3. Column 1 is the elapsed time in days. Plotted on the $Y$ axis is the third column (the total number of particles crossing the boundary by this time) divided by 1,000.

Figure 6.7-7. Propagation of Input Uncertainty in the Colloid Retardation Factor in Alluvium for Reversible Colloids to the Output Breakthrough Curves at the 18-km Boundary

\subsubsection{Flowing Interval Aperture in Volcanics}

The results from simulations that evaluate the effect of changes in flowing interval aperture in volcanics on the breakthrough curves are shown in Figure 6.7-8. When the flow aperture is at the lower end of the parameter distribution, $1.2 \times 10^{-5} \mathrm{~m}$ (Table 6.7-1), the retardation due to matrix diffusion is amplified so much that it overtakes the effects of increased velocities in the flowing interval. This results in no breakthrough observed during the 10,000 years considered. The reverse is true for the upper limit of the aperture, $41.7 \mathrm{~m}$, where there is very little diffusion, and breakthrough occurs somewhat earlier than in the base case (in 443 years rather than the base-case value of 705 years). 


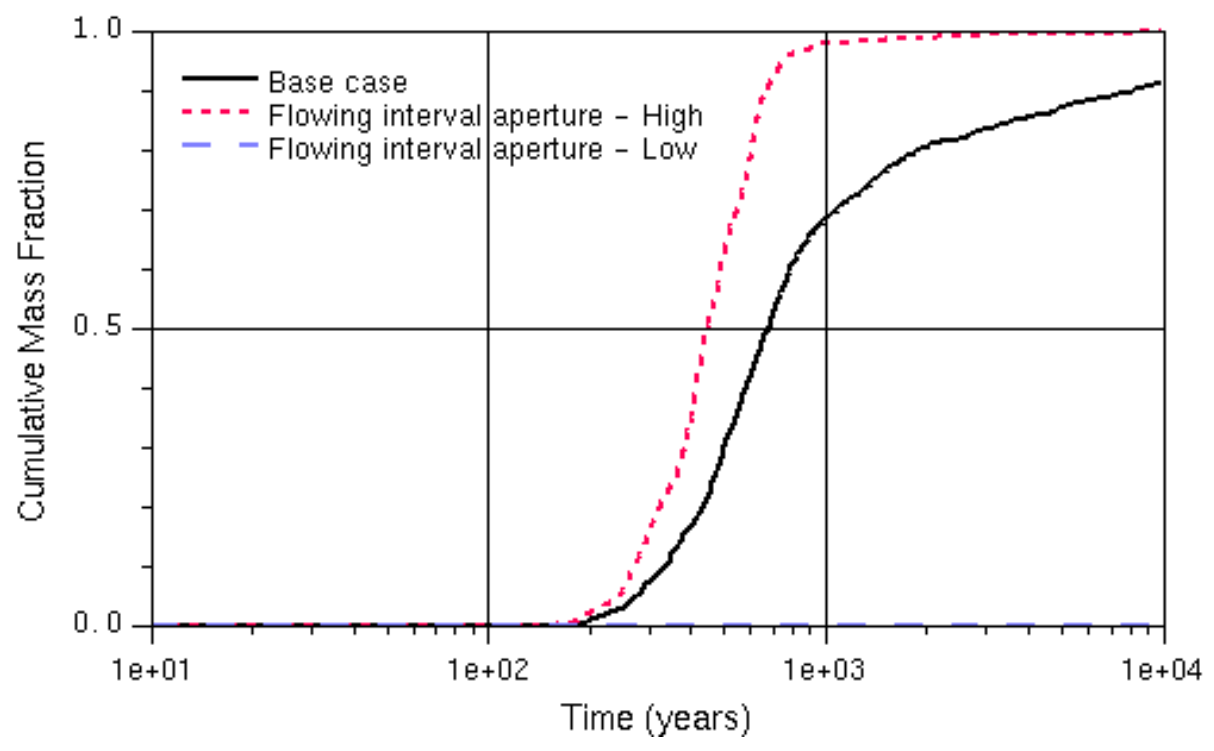

Output DTN: LA0309SK831231.001.

NOTES: Mass breakthrough curves and median transport times are for an instantaneous source, present-day climate, and do not include radionuclide decay. Particle source locations were distributed over the anticipated repository footprint.

The output results are stored in folder fi-aperture-h.d in file sens_fi-aperture-h.sptr3 and in folder fi-aperture-l.d in file sens_fi-aperture-l.sptr3 with the extension sptr3. Column 1 is the elapsed time in days. Plotted on the $Y$ axis is the third column (the total number of particles crossing the boundary by this time) divided by 1,000 .

Figure 6.7-8. Propagation of Input Uncertainty in the Flowing Interval Aperture in Volcanics to the Breakthrough Curves at the 18-km Boundary

\subsubsection{Effective Diffusion Coefficient in Volcanics}

The results from simulations that evaluate the effect of changes in effective diffusion coefficient in volcanics on the breakthrough curves are shown in Figure 6.7-9. The range of $5.0 \times 10^{-12}$ to $5.0 \times 10^{-10}\left(\mathrm{~m}^{2} / \mathrm{s}\right)$ in the input value results in the range of 520 to 2,100 years for output (50 percent breakthrough time). Considering that this parameter affects only the transport through the volcanics and not through that in the alluvium, it has a significant impact on the output. 


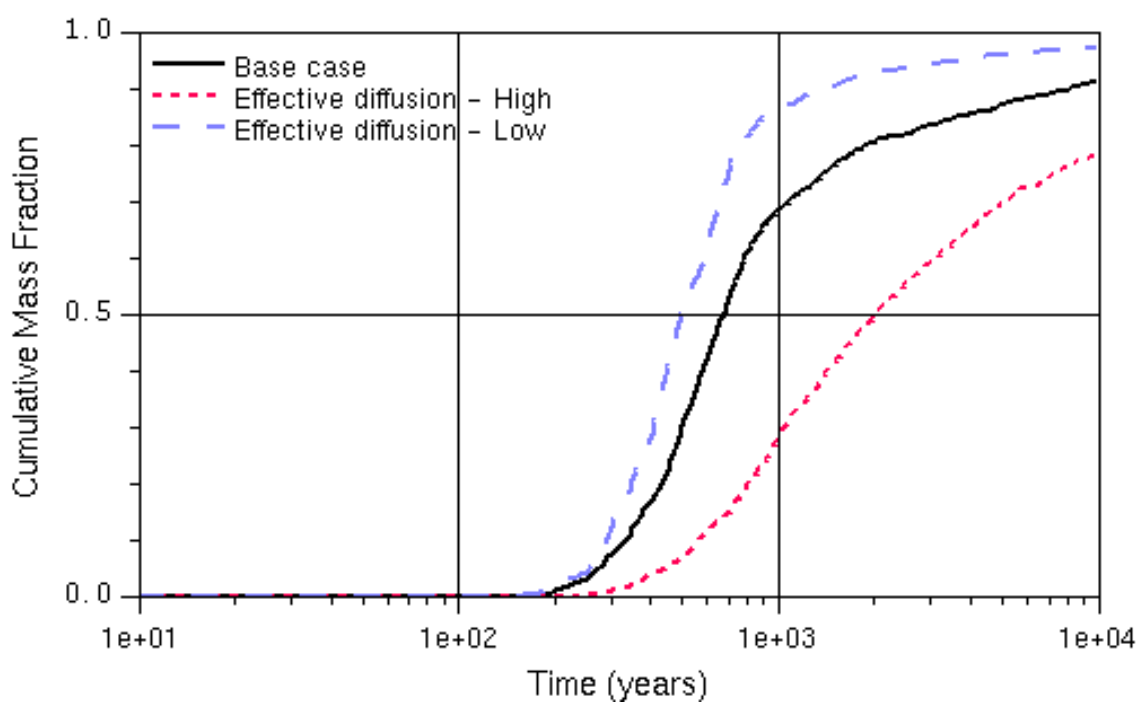

Output DTN: LA0309SK831231.001.

NOTES: Mass breakthrough curves and median transport times are for an instantaneous source, present-day climate, and do not include radionuclide decay. Particle source locations were distributed over the anticipated repository footprint.

The output results are stored in folder effdiffvol-h.d in file sens_effdiffvol-h.sptr3 and in folder effdiffvol-I.d in file sens_effdiffvol-I.sptr3. Column 1 is the elapsed time in days. Plotted on the $Y$ axis is the third column (the total number of particles crossing the boundary by this time) divided by 1,000.

Figure 6.7-9. Propagation of Input Uncertainty in the Effective Diffusion Coefficient in Volcanics to the Output Breakthrough Curves at the 18-km Boundary

\subsubsection{Matrix Sorption Coefficient in Volcanics}

The results from simulations that evaluate the effect of changes in matrix sorption coefficient in volcanics on the output breakthrough curves are shown in Figure 6.7-10. The results indicate that when the sorption coefficient is high $(10,000 \mathrm{~mL} / \mathrm{g})$, no breakthrough of the tracers is observed in 10,000 years. Thus, the volcanics could form a very effective barrier for those radionuclides that diffuse and adsorb in the matrix. The base-case simulation is the same as for the low-sorption case because the base-case value of the matrix sorption coefficient is 0 , which is the same as the lower limit value. 


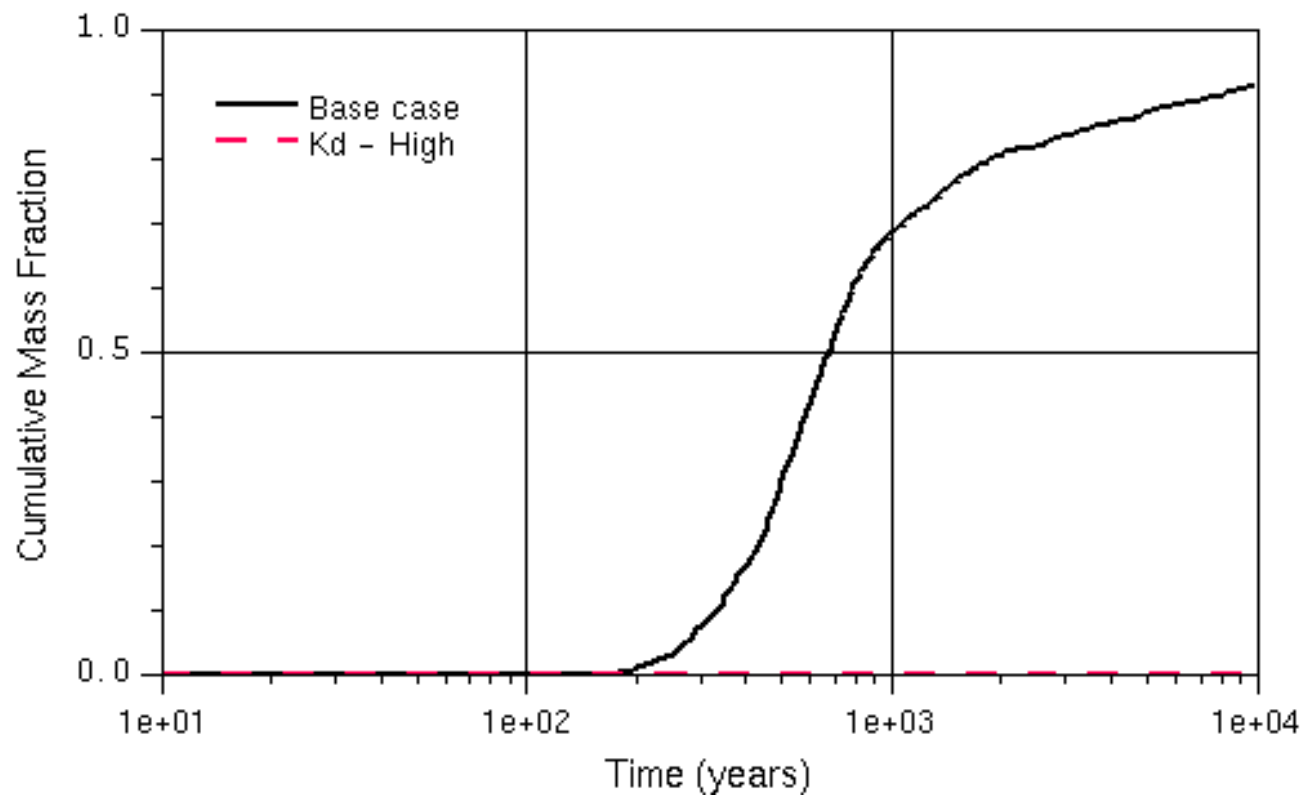

Output DTN: LA0309SK831231.001.

NOTES: Mass breakthrough curves and median transport times are for an instantaneous source, present-day climate, and do not include radionuclide decay. Particle source locations were distributed over the anticipated repository footprint.

The output results are stored in folder kd-volc-h.d in file sens_kd-v-h.sptr3. Column 1 is the elapsed time in days. Plotted on the $\mathrm{Y}$ axis is the third column (the total number of particles crossing the boundary by this time) divided by 1,000 .

Figure 6.7-10. Propagation of Input Uncertainty in the Matrix Sorption Coefficient in Volcanics to the Output Breakthrough Curves at the 18-km Boundary

\subsubsection{Retardation Factor in Volcanics for Radionuclides Attached Irreversibly to Colloids}

The results from simulations that evaluate the effect of changes in the colloid retardation factor on the transport of irreversibly sorbed radionuclides in volcanics on the output breakthrough curves are shown in Figure 6.7-11. As discussed in Section 6.4.2.6, these results include the retardation of the colloid particles due to reversible filtration in the porous media. The effect of colloids transported unretarded is applied in the TSPA-LA calculations after the breakthrough curves are calculated from the site-scale SZ transport model, and hence it is not considered here. For comparison, the base case, which considers nonsorbing radionuclides without any retardation, but includes matrix diffusion in the volcanic portion of the flow path, is also shown. Note that the retardation arises from the reversible filtration of the colloids in the alluvium. The range of uncertainty in the retardation factor of 6 to 794 translates into an output uncertainty range of 1,170 to greater than 10,000 years for the 50 percent breakthrough time. Recall that breakthrough time for the base case is 705 years. 


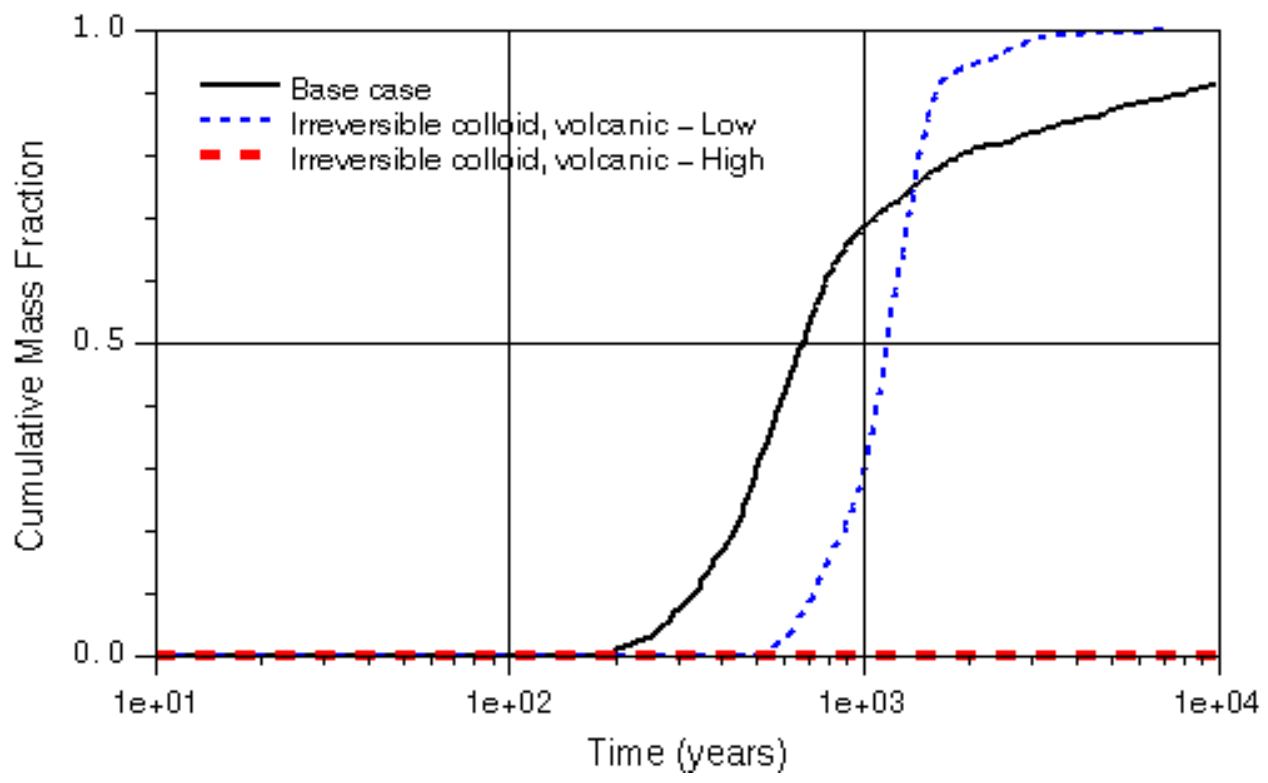

Output DTN: LA0309SK831231.001.

NOTES: Mass breakthrough curves and median transport times are for an instantaneous source, present-day climate, and do not include radionuclide decay. Particle source locations were distributed over the anticipated repository footprint.

Effect of colloids transported unretarded is applied in the TSPA-LA calculations after the breakthrough curves are calculated from the site-scale SZ transport model, and hence it is not considered here.

The output results are stored in folder coll-v-ir-h.d in file sens_coll-v-ir-h.sptr3 and in folder coll-v-ir-I.d in file sens_coll-v-ir-I.sptr3. Column 1 is the elapsed time in days. Plotted on the $Y$ axis is the third column (the total number of particles crossing the boundary by this time) divided by 1,000 .

Figure 6.7-11. Propagation of Input Uncertainty in the Colloid Retardation Factor in Volcanics for Irreversible Colloids to the Output Breakthrough Curves at the 18-km Boundary

\subsubsection{Reversible Sorption onto Colloids in the Volcanics}

As described in Section 6.4.2.6.2, transport of radionuclides attached reversibly to colloids is described by the distribution parameter $K_{c}$, which in turn is a product of the concentration of colloids in the groundwater and the sorption coefficient for the radionuclide onto the colloids. The results from simulations that evaluate the effect of changes in the distribution parameter $\mathrm{K}_{\mathrm{c}}$ for reversible sorption onto colloids in the volcanics are shown in Figure 6.7-12. Note that using Equations 80a, 80b, and 81, the modified sorption coefficient for this case has the same range of values as the original sorption coefficient in volcanics; that is, 0 to $10,000 \mathrm{~mL} / \mathrm{g}$ (Table 6.7-1). Thus, the results for the lower limiting case are identical to the base case, and those for the upper limiting case show no breakthrough at all within 10,000 years. 


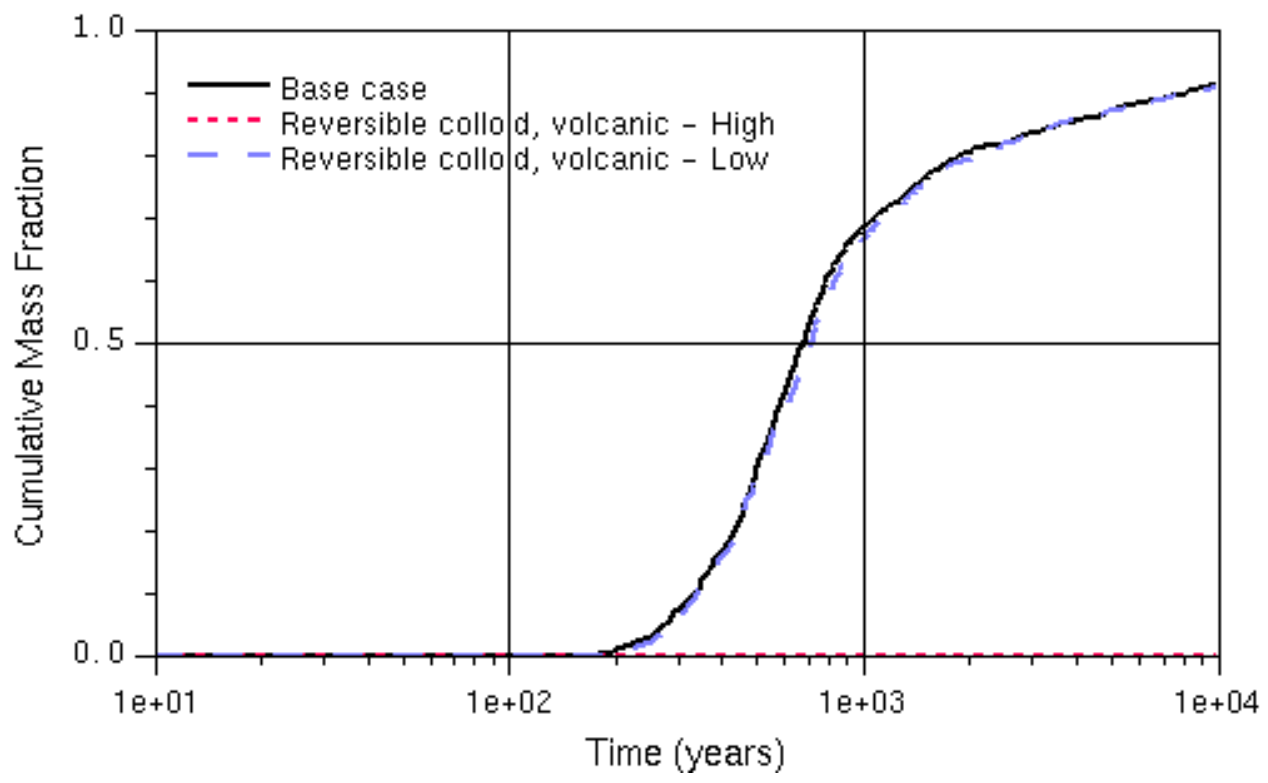

Output DTN: LA0309SK831231.001.

NOTES: Mass breakthrough curves and median transport times are for an instantaneous source, present-day climate, and do not include radionuclide decay. Particle source locations were distributed over the anticipated repository footprint.

The output results are stored in folder coll-v-rev-h.d in file sens_coll-v-rev-h.sptr3 and in folder coll-v-rev-l.d in file sens_coll-v-rev-I.sptr3. Column 1 is the elapsed time in days. Plotted on the $Y$ axis is the third column (the total number of particles crossing the boundary by this time) divided by 1,000.

Figure 6.7-12. Propagation of Input Uncertainty in the Distribution Parameter $\mathrm{Kc}$ in Volcanics for Reversible Colloids to the Output Breakthrough Curves at the 18-km Boundary

\subsubsection{Longitudinal Dispersivity}

The results from simulations that evaluate the effect of changes in longitudinal dispersivity on the output breakthrough curves are shown in Figure 6.7-13. In these simulations, the same longitudinal dispersivity values were applied to both the volcanics and the alluvium together. At the minimum value of dispersivity $(0.1 \mathrm{~m})$, the results with a breakthrough time of 664 years are only slightly different from the base case (which has a dispersivity value of $10 \mathrm{~m}$ ). This is because at low values of dispersivity, both the effects of spreading in the source term and diffusion tend to mask the effects of dispersion. At maximum values of dispersivity (2,000 m), an earlier low-concentration breakthrough is observed, which continues at a lower concentration relative to the base case for the duration of the simulation. The 50 percent breakthrough is delayed to 1,486 years. The high dispersivity case has an overall longer release time (start to end of release) than the other cases. 


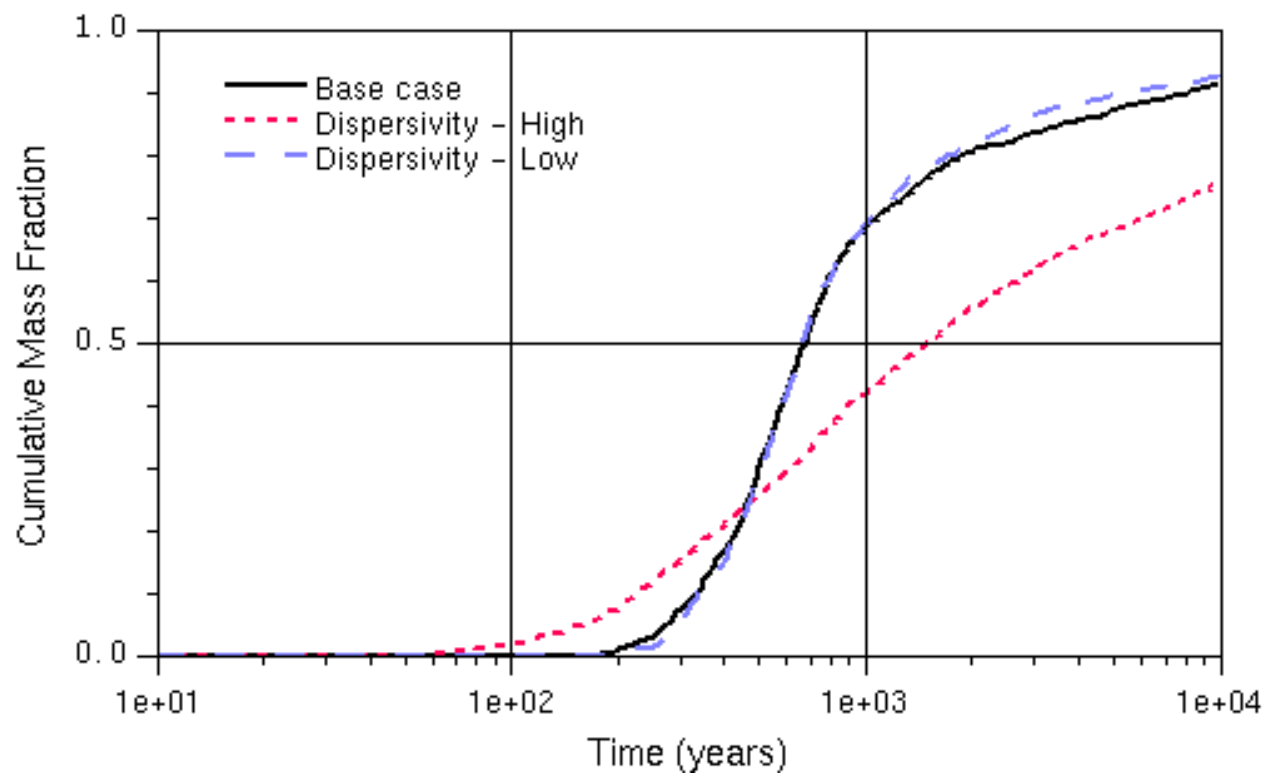

Output DTN: LA0309SK831231.001.

NOTES: Mass breakthrough curves and median transport times are for an instantaneous source, present-day climate, and do not include radionuclide decay. Particle source locations were distributed over the anticipated repository footprint.

The output results are stored in folder dispersivity-h.d in file sens_dispersivity-h.sptr3 and in folder dispersivity-I.d in file sens_dispersivity-I.sptr3. . Column 1 is the elapsed time in days. Plotted on the $Y$ axis is the third column (the total number of particles crossing the boundary by this time) divided by 1,000.

Figure 6.7-13. Propagation of Input Uncertainty in the Longitudinal Dispersivity to the Output Breakthrough Curves at the 18-km Boundary

\subsection{BARRIER CAPABILITY}

\subsubsection{Introduction}

This section presents transport model calculations designed to illustrate the function of the SZ barrier. These simulations explore in greater detail some of the key aspects of the system, important uncertain model parameters, physical properties, and boundaries to illustrate the functioning of the SZ as a barrier to radionuclide migration. Combined with the validation section (Section 7) and the analysis of model uncertainties (Section 8.3), this section describes the technical basis for the SZ barrier in the context of the Yucca Mountain waste disposal system.

In its simplest form, the SZ performs two functions in its role as a barrier to radionuclide migration: (1) It delays the transport of radionuclides from beneath the repository to the boundary of the accessible environment at $18 \mathrm{~km}$, and (2) it attenuates the concentration of radionuclides in the mobile water. By examining the processes of matrix diffusion, advection, and dispersion for various ranges of parameters, the role of various processes on the delay and dilution of radionuclides is presented. Also presented are intermediate breakthrough curves at the contact between the fractured volcanic tuffs and the alluvium to assess the relative importance of the two key hydrostratigraphic rock types. Because all parameters are estimated rather than known with certainty, a few key parameters in this section are varied to complement 
the results from Section 8.3. Finally, transport simulations for the fraction of radionuclides bound to colloids are also examined because of the importance of this process to radionuclide transport predictions in the SZ.

\subsubsection{Saturated Zone Subsystem Performance: Sorption in Volcanics and Alluvium}

Most radionuclides are expected to sorb to the rock, which should delay their arrival at the boundary of the accessible environment at $18 \mathrm{~km}$. Figure 6.8-1a also shows several simulations of sorbing radionuclides. The curve labeled "Matrix sorption" allows sorption in the matrix continuum of the volcanic tuffs and reflects a small matrix sorption coefficient of $1.3 \mathrm{~mL} / \mathrm{g}$. Including only this process yields a breakthrough curve similar to the base-case breakthrough curve but with significantly delayed transport times. The final two curves in the figure show the influence of sorption in the alluvium (either with or without sorption in the fractured volcanics). Travel times largely in excess of 10,000 years are predicted in the SZ alone for a sorption coefficient of $6.3 \mathrm{~mL} / \mathrm{g}$, meant to fall in the range of $K_{d}$ values for weakly sorbing radionuclides such as neptunium. It is seen from these curves that sorption in the alluvium can increase the transport time by orders of magnitude of even the weakly sorbing radionuclides such as neptunium. Note that the results presented in Figure 6.8-1a do not include the effects of colloidfacilitated transport; those are discussed in Section 6.8.5. Thus, for the base-case site-scale SZ transport model with the inclusion of sorption in the alluvium but excluding the effects of colloid facilitated transport, these results demonstrate that the SZ barrier provides a transport time delay on the order of the regulatory time scale of interest for the repository for all but the conservative or very weakly sorbing radionuclides.

\subsubsection{Saturated-Zone Subsystem Performance: Fractured Volcanic Tuffs and Alluvium}

To illustrate in more detail the function of the SZ, breakthrough curves were computed at an intermediate location in the model at a boundary defined by the transition from fractured volcanic tuffs to alluvium. Figure 6.8-1b shows breakthrough curves for the base-case and matrix sorption scenarios at the volcanic/alluvium boundary, which is approximately $10 \mathrm{~km}$ south of the southern boundary of the repository footprint. Comparing these simulations to the equivalent curves in Figure 6.8-1a, it is observed that the early parts of the breakthrough curves differ from each other, but the latter parts are very close to each other. This shows that alluvium plays a significant role for short transport times. By contrast, the tails of the breakthrough curves are due primarily to transport through fractures and matrix diffusion. Therefore, while the fractured volcanic tuffs provide significant delay for a fraction of the mass, the fastest moving portion of a radionuclide is controlled by transport through the alluvium. The reason for this result is that the continuum flow and transport through the bulk medium characterizes alluvium transport, in contrast to the fracture transport characterized by dual-porosity in the volcanics. Finally, based on the matrix sorption breakthrough curve in Figure 6.8-1b, sorption in the fractured tuffs is expected to provide significant transport-time delays, even without considering the subsequent transport through the alluvium. 


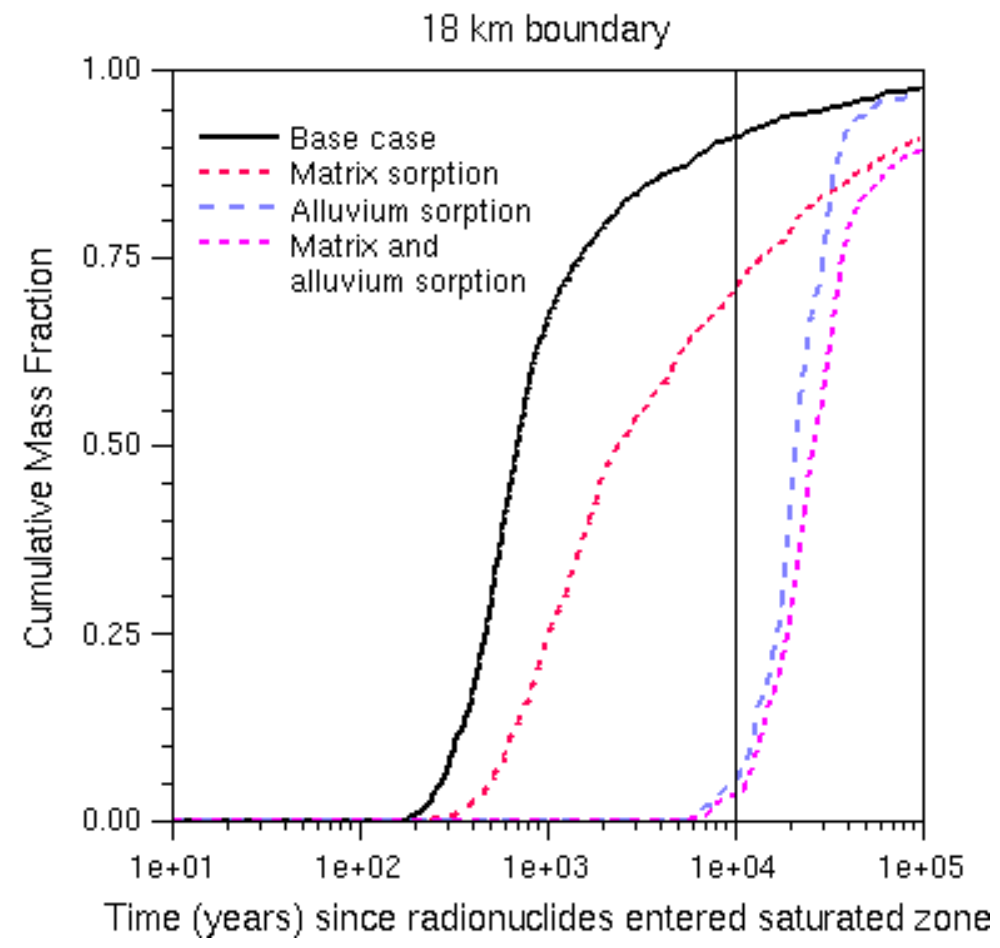

Output DTN : LA0403SK831231.001.

NOTE: Mass breakthrough curves and median transport times are for an instantaneous source, present-day climate, and do not include radionuclide decay. Particle source locations were distributed over the anticipated repository footprint.

Figure 6.8-1a. Breakthrough Curves for the Base Case, Conservative Radionuclide, and Sorbing Radionuclides: 18-km Boundary

\subsubsection{Saturated-Zone Sensitivity Analyses: Advection, Diffusion, and Dispersion}

The processes of advection, dispersion, and diffusion into the rock matrix all play key roles in the prediction of SZ barrier performance. In this section, the importance of these processes is illustrated by examining breakthrough curves for a variety of scenarios in which one or more parameters are changed to isolate a particular process. These results are presented in the context of SZ transport barrier performance, focusing on results relevant to the arrival times and dispersion of radionuclides. Figures 6.8-2a (18-km boundary) and 6.8-2b (volcanic/alluvium contact) show the breakthrough curves for a conservative radionuclide for the base case, a case with matrix diffusion but no hydrodynamic dispersion (referred to hereafter simply as diffusion and dispersion, respectively), and a case with dispersion but no diffusion. Both diffusion and dispersion result in the spreading of breakthrough curves at the 18-km boundary (or the volcanic/alluvium contact). The implication for radionuclide dilution is that any sharp pulse of high concentration reaching the SZ would be attenuated due to diffusion and dispersion by the time that mass reached the boundary of the accessible environment at $18 \mathrm{~km}$. Additional discussion of this point is provided in Section 6.8.6. 


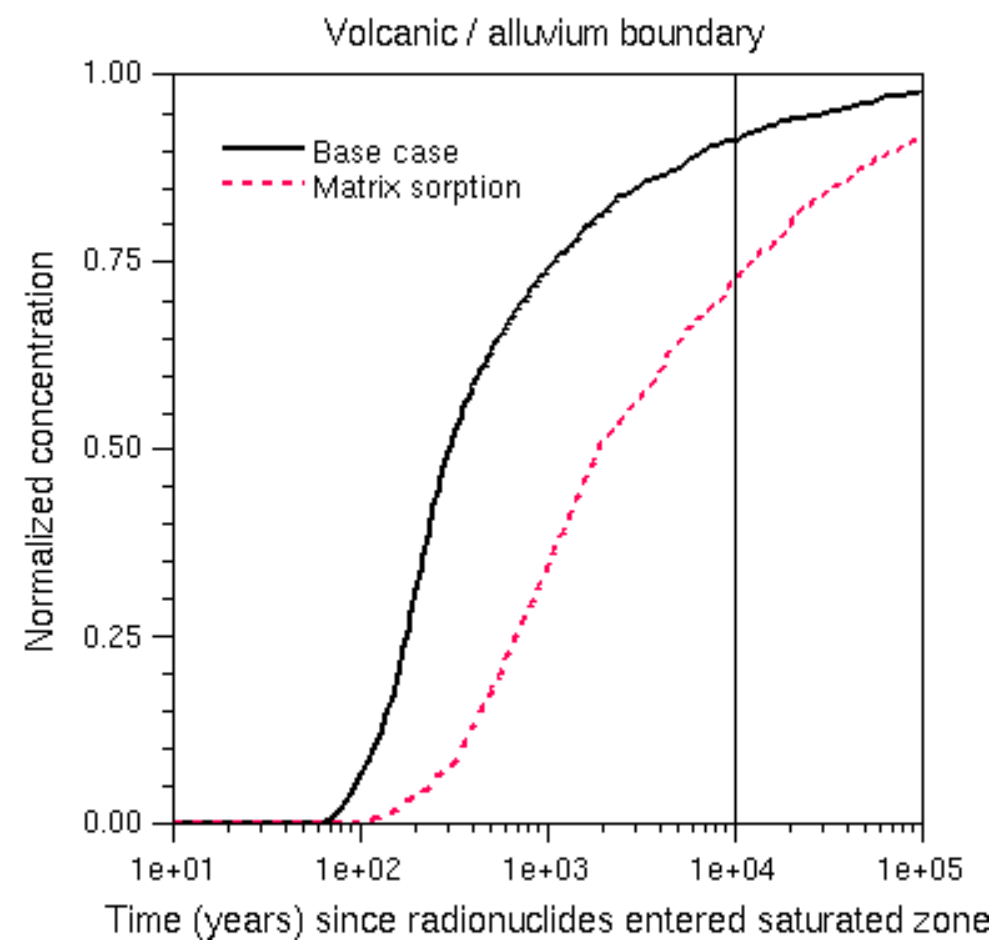

Output DTN: LA0403SK831231.001.

NOTE: Mass breakthrough curves and median transport times are for an instantaneous source, present-day climate, and do not include radionuclide decay. Particle source locations were distributed over the anticipated repository footprint.

Figure 6.8-1b. Breakthrough Curves for the Base Case, Conservative Radionuclides, and Sorbing Radionuclides: Volcanic/Alluvium Boundary

Regarding the relative importance of diffusion and dispersion in spreading the arrival time distribution, Figures 6.8-2a and 6.8-2b suggest that as long as diffusion in the volcanics matrix occurs, the additional spreading afforded by a dispersion mechanism is very small (note the close similarity of the base-case and "no dispersion" curves). By contrast, the no-diffusion breakthrough curve deviates significantly from the base case, showing the role of diffusion both for dispersing the mass and delaying the arrival times. One important factor to consider is that the dispersion being examined in this sensitivity analysis is only the hydrodynamic dispersion that occurs at scales smaller than the model grid block, as parameterized with the random-walk dispersion model. Larger-scale heterogeneities (e.g., hydrostratigraphic units of contrasting permeabilities, faults) are explicitly incorporated in the model. Therefore, because these large-scale dispersion mechanisms are "built into" the model, they are not turned off in the no-dispersion simulations presented here. Nevertheless, the breakthrough curve comparisons show the importance of diffusion as a mechanism for both delaying the transport times and spreading the distribution of the arrival times. Small-scale dispersion is relatively unimportant. 


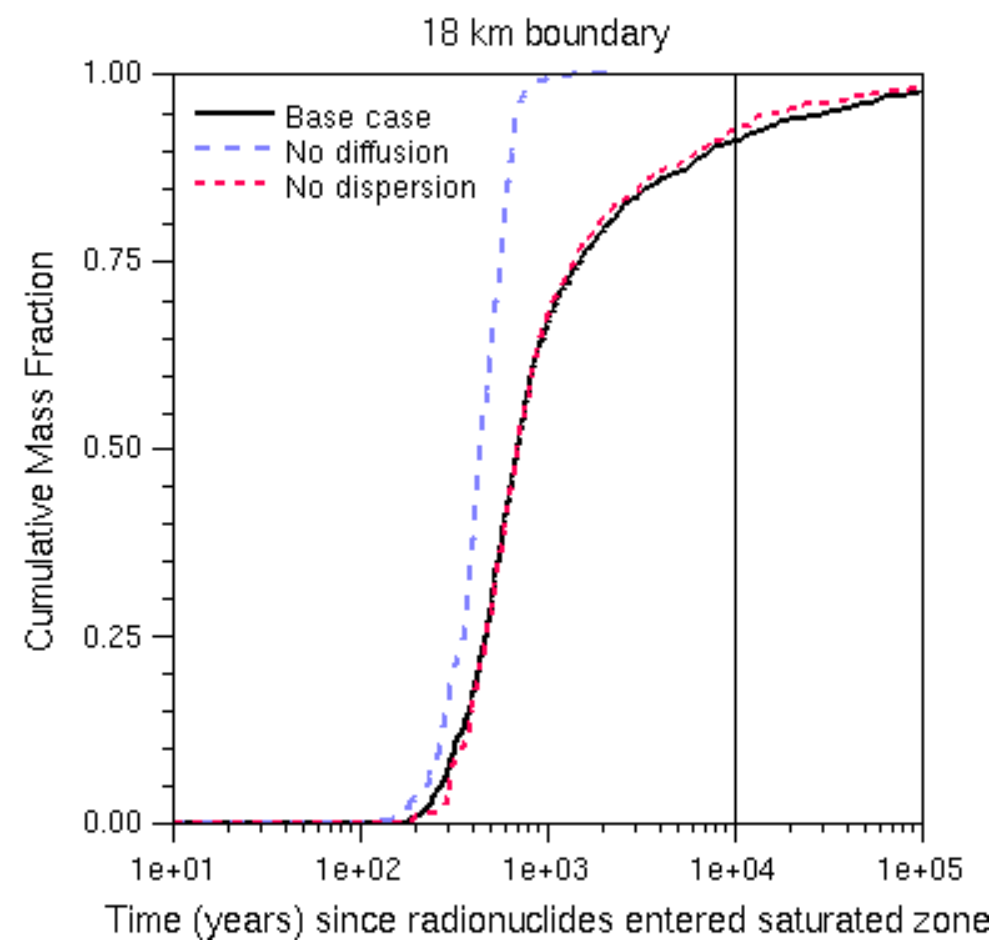

Output DTN: LA0307ZD831231.001.

NOTE: Mass breakthrough curves and median transport times are for an instantaneous source, present-day climate, and do not include radionuclide decay. Particle source locations were distributed over the anticipated repository footprint.

Figure 6.8-2a. Breakthrough Curves Comparing the Base Case, Nondispersive, and Nondiffusive Cases: 18-km Boundary

Another factor requiring examination is the spatial distribution of the contaminant source term. These breakthrough curves have been generated for a distributed release of contaminants, simulated by distributing a patch of particles throughout the repository footprint. There is a possibility that some of the spreading of arrival times is caused by the different starting locations of the particles, especially if there is a wide range of permeabilities and fluid fluxes directly beneath the repository. In contrast, if the radionuclide source term occurs due to the failure of only one or a few waste packages, the source term at the SZ would more closely resemble a point source. Figures 6.8-3a and 6.8-3b examine the role of the contaminant source in controlling the breakthrough curve by comparing the distributed source with one in which all particles are introduced at a single location near the center of the repository footprint. The breakthrough curve at the 18-km boundary (Figure 6.8-3a) for the case without diffusion is sharper than the one for the base case, and the difference between the curves is more pronounced at later times. This suggests that the spreading at early arrival times for nondiffusive transport is caused by the distributed source. However, diffusion into the rock matrix tends to mask this effect at later times. For example, in Figure 6.8-3b, a comparison of the base-case curves (solid black-distributed source; dashed green-point source) shows that apart from a slight difference in the first arrival times, the breakthrough curves track each other closely, suggesting that the details of the release location(s) at the repository footprint should have a relatively minor effect on the predicted breakthrough curve. 


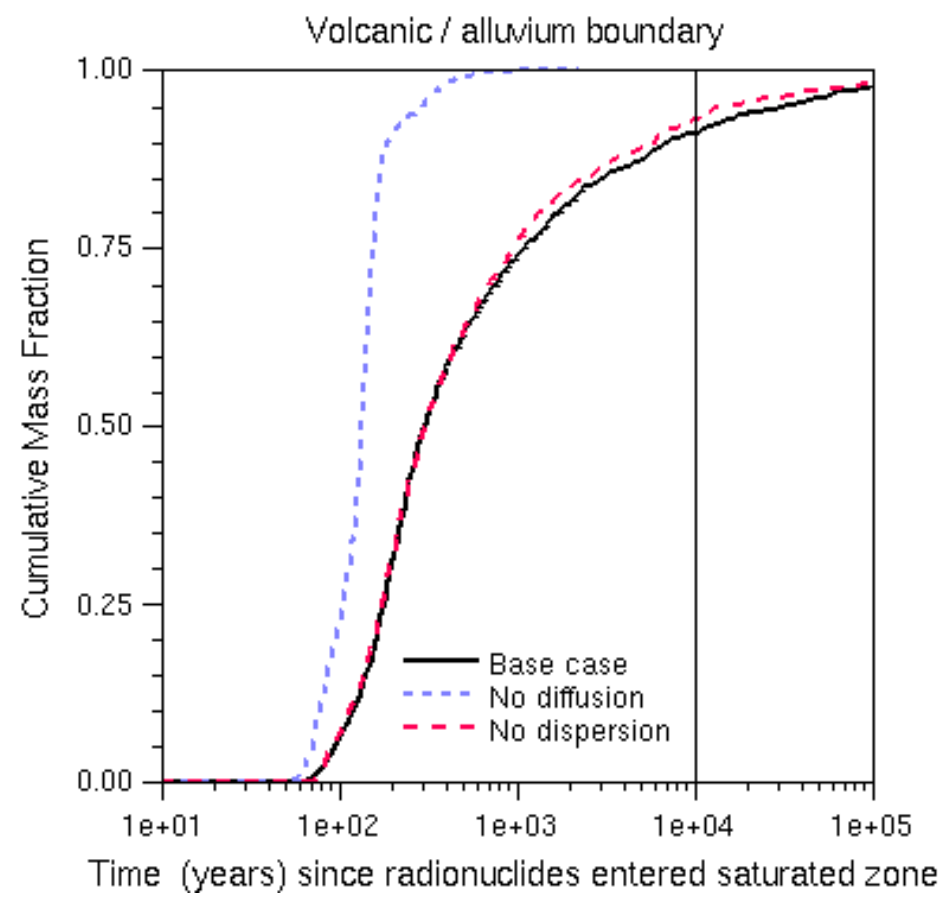

Output DTN: LA0307ZD831231.001.

NOTE: Mass breakthrough curves and median transport times are for an instantaneous source, present-day climate, and do not include radionuclide decay. Particle source locations were distributed over the anticipated repository footprint.

Figure 6.8-2b. Breakthrough Curves Comparing the Base Case, Nondispersive, and Nondiffusive Cases: Volcanic/Alluvium Boundary

Regarding advection, specific discharge is one of the most important uncertain parameters in the site-scale SZ transport model, owing to its first-order influence on solute velocity and the fact that available data and models can only provide relatively wide bounds on its estimated value. Figures 6.8-4a (18-km boundary) and 6.8-4b (volcanic/alluvium contact) show the predicted breakthrough curve for a conservative radionuclide over a broad range of values of specific discharge. This wide range of fluxes (Table 4-2) covers both the uncertainty in the specific discharge and the anticipated increases in groundwater flux caused by the change to a future, wetter climate. 


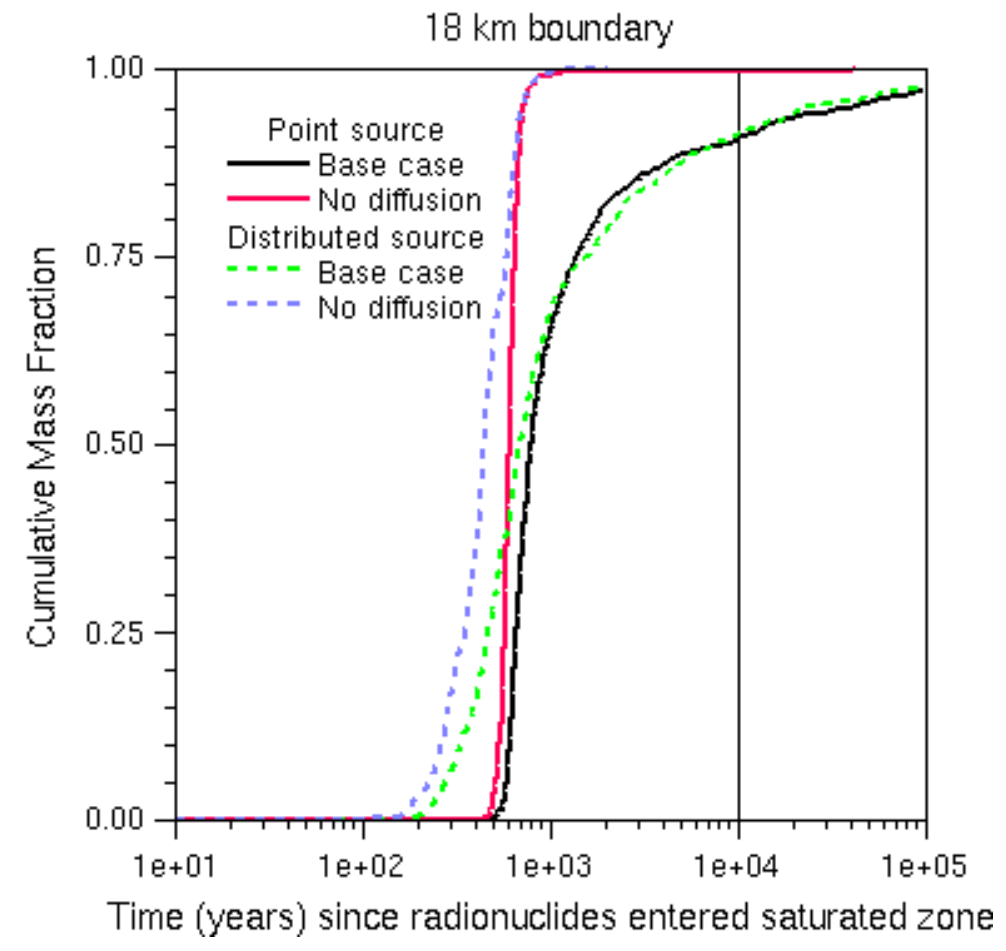

Output DTN: LA0307ZD831231.001.

NOTE: Mass breakthrough curves and median transport times are for an instantaneous source, present-day climate, and do not include radionuclide decay.

Figure 6.8-3a. Breakthrough Curves for the Base-Case Parameters with Point Source and Distributed Source for Input to the Saturated Zone: 18-km Boundary 


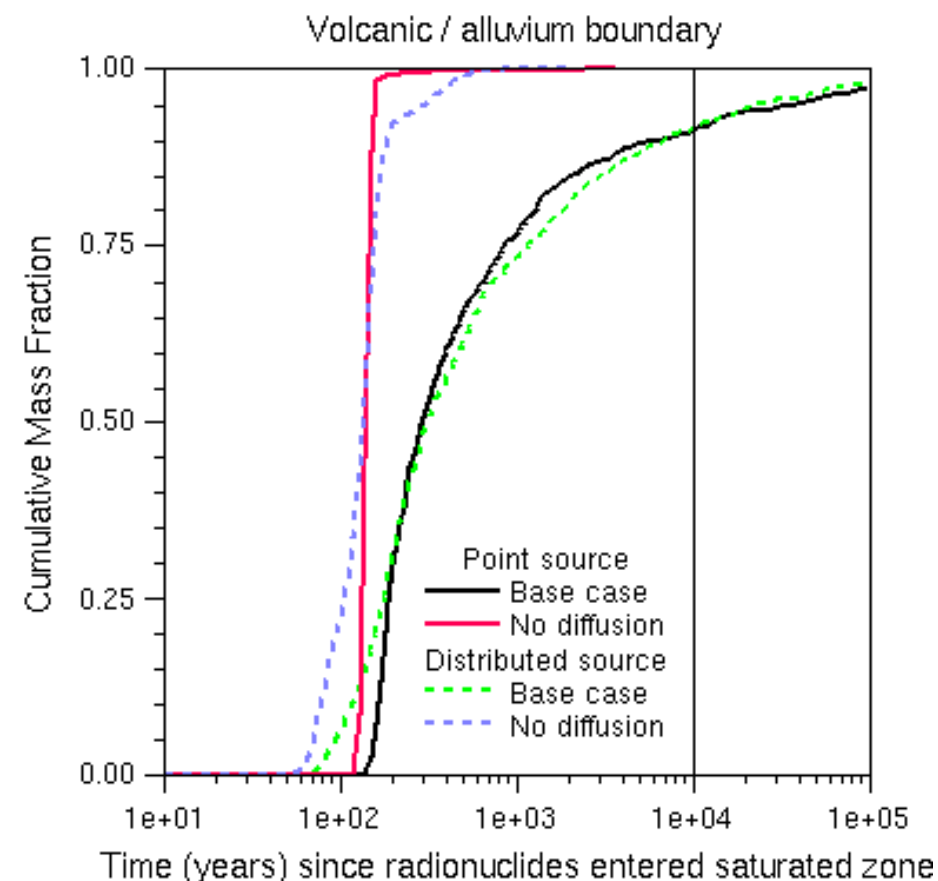

Output DTN: LA0307ZD831231.001.

NOTE: Mass breakthrough curves and median transport times are for an instantaneous source, present-day climate, and do not include radionuclide decay.

Figure 6.8-3b. Comparison of Breakthrough Curves for the Point Source and Distributed Source, Base Case, and Nondiffusive Case: Volcanic/Alluvium Boundary 


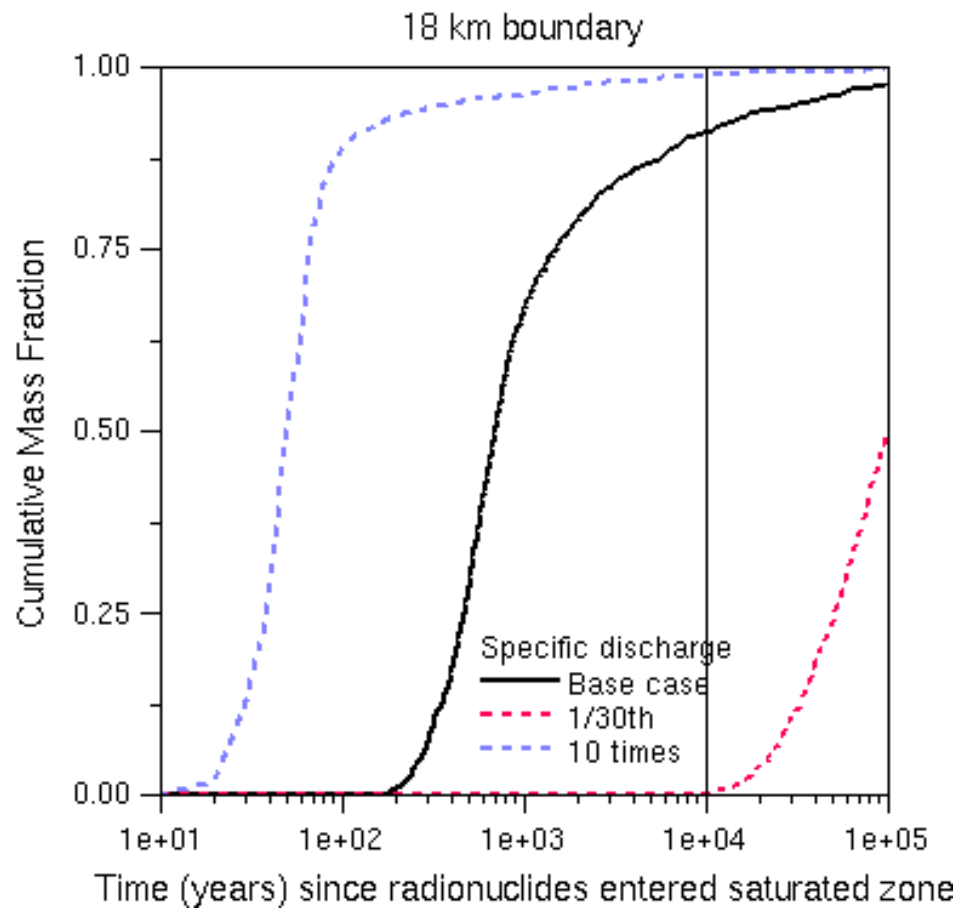

Output DTN: LA0307SK831231.002.

NOTE: Mass breakthrough curves and median transport times are for an instantaneous source, present-day climate, and do not include radionuclide decay. Particle source locations were distributed over the anticipated repository footprint.

Figure 6.8-4a. Breakthrough Curves for the Base Case and Cases with Lower and Higher Specific Discharge: 18-km Boundary 


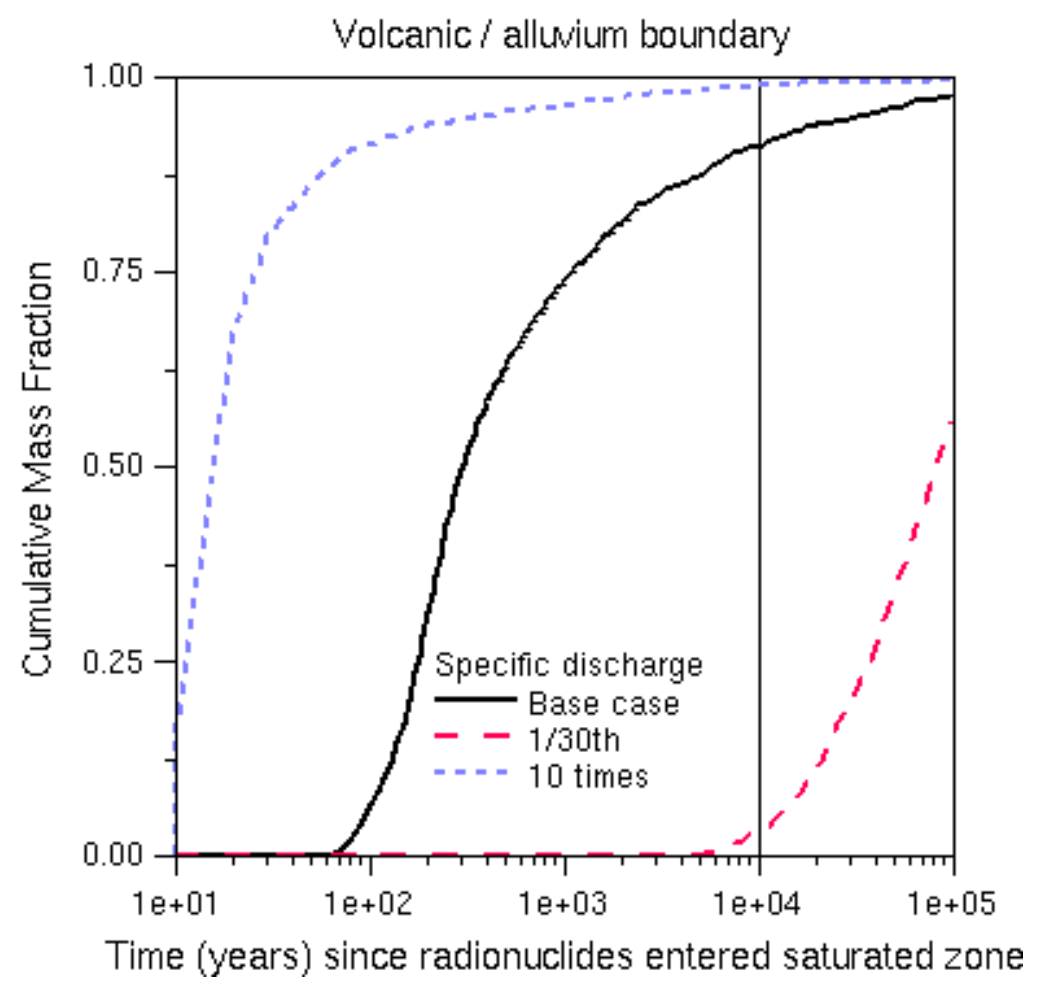

Output DTN: LA0307SK831231.002.

NOTE: Mass breakthrough curves and median transport times are for an instantaneous source, present-day climate, and do not include radionuclide decay. Particle source locations were distributed over the anticipated repository footprint.

Figure 6.8-4b. Breakthrough Curves for the Base Case and Cases with Lower and Higher Specific Discharge: Volcanic/Alluvium Boundary

\subsubsection{Colloid-Facilitated Transport in the Saturated Zone}

In the final set of simulations of this section, calculations are presented that illustrate the performance of the SZ barrier to retard the migration of radionuclides bound to colloids. Given that the most deleterious colloid-related effect on SZ performance is likely to be the mobility of otherwise immobile radionuclides via colloids, the focus of this section is on that process. For this reason, attention is also restricted to radionuclides irreversibly attached to colloids (termed "irreversible colloids" in the figures). These radionuclides do not diffuse into the volcanic matrix due to the large size of the colloids. Thus, the matrix diffusion coefficient is set to 0 in this simulation. Retardation occurs due to the reversible filtration of the colloids themselves in the alluvium and in the fractures within the volcanic units, modeled using a retardation factor that will be treated as a stochastic parameter in TSPA-LA calculations. The small fraction of the colloids that travels unretarded is considered explicitly in the TSPA-LA calculations and is not considered here. The colloid retardation factor distributions for the volcanics and the alluvium were constructed from numerous laboratory and field experiments. The construction of these distributions is described in detail in Saturated Zone Colloid Transport (BSC 2004 [DIRS 170006], Sections 6.4 and 6.5). Median values for the colloid retardation factor in alluvium of 33.9 and in the volcanics of 26 are used in the simulation, as given in Saturated Zone 
Flow and Transport Model Abstraction (BSC 2004 [DIRS 170042], Section 6.5.2; DTN: SN0310T0502103.009 [DIRS 168763]). Figure 6.8-5a shows the expected behavior of colloidal-species transport compared to the base-case aqueous species transport. First arrivals are predicted at about 5,000 years, while most of the inventory will arrive at the boundary of the accessible environment at $18 \mathrm{~km}$ later than 10,000 years. However, if larger specific discharge values were used, as would be the case for a future, wetter climate, the entire breakthrough curve would shift to transport times of earlier than 10,000 years. The difference between the aqueous and colloid breakthrough curves is attributable to reversible filtration of the colloids. Thus, the modeling suggests that for the fraction of the radionuclide inventory that reaches the SZ within the compliance time period, the SZ is expected to impart a significant transport-time delay, but perhaps not sufficient to prevent some of these radionuclides from reaching the biosphere. Finally, comparison of the colloid breakthrough curves of Figures 6.8-5a and 6.8-5b shows that transport of colloid-bound radionuclides in the volcanics accounts for about one fourth of the total transport time through the system.

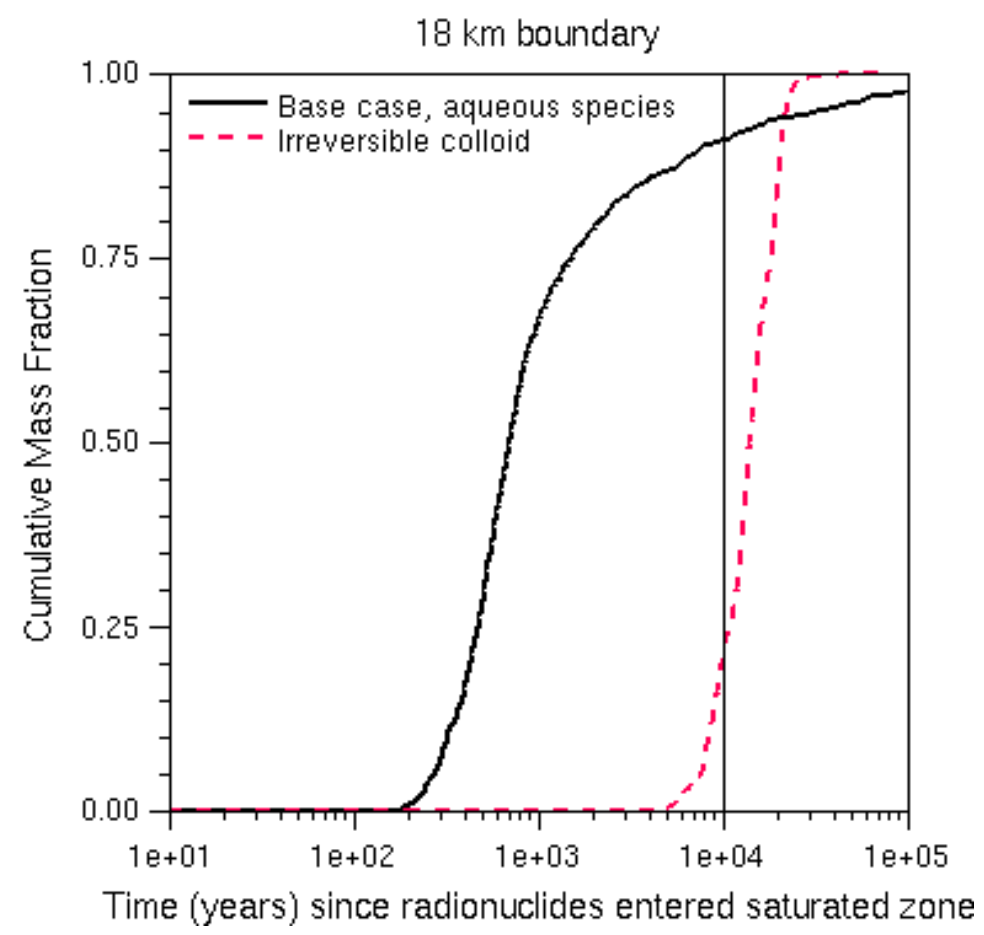

Output DTN: LA0307ZD831231.001.

NOTE: Mass breakthrough curves and median transport times are for an instantaneous source, present-day climate, and do not include radionuclide decay. Particle source locations were distributed over the anticipated repository footprint.

Figure 6.8-5a. Comparison of Breakthrough Curves for the Base Case and Radionuclides Irreversibly Attached to Colloids: $18-\mathrm{km}$ Boundary 


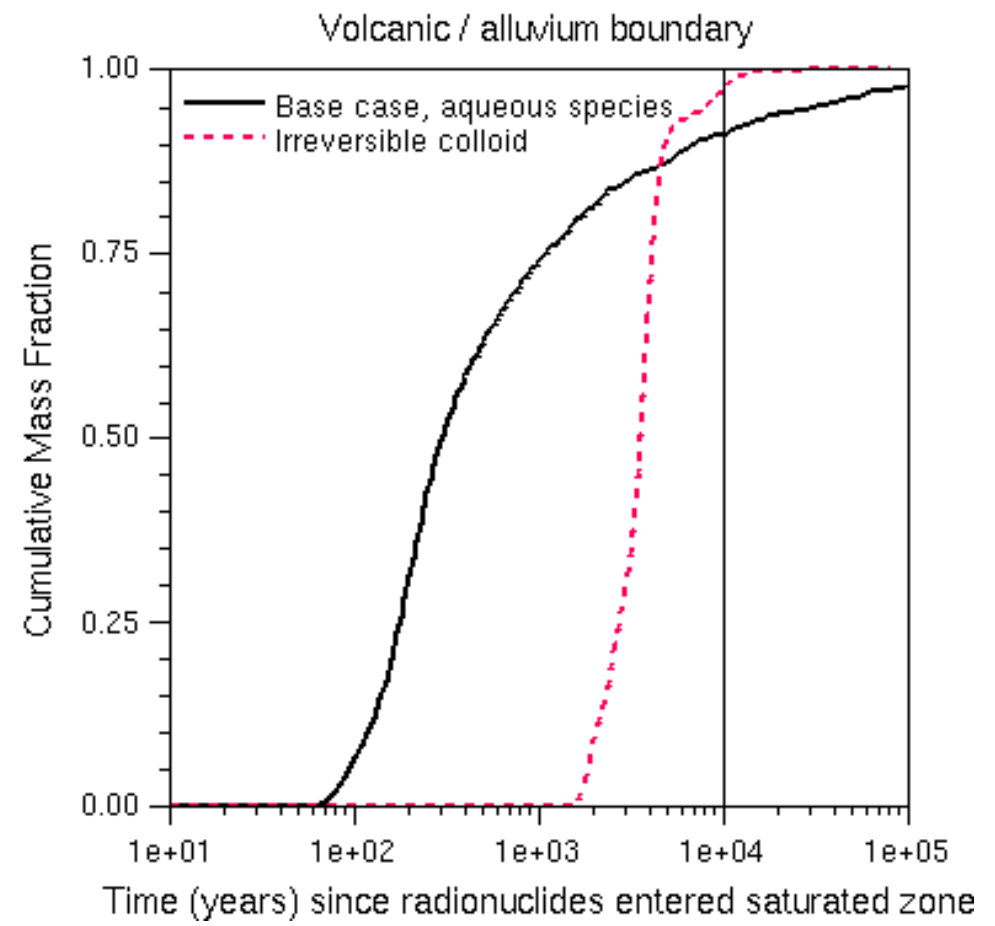

Output DTN: LA0307ZD831231.001.

NOTE: Mass breakthrough curves and median transport times are for an instantaneous source, present-day climate, and do not include radionuclide decay. Particle source locations were distributed over the anticipated repository footprint.

Figure 6.8-5b. Comparison of Breakthrough Curves for the Base Case and Radionuclides Irreversibly Attached to Colloids: Volcanic/Alluvium Boundary

\subsubsection{Discussion of Saturated-Zone Barrier Performance}

The two functions of the SZ barrier, which are the ability to delay the arrival and attenuate the radionuclides via the mechanisms of dispersion, diffusion and sorption, have been demonstrated in this section through a series of model simulations. The results and the discussion presented here are for the case of present day climate without water table rise (effects of alternate conceptual models resulting from water table rise due to possible future climate changes are discussed in Table 6.6-1a). The results presented here are for base-case transport property values (Table 6.4-2) unless explicitly noted otherwise. It is seen that sorption in the alluvium can increase the transport time by orders of magnitude of even the weakly sorbing radionuclides such as neptunium. Thus, for the base-case site-scale SZ transport model with the inclusion of sorption in the alluvium, transport times in excess of 10,000 years are expected within the SZ for all but the most weakly or nonsorbing radionuclides. For nonsorbing species, transport times on the order of 1,000 years are expected. Radionuclides irreversibly bound to colloids may be delayed by several thousand years, but the actual value is quite uncertain due to the multiplicative impact of uncertainties in the specific discharge and the effective filtration retardation factor of colloids. Transport times for radionuclides irreversibly bound to the fast fraction of colloids are expected to be on the order of 1,000 years, the same as those for the nonsorbing case. In essence, the SZ barrier provides a transport-time delay on the order of or 
greater than the regulatory time period of interest for many radionuclides, but not those expected to travel without significant retardation due to sorption or colloid filtration.

To understand the role played by the SZ barrier in attenuating radionuclides, the spread of arrival times at the boundary of the accessible environment at $18 \mathrm{~km}$ in the form of cumulative arrival-time distributions was examined, rather than simulating the ground water concentrations. The justification for this approach relates to the regulatory framework in which the modeling is being performed. Radionuclide mass flux is calculated by assuming that the entire annual radionuclide mass reaching the accessible environment is captured (10 CFR 63.332 (b)(2) [DIRS 156605]). The concentration is then calculated by assuming that this mass is uniformly distributed in the representative volume of groundwater that would be withdrawn annually (3,000 acre-feet per year as defined by 10 CFR 63.332(a)(3) [DIRS 156605]). Therefore, in situ concentrations are not relevant to barrier performance. The mass flux of radionuclides divided by the representative volume is the concentration of interest. Radionuclide mass flux at a boundary of the accessible environment at $18 \mathrm{~km}$ is a common metric used in studies of contaminant transport in groundwater (e.g., Dagan et al. 1992 [DIRS 163800], pp. 1369 to 1370). The approach taken here is patterned after the well-known solute mass flux approach. Hence, it is acceptable to use the particle-tracking model, although it has limitations requiring the use of a large number of input particles in applications where in situ concentrations are needed.

Nevertheless, attenuation of radionuclides during their transit from beneath the repository to the boundary of the accessible environment at $18 \mathrm{~km}$ can be treated qualitatively by recognizing that the spread of the arrival-time distribution is related to the ability of the SZ to dilute radionuclide concentrations. The approximate duration over which relative concentration in the breakthrough curves rise from 0 to 1 can be compared to the duration of a transient pulse of high concentration. If the latter is smaller than the former, then the SZ barrier will dilute the input pulse of high concentration to a lower value through the process of hydrodynamic dispersion and matrix diffusion. Thus, based on the breakthrough curves provided in this section, short-duration pulse of radionuclides of duration of about 100 years or less would become spread out in time within the SZ alone, to a high degree of certainty. Instantaneous output concentrations resulting from such a pulse will be significantly lower compared to the initial concentrations even though the cumulative mass breakthrough may be the same. This characteristic of the SZ barrier has implications on how the system is modeled for TSPA-LA. Specifically, it implies that short-time-scale, high-concentration pulses need not be simulated explicitly as long as the correct overall radionuclide mass is input into the model.

For reasons related to the discussion above, transverse dispersion, though included in the site-scale SZ transport model, was not examined in sensitivity studies of barrier performance because it is not likely to be important to the function of the barrier. Small transverse dispersivity values estimated for the SZ will spread radionuclides a short distance orthogonal to the principal transport direction. This would have a significant influence on the in situ concentration but not on the overall flow path through which the plume travels. Therefore, the mass flux reaching the boundary of the accessible environment will not be affected significantly. 


\section{VALIDATION}

The site-scale SZ transport model is designed to provide an analysis tool that facilitates understanding of solute transport in the aquifer beneath and downgradient from the repository. It is also a computational tool for performing radionuclide migration predictions in the SZ. For these predictions to be creditable, it must be demonstrated that the site-scale SZ transport model has been validated for its intended use. This statement means that there is established "confidence that a mathematical model and its underlying conceptual model adequately represents with sufficient accuracy the phenomenon, process, or system in question" (AP-SIII.10Q, Rev. 2, ICN 7, Models, Section 3.13).

The validation activities for the site-scale SZ transport model are carried out according to the Technical Work Plan For: Natural System - Saturated Zone Analysis and Model Report Integration (BSC 2004 [DIRS 171421], Work Package ASZM04) and Technical Work Plan for: Saturated Zone Flow and Transport Modeling and Testing (BSC 2003 [DIRS 163965], Section 2.3) (reference to the previous version of the technical work plan has been retained because it specifies the validation criteria to which this work was done. These criteria are not reproduced in the new version.) For validation of the site-scale SZ transport model, the technical work plan states that the Level II validation "will be achieved by satisfying the criteria listed in items a) through f) of Section 8 of AP-2.27Q, Planning for Science Activities, together with postmodel development validation methods based on corroborative field and laboratory data" (BSC 2003 [DIRS 163965], Section 2.3) The technical work plan specifies that postdevelopment validation of the transport model will include the following comparisons:

1. Quantitative comparison of predicted transit times from the repository footprint to the boundary of the accessible environment at $18 \mathrm{~km}$ with those derived from analyses of field hydrochemical and isotopic data. Validation will be considered acceptable if the range of model results is consistent with the range derived from the data. The distributions of transit times will be compared with groundwater ages to ensure that calculated breakthrough behavior is consistently faster than natural aging of the waters.

2. Qualitative comparison of predicted flow paths with flow paths from analysis of field hydrochemistry and isotopic data. Validation will be considered acceptable if the predicted flow paths starting at the repository footprint lie within the appropriate flow region inferred from the data.

As a result of the time and spatial scales involved and the fact that radionuclides cannot be used as tracers in field experiments, validation and confidence building for the site-scale SZ transport model relies on indirect data and inferences derived from technically related laboratory and field tests and natural analogues. Recognizing that the model is being used to perform probabilistic calculations in which parameter uncertainties are propagated through the model, the intent of the validation and confidence-building activities is to confirm that radionuclide parameters and processes operative at the Yucca Mountain site are adequately represented with sufficient accuracy in the site-scale SZ transport model. This confirmation is accomplished by a series of different approaches that include comparisons to analogue sites, model-data comparisons, and comparison with data published in referenced journals. 
The validation exercises performed here demonstrate that the parameters and processes selected have an experimental or observational basis and that the model-derived flow paths and transit times are consistent with field data. The model is validated for use in a stochastic analysis that establishes ranges of potential behavior of the SZ transport system. The site-scale SZ transport model is valid for simulating radionuclide transport in the SZ resulting in generation of radionuclide breakthrough curves several kilometers downstream from the source region. This model is intended for use with stochastic simulations using large uncertainty ranges for certain parameters such as specific discharge, fracture spacing and aperture, diffusion coefficient, and sorption coefficients. Care should be exercised in interpreting individual simulations for a single set of parameter values. Also, care should be exercised if the parameters used fall outside the range of parameter values given in this report.

\subsection{CONFIDENCE BUILDING DURING MODEL DEVELOPMENT TO ESTABLISH SCIENTIFIC BASIS AND ACCURACY FOR INTENDED USE}

Confidence building during model development was based on the available laboratory and analogue data as well as comparison with an independent site-scale groundwater flow model for the Yucca Mountain SZ (Winterle et al. 2003 [DIRS 163823], pp. 152 to 153). These data were not used for postdevelopment model validation, and only the comparison with the field hydrochemical and isotopic data were used as a postdevelopment method for satisfying the validation criteria as detailed in Section 7.2.

For Level II validation, the development of the model should be documented in accordance with the requirements of Section 5.3.2(b) of AP-SIII.10Q. The development of the site-scale SZ transport model was conducted according to the following criteria (italicized). The paragraphs following each criterion describe how it was satisfied.

1. Selection of input parameters and/or input data, and a discussion of how the selection process builds confidence in the model [AP-SIII.10Q, 5.3.2(b)(1) and AP-2.27Q Attachment 3, Level I (a)].

The inputs to the site-scale SZ transport model have all been obtained from controlled sources (Table 4.1), including discussion about selection of input and design parameters (Sections 4.1.2.1 through 4.1.2.18). Confidence building in submodel components of the site-scale SZ transport model was conducted through comparison of the conceptual model of SZ transport with the results of field tests conducted at YMP and analog sites to support transport parameters. These are presented in Section 7.1.2. Additional studies of input parameters based on observations at analogue sites are presented in Section 7.1.1, leading to increased confidence in the processes and parameters that are used in the transport model presented in this report. Thus, this requirement is considered satisfied.

2. Description of calibration activities, and/or initial boundary condition runs, and/or run convergences, simulation conditions set up to span the range of intended use and avoid inconsistent outputs, and a discussion of how the activity or activities build confidence in the model. Inclusion of a discussion of impacts of any nonconvergence runs [(AP-SIII.10Q 5.3.2(b)(2) and AP-2.27Q Attachment 3 Level I (e)]. 
The site-scale SZ transport model uses the calibrated SZ Flow model (DTN: LA0304TM831231.002 [DIRS 163788]) as a starting point. The SZ Flow Model is calibrated to available head data in the vicinity of Yucca Mountain. However, the radionuclide transport model itself has been developed in the absence of direct data on which to base predictions of radionuclide transport over the length and time scales of interest. Therefore, the transport model development does not include formal, numerical model calibration to data beyond that used for calibration of the flow model. Nevertheless, there are numerous lines of evidence available to provide confidence that the model, with associated parameters and uncertainty ranges, captures the range of uncertainty in radionuclide transport predictions from beneath the repository to the 18-km compliance boundary. Most field data sets provide confidence that the conceptual model for transport in the fractured volcanic tuff and the alluvium are appropriate for use in predicting SZ transport behavior. These tests and their use in building confidence in the model are presented in Section 7.3.

The site-scale SZ transport model generates breakthrough curves for a unit instantaneous source. Section 6.3, item 1 discusses the source term for the transport model. Section 6.3, item 9, and Section 6.4.2.3 discuss the transport boundary conditions downstream of the repository footprint. Sections 6.5 and 6.8 provide detailed discussion of various model results. As discussed in Section 6.4.2.3, particle tracking method of solving the advective-dispersion equation conserves mass and the method is free from the numerical convergence problems and numerical dispersion errors. Thus, this requirement is considered satisfied.

3. Discussion of the impacts of uncertainties to the model results including how the model results represent the range of possible outcomes consistent with important uncertainties. [(AP-SIII.10 Q 5.3.2(b)(3) and AP-2.27Q Attachment 3 Level 1 (d) and $(f)]$.

Discussion of model uncertainties is provided in Sections 6.7 and 8.3. Sensitivity of the output breakthrough curves to each of the uncertain input parameters is discussed in Sections 6.7.1.1 through 6.7.1.13.

4. Formulation of defensible assumptions and simplifications. [AP-2.27Q Attachment 3 Level I (b)].

Discussion of assumptions and simplifications are provided in Sections 5 and 6.3. The conceptual model of transport in the SZ and the components of the model are discussed in Section 6.3. As discussed in detail in Section 7.1.2, further confidence building in submodel components of the site-scale SZ transport model was conducted through comparison of the conceptual model of SZ transport with the results of field tests conducted at the C-wells complex, the ATC, and the CAMBRIC site on the NTS. Thus, this requirement is considered satisfied.

5. Consistency with physical principles, such as conservation of mass, energy, and momentum. [AP-2.27Q Attachment 3 Level I (c)] 
Consistency with physical principles is demonstrated by the conceptual and mathematical formulation in Sections 6.3 and 6.4, and the selection and use of the FEHM code in Section 3. The flow model is based on the principles of conservation of mass and energy, and Darcy's law. The transport model (Equation 1) is based on the principle of conservation of mass of the solutes. Thus, this requirement is considered satisfied.

\subsubsection{Analogue Studies to Support Transport Parameters}

The study of radionuclide transport parameters based on observations at analogue sites leads to increased confidence in the parameters that are used in the transport model presented in this report. The transport parameter values and processes for radionuclides of concern are sensitive to the site-specific geological and geochemical conditions. However, the site-scale SZ transport model is intended for use in making TSPA-LA predictions using a wide range of parameter input values that reflect uncertainty in the input. The analogue studies provide a qualitative comparison of the information with the parameters used in this model report. The analogue studies considered here are the NTS and uranium analogue sites-Uranium Mill Tailings Remedial Action sites and Los Alamos in the United States; El Borrocal in Spain; Palmottu, Finland; Alligator Rivers in Australia; Pocos de Caldas in Brazil; and Cigar Lake in Canada. Consistency between the transport parameters from the site-scale SZ transport model and those derived from analogue studies leads increased confidence in the selection and choice of ranges for the transport parameters.

\subsubsection{Studies Performed at the Nevada Test Site}

Discussed in this section are relevant information and insights gained from radionuclide migration studies performed at the NTS, which is the United States continental nuclear weapons testing site. As shown in Figure 7-1, Yucca Mountain and the NTS are near each other. The NTS results are relevant because the NTS geology and geochemistry are similar to those at Yucca Mountain. Between 1951 and 1992, 828 underground tests were conducted at the NTS at locations indicated in Figure 7-1 (Wolfsberg et al. 2002 [DIRS 162688]). In general, though not exclusively, tests were conducted below the water table, making radionuclide migration information potentially important to consider for the site-scale SZ transport model. Tests in Yucca Flat and Frenchman Flat were principally situated in Quaternary-aged alluvium, analogous to the alluvium downgradient from Yucca Mountain. In contrast, tests beneath Pahute Mesa were generally conducted in volcanic-rock aquifers, which is a useful analogue for the fractured volcanic tuffs beneath Yucca Mountain. Therefore, observations of radionuclide migration from these tests are useful corroborative information relevant to the main rock types in the flow path from beneath Yucca Mountain to the boundary of the accessible environment. 


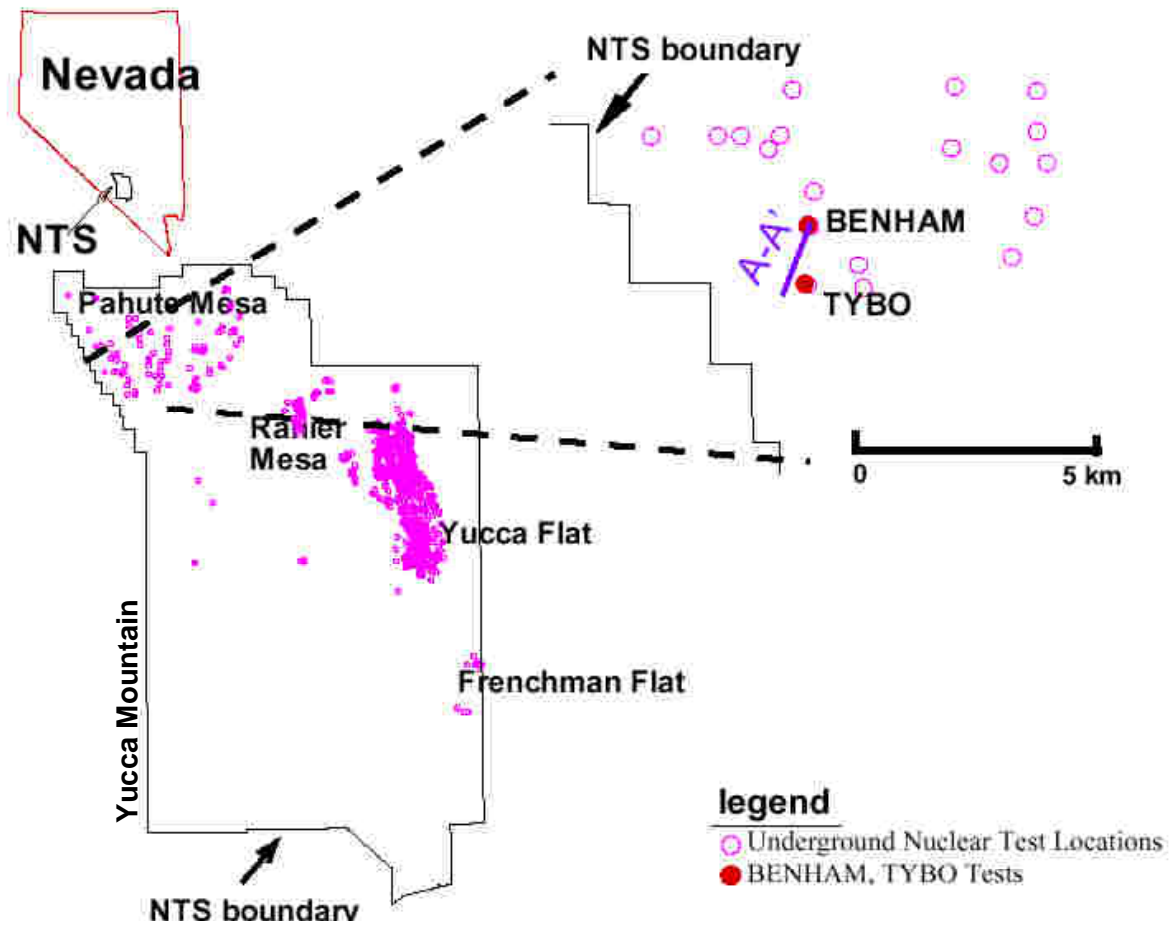

Source: Wolfsberg et al. 2002 (DIRS 162688), Figure 1-1.

Figure 7-1. Locations of Underground Nuclear Tests and the Specific Locations of the TYBO and BENHAM Tests at the Nevada Test Site

For several decades, radiological data have been gathered to examine the potential migration of radionuclides in groundwater away from underground nuclear tests. Smith summarizes the radiochemical investigations performed by the DOE Defense and Environmental Management Programs to characterize the current and potential future migration rates of radionuclides from these tests (2002 [DIRS 162687]). In addition to general conclusions about the relative mobility of radionuclides at the NTS, there are more site-specific results in both alluvium and volcanic-rock aquifers that provide points of reference for large-scale mobility under hydrogeologic conditions similar to the Yucca Mountain SZ. The next section summarizes the general conclusions, followed by detailed discussions on observations and testing at the CAMBRIC site and the ER-20-5 wells adjacent to the TYBO-BENHAM site. For the latter discussions, the NTS results are compared to the SZ transport conceptual model to demonstrate consistency with NTS data and analyses.

\subsection{General Conclusions from NTS Investigations}

To understand the similarities and differences between potential transport in the SZ beneath Yucca Mountain and migration away from an underground nuclear test, a brief conceptual description derived from Smith is provided (2002 [DIRS 162687], pp. 20 to 23). Transport from a nuclear test occurs in two steps: (1) a so-called "prompt transport" occurring over time scales of the nuclear detonation, and (2) groundwater transport occurring over a scale of years to centuries, depending on the flow regime. Prompt processes are generally thought to be as a plasma or gas through localized zones of failure in the rock. After this initial period, the system typically reverts over a period of years to a more ambient condition. However, the near-field 
system is permanently changed as a result of the nuclear detonation. Generally, a cavity is created containing a large inventory of radionuclides in the form of solidified glass. The interaction of the glass with groundwater results in a long-term source for radionuclides in the groundwater. Above the cavity, a so-called chimney of hydrologic-affected rock exists as a result of the underground explosion. If this chimney is of higher permeability than the surrounding rock, residual heat from the detonation can create a thermally buoyant water flow and radionuclide transport pathway up the chimney to zones of permeable rock in the aquifer. Then, groundwater flow under natural conditions transports radionuclides in the prevailing direction and at a velocity governed by the aquifer flow conditions.

Only the far-field flow and transport from underground tests are directly relevant to Yucca Mountain SZ transport. Therefore, data from downgradient wells provide the most useful information for the present study. However, the far-field observations contain inherent uncertainties due to the other transport processes (e.g., prompt transport and buoyant transport in the chimney). Additional indirect evidence on radionuclide mobility is also available by comparing the radionuclide concentrations in the cavity, chimney, and far-field fluids collected and summarized by Smith (2002 [DIRS 162687]). Caution must be exercised with cavity and chimney data because they can have unique thermal and geochemical conditions that could influence the speciation and sorption characteristics of the radionuclides. As a result, including radionuclide concentrations from cavities and chimneys could potentially introduce data that were collected under conditions that are not representative of the SZ beneath and downgradient of Yucca Mountain. Despite these limitations, the following summarizes Smith's conclusions on the relative mobility of radionuclides derived from cavity, chimney, and far-field radionuclide measurements (2002 [DIRS 162687], p. 31).

- The most mobile radionuclides at the NTS, traveling essentially unretarded compared to tritiated water $\left({ }^{3} \mathrm{H}\right)$, are ${ }^{14} \mathrm{C},{ }^{36} \mathrm{Cl},{ }^{85} \mathrm{Kr},{ }^{99} \mathrm{Tc}$, and ${ }^{129} \mathrm{I}$.

- By contrast, ${ }^{90} \mathrm{Sr},{ }^{137} \mathrm{Cs},{ }^{152} \mathrm{Eu},{ }^{154} \mathrm{Eu}$, and ${ }^{239} \mathrm{Pu}$ are relatively immobile, appearing in the cavity and chimney waters but generally not in the far field.

- Despite this conclusion, several radionuclides generally considered immobile, such as plutonium, sorb to natural colloids and appear to migrate over significant distances. For example, Kersting et al. showed that the concentration of plutonium observed in wells significantly downstream from the source was very small (approximately $10^{-14} \mathrm{M}$ ) and, therefore, concluded that only a very limited fraction of the plutonium associated with the test was mobile (1999 [DIRS 103282], p. 59). Additional details are provided in Section 7.1.1.1.3.

- Wolfsberg et al. report that cesium, strontium, and europium isotopes were also found in these wells in the presence of colloids (2002 [DIRS 162688], Section 7.7.3).

- Finnegan and Thompson detected ${ }^{237} \mathrm{~Np}$ in the same wells where the more reactive radionuclides listed above were found (2002 [DIRS 162695], pp. 13 to 14).

These results are qualitatively consistent with the site-scale SZ transport model conceptualization and parameter distributions. Sorption coefficients are set to zero for radionuclides found to be 
mobile at the NTS, most notably ${ }^{14} \mathrm{C},{ }^{36} \mathrm{Cl},{ }^{99} \mathrm{Tc}$, and ${ }^{129} \mathrm{I}$. The SZ base-case transport model represents the case of these radionuclides. For less-mobile radionuclides, such as ${ }^{90} \mathrm{Sr}$ and ${ }^{137} \mathrm{Cs}$, as discussed in Appendix A, relatively large sorption coefficients are recommended, and hence, significant retardation. The available CAMBRIC data, based on a lack of arrival of the radionuclides, can only lead to general conclusions regarding a plausible lower bound on $K_{d}$. But the data do not contradict the lower bound being higher, which is what is derived from the Lab data in Appendix A. Colloid-facilitated transport is included in the site-scale SZ transport model. Also it not possible to draw any conclusions from these data on following items: (1) upper limits on $K_{d} \mathrm{~S}$ and (2) differences in the $K_{d} \mathrm{~s}$ for different radionuclides. In general, the relative mobility of the different radionuclides included in the site-scale SZ transport model are consistent with the data available from the NTS radiological measurements.

\subsection{Radionuclide Transport in Alluvium (CAMBRIC Test)}

Transport from the CAMBRIC test, conducted in 1965 in the alluvium in Frenchman Flat, has been extensively studied in subsequent years to understand the rates of radionuclide migration in groundwater. Wells were drilled to obtain radionuclide concentrations in both the near field and far field, and a 16-year aquifer pump test was conducted to determine the migration rates through the alluvium from the test cavity to the pumping well under forced-gradient conditions. The pumping well RNM-2S was drilled $91 \mathrm{~m}$ south of the CAMBRIC test and screened from $16 \mathrm{~m}$ to $41 \mathrm{~m}$ below the bottom of the CAMBRIC cavity (Smith 2002 [DIRS 162687], p. 7). After about two years of pumping, essentially simultaneous breakthrough of ${ }^{3} \mathrm{H},{ }^{85} \mathrm{Kr},{ }^{36} \mathrm{Cl},{ }^{129} \mathrm{I}$, ${ }^{106} \mathrm{Ru}$, and ${ }^{99}$ Tc were observed. A tritium breakthrough curve published in Tompson et al. shows a peak concentration about 5.5 years after pumping started and a slow decline in concentrations thereafter, until the end of the test (1999 [DIRS 162686], Figure 35). By contrast, cations ${ }^{90} \mathrm{Sr}$, ${ }^{137} \mathrm{Cs},{ }^{152} \mathrm{Eu},{ }^{154} \mathrm{Eu}$, and ${ }^{239} \mathrm{Pu}$, though present in the cavity fluids, were not observed in the well during the 16 years of pumping (Smith 2002 [DIRS 162687], p. 7).

The lack of arrival of less-mobile radionuclides at the pumping well allows a lower bound to be placed on the retardation factor. As an order-of-magnitude estimate, lack of arrival after 16 years when the first arrival of tritium was about two years led to retardation factors of at least 8. Using the range of porosities of 0.1 to 0.38 reported in Thompson et al. (1999 [DIRS 162686], Table 20), this value of the retardation factor leads to $K_{\mathrm{d}}$ values between 5 and $10 \mathrm{~mL} / \mathrm{g}$, which are consistent with the ranges given in Appendix A for these radionuclides. Complications include a time-varying flow rate and analytical detection limit issues for plutonium (Tompson et al. 1999 [DIRS 162686], p. 152). Nevertheless, this minimum value of 8 can be compared to the retardation factors exceeding 1,000 based on models and laboratory data for ${ }^{90} \mathrm{Sr}$ and ${ }^{137} \mathrm{Cs}$ (Tompson et al. 1999 [DIRS 162686], Table 25). In short, breakthrough was not expected based on laboratory data and sorption models, and it did not occur in the field, which lends credence to the concept of retardation by sorption in the alluvium. However, a tight bound on the actual retardation factor is not possible from this field test.

\subsection{Radionuclide Transport in the Volcanics (TYBO-BENHAM Study)}

The interpretations presented in this section were obtained from a report by Wolfsberg et al., who performed a comprehensive analysis of transport at the NTS (2002 [DIRS 162688], Chapter 1). Underground tests called BENHAM (in 1968) and TYBO (in 1975) were conducted 
at Pahute Mesa in volcanic rocks beneath the water table (Figure 7-1). Subsequently, to investigate the potential migration of radionuclides, observation wells ER-20-5 \#1 and ER-20-5 \#3 were drilled and completed in the Topopah Spring welded tuff approximately $300 \mathrm{~m}$ southwest of TYBO. Topopah Spring is the same unit in which the TYBO test was carried out (Pawloski 1999 [DIRS 162685], p. 20). Sampling of these observation wells between 1996 and 1998 indicates elevated concentrations of isotopes of hydrogen, carbon, chlorine, strontium, technetium, iodine, cesium, cobalt, europium, americium, and plutonium (Wolfsberg et al. 2002 [DIRS 162688], Tables 1-1 and 1-2). Kersting et al. studied the low levels of plutonium found in the two observation wells in greater detail (1999 [DIRS 103282], p. 56). Plutonium was detected in ER-20-5 \#3 at approximately the elevation of the BENHAM working (detonation) point and in ER-20-5 \#1, approximately $500 \mathrm{~m}$ above the lava (Figure 7-2). The plutonium in both wells was found associated with colloidal material, and isotopic fingerprinting by Kersting et al. showed that the plutonium originated at BENHAM rather than TYBO, despite the closer proximity of the ER-20-5 wells to TYBO (1999 [DIRS 103282], p. 58).

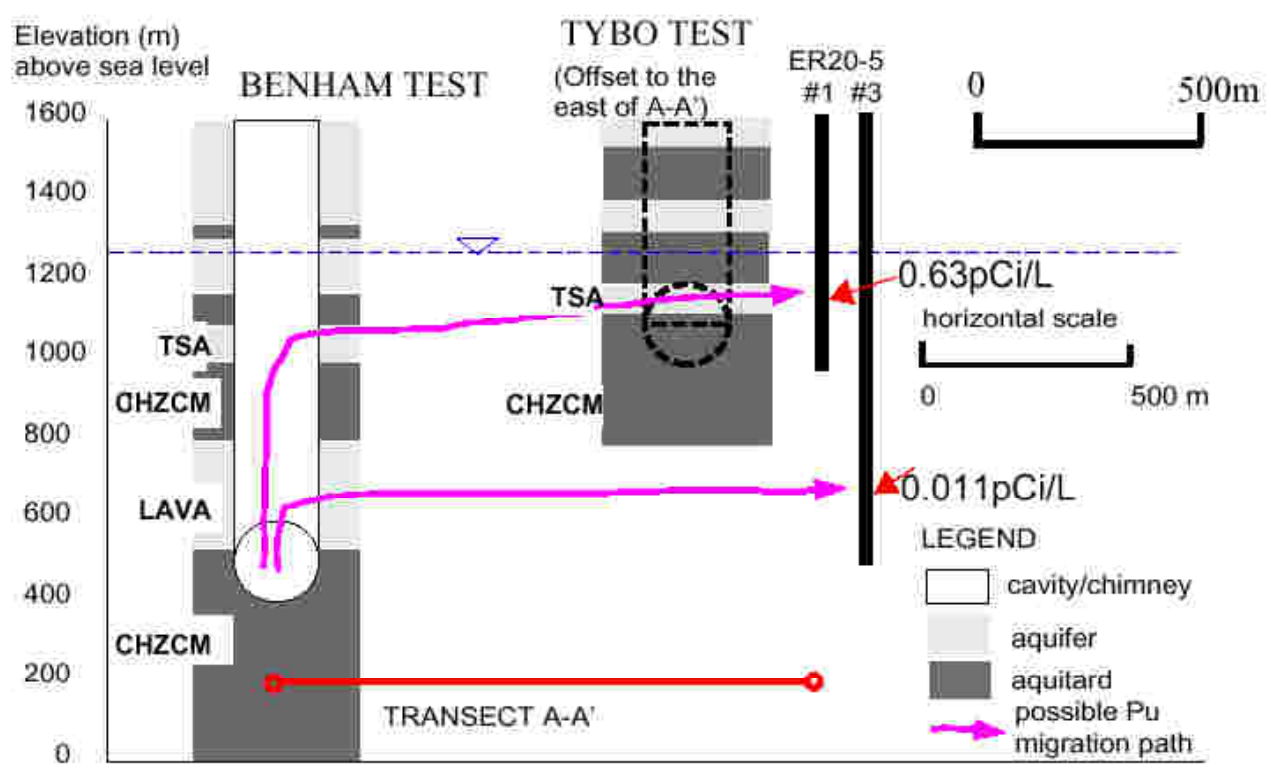

Source: Wolfsberg et al. 2002 [DIRS 162688], Figure 1-2.

Figure 7-2. Schematic of Possible Pathways from the BENHAM Test to the ER-20-5 Observation Wells

This observation suggests that plutonium, typically considered to be relatively immobile, migrated $500 \mathrm{~m}$ vertically and 1,300 m horizontally. Kersting et al. point out that it is unlikely that plutonium from BENHAM was transported via prompt injection (at the time of detonation) over the distances necessary for observation at the two separate ER-20-5 wells (1999 [DIRS 103282], p. 58). Further, because the plutonium detected in the ER-20-5 wells was entirely associated with colloids, plutonium migration via colloid-facilitated transport in groundwater is the most likely explanation. Wolfsberg et al. constructed an integrated model of the system based on the conceptual model depicted in Figure 7-2 (2002 [DIRS 162688], Chapter 1). That study concluded that for expected values for parameters, migration of small amounts of plutonium from BENHAM to the observation points is plausible in less than 30 years 
(Wolfsberg et al. 2002 [DIRS 162688], p. 8-3). Their colloid-facilitated transport model employed a kinetic model for the sorption of plutonium onto colloids. It was found that slow desorption of plutonium from the colloids is required to allow a small fraction of the mass to travel via colloids. In the site-scale SZ transport model, two mechanisms of colloid-facilitated transport are incorporated: One is a reversible sorption model, and the other is an irreversible sorption model for radionuclide attachment onto colloids. The only retardation mechanism for the latter is retardation of the colloids by filtration, which is modeled using a retardation-factor-type parameter. This latter approach is consistent with the field observation from the TYBO-BENHAM and ER-20-5 wells that a fraction of the inventory of otherwise immobile radionuclides is transported via colloids.

There is no way to fingerprint the source of the other radionuclides detected at these observation wells using isotope ratios. Nevertheless, the plutonium observations pointing to a BENHAM source can be used to hypothesize that the other radionuclides cited above also migrated 1,300 $\mathrm{m}$ horizontally from BENHAM, given that a groundwater transport pathway is implied by the plutonium data. For some of these other radionuclides, such as americium, colloid-facilitated transport is a likely mechanism; this explanation is adopted in the site-scale SZ transport model by treating americium using a colloid-facilitated transport model. Others, such as ${ }^{90} \mathrm{Sr}$ and ${ }^{137} \mathrm{Cs}$, are normally considered to be aqueous, sorbing radionuclides. Based on the available information, it is not possible to choose among the following explanations: (1) Colloids are also responsible for migration; (2) sorption is not effective due to limited contact with the rock during fracture flow; or (3) the source of these radionuclides is not BENHAM, but TYBO, and transport distances are much shorter. The site-scale SZ transport model includes wide uncertainty distributions for the values of fracture flow parameters and specific discharge, as well as those for sorption coefficient of strontium and cesium, thus allowing for relatively rapid transport of these radionuclides. The data reported here from the TYBO-BENHAM study are inconclusive on the issue of Cs being transported as a radionuclide that attaches reversibly to colloids

\subsubsection{Transport of Uranium at Natural Analogue Sites}

This section briefly summarizes information on transport of uranium from different analogue sites to determine their relevance to conditions present at Yucca Mountain. Based on monitoring programs at Uranium Mill Tailings Remedial Action sites across the U.S., under oxidizing conditions representative of the transport path in the SZ, uranium transports are considered as nonsorbing to weakly sorbing contaminants as shown in Natural Analogue Synthesis Report (BSC 2004 [DIRS 169218], Section 12.3). This finding is consistent with the conceptual and mathematical model presented in Section 6 and the ranges of $K_{d}$ values developed for uranium in Appendix A.

A study of natural uranium ore bodies at El Berrocal in Spain as shown in Natural Analogue Synthesis Report (BSC 2004 [DIRS 169218], Section 12.4.2.1) and a study of radionuclide migration in fractured gneisses and migmatites at Palmottu, Finland, Natural Analogue Synthesis Report, (BSC 2004 [DIRS 169218], Section 12.4.2.2) led to the conclusion that effects of matrix diffusion are seen in up to several tens of millimeters of rock matrix adjacent to fracture surfaces. These studies also showed that reactive processes between the radionuclides and the rock matrix effectively immobilized the radionuclides. 
In studies at Cigar Lake, Canada; Alligator Rivers, Australia; and Pocos de Caldas, Brazil, it was found that uranium, thorium, and rare-earth elements transported in association with colloids as shown in Natural Analogue Synthesis Report (BSC 2004 [DIRS 169218], Section 12.4.3). Similar conclusions were reached in studies at the Nevada Test Site and Los Alamos in the United States as presented in Natural Analogue Synthesis Report(BSC 2004 [DIRS 169218], Section 12.4.3). These studies support the inclusion of colloid facilitated transport in the sitescale SZ transport model.

\subsubsection{Submodel Components}

Confidence building in submodel components of the site-scale SZ transport model was conducted through comparison of the conceptual model of SZ transport with the results of field tests conducted at the C-wells complex, the ATC, and the CAMBRIC site on the NTS. At the C-wells complex, which is located approximately $2 \mathrm{~km}$ southeast of the high-level radioactive waste repository footprint at Yucca Mountain, a series of conservative and reactive tracer tests were conducted in the various hydrogeologic units, principally fractured volcanic tuffs. Tracer tests were conducted separately in Bullfrog Tuff and Prow Pass Tuff units to study contaminant transport in different fractured volcanic media presented in Saturated Zone In-Situ Testing (BSC 2004 [DIRS 170010], Section 6.3). The tracer tests conducted at the C-wells are used to identify the important transport processes for the fractured volcanics. Conceptualization of transport processes for the alluvium was also based on field tests conducted at the NC-EWDP-19D1 wells and at the CAMBRIC site on the NTS. At the NC-EWDP-19D1 wells, three single-well, injection-withdrawal tests were conducted in the saturated alluvium. In each of the three tracer tests, two nonsorbing solute tracers with different diffusion coefficients were simultaneously injected. Detailed information on the identification of the various transport processes is provided in Saturated Zone In-Situ Testing (BSC 2003 [DIRS 170010], Sections 6.3 and 6.5). Summary level discussions of the conceptual model elements follow in Sections 7.1.2.1 to 7.1.2.9 of this report. More lengthy discussions in Section 6 give added detail to the inclusion of the following in the site-scale SZ transport model:

- Advection in the volcanics (see Section 6.3, item number 2)

- Dispersion in the volcanics (see Section 6.3, item number 2)

- Matrix diffusion in the volcanics (see Section 6.3, item number 3)

- Sorption in the volcanics (see Section 6.3, item number 4)

- Colloid-facilitated transport in the volcanics (see Section 6.3, item number 5)

- Advection in the alluvium (see Section 6.3, item number 6)

- Diffusion and dispersion in the alluvium (see Section 6.3, item number 6)

- Sorption in the alluvium (see Section 6.3, item number 7)

- Colloid-facilitated transport in the alluvium (see Section 6.3, item number 8).

\subsubsection{Advection Through Fractures in the Volcanics}

Figure 7-3 shows the results of a cross-hole tracer test in the Bullfrog Tuff member of the Crater Flat Group for the three soluble tracers: PFBA, bromide, and lithium (a weakly sorbing tracer). The injection and production wells were separated by approximately $30 \mathrm{~m}$ at the test depth as presented in Saturated Zone In-Situ Testing (BSC 2004 [DIRS 170010], Table C-6). The fractional recovery for PFBA and bromide was 0.69 and for lithium was 0.39 as shown in 
Saturated Zone In-Situ Testing (BSC 2004 [DIRS 170010] Appendix D4.5). This is consistent with the interpretation of lithium as a sorbing tracer. Tracer breakthrough curves are plotted as normalized concentration versus time (normalization is performed by dividing by the tracer mass injected) at the production well so that they can be compared directly to one another. The most prevalent feature in these breakthrough curves is the presence of multiple peaks. This result was due to the presence of two advective pathways between the wells and the particular means for injecting the tracer mixture, which gave rise to two distinct flow paths of overlapping transport times. This feature is a site-specific detail due to the local hydrologic conditions. The general behavior of the system implied by the breakthrough curves in Figure 7-3 is that advection occurs primarily in the fractures. Rapid travel velocities between the wells (solute breakthrough within the first 10 hours of the test) are consistent with the conceptual model element of fracture flow through the volcanics. This test was conducted under forced gradient conditions discussed in Saturated Zone In-Situ Testing (BSC 2004 [DIRS 170010], Sections D.4.3 and D.4.5) and the solute velocities were expected to be much higher than the natural gradient conditions. By contrast, advection in the matrix would have resulted in much longer transport times. Therefore, these observations lend strong support to the use of a fracture-flow model for advection in the volcanics.

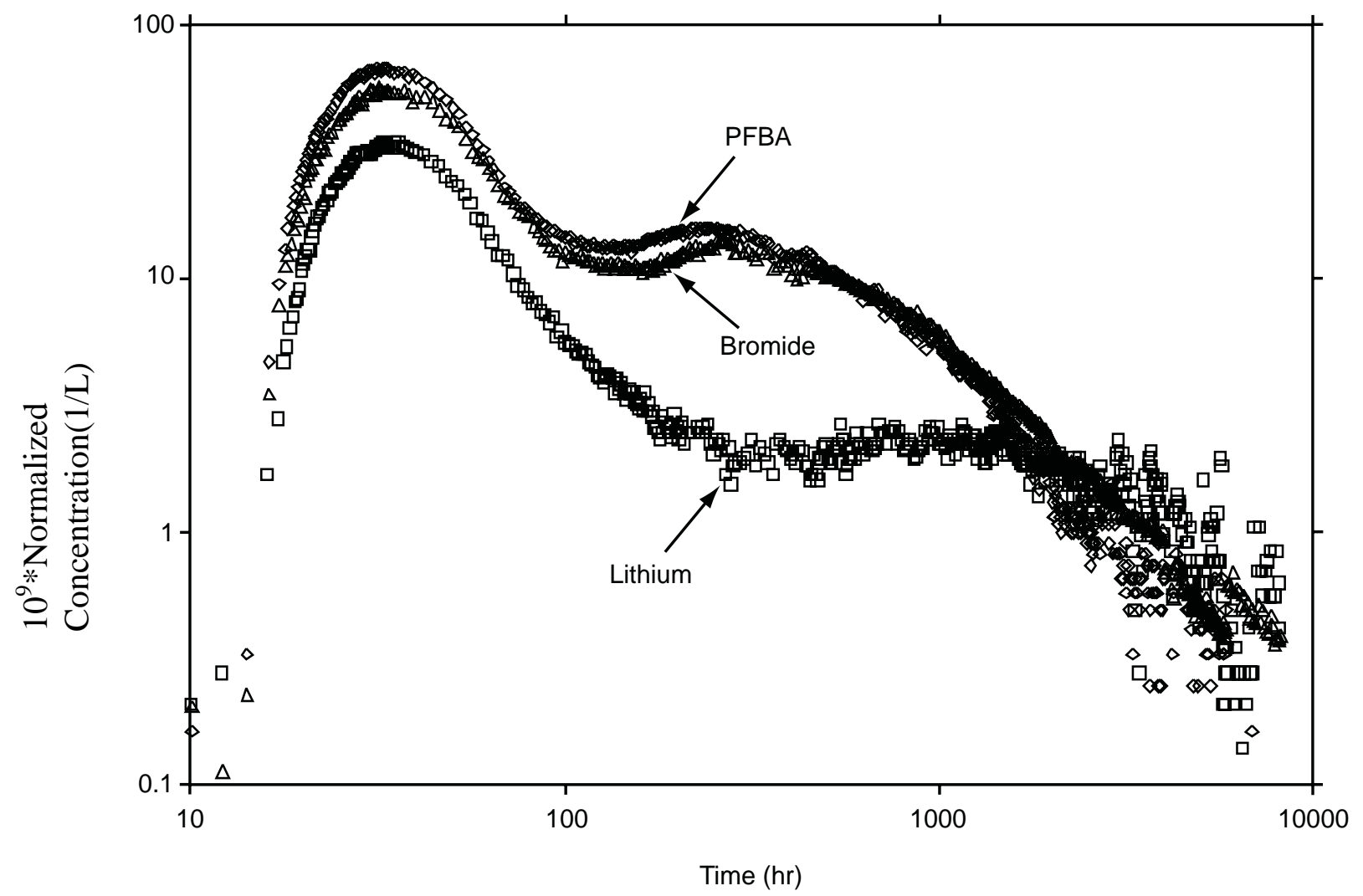

Source: Saturated Zone In-Situ Testing (BSC 2004 [DIRS 170010], Figure D-19).

NOTE: Log-log scales are used for the axes so that the bimodal nature of the tracer responses can be seen more clearly.

Figure 7-3. Normalized Tracer Concentrations Versus Time in the Bullfrog Tuff Tracer Test Conducted from October 1996 to September 1997 


\subsubsection{Dispersion in the Volcanics}

A computer modeling analysis of the C-wells test has been used to derive field-scale transport parameters for longitudinal dispersivity. A plot of the longitudinal dispersivity values as a function of test scale for several NTS fractured-rock, tracer-test programs is shown in Figure 7-4. The plot indicates that the longitudinal dispersivity increases with test scale, the range of the test scale going from less than $1 \mathrm{~m}$ to more than $100 \mathrm{~m}$ (Leap and Belmonte 1992 [DIRS 156838], pp. 87 to 95). Figure 7-5 shows the range of longitudinal dispersivities as a function of scale derived from the C-wells multiple-tracer tests (darkened area) superimposed on a plot of dispersivity versus scale prepared by Neuman (1990 [DIRS 101464], Figure 3). Note that the lower end of the range of length scales associated with the darkened area corresponds to the interwell separation in the tracer tests and the upper end corresponds to the test interval thickness (used as an upper bound for the transport distance). The range of longitudinal dispersivities derived from the $\mathrm{C}$-wells tests and the scale dependence of longitudinal dispersivity in the tests plotted in Figure 7-4 are consistent with the understanding developed in the literature. This result lends strong support to the use of a longitudinal dispersivity model.

There is no site-specific information available to select the transverse dispersivity in the fractured volcanics. Values were chosen based on the Saturated Zone Flow and Transport Expert Elicitation Project (CRWMS M\&O 1998 [DIRS 100353], pp. 3-11 and LG-11 to LG-14).

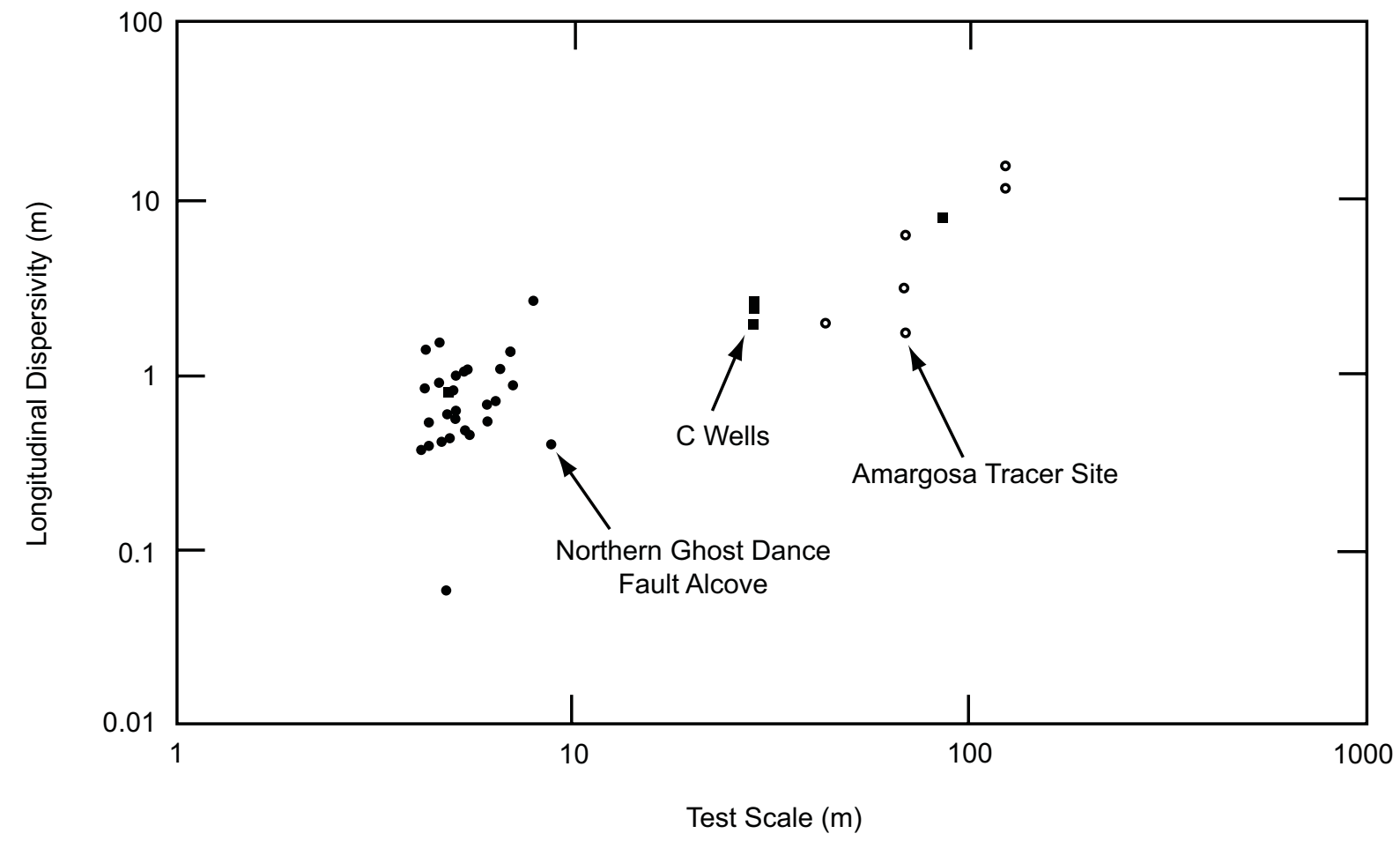

Source: Saturated Zone In-Situ Testing (BSC 2004 [DIRS 170010], Figure E-39).

Figure 7-4. Longitudinal Dispersivity as a Function of Test Scale in Several Tracer Tests Conducted in the Vicinity of Yucca Mountain 


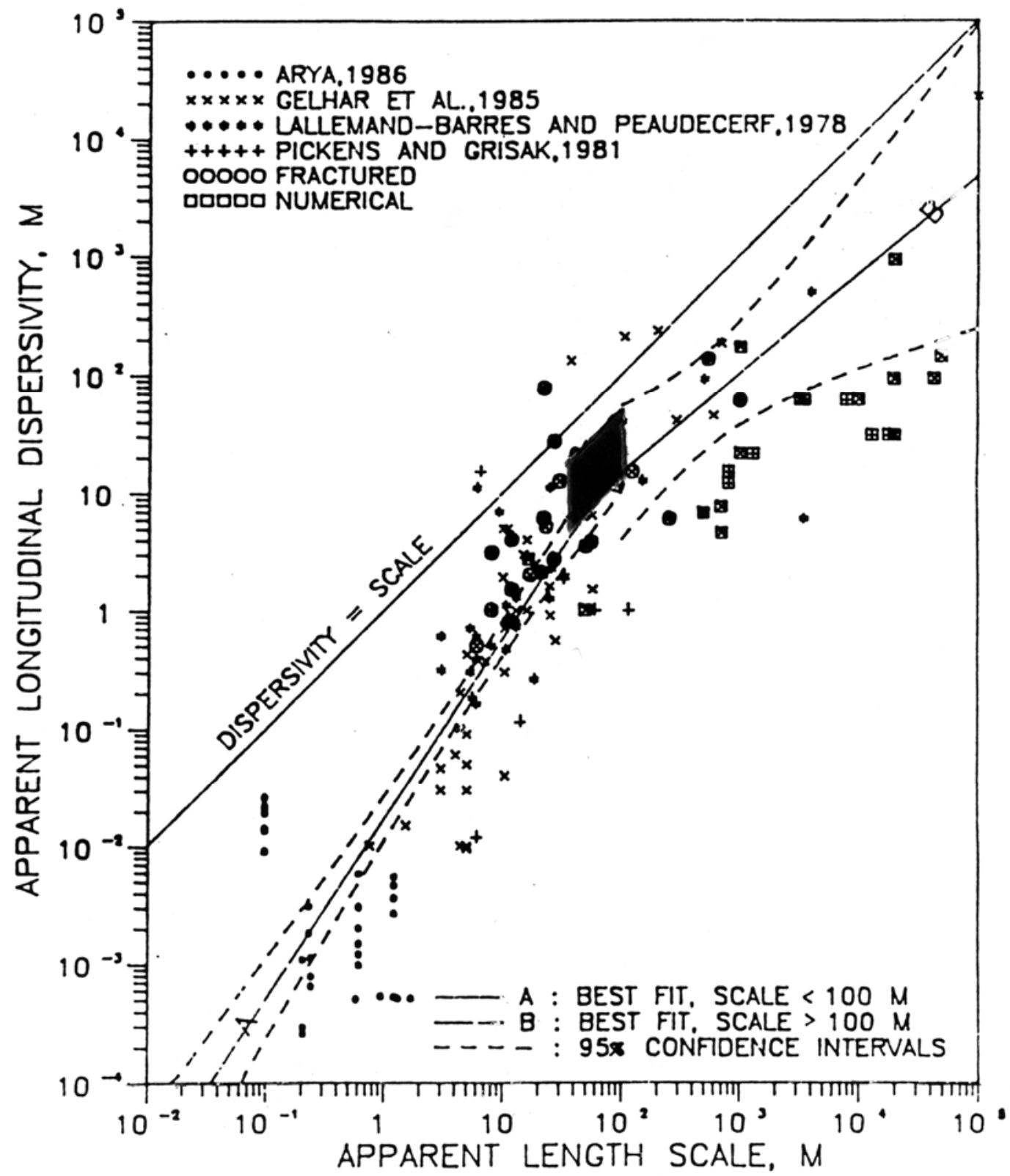

Source: Saturated Zone In-Situ Testing (BSC 2004 [DIRS 170010], Figure E-40).

NOTE: The darkened box shows the range of values derived from the multiple-tracer tests. The right edge of the box corresponds to the interwell separation distance, and the left edge of the box corresponds to the test interval thickness (taken to be the upper limit of transport distance).

Figure 7-5. Plot of Longitudinal Dispersivity Versus Length Scale Showing the Range of C-Wells Values Derived from Interpretations of the Prow Pass and Bullfrog Multiple-Tracer Tests 


\subsubsection{Matrix Diffusion in the Volcanics}

For the purposes of validating the matrix-diffusion model for the fractured volcanics, the critical result from the $\mathrm{C}$-wells tests is the relative heights of the peaks for the various tracers shown in Figure 7-3. For the conservative tracers, the fact that the tracer with the lower molecular diffusion coefficient (PFBA) exhibited a higher peak concentration is consistent with the dual-porosity model in which tracer travels in the fractures but also diffuses into the rock matrix.

When using a dual-porosity medium, as was done in this study, nonuniqueness of tracer test interpretations is an issue. For instance, long tails in tracer responses can be interpreted as being the result of either large longitudinal dispersion or significant matrix diffusion. In addition, at short time and distance scales, there may be a significant influence of diffusion into stagnant-free water within fractures in addition to "true" matrix diffusion. Thus, matrix-diffusion parameters obtained from laboratory tracer experiments should be used cautiously when predicting contaminant migration at larger scales in fractured media. Nevertheless, in the multiple-tracer tests, nonuniqueness of interpretations was minimized by simultaneously fitting the tracer responses using known ratios of diffusion coefficients as constraints on the relative matrix diffusion of different tracers. The method of interpreting the field test data is given in detail in Saturated Zone In-Situ Testing (BSC 2004 [DIRS 170010], Section 6.3.) and the parameter values obtained from the data fit are reported in Saturated Zone In-Situ Testing (BSC 2004 [DIRS 170010], Tables D-2, D-3, D-7, and D-10). The mass transfer coefficients and fracture aperture values given in the above reference along with the base-case matrix porosity given in Table 4-2 (0.15 to 0.25) lead to matrix diffusion coefficient values in the range of $1.6 \times 10^{-8} \mathrm{~m}^{2} / \mathrm{s}$ to $8 \times 10^{-13} \mathrm{~m}^{2} / \mathrm{s}$, which overlaps with the range given in Table 4-2. Rationale for the selection of the range of diffusion coefficient values given in Table 4-2 are provided in Section 4.1.2.10 and in Saturated Zone Flow and Transport Model Abstraction (BSC 2004 [DIRS 170042], Section 6.5.2.6).

The intent of the present discussion is to build confidence in the conceptual model of diffusive mass transfer in the volcanics. All the C-wells test results discussed in Saturated Zone In-Situ Testing (BSC 2004 [DIRS 170010], Section 6.3.) are consistent with diffusive mass transfer having a strong influence on the migration of solutes in fractured volcanic tuffs. Therefore, the field evidence strongly supports the use of a matrix-diffusion model as opposed to a single-continuum model for transport in the fractured volcanics.

\subsubsection{Sorption in the Volcanics}

As with matrix diffusion, sorption can also be observed in the C-wells tests by examining the reactive tracer shown in Figure 7-3. Lithium, the sorbing tracer, exhibits further attenuation but a similar arrival time as the two conservative tracers. Note that a matrix-diffusion model explains this observation more completely than a single-continuum model, which would predict delayed arrival times for a sorbing tracer in addition to attenuation. Using the dual-porosity equivalent continuum model, sorption within fractures leads to a delayed peak time along with attenuation, whereas sorption in matrix but not within fractures leads to the same peak arrival time but with attenuated response (Robinson 1994 [DIRS 101154], Figure 7). Thus, the field observed response of lithium is indicative of sorption within the volcanic matrix and not within the fractures on the scale of the C-wells test (tens of meters). Sorption coefficients were 
measured in the laboratory and compared to field-estimated values of $K_{d}$. The lithium $K_{d}$ values estimated from the field tracer tests are in the same range but consistently higher than the corresponding $K_{d}$ values measured at the lowest lithium concentrations in the laboratory. These results suggest that the use of laboratory-derived $K_{d}$ values to predict sorbing species transport in the saturated fractured tuffs near the C-wells location would tend to under-predict the amount of sorption experienced by the species in the field. The fact that the field $K_{d}$ values tended to be greater than the laboratory $K_{d}$ values suggests that lithium may have come into contact with alteration minerals in the field that were not present or were depleted in the lab rock samples. Any loosely adhering alteration minerals (e.g., clays) that may have been present in the core samples would very likely have been lost during crushing and sieving of the material when it was prepared for the batch-sorption experiments given in Saturated Zone In-Situ Testing (BSC 2004 [DIRS 170010], Section D.4.8.4). Nevertheless, given the consistency of the breakthrough curves with sorption and the field estimates of $K_{d}$ yielding similar values to those in the laboratory (which are considered conservative for PA because the values from the field tests are generally higher), the available data strongly support the use of a $K_{d}$-based matrix sorption model for the fractured volcanics. Broad ranges of values for $K_{d}$ are being used in the TSPA-LA analysis (Appendix C, Table C-14) to account for the uncertainties in determining this parameter.

\subsubsection{Colloid-Facilitated Transport in the Volcanics}

The approach used in the site-scale SZ transport model for colloid-facilitated transport is presented in Section 6.4.2.6 and Table 6.4-1.

\subsection{Radionuclides Attached Irreversibly to the Colloids}

The radionuclides attached irreversibly to colloids transport in a manner identical to the colloids. Tests were conducted at the C-wells to study the transport of colloids. In addition to the conservative and reactive tracers, 360-nm-diameter carboxylate-modified polystyrene microspheres were injected in the Bullfrog tracer test to examine how finite-sized particles transport through the fractured tuffs. These microspheres are meant to provide insight into the potential behavior of colloids transporting in the groundwater. Figure 7-6 shows a comparison of the microsphere breakthrough curve with the PFBA tracer. Microspheres do indeed transport through the medium at the scale of this test, but the concentrations are attenuated compared to a conservative solute. The fractional recovery of the microspheres is 0.145 compared to 0.69 for the PFBA as discussed in Saturated Zone In-Situ Testing (BSC 2004 [DIRS 170010] Section D.4.5). This result is probably due to filtration effects in the medium, although the possibility of settling cannot be ruled out. The site-scale SZ transport model applies a reversible-filtration model to simulate colloid-facilitated transport. The retardation factors estimated from the colloid attachment and resuspension rates (for tests that showed measurable microsphere recovery) given in Tables D-8 and D-9 of Saturated Zone In-Situ Testing (BSC 2004 [DIRS 170010]) are in the range of about 6 to 794, which is the range given in Table 4-2 of this report. The data in Figure 7-6 strongly support the concept of a filtration component of the conceptual model. 


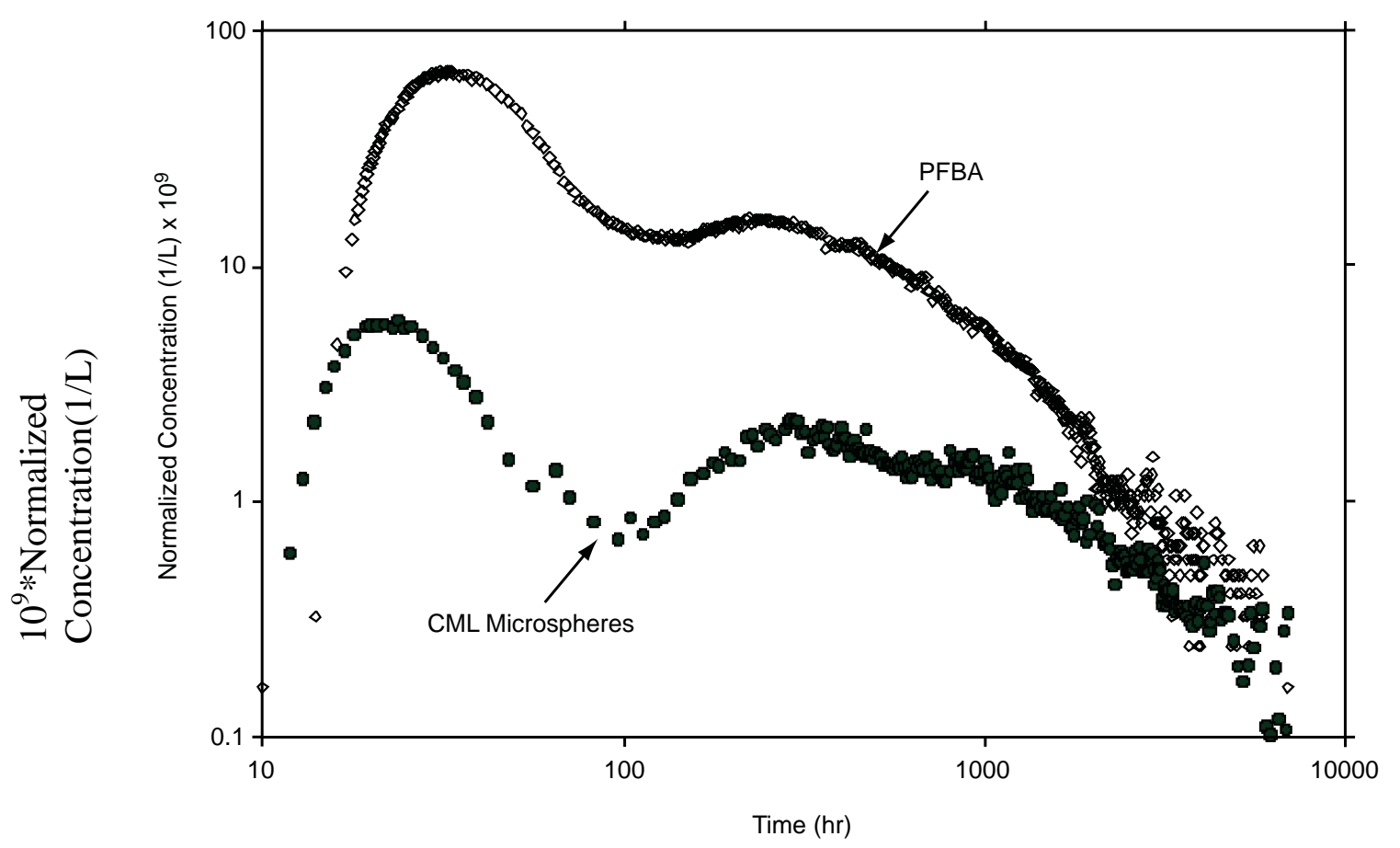

Source: Saturated Zone In-Situ Testing (BSC 2004 [DIRS 170010], D-20).

NOTE: Log-log scales are used for the axes so that the bimodal nature of the tracer responses can be seen more clearly.

Figure 7-6. Normalized Concentrations of PFBA and 360-nm-Diameter Carboxylate-Modified Polystyrene Latex Microspheres in the Bullfrog Tuff Tracer Test

\subsection{Radionuclides Attached Reversibly to the Colloids}

Published work from literature is described in this section to build confidence in the conceptual model for the transport of the radionuclides attached reversibly to the colloids. Early models considered equilibrium partitioning of contaminants between the solute phase, colloid surfaces, and media surfaces (Hwang et al. 1989 [DIRS 165931], p. 600; Smith and Degueldre 1993 [DIRS 144658], pp. 145 to 150; and Grindrod 1993 [DIRS 165928], pp. 171 to 175). These efforts focused on describing transport through fractured media. Corapcioglu and Jiang (1993 [DIRS 105761], pp. 2217 to -2221) introduced a numerical model with first order reversible rate expressions describing colloid attachment and detachment to media surfaces. Their model (with some adjustments) was later used to describe $\mathrm{Cs}^{137}$ transport facilitated by silica colloids through glass bead columns (Noell et al. 1998 [DIRS 106920], pp. 48 to 52). Ibaraki and Sudicky (1995 [DIRS 109297], pp. 2948 to 2951; and 1995 [DIRS 165930], pp. 2961 to 2964) were the first to implement kinetic expressions in a model to explicitly describe colloid-facilitated contaminant transport in discrete fractures and fracture networks, although their approach was essentially mathematically identical to that of Corapcioglu and Jiang (1993 [DIRS 105761], pp. 2217 to 2221). Oswald and Ibaraki (2001 [DIRS 165961], pp. 217 to 218) later extended the model to account for matrix diffusion of colloids, a phenomenon that was observed in laboratory experiments conducted in a fractured saprolite of high matrix porosity (the exclusion of this phenomenon from the site-scale SZ transport model, as discussed in Section 6.3, leads to potentially shorter transit time predictions). 


\subsubsection{Advection in the Alluvium}

\subsection{Alluvial Tests at NC-EWDP-19D1}

Three single-well injection-withdrawal tracer tests were conducted in the saturated alluvium at NC-EWDP-19D1 between December 2000 and April 2001. In each of the three tracer tests, two nonsorbing solute tracers with different diffusion coefficients were simultaneously injected (a halide and a fluorinated benzoic acid dissolved in the same solution). The three tests were conducted in essentially the same manner except for the time that was allowed to elapse between the cessation of tracer and chase-water injection and the initiation of pumping-that is, the so-called "rest" or "shut-in" period. The rest period was systematically varied from approximately 0.5 hour to approximately 2 days and to approximately 30 days in the tests to vary the time allowed for tracers to diffuse into stagnant water in the flow system and for the tracers to migrate with the natural groundwater flow. Test interpretations were based on comparing the responses of the different tracers in the same test as well as the responses of similar tracers in the different tests. Differences in the responses of the two tracers injected in the same test provided information on diffusion into stagnant water in the system, whereas differences in the responses of tracers injected in different tests (after correcting for the effects of diffusion) provided information on tracer drift during the rest periods of the tests. Because the three tracer tests used different drift durations, a comparison of the results, combined with an idealized model of the groundwater flow behavior near the well, could be used to estimate the specific discharge. Values ranging from 1.2 to $9.4 \mathrm{~m} / \mathrm{y}$, depending on conceptual model and parameter uncertainties, were obtained from Saturated Zone In-Situ Testing (BSC 2004 [DIRS 170010], Table G-8). This range is in agreement with the values calculated in the calibrated site-scale SZ flow model as presented in Saturated Zone Site-Scale Flow Model (BSC 2004 [DIRS 170037], Section 6.6.2.4) and the range of input values used in this report (Table 4-2). The site-scale SZ transport model is intended for use in making TSPA-LA predictions using a wide range of parameter input values that reflect uncertainty in the input. Hence, a comparison of the range of values is considered sufficient. This result lends strong support to the process of advection in the alluvium.

\subsection{CAMBRIC (NTS)}

Velocities of movement under forced-gradient conditions used in the CAMBRIC experiment are not directly relevant to natural-gradient flow and transport at Yucca Mountain. However, modeling analyses performed by Tompson et al. (1999 [DIRS 162686], Chapter 10) for the NTS Environmental Restoration (ER) Project provide information on relevant hydrologic and transport parameter estimates for alluvium at the site. Using a heterogeneous distribution of permeability and constant porosities ranging from 0.1 to 0.38 (Tompson et al. 1999 [DIRS 162686], Table 20), Tompson showed that a continuum model, after calibration, was able to match the tritium breakthrough curve well (Tompson et al. 1999 [DIRS 162686], Figure 40). Note that this range of porosities compares favorably with the uncertainty range of 0.02 to 0.3 for the effective alluvium porosity in Table 4-2 of this report. In a simulation of tracer migration in which the fluid extraction rate is specified, the key parameters controlling the breakthrough curve are the effective porosity, which controls the mean arrival time, and the correlation length of the heterogeneity, which controls the macrodispersive spreading of the breakthrough curve. In the site-scale SZ transport model, porosity and permeability are taken to be uniform within hydrogeologic units, and a macrodispersion model governs the dispersion. The NTS and 
YMP models are both continuum models, so the mean arrival time is controlled by the effective porosity. The models handle dispersion differently, which does not affect the mean arrival time. The fact that both are continuum models means that effective porosity values derived by Tompson et al. (1999 [DIRS 162686], Table 20) should be relevant to the site-scale SZ transport model. The CAMBRIC test and modeling of Tompson et al. (1999 [DIRS 162686], Chapter 10), therefore, confirms that a continuum model with porosity values of the same order of magnitude as the small-scale, measured porosity of the alluvial material is valid.

\subsubsection{Diffusion and Dispersion in the Alluvium}

Figure 7-7 shows a representative result from one of the single-well injection-withdrawal tracer experiments. The normalized tracer responses for two solutes and microspheres are shown. The two solute tracers had essentially identical responses (within experimental error) in this test (and other similar tests carried out in this interval). Flow interruptions during the tailing portions of the two longer tests (not shown) provided additional evidence for minimal diffusive mass transfer in the aquifer. The results here suggest that, in contrast to the fractured tuffs, the alluvium exhibits more of a continuum model behavior with less diffusion into stagnant fluid, such as the pore water of low-permeability material. This result may be scale-dependent such that, over longer transport times, diffusion becomes a more important factor. Nevertheless, these results support the conservative conceptual model of single-continuum transport with little or no diffusion into stagnant regions.

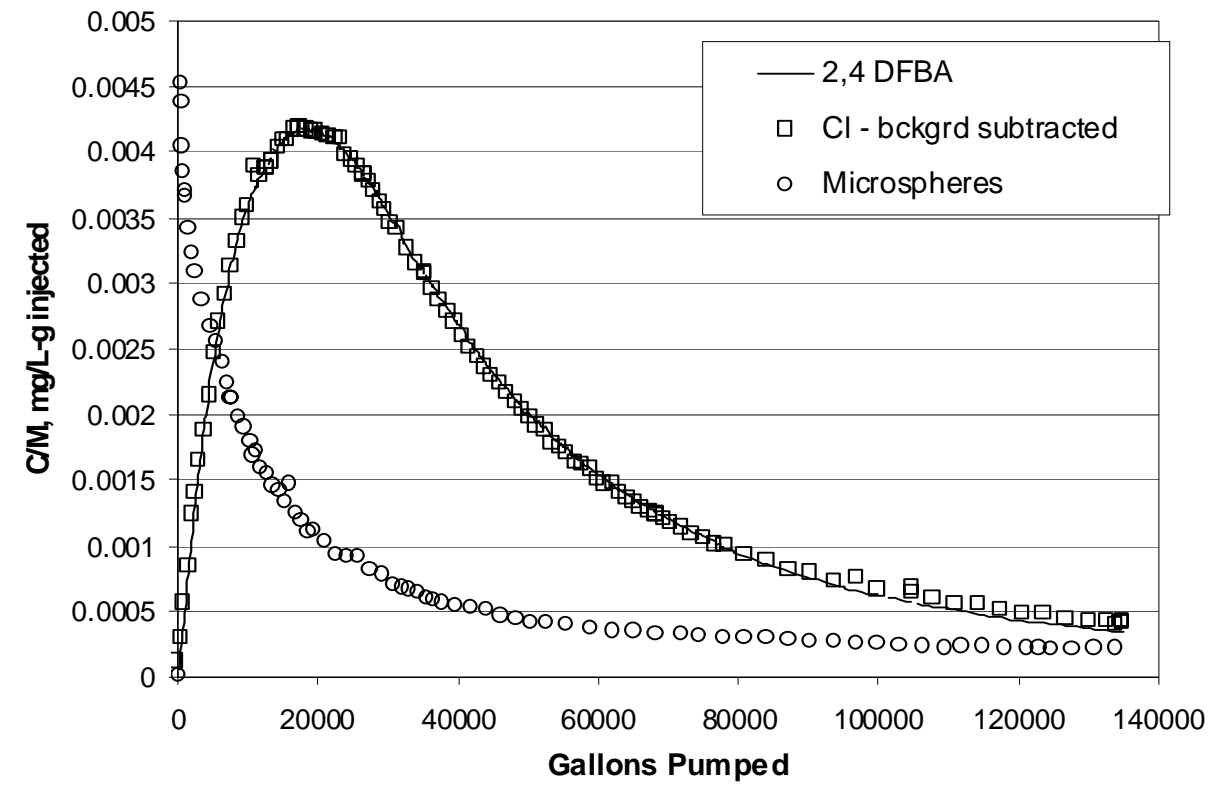

Source: Saturated Zone In-Situ Testing (BSC 2004 [DIRS 170010], Figure G-18).

NOTE: Microspheres were 640-nm-diameter carboxylate-modified polystyrene latex spheres tagged with a ultraviolet-excited fluorescent dye for detection.

Figure 7-7. Normalized Concentrations of Tracers in Production Water from NC-EWDP-19D1 as a Function of Gallons Pumped After a Rest Period of Approximately 0.5 Hour 
Using a semianalytical method, data from the NC-EWDP-19D1 test were used to provide an estimate of $5 \mathrm{~m}$ for the longitudinal dispersivity shown in Saturated Zone In-Situ Testing (BSC 2004 [DIRS 170010], Section G4.2.4) along with a porosity of 10 percent and a transverse dispersivity of $2 \mathrm{~m}$, the scale of the test being estimated around $8 \mathrm{~m}$. Also, the literature is full of information on dispersivity values in alluvial aquifers. Neuman (1990 [DIRS 101464], Figures 1 to 3) compiles a comprehensive list of dispersivity values in porous media including alluvium at different scales (Figure 7-5). Using scientific judgment, one can infer that values for dispersivity from the literature are applicable to the alluvial system at Yucca Mountain. All these considerations lend strong support to the conceptual model of dispersion in the alluvium. Broad ranges of values for dispersivities are being used in the TSPA-LA analysis and presented in Saturated Zone Flow and Transport Model Abstraction (BSC 2004 [DIRS 170042], Section 6.5.2.9) to account for the uncertainties in determining this parameter.

\subsubsection{Sorption in the Alluvium}

No site-specific field data are available to confirm that sorption occurs in the alluvium downgradient from Yucca Mountain. Laboratory-scale experimental results are available, however. Figure 7-8 presents the results of several column transport experiments using groundwater and alluvium obtained from the site of the ATC Well NC-EWDP-19D1. These experiments involved injecting lithium bromide as pulses at three different concentrations spanning the range of Li concentrations expected in the field.

Examination of the results (Figure 7-8) indicates that the data are consistent with a model that includes sorption. This result suggests that sorption is occurring in the columns. The issue of lack of site-specific field evidence is mitigated by two considerations. First, transport field tests using sorbing tracers in similar hydrogeologic settings have shown that sorption does occur in such systems (LeBlanc et al. 1991 [DIRS 163781], p. 905; Hess et al. 2002 [DIRS 163780], pp. 36-6 and 36-14; Thorbjarnarson and Mackay 1994 [DIRS 163782], pp. 413 to 414). Second, as demonstrated earlier, the fractured volcanic tuffs were shown to sorb tracers in the C-wells tracer experiments. These rocks have a similar mineralogic content as the alluvium and a similar fluid geochemistry. Therefore, demonstrated sorption in the fractured tuffs provides a line of site-specific field evidence in favor of sorption in the alluvium. All of these considerations lend strong support to the validity of the conceptual model of sorption in the alluvium. Broad ranges of values for sorption coefficients in alluvium are being used in TSPA-LA analysis as discussed in Saturated Zone Flow and Transport Model Abstraction (BSC 2004 DIRS 170042], Section 6.5.2.8) to account for the uncertainties in determining this parameter.

\subsubsection{Colloid-Facilitated Transport in the Alluvium}

The approach used in the site-scale SZ transport model for colloid-facilitated transport is presented in Section 6.4.2.6 and Table 6.4-1. 

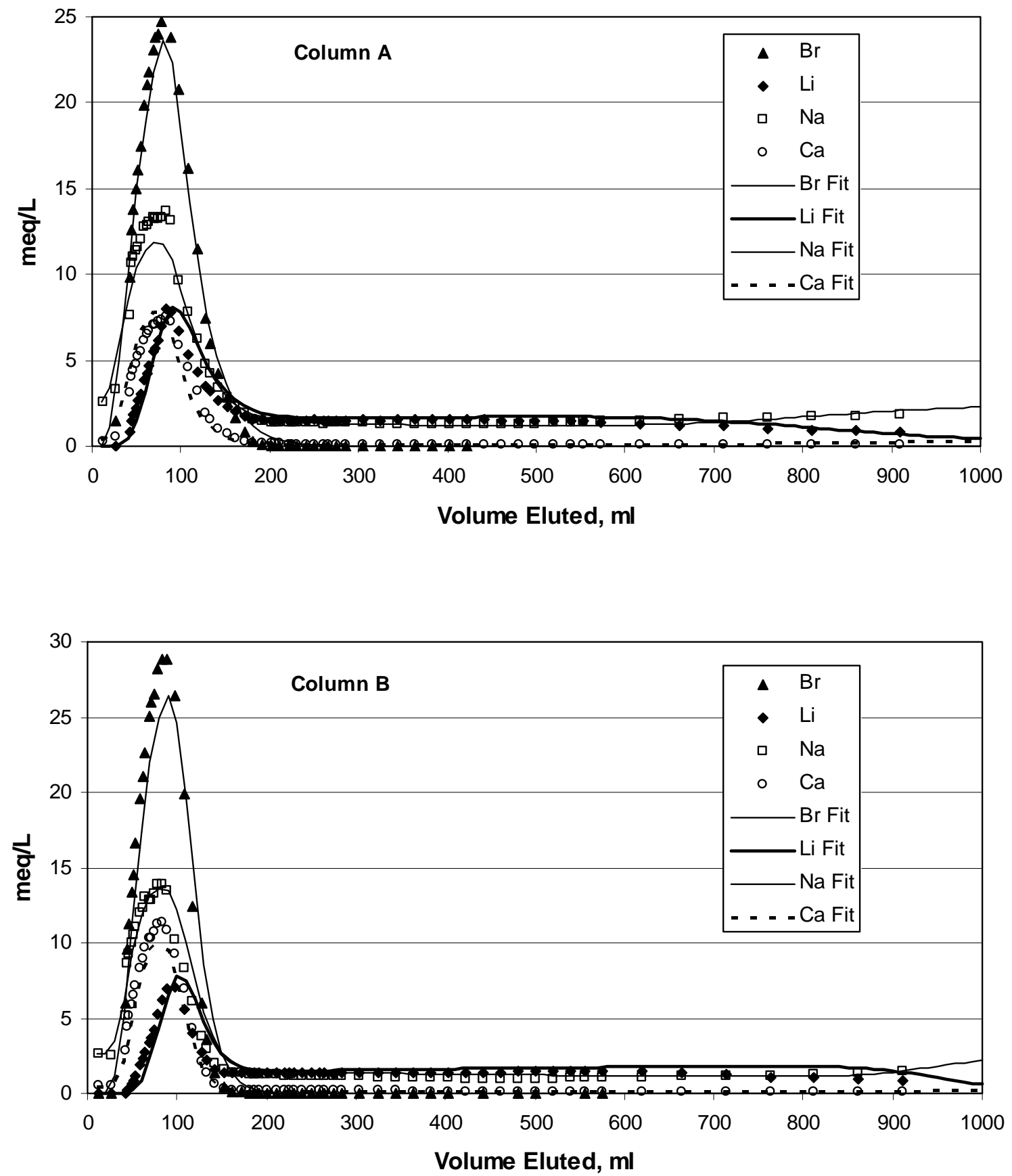

Source: Saturated Zone In-Situ Testing (BSC 2004 [DIRS 170010], Figure H-4 (model).

Figure 7-8. Column Data (Concentration in the units of milli-equivalent/liter) and MULTRAN Fits for Experiments with a LiBr Injection Concentration of $0.0275 \mathrm{M}$ 


\subsection{Radionuclides Attached Irreversibly to the Colloids}

The radionuclides attached irreversibly to colloids get transported along with the colloid particles. Tests were conducted at the C-wells to study the transport of colloids. The microsphere results shown in Figure 7-7 provide information on colloid filtration and detachment rates in the alluvial flow system. Qualitatively, compared to the conservative aqueous tracers, a much earlier breakthrough of microspheres in the withdrawal portion of the test was observed, followed by a very long tail. These observations are consistent with a model in which colloidal-sized particles undergo filtration in the alluvium. Filtration holds some microspheres up very close to the borehole. Upon pumping the aquifer, those microspheres close to the borehole are produced, essentially, instantly. Borehole effects could influence the early return of the microspheres as well. The fraction of microspheres that traveled farther into the medium undergoes filtration during their return, resulting in the long tail. The tail could be influenced by plume drift and aquifer heterogeneities. This qualitative evidence lends support to the conceptual model of filtration of colloids in the alluvium.

\subsection{Radionuclides Attached Reversibly to the Colloids}

The discussion in Section 7.1.2.5.2 also applies to the alluvium.

\subsubsection{Comparison of Flow Paths and Transit Times Against Those Computed by an Independent Site-Scale Model}

The NRC developed an independent site-scale groundwater flow model of the Yucca Mountain SZ (Winterle et al. 2003 [DIRS 163823]). Two different flow models of the flow system were considered. They differed in the material geometries, the number of material zones, and the permeability values assigned to each material type. Each model was calibrated to the measured heads by a trial and error process. Particle flow paths and transport times to the boundary of the accessible environment at $18 \mathrm{~km}$ (10 CFR 63.302 [DIRS 156605]) were calculated from the model using advection only, excluding dispersion, diffusion, and retardation. The first model predicted flow paths starting to the east but shortly turning southward and traveling more or less directly southward (Winterle et al. 2003 [DIRS 163823], Figure 4). The particle transport times predicted by this model ranged from 1,800 to 110,000 years (Winterle et al. 2003 [DIRS 163823], Figure 5). The second model showed flow paths moving farther east before swinging south (Winterle et al. 2003 [DIRS 163823], Figure 8). This model predicted flow times in the range of 7,100 years to 205,000 years (Winterle et al. 2003 [DIRS 163823], p. 153).

The general trend of flow paths reported in the Winterle et al. (2003 [DIRS 163823], Figures 4 and 8) study, starting eastward and then moving southward to the boundary of the accessible environment, compares favorably with the flow paths in Figure 7-9b of this report, although the latter show a more westward component in the southern part of the model compared to the Winterle et al. (2003 [DIRS 163823], Figure 8) study. Although the model described in this report does not predict the upper end of the transport times reported by Winterle et al. (2003 [DIRS 163823], p.153), the range of transit times reported here overlaps with those reported by them. This result corroborates the site-scale SZ transport model. 


\subsection{POST-DEVELOPMENT VALIDATION TO SUPPORT THE SCIENTIFIC BASIS OF THE MODEL}

Numerical results from the site-scale SZ transport model have been compared both to data and to independent models to provide confidence that, when combining the submodels with appropriate geologic-, hydrologic-, and boundary-condition information, the overall model is consistent with available observational data. Specifically, the flow pathways from the model are compared to hydrochemistry data (Section 7.2.1), and the transit times computed from the site-scale SZ transport model are compared to ${ }^{14} \mathrm{C}$ data at the field scale (Section 7.2.2).

\subsubsection{Comparison of Flow Paths Against Those Deduced from Hydrochemistry Data}

Flow paths of tracer particles were calculated for the base-case transport model. The particles were started in the vicinity of the repository footprint and allowed to transport downstream to the boundary of the accessible environment at $18 \mathrm{~km}$. The transport parameter values used in this model calculation are given in Table 6.4-2 of this report. The results are shown in Figure 7-9b. Flow paths deduced from the hydrochemistry data are shown in Figure 7-9 (a and b) are given in Saturated Zone Site-Scale Flow Model (BSC 2004 [DIRS 170037], Section A6.6.11). Chemical and isotopic compositions were measured for groundwater samples taken from a number of wells in the area of the site-scale SZ flow model. As explained in detail in Saturated Zone Site-Scale Flow Model (BSC 2004 [DIRS 170037], Section A6.6.11), graphical analysis was done of the variations in the hydrochemical concentrations and isotopic ratios to estimate plausible flow lines. Of particular interest are the flow paths labeled \# 2 and \#7 in Figure 7-9 (a and b) from this analysis. Flow path 7, which is derived from hydrochemistry data, originates in the vicinity of the repository footprint and generally overlaps the model-calculated flow paths as seen in Figure 7-9b. Flow path 2 is also of interest here, although it originates northeast of the repository, because it closely bounds flow path \#7 to the east. Note that the flow path 9 (shown by broad dashed line in Figure 7-9a going from east to west), which appears to cut across the flow paths 2 and 7 in the two-dimensional figure, actually represents regional underflow in the deep carbonate aquifer that underlies the volcanics and the alluvium in the SZ model area, and thus does not interfere with the flow paths 2 and 7.

Flow path 2 traces the movement of groundwater from the Fortymile Canyon area southward along the axis of Fortymile Wash into the Amargosa Desert. This pathway is drawn on the basis of similar anion and cation concentrations along the flow path and dissimilarities to regions to the east and west. Further details are presented in Saturated Zone Site-Scale Flow Model (BSC 2004 [DIRS 170037], Section A6.6.11).

Flow path 7 traces the movement of groundwater from northern Yucca Mountain southeastward toward wells in the Dune Wash area and then southwestward along the western edge of the Fortymile Wash shown in Saturated Zone Site-Scale Flow Model (BSC 2004 [DIRS 170037], Section A6.6.11). High ${ }^{234} \mathrm{U}^{238} \mathrm{U}$ activity ratios and low $\mathrm{Cl}^{-}, \mathrm{SO}_{4}{ }^{2-}, \delta D$ and $\delta^{18} \mathrm{O}$ values characterize this flow path. A detailed discussion is presented in Saturated Zone Site-Scale Flow Model (BSC 2004 [DIRS 170037], Section A6.6.11). 

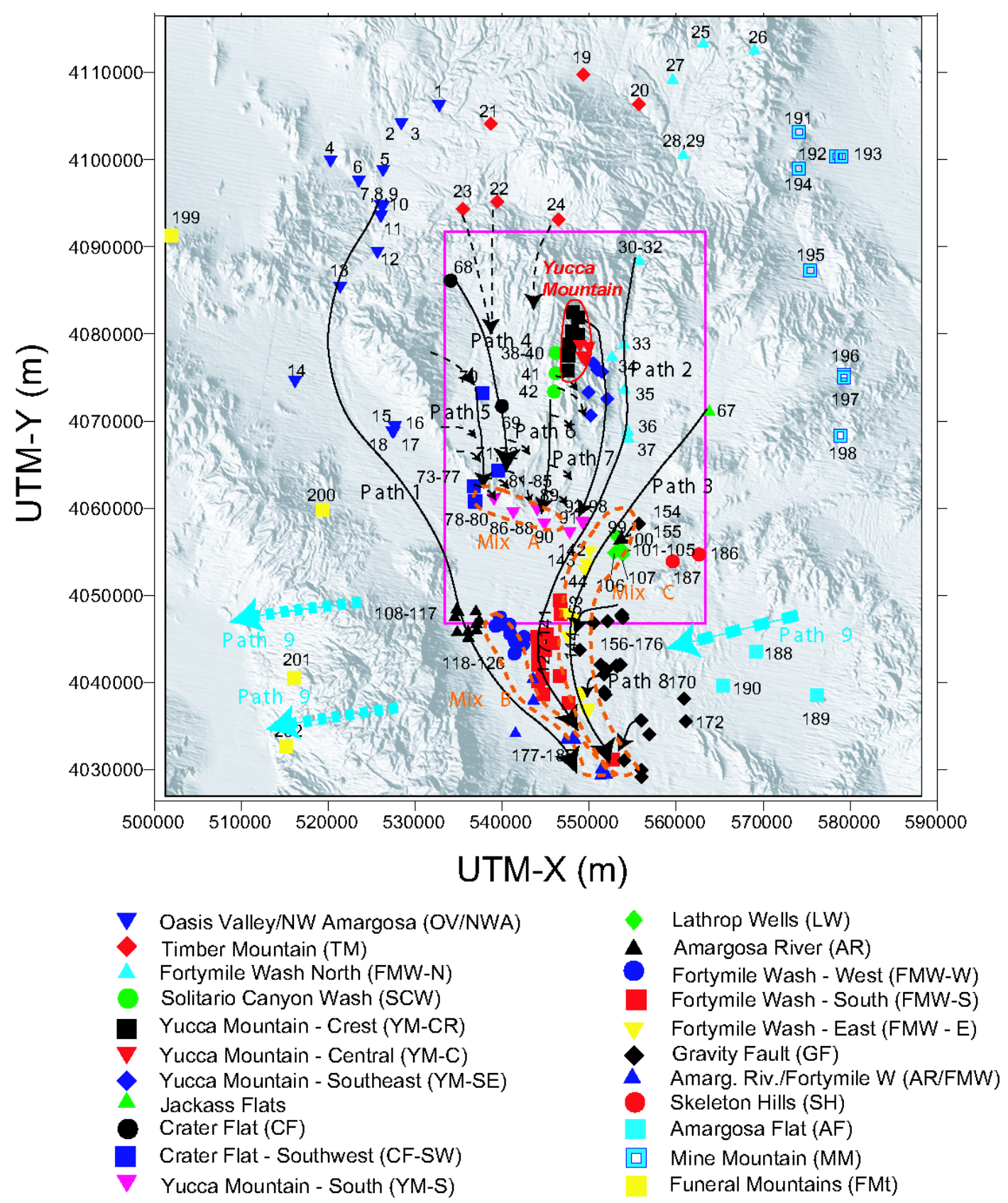

Discussion of flow pathways and reference to sample numbers are given in ANL-NBS-HS-000021, Rev 01

Source: Saturated Zone Site-Scale Flow Model (BSC 2004 [DIRS 170037], Figure A6-62.)

${ }^{1}$ Colored symbols in the figure represent details of geochemical data, not directly relevant to the present discussion. Hence, for the sake of clarity, the detailed legend is not included here; see the source of the figure for details.

${ }^{2}$ Flow path 9 shown by broad dotted blue arrows pointing easterly is flow through deep regional aquifer and does not interfere with the flow paths 1 through 8 in the shallow volcanics and alluvium.

Figure 7-9a. Transport Pathways Deduced from Hydrochemistry 


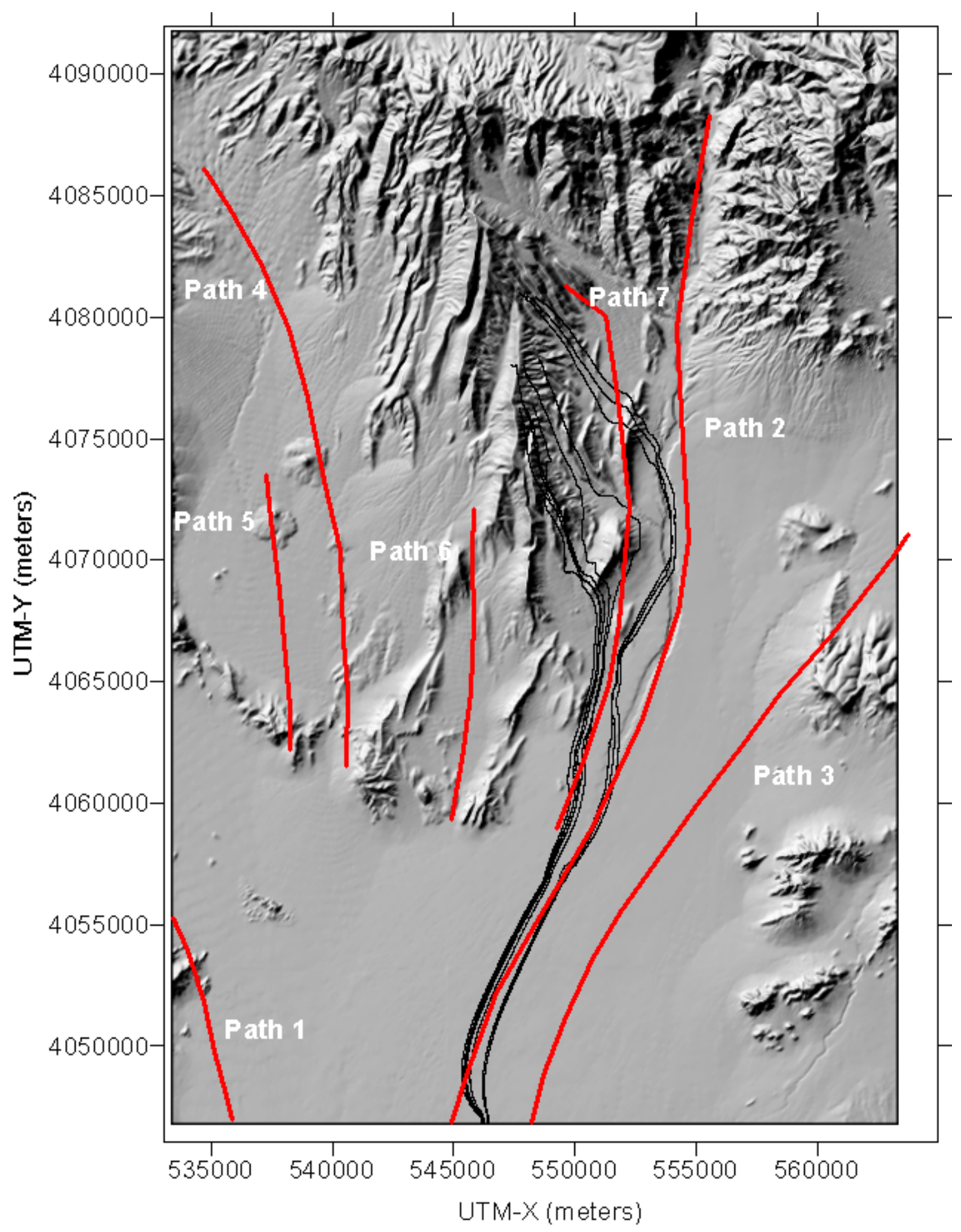

Source: Output DTN: LA0307SK831231.001.

Figure 7-9b. Transport Pathways Deduced from Hydrochemistry Data (in red, enlarged from Figure 7-9a) Overlaying Flow Paths Calculated from the Site-Scale SZ Transport Model (in black) for Tracer Particles Starting at the Repository Footprint 
The flow paths deduced from the geochemistry data are qualitative in nature and denote broad areas of flow continuity inferred from available data, rather than specific streamlines. They are meant to represent broad flow directions and not the detailed variations that can be seen in a streamline computed from the model. It is seen in Figure 7-9b that the model streamlines originating at the repository footprint follow the general orientation and remain within the flow regions defined by the Flow paths 7 and 2 deduced from the geochemistry data. Hence, this validation is considered acceptable as defined in the technical work plan (BSC 2003 [DIRS 163965], Section 2.3, second validation criteria)

\subsubsection{Comparison of Transit Times Against Those Deduced from ${ }^{14} \mathrm{C}$ Data}

The radioactive isotope of carbon, ${ }^{14} \mathrm{C}$, is produced in the atmosphere primarily by the interaction of cosmic rays with the atmospheric ${ }^{14} \mathrm{~N}$. ${ }^{14} \mathrm{C}$ decays with a half-life of 5,730 years. ${ }^{14} \mathrm{C}$ is rapidly incorporated into the atmospheric $\mathrm{CO}_{2}$ and becomes available for terrestrial processes including that of dissolution into atmospheric precipitation as presented in Saturated Zone Site-Scale Flow Model (BSC 2004 [DIRS 170037], Section A6.6.1.2.2). ${ }^{14} \mathrm{C}$ enters the SZ groundwater through recharge and is transported principally as bicarbonate as given in Saturated Zone Site-Scale Flow Model (BSC 2004 [DIRS 170037], Section A6.6.1.2.2) as a nonsorbing species. Estimates of groundwater age are obtained from the measured ${ }^{14} \mathrm{C}$ activity, corrected for possible water/rock interactions are given in Saturated Zone Site-Scale Flow Model (BSC 2004 [DIRS 170037], Section A6.6.1.2.2). In interpreting these age estimates, it must be noted that the possibility of a fraction of younger water mixing with older water leading to the apparent age as determined from the ${ }^{14} \mathrm{C}$ activity cannot be ruled out with complete certainty. Radiometric dates in geochemically open systems are mixed dates that can be used for estimating upper and lower bounds of the groundwater ages. Hence, the values obtained from ${ }^{14} \mathrm{C}$ activity data should be interpreted as representing the likely range of groundwater ages, without excluding some probability of groundwater ages having values outside the indicated range.

Data from water samples taken from 7 wells in the vicinity of Yucca Mountain were analyzed for ${ }^{14} \mathrm{C}$ activity as shown in Saturated Zone Site-Scale Flow Model (BSC 2004 [DIRS 170037], Section A6.6.6.6.2) and the resulting ages are reported to lie in the range of 11,430 years to 16,390 years as presented in Saturated Zone Site-Scale Flow Model (BSC 2004 [DIRS 170037], Table A6.-9). These ages reflect the time from atmospheric precipitation to the present and thus include transport time through the $\mathrm{UZ}$ as well as the SZ, along with the residence time within SZ. Thus, they are not direct indicators of transit times in the SZ, but they do provide a plausible upper bound on the SZ transit time.

Groundwater transit times can also be inferred from measured ${ }^{14} \mathrm{C}$ activity from water samples taken from wells that are inferred to lie along a flow path and where the geochemical compositions of waters suggests that flow paths exist between the two wells as given in Saturated Zone Site-Scale Flow Model (BSC 2004 [DIRS 170037], Section A6.6.8.10). Data from two wells were used: UE-25 WT\#3 and NC-EWDP-19D, both of which are downstream of the repository footprint, and lie on the interpreted flow path 7 that starts at Yucca Mountain and moves downstream in an overall southwardly direction (Figure 7-9a). The approximate distance between the two wells is $15 \mathrm{~km}$ as shown in Saturated Zone Site-Scale Flow Model (BSC 2004 [DIRS 170037], Section A6.6.9.1). The ${ }^{14} \mathrm{C}$ transport times lie in the range of 0 (or negative values) years to 3,110 years over the sampled depths shown in Saturated Zone Site-Scale Flow 
Model (BSC 2004 [DIRS 170037], Section A6.6.9, Table A6-13). The zero or negative values, as discussed in Saturated Zone Site-Scale Flow Model (BSC 2004 [DIRS 170037], Section A6.6.9.1), likely result from experimental uncertainties, and are not considered in this analysis. The data are clustered around two ranges corresponding to different sampling intervals: one in the interval of 188 to 535 years and the other in the interval of 1,601 to 3,110 years. Using the interwell distance of $15 \mathrm{~km}$ as stated above, these ranges translate into groundwater velocity ranges of 80 to $28 \mathrm{~m} /$ year and 9.4 to $4.8 \mathrm{~m} /$ year. Using a nominal distance of $18 \mathrm{~km}$ from the repository footprint to the boundary of the accessible environment at $18 \mathrm{~km}$ and taking constant velocities along the flow path, these velocities lead to transit time ranges of 226 to 643 years and 1,915 to 3,750 years. As discussed in the previous paragraph, in interpreting these transit time estimates, it must be noted that the possibility of a fraction of younger water mixing with older water leading to the apparent age as determined from the ${ }^{14} \mathrm{C}$ activity cannot be ruled out with complete certainty. Hence, the values obtained from ${ }^{14} \mathrm{C}$ activity data should be interpreted as representing the likely range of groundwater transport times, without excluding a small probability of groundwater ages having values outside the indicated range.

There are two simplifications built into the above estimates (in addition to the ${ }^{14} \mathrm{C}$ data interpretation as discussed in Saturated Zone Site-Scale Flow Model (BSC 2004 [DIRS 170037], Sections A6.6.8 and A6.6.9): one is that in calculating velocities from the transport time estimates, a nominal travel distance is used equal to the well separation, and the second is that a constant velocity is used along the entire flow path. Regarding the first simplification, using a nominal distance instead of the distance along the actual flow path, details of the tortuousness of the flow path are neglected. In actuality, the distance along the flow path will be somewhat greater than the interwell spacing, and thus the calculated velocity will be somewhat less than the actual velocity. However, the influence of this simplification on the estimated transport time to the 18-km boundary is somewhat offset by the fact that the actual travel distance along the flow path (including the segment beyond the well UE-25 WT\#3) is also replaced by the somewhat lower value of $18 \mathrm{~km}$, thus a ratio is taken of two numbers that are both somewhat lower than the actual values in the field. Secondly, in using a constant velocity along the flow path from the well UE-25 WT\#3 to the boundary of the accessible environment at $18 \mathrm{~km}$, an average of velocities in the volcanics and the alluvium is applied to the additional distance of $3 \mathrm{~km}$ that is traveled within the alluvium from the well NC-EWDP-19D to the boundary of the accessible environment. Considering that the porosity in alluvium is many orders of magnitude greater than the volcanic rocks, the effect of this simplification could be quite large. This would result in the estimated upper limit of 3,750 years being an underestimation of the actual transport time.

Transport model simulations were conducted to evaluate the propagation of uncertainty in the input parameter values to the output breakthrough curves, as documented in Section 6.7 of this report. Starting with the base-case parameter values (Section 6), scenarios were constructed by considering one parameter at a time, using the upper and lower limit of each parameter value (Table 6.7-1). The parameters considered include specific discharge, horizontal anisotropy ratio in permeability, effective porosity in the alluvium, flowing interval aperture in the volcanics, effective diffusion coefficient in the volcanics, and the longitudinal dispersivity. Reactive transport parameters were also considered in the analysis described in Section 6.7 but are not considered here, as they are not relevant to ${ }^{14} \mathrm{C}$ transport. The time at which 50 percent of the injected particles broke through the boundary of the accessible environment at $18 \mathrm{~km}$ downstream from the repository footprint is given in Table 6.7-1. 
It can be seen from Table 6.7-1 that most of the nonsorbing cases (Case numbers 1, 2, 3, 5, 8, 9, and 13) considered to predict transport times within the range estimated on the basis of the ${ }^{14} \mathrm{C}$ hydrochemistry data discussed on the previous page of 226 to 3,750 years. The base-case transport model leads to a transit time of 705 years, well within the range predicted by hydrochemistry data. This validation is considered acceptable, as defined in the technical work plan (BSC 2003 [DIRS 163965], Section 2.3, first bullet of validation criteria) (reference to the previous version of the technical work plan has been retained because it specifies the validation criteria to which this work was done). (These criteria are not reproduced in the new version.) There are three outlier cases-using the limits of data interpretation discussed in the first paragraph of this section, and as discussed below, they are considered to be within the requirements of the model validation criteria:

- The case of maximum specific discharge leads to a transport time of 50 years, which is lower than the lower limit of 226 years calculated above. This case is within the criteria specified in the technical work plan (BSC 2003 [DIRS 163965], Section 2.3, first bullet of validation criteria) (reference to the previous version of the technical work plan has been retained because it specifies the validation criteria to which this work was done). (These criteria are not reproduced in the new version.)

- The case of minimum specific discharge leads to a transport time greater than 10,000 years, which is greater than the upper limit of 3,750 years calculated from the ${ }^{14} \mathrm{C}$ data. As noted in the first paragraph of this section, the value of 3,600 years is an estimate of the upper bound in the range of inferred transit times, and longer transport times cannot be completely excluded from consideration, based on the available data.

- A transit time of 3,750 years would result from a specific discharge of approximately $0.14 \mathrm{~m} /$ year. The rationale for choosing a lower value of $0.02 \mathrm{~m} /$ year as the bounding value of the uncertainty distribution is based on Saturated Zone Flow and Transport Expert Elicitation Project (CRWMS M\&O 1998 [DIRS 100353], Figures 3-2a through 3-2e) and field data at the ATC as shown in Saturated Zone In-Situ Testing (BSC 2004 [DIRS 170010], Section 6.5). The rationale is discussed in Saturated Zone Flow and Transport Model Abstraction (BSC 2004 [DIRS 170042], Section 6.5.2.1), and as seen in Figure 6-7 of that report, the uncertainty distribution at the low values of the specific discharge multiplier tapers off to 0 at a value of 1/30, and the cumulative probability of finding a value of specific discharge multiplier less than 1/4 (corresponding to the approximate transport time of 3,750 years) is low (about 0.08), and only a few stochastically sampled values are expected to fall below the factor of 1/4.

- The case of a minimum value of flowing-interval aperture, which corresponds to the product of the minimum value of flowing-interval porosity and the minimum value of flowing-interval spacing, leads to a transit time greater than 10,000 years, which is greater than the upper limit of 3,750 years calculated from the ${ }^{14} \mathrm{C}$ data. As noted in the first paragraph of this section, the value of 3,750 years is an estimate of the upper bound in the range of inferred transit times, and longer transport times cannot be completely excluded from consideration, based on the available data. 
A transit time of 3,750 years would result from a flowing-interval aperture of approximately $2 \times 10^{-4} \mathrm{~m}$. The flowing interval aperture is calculated as the product of the flowing interval spacing and the flowing interval porosity. The rationale for choosing a lower value of $1.2 \times 10^{-5} \mathrm{~m}$ as the limiting value of the uncertainty distribution, which corresponds to the lower limit $1.2 \mathrm{~m}$ for spacing and $10^{-5}$ for porosity, is discussed in detail Saturated Zone Flow and Transport Model Abstraction (BSC 2004 [DIRS 170042], Sections 6.5.2.4 and 6.5.2.5). As seen in Figure 6-13 of that report, the uncertainty distribution at the low values of the porosity tapers off to zero at about $10^{-5}$, and Figure 6-12 of that report shows that the interval spacing tapers off to zero at about $1.2 \mathrm{~m}$. Hence, the probability of finding a value of aperture less than $2 \times 10^{-4} \mathrm{~m}$ (corresponding to the approximate transport time of 3,750 years) is low, less than approximately 5 percent. Thus, only a few stochastically sampled values are expected to fall below the validated lower limit of about $2 \times 10^{-4} \mathrm{~m}$.

\subsection{VALIDATION SUMMARY}

The site-scale SZ transport model has been validated by applying acceptance criteria based on an evaluation of the model's relative importance to the potential performance of the repository system. All validation requirements defined in Section 2.3 of the Technical Work Plan for: Saturated Zone Flow and Transport Modeling and Testing have been fulfilled, including corroboration of model results with field data (BSC 2003 [DIRS 163965]). Activities requirements for confidence building during model development have also been satisfied. The model development activities and postdevelopment validation activities described establish the scientific bases for the site-scale SZ transport model. Therefore, the site-scale SZ transport model used in this model report is considered to be sufficiently accurate and adequate for the intended purpose and to the level of confidence required by the model's relative importance to the potential performance of the repository. 


\section{CONCLUSIONS}

The site-scale SZ transport model is the culmination of efforts incorporating geologic, hydrologic, and geochemistry data from laboratory and field-testing with theoretical models of radionuclide transport into a coherent representation of transport through the SZ near Yucca Mountain (Sections 6.3 and 6.4) This model uses as its basis the calibrated SZ site-scale flow model, described in the model and analysis report Saturated Zone Site-Scale Flow Model (BSC 2004 [DIRS 170037], Section 6.6).

The site-scale SZ transport model matches field data both quantitatively and qualitatively. These data include transit times derived from ${ }^{14} \mathrm{C}$ (Section 7.2.2) and flow paths inferred from $\mathrm{Cl}^{-}$and $\mathrm{SO}_{4}{ }^{-2}$ hydrochemical data (Section 7.2.1). The base-case transport model (for present-day climate, not include radionuclide decay, for an instantaneous source) leads to the breakthrough curve (Figure 6.5-1) at the boundary of the accessible environment at $18 \mathrm{~km}$ (10 CFR 63.302 [DIRS 156605]) corresponding to a breakthrough time at 50 percent concentration of 705 years.

The analysis of $K_{d}$ data (Appendix A) and the modeling to upscale these data to a 500-m grid (Appendix C), combined with expert judgment (Appendix C) lead to the $K_{d}$ distributions presented in Table C-14.

When using the site-scale SZ transport model for TSPA-LA calculations, there are limitations that must be noted with regard to the following:

- Input parameter values/ranges. The transport model is intended for use with stochastic simulations using large uncertainty ranges for particular parameters such as specific discharge, fracture spacing and aperture, diffusion coefficient, and sorption coefficients. Care should be exercised in interpreting individual simulations for single sets of parameter values. Also, care should be exercised if the parameters used fall outside the range of parameter values (Table 4-2) or outside the range established by model validation (Section 7.2).

- Useable path-line distances. The flow field underlying the site-scale SZ transport model is based on the dual-porosity, effective-continuum approach requiring large grid blocks that effectively average fracture, rock matrix, and alluvium properties. Also, the parallel fracture model used to model advection/diffusion in the volcanics is valid only for grid-block sizes much larger than the mean flowing-interval spacing of $20 \mathrm{~m}$. It is recommended in Saturated Zone Site-Scale Flow Model (BSC 2004 [DIRS 170037], Section 8) that to produce meaningful results, the flow path should be long compared to the grid-block size, and a minimum distance of $2 \mathrm{~km}$ is recommended for path lines used in PA calculations. 
- Sufficient number of input particles for particle tracking. Radionuclide transport is implemented in the site-scale SZ transport model, using particle tracking with a randomwalk method (Sections 6.4.2.3 and 6.4.2.4). In order to obtain reproducible results, a sufficient number of particles must be input to the model. The base-case transport model utilizes 1,000 input particles (Output DTN: LA0306SK831231.001), visually judged as sufficient for obtaining a smooth breakthrough curve at the boundary of the accessible environment at $18 \mathrm{~km}$ (Figure 6.5-1). However, a larger number of input particles may be required depending on the purpose of the model use.

\subsection{SUMMARY OF MODELING ACTIVITIES}

Available hydrogeology, hydrochemistry, field, and laboratory data on transport processes were reviewed to form a conceptual model of the transport processes of importance to the site-scale SZ region (Sections 6.3 and 7.1). Available data on sorption of radionuclides were synthesized to develop distributions for sorption parameters (Appendices A and C). A mathematical formulation of the conceptual model was developed (Section 6.5) and incorporated in the FEHM numerical code. A calibrated numerical model of groundwater flow, the SZ site-scale flow model, Saturated Zone Site-Scale Flow Model (BSC 2004 [DIRS 170037], Section 6.6) was taken as the basis for transport calculations. A series of validation and confidence-building activities was completed (Section 7). Finally, results of this model were provided (Output DTN: LA0306SK831231.001), and the associated uncertainties were discussed (Section 8.3).

\subsubsection{Hydrogeologic Setting and Conceptual Flow Model}

The discussion of hydrogeologic setting and the conceptual flow model in this subsection is presented for the case of completeness only. Details are given in BSC 2004 [DIRS 170037]. Yucca Mountain is located about $150 \mathrm{~km}$ northwest of Las Vegas, Nevada, in the Great Basin section of the Basin and Range province. Yucca Mountain consists of a group of north-trending block-faulted ridges composed of volcanic rocks bounded by basins composed of volcanic rocks, alluvium, underlain by a thick carbonate aquifer. Yucca Mountain is part of the Alkali Flat-Furnace Creek subbasin of the Death Valley groundwater basin. The water table in the vicinity of the repository footprint is deep, as much as $750 \mathrm{~m}$ below the surface. The SZ occurs in thick ash-flow and ash-fall tuffs, underlain by the regional carbonate aquifer.

The general conceptual model of SZ flow in the model area is that groundwater flows southward from recharge areas in the north and at higher elevations, through the Tertiary volcanic rocks, toward the Amargosa Desert. Water inputs to the site-scale model include inflow along the northern boundary, recharge from precipitation at higher elevations, and recharge from surface runoff in the Fortymile Wash area. In the northern portion of the model, the flow occurs through the fractured volcanic rocks underlain by the carbonate aquifer. Toward the southern portion of the model area, the flow enters the valley-fill alluvium. Discharge from the model occurs via the alluvium, across the model boundary to the south and southeast, and through pumping of wells in the Amargosa Valley. 
The overall groundwater flow is modeled as a 3-D steady-state system. A confined aquifer solution is used for the flow model. The dual-porosity, effective-continuum approach is used to model flow and transport, which averages fracture and surrounding rock properties in a given grid block, with the modification that several of the important fault zones are included as explicit features.

\subsubsection{Conceptual Model of Transport}

The flow paths from the repository site to the boundary of the accessible environment at $18 \mathrm{~km}$ begin in the volcanic tuffs below the repository footprint and end in the alluvium of the Amargosa Valley (Figure 6.5-2). Within the SZ, radionuclides are transported in the upper-few-hundred meters below the water table with pore water that flows subhorizontally in a southwardly direction through the highly fractured portions of the tuff, leading to the alluvium in the south (Section 6.3). The components of the conceptual model are: (1) radionuclides enter the SZ via fluids percolating through the unsaturated zone (UZ) below the repository site. Within the SZ, they are transported with the groundwater that flows subhorizontally in a southerly or southeasterly direction; (2) the radionuclides advect and disperse with the groundwater through the fractured portions of the tuffs near the water table; (3) fluid flow occurs preferentially within the flowing intervals, whereas stagnant fluid resides in the rock matrix. Solutes diffuse in and out of fluid within the rock matrix that is essentially stagnant; (4) sorption reactions occur in volcanics between the rock matrix and some of the radionuclides, tending to retard the transport of these radionuclides; (5) radionuclides can undergo colloid-facilitated transport in the volcanics; (6) the radionuclides advect and disperse with the groundwater through the alluvium; (7) sorption reactions occur in alluvium between the rock and some of the radionuclides, tending to retard the transport of these radionuclides; (8) radionuclides can undergo colloid-facilitated transport in alluvium; and (9) the radionuclides reaching the model boundary, which is downstream from the boundary to the accessible environment at $18 \mathrm{kms}$ downstream of the repository footprint are removed from the model.

The mathematical basis (and the associated numerical approaches) of the site-scale SZ transport model is designed to incorporate the important transport processes in an efficient numerical code to compute radionuclide breakthrough curves and transit times for use in TSPA-LA simulations (Section 6.4). A particle-tracking approach is used to compute solute trajectories and transport times, combined with a random-walk model to incorporate dispersion. A semianalytical method is used for including retardation due to diffusion and sorption, as well as colloid-facilitated transport in the random-walk model.

\subsubsection{Model Validation and Confidence Building}

Recognizing that the site-scale SZ transport model is being used to perform probabilistic calculations in which parameter uncertainties are propagated through the model, the intent of validation was to confirm the radionuclide parameters and processes included in the model. Confidence in the results of the model was built by a series of different approaches (Section 7.1) that included: (1) comparisons to analogue sites, (2) model-data comparisons, (3) comparison with data published in referenced journals, and (4) comparison to an independent site-scale groundwater flow model for the Yucca Mountain SZ. The data used in the confidence building for the relevant transport parameters (e.g., sorption coefficient), submodel processes 
(e.g., advection, sorption), and site-scale model processes (e.g., flow pathways, transit times) were based on laboratory testing, field tests, natural analogue sites, and expert elicitations. The model was validated by postdevelopment comparison of model transit times with those inferred from ${ }^{14} \mathrm{C}$ data (Section 7.2.2) and qualitative comparison of flow paths predicted by the model and those inferred from the hydrochemistry and isotopic data (Section 7.2.1).

\subsection{OUTPUTS}

\subsubsection{Technical Output}

The technical output of this report is composed of (1) the site-scale SZ transport model and associated input and output files (base-case transport files); and (2) SZ Distribution Coefficients $\left(K_{d} \mathrm{~S}\right)$ data for uranium, neptunium, plutonium, cesium, americium, protactinium, strontium, thorium, radium, carbon, technetium, and iodine (Table C-14). The output breakthrough curves and transport times will be integrated into the SZ flow and transport abstractions model for use in the TSPA-LA calculations.

\subsubsection{Developed Output Listed by Data Tracking Number}

The outputs associated with the site-scale SZ transport model are listed in Table 8.2-1.

Table 8.2-1. Output Data

\begin{tabular}{|c|c|c|}
\hline \multicolumn{3}{|c|}{ Transport Model } \\
\hline Data Description & Source & Data Tracking Number \\
\hline $\begin{array}{l}\text { Saturated zone distribution coefficient }\left(K_{d}\right) \text { data } \\
\text { for } \mathrm{U}, \mathrm{Np}, \mathrm{Pu}, \mathrm{Cs}, \mathrm{Am}, \mathrm{Pa}, \mathrm{Sr}, \mathrm{Th} \text {, and } \mathrm{Ra}\end{array}$ & Appendices $\mathrm{A}$ and $\mathrm{C}$ & LA0310AM831341.002 \\
\hline $\begin{array}{l}\text { Type curve data for FEHM macro 'sptr' based on } \\
\text { Sudicky and Frind solution }\end{array}$ & Section 6 & LA0302RP831228.001 \\
\hline $\begin{array}{l}\text { Modeling calculations of radionuclide sorption via } \\
\text { surface-complexation reactions and solubilities of } \\
\mathrm{U}, \mathrm{Sr}, \mathrm{Ba} \text { in water }\end{array}$ & Appendix A & $\begin{array}{l}\text { LA0407AM831343.001 } \\
\text { Files contained in the } \\
\text { directory "output" }\end{array}$ \\
\hline $\begin{array}{l}\text { Files for FEHM } \vee 2.20 \text { for site-scale SZ transport } \\
\text { model, FEHM files for base case }\end{array}$ & Section 6.4 .3 and Section 6.5 & LA0306SK831231.001 \\
\hline $\begin{array}{l}\text { Files for FEHM V } 2.20 \text { for evaluating barrier } \\
\text { capabilities }\end{array}$ & Section 6.8 & LA0307ZD831231.001 \\
\hline $\begin{array}{l}\text { Files for FEHM V } 2.20 \text { for comparison of flow } \\
\text { paths against those from hydrochemistry data }\end{array}$ & Section 7.41 & LA0307SK831231.001 \\
\hline Files for FEHM V 2.20 for specific discharge & Section 6.4 .3 and Section 6.5 & LA0307SK831231.002 \\
\hline $\begin{array}{l}\text { Files for FEHM V } 2.20 \text { for evaluating propagation } \\
\text { of uncertainty in the input parameter values }\end{array}$ & Section 6.7 & LA0309SK831231.001 \\
\hline $\begin{array}{l}\text { Files for GSLIB for generating stochastic } \\
\text { realizations of rock types from mineralogic data }\end{array}$ & Appendix C & LA0309RP831321.001 \\
\hline $\begin{array}{l}\text { Files FEHM } \vee 2.20 \text { for calculation of effective } K_{d} \\
\text { distribution for } U\end{array}$ & Appendix C & LA0309RP831341.004 \\
\hline $\begin{array}{l}\text { Files FEHM V } 2.20 \text { for calculation of effective } K_{d} \\
\text { distribution for } \mathrm{Cs}\end{array}$ & Appendix C & LA0309RP831341.001 \\
\hline
\end{tabular}


Table 8.2-1. Output Data (Continued)

\begin{tabular}{|l|l|l|}
\hline \multicolumn{2}{|c|}{ Transport Model } \\
\hline \multicolumn{1}{|c|}{ Data Description } & \multicolumn{1}{|c|}{ Source } & Data Tracking Number \\
\hline $\begin{array}{l}\text { Files FEHM } \vee 2.20 \text { for calculation of effective } K_{d} \\
\text { distribution for Np }\end{array}$ & Appendix C & LA0309RP831341.002 \\
\hline $\begin{array}{l}\text { Files FEHM } \vee 2.20 \text { for calculation of effective } K_{d} \\
\text { distribution for Pu }\end{array}$ & Appendix C & LA0309RP831341.003 \\
\hline $\begin{array}{l}\text { Files for FEHM } \vee 2.20 \text { for site-scale SZ transport } \\
\text { model, FEHM files for matrix sorption }\end{array}$ & Section 6.4.3 and Section 6.5 & LA0403SK831231.001 \\
\hline
\end{tabular}

\subsection{OUTPUT UNCERTAINTY}

Model-form uncertainty is explicitly addressed by discussing alternative conceptual models in Section 6.6 of this report. There are uncertainties associated with scaling parameter values from the scale of measurements to the scale of interest. Much of the data used for deriving parameter values in this report are from laboratory or field experiments conducted on spatial and temporal scales much smaller than those in the site-scale SZ model. Geological formations are inherently inhomogeneous, and they embed fractures, faults, and other heterogeneities on a variety of scales. Spatial correlations over some length scales often exist. Thus, it is difficult to extrapolate the measurements on a small scale to the SZ site scale. Accommodations are made for these uncertainties by choosing wide distributions of input parameters (Table 4-2) for TSPA analysis (BSC 2004 [DIRS 170042], Section 6.5.2).

Epistemic uncertainty is explicitly described by using probability distributions for appropriate model parameters. Expected uncertainty ranges for the various transport parameters are discussed in Section 4.1.2 and summarized in Table 4-2 of this report. The development and discussion of the $K_{\mathrm{d}}$ parameters needed for modeling reactive transport and the $K_{\mathrm{c}}$ parameters needed for modeling colloid-facilitated transport are presented in Appendices A, B, and C of this report. The probability distributions for all other model parameters are presented and discussed in the model report Saturated Zone Flow and Transport Model Abstraction (BSC 2004 [DIRS 170042], Section 6.5.2). These uncertainties are propagated through the model to the output breakthrough curves, presented in Section 6.7 of this report. Radionuclide transit times are most sensitive to groundwater-specific discharge. Other parameters of importance to the breakthrough times are matrix diffusion, the sorption coefficient in the volcanics as well as the alluvium, the effective fracture porosity in the volcanics (as reflected in the flowing interval aperture and spacing), the effective porosity in the alluvium, and the sorption coefficients for reversible colloids and retardation factor for irreversible colloids in the volcanics and alluvium. Quantification of the sensitivity of the model output breakthrough curves for various radionuclides of concern to parameter uncertainties are further evaluated in the model report Saturated Zone Flow and Transport Model Abstraction (BSC 2004 [DIRS 170042], Sections 6.7 and 8).

\subsection{HOW THE ACCEPTANCE CRITERIA ARE ADDRESSED}

The following information describes how this analysis addresses the acceptance criteria in the YMRP (NRC 2003 [DIRS 163274], Section 2.2.1.3.9.3). Only those acceptance criteria that are applicable to this report (Section 4.2) are discussed. In most cases, the applicable acceptance 
criteria are not addressed solely by this report; rather, the acceptance criteria are fully addressed when this report is considered in conjunction with other analysis and model reports that describe flow and transport in the SZ. Compliance with the procedures was determined through QA audits and other oversight activities.

\section{Acceptance Criteria from Section 2.2.1.3.9.3, Radionuclide Transport in the Saturated Zone}

\section{Acceptance Criterion 1: System Description and Model Integration Are Adequate}

Subcriterion (1): The model described in this model report incorporates or considers known Yucca Mountain physical phenomena and couplings affecting radionuclide transport through the SZ. Sections 6.1 through 6.8 describe the main SZ transport processes that were included to construct an efficient model to be used in the abstraction process and TSPA-LA. The primary assumptions incorporated in the model (Section 5) are: (1) Sorption on individual fracture surfaces is not included; (2) Sorption coefficients assume that conditions are oxidizing in groundwater; and (3) For $\mathrm{Am}, \mathrm{Cs}, \mathrm{Pu}, \mathrm{Pa}, \mathrm{Sr}$, and Th, sorption coefficients are assumed to be those corresponding to the values measured on samples of devitrified crushed tuff. These assumptions are consistent with the model (Section 6.3) and with those made in reports supporting other abstractions prepared for Yucca Mountain and the assumptions are appropriate for describing a conservative model of transport in the SZ (Sections 5 and 6.3).

Subcriterion (2): This report provides extensive descriptions of the aspects of hydrology, geology, geochemistry, physical phenomena, and couplings that are considered and/or included in the site-scale model (see Sections 6.2 for FEPs and 6.3 for model development). Conditions and assumptions supporting the abstraction of radionuclide transport in the SZ are readily identified in Sections 5, 6.3, 6.4, and 6.6 and they are consistent with the body of data incorporated in the report (Section 4.1). Changes in transport due to aspects of sorption and matrix diffusion are considered and are either specifically included in the model or discarded due to nonconservatism (Sections 6.2, 6.3, and 6.6).

Subcriterion (3): The assumptions, technical bases, data, and models employed in this site-scale model are appropriate due to their inclusion of major factors affecting transport of radionuclides in the SZ (Sections 4.1, 6.1, 6.3, and 6.6) and are consistent with other related Yucca Mountain abstractions. The site-scale SZ model described in this report is used directly in the model report Saturated Zone Flow and Transport Model Abstraction (BSC 2004 [DIRS 170042]) for generating a set of radionuclide breakthrough curves at the accessible environment for use in the TSPA-LA simulations of radionuclide release to the biosphere. The descriptions and technical bases in this report (detailed in Sections 6 and 7) provide transparent and traceable support for the abstraction of radionuclide transport in the SZ through detailed descriptions of assumptions, phenomena affecting transport, and numerical methods employed.

Subcriterion (5): This model report addresses the SZ FEPs pertaining to the site-scale SZ transport that are included for TSPA-LA (Table 6.2-1). Saturated Zone FEPs that were excluded for TSPA-LA are described in Features, Events, and Processes in SZ Flow and Transport (BSC 2004 [DIRS 170013]. Table 6.2-1 provides a list of FEPs that are relevant to this model analysis in accordance with their assignment in the LA FEP list (DTN: MO0407SEPFEPLA.000 [DIRS 170760]). 
Subcriterion (6): This report was developed using processes and procedures consistent with the QARD, which commits to these NUREGs and with AP-SIII.10Q, Models, which implements those commitments for model reports.

\section{Acceptance Criterion 2: Data Are Sufficient for Model Justification}

Subcriterion (1): Input parameters to this transport model are radionuclide release locations, dispersivities in the volcanics, matrix porosity, matrix diffusion coefficient, sorption distribution coefficient $K_{d}$ in the matrix, flowing interval porosity, flowing interval spacing, retardation factor in the flowing interval, effective porosity of the alluvium, dispersivities in the alluvium, and the $K_{d}$ value in the alluvium. Their base-case values and range of values are listed in Table 6.4-2 in Section 6.4.3 and in Table 4-2 in Section 4. Adequate justifications for the average or range of values chosen and descriptions of how the data comprising these values were interpreted and used are presented in detail in Sections 6.3 and 6.4.

Subcriterion (3): Data employed in this model and results were developed from Yucca Mountain borehole data, Yucca Mountain and other related field experiments, laboratory experiments, natural analogue research, and current, accepted technical information as shown in Table 4-1. The documents cited as sources in Table 4-1 provide detailed descriptions of the methods used in generating the data. Discussions of model uncertainties are included in Section 6.7. Sensitivity of the output breakthrough curves to each of the uncertain input parameters is discussed in Sections 6.7.1.1 through 6.7.1.13.

\section{Acceptance Criterion 3: Data Uncertainty Is Characterized and Propagated Through the Model Abstraction}

Subcriterion (1): Site-scale SZ transport model parameters are listed in Table 6.4-2 in Section 6.4.3 and in Table 4-2 in Section 4. Sections 6.3, 6.4, and 6.6 provide technically defensible arguments for their adoption in this model. Those sections also discuss uncertainties in the parameters and nonconservatisms that might be introduced if other parameters or approaches were adopted. Also, the formulation of the model incorporates numerous conservative approaches that should prevent an underestimate of the risk estimate. This conclusion is supported by the validation process that employed analogue studies (Section 7.2) to support the chosen transport parameters and the submodel components.

Subcriterion (2): Sources of flow and transport parameters for various nuclides important to waste isolation are given in Table 4-1 (Section 4.1). These parameters are based on Yucca Mountain borehole data, Yucca Mountain and other related field experiments, laboratory experiments, natural analogue research, and current, accepted technical information as shown in Table 4-1 and in assumption number 3 in Table 5-1 for the sorption coefficients of radionuclides Am, Cs, Pu, Pa, Sr, and Th in alluvium. The model was validated by various methods including: (1) analogue studies from the NTS (Section 7.1.1.1) and uranium transport at natural analogue sites (Section 7.1.1.2); (2) comparison of flow paths against those deduced from hydrochemistry data (Section 7.2.1); and (3) comparison of transit times against those deduced from ${ }^{14} \mathrm{C}$ data (Section 7.2.2). The submodel components were validated by numerous methods described in Sections 7.1.2.1 through 7.1.2.9. 
Subcriterion (4): Effective diffusion coefficients, matrix sorption coefficients, dispersivity coefficients, and colloid retardation factors in volcanics and alluvium employed in this model are consistent with aquifer properties. Their parametric values and data sources are shown in Table 4-2. These sources are primarily documents describing Yucca Mountain aquifer characteristics taken from borehole data and experiments. Further, the above parameters are taken to be stochastic variables sampled from broad distributions to allow for uncertainties in the aquifer characteristics and the influence of these uncertainties on the parameter values.

Subcriterion (5): Uncertainty in representation of radionuclide transport is adequately represented by adopting conservative positions on mechanisms and alternate models included in this site-scale SZ transport model. An example is that sorption on individual fracture surfaces was not included in the model because it would tend to retard radionuclide transport and the mineral distributions in fractures as opposed to the matrix are not well known. Another example is oxidizing conditions were included along the entire flow path because they tend to lead to lower values of sorption coefficients (Section 6.3, Item 4). Section 6.6 discusses alternative conceptual models that were screened out because they lead to transit times greater than those calculated by the site-scale SZ transport model. Discussions of model uncertainties are included in Section 6.7. Sensitivity of the output breakthrough curves to each of the uncertain input parameters is discussed in Sections 6.7.1.1 through 6.7.1.13.

Subcriterion (6): Sufficient data did not exist hence the process of expert elicitation was used for determining the range of values of the specific discharge multiplier (Section 4.1.2.1) and dispersivities (Sections 4.1.2.16, 4.1.2.17, and 4.1.2.18), as well as the distribution functions for sorption coefficients of various radionuclides (Appendix C). Further, because of the time and spatial scales involved and the fact that radionuclides cannot be used as tracers in field experiments, confidence-building activities during model development (Section 7.1) as well as postdevelopment validation (Section 7.2) were conducted to support the definition of parameter values and the conceptual models (Sections 6.3, and 6.4) used in this report.

\section{Acceptance Criterion 4: Model Uncertainty Is Characterized and Propagated Through the Model Abstraction}

Subcriterion (1): Alternative conceptual models are discussed in detail in Section 6.6. The alternative conceptual models fall into two classes: (1) Those that are screened out because they lead to transit times greater than those calculated by the site-scale SZ transport model (Table 6.6-1a); and (2) alternative conceptual models that are implicitly included in the site-scale SZ transport model through the range of uncertainty in parameter values (see Table 6.4-1b). The key components, screening assessment, and basis for the assessment are presented in Tables 6.6-1a and 6.6-1b. The screening assessment and basis discuss the model approach, assumptions, results, and whether the alternative conceptual model is included implicitly or explicitly in the model supporting the abstraction of SZ radionuclide transport.

Subcriterion (2): Discussions of conceptual model uncertainties are included in Section 6.6 and 6.7. Sensitivity of the output breakthrough curves to each of the uncertain input parameters is discussed in Sections 6.7.1.1 through 6.7.1.13. Expected uncertainty ranges for transport parameters is discussed in Sections 4.1 and 6.7. Although the model is meant to represent the SZ transport accurately, for the case of those alternative conceptual models that could not be 
included in the model, the transport model was selected such that it resulted in transit times faster than those expected for the alternative conceptual model (Section 6.6). Uncertainties associated with scaling parameter values from the scale of measurements to the scale of interest are discussed in Section 6.7. Sensitivity of the output breakthrough curves to each of the uncertain input parameters was investigated by considering the upper and lower levels of each parameter individually (see Section 6.7 and Table 6.7-1).

Subcriterion (3): The uncertainties related to the $K_{d}$ parameters needed for modeling reactive transport, the $K_{\mathrm{c}}$ parameters needed for modeling colloid-facilitated transport, and the probability distributions for all other model parameters are propagated through the model to the output breakthrough curves, presented in Section 6.7 of this report. Through analysis of sensitivity of the output breakthrough curves to each of the uncertain input parameters (see Section 6.7 and Table 6.7-1), the uncertainty of the site characterization data, experiments, measurements, and studies is incorporated. The model formulation results in conservative choices of parameters and approaches that result in relatively faster transit times for transport of radionuclides than otherwise would exist. Therefore, this model does not contribute to an under-representation of the risk estimate.

Subcriterion (4): Alternative conceptual models are discussed in detail in Section 6.6 and are consistent with available and current scientific knowledge. The results and limitations of each of the models and approaches are presented in Tables 6.6-1a and 6.6-1b. The screening assessment and basis in those tables discuss approach, assumptions, supporting data, and scientific knowledge associated with the model. For example, "water table rise" considers potential future glacial climatic conditions that could occur as is discussed in the model in Section 6.6.5. Other implicitly included alternative conceptual models in Table 6.6-1b are analyzed in Appendices A through D. 


\section{INTENTIONALLY LEFT BLANK}




\section{INPUTS AND REFERENCES}

The following is a list of the references cited in this document. Column 2 represents the unique six digit numerical identifier (the Document Input Reference System number), which is placed in the text following the reference callout (e.g., BSC 2004 [DIRS 161340]). The purpose of these numbers is to assist in locating a specific reference. Multiple sources by the same author (e.g., BSC 2004) are sorted alphabetically by title.

\subsection{DOCUMENTS CITED}

Allard, B. 1982. Sorption of Actinides in Granitic Rock. SKB TR-82-21.

104512

Stockholm, Sweden: Svensk Kärnbränsleförsörjning A.B. TIC: 205892.

Allard, B. and Beall, G.W. 1979. "Sorption of Americium on Geologic Media."

147235

Journal of Environmental Health, A14, (6), 507-518. New York, New York:

Marcel Dekker. TIC: 224102.

Allard, B.; Beall, G.W.; and Krajewski, T. 1980. "The Sorption of Actinides in

104410

Igneous Rocks.” Nuclear Technology, 49, (3), 474-480. La Grange Park, Illinois:

American Nuclear Society. TIC: 245772.

Allard, B.; Olofsson, U.; Torstenfelt, B.; and Kipatsi, H. 1983. Sorption Behaviour

162982

of Well-Defined Oxidation States. SKB TR-83-61. Stockholm, Sweden: Svensk

Kärnbränsleförsörjning A.B. TIC: 206122.

Altman, W.D.; Donnelly, J.P.; and Kennedy, J.E. 1988. Qualification of Existing

103750

Data for High-Level Nuclear Waste Repositories: Generic Technical Position.

NUREG-1298. Washington, D.C.: U.S. Nuclear Regulatory Commission. TIC:

200652.

Altman, W.D.; Donnelly, J.P.; and Kennedy, J.E. 1988. Peer Review for High-Level

103597

Nuclear Waste Repositories: Generic Technical Position. NUREG-1297.

Washington, D.C.: U.S. Nuclear Regulatory Commission. TIC: 200651.

Anderson, M.P. 1979. "Using Models to Simulate the Movement of Contaminants

Through Groundwater Flow Systems.” CRC Critical Reviews in Environmental

Control. Pages 97-156. Boca Raton, Florida: CRC Press. TIC: 224010.

Bahr, J.M. and Rubin, J. 1987. "Direct Comparison of Kinetic and Local

Equilibrium Formulations for Solute Transport Affected by Surface Reactions.”

Water Resources Research, 23, (3), 438-452. Washington, D.C.: American

Geophysical Union. TIC: 246894. 
Beall, G.W.; Lee, W.W.-L.; and Van Luik, A.E. 1986. "Americium Speciation and 162983 Distribution Coefficients in a Granitic Ground Water.” Scientific Basis for Nuclear Waste Management IX, Symposium held September 9-11, 1985, Stockholm, Sweden. Werme, L.O., ed. 50, 501-508. Pittsburgh, Pennsylvania: Materials Research Society. TIC: 203664.

Bear, J. 1972. Dynamics of Fluids in Porous Media. Environmental Science Series. Biswas, A.K., ed. New York, New York: Elsevier. TIC: 217356.

Bedinger, M.S.; Sargent, K.A.; Langer, W.H.; Sherman, F.B.; Reed, J.E.; and Brady, B.T. 1989. Studies of Geology and Hydrology in the Basin and Range Province, Southwestern United States, for Isolation of High-Level Radioactive Waste-Basis of Characterization and Evaluation. U.S. Geological Survey Professional Paper 1370-A. Washington, D.C.: U.S. Government Printing Office. ACC: NNA.19910524.0125.

Berry, J.A.; Hobley, J.; Lane, S.A.; Littleboy, A.K.; Nash, M.J.; Oliver, P.; Smith-Briggs, J.L.; and Williams, S.J. 1989. "Solubility and Sorption of Protactinium in the Near-Field and Far-Field Environments of a Radioactive Waste Repository.” Analyst, 114, 339-347. Cambridge, England: Royal Society of Chemistry. TIC: 247004.

Broxton, D.E.; Warren, R.G.; Hagan, R.C.; and Luedemann, G. 1986. Chemistry of Diagenetically Altered Tuffs at a Potential Nuclear Waste Repository, Yucca Mountain, Nye County, Nevada. LA-10802-MS. Los Alamos, New Mexico: Los Alamos National Laboratory. ACC: MOL.19980527.0202.

BSC (Bechtel SAIC Company) 2002. Guidelines for Developing and Documenting Alternative Conceptual Models, Model Abstractions, and Parameter Uncertainty in the Total System Performance Assessment for the License Application.

TDR-WIS-PA-000008 REV 00 ICN 01. Las Vegas, Nevada: Bechtel SAIC Company. ACC: MOL.20020904.0002.

BSC 2002. Radionuclide Screening. ANL-WIS-MD-000006 REV 01. Las Vegas, Nevada: Bechtel SAIC Company. ACC: MOL.20020923.0177.

BSC 2003. Technical Work Plan for: Saturated Zone Flow and Transport Modeling and Testing. TWP-NBS-MD-000002 REV 01. Las Vegas, Nevada: Bechtel SAIC Company. ACC: DOC.20030618.0021.

BSC 2004. Analysis of Hydrologic Properties Data. ANL-NBS-HS-000042, Rev. 00. Las Vegas, Nevada: Bechtel SAIC Company.

BSC 2004. Dissolved Concentration Limits of Radioactive Elements.

156269

129676

100023

158794

ANL-WIS-MD-000010, Rev. 03. Las Vegas, Nevada: Bechtel SAIC Company. 
BSC 2004. Features, Events, and Processes in SZ Flow and Transport.

170013 ANL-NBS-MD-000002, Rev. 03. Las Vegas, Nevada: Bechtel SAIC Company.

BSC 2004. Hydrogeologic Framework Model for the Saturated Zone Site Scale

170008 Flow and Transport Model. MDL-NBS-HS-000024, Rev. 00. Las Vegas, Nevada: Bechtel SAIC Company.

BSC 2004. In-Package Chemistry Abstraction. ANL-EBS-MD-000037, Rev. 03. Las Vegas, Nevada: Bechtel SAIC Company.

BSC 2004. Mineralogic Model (MM3.0) Report. MDL-NBS-GS-000003 REV 01. Las Vegas, Nevada: Bechtel SAIC Company. ACC: DOC.20040908.0006.

BSC 2004. Natural Analogue Synthesis Report. TDR-NBS-GS-000027 REV 01. Las Vegas, Nevada: Bechtel SAIC Company. ACC: DOC.20040524.0008.

BSC 2004. Probability Distribution for Flowing Interval Spacing. ANL-NBS-MD-000003, Rev. 01. Las Vegas, Nevada: Bechtel SAIC Company.

BSC 2004. Q-List. 000-30R-MGR0-00500-000-000 REV 00. Las Vegas, Nevada: Bechtel SAIC Company. ACC: ENG.20040721.0007.

BSC 2004. Recharge and Lateral Groundwater Flow Boundary Conditions for the Saturated Zone Site-Scale Flow and Transport Model. ANL-NBS-MD-000010, Rev. 01. Las Vegas, Nevada: Bechtel SAIC Company.

BSC 2004. Rock Properties Model. MDL-NBS-GS-000004 REV 01. Las Vegas, Nevada: Bechtel SAIC Company. ACC: DOC.20040915.0011.

BSC 2004. Saturated Zone Colloid Transport. ANL-NBS-HS-000031, Rev. 02. Las Vegas, Nevada: Bechtel SAIC Company.

BSC 2004. Saturated Zone Flow and Transport Model Abstraction. 
BSC 2004. Waste Form and In-Drift Colloids-Associated Radionuclide 170025 Concentrations: Abstraction and Summary. MDL-EBS-PA-000004, Rev. 01. Las Vegas, Nevada: Bechtel SAIC Company.

BSC 2004. Water-Level Data Analysis for the Saturated Zone Site-Scale Flow and Transport Model. ANL-NBS-HS-000034, Rev. 02. Las Vegas, Nevada: Bechtel SAIC Company.

Burbey, T.J. and Wheatcraft, S.W. 1986. Tritium and Chlorine-36 Migration from a Nuclear Explosion Cavity. DOE/NV/10384-09. Reno, Nevada: University of Nevada, Desert Research Institute, Water Resources Center. TIC: 201927.

Burnett, R.D. and Frind, E.O. 1987. "Simulation of Contaminant Transport in Three Dimensions, 2. Dimensionality Effects.” Water Resources Research, 23, (4), 695-705. Washington, D.C.: American Geophysical Union. TIC: 246359.

Canori, G.F. and Leitner, M.M. 2003. Project Requirements Document. 166275 TER-MGR-MD-000001 REV 02. Las Vegas, Nevada: Bechtel SAIC Company. ACC: DOC.20031222.0006.

Castor, S.B.; Tingley, J.V.; and Bonham, H.F., Jr. 1994. "Pyritic Ash-Flow Tuff, Yucca Mountain, Nevada.” Economic Geology, 89, 401-407. El Paso, Texas: Economic Geology Publishing. TIC: 234278.

Chipera, S.J. and Bish, D.L. 1989. Quantitative X-Ray Diffraction Analyses of Samples Used for Sorption Studies by the Isotope and Nuclear Chemistry Division, Los Alamos National Laboratory. LA-11669-MS. Los Alamos, New Mexico: Los Alamos National Laboratory. ACC: NNA.19890414.0062.

Chipera, S.J.; Vaniman, D.T.; Carlos, B.A.; and Bish, D.L. 1995. Mineralogic Variation in Drill Core UE-25 UZ\#16, Yucca Mountain, Nevada. LA-12810-MS. Los Alamos, New Mexico: Los Alamos National Laboratory. ACC: NNA.19940427.0099.

Corapcioglu, M.Y. and Jiang, S. 1993. “Colloid-Facilitated Groundwater Contaminant Transport.” Water Resources Research, 29, (7), 2215-2226. Washington, D.C.: American Geophysical Union. TIC: 222362.

Cotton, F.A. and Wilkinson, G. 1980. Advanced Inorganic Chemistry: A 101584 Comprehensive Text. 4th Edition. New York, New York: John Wiley \& Sons. TIC: 217739.

CRWMS (Civilian Radioactive Waste Management System) M\&O (Management 100328 and Operating Contractor) 1997. Report of Results of Hydraulic and Tracer Tests at the C-Holes Complex. Deliverable SP23APM3. Las Vegas, Nevada: CRWMS M\&O. ACC: MOL.19971024.0074. 
CRWMS M\&O 1998. Saturated Zone Flow and Transport Expert Elicitation

100353

Project. Deliverable SL5X4AM3. Las Vegas, Nevada: CRWMS M\&O. ACC:

MOL.19980825.0008.

CRWMS M\&O 2000. Modeling Sub Gridblock Scale Dispersion in

152259

Three-Dimensional Heterogeneous Fractured Media (S0015).

ANL-NBS-HS-000022 REV 00 ICN 01. Las Vegas, Nevada: CRWMS M\&O.

ACC: MOL.20001107.0376.

CRWMS M\&O 2000. Total System Performance Assessment for the Site

153246

Recommendation. TDR-WIS-PA-000001 REV 00 ICN 01. Las Vegas, Nevada: CRWMS M\&O. ACC: MOL.20001220.0045.

Dagan, G.; Cvetkovic, V.; and Shapiro, A. 1992. "A Solute Flux Approach to

163800

Transport in Heterogeneous Formations 1. The General Framework.” Water

Resources Research, 28, (5), 1369-1376. Washington, D.C.: American Geophysical

Union. TIC: 254598.

Daniels, W.R.; Wolfsberg, K.; Rundberg, R.S.; Ogard, A.E.; Kerrisk, J.F.; Duffy, C.J.; Newton, T.W.; Thompson, J.L.; Bayhurst, B.P.; Bish, D.L.; Blacic, J.D.; Crowe, B.M.; Erdal, B.R.; Griffith, J.F.; Knight, S.D.; Lawrence, F.O.; Rundberg, V.L.; Skyes, M.L.; Thompson, G.M.; Travis, B.J.; Treher, E.N.; Vidale, R.J.; Walter, G.R.; Aguilar, R.D.; Cisneros, M.R.; Maestas, S.; Mitchell, A.J.; Oliver, P.Q.; Raybold, N.A.; and Wanek, P.L. 1982. Summary Report on the Geochemistry of Yucca Mountain and Environs. LA-9328-MS. Los Alamos, New Mexico: Los Alamos National Laboratory. ACC: NNA.19870406.0243.

de Marsily, G. 1986. Quantitative Hydrogeology: Groundwater Hydrology for Engineers. San Diego, California: Academic Press. TIC: 208450.

Ding, M.; Reimus, P.W.; Ware, S.D.; and Meijer, A. 2003. "Experimental Studies of Radionuclide Migration in Yucca Mountain Alluvium.” Proceedings of the 10th International High-Level Radioactive Waste Management Conference (IHLRWM), March 30-April 2, 2003, Las Vegas, Nevada. Pages 126-135. La Grange Park, Illinois: American Nuclear Society. TIC: 254559.

Dixit, S. and Van Cappellen, P. 2002. "Surface Chemistry and Reactivity of Biogenic Silica.” Geochimica et Cosmochimica Acta, 66, (14), 2559-2568. New York, New York: Pergamon. TIC: 254531.

DOE (U.S. Department of Energy) 1997. Regional Groundwater Flow and Tritium Transport Modeling and Risk Assessment of the Underground Test Area, Nevada Test Site, Nevada. DOE/NV-477. Las Vegas, Nevada: U.S. Department of Energy. ACC: MOL.20010731.0303. 
Erickson, J.R. and Waddell, R.K. 1985. Identification and Characterization of

105279 Hydrologic Properties of Fractured Tuff Using Hydraulic and Tracer Tests--Test Well USW H-4, Yucca Mountain, Nye County, Nevada. Water-Resources Investigations Report 85-4066. Denver, Colorado: U.S. Geological Survey. ACC: NNA.19890713.0211.

Finnegan, D.L. and Thompson, J.L. 2002. Laboratory and Field Studies Related to Radionuclide Migration at the Nevada Test Site in Support of the Underground Test Area and Hydrologic Resources Management Projects. LA-13919-MS. Los Alamos, New Mexico: Los Alamos National Laboratory. ACC: MOL.20030926.0011.

Flint, L.E. 1998. Characterization of Hydrogeologic Units Using Matrix Properties, Yucca Mountain, Nevada. Water-Resources Investigations Report 97-4243. Denver, Colorado: U.S. Geological Survey. ACC: MOL.19980429.0512.

Freeze, R.A. and Cherry, J.A. 1979. Groundwater. Englewood Cliffs, New Jersey: Prentice-Hall. TIC: 217571.

Gardiner, C.W. 1997. Handbook of Stochastic Methods for Physics, Chemistry and the Natural Sciences. 2nd Edition. 442. New York, New York: Springer-Verlag. TIC: 247770.

Gelhar, L.W. 1997. "Perspectives on Field-Scale Application of Stochastic 145122 Subsurface Hydrology.” Subsurface Flow and Transport: A Stochastic Approach. Dagan, G. and Neuman, S.P., eds. Pages 157-176. New York, New York: Cambridge University Press. TIC: 247805.

Gelhar, L.W. and Axness, C.L. 1983. "Three-Dimensional Stochastic Analysis of Macrodispersion in Aquifers.” Water Resources Research, 19, (1), 161-180. Washington, D.C.: American Geophysical Union. TIC: 222815.

Grindrod, P. 1993. The Impact of Colloids on the Migration and Dispersal of Radionuclides within Fractured Rock. 13. 1-4. 167-181. New York, New York: Elsevier. TIC: 222399.

Hess, K.M.; Davis, J.A.; Kent, D.B.; and Coston, J.A. 2002. “Multispecies Reactive 163780 Tracer Test in an Aquifer with Spatially Variable Chemical Conditions, Cape Cod, Massachusetts: Dispersive Transport of Bromide and Nickel.” Water Resources Research, 38, (8), 36-1 to 36-17. Washington, D.C.: American Geophysical Union. TIC: 254599.

Hummel, W.; Berner, U.; Curti, E.; Pearson, F.J.; and Thoenen, T. 2002. Nagra/PSI Chemical Thermodynamic Data Base 01/01. Parkland, Florida: Universal Publishers. TIC: 253421. 
Hwang, Y.; Chambré, P.L.; Lee, W.W.-L.; and Pigford, T.H. 1989. “Analytic 165931 Studies of Colloid Transport in Fractured Porous Media.” Scientific Basis for Nuclear Waste Management XIII, Symposium held November 27-30, 1989, Boston, Massachusetts. Oversby, V.M. and Brown, P.W., eds. 176, 599-605. Pittsburgh, Pennsylvania: Materials Research Society. TIC: 203658.

Ibaraki, M. and Sudicky, E.A. 1995. "Colloid-Facilitated Contaminant Transport in Discretely Fractured Porous Media 1. Numerical Formulation and Sensitivity Analysis.” Water Resources Research, 31, (12), 2945-2960. Washington, D.C.: American Geophysical Union. TIC: 245719.

Ibaraki, M. and Sudicky, E.A. 1995. "Colloid-Facilitated Contaminant Transport in 165930 Discretely Fractured Porous Media 2. Fracture Network Examples.” Water Resources Research, 31, (12), 2961-2969. Washington, D.C.: American Geophysical Union. TIC: 252318.

Karasaki, K.; Landsfeld, M.; and Grossenbacher, K. 1990. "Building of a Conceptual Model at the UE25-c Hole Complex.” High Level Radioactive Waste Management, Proceedings of the International Topical Meeting, Las Vegas, Nevada, April 8-12, 1990. 2, 811-817. La Grange Park, Illinois: American Nuclear Society. TIC: 202058.

Keeney-Kennicutt, W.L. and Morse, J.W. 1985. "The Redox Chemistry of $\mathrm{Pu}(\mathrm{V}) \mathrm{O}$ \{superscript + \} \{ subscript 2 \} Interaction with Common Mineral Surfaces in Dilute Solutions and Seawater.” Geochimica et Cosmochimica Acta, 49, (12), 2577-2588. New York, New York: Pergamon. TIC: 237000.

Kersting, A.B.; Efurd, D.W.; Finnegan, D.L.; Rokop, D.J.; Smith, D.K.; and Thompson, J.L. 1999. "Migration of Plutonium in Ground Water at the Nevada Test Site.” Nature, 397, (6714), 56-59. London, England: Macmillan Journals. TIC: 243597.

Kersting, A.P. and Reimus, P.W., eds. 2003. Colloid-Facilitated Transport of 162421 Low-Solubility Radionuclides: A Field, Experimental, and Modeling Investigation. UCRL-ID-149688. Livermore, California: Lawrence Livermore National Laboratory. TIC: 254176.

Kotra, J.P.; Lee, M.P.; Eisenberg, N.A.; and DeWispelare, A.R. 1996. Branch 100909 Technical Position on the Use of Expert Elicitation in the High-Level Radioactive Waste Program. NUREG-1563. Washington, D.C.: U.S. Nuclear Regulatory Commission. TIC: 226832.

LaBolle, E.M.; Fogg, G.E.; and Tompson, A.F.B. 1996. "Random-Walk Simulation 105039 of Transport in Heterogeneous Porous Media: Local Mass-Conservation Problem and Implementation Methods.” Water Resources Research, 32, (3), 583-593. Washington, D.C.: American Geophysical Union. TIC: 245563. 
Langmuir, D. 1997. Aqueous Environmental Geochemistry. Upper Saddle River,

Langmuir, D. and Herman, J.S. 1980. "The Mobility of Thorium in Natural Waters at Low Temperatures.” Geochimica et Cosmochimica Acta, 44, 1753-1766. New York, New York: Pergamon Press. TIC: 237029.

LANL (Los Alamos National Laboratories) 1984. Quality Assurance Audit of Los Alamos National Laboratories (LANL) Nevada Nuclear Waste Storage Investigations (NNWSI) Project. Los Alamos, New Mexico: Los Alamos National Laboratories. ACC: NNA.19870317.0482.

LANL 2003. Validation Test Plan (VTP) for the FEHM Application Version 2.20. SDN: 10086-VTP-2.20-01. Los Alamos, New Mexico: Los Alamos National Laboratory. ACC: MOL.20030314.0010.

Leap, D.I. and Belmonte, P.M. 1992. "Influence of Pore Pressure on Apparent Dispersivity of a Fissured Dolomitic Aquifer.” Ground Water, 30, (1), 87-95. Worthington, Ohio: Water Well Journal Publishing. TIC: 239275.

LeBlanc, D.R.; Garabedian, S.P.; Hess, K.M.; Gelhar, L.W.; Quadri, R.D.; 171444 Stollenwerk, K.G.; and Wood, W.W. 1991. "Large-Scale Natural Gradient Tracer Test in Sand and Gravel, Cape Cod, Massachusetts 1. Experimental Design and Observed Tracer Movement.” Water Resources Research, 27, (5), 895-910. Washington, D.C.: American Geophysical Union. TIC: 254593.

Lichtner, P.C.; Kelkar, S.; and Robinson, B. 2002. “New Form of Dispersion 164150 Tensor for Axisymmetric Porous Media with Implementation in Particle Tracking." Water Resources Research, 38, (8), 21-1 through 21-16. Washington, D.C.: American Geophysical Union. TIC: 254597.

Lieser, K.H. and Muhlenweg, U. 1988. "Neptunium in the Hydrosphere and in the Geosphere, I. Chemistry of Neptunium in the Hydrosphere and Sorption of Neptunium from Groundwaters on Sediments Under Aerobic and Anaerobic Conditions.” Radiochimica Acta, 43, 27-35. Munchen, Germany: R. Oldenbourg Verlag. TIC: 236783.

Lindberg, R.D. and Runnells, D.D. 1984. “Ground Water Redox Reactions: An Analysis of Equilibrium State Applied to Eh Measurements and Geochemical Modeling.” Science, 225, 925-927. Washington, D.C.: American Association for the Advancement of Science. TIC: 224111.

Los Alamos Scientific Laboratories 1979. Audit of Los Alamos Scientific Laboratories Quality Assurance Program Plan for Nevada Nuclear Waste Storage Investigations. Audit 79-5. Los Alamos, New Mexico: Los Alamos Scientific Laboratories. ACC: HQZ.19880629.553-1. 
Luckey, R.R.; Tucci, P.; Faunt, C.C.; Ervin, E.M.; Steinkampf, W.C.; D'Agnese, 100465 F.A.; and Patterson, G.L. 1996. Status of Understanding of the Saturated-Zone Ground-Water Flow System at Yucca Mountain, Nevada, as of 1995. Water-Resources Investigations Report 96-4077. Denver, Colorado: U.S. Geological Survey. ACC: MOL.19970513.0209.

Maloszewski, P. and Zuber, A. 1985. "On the Theory of Tracer Experiments in 148312 Fissured Rocks with a Porous Matrix.” Journal of Hydrology, 79, 333-358. Amsterdam, The Netherlands: Elsevier. TIC: 222390.

Marmier, N.; Delisée, A.; and Fromage, F. 1999. "Surface Complexation Modeling 162986 of $\mathrm{Yb}(\mathrm{III})$ and $\mathrm{Cs}(\mathrm{I})$ Sorption on Silica.” Journal of Colloid and Interface Science, 212, (2), 228-233. New York, New York: Academic Press. TIC: 254530.

Neuman, S.P. 1990. "Universal Scaling of Hydraulic Conductivities and Dispersivities in Geologic Media.” Water Resources Research, 26, (8), 1749-1758. Washington, D.C.: American Geophysical Union. TIC: 237977.

Neuman, S.P.; Winter, C.L.; and Newman, C.M. 1987. "Stochastic Theory of 147577 Field-Scale Fickian Dispersion in Anisotropic Porous Media.” Water Resources Research, 23, (3), 453-466. Washington, D.C.: American Geophysical Union. TIC: 225294.

Nitsche, H.; Gatti, R.C.; Standifer, E.M.; Lee, S.C.; Müller, A.; Prussin, T.; Deinhammer, R.S.; Maurer, H.; Becraft, K.; Leung, S.; and Carpenter, S.A. 1993. Measured Solubilities and Speciations of Neptunium, Plutonium, and Americium in a Typical Groundwater (J-13) from the Yucca Mountain Region. LA-12562-MS. Los Alamos, New Mexico: Los Alamos National Laboratory. ACC: NNA.19930507.0136.

Nitsche, H.; Roberts, K.; Prussin, T.; Muller, A.; Becraft, K.; Keeney, D.; 100163 Carpenter, S.A.; and Gatti, R.C. 1995. Measured Solubilities and Speciations from Oversaturation Experiments of Neptunium, Plutonium and Americium in UE25p\#1 Well Water from the Yucca Mountain Region. LA-12563-MS. Los Alamos, New Mexico: Los Alamos National Laboratory. ACC: MOL.19951006.0171.

Noell, A.L.; Thompson, J.L.; Corapcioglu, M.Y.; and Triay, I.R. 1998. “The Role of Silica Colloids on Facilitated Cesium Transport Through Glass Bead Columns and Modeling.” Journal of Contaminant Hydrology, 31, (1-2), 23-56. Amsterdam, The Netherlands: Elsevier. TIC: 245510.

NRC (U.S. Nuclear Regulatory Commission) 2003. Yucca Mountain Review Plan, Final Report. NUREG-1804, Rev. 2. Washington, D.C.: U.S. Nuclear Regulatory Commission, Office of Nuclear Material Safety and Safeguards. TIC: 254568. 
Ogard, A.E. and Kerrisk, J.F. 1984. Groundwater Chemistry Along Flow Paths

100783

Between a Proposed Repository Site and the Accessible Environment.

LA-10188-MS. Los Alamos, New Mexico: Los Alamos National Laboratory. ACC:

HQS.19880517.2031.

Oswald, J.G. and Ibaraki, M. 2001. "Migration of Colloids in Discretely Fractured

165961

Porous Media: Effect of Colloidal Matrix Diffusion.” Journal of Contaminant

Hydrology, 52, (1-4), 213-244. New York, New York: Elsevier. TIC: 255089.

Pabalan, R.T.; Turner, D.R.; Bertetti, F.P.; and Prikryl, J.D. 1998.

162987

"Uranium\{superscript VI\} Sorption onto Selected Mineral Surfaces, Key

Geochemical Parameters.” Adsorption of Metals by Geomedia. Jenne, E.A., ed.

Pages 99-130. New York, New York: Academic Press. TIC: 239504.

Parkhurst, D.L. 1995. User's Guide to PHREEQC—A Computer Program for

142177

Speciation, Reaction-Path, Advective-Transport, and Inverse Geochemical Calculations. Water-Resources Investigations Report 95-4227. Lakewood, Colorado: U.S. Geological Survey. TIC: 248314.

Pawloski, G.A. 1999. Development of Phenomenological Models of Underground Nuclear Tests on Pahute Mesa, Nevada Test Site-BENHAM and TYBO.

UCRL-ID-136003. Livermore, California: Lawrence Livermore National Laboratory. ACC: MOL.20030926.0012.

Penrose, W.R.; Polzer, W.L.; Essington, E.H.; Nelson, D.M.; and Orlandini, K.A. 1990. "Mobility of Plutonium and Americium Through a Shallow Aquifer in a Semiarid Region.” Environmental Science \& Technology, 24, 228-234.

Washington, D.C.: American Chemical Society. TIC: 224113.

Peters, R.R.; Klavetter, E.A.; Hall, I.J.; Blair, S.C.; Heller, P.R.; and Gee, G.W. 1984. Fracture and Matrix Hydrologic Characteristics of Tuffaceous Materials from Yucca Mountain, Nye County, Nevada. SAND84-1471. Albuquerque, New Mexico: Sandia National Laboratories. ACC: NNA.19900810.0674.

Pollock, D.W. 1988. "Semianalytical Computation of Path Lines for Finite-Difference Models.” Ground Water, 26, (6), 743-750. Worthington, Ohio: National Water Well Association. TIC: 226464.

Poreh, M. 1965. "The Dispersivity Tensor in Isotropic and Axisymmetric 162685 Mediums.” Journal of Geophysical Research, 70, (16), 3909-3913. Washington, D.C.: American Geophysical Union. TIC: 254595.

Reimus, P.W.; Haga, M.J.; Humphrey, A.R.; Counce, D.A.; Callahan, T.J.; and Ware, S.D. 2002. Diffusion Cell and Fracture Transport Experiments to Support Interpretations of the BULLION Forced-Gradient Experiment. LA-UR-02-6884. Los Alamos, New Mexico: Los Alamos National Laboratory. TIC: 253859. 
Reimus, P.W.; Ware, S.D.; Benedict, F.C.; Warren, R.G.; Humphrey, A.; Adams, A.; Wilson, B.; and Gonzales, D. 2002. Diffusive and Advective Transport of $\mathrm{H}^{3}$, $C^{14}$, and $T_{c}^{99}$ in Saturated, Fractured Volcanic Rocks from Pahute Mesa, Nevada. LA-13891-MS. Los Alamos, New Mexico: Los Alamos National Laboratory. TIC: 253905.

Robinson, B.A. 1994. “A Strategy for Validating a Conceptual Model for Radionuclide Migration in the Saturated Zone Beneath Yucca Mountain.”

Radioactive Waste Management and Environmental Restoration, 19, (1-3), 73-96. Yverdon, Switzerland: Harwood Academic Publishers. TIC: 222513.

Robinson, B.A.; Wolfsberg, A.V.; Viswanathan, H.S.; Bussod, G.Y.; Gable, C.W.; and Meijer, A. 1997. The Site-Scale Unsaturated Zone Transport Model of Yucca Mountain. Milestone SP25BM3. Los Alamos, New Mexico: Los Alamos National Laboratory. ACC: MOL.19980203.0570.

Rundberg, R.S.; Ogard, A.E.; and Vaniman, D.T., eds. 1985. Research and 101355 Development Related to the Nevada Nuclear Waste Storage Investigations, April 1June 30, 1984. LA-10297-PR. Los Alamos, New Mexico: Los Alamos National Laboratory. ACC: NNA.19920922.0018.

Rundberg, R.S.; Partom, I.; Ott, M.A.; Mitchell, A.J.; and Birdsell, K. 1987. Diffusion of Nonsorbing Tracers in Yucca Mountain Tuff. Milestone R524. Los Alamos, New Mexico: Los Alamos National Laboratory. ACC: NNA.19930405.0074.

Smith, D.K. 2002. Evaluation of the Radiochemistry of Near-Field Water Samples at the Nevada Test Site Applied to the Definition of a Hydrologic Source Team. UCRL-ID-149049. Livermore, California: Lawrence Livermore National Laboratory. ACC: MOL.20030926.0013.

Smith, P.A. and Degueldre, C. 1993. "Colloid-Facilitated Transport of 144658

Radionuclides Through Fractured Media.” Journal of Contaminant Hydrology, 13, 143-166. Amsterdam, The Netherlands: Elsevier. TIC: 224863.

Steinborn, T.L. 2002. Data Qualification Report: Mineralogy Data for Use on the Yucca Mountain Project. TDR-NBS-HS-000005 REV 00. Las Vegas, Nevada: Bechtel SAIC Company. ACC: MOL.20020807.0442.

Stumm, W. and Morgan, J.J. 1981. Aquatic Chemistry, An Introduction 100829 Emphasizing Chemical Equilibria in Natural Waters. 2nd Edition. New York, New York: John Wiley \& Sons. TIC: 208448.

Sudicky, E.A. and Frind, E.O. 1981. "Carbon 14 Dating of Groundwater in 148342 Confined Aquifers: Implications of Aquitard Diffusion.” Water Resources Research, 17, (4), 1060-1064. Washington, D.C.: American Geophysical Union. TIC: 247712. 
Sudicky, E.A. and Frind, E.O. 1982. "Contaminant Transport in Fractured Porous

105043 Media: Analytical Solutions for a System of Parallel Fractures.” Water Resources Research, 18, (6), 1634-1642. Washington, D.C.: American Geophysical Union. TIC: 217475.

Tang, D.H.; Frind, E.O.; and Sudicky, E.A. 1981. "Contaminant Transport in 101160 Fractured Porous Media: Analytical Solution for a Single Fracture.” Water Resources Research, 17, (3), 555-564. Washington, D.C.: American Geophysical Union. TIC: 225358.

Thorbjarnarson, K.W. and Mackay, D.M. 1994. “A Forced-Gradient Experiment on Solute Transport in the Borden Aquifer. 3. Nonequilibrium Transport of the Sorbing Organic Compounds.” Water Resources Research, 30, (2), 401-419. Washington, D.C.: American Geophysical Union. TIC: 252320.

Tompson, A.F.B.; Bruton, C.J.; and Pawloski, G.A., eds. 1999. Evaluation of the Hydrologic Source Term from Underground Nuclear Tests in Frenchman Flat at the Nevada Test Site: The CAMBRIC Test. UCRL-ID-132300. Livermore, California: Lawrence Livermore National Laboratory. ACC: MOL.20030926.0014.

Tompson, A.F.B.; Vomvoris, E.G.; and Gelhar, L.W. 1987. Numerical Simulation of Solute Transport in Randomly Heterogeneous Porous Media: Motivation, Model Development, and Application. UCID 21281. Livermore, California: Lawrence Livermore National Laboratory. ACC: MOL.19950131.0007.

Triay, I.R.; Birdsell, K.H.; Mitchell, A.J.; and Ott, M.A. 1993. "Diffusion of Sorbing and Non-Sorbing Radionuclides.” High Level Radioactive Waste Management, Proceedings of the Fourth Annual International Conference, Las Vegas, Nevada, April 26-30, 1993. 2, 1527-1532. La Grange Park, Illinois: American Nuclear Society. TIC: 208542.

Triay, I.R.; Cotter, C.R.; Huddleston, M.H.; Leonard, D.E.; Weaver, S.C.; Chipera, S.J.; Bish, D.L.; Meijer, A.; and Canepa, J.A. 1996. Batch Sorption Results for Neptunium Transport Through Yucca Mountain Tuffs. LA-12961-MS. Los Alamos, New Mexico: Los Alamos National Laboratory. ACC: MOL.19980924.0050.

Triay, I.R.; Furlano, A.C.; Weaver, S.C.; Chipera, S.J.; and Bish, D.L. 1996. Comparison of Neptunium Sorption Results Using Batch and Column Techniques. LA-12958-MS. Los Alamos, New Mexico: Los Alamos National Laboratory. ACC: MOL.19980924.0049.

Triay, I.R.; Meijer, A.; Cisneros, M.R.; Miller, G.G.; Mitchell, A.J.; Ott, M.A.; Hobart, D.E.; Palmer, P.D.; Perrin, R.E.; and Aguilar, R.D. 1991. "Sorption of Americium in Tuff and Pure Minerals Using Synthetic and Natural Groundwaters.” Radiochimica Acta, 52/53, 141-145. München, Germany: R. Oldenbourg Verlag. TIC: 222704. 
Triay, I.R.; Meijer, A.; Conca, J.L.; Kung, K.S.; Rundberg, R.S.; Strietelmeier, 100422 B.A.; and Tait, C.D. 1997. Summary and Synthesis Report on Radionuclide Retardation for the Yucca Mountain Site Characterization Project. Eckhardt, R.C., ed. LA-13262-MS. Los Alamos, New Mexico: Los Alamos National Laboratory. ACC: MOL.19971210.0177.

Turin, H.J.; Groffman, A.R.; Wolfsberg, L.E.; Roach, J.L.; and Strietelmeier, B.A. 2002. "Tracer and Radionuclide Sorption to Vitric Tuffs of Busted Butte, Nevada." 164633 Applied Geochemistry, 17, (6), 825-836. New York, New York: Pergamon. TIC: 254046.

Turner, D.R.; Pabalan, R.T.; and Bertetti, F.P. 1998. "Neptunium(V) Sorption on Montmorillonite: An Experimental and Surface Complexation Modeling Study.” Clays and Clay Minerals, 46, (3), 256-269. Boulder, Colorado: Clay Minerals Society. TIC: 254532.

USGS (U.S. Geological Survey) n.d. Bulk Density. Denver, Colorado: U.S.

154495 Geological Survey. ACC: NNA.19940406.0076.

Valocchi, A.J. 1985. "Validity of the Local Equilibrium Assumption for Modeling Sorbing Solute Transport Through Homogeneous Soils.” Water Resources Research, 21, (6), 808-820. Washington, D.C.: American Geophysical Union. TIC: 223203.

Waddell, R.K.; Robison, J.H.; and Blankennagel, R.K. 1984. Hydrology of Yucca Mountain and Vicinity, Nevada-California--Investigative Results Through Mid-1983. Water-Resources Investigations Report 84-4267. Denver, Colorado: U.S. Geological Survey. ACC: NNA.19870406.0343.

Wen, X-H. and Gomez-Hernandez, J.J. 1996. "The Constant Displacement Scheme for Tracking Particles in Heterogeneous Aquifers.” Ground Water, 34, (1), 135-142. Worthington, Ohio: Water Well Journal Publishing. TIC: 246656.

Wilson, M.L.; Gauthier, J.H.; Barnard, R.W.; Barr, G.E.; Dockery, H.A.; Dunn, E.; Eaton, R.R.; Guerin, D.C.; Lu, N.; Martinez, M.J.; Nilson, R.; Rautman, C.A.; Robey, T.H.; Ross, B.; Ryder, E.E.; Schenker, A.R.; Shannon, S.A.; Skinner, L.H.; Halsey, W.G.; Gansemer, J.D.; Lewis, L.C.; Lamont, A.D.; Triay, I.R.; Meijer, A.; and Morris, D.E. 1994. Total-System Performance Assessment for Yucca Mountain - SNL Second Iteration (TSPA-1993). SAND93-2675. Executive Summary and two volumes. Albuquerque, New Mexico: Sandia National Laboratories. ACC:

NNA.19940112.0123.

Winterle, J.R.; Claisse, A.; and Arlt, H.D. 2003. “An Independent Site-Scale 163823 Groundwater Flow Model for Yucca Mountain.” Proceedings of the 10th International High-Level Radioactive Waste Management Conference (IHLRWM), March 30-April 2, 2003, Las Vegas, Nevada. Pages 151-158. La Grange Park, Illinois: American Nuclear Society. TIC: 254559. 
Wolfsberg, A.; Glascoe, L.; Lu, G.; Olson, A.; Lichtner, P.; McGraw, M.; Cherry, 162688 T.; and Roemer, G. 2002. TYBO/BENHAM: Model Analysis of Groundwater Flow and Radionuclide Migration from Underground Nuclear Tests in Southwestern Pahute Mesa, Nevada. LA-13977. Los Alamos, New Mexico: Los Alamos National Laboratory. ACC: MOL.20030926.0009.

Zheng, C. and Bennett, G.D. 1995. Applied Contaminant Transport Modeling, Theory and Practice. New York, New York: Van Nostrand Reinhold. TIC: 249865.

Zyvoloski, G.A.; Robinson, B.A.; Dash, Z.V.; and Trease, L.L. 1997. Summary of the Models and Methods for the FEHM Application-A Finite-Element Heat- and Mass-Transfer Code. LA-13307-MS. Los Alamos, New Mexico: Los Alamos National Laboratory. TIC: 235587.

\subsection{CODES, STANDARDS, REGULATIONS, AND PROCEDURES}

10 CFR 63. Energy: Disposal of High-Level Radioactive Wastes in a Geologic Repository at Yucca Mountain, Nevada. Readily available.

AP-2.22Q, Rev. 1, ICN 0. Classification Analyses and Maintenance of the Q-List. Washington, D.C.: U.S. Department of Energy, Office of Civilian Radioactive Waste Management. ACC: DOC.20030930.0002.

AP-SI.1Q, Rev. 5, ICN 2. Software Management. Washington, D.C.: U.S. Department of Energy, Office of Civilian Radioactive Waste Management. ACC: DOC.20030902.0003.

AP-2.27Q, Rev. 1, ICN 4. Planning for Science Activities. Washington, D.C.: U.S. Department of Energy, Office of Civilian Radioactive Waste Management. ACC: DOC.20040610.0006.

AP-SIII.2Q, Rev. 1, ICN 2. Qualification of Unqualified Data. Washington, D.C.: U.S. Department of Energy, Office of Civilian Radioactive Waste Management. ACC: DOC.20040127.0008.

AP-SIII.10Q, Rev. 2, ICN 7. Models. Washington, D.C.: U.S. Department of Energy, Office of Civilian Radioactive Waste Management.

ACC: DOC.20040920.0002.

LANL-INC-DP-86, R0. Sorption and Desorption Determinations by a Batch Sample Technique for the Dynamic Transport Task. Los Alamos, New Mexico: Los Alamos National Laboratory. ACC: NNA.19920416.0158.

LA-9331-MS. Los Alamos National Laboratory Quality Assurance Plan and Procedures for the Nevada Nuclear Waste Storage Investigations. Los Alamos, New Mexico: Los Alamos National Laboratory. ACC: MOL.19980716.0258. 
LP-SI.11Q-BSC REV 0, ICN 1. Software Management. Washington, D.C.: U.S. Department of Energy, Office of Civilian Radioactive Waste Management. ACC: DOC.20041005.0008.

QMR 1, Rev. 2. Quality Assurance Management and Planning. Los Alamos, New Mexico: Los Alamos National Laboratory. ACC: MOL.20040708.0326.

QMI 1-1, Rev. 2. Quality Program Management. Los Alamos, New Mexico: Los Alamos National Laboratory. ACC: MOL.20040708.0327.

QMR 9, Rev. 1. Control and Calibration of Standards and Measuring and Testing Equipment. Los Alamos, New Mexico: Los Alamos National Laboratory. ACC: MOL.20040708.0328.

QMI 9-1, Rev. 2. Acquisition and Calibration Control of Standards and Measuring and Testing Equipment. Los Alamos, New Mexico: Los Alamos National Laboratory. ACC: MOL.20040708.0329

QMR 12, Rev. 1. Corrective Action, Los Alamos, New Mexico: Los Alamos National Laboratory. ACC: MOL.20040708.0330.

QMI 15-1, Rev. 1 Quality Audits Procedures. Los Alamos, New Mexico: Los Alamos National Laboratory. ACC: MOL.20040708.0332.

TWS-CMBQA-QP-02, R1. Quality Assurance Program Index. Los Alamos, New Mexico: Los Alamos National Laboratory. ACC: NNA.19891030.0032.

TWS-CMBQA-QP-02, R2. Quality Assurance Program Index. Los Alamos, New Mexico: Los Alamos National Laboratory. ACC: NNA.19891030.0033.

TWS-CMBQA-QP-02, R4. Quality Assurance Program Index. Los Alamos, New Mexico: Los Alamos National Laboratory. ACC: NNA.19891030.0035.

TWS-CNC-11-DP-05, R0. Sorption, Desorption Ratio Determinations of Geologic Materials by a Batch Method. Los Alamos, New Mexico: Los Alamos National Laboratory. ACC: NNA.19900713.0290.

TWS-CNC-DP-05, R1. Sorption, Desorption Ratio Determinations of Geologic Materials by a Batch Method. Los Alamos, New Mexico: Los Alamos National Laboratory. ACC: NNA.19870430.0094.

TWS-CNC-WP-03, R4. Tuff Experiments-Sorption Ratios and Migration Measurements. Los Alamos, New Mexico: Los Alamos National Laboratory. ACC: NNA.19891030.0062. 
TWS-INC-DP-05, R2. Sorption, Desorption Ratio Determinations of Geologic Materials by a Batch Method. Los Alamos, New Mexico: Los Alamos National Laboratory. ACC: NNA.19900809.0031.

TWS-QI-1, R0. Quality Assurance Program Index. Los Alamos, New Mexico: Los Alamos National Laboratory. ACC: MOL.19980924.0109.

\subsection{SOURCE DATA, LISTED BY DATA TRACKING NUMBER}

LA000000000086.002. Mineralogic Variation in Drill Core UE-25 UZ\#16 Yucca 107144 Mountain, Nevada. Submittal date: 03/28/1995.

LA0003JC831362.001. Preliminary Matrix Diffusion Coefficients for Yucca Mountain Tuffs. Submittal date: 4/10/2000.

LA0109MD831341.001. Adsorption of NP-237 in Three Types of Alluvium as a Function of Time and Stratigraphic Position. Submittal date: 09/14/2001.

LA0109MD831341.002. Adsorption of TC-99 in Three Types of Alluvium as a 156871 Function of Time and Stratigraphic Position. Submittal date: 09/14/2001.

LA0206AM831234.001. Eh-pH Field Measurements on Nye County EWDP Wells. 160051 Submittal date: 06/21/2002.

LA0206AM831234.002. Geochemical Field Measurements on Nye County EWDP 163852 Wells. Submittal date: 06/21/2002.

LA0301AA831352.001. Experiments on Pu(V)-Colloid Transport in Columns Packed with Material from Nye County Borehole 19D, Zone 4. Submittal date: 162433 01/23/2003.

LA0301PR831361.003. Breakthrough Curves of Tritium, Plutonium, and Various 162435 Colloids in Saturated UE20C Fractured Cores from the Nevada Test Site. Submittal date: $01 / 22 / 2003$.

LA0301PR831361.004. Breakthrough Curves of Tritium, Plutonium, and Various Colloids in Saturated PM-1 and PM-2 Fractured Cores from the Nevada Test Site. Submittal date: 01/22/2003.

LA0302HV831361.001. A Study of Kinetic Reaction Rates for Plutonium Sorption in Devitrified Tuffs. Submittal date: 02/10/2003.

LA0302MD831341.001. Iodine-129 Sorption in Alluvium from NCEWDP Wells 164956 19IM1A, 10SA, and 22SA Under Ambient Conditions. Submittal date: 02/13/2003. 
LA0302MD831341.002. Technetium-99 Sorption in Alluvium from NCEWDP

164957 Wells 19IM1A, 10SA, and 22SA Under Ambient Conditions. Submittal date: 02/11/2003.

LA0302MD831341.003. Neptunium-237 Sorption in Alluvium from NC-EWDP 163784 Wells 19IM1A, 10SA, and 22SA Under Ambient Conditions. Submittal date: 02/11/2003.

LA0302MD831341.004. Uranium Sorption in Alluvium from NC-EWDP Wells 163785 19IM1A, 10SA, and 22SA Under Ambient Conditions. Submittal date: 02/11/2003.

LA0303HV831352.002. Colloid Retardation Factors for the Saturated Zone 163558 Fractured Volcanics. Submittal date: 03/31/2003.

LA0303HV831352.004. Colloid Retardation Factors for the Saturated Zone 163559 Alluvium. Submittal date: 03/31/2003.

LA0304TM831231.002. SZ Site-Scale Flow Model, FEHM Files for Base Case. 163788 Submittal date: 04/14/2003.

LA0305AM831341.001. 1977 to 1987 Sorption Measurements of AM, BA, CS, NP, PU, PA, SR, TH, and U with Yucca Mountain Tuff Samples. Submittal date: 05/21/2003.

LA0310AM831341.001. Sorption/Desorption Measurements of Cesium on Yucca Mountain Tuff. Submittal date: 10/21/2003.

LA0401PR831361.001. Column Studies Using G4-268 Devitrified Tuff with J-13 Well Water and Radionuclides (H-3 and Pu-239). Submittal date: 01/13/2004.

LA0407AM831341.001. Batch Sorption Coefficient Data for Barium on Yucca 170623 Mountain Tuffs in Representative Water Compositions. Submittal date: 07/12/2004.

LA0407AM831341.002. Batch Sorption Coefficient Data for Cesium on Yucca 170621 Mountain Tuffs in Representative Water Compositions. Submittal date: 07/12/2004.

LA0407AM831341.003. Batch Sorption Coefficient Data for Strontium on Yucca Mountain Tuffs in Representative Water Compositions. Submittal date: 07/12/2004.

LA0407AM831341.004. Batch Sorption Coefficient Data for Neptunium on Yucca Mountain Tuffs in Representative Water Compositions. Submittal date: 07/12/2004. 
LA0407AM831341.005. Batch Sorption Coefficient Data for Plutonium on Yucca 170625 Mountain Tuffs in Representative Water Compositions. Submittal date:

07/12/2004.

LA0407AM831341.006. Batch Sorption Coefficient Data for Uranium on Yucca

170628 Mountain Tuffs in Representative Water Compositions. Submittal date:

07/12/2004.

LA9907AM831234.009. Flow-through Cell Measurements for NC-EWDP-01S, NC-EWDP-03S, NC-EWDP-09SX, 5/17/99, 5/18/99, 5/19/99, 5/20/99. Submittal date: $01 / 27 / 2000$.

LA9907AM831234.011. Flow-Through Cell Measurements for AD-2, 10-Jun-99. Submittal date: 01/27/2000.

LAAM831311AQ98.007. Flow-Thru Cell and Static Measurements at UE-25 WT\#3, 22-Jun-98. Submittal date: 09/14/1998.

LAAM831311AQ98.010. Static Measurements for UE-25 WT\#17, 01 Jul 98.

149522

Submittal date: 09/14/1998.

LADV831321AQ99.001. Quantitative XRD Results for the USW SD-6 and USW WT-24 Drill Core Samples. Submittal date: 04/16/1999.

LAJC831321AQ98.005. Quantitative XRD Results for Drill Core USW SD-7, USW SD-9, USW SD-12 and UE-25 UZ\#16. Submittal date: 10/27/1998.

LASC831321AQ98.001. Results of Real-Time Analysis for Erionite in Drill Hole USWWT-24, Yucca Mountain, Nevada. Submittal date: 02/10/1998.

MO0006J13WTRCM.000. Recommended Mean Values of Major Constituents in J-13 Well Water. Submittal date: 06/07/2000.

MO0007MAJIONPH.011. Major Ion Content of Groundwater from Selected Yucca Mountain Project Boreholes Extracted from ANL-NBS-HS-000021, Geochemical and Isotopic Constraints on Groundwater Flow Directions, Mixing and Recharge at Yucca Mountain, Nevada. Submittal date: 07/27/2000.

MO0010CPORGLOG.002. Calculated Porosity from Geophysical Logs Data from “Old 40" Boreholes. Submittal date: 10/16/2000.

MO0101XRDDRILC.002. XRD Analyses of Drill Core from Boreholes UE-25 A\#1 and USW G-2. Submittal date: 01/26/2001.

MO0101XRDMINAB.001. XRD Analyses of Drill Core from Boreholes 109044 109004 109047 151029 151524 UE-25B\#1, USW G-1, USW G-3, USW GU-3, and USW G-4. Submittal date: 01/26/2001. 
MO0105GPLOG19D.000. Geophysical Log Data from Borehole NC EWDP 19D. 163480 Submittal date: 05/31/2001.

MO0106XRDDRILC.003. XRD Analyses of Drill Core from Borehole USW H-6. 163797 Submittal date: 06/08/2001.

MO0109HYMXPROP.001. Matrix Hydrologic Properties Data. Submittal date: 155989 09/17/2001.

MO0403SPAYMPR3.000. YMP_R3.DAT Thermodynamic Database for 170937 PHREEQC. Submittal date: 03/12/2004.

MO0407SEPFEPLA.000. LA FEP List. Submittal date: 07/20/2004.

170760

MO0408K8313211.000. Mineralogic Variation in Drill Holes. Submittal date:

171437 08/27/2004.

SN0004T0501399.003. Statistical Summary of Porosity Data. Submittal date: 04/13/2000.

SN0306T0504103.005. Revised Groundwater Colloid Mass Concentration Parameters for TSPA (Total System Performance Assessment). Submittal date: 06/30/2003.

SN0306T0504103.006. Revised Sorption Partition Coefficients (Kd Values) for 164131 Selected Radionuclides Modeled in the TSPA (Total System Performance Assessment). Submittal date: 06/30/2003.

SN03010T0502103.009. Revised Saturated Zone Transport Abstraction Model Uncertain Inputs. Submittal date: 10/09/2003.

\subsection{OUTPUT DATA, LISTED BY DATA TRACKING NUMBER}

LA0302RP831228.001. Type Curve Data for FEHM Macro “SPTR” Based on Sudicky and Frind Solution. Submittal date: 02/11/2003.

LA0306SK831231.001. SZ Site-Scale Transport Model, FEHM Files for Base Case. Submittal date: 06/25/2003.

LA0307SK831231.001. Files for FEHM V 2.20 for Comparison of Flow Paths Against Those From Hydrochemistry Data. Submittal date: 07/31/2003.

LA0307SK831231.002. Files for FEHM V 2.20 for Specific Discharge. Submittal date: $07 / 31 / 2003$.

LA0309SK831231.001. Files for FEHM V 2.20 for Evaluating Propagation of Uncertainty in the Input Parameter Values. Submittal date: 07/31/2003. 
LA0307ZD831231.001. Files for FEHM V 2.20 for Evaluating Barrier Capabilities. Submittal date: 07/31/2003.

LA0309RP831321.001. Calculation of the Rock Type Distributions, SZ Transport Model. Submittal date: 09/23/2003.

LA0309RP831341.001. Calculation of Kd Distributions for Cesium. Submittal date: $09 / 23 / 2003$.

LA0309RP831341.002. Calculation of Kd Distributions for Neptunium. Submittal date: 09/30/2003.

LA0309RP831341.003. Calculation of Kd Distributions for Plutonium. Submittal date: $09 / 30 / 2003$.

LA0309RP831341.004. Calculation of Kd Distributions for Uranium. Submittal date: $09 / 29 / 2003$.

LA0310AM831341.002. Saturated Zone Distribution Coefficients $\left(K_{\mathrm{d}} \mathrm{s}\right)$ for U, Np, $\mathrm{Pu}, \mathrm{Cs}, \mathrm{Am}, \mathrm{Pa}, \mathrm{Sr}$, Th, Ra, C, Tc, and I. Submittal date: 10/21/2003.

LA0403SK831231.001. Sz Transport Model, Fehm Files For Natural Barrier

Transport Calculations For Matrix Sorption . Submittal date: 03/11/2004.

LA0407AM831343.001. Modeling Calculations of Radionuclide Sorption via Surface-Complexation Reactions. Submittal date: 07/29/2004.

\subsection{SOFTWARE CODES}

BSC 2001. Software Code: PHREEQC. V2.3. PC, LINUX, Windows 95/98/NT,

155323 Redhat 6.2. 10068-2.3-00.

LANL (Los Alamos National Laboratory) 2002. Software Code: RELAP. V2.0. PC, Windows 2000/NT. 10551-2.0-00.RELAP. V2.0. PC, Windows 2000/NT. 10551-2.0-00.

LANL 2003. Software Code: FEHM. V2.20. SUN, PC. 10086-2.20-00. FEHM. 161725 V2.20. SUN, PC. 10086-2.20-00.

LANL 2003. Software Code: FRACT_p. V1.0. PC, LINUX 2.4.18. 11009-1.000.FRACT_p. V1.0. PC, LINUX 2.4.18. 11009-1.0-00.

164509

LBNL (Lawrence Berkeley National Laboratory) 2000. Software Code: GSLIB. V1.0GAMV3V1.201. SUN w/Unix OS. 10398-1.0GAMV3V1.201-00. GSLIB. V1.0GAMV3V1.201. SUN w/Unix OS. 10398-1.0GAMV3V1.201-00. 
SNL (Sandia National Laboratories) 2000. Software Code: GSLIB V2.0 MSISIM. 149114 V2.0. 10098-2.0MSISIMV2.0-00. GSLIB V2.0 MSISIM. V2.0. 10098-2.0MSISIMV2.0-00.

SNL 2000. Software Routine: calc_cdf.c. V1.0. ANL-NBS-HS-000022.calc_cdf.c. 149117 V1.0. ANL-NBS-HS-000022.

SNL 2002. Software Code: GS2FEHM.C. V 1.0. SUN, SunO.S. 5.7. 10923-1.0-00. 163837 GS2FEHM.C. V 1.0. SUN, SunO.S. 5.7. 10923-1.0-00.

SNL 2002. Software Code: cr8sptr.c. V 2.0. SUN, SunO.S. 5.7. 10927-2.0-00. 163836 cr8sptr.c. V 2.0. SUN, SunO.S. 5.7. 10927-2.0-00. 


\section{INTENTIONALLY LEFT BLANK}




\section{APPENDIX A \\ TECHNICAL BASIS FOR SORPTION-COEFFICIENT PROBABILITY DISTRIBUTION FUNCTIONS}




\section{A1. INTRODUCTION}

This appendix provides the bases for the derivation of sorption-coefficient probability distributions used in the SZ transport model. These distributions (Tables A-4 and A-5) in conjunction with the analysis presented in Appendix $\mathrm{C}$ lead to the recommended composite distributions for $K_{d}$ S (Tables C-14 and A-5) that form a technical product output of this report (output DTN: LA0310AM831341.002). The data needed as inputs to the analysis presented in this appendix are treated as being "direct input" to the site-scale SZ transport model. The sorption-coefficient data on which the distributions are based were obtained in laboratory experiments in which representative rock samples from the Yucca Mountain site were contacted with groundwaters (or simulated groundwaters) representative of the site, spiked with one or more of the elements of interest. Sorption experiments were carried out as a function of rock type, time, element concentration, atmospheric composition, grain size, and temperature. In some cases, the solids remaining from sorption experiments were contacted with unspiked groundwater in desorption experiments. The sorption and desorption experiments together provide information on the equilibration rates of the sorption reactions.

For elements that sorb primarily through surface-complexation reactions, the experimental data are augmented with the results of modeling calculations using PHREEQC (PHREEQC V2.3, STN: 10068-2.3-00) (BSC 2001 [DIRS 155323]) with the thermodynamic input data file YMP_R3.DAT (DTN: MO0403SPAYMPR3.000 [DIRS 170937]). The inputs for the modeling calculations include groundwater compositions, rock surface areas, binding constants for the elements of interest, and thermodynamic data for solid and solution species (Table 4-1). These modeling calculations provide a basis for interpolation and extrapolation of the experimentally derived sorption-coefficient data set.

The primary controls on sorption behavior of the elements of interest in the SZ at Yucca Mountain include the detailed characteristics of mineral surfaces in the rock units through which water flows in the SZ, the detailed chemistry of groundwater in the SZ, the sorption behavior of each element, and the concentrations of the various radionuclides in the waters. These parameters will be discussed in the following sections.

As noted in the model report Saturated Zone Flow and Transport Model Abstraction (BSC 2004 [DIRS 170042]), the SZ transport model uses a stochastic approach to calculate the transport rates of radionuclides of interest. In this approach, each realization (i.e., calculation) selects a single value for the sorption coefficient of each radionuclide from a probability distribution that represents the range of sorption-coefficient values that could be encountered in the saturated volcanic section or in the alluvial section. This appendix provides probability distributions for each element of interest on the two major rock types (devitrified and zeolitic tuff) found in the saturated volcanic section. It also provides probability distributions for sorption coefficients in alluvium. The influence of expected variations in water chemistry, radionuclide concentrations, and variations in rock surface properties within one of the major rock types or alluvium were incorporated into the probability distributions. The distributions types were based in part on actual experimental data and in part on professional judgment regarding the impact of variables not considered in the experimental program on the distributions. For example, there are potential impacts from variations in water compositions outside those addressed in the experimental program (e.g., Eh, variations in surface areas, variations in mineralogical composition, etc.) In 
the case of mineralogical composition, an attempt was made to emphasize those experiments that used samples closest to the mean composition of the hydrologic units as defined in the transport model. Thus, experimental data points for devitrified tuff samples containing significant quantities of clays were discounted, leading to more conservative estimates of $K_{d}$.

This analysis of sorption coefficients focuses on room temperature conditions. Available data show little statistical variation in the sorption coefficient values up to $80^{\circ} \mathrm{C}$ (DTN: LA0407AM831341.002 [DIRS 170621]) and LA0407AM831341.003 [DIRS 170626]. Temperatures in the SZ are expected to be in the neighborhood of $35^{\circ} \mathrm{C}$ Saturated Zone SiteScale Flow Model (BSC 2004 [DIRS 170037], Table 7-9). Hence, sorption coefficient values at elevated temperatures are not discussed in this model report.

\section{A2. AQUIFER MATRIX COMPOSITIONS ALONG MOST PROBABLE TRANSPORT PATHWAYS}

Sources of data on aquifer matrix compositions in the SZ are provided in Appendix C, Table C-3. These data have been incorporated into Mineralogic Model (MM3.0) Report (BSC 2004 [DIRS 170031]). There are two dominant rock types in the SZ along potential flow paths in volcanics to the 18-km boundary: devitrified tuff and zeolitic tuff. Devitrified tuff is composed primarily of silica (quartz and cristobalite) and alkali feldspar. It may also contain minor to trace amounts of mica, hematite, calcite, tridymite, kaolinite, and hornblende and minor amounts (less than 25 percent) of smectite and/or zeolite. For the purposes of this analysis, sorption-coefficient distributions for devitrified tuff are based on data obtained on samples that are composed primarily of silica phases and feldspar with only trace amounts of other minerals. Although devitrified tuff samples that contain significant amounts (more than 5 percent) of clays or zeolites generally have higher sorption coefficients than samples that do not, the distribution of these types of altered rocks along the flow path is not well enough established to reliably incorporate these rock types into the sorption-coefficient probability distributions. Sorption-coefficient distributions for zeolitic tuff use only samples that contain more than 50 percent zeolite, with the balance made up of silica phases, alkali feldspar, clays, and/or glass.

The total number of samples of each rock type used in the analysis in this appendix included 38 devitrified tuffs and 34 zeolitic tuffs. Not all of these samples were obtained from the SZ. Nonetheless, these samples are taken to be representative of devitrified and zeolitic tuffs in the SZ at Yucca Mountain.

As explained further, surface areas for the sorption substrates are an input to PHREEQC calculations. In the modeling, surface sites on devitrified tuff were taken to be equivalent to surface sites on silica, $2.8 \mathrm{~m}^{2} / \mathrm{g}$ (Allard et al. 1980 [DIRS 104410]). For comparison, surface-area analyses for 20 tuff samples from Triay et al. (1996 [DIRS 101023], p. 62) are presented in Table A-1. $2.8 \mathrm{~m}^{2} / \mathrm{g}$ falls towards the lower end of the values represented in this table. The use of a lower end value for the surface area leads to conservative estimates of the sorption coefficients, since a larger surface areas leads to larger sorption coefficients. Similarly, a representative value of $28 \mathrm{~m}^{2} / \mathrm{g}$ is used for zeolitic tuff, which also falls towards the lower end of the values in Table A-1. 
Table A-1. Surface Areas $\left(\mathrm{m}^{2} / \mathrm{g}\right)$ for Yucca Mountain Tuffs

\begin{tabular}{|c|c|c|c|c|}
\hline Borehole & Not Crushed & Dry Sieved & $\begin{array}{c}\text { Wet Sieved With } \\
\text { J-13 Water }\end{array}$ & $\begin{array}{l}\text { Wet Sieved With } \\
\text { UE-25 p\#1 Water }\end{array}$ \\
\hline USW G1-732 & 2.1 & 2.7 & 2.6 & 3.3 \\
\hline USW G1-1936 & 4.5 & 4.9 & 3.6 & 3.7 \\
\hline USW G4-270 & 2 & 6.4 & 5.1 & 5 \\
\hline USW G4-275 & 2.9 & 4.5 & & \\
\hline USW G4-2570 & 2.8 & 3.6 & 2.9 & 2.8 \\
\hline USW GU3-747 & 2.2 & 2.9 & 2.8 & 2.8 \\
\hline USW GU3-2325 & 1.8 & 2.5 & 2.2 & 2.5 \\
\hline Average & 2.6 & 3.9 & 3.2 & 3.4 \\
\hline USW G1-1405 & 32 & 28 & 26 & 31 \\
\hline USW G2-1813 & & & 34 & \\
\hline USW G2-1951 & & & 66 & \\
\hline USW GU3-1992 & & & 32 & \\
\hline USW G4-1506 & 22 & 30 & 27 & 25 \\
\hline USW G4-1529 & 37 & 21 & 22 & 31 \\
\hline USW G4-1530 & 40 & 41 & & \\
\hline USW G4-1625 & 28 & 27 & 28 & 33 \\
\hline USW G4-1772 & 23 & 22 & 23 & 23 \\
\hline USW G4-2077 & 19 & 18 & & \\
\hline Average & 28.7 & 26.7 & 32.3 & 28.6 \\
\hline USW G2-767 & 0.89 & 1.1 & 0.62 & 0.87 \\
\hline USW GU3-1249 & 0.52 & 0.92 & 0.99 & 0.87 \\
\hline USW GU3-1407 & 1.7 & 3.3 & 3 & 3.2 \\
\hline Average & 1.0 & 1.8 & 1.5 & 1.6 \\
\hline
\end{tabular}

The list of radionuclides for which sorption-coefficient data are required was derived in the analysis and modeling report Radionuclide Screening (BSC 2002 [DIRS 160059], Table 10). The list includes isotopes of americium, cesium, neptunium, protactinium, plutonium, radium, strontium, thorium, and uranium. Because different isotopes of a given (heavy) element behave the same in chemical reactions, this discussion will focus on this list of elements.

Sorption coefficients for the radionuclides-of-interest are often a function of the concentrations of the radionuclides present in solution (i.e., sorption isotherms are often non-linear). Thus, sorption experiments have been carried out as a function of radionuclide concentration. As the radionuclide concentrations are increased in the experiments, an upper limit is eventually reached for most elements-of-interest where a pure phase incorporating the element of interest becomes oversaturated and can precipitate out of solution. This concentration is known as the solubility limit for the element of interest in the experimental system. Because sorption experiments have been carried out by the Project up to and, in some cases above, the solubility limit for most elements-of-interest, the ranges of sorption coefficients obtained reflect this dependency. The only element-of-interest for which the experimental concentrations did not approach a solubility limit was cesium. The solubility of cesium is very high (Section A7.2). 
Thus, the sorption coefficient probability distributions for cesium must be calibrated to the cesium concentrations expected in the SZ.

A sorption experiment in which the final concentration of a radionuclide-of-interest in solution is in excess of a solubility limit should not be used to derive a sorption coefficient. The reason for this is that the concentration of the radionuclide-of-interest in the solid phase in this situation could represent a combination of the amount sorbed onto the solid phase (i.e., rock) and the amount of the pure phase precipitated out of solution. A sorption coefficient is supposed to reflect only sorption reactions.

As part of the evaluation of the sorption coefficient data obtained by the Project for the various elements-of-interest and water/rock combinations, the calculated final solution concentrations of the radionuclide-of-interest in each sorption experiment was compared against a solubility limit that reflected the experimental conditions (i.e., water composition, temperature, atmosphere). Experiments in which calculated final solution concentrations of the radionuclide-of-interest exceeded these solubility limits were not used in the derivation of sorption coefficient probability distribution functions. The solubility limits used in this analysis were eitherobtained from solubility experiments carried out by the Project or from calculations using the geochemical code PHREEQC.

For solubility limits obtained from solubility experiments using waters from the site (i.e., J-13 and p\#1), this approach is adequate. For the solubility limits not based on solubility experiments with site waters, a potential problem can arise with this approach. The problem results from the fact that the waters used in the sorption experiments (i.e., J-13, p\#1) contain natural concentrations of some of the elements-of-interest. For example, J-13 and p\#1 water contain background concentrations of the elements-of-interest barium, cesium, protactinium, strontium, and thorium. During sorption experiments, this background is sorbed to the solid phase along with the radioactive isotopes used in the experiments. Thus, the actual concentrations of these elements-of-interest were higher in these sorption experiments than the concentrations calculated only on the basis of the activity of the radioactive isotopes used in the experiments. The problem arises mainly in sorption experiments carried out at final solution concentrations that were just below the solubility limit based only on the concentration of the radioactive isotope. When the natural element concentrations are added to the concentrations of the radioactive isotope, some of these solutions could actually have been oversaturated with a pure solid phase of the element-of-interest.

Normally, it would be expected that the precipitation of a pure phase would result in a higher sorption coefficient than would be the case if such a phase had not precipitated. However, as discussed below in the sections on individual elements, the experimental data do not reflect this expectation. In fact, experiments in which final solution concentrations exceeded solubility limits (i.e., oversaturated solutions) generally resulted in lower sorption coefficients than experiments with undersaturated solutions. A likely reason for this observed behavior is that the nucleation and/or precipitation rates of the appropriate pure solid phases were slow enough under the conditions used in the experiments so that no solid precipitates were formed. Alternatively, the pure-phase precipitates did form but were too fine-grained to be removed from solution by the techniques used to separate the solid and liquid phases at the end of each experiment. 
There is another aspect of natural element "background" that is worth mention. That is the fact that those elements-of-interest that have a natural background generally had final solution concentrations in the sorption experiments carried out by the Project that are higher than those calculated only on the basis of the radioactive isotope concentration used in the experiments even in undersaturated solutions. In those cases where the sorption isotherm for the element-ofinterest is non-linear, the presence of the natural background concentration in the sorption experiments would generally result in lower sorption coefficients than would be the case in the absence of the natural background concentrations.

Thus, in the derivation of sorption coefficient probability distributions for TSPA, the inclusion of sorption coefficient data from experiments containing natural background concentrations generally results in an underestimation of retardation potential.

Because experiments have been carried out at concentrations up to the solubility limit for most elements of interest, the experimental results reflect this dependency. The only element for which the experimental concentrations did not approach a solubility limit was cesium. The solubility of cesium compounds is very high (Section A7.2). Thus, the sorption-coefficient probability distributions for cesium must be calibrated to the cesium concentrations expected in the SZ.

\section{A3. WATER COMPOSITIONAL RANGES ALONG TRANSPORT PATHWAYS}

The chemistry of water in the SZ along potential flow paths to the accessible environment is discussed in Saturated Zone Site-Scale Flow Model (BSC 2004 [DIRS 170037] Table A6-3). In the SZ, there are two rather distinct water types in the ambient system. One is typified by water from well UE-25 J-13 (J-13), located on the east side of Fortymile Wash. The other is from well UE-25 p\#1 (p\#1), located near the southern entrance to the Exploratory Shaft Facility.

The J-13 and p\#1 waters were used in sorption experiments as end-member compositions intended to bracket the impact of water composition on sorption coefficients. Table A-2a presents these data for a sample from the well UE-25 J-13, a composite water sample from the volcanic portion of the well UE-25 p\#1, and a carbonate portion of UE-25 p\#1. Comparative information on range of concentrations of the same chemical constituents from other wells in the area is presented in Table A-2b. It can be seen from these tables that for most constituents, these two water compositions approximately bracket the compositions of other wells both in volcanics and alluvium along the potential flow paths to the accessible environment. The $\mathrm{pH}$ of the waters from alluvium wells ranges outside that given by J-13 and p\#1; however, this is accommodated in the experiments, as seen in Section A7, where water $\mathrm{pH}$ ranged up to 8.4. 
The water compositions used in modeling studies were those reported in Table A-2a.

As will become evident in the discussions of the sorption behavior of individual elements given below, variations in water chemistry can have a significant impact on the sorption behavior of a limited number of elements, particularly uranium and neptunium. To constrain these potential impacts, modeling studies with PHREEQC with the thermodynamic input data file YMP_R3.DAT (DTN: MO0403SPAYMPR3.000 [DIRS 170937]) were carried out as discussed further.

Because carbonate water from the $\mathrm{p} \# 1$ well was not available to the experimental program at all times, a synthetic p\#1 water was developed. This water (Table A-2b) was primarily intended to have a bicarbonate concentration similar to that found in UE-25 p\#1 (carbonate). It was used in experiments with uranium, neptunium, and plutonium because the solution and sorption behavior of these elements is sensitive to the bicarbonate and carbonate concentrations in solution (Nitsche et al. 1993 [DIRS 155218], pp. 54, 60, and 78, and Nitsche et al. 1995 [DIRS 100163], p. 39).

Table A-2a. Compositions of Waters from UE-25 J-13 and UE-25 p\#1

\begin{tabular}{|l|c|c|c|}
\hline \multicolumn{1}{|c|}{ Chemical Constituent } & $\begin{array}{c}\text { UE-25 } \\
\text { J-13 Water }\end{array}$ & $\begin{array}{c}\text { UE-25 } \mathbf{p \# 1 ~ ( v o l c a n i c s ) ~} \\
\text { Water (mg/L) }\end{array}$ & $\begin{array}{c}\text { UE-25 p\#1 (Carbonate) } \\
\text { Water (mg/L) }^{\mathbf{a}}\end{array}$ \\
\hline $\mathrm{Ca}^{2+}$ & 11.5 & 37 & 87.8 \\
\hline $\mathrm{Mg}^{2+}$ & 1.76 & 10 & 31.9 \\
\hline $\mathrm{Na}^{+}$ & 45 & 92 & 171 \\
\hline $\mathrm{K}^{+}$ & 5.3 & 5.6 & 13.4 \\
\hline $\mathrm{SiO}_{2}$ & 64.2 & 49 & 64.2 \\
\hline $\mathrm{Cl}^{-}$ & 6.4 & 13 & 37 \\
\hline $\mathrm{F}^{-}$ & 2.1 & 3.4 & 3.5 \\
\hline $\mathrm{SO}_{4}{ }^{2-}$ & 18.1 & 38 & 129 \\
\hline $\mathrm{HCO}_{3}{ }^{-}$ & $128^{\mathrm{b}}$ & 344 & 666 \\
\hline $\mathrm{CO}_{3}{ }^{2-}$ & 0.0 & 0.0 & 0 \\
\hline $\mathrm{pH}$ & 6.9 & 6.8 & 6.7 \\
\hline
\end{tabular}

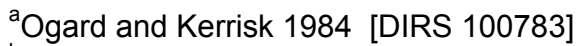

${ }^{\mathrm{b}}$ Computed from other data in this table.

'MO0007MAJIONPH.011 [DIRS 151524] 
Table A-2b. Comparative Information on the Range of Concentrations From Wells in the SZ Transport Model Area

\begin{tabular}{|l|c|c|l|}
\hline $\begin{array}{c}\text { Chemical } \\
\text { Constituent }\end{array}$ & $\begin{array}{c}\text { Range of Concentrations in } \\
\text { Downgradient Wells in Volcanics } \\
(\mathbf{m g} / \mathbf{L})^{\mathbf{a}}\end{array}$ & $\begin{array}{c}\text { Range of Concentrations in } \\
\text { Downgradient Wells in Alluvium } \\
(\mathbf{m g} / \mathbf{L})^{\mathbf{a}}\end{array}$ & $\begin{array}{c}\text { Synthetic } \mathbf{p \# 1} \\
\text { Water (mg/L) }\end{array}$ \\
\hline $\mathrm{Ca}^{2+}$ & $0.8-37$ & $0.8-20.3$ & \\
\hline $\mathrm{Mg}^{2+}$ & $<0.1-10$ & $0.0-7.7$ & \\
\hline $\mathrm{Na}^{+}$ & $38-120$ & $57.9-180.5$ & $261^{\mathrm{b}}$ \\
\hline $\mathrm{K}^{+}$ & $1.1-8.9$ & $1.8-5.5$ & \\
\hline $\mathrm{SiO}_{2}$ & $36-57$ & $40.5-61$ & \\
\hline $\mathrm{Cl}^{-}$ & $6.0-13$ & $5.6-18$ & \\
\hline $\mathrm{F}^{-}$ & $1.0-6.7$ & $1.6-4.2$ & $691^{\mathrm{b}}$ \\
\hline $\mathrm{SO}_{4}{ }^{2-}$ & $14-38$ & $18.7-61.7$ & \\
\hline $\mathrm{HCO}_{3}{ }^{-}$ & $107-344$ & $110-255.5$ & \\
\hline $\mathrm{CO}_{3}{ }^{2-}$ & 0.0 & $0.0-23.5$ & - \\
\hline $\mathrm{pH}^{\mathrm{Eh}}$ & $6.8-8.4$ & $7.5-8.9$ & \\
\hline
\end{tabular}

${ }^{\mathrm{a} B S C} 2004$ ([DIRS 170037], Table A6-1)

${ }^{\mathrm{b}}$ Triay et al. (1996 [DIRS 101024], p. 4)

\section{A4. EXPERIMENTAL TECHNIQUES}

The basic technique for the laboratory determination of sorption coefficients involved the contact of a groundwater sample, spiked with the radionuclide of interest, with a crushed sample of tuff or alluvium (Daniels et al. 1982 [DIRS 105803]). The rock sample was generally obtained as a core sample. The core was crushed in a jaw crusher and subsequently sieved to a selected grain-size fraction. The sieving process was usually carried out under water. Initially, several different grain-size fractions were used in the experiments. With experience, it was concluded that the 75- to 500- $\mu \mathrm{m}$ fraction was the most appropriate for use in these types of experiments. Results for samples that included all sizes below a certain grain size (e.g., less than $35 \mu \mathrm{m}$ ) tended to produce higher sorption-coefficient values than the 75 - to $500-\mu \mathrm{m}$ fraction.

Because the tuffs are very fine grained (i.e., crystal sizes on the order of 10-20 $\mu \mathrm{m}$ ), crushing would not produce significant increases in mineral surface area. Mineral fractionation is the process by which certain minerals, such as clays, may become enriched in a very fine-grain size fraction due to sieving effects. Mineralogic analysis of different mineral fractions, including fines, indicates some mineral fractionation has occurred (Chipera and Bish 1989 [DIRS 101374], Appendix A). Thus, this fractionation may be the cause of the enhanced sorption observed in the fractions that include fines.

One gram of crushed rock material (usually the 75 to $500-\mu$ m fraction) was added to a test tube with $20 \mathrm{~mL}$ groundwater spiked with the radionuclide(s) of interest. The test tube was put on a shaker table for a predetermined period to allow reaction to occur. After the predetermined time had passed, the solution was separated from the solid phase by either centrifugation or filtration. Centrifugation was preferred for those elements thought to have an affinity for the filter medium. The separations were not always perfect due to various experimental constraints. In some cases, the solid fraction was separately counted. A sorption coefficient was usually calculated from the 
difference between the initial and final solution concentrations. Corrections were generally made for sorption onto the surface of the test tube during the equilibration (shaking) period.

Some potential sources of errors and experimental artifacts that may pertain to the sorption coefficients include weighing errors, counting errors, errors resulting from solutions being oversaturated with the element of interest, errors from imperfect solid/liquid separations, errors from inaccurate correction for sorption onto container walls, recording errors, transcription errors, inadvertent laboratory errors, and calculation errors. These errors cannot be quantitatively assessed. However, their existence will become apparent in the scatter of the data on diagrams presented in Section A7.

\section{A5. APPROACH TO THE DERIVATION OF SORPTION-COEFFICIENT RANGES FOR MAJOR ROCK TYPES IN THE YUCCA MOUNTAIN FLOW SYSTEM}

The derivation of sorption-coefficient probability distributions for the elements of interest on the major rock types in Yucca Mountain involves both an evaluation of available experimental data and sorption modeling. Experimental data are used to evaluate the impact of variations in (1) rock sorption properties (within each of the two major groupings), (2) radionuclide concentrations, (3) sorption kinetics, and (4) water chemistry, on sorption coefficients for the elements of interest.

The radionuclides of interest are divided into three groups of radioelements. For the first group, including americium, neptunium, plutonium, thorium, and uranium, experimental data are used to evaluate the impact of radionuclide concentrations, sorption kinetics, and variations in water chemistry on sorption coefficients. Surface-complexation modeling is used to further evaluate the impact of variations in water chemistry and surface area on sorption coefficients. The surface-complexation models used in this analysis are based on the code PHREEQC, using the thermodynamic input data file YMP_R3.DAT (DTN: MO0403SPAYMPR3.000 [DIRS 170937]). The binding constants required for surface-complexation modeling are either obtained from the literature or derived from experimental data involving sorption of the radioelement on silica.

In the second group of elements, including cesium, protactinium, radium, and strontium, the ranges of sorption-coefficient values for the major rock types are derived directly from the available experimental data and the ranges for environmental variables expected in the transport system. Although it would be preferable to have a theoretical model to evaluate the impacts of variations in water chemistry and rock chemistry on sorption coefficients for these radionuclides, there are not sufficient data available to properly constrain such a model.

For the third group, including carbon, iodine, and technetium, the sorption coefficient is taken to be zero in the volcanic rocks and in the alluvium. This approach is taken due to the lack of available data and leads to model breakthrough times longer than the transit times that would result in the presence of sorption of these radionuclides on to the rock surfaces. 


\section{A6. SURFACE-COMPLEXATION MODELING}

The PHREEQC surface-complexation model used in this analysis is a nonelectrostatic model (Parkhurst 1995 [DIRS 142177], pp. 10-11). Inputs required for modeling include deprotonation constants, binding constants for elements of interest, total site concentrations, water chemistry, and a thermodynamic database for solution species and solids. Because the tuffs contain up to 76 weight percent silica (Broxton et al. 1986 [DIRS 100023], p. 39), a silica surface was used to represent the mineral surfaces in the tuffs. The surface-complexation models included the effects of competition from common constituents in the rock such as calcium, magnesium, sodium, potassium, and aluminum. Deprotonation constants for silica were obtained from Dixit and Van Cappellen (2002 [DIRS 162985], p. 2565). Binding constants were obtained from Dixit and Van Cappellen (2002 [DIRS 162985], p. 2565) for aluminum on silica, and from Marmier et al. (1999 [DIRS 162986], p. 228) for sodium on silica. The total site concentrations were obtained from surface area (Section A2 and Table A-1), and a site density of 2.3 sites $/ \mathrm{nm}^{2}$ is used as recommended by Pabalan et al. (1998 [DIRS 162987], p. 124). Pabalan was the source for uranium binding constants on silica. The binding constants were used in surface complexation modeling calculations to evaluate the impact of variations in water chemistry (e.g., $\mathrm{pH}, \mathrm{J}-13$ versus $\mathrm{p \# 1)}$ on uranium sorption coefficients). The thermodynamic data used for the modeling was from file YMP_R3.DAT (DTN: MO0403SPAYMPR3.000 [DIRS 170937]).

\section{A7. DISCUSSIONS AND ANALYSIS OF EXPERIMENTAL SORPTION-COEFFICIENT DATA AND SURFACE-COMPLEXATION MODELING}

The data and modeling results for each element are discussed in separate sections, arranged in alphabetical order.

\section{A7.1 AMERICIUM}

According to Nitsche et al. (1993 [DIRS 155218], p. 78), the solubility of americium in J-13 water is controlled by $\mathrm{AmOH}(\mathrm{CO})_{3}$.

At $25^{\circ} \mathrm{C}$ and a $\mathrm{pH}$ of 8.5 , the solubility is $2.4( \pm 1.9) \times 10^{-9} \mathrm{M} / \mathrm{L}$. At $25^{\circ} \mathrm{C}$ and a $\mathrm{pH}$ of 7.0 , the solubility is $1.2( \pm 0.3) \times 10^{-9} \mathrm{M} / \mathrm{L}$.

\section{A7.1.1 Devitrified Tuff}

Sorption coefficients $\left(K_{d}\right)$ measured on devitrified tuffs are plotted versus calculated final solution concentrations in Figure A-1. The points identified by the term "sorption" refer to experiments in which J-13 water spiked with americium was contacted with devitrified tuff. The points identified by the term "desorption" refer to experiments in which the solid remaining after the "sorption" step was contacted with unspiked J-13 water. The steep negative slopes evident at the higher concentrations for individual samples reflect a mass balance constraint. That is, the final solution concentration is calculated using the starting solution concentration and the measured sorption coefficient. This approach forces a linear dependence of the sorption coefficient on the final solution concentration. The offset between the points for the various samples primarily reflects different starting concentrations. 
The positions of samples along a slope reflect variations in the degree of equilibration among various experiments on the same sample with the same starting concentration. When the sorption experiments are initiated, these trends start on the abscissa at the initial concentration. As the experiment duration is increased, americium is sorbed to the solid phase, the solution concentration decreases correspondingly, and the value of $K_{d}$ increases. At equilibrium, the solution concentration remains constant with time and the $K_{d}$ is at a maximum value. For desorption experiments, the trend is reversed. As the duration of a desorption experiment increases, the solution concentrations increase as americium is released from the solid, and the value of $K_{d}$ decreases. At equilibrium, the $K_{d}$ value obtained from a desorption experiment should approach the $K_{d}$ value obtained from the associated sorption experiment unless the isotherm is quite nonlinear, in which case, the trends could be offset. The offset would be due to a different total americium concentration in the sorption and desorption experiments. The $K_{d}$ value on which the two trends converge is considered the equilibrium value.

As shown in Figure A-1, the calculated final solution concentrations were higher than the solubility of $\mathrm{AmOH}(\mathrm{CO})_{3}$ in numerous experiments with samples JA-32 and YM-54. Thus, the results for these experiments must be discounted. For the remaining experiments, the sorption coefficients range from 1,000 to more than $10,000 \mathrm{~mL} / \mathrm{g}$. In one sample (GU3-688), americium concentrations were analyzed by isotope dilution mass spectrometry (Triay et al. 1991 [DIRS 104129], p. 142-144). This technique has higher sensitivity than the (radioactivity) counting techniques normally used, thereby allowing the use of lower americium concentrations. The result obtained for this sample is in the range of the results obtained for the other samples.

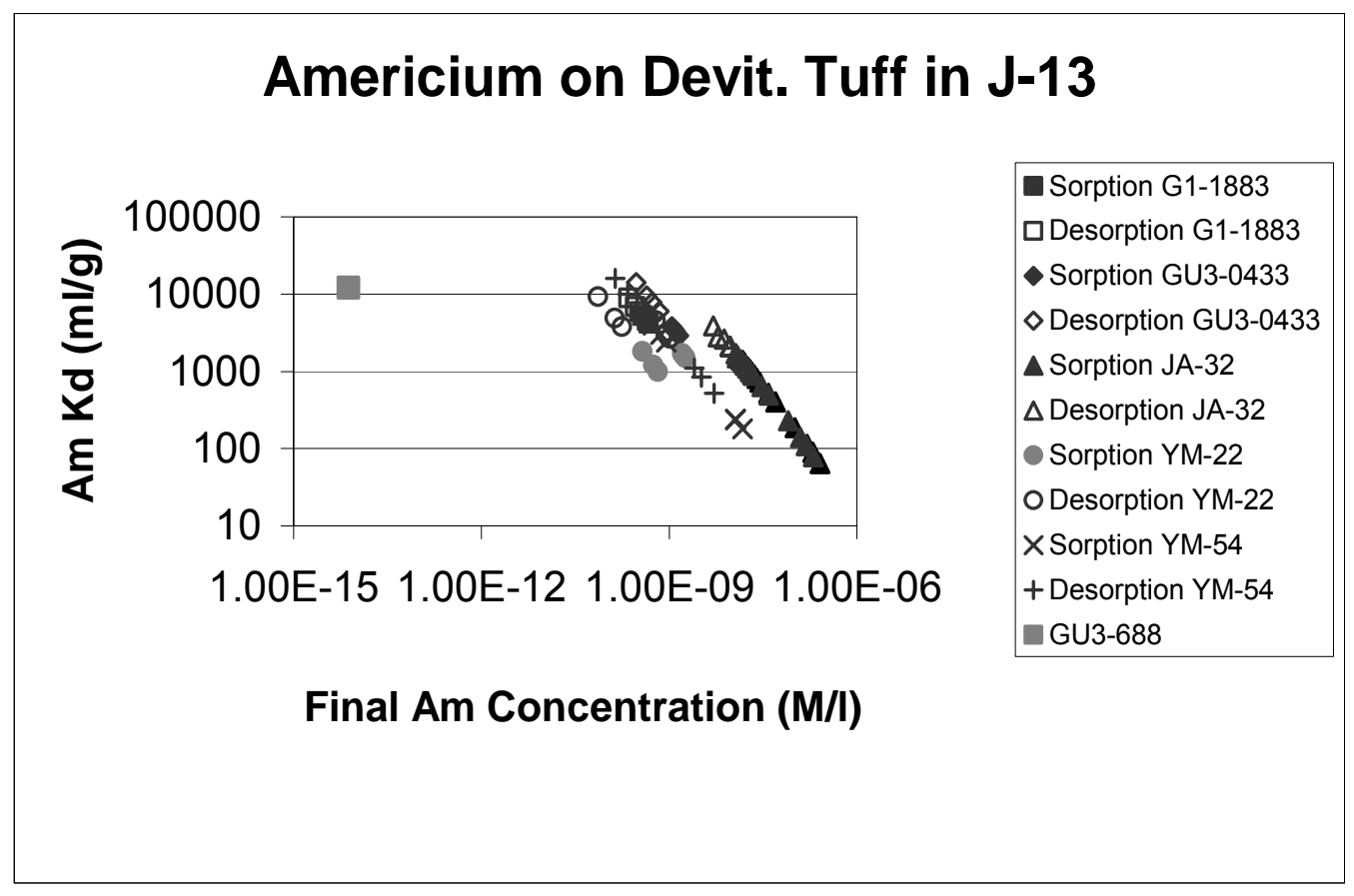

Source DTN: LA0305AM831341.001 [DIRS 163789].

Figure A-1. Americium Sorption Coefficients on Devitrified Tuff Versus Calculated Final Americium Concentration in Solution 
The sorption coefficients derived from desorption experiments are generally larger than those derived from the sorption experiments on a given sample (Figure A-1). This result could be due to slow desorption kinetics or due to a nonlinear isotherm.

Americium sorption coefficients are plotted versus duration of the sorption and desorption experiments in Figure A-2. The trend in the data (ignoring data from samples JA-32 and YM-54) suggests that americium sorption-coefficient values are not very sensitive to the duration of the experiments except in those experiments with very short durations. Thus, the kinetics of the americium sorption reactions appear to be relatively fast.

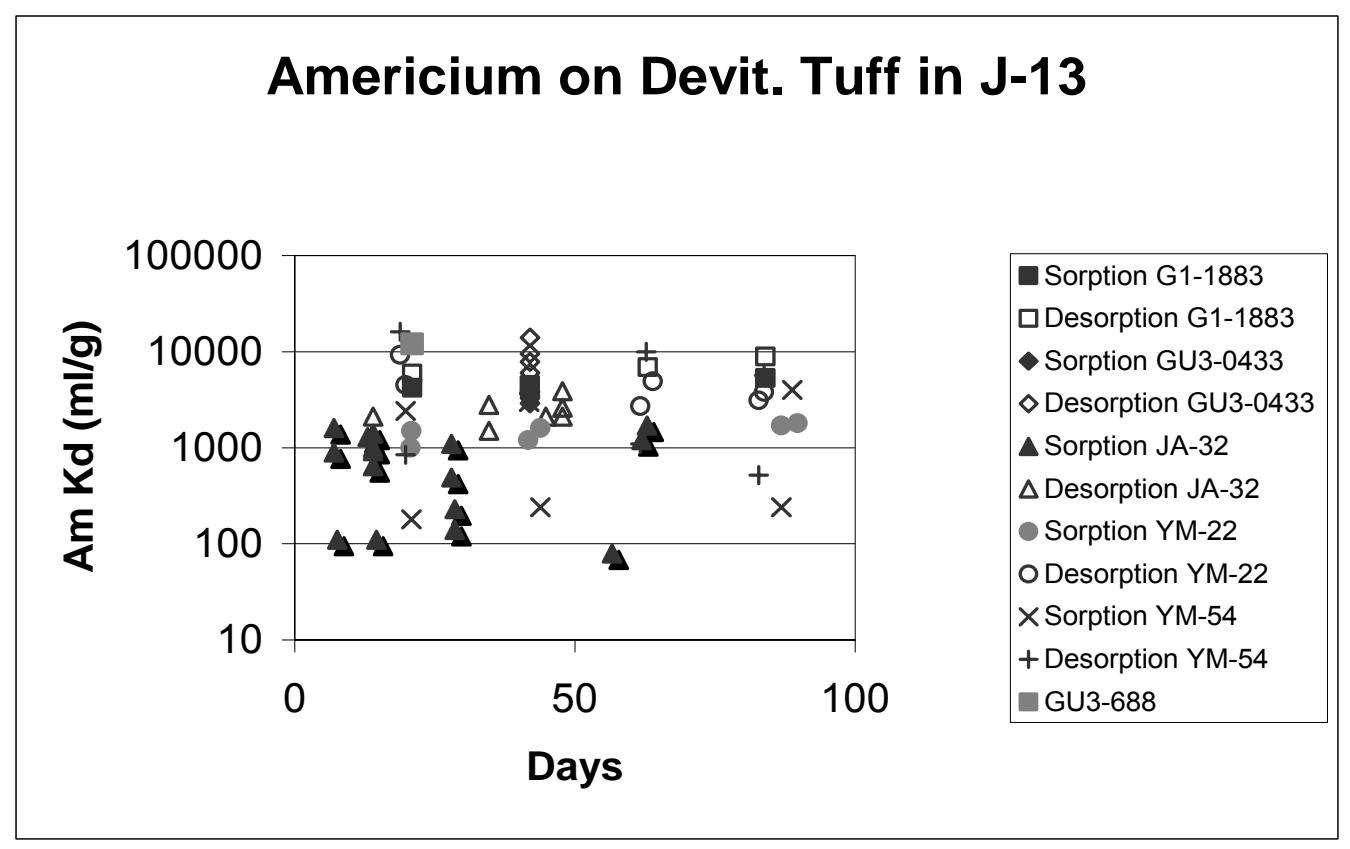

Source DTN: LA0305AM831341.001 [DIRS 163789].

Figure A-2. Americium Sorption Coefficients for Devitrified Tuff as a Function of Experiment Duration

The effects of variations in water chemistry on americium sorption coefficients have not been tested experimentally except for variations in solution $\mathrm{pH}$. All the americium sorption and desorption experiments carried out with Yucca Mountain samples used J-13 water. Americium sorption and desorption coefficients in J-13 water are plotted versus pH in Figure A-3. It is evident that there is no clear trend among the data points plotted. The variations in sorption coefficients observed in multiple experiments with the same rock sample could reflect (radioactivity) counting statistics, long-term stability of the counting equipment, sorption kinetics, imperfect separation of the solid and liquid phases, and/or the consistency of adsorption of americium to the walls of the experimental containers. Counting statistics are generally a small percentage of the measured sorption coefficient. Sorption kinetics may explain some of the scatter but not all of it. The other factors are likely significant, but they cannot be quantified with the available data. 


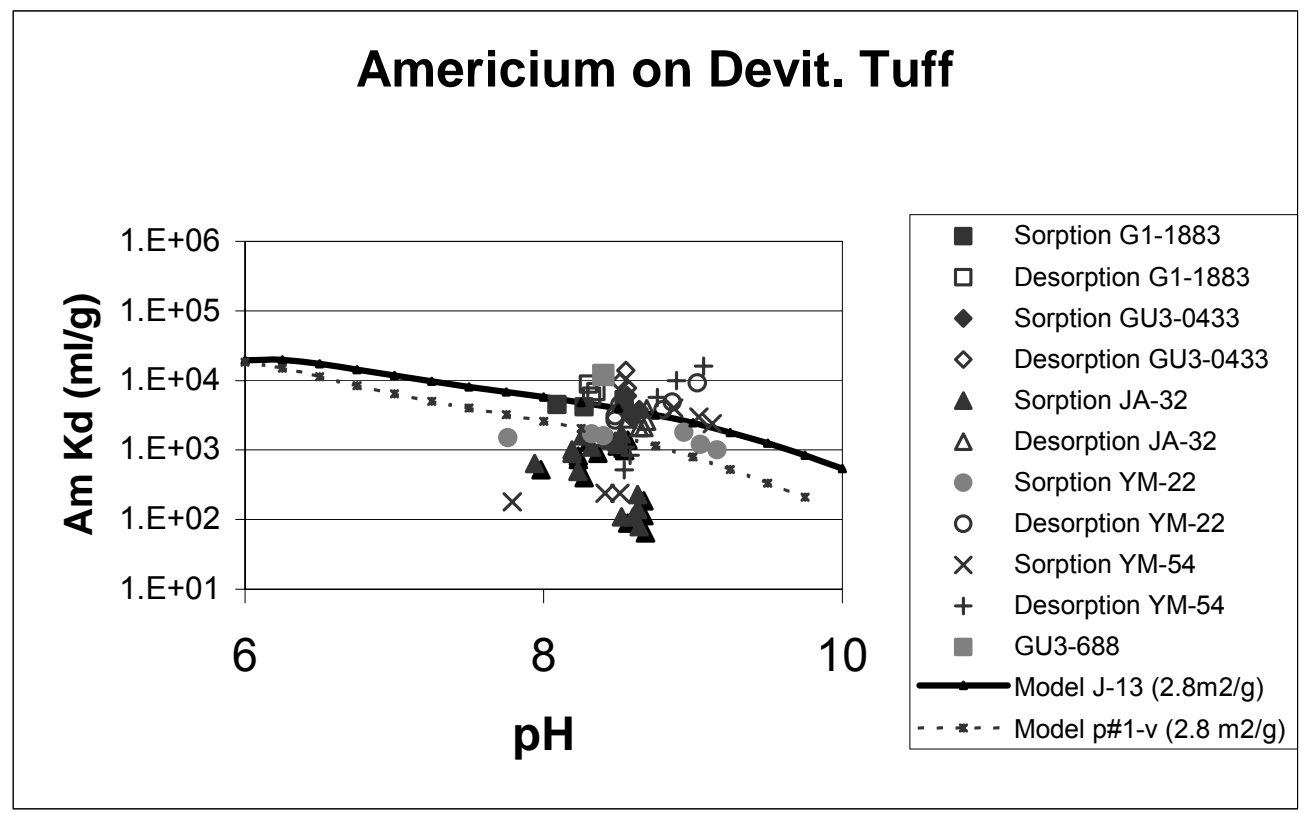

Source DTN: LA0305AM831341.001 [DIRS 163789].

NOTE: Model curves derived with PHREEQC surface-complexation modeling are also shown (output DTN: LA0306AM831343.001 file output/Amsij13.pun).

Figure A-3. Americium Sorption Coefficients on Devitrified Tuff Versus pH

To gauge the potential impact of variations in water chemistry on americium sorption coefficients, surface complexation modeling was carried out with PHREEQC with the thermodynamic input data file YMP_R3.DAT (DTN: MO0403SPAYMPR3.000 [DIRS 170937]). In the modeling, surface sites on devitrified tuff were taken to be equivalent to surface sites on silica, $2.8 \mathrm{~m}^{2} / \mathrm{g}$ (Allard et al. 1980 [DIRS 104410]). Binding constants for americium species on silica were derived by fitting data presented by Beall et al. (1986 [DIRS 162983], p. 502) for the sorption of americium onto quartz (2.8 $\mathrm{m}^{2} / \mathrm{g}$; Allard et al. 1980 [DIRS 104410], p. 478) in Oak Ridge National Laboratory Standard Water (Allard and Beall (1979 [DIRS 147235] p. 511). This water is similar in composition to J-13. (Allard 1980 was the source for the surface area $\left(\mathrm{m}^{2} / \mathrm{g}\right)$ of silica samples used in experiments reported in Beall et al. (1986). This surface area data was needed to do surface complexation modeling for americium. The surface complexation modeling for americium is needed to evaluate the impact of variations in water chemistry on sorption coefficients for americium. The project has americium sorption coefficient data only with J-13 water compositions). As shown in Figure A-4, the sorption coefficients for americium on quartz (Beall et al. 1986 [DIRS 162983], p. 502) as a function of pH could be fit very well using two surface reactions involving an americium sulfate complex and an americium carbonate complex. (Beall et al. 1986 was the source of americium sorption coefficients used in surface complexation modeling for americium. The data reported in this paper were used to derive surface complexation binding constants for americium on silica. These constants were used in additional modeling to evaluate the impact of variations in water chemistry on americium sorption coefficients. The project has americium sorption data only for J-13 water composition.) These reactions are listed in Table A-3. 


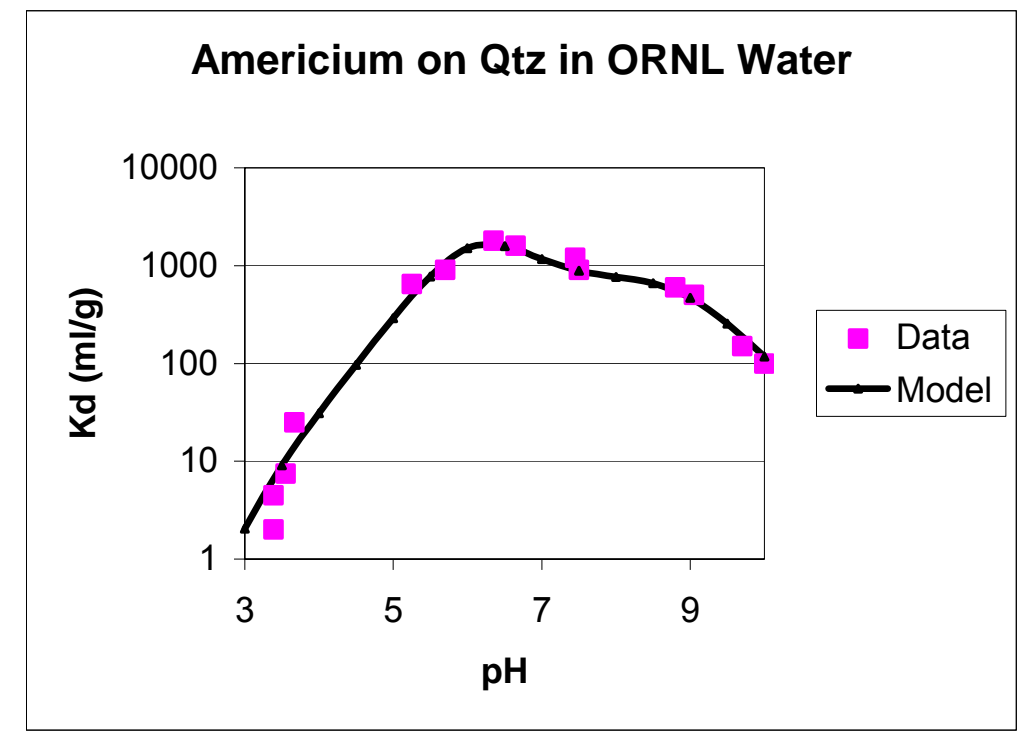

Data: Beall et al. 1986 [DIRS 162983].

Model: Output DTN: LA0306AM831343.001 file output/Amornl5.pun.

NOTE: Model curves derived with PHREEQC surface-complexation modeling are also shown (output DTN: LA0306AM831343.001 file output/Amornl5.pun).

Figure A-4. Americium Sorption Coefficients on Quartz from Beall et al. and Model Fit

Table A-3. Surface Complexation Reactions for Americium

\begin{tabular}{|l|l|}
\hline \multicolumn{1}{|c|}{ Reaction } & Log $K$ \\
\hline $\mathrm{SiOH}+\mathrm{Am}^{3+}+\mathrm{SO}_{4}{ }^{2-} \leftrightarrow \mathrm{SiOAmSO}_{4}+\mathrm{H}^{+}$ & 5.5 \\
\hline $\mathrm{SiOH}+\mathrm{Am}^{3+}+\mathrm{CO}_{3}{ }^{2-} \leftrightarrow \mathrm{SiOAmCO}_{3}+\mathrm{H}^{+}$ & 6.5 \\
\hline
\end{tabular}

Using a surface area of $2.8 \mathrm{~m}^{2} / \mathrm{g}$, americium sorption coefficients were calculated for "devitrified tuff” in J-13 water as a function of $\mathrm{pH}$. The model curves are shown in Figure A-3. The fact that the model sorption coefficient for $\mathrm{J}-13$ at a $\mathrm{pH}$ value of 8.5 lies in the middle of the range of experimental values provides confidence that the model is reasonable. The model curves move up or down linearly with surface area. Thus, at least part of the range of experimental values could reflect variations in devitrified tuff surface areas.

The model curve shown in Figure A-3 indicates that sorption coefficients for americium will increase with decreasing $\mathrm{pH}$ in the $\mathrm{pH}$ range from 7.0 to 9.0. Thus, in terms of $\mathrm{pH}$, the experimentally derived sorption coefficients are at the low end of the range of coefficients to be expected in the SZ. The effect of variation in major ion chemistry of groundwater is shown by the curve calculated for Borehole p\#1 groundwater. Americium sorption coefficients calculated using p\#1 water are similar to those calculated using J-13 water. Thus, variations in water chemistry are not expected to have a major impact on americium sorption coefficients. 
On the basis of the experimental data and model curves plotted in Figure A-3, the range of americium sorption coefficients expected for devitrified tuffs in the saturated volcanic section at Yucca Mountain is selected as 1,000 to $10,000 \mathrm{~mL} / \mathrm{g}$. The probability distribution type selected is a truncated normal distribution with a mean of $5,500 \mathrm{~mL} / \mathrm{g}$ and standard deviation of $1,500 \mathrm{~mL} / \mathrm{g}$. The data for JA-32 and YM-54 were discounted in derivation of the distribution because the final solutions in the experiments with these samples were oversaturated with $\mathrm{AmOH}(\mathrm{CO})_{3}$.

\section{A7.1.2 Zeolitic Tuff}

The measured sorption coefficients for zeolitic tuff are plotted versus calculated final solution concentrations in Figure A-5. The calculated final solution concentrations were higher than the solubility of $\mathrm{AmOH}(\mathrm{CO})_{3}$ in essentially all the experiments with sample JA-18. Therefore, the sorption coefficients obtained for this sample are discounted in the derivation of the sorption-coefficient probability distribution for americium on zeolitic tuff. Sorption experiments with sample G4-1952 were close to saturation with $\mathrm{AmOH}(\mathrm{CO})_{3}$. The sorption coefficients obtained for this sample will be used in the derivation of the distribution.

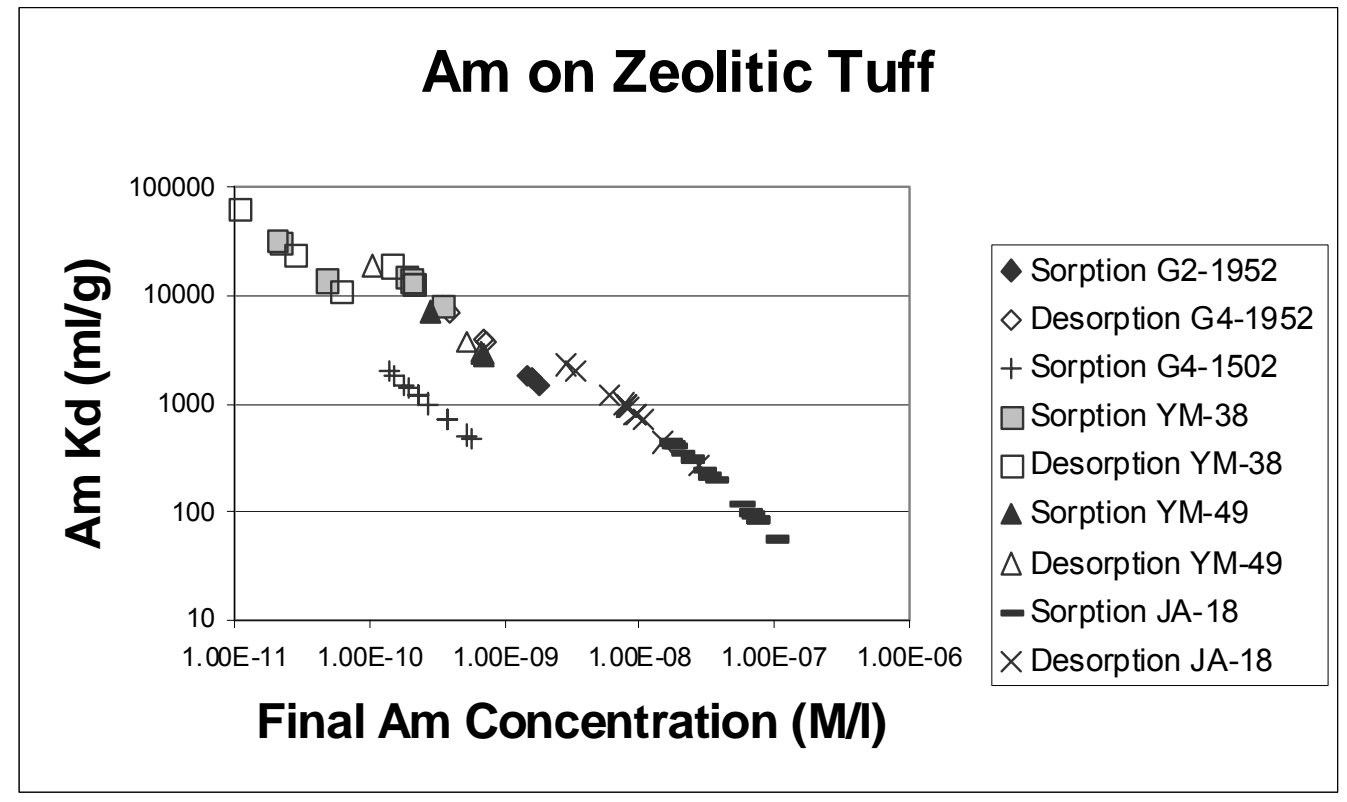

Source DTN: LA0305AM831341.001 [DIRS 163789].

Figure A-5. Americium Sorption Coefficients on Zeolitic Tuff Versus Calculated Final Americium Concentration in Solution

Americium sorption coefficients for zeolitic tuff are plotted versus duration of the sorption and desorption experiments in Figure A-6. As with the devitrified tuffs, the trend in the data (excluding sample JA-18) suggests americium sorption-coefficient values obtained in experiments with solutions undersaturated with $\mathrm{AmOH}(\mathrm{CO})_{3}$ are not very sensitive ( $<10$ times) to the duration of the experiments except in those experiments with short durations (e.g., sample G4-1502). Thus, the kinetics of the americium sorption reactions on zeolitic tuff appear to be relatively fast. 


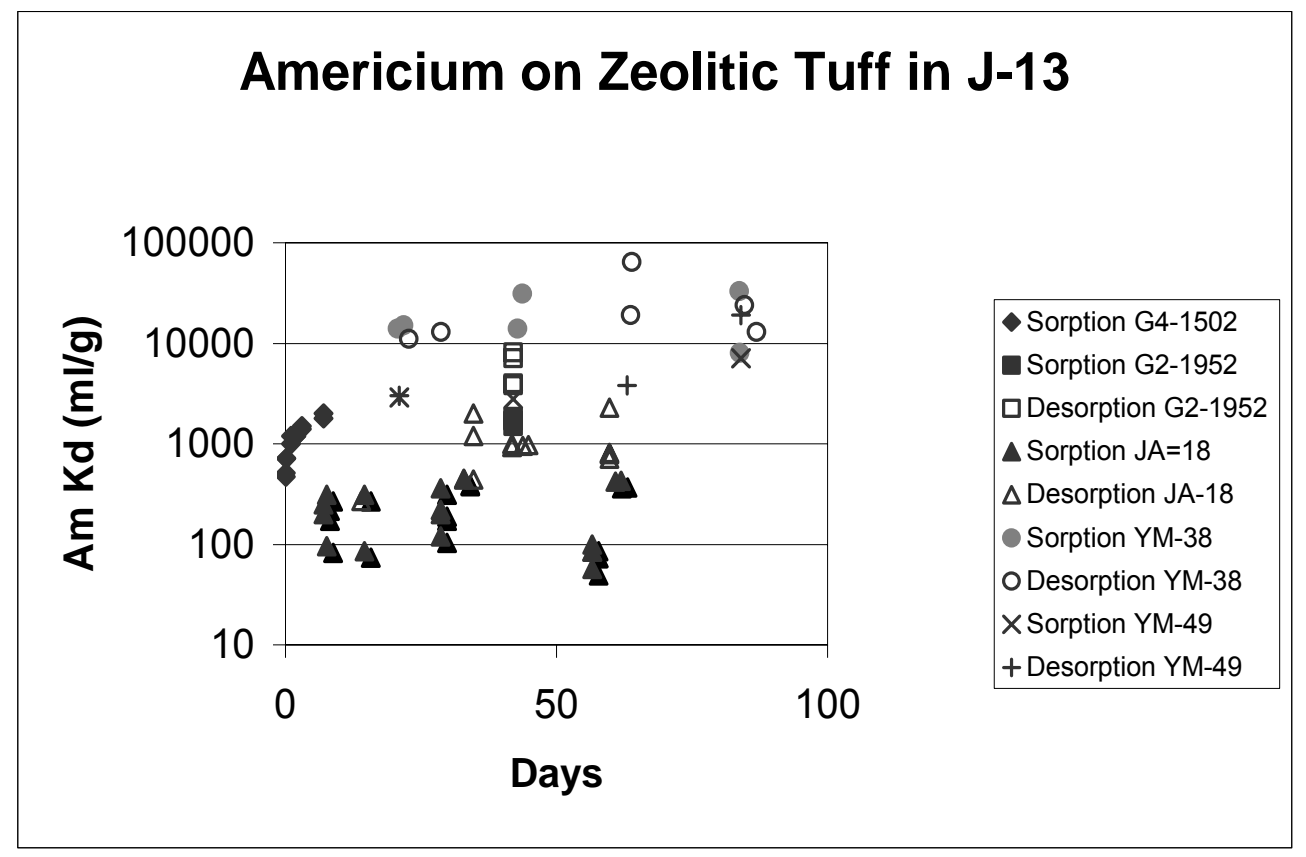

Source DTN: LA0305AM831341.001 [DIRS 163789].

Figure A-6. Americium Sorption Coefficients for Zeolitic Tuff as a Function of Duration of Sorption and Desorption Experiments

The surface complexation model developed for zeolitic tuff is similar to the model developed for devitrified tuff except that a surface area of $28 \mathrm{~m}^{2} / \mathrm{g}$ was used as a representative value (Table A-1 of this report). The model sorption coefficient at a pH of 8.5 lies at the high end of the range of experimental values (Figure A-7). It might be expected that the sorption coefficients for zeolitic tuffs would be on the order of 10 times larger than those for devitrified tuffs to reflect the factor of 10 increase in surface area. The fact that the americium sorption coefficients obtained for zeolitic tuffs are not, on average, a factor of 10 larger than those measured in devitrified tuffs suggests not all the surface area in the zeolitic tuffs may be accessible to americium. Alternatively, the binding sites in the interior of zeolite crystals may not have a high affinity for americium species.

As with devitrified tuff, the model curves for zeolitic tuffs shown in Figure A-7 indicate that sorption coefficients for americium will increase with decreasing $\mathrm{pH}$ in the $\mathrm{pH}$ range from 7.0 to 9.0. Thus, in terms of $\mathrm{pH}$, the experimentally derived sorption coefficients are at the low end of the range of coefficients to be expected in the SZ. The effect of variation in major ion chemistry of groundwater is shown by the curve calculated for Borehole $\mathrm{p \# 1}$ groundwater. Americium sorption coefficients calculated using p\#1 water are similar to the coefficients calculated using J-13 water. 


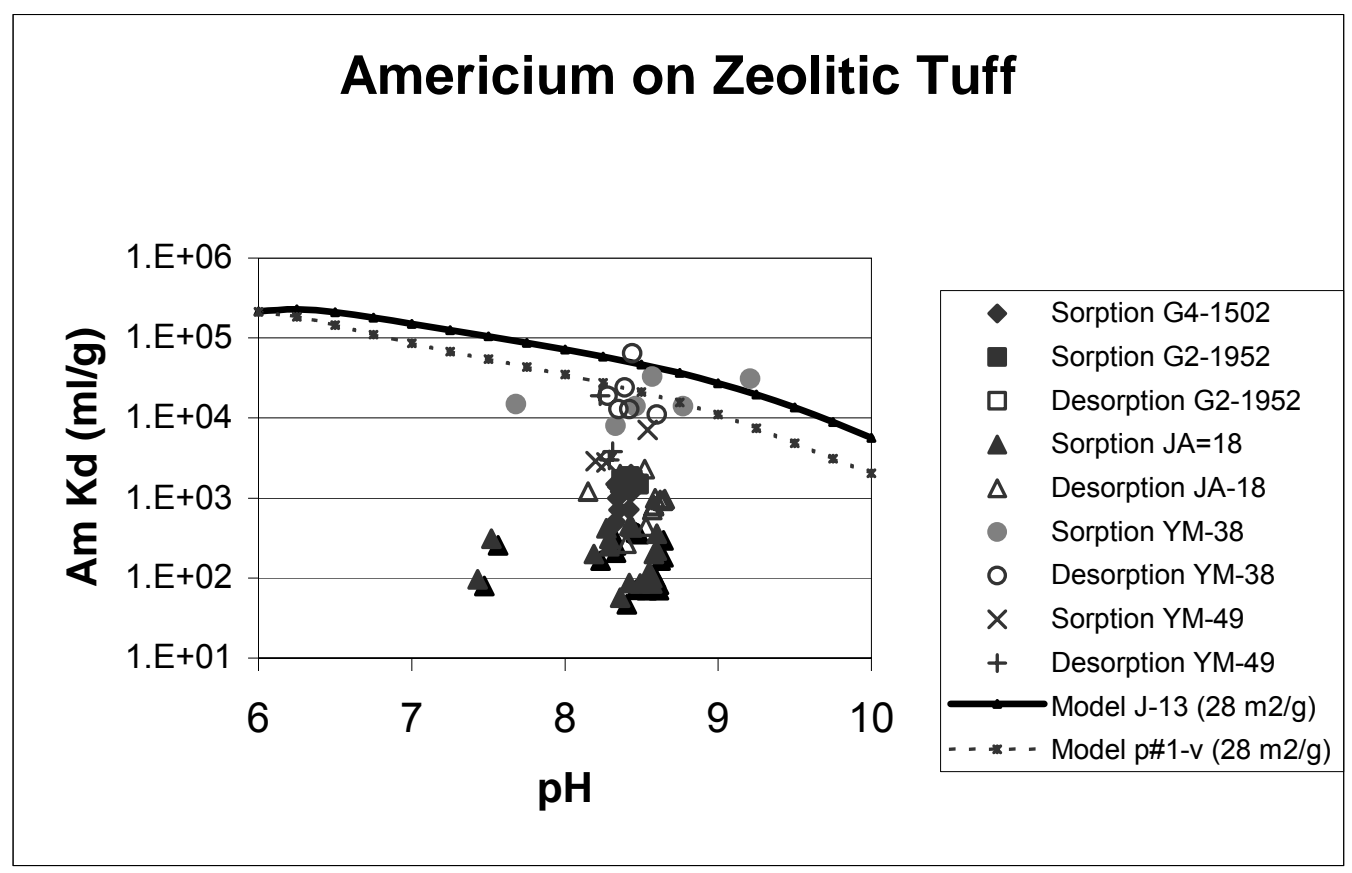

Source DTN: LA0305AM831341.001 [DIRS 163789].

NOTE: Model curves derived with PHREEQC surface-complexation modeling are also shown (DTN: LA0306AM831343.001 file output/ Amzeop1.pun).

Figure A-7. Americium Sorption Coefficient Data and Modeling Results for Zeolitic Tuff as a Function of $\mathrm{pH}$

On the basis of the experimental data and model curves plotted in Figure A-7, the range of americium sorption coefficients selected for zeolitic tuffs in the saturated volcanic section at Yucca Mountain is 1,000 to $10,000 \mathrm{~mL} / \mathrm{g}$. The probability distribution type selected is a truncated normal distribution with the mean of $5,500 \mathrm{~mL} / \mathrm{g}$ and the standard deviation of $1,500 \mathrm{~mL} / \mathrm{g}$. The upper end of this distribution could have been set at $100,000 \mathrm{~mL} / \mathrm{g}$ instead of $10,000 \mathrm{~mL} / \mathrm{g}$ based on the available data and modeling results. However, the $10,000 \mathrm{~mL} / \mathrm{g}$ value is large enough to effectively keep americium from being transported over the regulatory time frame. In addition, by using an upper limit of $10,000 \mathrm{~mL} / \mathrm{g}$, the same distribution can be used for devitrified and zeolitic tuffs. The data for sample JA-18 were discounted in the derivation of the distribution because the final solutions in the experiments with this sample were oversaturated with $\mathrm{AmOH}(\mathrm{CO})_{3}$.

\section{A7.1.3 Alluvium}

The probability distribution for devitrified tuff will be used as a default for the americium sorption-coefficient probability distribution in alluvium. Alluvium along the flow path is composed largely of disaggregated tuffaceous materials. Because devitrified tuff makes up a major portion of the volcanic units exposed at the surface, it should be a major component in alluvium. In addition, clays and other secondary minerals are enriched in alluvial materials. These characteristics should result in higher sorption coefficients for alluvial materials compared to intact devitrified tuff. 


\section{A7.2 CESIUM}

The solubility of cesium in $\mathrm{J}-13$ water at $25^{\circ} \mathrm{C}$ is very high. In fact, cesium concentrations in Yucca Mountain groundwaters will not have a solubility limitation (BSC 2004 [DIRS 169425], Section 6.17).

\section{A7.2.1 Devitrified Tuff}

Experimentally derived sorption coefficients for cesium on devitrified tuff are plotted against the calculated final cesium concentrations of the experiments in Figure A-8. The data points are separated into groups on the basis of when the experiments were carried out (pre-1990 = "old" and post-1990 = "new"), water type, and on whether the sorption coefficient was determined from a sorption or a desorption experiment. There are also data for sorption isotherms on samples YM-22 and G1-2840.

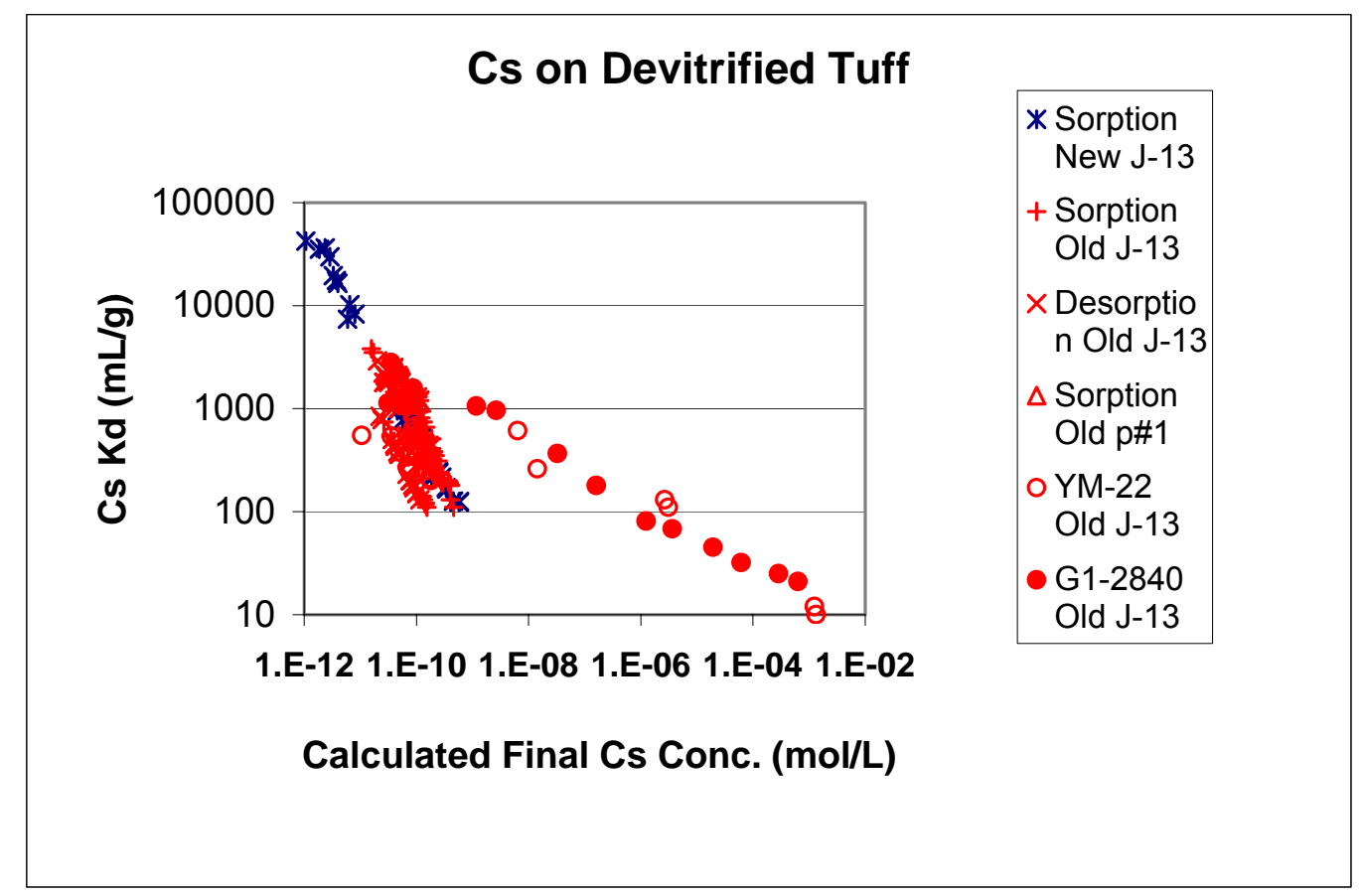

Source DTNs: $\quad$ LA0305AM831341.001 [DIRS 163789]; LA0407AM831341.002 [DIRS 170621].

Figure A-8. Cesium Sorption Coefficients on Devitrified Tuff Versus Calculated Final Cesium Concentration in Solution 
Sorption coefficients obtained in "new" experiments lie within the range defined by the "old" experiments at similar cesium concentrations. Although most of the sorption experiments resulted in $K_{d}$ values greater than $100 \mathrm{~mL} / \mathrm{g}$, these experiments were carried out at cesium concentrations below $1.0 \times 10^{-6} \mathrm{M} / \mathrm{L}$. At higher cesium concentrations, the $K_{d}$ values obtained were between 10 and $100 \mathrm{~mL} / \mathrm{g}$. Nonlinear sorption isotherms were obtained for samples YM-22 and G1-2840 in J-13 water. The data for sample G1-2840 were fit using a Freundlich equation as shown in Figure A-9. Sorption coefficients obtained in experiments with $\mathrm{p \# 1}$ water fall in the middle of the cluster of points in Figure A-8 near $1 \times 10^{-10} \mathrm{M} / \mathrm{L}$. Thus, there is little or no impact of variations in water chemistry on cesium sorption coefficients on devitrified tuff.

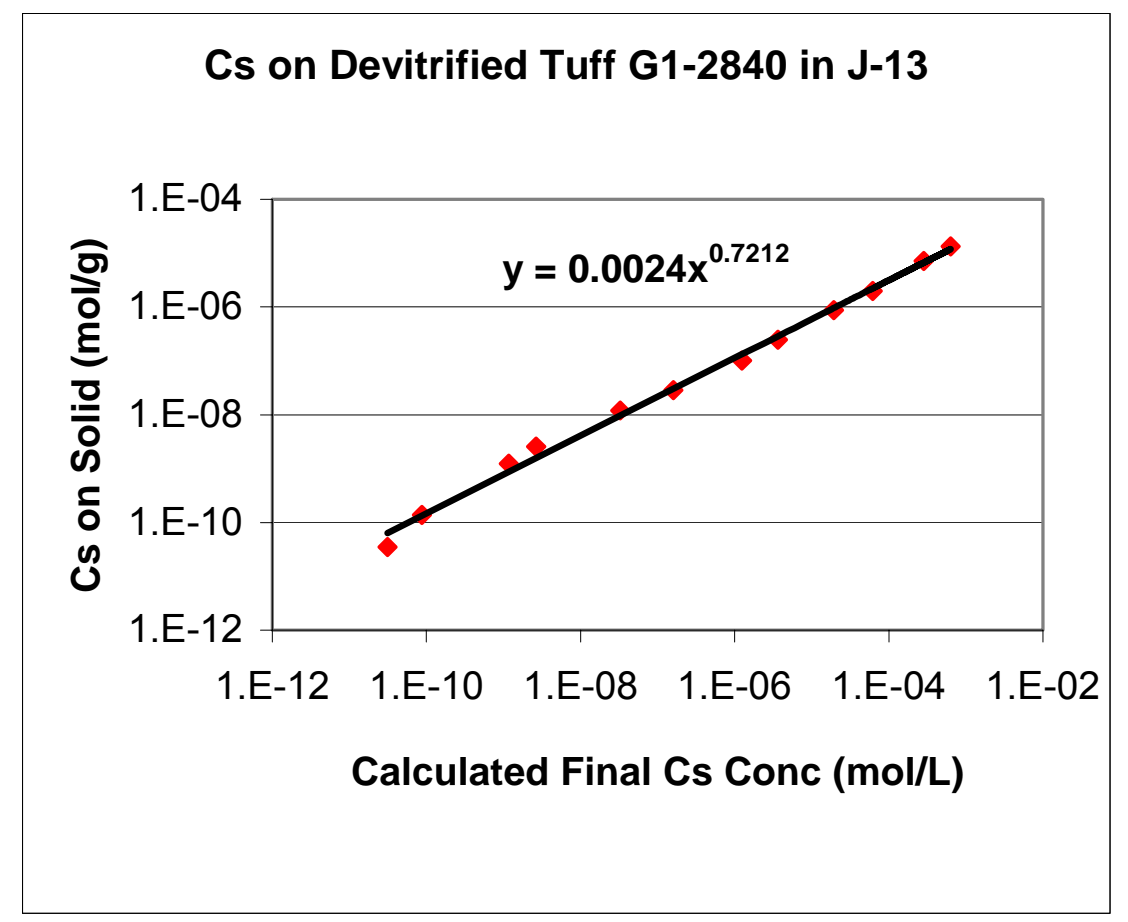

Source DTNs: LA0407AM831341.002 [DIRS 170621]; LA0305AM831341.001 [DIRS 163789].

Figure A-9. Freundlich Isotherm Fit to Sorption-Coefficient Data for Cesium on Devitrified Tuff Sample G1-2840 in J-13 Water

The effects of experiment duration on the cesium sorption coefficients for devitrified tuff are shown in Figure A-10. The large range in sorption coefficients obtained at a given duration (e.g., 21 days) mainly reflects variations in cesium solution concentrations, although variations in ion-exchange capacities may also contribute to the range. The range of sorption-coefficient values is fairly constant with duration when the results for samples YM-22 and G1-2840 are excluded. This range extends from just above $100 \mathrm{~mL} / \mathrm{g}$ to above $1,000 \mathrm{~mL} / \mathrm{g}$. The constancy indicates cesium sorption reactions are fast. 


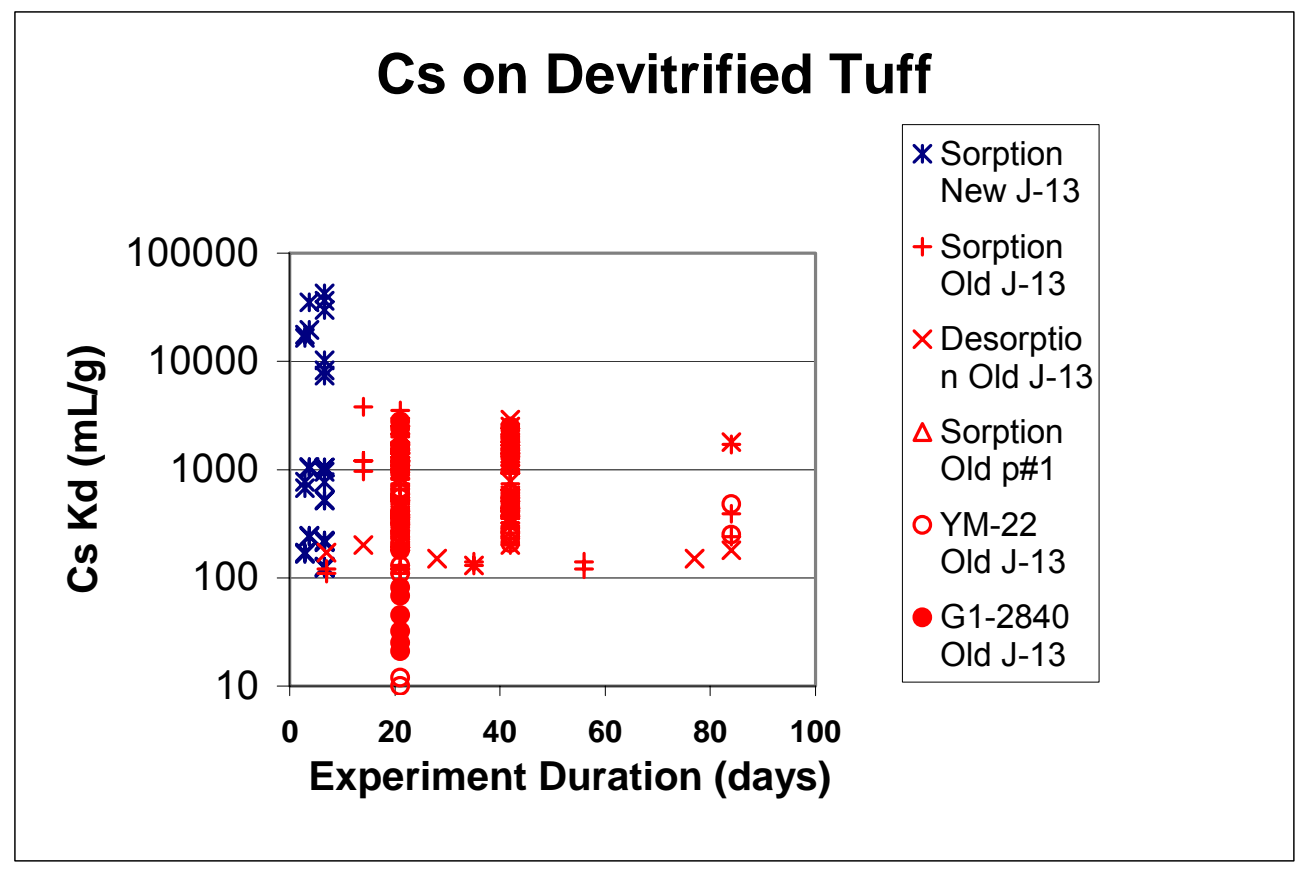

Source DTNs: LA0305AM831341.001 [DIRS 163789]; LA0407AM831341.002 [DIRS 170621].

Figure A-10. Cesium Sorption Coefficients on Devitrified Tuff Versus Experiment Duration for Sorption (Forward) and Desorption (Backward) Experiments

The cesium sorption-coefficient probability distribution derived for devitrified tuff in the SZ is a truncated normal distribution with a range of 100 to $1,000 \mathrm{~mL} / \mathrm{g}$, mean value of $728 \mathrm{~mL} / \mathrm{g}$, and a standard deviation of $464 \mathrm{~mL} / \mathrm{g}$. The low end of the chosen range was selected based on the minimum value observed in experiments with solution concentrations less than $10^{-7} \mathrm{M} / \mathrm{L}$. The upper end of the distribution was chosen as a minimum upper limit given the potential impacts of low cesium solution concentrations and high ion-exchange capacities.

\section{A7.2.2 Zeolitic Tuff}

Experimentally derived sorption coefficients for cesium on zeolitic tuff are plotted against the calculated final cesium concentrations of the experiments in Figure A-11. The data points are separated into groups on the basis of when the experiments were carried out (pre-1990 = "old" and post-1990 = "new"), water type, and on whether the sorption coefficient was determined from a sorption or a desorption experiment. There are also data for a sorption isotherm on sample YM-38.

The range of measured cesium sorption coefficients for zeolitic tuffs is 3,500 to 72,000 mL/g. A sorption isotherm was obtained for sample YM-38 in J-13 water. As shown in Figure A-12, the isotherm is nearly linear. Sorption coefficients obtained in experiments with Borehole p\#1 water fall at the lower end of the range of values obtained for experiments with $\mathrm{J}-13$ water (Figure A 1-1). Thus, there is some impact of variations in water chemistry on cesium sorption coefficients on zeolitic tuff, although this impact is minor. 


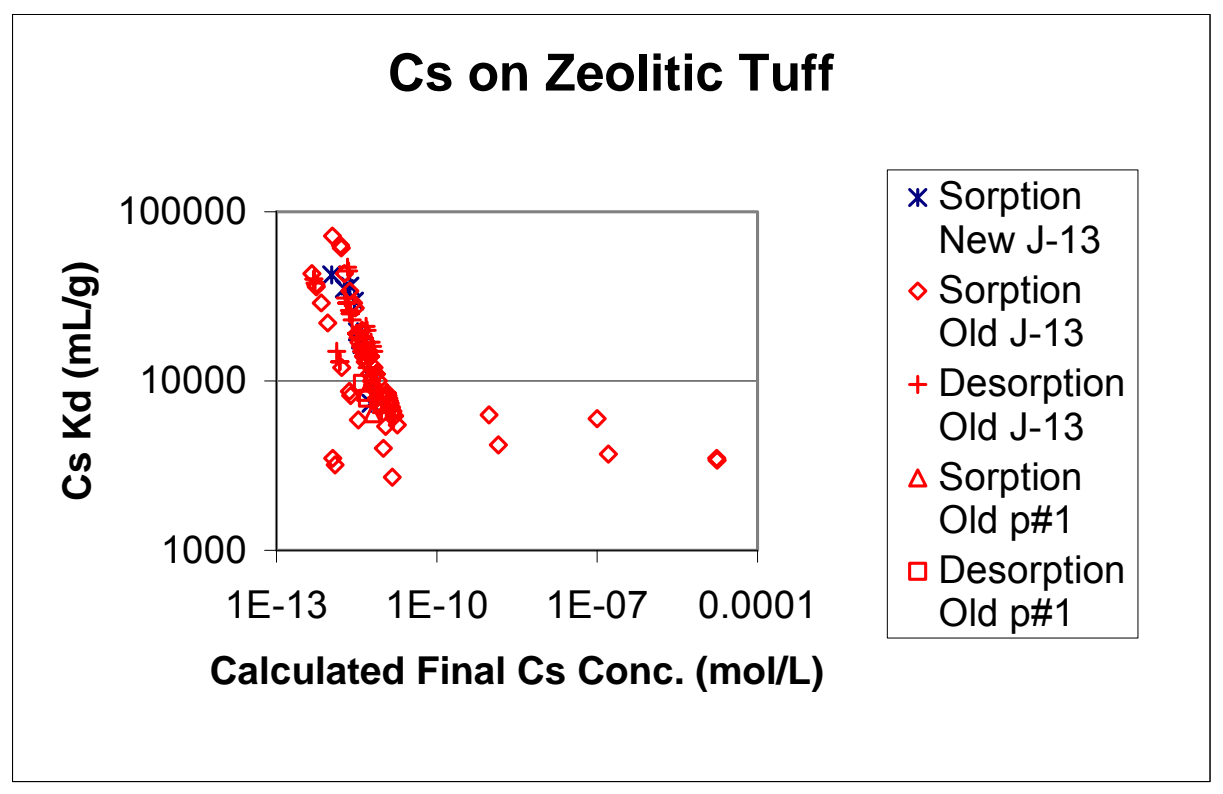

Source DTNs: LA0305AM831341.001 [DIRS 163789]; LA0407AM831341.002 [DIRS 170621].

Figure A-11. Cesium Sorption Coefficients on Zeolitic Tuff Versus Calculated Final Cesium Concentration in Solution

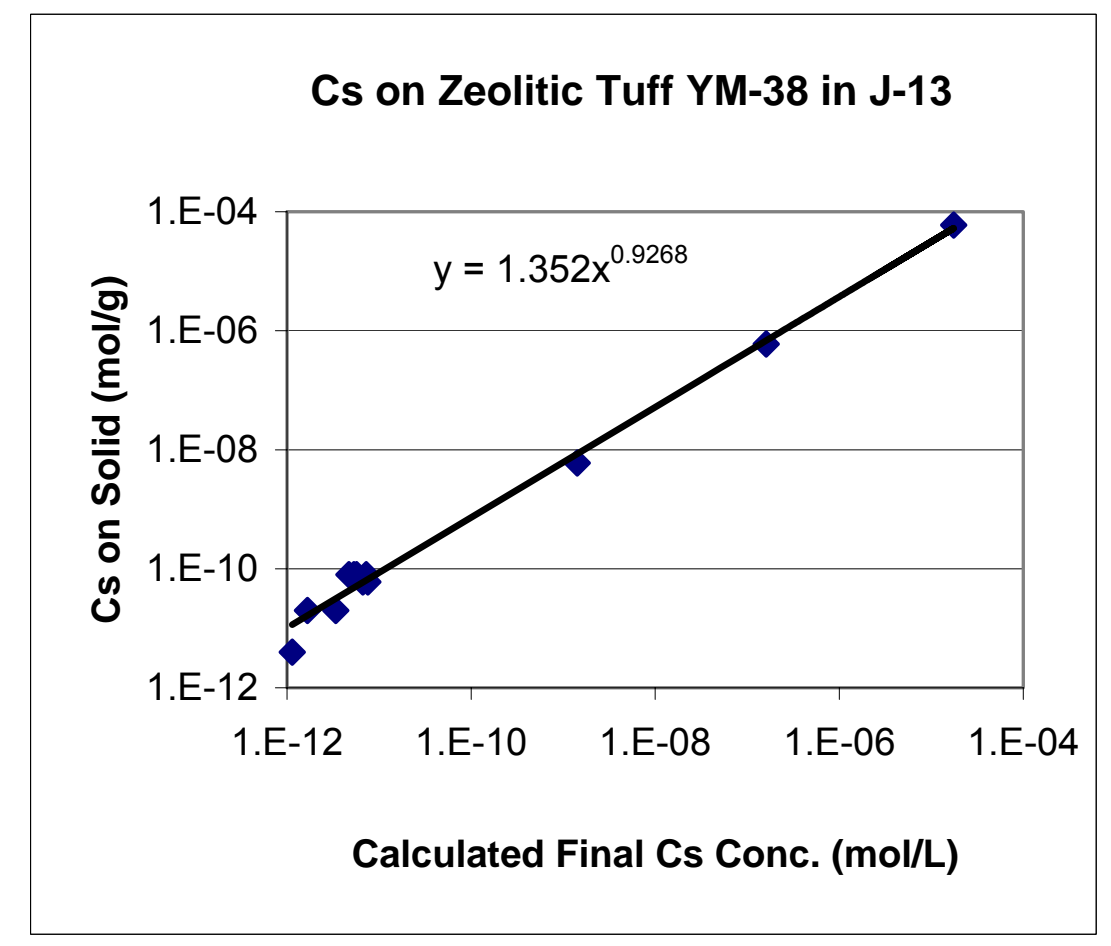

Source DTN: LA0305AM831341.001 [DIRS 163789].

Figure A-12. Freundlich Isotherm Fit to Sorption-Coefficient Data for Sample YM-38 in J-13 Water 
The effects of experiment duration on the value of $K_{d}$ for cesium on zeolitic tuff are shown in Figure A-13. The large range in sorption coefficients obtained at a given duration mainly reflects variations in cesium solution concentrations, although there must also be some contribution from variations in ion-exchange capacities of the zeolitic tuff samples used in the experiments. The range of sorption-coefficient values is fairly consistent with duration. For example, the range of sorption-coefficient values for the 3.6-day experiments is similar to the range for the 42-day experiments. This result indicates the sorption reaction kinetics are fast.

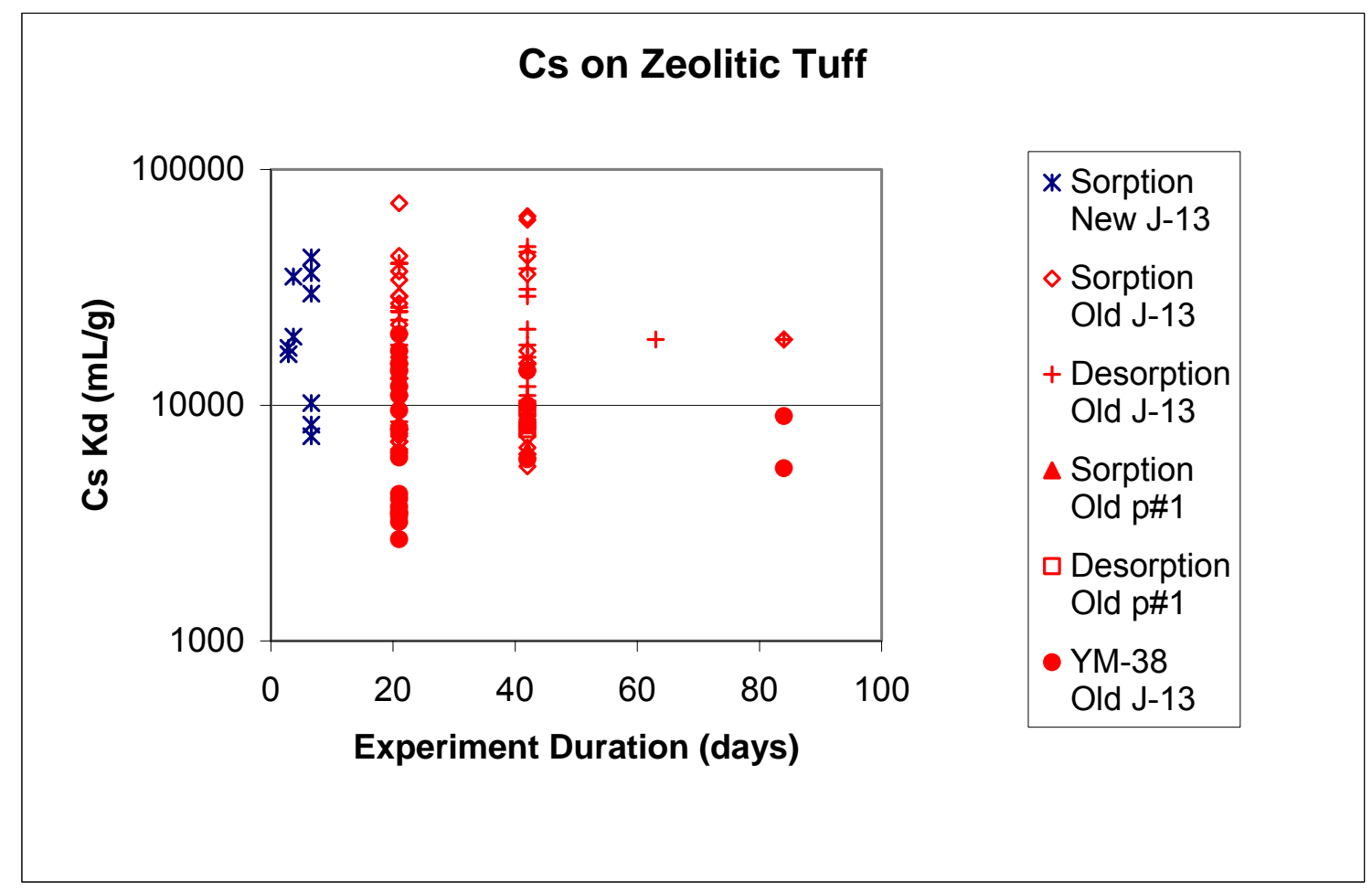

Source DTNs: LA0305AM831341.001 [DIRS 163789]; LA0407AM831341.002 [DIRS 170621].

Figure A-13. Cesium Sorption Coefficients on Zeolitic Tuff Versus Experiment Duration for Sorption (Forward) and Desorption (Backward) Experiments

The cesium sorption-coefficient probability distribution selected for zeolitic tuff in the SZ is an exponential distribution with a range of 4,000 to $42,000 \mathrm{~mL} / \mathrm{g}$, a mean of $16,942 \mathrm{~mL} / \mathrm{g}$, and a standard deviation of $14,930 \mathrm{~mL} / \mathrm{g}$. The low end of the chosen range was selected based on the minimum value observed in the available experiments, including those with p\#1 water. The upper end of the distribution was chosen as a minimum upper limit given the potential impacts of lower cesium solution concentrations and higher ion-exchange capacities than those encountered in the experimental program. 


\section{A7.2.3 Alluvium}

The probability distribution for devitrified tuff will be used as a default for the cesium sorption-coefficient probability distribution in alluvium. Alluvium along the flow path is composed largely of disaggregated tuffaceous materials. Because devitrified tuff makes up a major portion of the volcanic units exposed at the surface, it should be a major component in alluvium. In addition, clays and other secondary minerals are enriched in alluvial materials. These characteristics should result in higher sorption coefficients for alluvial materials compared to intact devitrified tuff.

\section{A7.3 NEPTUNIUM}

According to Nitsche et al. (1993 [DIRS 155218] Table VI), the solubility of neptunium in $\mathrm{J}-13$ water at $25^{\circ} \mathrm{C}$ and a $\mathrm{pH}$ of 8.5 is $4.4 \times 10^{-5} \mathrm{M} / \mathrm{L}$, and the solubility-controlling solid is $\mathrm{Np}_{2} \mathrm{O}_{5}$ under oxidizing conditions (Eh $>180 \mathrm{mV}$ ) (Eh is the oxidation/reduction potential normalized to the standard hydrogen electrode). At a $\mathrm{pH}$ of 6.9 , the solubility is higher at $1.3 \times 10^{-4} \mathrm{M} / \mathrm{L}$ under oxidizing conditions (Eh $>250 \mathrm{mV}$ ). The solubility of neptunium in Borehole p\#1 water at $25^{\circ} \mathrm{C}$ and a $\mathrm{pH}$ of 8.5 is 7.0$) \times 10^{-6} \mathrm{M} / \mathrm{L}$ and $4.7( \pm 0.4) \times 10^{-5} \mathrm{M} / \mathrm{L}$ at $25^{\circ} \mathrm{C}$ and a pH of 7.0, according to Nitsche et al. (1995 [DIRS 100163], Table A).

\section{A7.3.1 Devitrified Tuff}

The results of sorption experiments with devitrified tuff are shown in Figure A-14. Some of the experiments with $\mathrm{J}-13$ water had final neptunium concentrations above $7.0 \times 10^{-6} \mathrm{M} / \mathrm{L}$. Thus, the results for these experiments should be discounted because the experiments could have been oversaturated with $\mathrm{Np}_{2} \mathrm{O}_{5}$. All but four of the experiments with synthetic p\#1 water had final neptunium solution concentrations less than $2.5 \times 10^{-5} \mathrm{M} / \mathrm{L}$. The results for the four experiments with oversaturated final solutions will be discounted. The remaining data points, plotted in Figure A-15, are suggestive of a dependence of the sorption coefficient on the final neptunium solution concentration.

Neptunium sorption experiments carried out as a function of experiment duration are shown in Figure A-16. There is a significant difference between the results for "old" and "new" experiments with the "old" results generally having higher values than the "new" results. The most straightforward explanation is that the difference is due to the fact that the "old" results represent experiments with longer durations than the "new" results. Within the "old" data points, the sorption-coefficient values appear to reach a steady-state level after 42 days. 


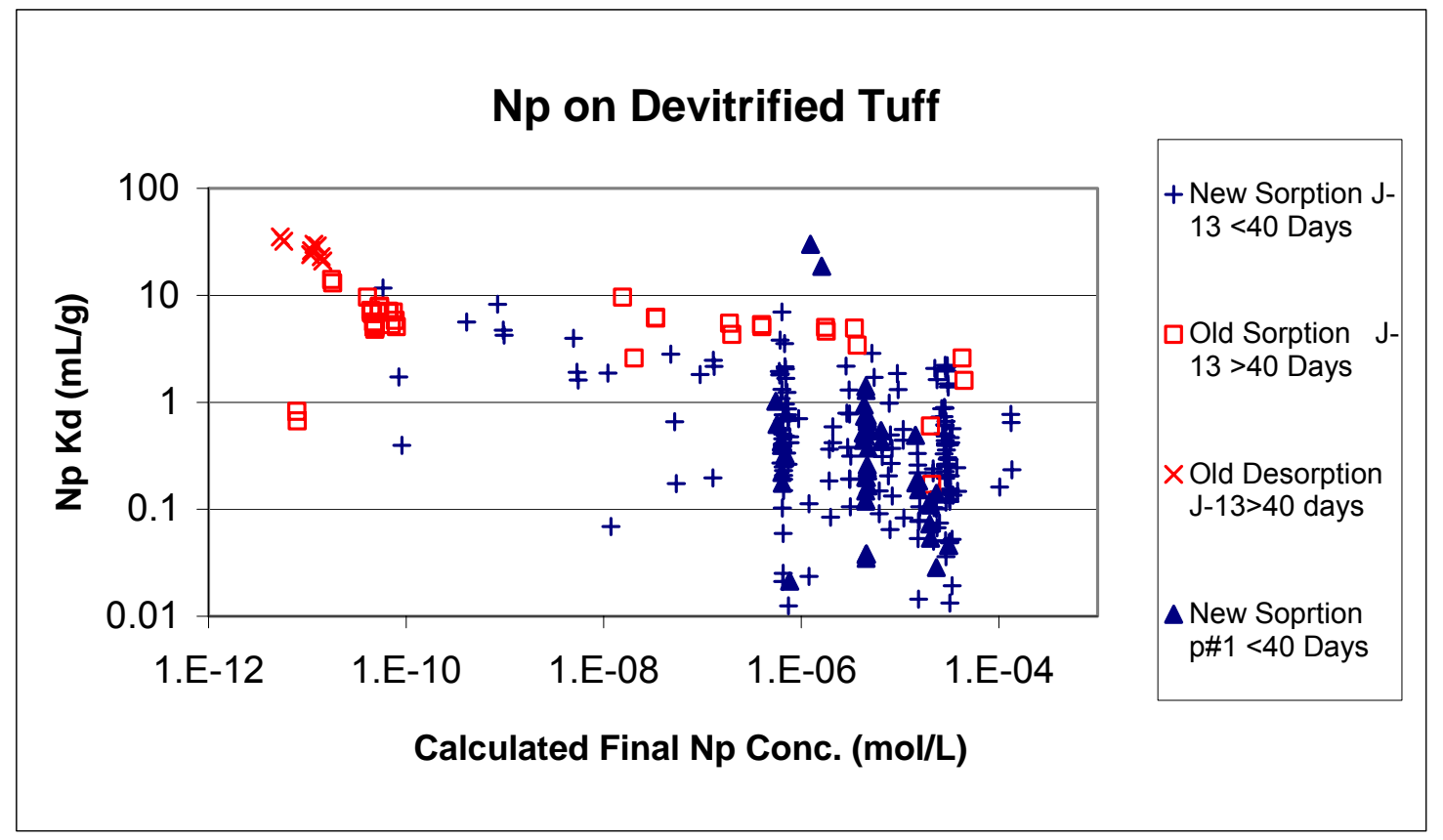

Source DTNs: LA0305AM831341.001 [DIRS 163789]; LA0407AM831341.004 [DIRS 170622].

Figure A-14. Neptunium Sorption Coefficients on Devitrified Tuff Versus Calculated Final Neptunium Concentration in Solution

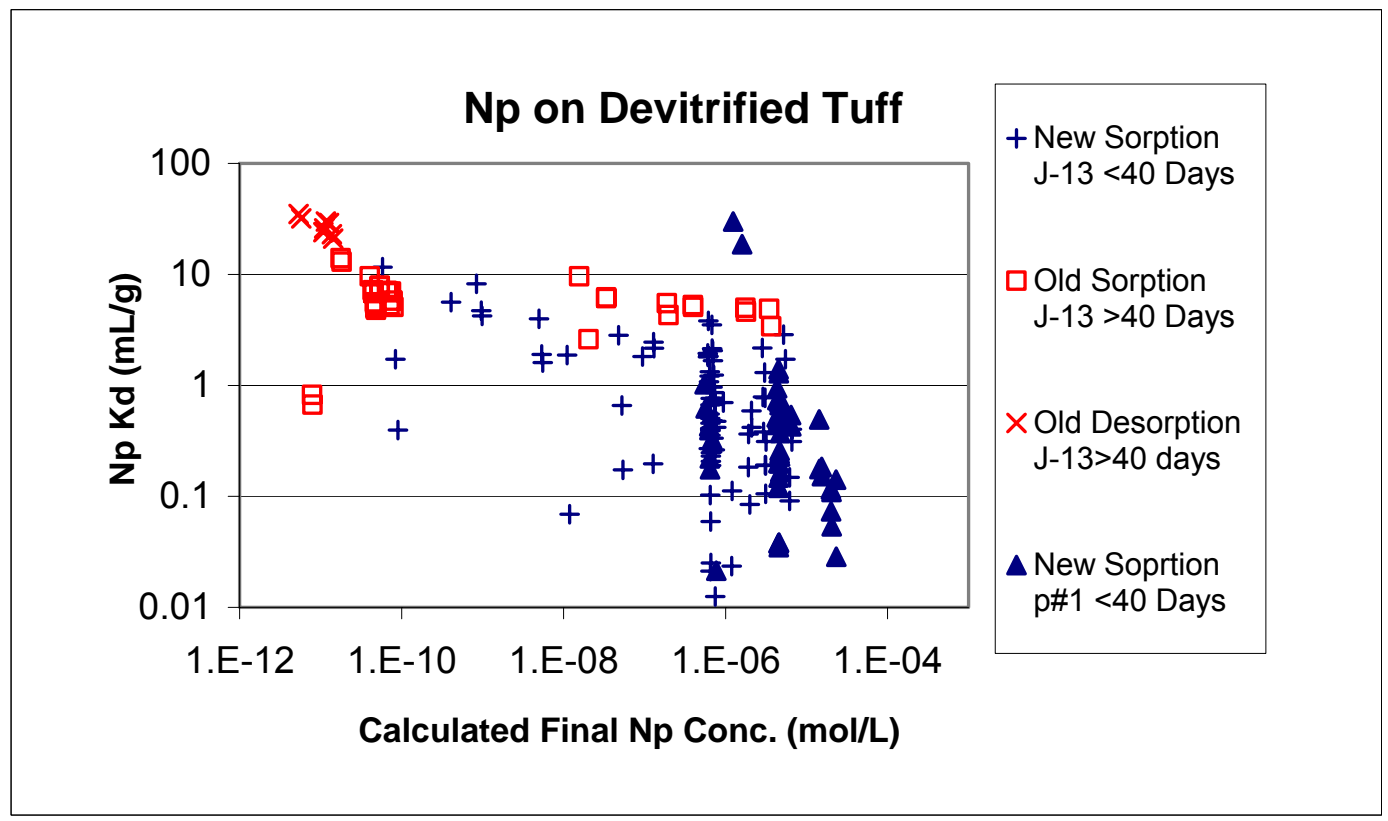

Source DTNs: LA0305AM831341.001 [DIRS 163789]; LA0407AM831341.004 [DIRS 170622].

NOTE: Experiments oversaturated with $\mathrm{Np}_{2} \mathrm{O}_{5}$ have been omitted.

Figure A-15. Neptunium Sorption Coefficients on Devitrified Tuff Versus Calculated Final Neptunium Concentration in Solution 


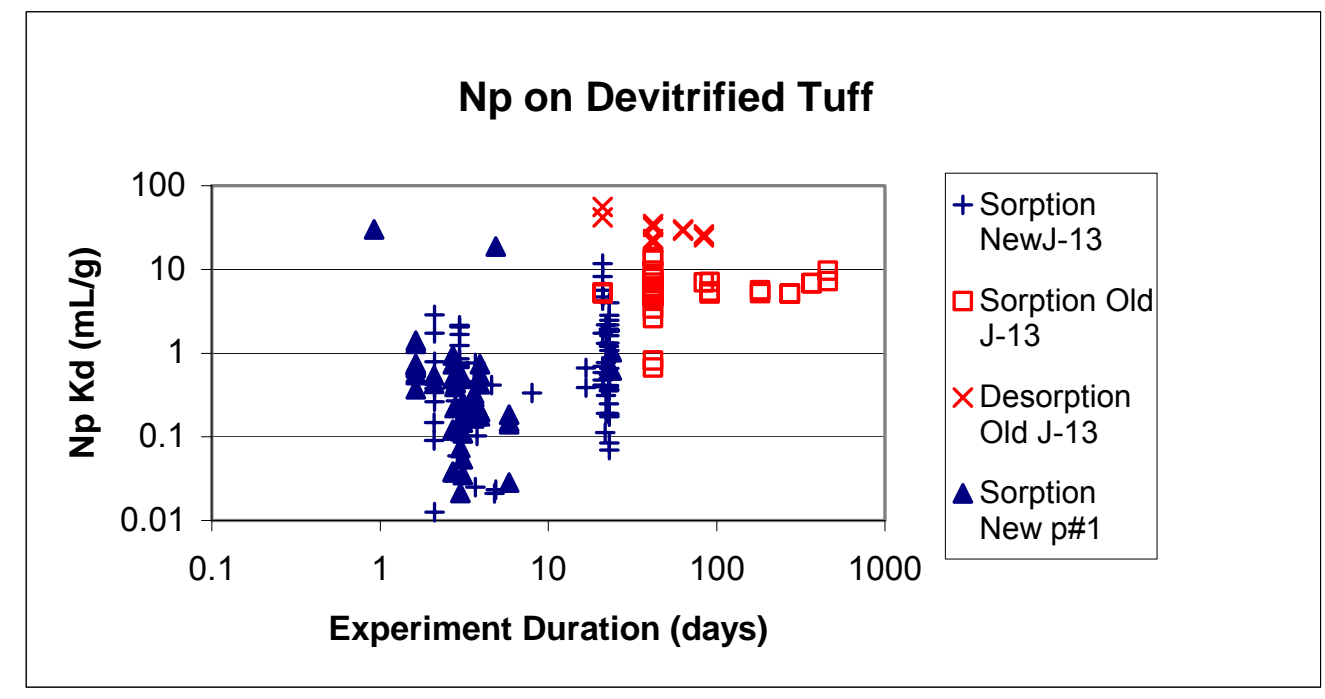

Source DTNs: LA0305AM831341.001 [DIRS 163789]; LA0407AM831341.004 [DIRS 170622].

NOTE: Experiments oversaturated with $\mathrm{Np}_{2} \mathrm{O}_{5}$ have been omitted.

Figure A-16. Neptunium Sorption Coefficients on Devitrified Tuff Versus Experiment Duration for Sorption (Forward) and Desorption (Backward) Experiments

The impact of variations in $\mathrm{pH}$ on neptunium coefficients on devitrified tuffs is shown in Figure A-17. There is a lot of scatter in the "new" data, and there do not appear to be clear positive or negative trends among these data points. Nor does there appear to be much difference between the results for J-13 water and synthetic p\#1 water. The "old" data points are more consistent and show very little dependence of the neptunium sorption coefficient on $\mathrm{pH}$ except at $\mathrm{pH}$ values less than 7.0. Curves calculated from the PHREEQC surface complexation model with the thermodynamic input data file YMP_R3.DAT (DTN: MO0403SPAYMPR3.000 [DIRS 170937]) for “devitrified tuff” in J-13 and p\#1 waters are also plotted. The neptunium binding constants on silica were obtained from Turner et al. (1998 [DIRS 162989], p. 264). The J-13 curve lies between the "old” sorption and desorption points. This result suggests the curve may reflect the equilibrium values of neptunium sorption coefficients on devitrified tuff better than the experimental data. In this interpretation, the sorption data points reflect experiments that have not reached an equilibrium state. 


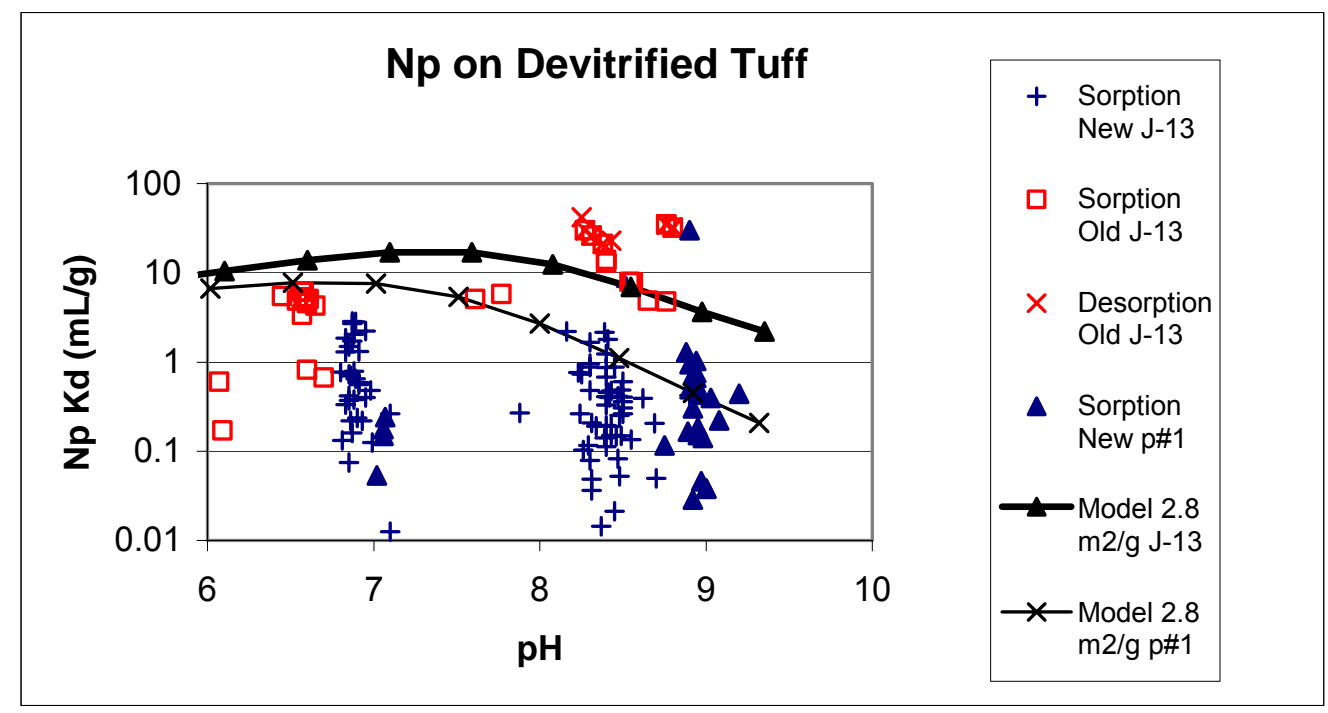

Source DTNs: LA0305AM831341.001 [DIRS 163789]; LA0407AM831341.004 [DIRS 170622].

NOTE: PHREEQC model results for J-13 and p\#1 waters are also plotted (DTN: LA0306AM831343.001. Data from file output/Npsij13.pun).

Figure A-17. Neptunium Sorption Coefficients on Devitrified Tuff in J-13 and Synthetic p\#1 Waters Versus Solution pH in Sorption (Forward) and Desorption (Backward) Experiments

The neptunium sorption-coefficient probability distribution selected for devitrified tuff in the SZ is an exponential distribution with a range of 0 to $2 \mathrm{~mL} / \mathrm{g}$ and a mean of $0.69 \mathrm{~mL} / \mathrm{g}$, and a standard deviation of $0.707 \mathrm{~mL} / \mathrm{g}$. The low end of the chosen range is selected based on the minimum value obtained in short-term experiments (up to 21 days). The experiments on which this value is based do not appear in the graphs because the graphs use logarithmic coordinates. The upper end of the distribution was chosen as a minimum upper limit with a neptunium concentration near the solubility limit (Figure A-15) with emphasis on results from experiments with $\mathrm{pH}$ near 7.0. Experiments with $\mathrm{pH}$ near 7.0 were emphasized because the $\mathrm{pH}$ values of SZ waters in volcanic units downgradient from the repository cluster near 7.0 as shown in Saturated Zone Site-Scale Flow Model (BSC 2004 [DIRS 170037] Table A6-3). It is acknowledged that a higher limit could be selected for the upper end of the distribution based on the available data.

\section{A7.3.2 Zeolitic Tuff}

Sorption coefficients on zeolitic tuff are shown as a function of calculated final solution concentration in Figure A-18. Many of the experiments with J-13 water had final neptunium concentrations above $7.0 \times 10^{-6} \mathrm{M} / \mathrm{L}$. Thus, the results for these experiments should be discounted because the experiments could have been oversaturated with $\mathrm{Np}_{2} \mathrm{O}_{5}$. Many of the experiments with synthetic p\#1 water had final neptunium solution concentrations close to or greater than $2.5 \times 10^{-5} \mathrm{M} / \mathrm{L}$. The results for these experiments must also be discounted. 


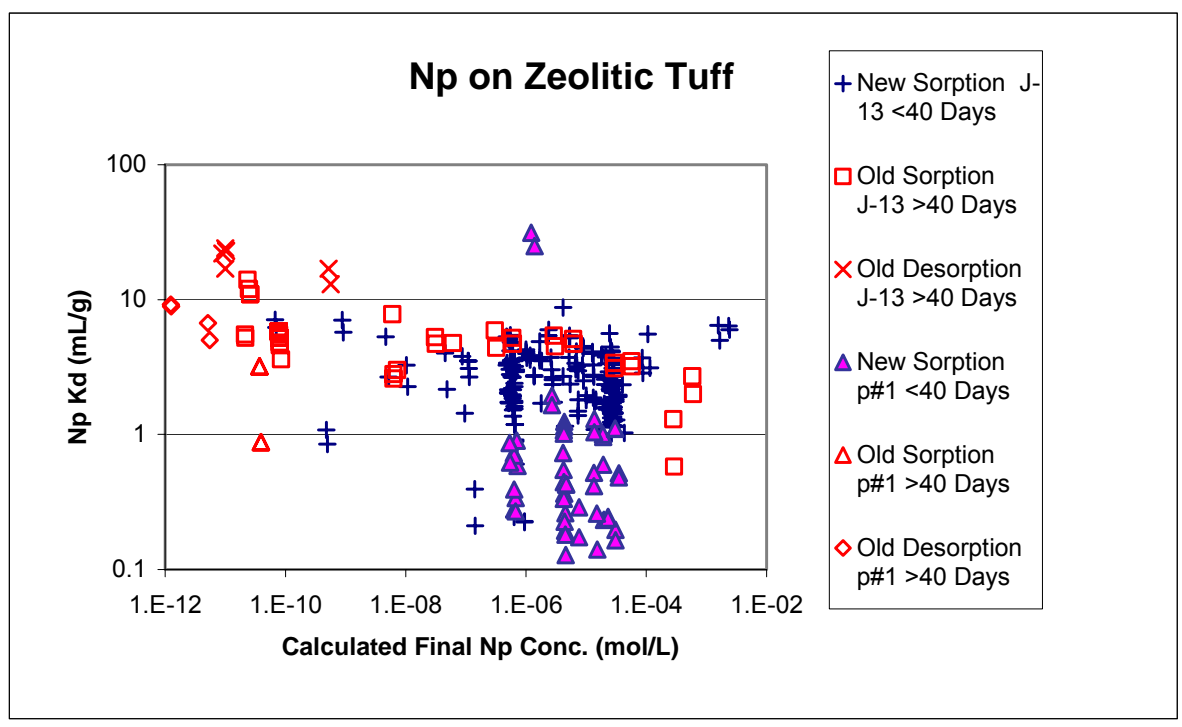

Source DTNs: $\quad$ LA0305AM831341.001 [DIRS 163789]); LA0407AM831341.004 [DIRS 170622].

Figure A-18. Neptunium Sorption Coefficients on Zeolitic Tuff Versus Calculated Final Neptunium Concentration in Solution

Removal of the data from experiments that involved oversaturation leads to the results shown in Figure A-19. With these points removed, there is essentially no dependence of sorption coefficient on calculated final solution concentration for J-13 experiments. However, it is clear that there is a dependence of sorption coefficient on water chemistry in the short-term experiments. If the solubility of neptunium in synthetic p\#1 water is less than the solubility in actual p\#1 water, this dependence on water chemistry may not be real.

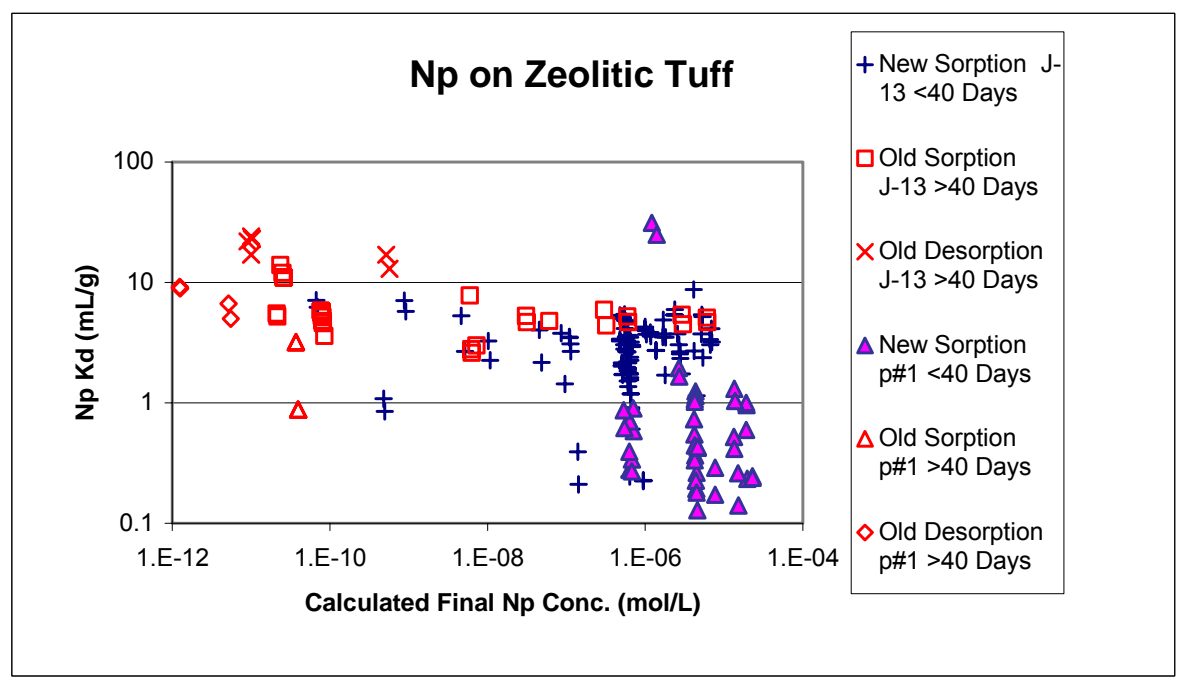

Source DTNs: LA0305AM831341.001 [DIRS 163789]; LA0407AM831341.004 [DIRS 170622].

Figure A-19. Neptunium Sorption Coefficients on Zeolitic Tuff Versus Calculated Final Neptunium Concentration in Solution with Data from Oversaturated Experiments Removed 
Neptunium sorption experiments carried out as a function of experiment duration are shown in Figure A-20. There is a significant difference between the results for "old" and "new" experiments with the "old" results generally having higher values than the "new" results. The most straightforward explanation is that the difference is because the "old" results represent experiments with longer durations than the "new" results. Within the "old" data points, the sorption-coefficient values appear to reach a steady-state level after 42 days.

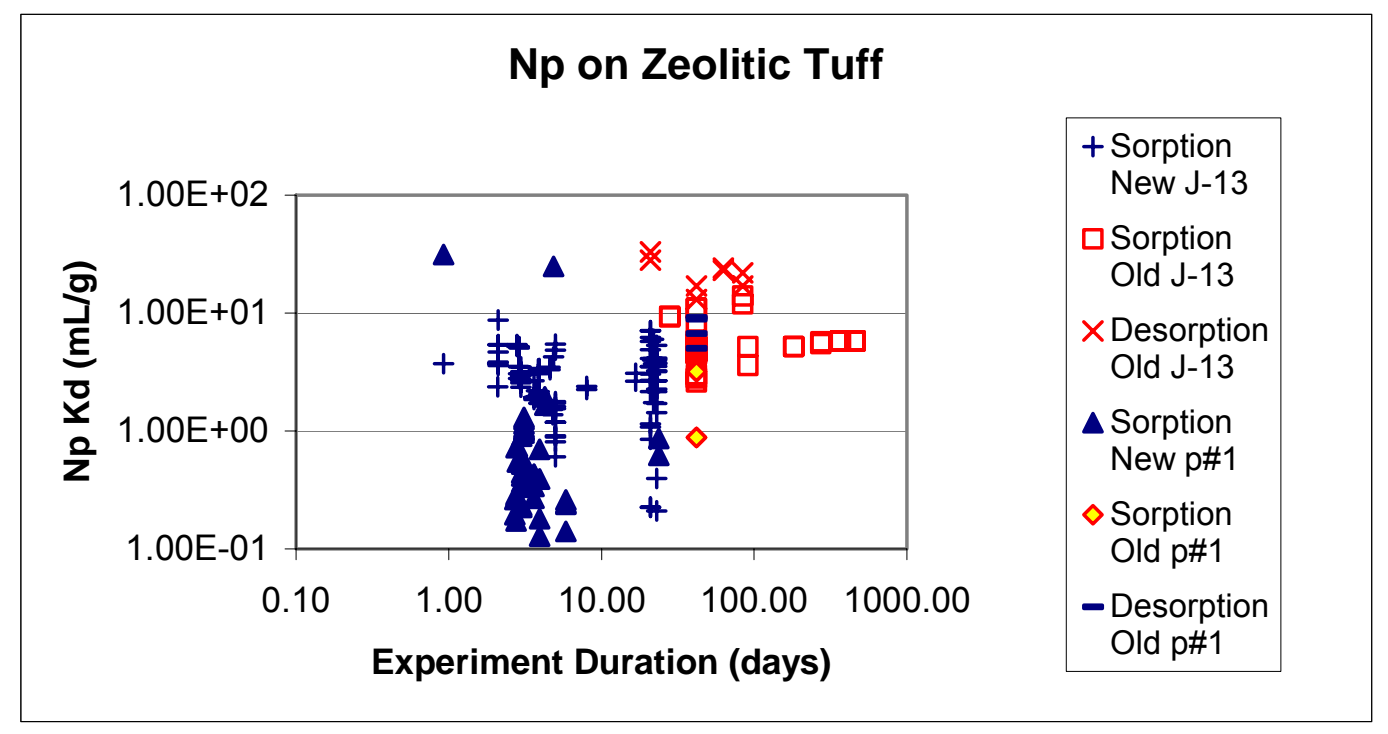

Source DTNs: LA0305AM831341.001 [DIRS 163789]; LA0407AM831341.004 [DIRS 170622].

NOTE: Oversaturated experiments have been omitted.

Figure A-20. Neptunium Sorption Coefficients on Zeolitic Tuff Versus Experiment Duration for Sorption (Forward) and Desorption (Backward) Experiments

The impact of variations in $\mathrm{pH}$ on neptunium coefficients for sorption on devitrified tuffs is shown in Figure A-21. There is a lot of scatter in the "new" data, and there do not appear to be clear positive or negative trends among these data points. Nor does there appear to be much difference between the results for J-13 water and synthetic p\#1 water. The "old" data points are more consistent and show very little dependence of the neptunium sorption coefficient on $\mathrm{pH}$ except at $\mathrm{pH}$ values less than 7.0.

The neptunium sorption-coefficient probability distribution selected for zeolitic tuff in the SZ is a truncated normal distribution with a range of 0 to $6 \mathrm{~mL} / \mathrm{g}$, a mean of $2.88 \mathrm{~mL} / \mathrm{g}$, and a standard deviation of $1.47 \mathrm{~mL} / \mathrm{g}$. The low end of the chosen range is selected based on the minimum value obtained in short-term experiments (up to 21 days). The upper end of the distribution was chosen as a minimum upper limit with a neptunium concentration near the solubility limit (Figure A-19) with emphasis on results from experiments at $\mathrm{pH}$ values near 7.0. It is acknowledged that a higher limit could be selected for the upper end of the distribution based on the available data. 


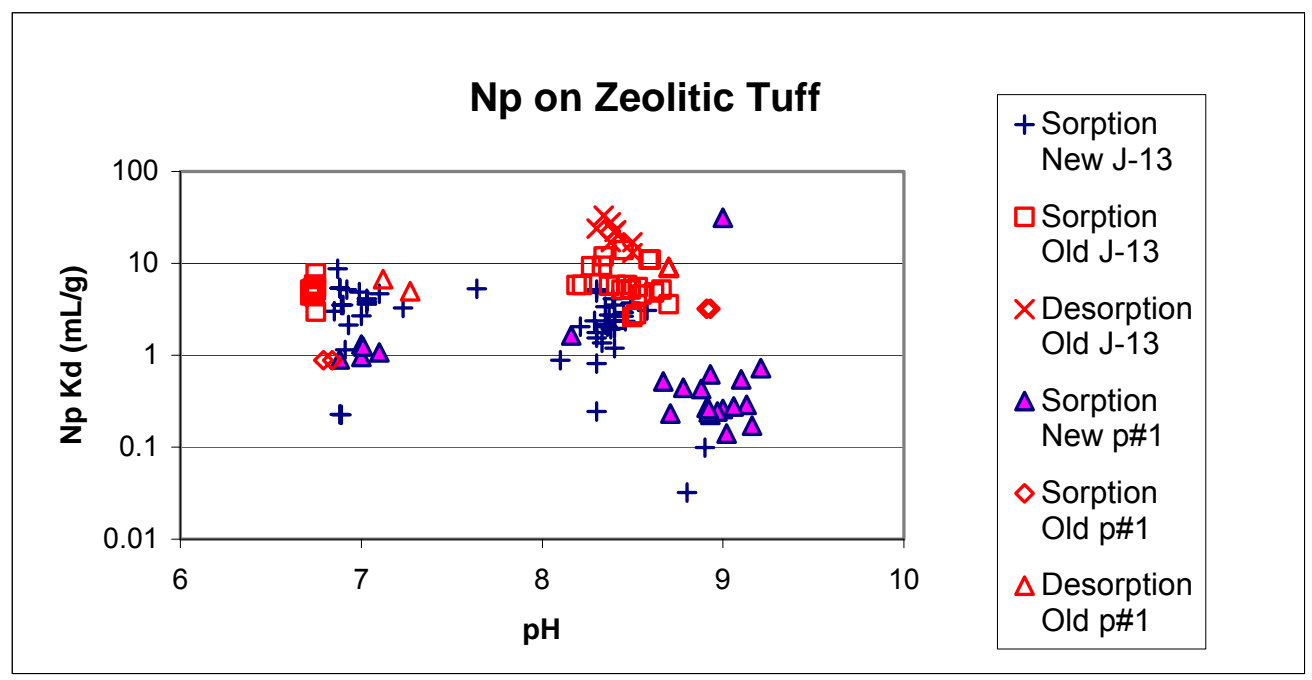

Source DTNs: LA0305AM831341.001 [DIRS 163789]; LA0407AM831341.004 [DIRS 170622].

NOTE: Oversaturated experiments have been omitted.

Figure A-21. Neptunium Sorption Coefficients on Devitrified Tuff in J-13 Water and Synthetic p\#1 Water Versus Solution pH in Sorption (Forward) and Desorption (Backward) Experiments

\section{A7.3.3 Alluvium}

Sorption coefficients have been measured in batch experiments from core obtained from three wells drilled into the alluvium (DTN: LA0302MD831341.003 [DIRS 163784]): Borehole NC-EWDP-10SA to the north, NC-EWDP-22SA in the middle, and NC-EWDP-19IM1A to the south, spaced between 4 to $5 \mathrm{~km}$ apart. Samples were taken below the water table from a $5-\mathrm{ft}$ interval at two depths in wells NC-EWDP-19IM1A and NC-EWDP-10SA and at six depths in well NC-EWDP-22SA. For each core sample, batch experiments were run on three particle sizes: 500 to 2,000 $\mu \mathrm{m}, 75$ to $500 \mu \mathrm{m}$, and $<75 \mu \mathrm{m}$. The $K_{d}$ values observed on the third particle size fraction were noticeably larger than the other two size fractions, probably because of mineral sorting effects. The $K_{d}$ values for this size fraction were not considered further. Several replicates of each batch experiment were run so that a sample standard deviation could be obtained for each size fraction. Twelve of the 20 samples had a sample coefficient of variation $<0.1$; six had a coefficient of variation $<0.22$; and two had a coefficient of variation $<0.7$.

The cell size in the three-dimensional FEHM (FEHM V2.20, STN: 10086-2.20-00) (LANL 2003 [DIRS 161725]) model is $500 \mathrm{~m} \times 500 \mathrm{~m} \times \sim 10 \mathrm{~m}$ near the water table in the alluvium. Hence, the $K_{d}$ parameter in the model represents a mean value, and the uncertainty distribution needs to represent the uncertainty about the mean on this large scale, not the local variance of the smallscale experiments. To avoid overly weighting the 12 samples from well 22SA, the mean $K_{d}$ value was evaluated for each well (i.e., the $K_{d}$ values obtained from any one well were similar and, thus, suggested some correlation). The coefficients of variation for these means were of the same order of magnitude as those for the samples (i.e., $<0.16$ ), and so the small errors in any one experiment could be neglected. The three mean $K_{d}$ values were taken to represent the best estimate of the $K_{d}$ for any one cell in the FEHM model. According to the Law of Large Numbers, the means should be normally distributed; however, since the number of samples is 
small (i.e., 3), the Student-t distribution was used to correctly account for the error in estimating the standard deviation. The $K_{d}$ value for $\mathrm{Np}$ were taken to be described by a t distribution with the following statistics: $\mu \mathrm{Np}=6.3 \mathrm{~mL} / \mathrm{g}, \sigma \mathrm{Np}=0.81 \mathrm{~mL} / \mathrm{g}$.

Although the t distribution was thought to correctly capture the spatial uncertainty, the question about the representativeness of the experiment does introduce a small amount of additional uncertainty. For example, other sources of uncertainty that may not have been completely represented in the experiments included potential competitive effects among radionuclides, different adsorption behavior between different oxidation states of the radionuclides, impact of climatic change, variability in water composition and rock types, and complexation by organic ligands. The probability that these uncertainties were not properly represented by the experiment was thought to be less than 10 percent. Nonetheless, to accommodate for this possibility, the tails of the distribution were extended from the approximate bounds of the $t$ distribution $( \pm 3 \sigma)$ to the maximum and minimum experimental values observed. A 10 percent probability was assigned to these tails. This adjustment was implemented by using a piecewise-uniform cumulative distribution. For neptunium, the minimum experimentally observed value was $1.8 \mathrm{~mL} / \mathrm{g}$. Five percent probability was uniformly distributed between $1.8 \mathrm{~mL} / \mathrm{g}$ and the lower bound of the $\mathrm{t}$ distribution at $4.0 \mathrm{~mL} / \mathrm{g}$. Since the $\mathrm{t}$ distribution had such little variation, it was reasonably represented by a uniform distribution; hence, 90 percent probability was uniformly distributed between $4.0 \mathrm{~mL} / \mathrm{g}$ and the upper bound of the $\mathrm{t}$ distribution at $8.7 \mathrm{~mL} / \mathrm{g}$. The remaining 5 percent probability was uniformly distributed between $8.7 \mathrm{~mL} / \mathrm{g}$ and the maximum experimentally observed value of $13 \mathrm{~mL} / \mathrm{g}$.

\section{A7.4 PLUTONIUM}

According to Nitsche et al. (1993 [DIRS 155218], p. 54), the solubility of plutonium in J-13 water at $25^{\circ} \mathrm{C}$ and a pH of 8.4 is $2.9( \pm 0.8) \times 10^{-7} \mathrm{M} / \mathrm{L}$ and is not very sensitive to $\mathrm{pH}$ over the range from 7.0 to 8.4. The solubility of plutonium in $\mathrm{p \# 1}$ water at $25^{\circ} \mathrm{C}$ and a $\mathrm{pH}$ of 8.5 is 1.0 $( \pm 0.1) \times 10^{-6} \mathrm{M} / \mathrm{L}$ and $4.5( \pm 0.4) \times 10^{-7} \mathrm{M} / \mathrm{L}$ at $25^{\circ} \mathrm{C}$ and a $\mathrm{pH}$ of 7.0 according to Nitsche et al. (1995 [DIRS 100163], p. 39). Thus, the solubility of plutonium in J-13 is somewhat lower than it is in $\mathrm{p} \# 1$ water.

\section{A7.4.1 Devitrified Tuff}

The experimentally derived sorption coefficients for plutonium on devitrified tuff are plotted against the calculated final plutonium concentrations of the experiments in Figure A-22. The data points are separated into groups on the basis of when the experiments were carried out (pre-1990 = "old" and post-1990 = "new"), water chemistry, experiment duration, and on whether the sorption coefficient was determined from a sorption or a desorption experiment. The plotted data indicate that the "new" and "old" data show similar ranges of $K_{d}$ values, that the longer-term experiments generally yield higher sorption-coefficient values than the shorter-term experiments, and that desorption experiments yield higher sorption-coefficient values than sorption experiments. These points are discussed in greater detail in the following paragraphs. 
The maximum calculated final plutonium concentration plotted in Figure A-22 is slightly less than the solubility determined by Nitsche et al. (1993 [DIRS 155218], p. 54) for plutonium in J-13 water. Thus, the plutonium sorption coefficients plotted in Figure A-22 reflect solutions that were undersaturated with the solid plutonium phase precipitated in the experiments reported by Nitsche et al. (1993 [DIRS 155218], p. 54).

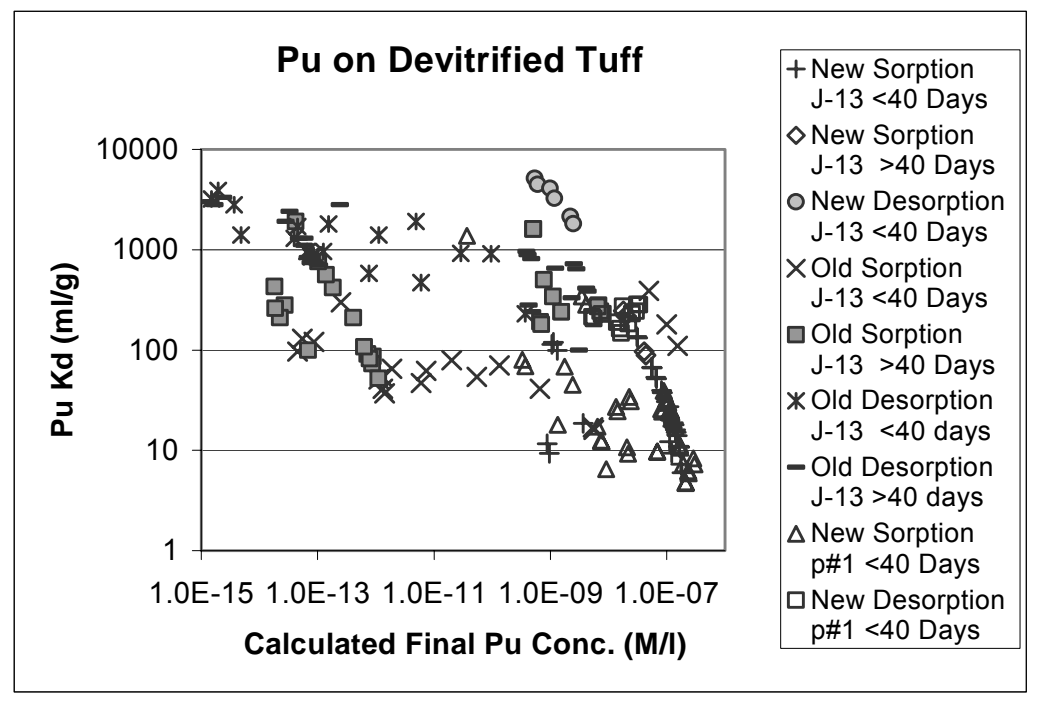

Source DTNs: LA0305AM831341.001 [DIRS 163789]; LA0407AM831341.005 [DIRS 170625].

Figure A-22. Plutonium Sorption Coefficients on Devitrified Tuff Versus Calculated Final Plutonium Concentration in Solution

The effect of the solution plutonium concentration on the sorption-coefficient value obtained is shown more clearly in Figure A-23. Data are plotted for two devitrified tuff samples that contain only trace amounts of secondary phases (e.g., clays, zeolites). In addition, plotted data are restricted to 21-day experiment durations and a $\mathrm{pH}$ value near 8.4. As is evident in the figure, within the errors of the analyses, the measured plutonium sorption coefficients are nearly independent of the final plutonium solution concentration.

The effect of experiment duration on the plutonium $K_{\mathrm{d}}$ value is shown in Figure A-24. As expected, the $K_{\mathrm{d}}$ values for sorption experiments increase with increasing duration, and the $K_{\mathrm{d}}$ values for desorption experiments decrease with increasing duration. However, the increase in the sorption values is much greater than the decrease in desorption values over the time frame of the experiments. This result may reflect the reduction of plutonium in the +5 and/or +6 oxidation states to plutonium in the +4 oxidation state on the mineral surfaces present in devitrified tuff, as discussed in greater detail below. The trends in the sorption and desorption data points suggest they would converge to values somewhere between 100 and 1,000 mL/g. Based on the data plotted, such convergence would require more than 100 days. Note that the "old" sorption data points exceeding $1,000 \mathrm{~mL} / \mathrm{g}$ are for samples that contain significant amounts of clay or zeolite. For this reason, these data are discounted in the derivation of the sorption-coefficient probability distribution. 


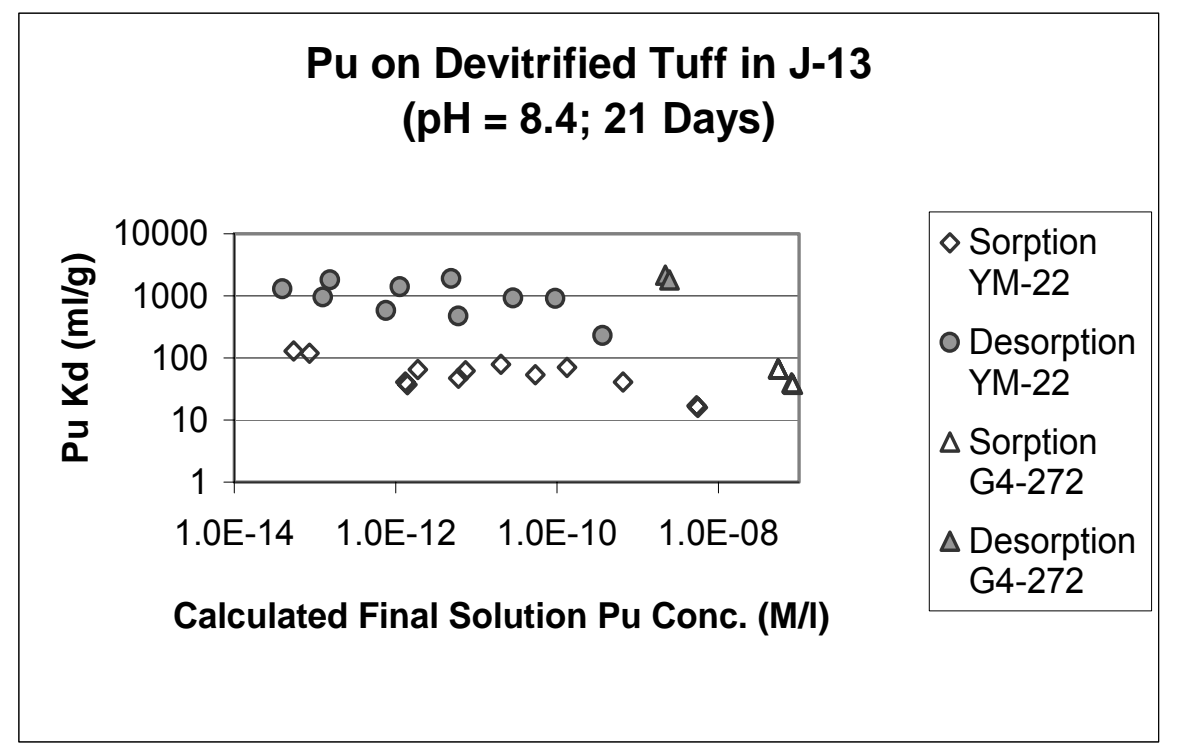

Source DTNs: $\quad$ LA0407AM831341.005 [DIRS 170625]; LA0305AM831341.001 [DIRS 163789].

Figure A-23. Plutonium Sorption Coefficients Versus Calculated Final Plutonium Solution Concentration (M/L) for Experiments with Samples YM-22 and G4-272

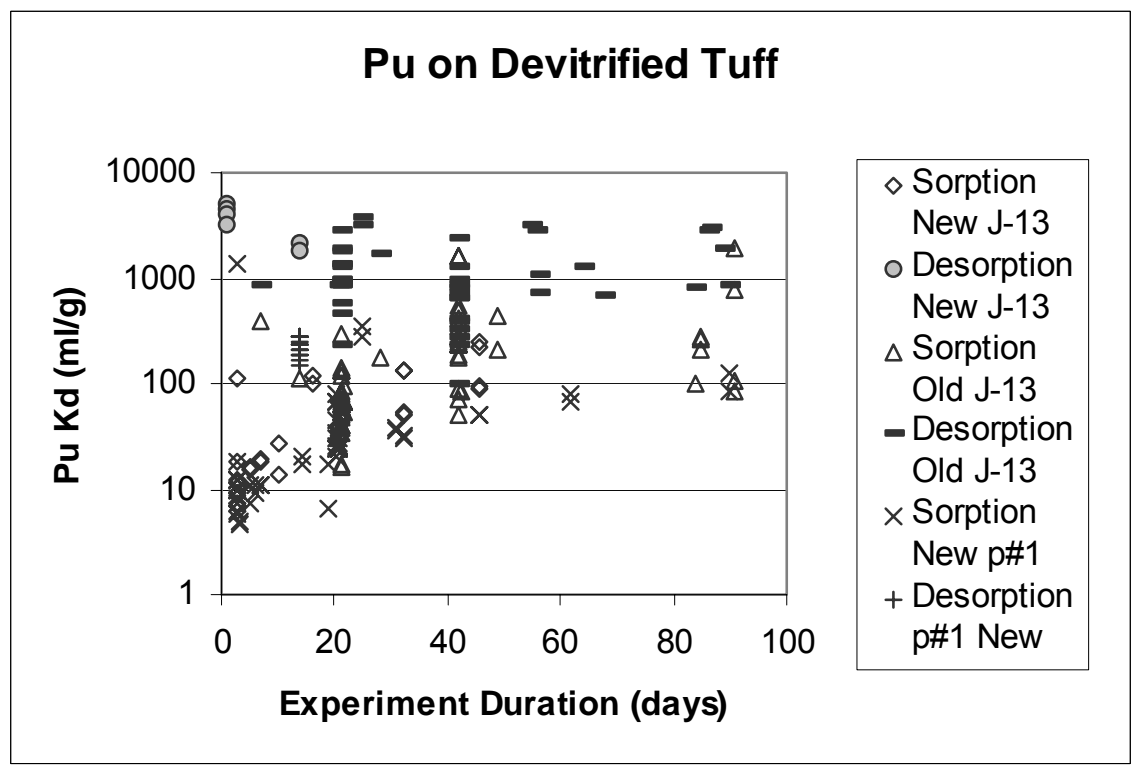

Source DTNs: LA0305AM831341.001 [DIRS 163789]; LA0407AM831341.005 [DIRS 170625].

Figure A-24. Plutonium Sorption Coefficients on Devitrified Tuff Versus Experiment Duration for Sorption (Forward) and Desorption (Backward) Experiments

The impact of variations in $\mathrm{pH}$ on plutonium sorption coefficients on devitrified tuffs is shown in Figure A-25. There is a lot of scatter in the data, and there do not appear to be clear positive or negative trends in any of the data groupings. 


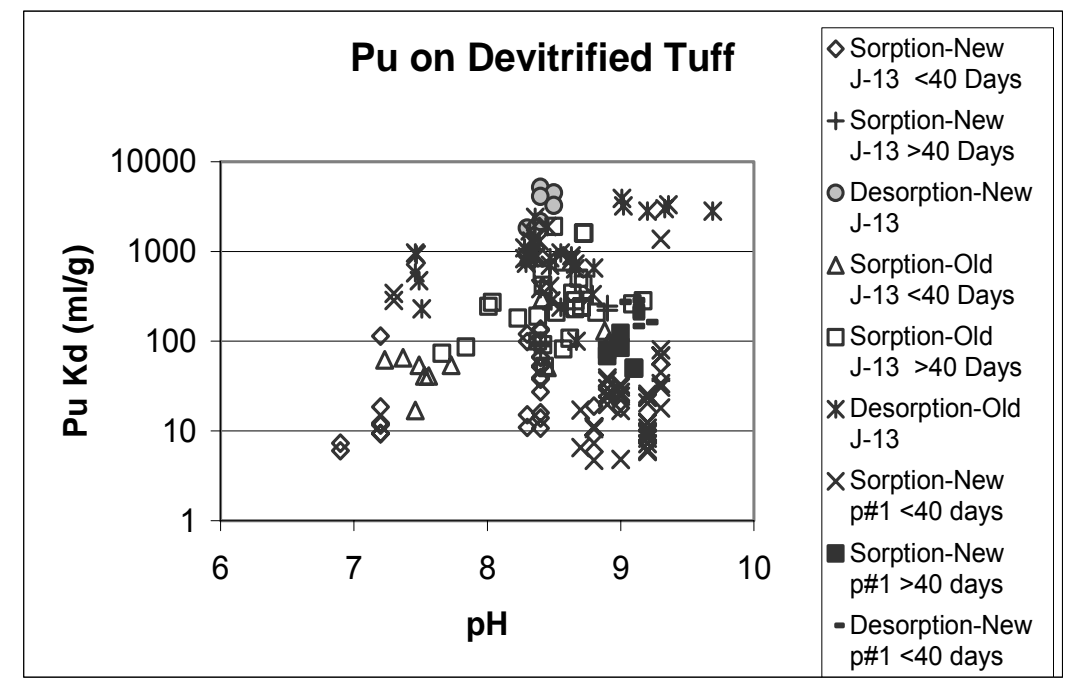

Source DTNs: LA0305AM831341.001 [DIRS 163789]; LA0407AM831341.005 [DIRS 170625].

NOTE: Experiments lasting 40 days or more are plotted separately from experiments lasting less than 40 days.

Figure A-25. Plutonium Sorption Coefficients on Devitrified Tuff in J-13 Water and Synthetic p\#1 Water Versus Solution pH in Sorption (Forward) and Desorption (Backward) Experiments

The lack of clear trends is also evident when the results of short-term experiments ( $<40$ days) are removed from the data set. This outcome is shown in Figure A-26 in which only the longer-term data are plotted.

The impact of variations in the major ion composition of groundwaters is also shown in Figures A-25 through A-26. Although there is a limited set of experiments with synthetic p\#1 water, the results are within the range of the results obtained in experiments with $\mathrm{J}-13$ water. Thus, there is no clear evidence of an impact of water chemistry variations on plutonium sorption coefficients in devitrified tuff.

A major factor not explicitly accounted for in the experimental program is the impact of variations in the Eh of Yucca Mountain groundwaters on plutonium sorption coefficients. The laboratory experiments upon which the data discussed in this section are based were invariably conducted under oxidizing conditions because the waters used in the experiments contained dissolved oxygen (DO) and were in contact with the atmosphere. Nitsche et al. (1993 [DIRS 155218], pp. 60-61) found that plutonium dissolved in J-13 water is present predominantly in the +5 and +6 oxidation states. If plutonium in the +5 and +6 oxidation states behaves similarly to neptunium +5 and uranium +6 , respectively, as has been suggested by many investigators (e.g., Keeney-Kennicutt and Morse 1985 [DIRS 106313], pp. 2577-2578), then small values $(<10 \mathrm{~mL} / \mathrm{g}$ ) would be expected for plutonium sorption coefficients under oxidizing conditions (see the sections on neptunium and uranium). The fact that plutonium sorption coefficients measured under oxidizing conditions are up to 2 to 3 orders of magnitude larger than expected (Figure A-26) suggests that either plutonium +5 and +6 do not behave like neptunium +5 and uranium +6 in sorption reactions or plutonium is reduced to the +4 oxidation state on rock/mineral surfaces. Data presented by Keeney-Kennicutt and Morse (1985 [DIRS 106313], p. 2577) support the latter alternative. 


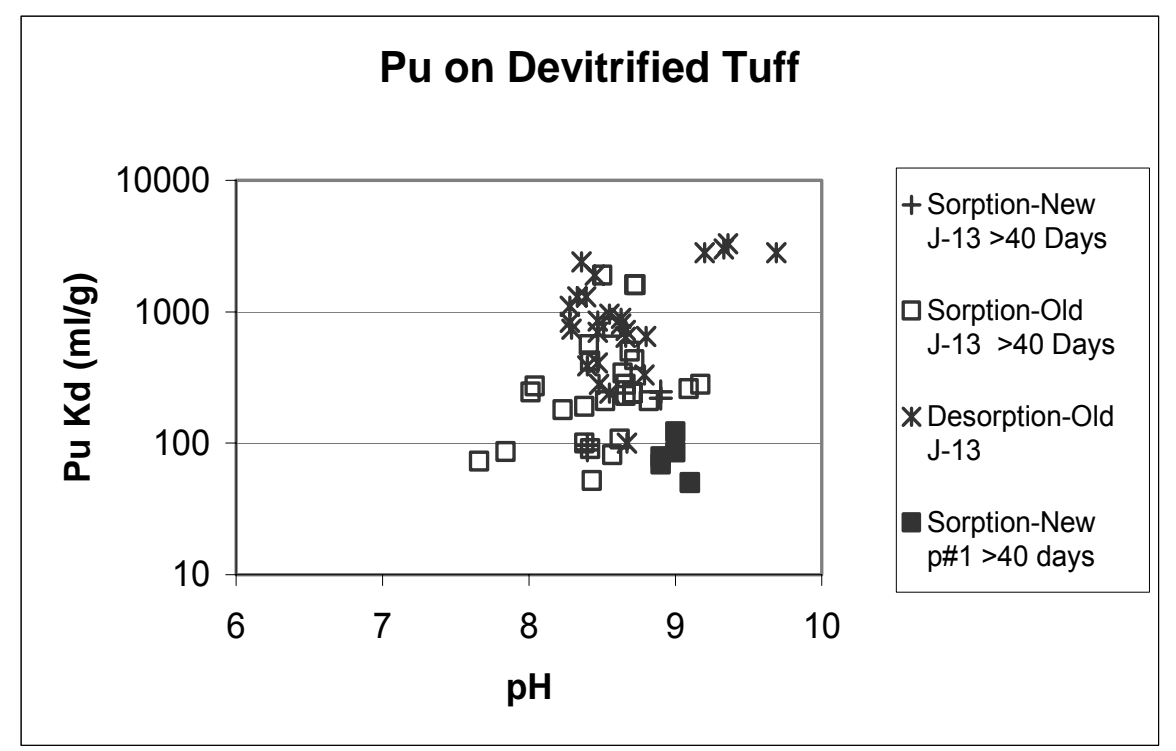

Source DTNs: $\quad$ LA0305AM831341.001 [DIRS 163789]; LA0407AM831341.005 [DIRS 170625].

Figure A-26. Plutonium Sorption Coefficients on Devitrified Tuff in J-13 Water and Synthetic p\#1 Water Versus Solution pH in Sorption (Forward) and Desorption (Backward) Experiments with Durations Greater than 40 Days

To further pursue the latter alternative, a PHREEQC model with the thermodynamic input data file YMP_R3.DAT (DTN: MO0403SPAYMPR3.000 [DIRS 170937]) was developed to calculate plutonium sorption coefficients as a function of Eh. To develop this model, binding constants for neptunium +5 and uranium +6 species were used for plutonium +5 and plutonium +6 species, respectively. For plutonium +4 , binding constants were derived by fitting experimental data for plutonium sorption on quartz in artificial groundwater published by Allard (1982 [DIRS 104512], p. 61). This water composition has somewhat reducing characteristics so that plutonium +4 would be expected to be sorbed onto the solid phase. The sorption coefficients calculated with this model using J-13 water are plotted as a function of Eh in Figure A-27. The curves plotted in that figure suggest that at Eh values less than $500 \mathrm{mV}$, plutonium is dominantly in the +4 oxidation state in the solution and on the solid phase. This Eh value is higher than the highest value measured in Yucca Mountain groundwater (DTN: LA0206AM831234.001 [DIRS 160051] and LA0206AM831234.002 [DIRS 163852]). This result implies that plutonium +4 will be the dominant oxidation state sorbed in most Yucca Mountain groundwaters. At higher Eh values, plutonium in solution will become progressively oxidized to plutonium +5 and eventually to plutonium +6 . The end points of the curves (high Eh) plotted in Figure A-27 represent the Eh values expected when the water is in contact with the atmosphere. In the $\mathrm{p} \# 1$ water composition, the lower ends of the curves are shifted downward somewhat to lower $K_{d}$ values at a given Eh value (not shown). 


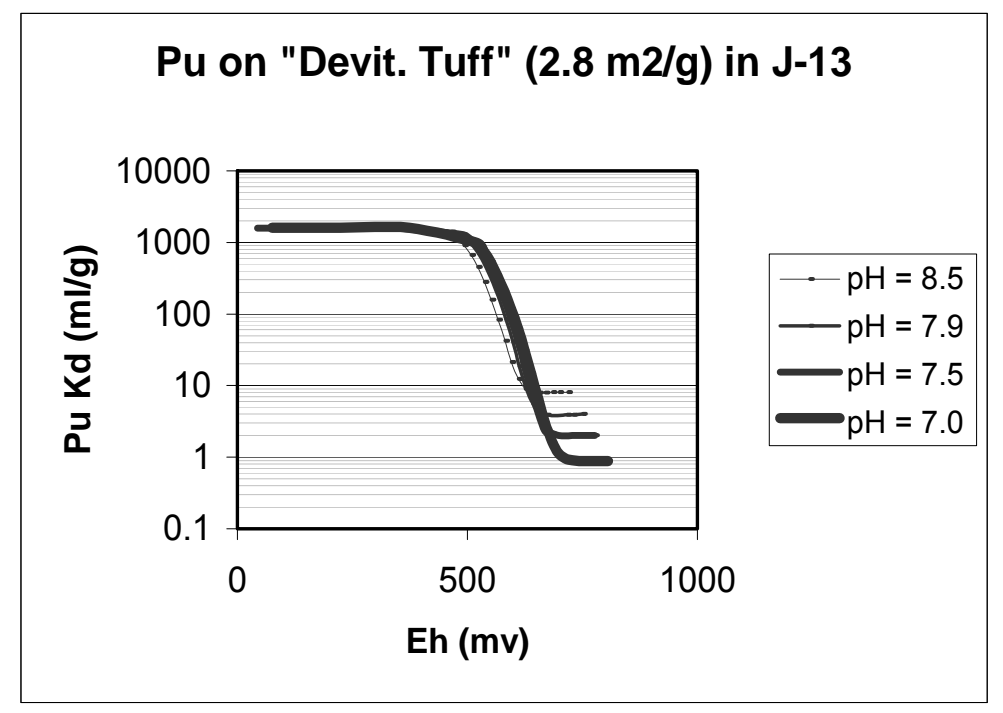

Output DTN： LA0306AM831343.001 files output/pudtfo70, pudt.fo75 pudtfo80 and pudtfo85.

NOTES: PHREEQC V2.3 (DIRS 155323) was used. Separate curves are shown for different pH values.

Figure A-27. Plutonium Sorption Coefficients Versus Eh as Predicted by PHREEQC Model

The large range in plutonium sorption coefficients measured in devitrified tuffs (e.g., Figure A-26) could be explained if plutonium is present in more than one oxidation state on the rock/mineral surfaces in the devitrified tuffs. However, in the absence of definitive data on the oxidation state of plutonium on rock/mineral surfaces, the experimentally determined sorption-coefficient values will be used to derive the probability distributions.

The plutonium sorption-coefficient probability distribution selected for devitrified tuff in the SZ is a beta distribution with a range of 50 to $300 \mathrm{~mL} / \mathrm{g}$, mean of $100 \mathrm{~mL} / \mathrm{g}$, and a standard deviation of $15 \mathrm{~mL} / \mathrm{g}$. The low end of the chosen range is selected based on the minimum value observed in long-term experiments ( $>40$ days), but it also captures the potential impacts of variations in surface areas among samples used in the experiments, variations in water chemistry, and variations in plutonium concentrations up to the solubility limit. The upper end of the distribution was chosen as a minimum upper limit given the potential impacts of sorption kinetics in the long term. The fact that sorption coefficients derived from desorption experiments were, on average, greater in value than those obtained from sorption experiments (Figure A-26) indicates that equilibrium had not been reached. In longer time frames, the coefficients derived from desorption experiments may continue to decrease whereas the coefficients from sorption experiments continue to increase. The upper limit was selected in acknowledgement of this possibility. In addition, the impacts of variations in sample surface areas, waters chemistry, and plutonium solution concentrations were considered in choosing the upper limit value. 


\section{A7.4.2 Zeolitic Tuff}

The experimentally derived sorption coefficients for plutonium on zeolitic tuff are plotted against the calculated final plutonium concentrations in Figure A-28. As before, the data points are separated into groups on the basis of when the experiments were carried out (pre-1990 = "old" and post-1990 = "new"), water chemistry, experiment duration, and on whether the sorption coefficient was determined from a sorption or a desorption experiment. The plotted data indicate that there is no clear trend of plutonium $K_{\mathrm{d}}$ values with solution concentration. Most of the data from sorption experiments plot between sorption-coefficient values of 100 and 1,000 mL/g. A series of "old" experiments at concentrations between $10^{-7}$ and $10^{-9} \mathrm{M} / \mathrm{L}$ yielded sorption-coefficient values less than $100 \mathrm{~mL} / \mathrm{g}$. However, the same rock type, when used in "new" experiments, yielded a series of sorption-coefficient values $>100 \mathrm{~mL} / \mathrm{g}$. Why there is a difference of almost a factor of 10 between these two sets of data is not known with certainty. Part of the answer lies in the fact that, on average, the "old" data points represent shorter duration experiments than the "new" data points. However, other factors are likely involved. For example, the oxidation state of plutonium in the starting solution may play a part.

As with devitrified tuffs, the plutonium sorption coefficients for zeolitic tuff plotted in Figure A-28 reflect solutions that were undersaturated, with the plutonium phase precipitated in the experiments conducted by Nitsche et al. (1993 [DIRS 155218], p. 54, and 1995 [DIRS 100163], p. 39).

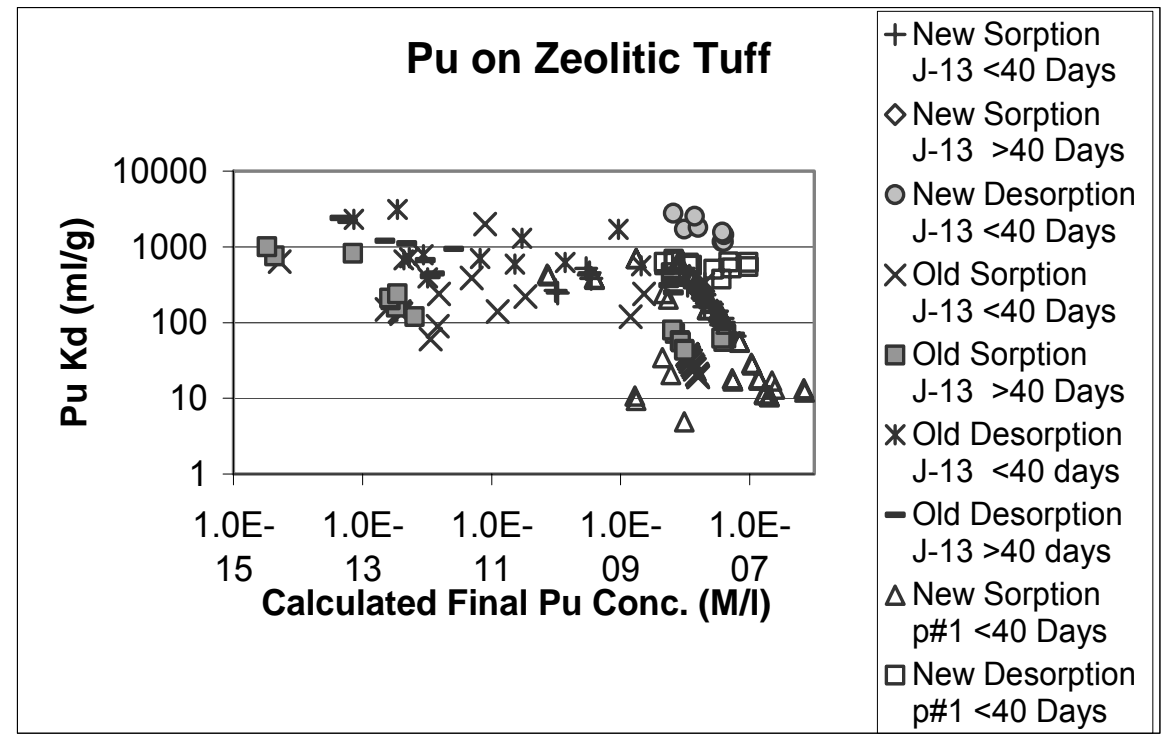

Source DTNs: LA0305AM831341.001 [DIRS 163789; LA0407AM831341.005 [DIRS 170625].

Figure A-28. Plutonium Sorption Coefficients on Zeolitic Tuff Versus Calculated Final Plutonium Concentration in Solution 
The effects of experiment duration on the plutonium $K_{\mathrm{d}}$ values for zeolitic tuff are shown in Figure A-29. As expected, the $K_{\mathrm{d}}$ values for sorption experiments increase with increasing duration and the values for desorption experiments decrease with increasing duration, at least in short-term experiments. Interestingly, the decrease in longer-term desorption experiments is not very pronounced. The trends in the sorption and desorption data points suggest they would converge to values somewhere between 100 and $1,000 \mathrm{~mL} / \mathrm{g}$. This result is similar to the range of values predicted for devitrified tuffs. Thus, the higher surface areas of zeolitic tuffs compared to devitrified tuffs (approximately 10 times) do not appear to result in higher sorption values. This effect was also observed by Pabalan et al. (1998 [DIRS 162987], p. 113) in experiments with uranium sorption on zeolite. The cause for the large range of values obtained at a given value for experiment duration is not known but may be due largely to variations in the oxidation state of plutonium in the starting solutions. However, variations in surface areas and surface chemistry among the samples used in the experiments must also contribute to the range observed.

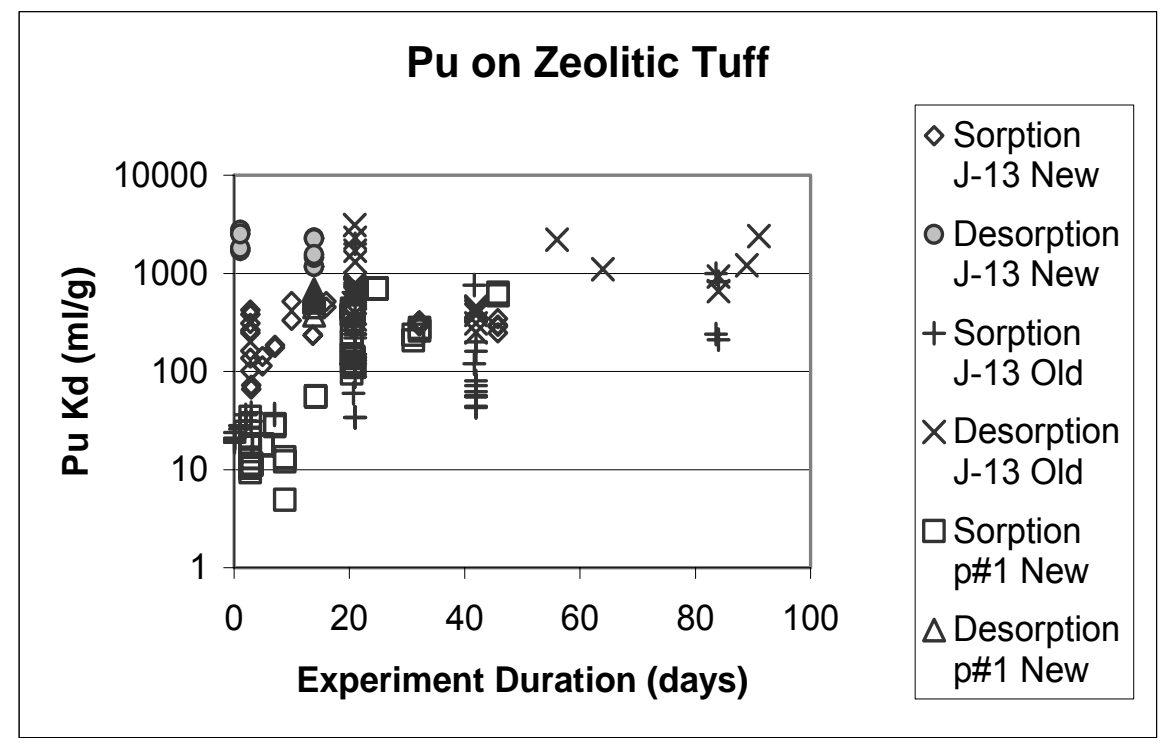

Source DTNs: LA0305AM831341.001 [DIRS 163789]; LA0407AM831341.005 [DIRS 170625].

Figure A-29. Plutonium Sorption Coefficients on Zeolitic Tuff Versus Experiment Duration for Sorption (Forward) and Desorption (Backward) Experiments

The impact of variations in $\mathrm{pH}$ on plutonium sorption coefficients on devitrified tuffs is shown in Figure A-30. Although there is a lot of scatter in the data, there do not appear to be clear positive or negative trends among any of the data groupings. 


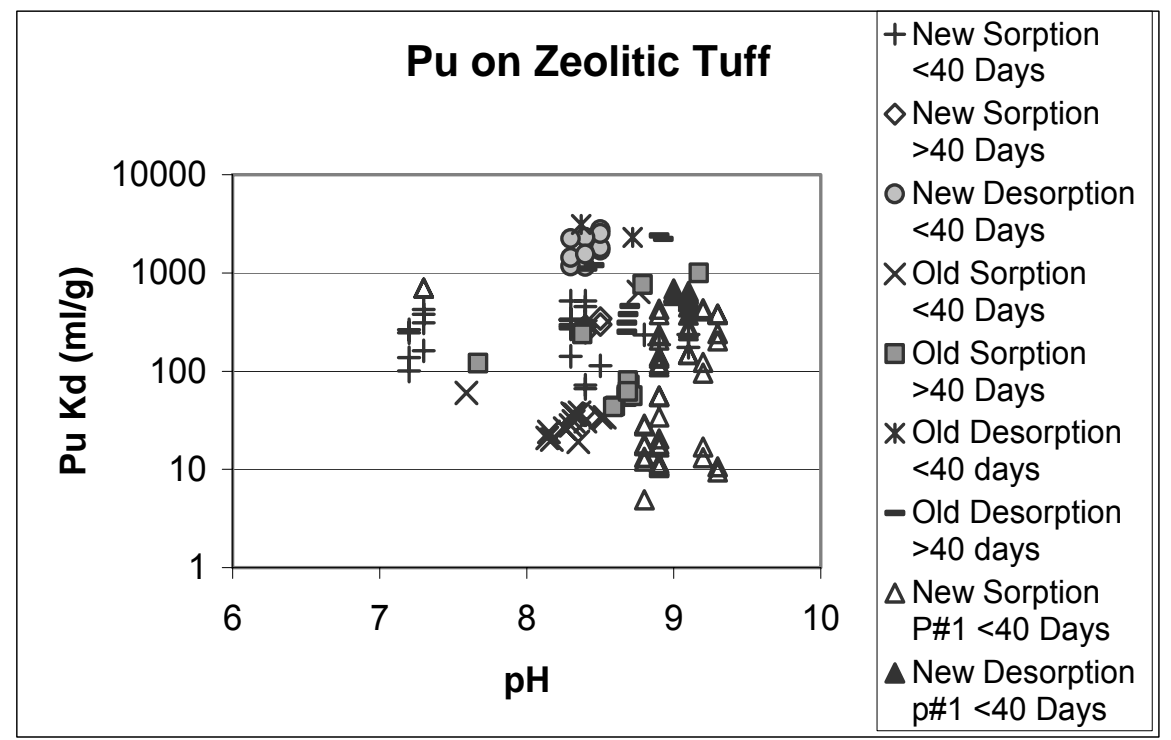

Source DTNs: LA0305AM831341.001 [DIRS 163789]; LA0407AM831341.005 [DIRS 170625].

Figure A-30. Plutonium Sorption Coefficients on Zeolitic Tuff in J-13 Water and Synthetic p\#1 Water Versus Solution pH in Sorption (Forward) and Desorption (Backward) Experiments with Durations Greater than 40 Days

The impact of variations in the major ion composition of groundwaters is also shown in Figure A-30. Although there is a limited set of experiments with synthetic p\#1 water, the results are largely within the range of the results obtained in experiments with $\mathrm{J}-13$ water. Thus, there is no clear evidence of an impact of water chemistry variations on plutonium sorption coefficients in devitrified tuff.

The plutonium sorption-coefficient probability distribution derived for zeolitic tuff in the SZ is the same as the distribution derived for devitrified tuff because the experimentally derived sorption-coefficient values show similar ranges as a function of variations in surface areas among samples used in the experiments, variations in water chemistry, and variations in plutonium concentrations up to the solubility limit.

\section{A7.4.3 Alluvium}

The probability distribution for devitrified tuff will be used as a default for the plutonium sorption-coefficient probability distribution in alluvium. Alluvium along the flow path is composed largely of disaggregated tuffaceous materials. Because it has been disaggregated, the surface area of alluvial material should be higher than that of devitrified tuff. In addition, clays and other secondary minerals are enriched in alluvial materials. These characteristics should result in higher sorption coefficients for plutonium in alluvial materials compared to intact devitrified tuff. 


\section{A7.5 PROTACTINIUM}

Protactinium is very insoluble in waters of the type found at Yucca Mountain. The best estimates of protactinium solubility in these waters range from $10^{-15}$ to $10^{-13} \mathrm{M} / \mathrm{L}$ (Berry et al. 1989 [DIRS 144728], p. 346).

\section{A7.5.1 Devitrified Tuff}

The oxidation state of protactinium is +5 in groundwaters of the type found at Yucca Mountain (Cotton and Wilkinson 1980 [DIRS 101584], pp. 1092-1093). No sorption-coefficient data have been obtained for protactinium on devitrified tuffs from Yucca Mountain. Allard et al. (1983 [DIRS 162982], p. 12) have reported protactinium sorption-coefficient data for experiments with a silica sample having a surface area similar to that measured for devitrified tuffs. The solution composition used in the experiments was $0.01 \mathrm{M} / \mathrm{L} \mathrm{NaClO}_{4}$. The initial protactinium concentration used in all experiments was $4.0 \times 10^{-12} \mathrm{M} / \mathrm{L}$. Allard's data indicate that protactinium sorption coefficients vary substantially (approximately 2 orders of magnitude) as a function of $\mathrm{pH}$, as shown in Figure A-31. The cause for this variation in sorption coefficients with $\mathrm{pH}$ is unknown. Over the $\mathrm{pH}$ range expected in SZ waters at Yucca Mountain (7-8.5), the sorption coefficients reported by Allard et al. (1983 [DIRS 162982], p. 12) range from approximately 7,500 to $20,000 \mathrm{~mL} / \mathrm{g}$. The results for 6 -hour experiments were similar to the results for experiments lasting up to 6 weeks. Thus, sorption kinetics for protactinium sorption reactions appear to be fast. Allard et al. (1983 [DIRS 162982], p. 12) reported results for alumina that were very similar to the results they reported for silica.

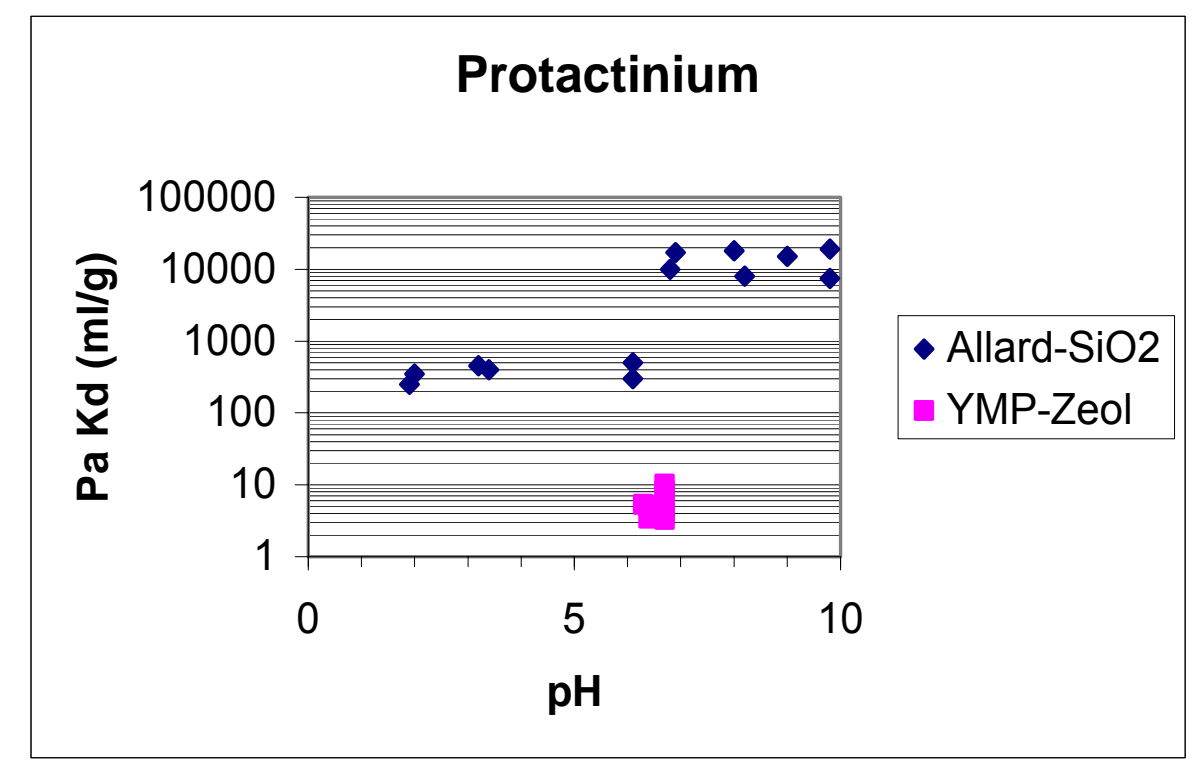

Source: DTN: LA0305AM831341.001 [DIRS 163789]; Allard et al. 1983 ([DIRS 162982], Figure 6]).

Figure A-31. Protactinium Sorption Coefficients Versus pH 
Hydrolysis reactions appear to dominate the solution chemistry of protactinium. The hydrolysis reactions of pentavalent protactinium in water are very complex even in relatively acidic solutions (Cotton and Wilkinson 1980 [DIRS 101584], pp. 1091 to 1093). In near-neutral solutions, the hydrolysis behavior of protactinium is essentially unknown. However, because hydrolysis reactions appear to dominate the solution behavior of protactinium, changes in the major ion chemistry of groundwaters are not expected to impact the sorption behavior of protactinium. Unfortunately, insufficient thermodynamic data are available to model protactinium sorption behavior using a surface complexation model.

The sorption-coefficient range for americium was used as a default for protactinium (i.e., 1,000 to $10,000 \mathrm{~mL} / \mathrm{g}$ ). This range is well within the range reported by Allard et al. (1983 [DIRS 162982], p. 12) for silica and alumina at values of $\mathrm{pH}>7.0$. As with americium sorption coefficients, a truncated normal distribution was selected with a mean of 5,500 mL/g and a standard deviation of $1,500 \mathrm{~mL} / \mathrm{g}$.

Corroboration of this range of sorption-coefficient values is provided by protactinium sorption experiments performed by Berry et al. (1989 [DIRS 144728], p. 347). These authors report a range of 1,000 to $1,000,000 \mathrm{~mL} / \mathrm{g}$ for protactinium sorption coefficients for rock samples, including sandstone, shale, granite, and clay in contact with natural groundwaters.

\section{A7.5.2 Zeolitic Tuff}

Sorption coefficients for protactinium on zeolitic tuffs from Yucca Mountain in J-13 water were reported by Rundberg et al. (1985 [DIRS 101355], p. 63). The reported sorption coefficients ranged from 3.3 to $10.1 \mathrm{~mL} / \mathrm{g}$ (Figure A-31). The initial solution concentrations in the experiments ranged from $1 \times 10^{-11}$ to $5 \times 10^{-14}$. The initial solution concentration used by Allard et al. (1983 [DIRS 162982], p. 6) was in the low end of this range. Thus, initial solution concentration does not appear to explain the difference between the results of Allard et al. (1983 [DIRS 162982], p. 12) and the results on Yucca Mountain samples. It is possible that the results on Yucca Mountain samples reflect oversaturation due to coprecipitation of protactinium with some other easily hydrolyzed species. This explanation requires that the precipitates were colloidal in size and, therefore, not efficiently removed from the final solutions.

The $\mathrm{pH}$ values of the final solutions in the experiments on Yucca Mountain samples were in the range of 6.3 to 6.7. These $\mathrm{pH}$ values are below the range expected in SZ waters at Yucca Mountain. Thus, the reported sorption coefficients do not directly apply to conditions in the SZ at Yucca Mountain. As shown in Figure A-31, there appears to be an adsorption edge at a $\mathrm{pH}$ close to 6.8 , and sorption coefficients increase by approximately 2 orders of magnitude at values of $\mathrm{pH}>6.8$. The cause for this increase is not known but is likely related to hydrolysis reactions.

Because zeolitic tuffs have greater surface area than devitrified tuffs, the sorption-coefficient distribution for devitrified tuff is used as a default for zeolitic tuff. This approach will lead to conservative predictions of protactinium transport rates. 


\section{A7.5.3 Alluvium}

The probability distribution for devitrified tuff will be used as a default for the protactinium sorption-coefficient probability distribution in alluvium. Alluvium along the flow path is composed largely of disaggregated tuffaceous materials. Because devitrified tuff makes up a major portion of the volcanic units exposed at the surface, it should be a major component in alluvium. In addition, clays and other secondary minerals are enriched in alluvial materials. The presence of these minerals should result in higher sorption coefficients in alluvial materials compared to intact devitrified tuff.

\section{A7.6 RADIUM}

The chemistry of the element radium is very similar to that of the element barium (Cotton and Wilkinson 1980 [DIRS 101584], p. 426). Because barium has a radioactive isotope that is more readily measured by gamma counting than the radium isotopes, barium was used to measure sorption coefficients for radium. A limited number of experiments were performed with radium to confirm its sorption behavior relative to barium.

Barite is the solubility-controlling solid for barium in Yucca Mountain groundwaters. The solubility of barite (barium sulfate) obtained from PHREEQC modeling with the thermodynamic input data file YMP_R3.DAT (DTN: MO0403SPAYMPR3.000 [DIRS 170937]) in J-13 water at $25^{\circ} \mathrm{C}$ is $1.0 \times 10^{-6} \mathrm{M} / \mathrm{L}$ (DTN: LA0306AM831343.001, File output/Usat.pun). The solubility of barite in $\mathrm{p} \# 1$ water at $25^{\circ} \mathrm{C}$ is $3.1 \times 10^{-7}$ to $3.3 \times 10^{-7} \mathrm{M} / \mathrm{L}$. At $25^{\circ} \mathrm{C}$, the solubility of radium sulfate is $3.3 \times 10^{-7} \mathrm{M} / \mathrm{L}$ in J-13 water (DTN: LA0306AM831343.001, File output/Usat.pun ).

\section{A7.6.1 Devitrified Tuff}

The experimentally derived sorption coefficients for barium on devitrified tuff are plotted against the calculated final barium concentrations of the experiments in Figure A-32. The data points are separated into groups on the basis of when the experiments were carried out (pre $1990=$ "old" and post-1990 = "new"), water chemistry, experiment duration, and on whether the sorption coefficient was determined from a sorption or a desorption experiment.

The calculated final concentration of barium in the experiments with $\mathrm{J}-13$ water was below saturation with barite in all but one experiment (Figure A-32). The results for this one experiment will be omitted from further consideration. The calculated final barium concentrations in experiments with $\mathrm{p \# 1}$ water are all lower than the barite saturation value of $2.0 \times 10^{-7} \mathrm{M} / \mathrm{L}$. Thus, oversaturation was not an issue in the barium sorption experiments with p\#1 water. Similarly, the radium solution concentrations were all lower than the radium sulfate saturation value in J-13 water. Thus, these experiments were undersaturated with radium sulfate. 


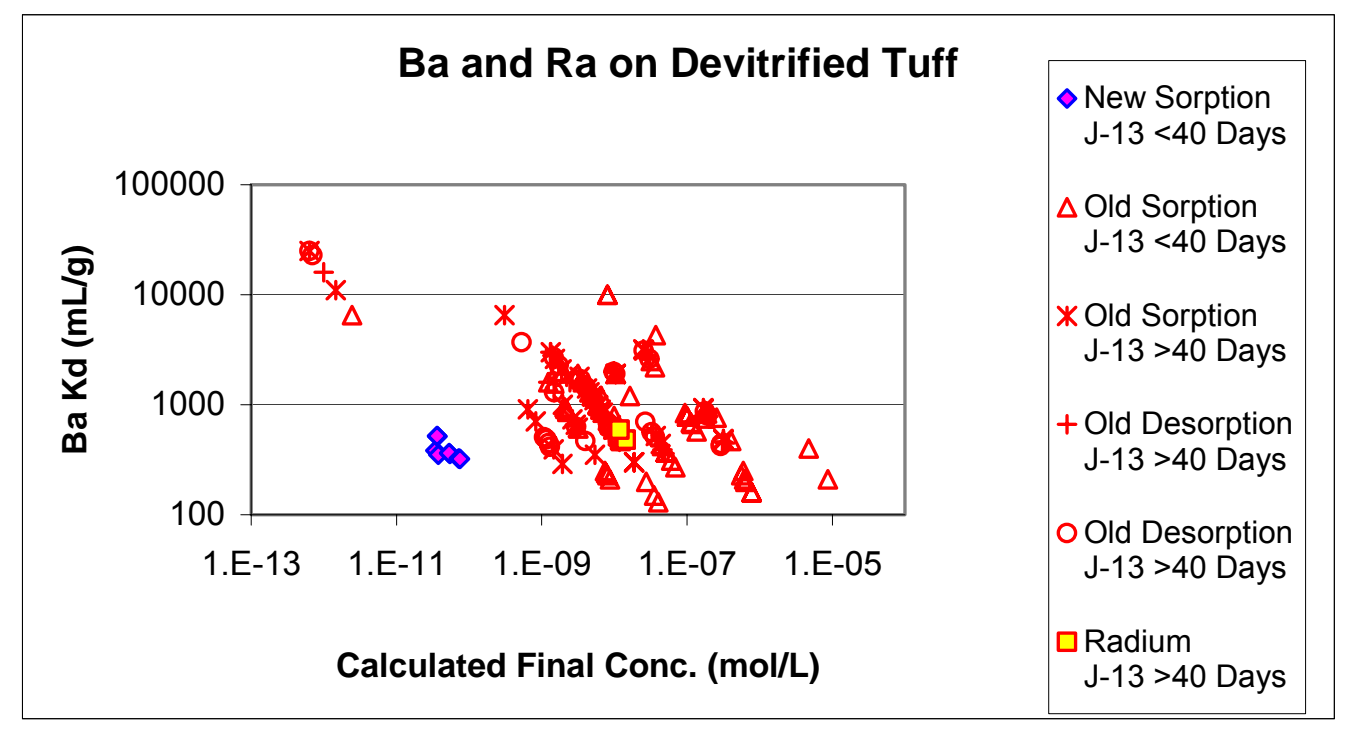

Source DTNs: LA0305AM831341.001 [DIRS 163789]; LA0407AM831341.001 [DIRS 170623].

Figure A-32. Barium and Radium Sorption Coefficients on Devitrified Tuff Versus Calculated Final Barium or Radium Concentrations in Solution

The data plotted in Figure A-32 show a general increase in sorption coefficient with decreasing concentration. This result suggests the isotherms for barium sorption onto devitrified tuff are nonlinear. The impact of this nonlinearity must be included in the sorption-coefficient probability distribution.

The effects of experiment duration on the barium $K_{d}$ value for devitrified tuff are shown in Figure A-33. The large range in sorption coefficients obtained at a given duration reflects variations in grain size of the crushed-tuff samples used in the experiments, variations in solution concentrations, variations in surface chemistry, and analytical errors and artifacts. Experiments with crushed-tuff samples that include the fines (e.g., $<30 \mu \mathrm{m}$ ) often have sorption coefficients that are larger than samples from which the fines have been removed (e.g., 75-500 $\mu \mathrm{m}$ ). This result is partly due to the higher surface area of samples with fines and partly due to mineral fractionation. Mineral fractionation can occur during the sieving process and cause the preferential concentration of very fine-grained minerals (e.g., clays) in the fine fraction.

Figure A-34 shows the effects of experiment duration on sorption coefficients for samples with the fines removed. The few sorption coefficients with values near $10,000 \mathrm{~mL} / \mathrm{g}$ represent experiments with very low solution concentrations (Figure A-34). For the remaining experiments, the total range of sorption-coefficient values is substantially reduced. Further, it appears likely the range would converge to a range between 100 and 1,000 mL/g with increasing duration. The horizontal trend among data points at durations other than 21 and 42 days suggests barium and radium sorption reactions are relatively fast. 


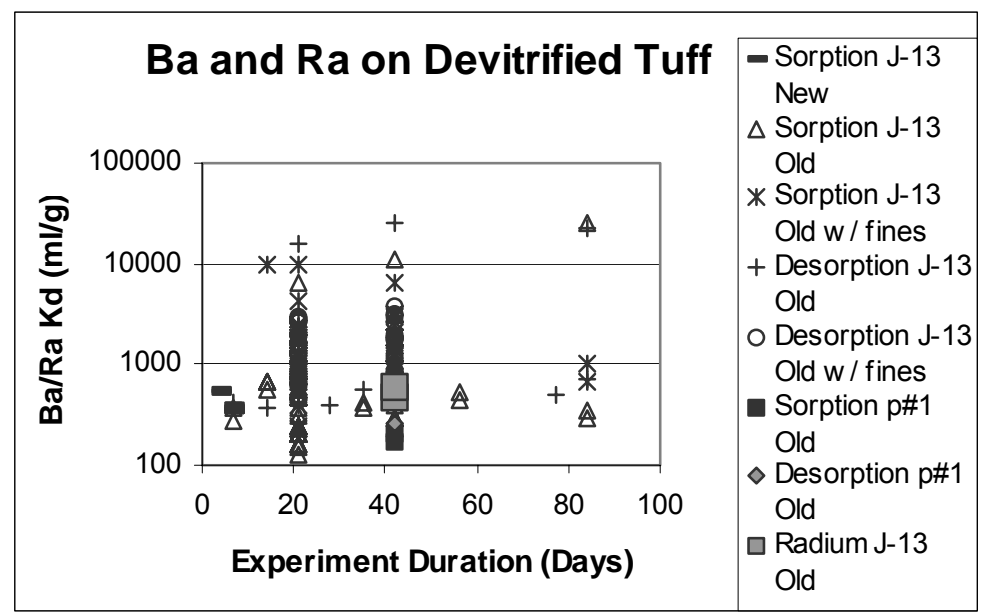

Source DTNs: LA0305AM831341.001 [DIRS 163789]; LA0407AM831341.001 [DIRS 170623].

Figure A-33. Barium and Radium Sorption Coefficients on Devitrified Tuff Versus Experiment Duration for Sorption (Forward) and Desorption (Backward) Experiments

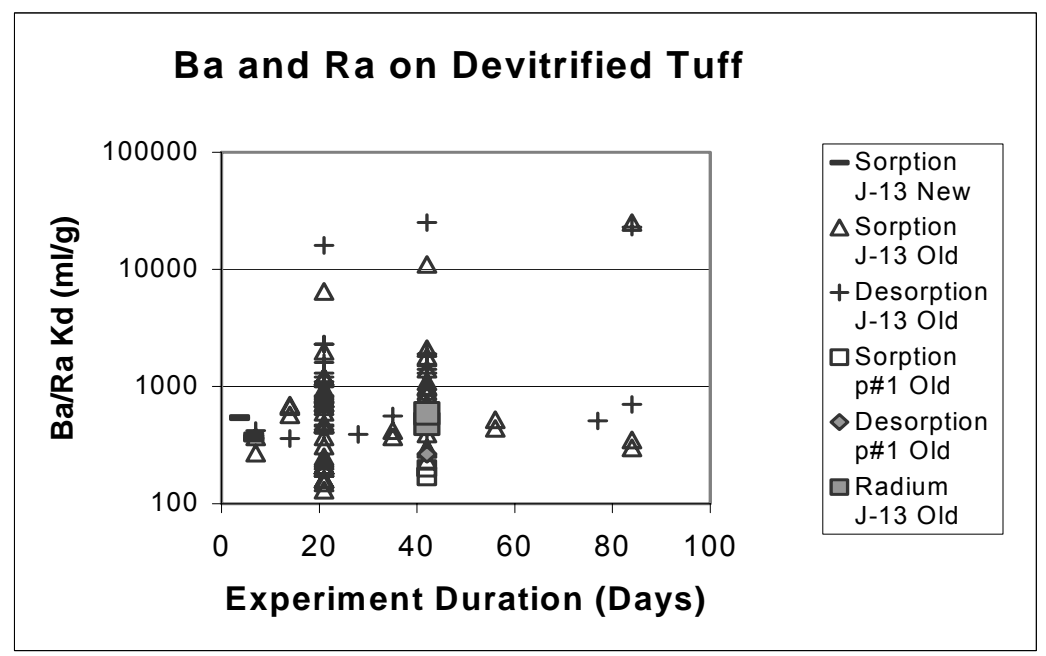

Source DTNs: LA0305AM831341.001 [DIRS 163789]; LA0407AM831341.001 [DIRS 170623].

Figure A-34. Barium and Radium Sorption Coefficients on Devitrified Tuff Versus Experiment Duration for Sorption (Forward) and Desorption (Backward) Experiments

The impact of variations in the major ion composition of groundwaters is also shown in Figures A-33 and A-34. Although there is a limited set of experiments with p\#1 water, the sorption coefficients obtained (at 42 days) fall at the low end of the range of results for J-13 water. Thus, there is some impact of water chemistry, although it is substantially less than an order of magnitude. 
The radium sorption-coefficient probability distribution derived for devitrified tuff in the SZ is a uniform distribution with a range of 100 to $1,000 \mathrm{~mL} / \mathrm{g}$. The low end of the chosen range was selected based on the minimum value observed in long-term experiments ( $>40$ days) and potential impacts of variations in water chemistry and surface areas among devitrified tuffs at Yucca Mountain. Because there are experiments within the data set that have solution concentrations close to saturation with a barium/radium sulfate, the effect of nonlinear isotherms is incorporated into the distribution. The upper end of the distribution was chosen as a minimum upper limit given the potential impacts of sorption kinetics, radium solution concentrations, and surface areas.

\section{A7.6.2 Zeolitic Tuff}

The experimentally derived sorption coefficients for barium and radium on zeolitic tuff are plotted against the calculated final barium or radium concentrations in Figure A-35. As before, the data points are separated into groups on the basis of when the experiments were carried out (pre-1990 = "old" and post-1990 = "new"), experiment duration, and on whether the sorption coefficient was determined from a sorption or a desorption experiment. The plotted data indicate that there is no clear trend of barium $K_{\mathrm{d}}$ values with solution concentration. Essentially all the sorption coefficients exceed a value of $10,000 \mathrm{~mL} / \mathrm{g}$ and some desorption experiments approach values of $1,000,000 \mathrm{~mL} / \mathrm{g}$. The radium results are in the range of the results for barium, confirming the similar sorption behavior of these two elements. As with devitrified tuffs, the barium and radium sorption coefficients for zeolitic tuff plotted in Figure A-35 reflect solutions that were undersaturated with barium and radium sulfate. The sorption coefficients obtained with $\mathrm{p} \# 1$ water are well within the range defined by the experiments with J-13 water. Thus, there does not appear to be a clear impact of variations in groundwater chemistry.

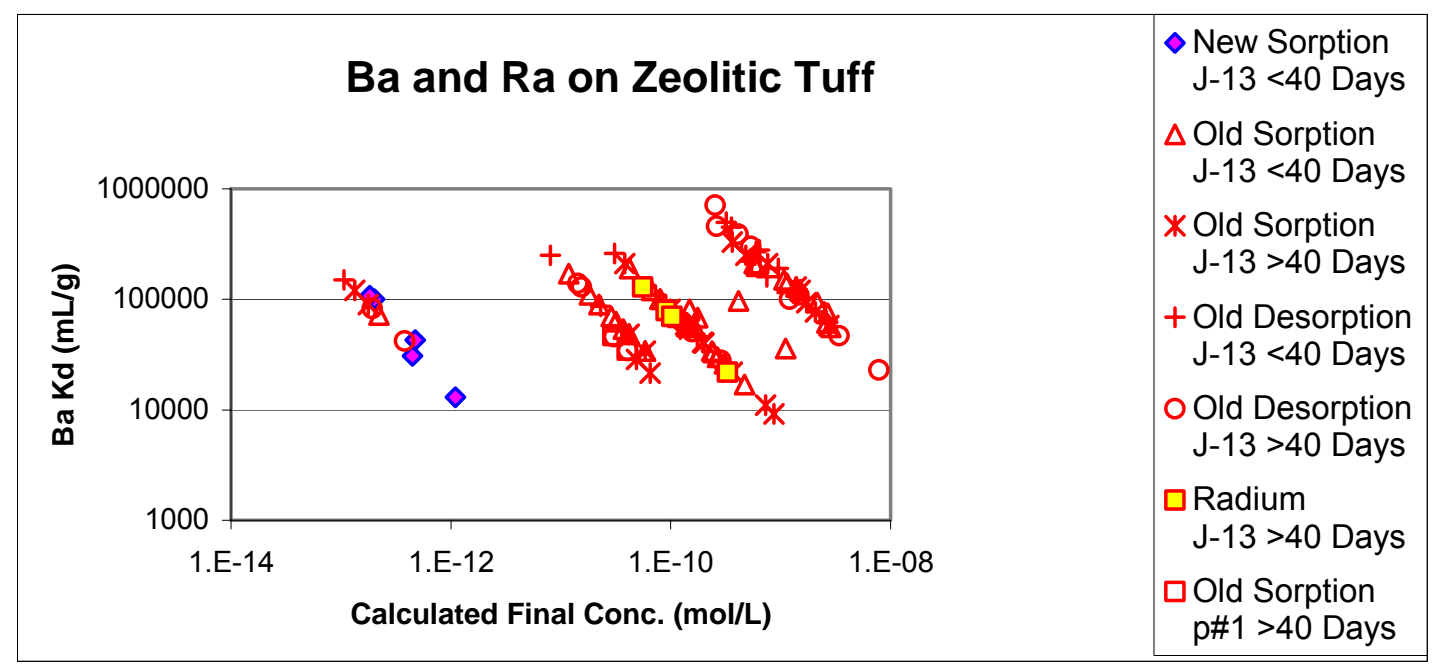

Source DTNs: LA0305AM831341.001 [DIRS 163789]; LA0407AM831341.001 [DIRS 170623].

Figure A-35. Barium and Radium Sorption Coefficients on Zeolitic Tuff Versus Calculated Final Barium or Radium Concentrations in Solution 
The data plotted in Figure A-36 also indicate that there is no clear trend of barium $K_{d}$ values with solution concentration. Data were obtained for a sorption isotherm on sample YM-38 (Figure A-36). As indicated by the exponent in the equation on the diagram, the calculated isotherm is essentially linear.

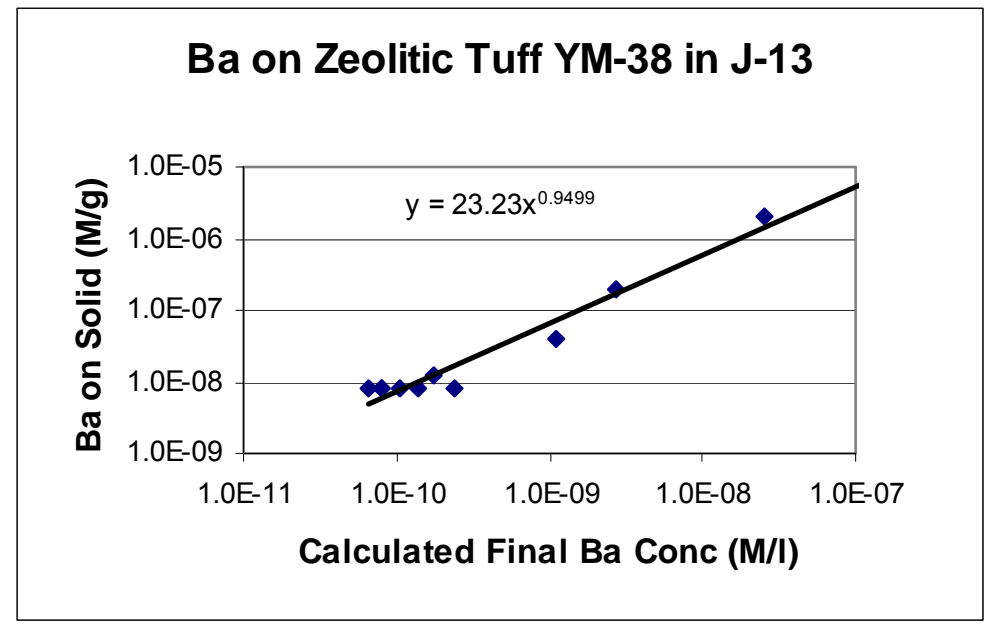

Source DTN: LA0305AM831341.001 [DIRS 163789].

Figure A-36. Isotherm Diagram for Barium Sorption on Zeolitic Tuff Sample YM-38 in J-13 Water

The effects of experiment duration on the barium $K_{d}$ values for zeolitic tuff are shown in Figure A-37. The barium and radium sorption reactions are quite fast. This characteristic is indicated by the fact that the range of values obtained in 6-day experiments (Figure A-37) is similar to the range of values obtained in 84-day experiments.

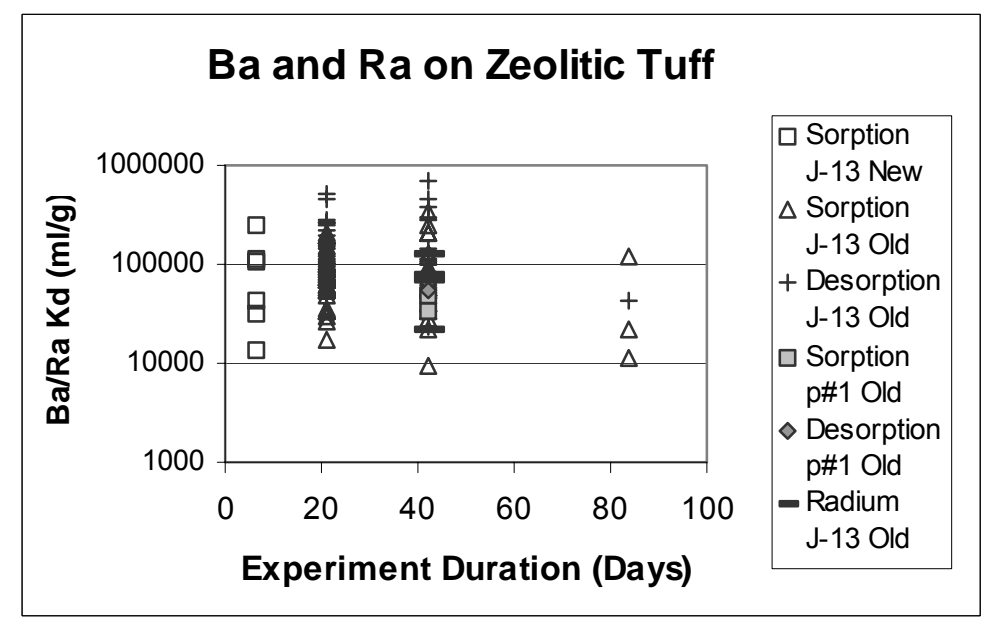

Source DTNs: LA0305AM831341.001 [DIRS 163789]; LA0407AM831341.001 [DIRS 170623].

Figure A-37. Barium and Radium Sorption Coefficients on Zeolitic Tuff in J-13 Water Versus Experiment Duration for Sorption (Forward) and Desorption (Backward) Experiments 
The impact of variations in the major ion composition of groundwaters is also shown in Figure A-37. Although there is a limited set of experiments with synthetic p\#1 water, the results are largely within the range of the results obtained in experiments with $\mathrm{J}-13$ water. Thus, there is no clear evidence of an impact of water chemistry variations on barium/radium sorption coefficients in zeolitic tuff.

The radium sorption-coefficient probability distribution derived for zeolitic tuff in the SZ is a truncated log-normal distribution with a range of 1,000 to $250,000 \mathrm{~mL} / \mathrm{g}$, a mean of $100,000 \mathrm{~mL} / \mathrm{g}$, and a standard deviation of $31,420 \mathrm{~mL} / \mathrm{g}$. The low end of the chosen range was selected based on the minimum value observed in long-term experiments ( $>40$ days) and potential impacts of variations in water chemistry and ion-exchange capacities among zeolitic tuffs at Yucca Mountain. The upper end of the distribution was chosen to address the potential for low radium solution concentrations and high ion-exchange capacities along the expected transport pathways.

Because zeolitic tuffs have greater surface area than devitrified tuffs, the sorption-coefficient distribution for devitrified tuff is used as a default for the radium sorption in the volcanic tuffs. This approach will lead to conservative predictions of radium transport rates.

\section{A7.6.3 Alluvium}

The probability distribution for devitrified tuff will be used as a default for the radium sorption-coefficient probability distribution in alluvium. Alluvium along the flow path is composed largely of disaggregated tuffaceous materials. Because devitrified tuff makes up a major portion of the volcanic units exposed at the surface, it should be a major component in alluvium. In addition, clays and other secondary minerals are enriched in alluvial materials. The presence of these minerals should result in higher sorption coefficients in alluvial materials compared to intact devitrified tuff.

\section{A7.7 STRONTIUM}

The solubility of strontium obtained from PHREEQC modeling with the thermodynamic input data file YMP_R3.DAT (DTN: MO0403SPAYMPR3.000 [DIRS 170937]) in J-13 water at $25^{\circ} \mathrm{C}$ ranges from $3.0 \times 10^{-5} \mathrm{M} / \mathrm{L}$ at a $\mathrm{pH}$ of 7.1 to $1.6 \times 10^{-6} \mathrm{M} / \mathrm{L}$ at a $\mathrm{pH}$ of 8.5 and the solubility controlling phase is strontianite (strontium carbonate). The solubility of strontianite in p\#1 water at $25^{\circ} \mathrm{C}$ ranges from $1.5 \times 10^{-5} \mathrm{M} / \mathrm{L}$ at a $\mathrm{pH}$ of 7.1 to $9.7 \times 10^{-7} \mathrm{M} / \mathrm{L}$ at a $\mathrm{pH}$ of 8.66 (output DTN: LA0306AM831343.001 file output/Usat.pun.).

\section{A7.7.1 Devitrified Tuff}

The experimentally derived sorption coefficients for strontium on devitrified tuff are plotted against the calculated final strontium concentrations of the experiments in Figure A-38. The data points are separated into groups on the basis of when the experiments were carried out (pre-1990 = "old" and post-1990 = "new"), water type, and on whether the sorption coefficient was determined from a sorption or a desorption experiment. There are also data for a sorption isotherm on sample G1-2840. 


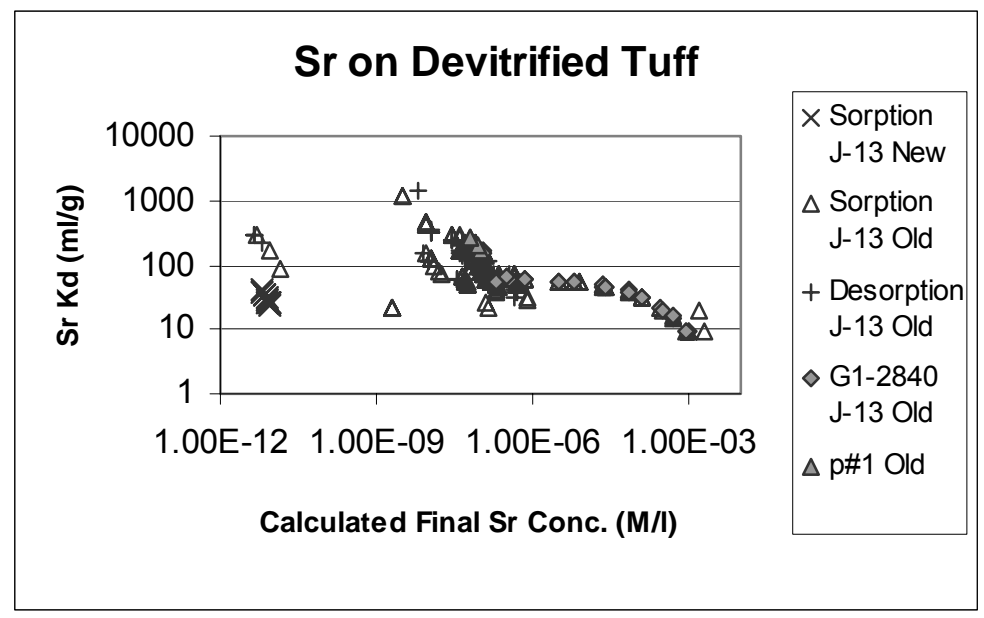

Source DTNs: LA0305AM831341.001 [DIRS 163789]; LA0407AM831341.003 [DIRS 170626].

Figure A-38. Strontium Sorption Coefficients on Devitrified Tuff Versus Calculated Final Strontium Concentration in Solution

Most of the calculated final solution concentrations for the sorption experiments are below saturation with strontianite in J-13. The data points with concentrations greater than $1.0 \times 10^{-6} \mathrm{M} / \mathrm{L}$ reflect experiments that were oversaturated with strontianite. Thus, the sorption coefficients obtained in these experiments will not be used in the derivation of the strontium sorption-coefficient probability distribution. The calculated final strontium concentrations used in experiments with p\#1 water are all lower than the saturation value. Thus, oversaturation was not an issue in the strontium sorption experiments with $\mathrm{p} \# 1$ water.

A sorption "isotherm" was obtained for sample G1-2840 in J-13 water. The "isotherm" was linear below a strontium-85 concentration of approximately $5 \times 10^{-5} \mathrm{M}$ as shown in Figure A-38. At higher concentrations, the final solutions were oversaturated with strontianite. Thus, these data points should not be used to construct an isotherm. Interestingly, if strontianite had precipitated in these experiments, it would be expected that the sorption coefficients would increase with increasing strontium concentrations. However, the data in Figure A-38 indicate sorption coefficients decreased with increasing solution concentrations. This suggests strontianite was not precipitated from solution for some reason. A possible explanation is that the nucleation or precipitation rates of strontianite are very slow relative to the duration of the experiments (21 days). Alternatively, strontianite could have nucleated but was sufficiently fine grained so that it remained with the solution phase during the process used to separate the solid and liquid phases in the experiments.

The $K_{\mathrm{d}}$ value in the linear portion of the "isotherm" in Figure A-38 is at the low end of the range of strontium sorption coefficients obtained with $\mathrm{J}-13$ and p\#1 waters. This suggests isotherms for the other samples plotted in Figure A-38 would lie at higher $K_{\mathrm{d}}$ values compared to the "isotherm" for sample G1-2840. The sorption coefficients obtained in experiments with p\#1 water fall in the middle of the range of values obtained for experiments with $\mathrm{J}-13$ water. Thus, there is little or no impact of variations in water chemistry on strontium sorption coefficients on devitrified tuff. The effects of experiment duration on values of the strontium $K_{\mathrm{d}}$ for devitrified tuff are shown in Figure A-39. The large range in sorption coefficients obtained at 
a given duration reflects variations in grain size of the crushed-tuff samples used in the experiments, variations in solution strontium concentrations, variations in surface chemistry, and analytical error and artifacts. Experiments with crushed-tuff samples that include the fines (e.g., particle size $<30 \mu \mathrm{m}$ ) usually have sorption coefficients that are larger than samples from which the fines have been removed (e.g., particle sizes $75-500 \mu \mathrm{m}$ ). This result is partly due to the higher surface area of samples with fines and partly due to mineral fractionation. Mineral fractionation can occur during the sieving process and cause the preferential concentration of very fine-grained minerals (e.g., clays) in the fine fraction.

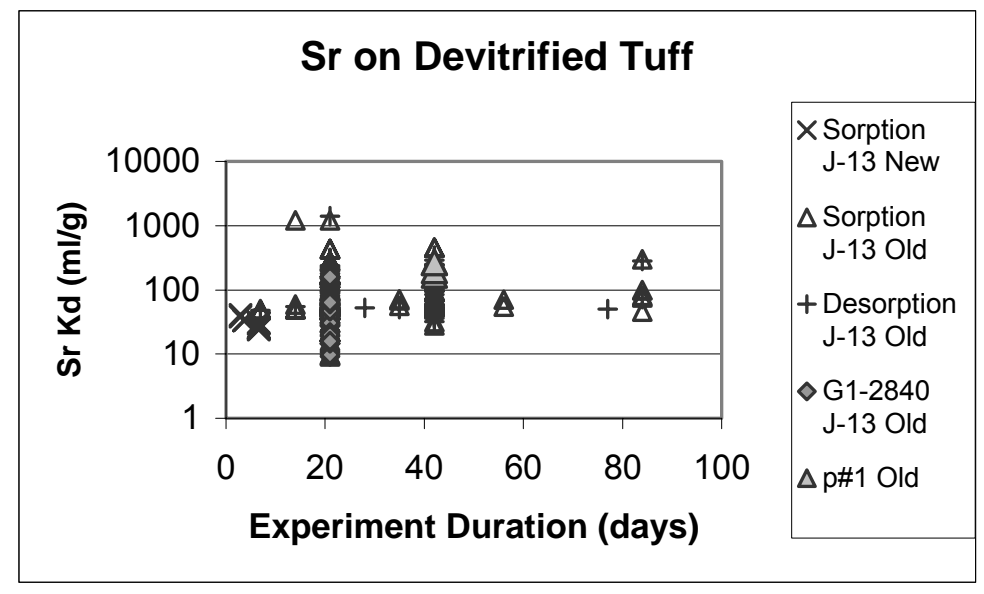

Source DTNs: LA0305AM831341.001 [DIRS 163789]; LA0407AM831341.003 [DIRS 170626].

NOTE: Data points include experiments in which fines were not removed from the samples.

Figure A-39. Strontium Sorption Coefficients on Devitrified Tuff Versus Experiment Duration for Sorption (Forward) and Desorption (Backward) Experiments

Figure A-40 shows the effects of duration on sorption coefficients for crushed-tuff samples with the fines removed. The total range of sorption-coefficient values is reduced compared to Figure A-39. Further, it appears likely this range would converge to values between 50 and $500 \mathrm{~mL} / \mathrm{g}$ with increasing duration. The horizontal trend among data points at durations other than 21 and 42 days suggests strontium sorption reactions are relatively fast.

The strontium sorption-coefficient probability distribution derived for devitrified tuff in the SZ is a uniform distribution with a range of 20 to $400 \mathrm{~mL} / \mathrm{g}$. The low end of the chosen range was selected based on the minimum value observed in long-term experiments ( $>40$ days) and potential impacts of variations in water chemistry and surface areas among devitrified tuffs at Yucca Mountain. Because there are experiments within the data set that have solution concentrations close to saturation with a strontium carbonate, the effect of nonlinear isotherms is incorporated into the distribution. The upper end of the distribution was chosen as a minimum upper limit given the potential impacts of strontium solution concentrations and surface areas. 


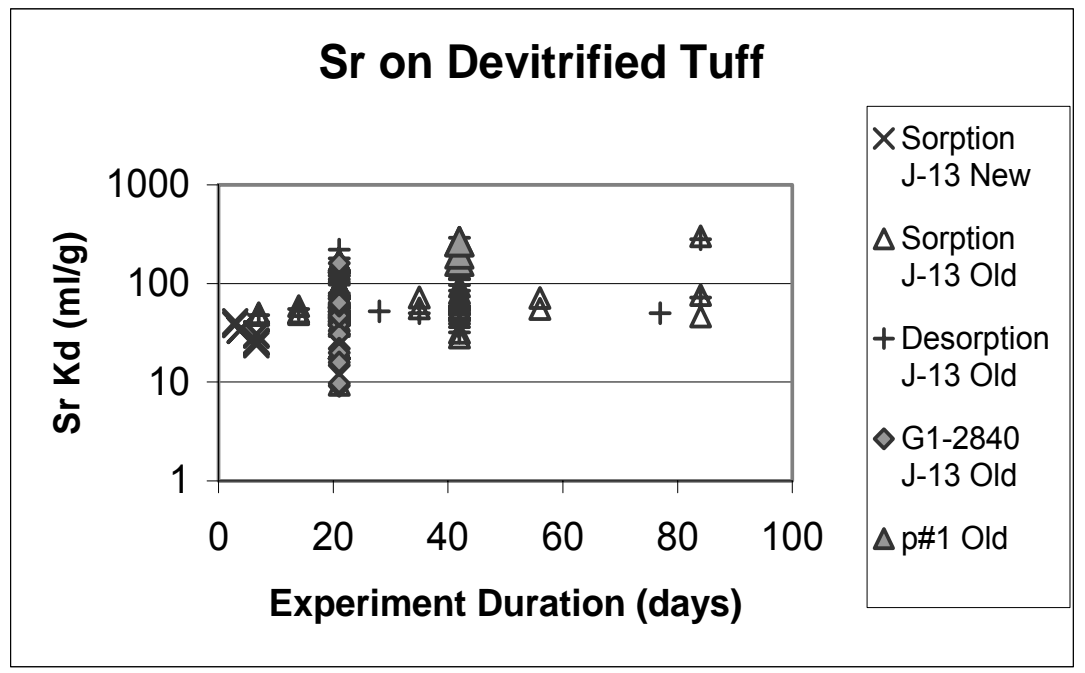

Source DTNs: LA0305AM831341.001 [DIRS 163789]; LA0407AM831341.003 [DIRS 170626].

NOTE: Samples containing fines fraction are removed from the figure.

Figure A-40. Strontium Sorption Coefficients on Devitrified Tuff Versus Experiment Duration for Sorption (Forward) and Desorption (Backward) Experiments with Reduced Range

\section{A7.7.2 Zeolitic Tuff}

The experimentally derived sorption coefficients for strontium on zeolitic tuff are plotted against the calculated final strontium concentrations of the experiments in Figure A-41. The data points are separated into groups on the basis of when the experiments were carried out (pre-1990 = "old" and post-1990 = "new"), water type, whether or not the solid phase included fines, and whether the sorption coefficient was determined from a sorption or a desorption experiment.

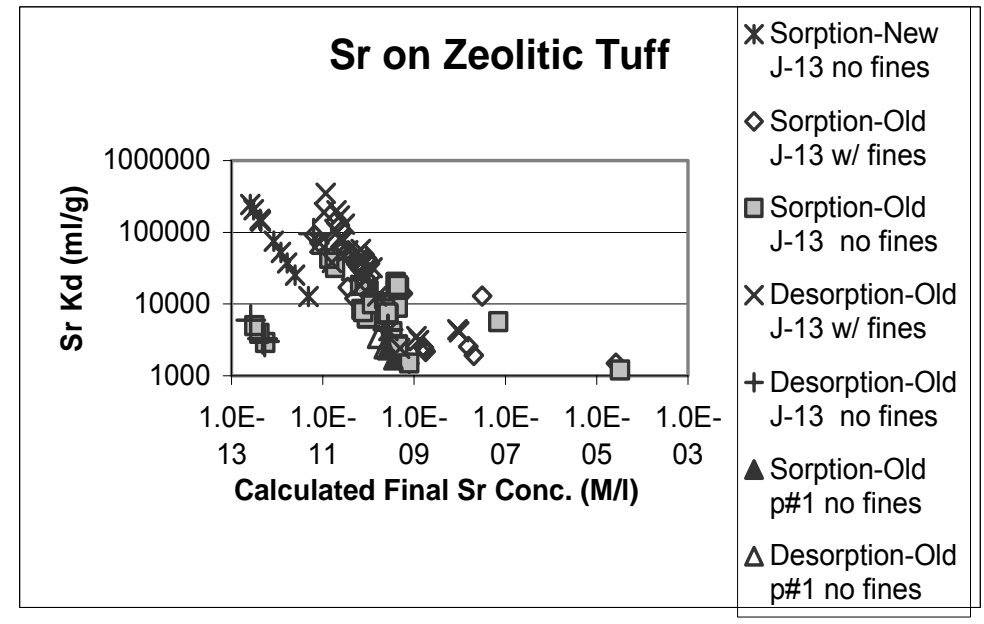

Source DTNs: LA0305AM831341.001 [DIRS 163789]; LA0407AM831341.003 [DIRS 170626].

Figure A-41. Strontium Sorption Coefficients on Zeolitic Tuff Versus Calculated Final Strontium Concentration in Solution 
All except two of the calculated final solution concentrations were below the saturation level with strontianite. The calculated final strontium concentrations used in experiments with p\#1 water are all lower than the saturation value. Thus, oversaturation was not an issue in most of the strontium sorption experiments.

The few sorption coefficients obtained with $\mathrm{p} \# 1$ water tend to lie at the low end of the range of values shown in Figure A-41. This result suggests variations in water composition will have an impact on the strontium sorption coefficient for zeolitic tuff.

Sorption experiments were carried out at a number of different starting concentrations to obtain an isotherm for sample YM-38. As shown in Figure A-42, the sorption coefficients obtained in these experiments are not consistent with a simple relationship between strontium concentration and sorption coefficient. Nonetheless, the sorption coefficients plotted in Figure A-42 were obtained over a range of strontium concentrations. Thus, the concentration dependence of the sorption coefficient is included in the data set.

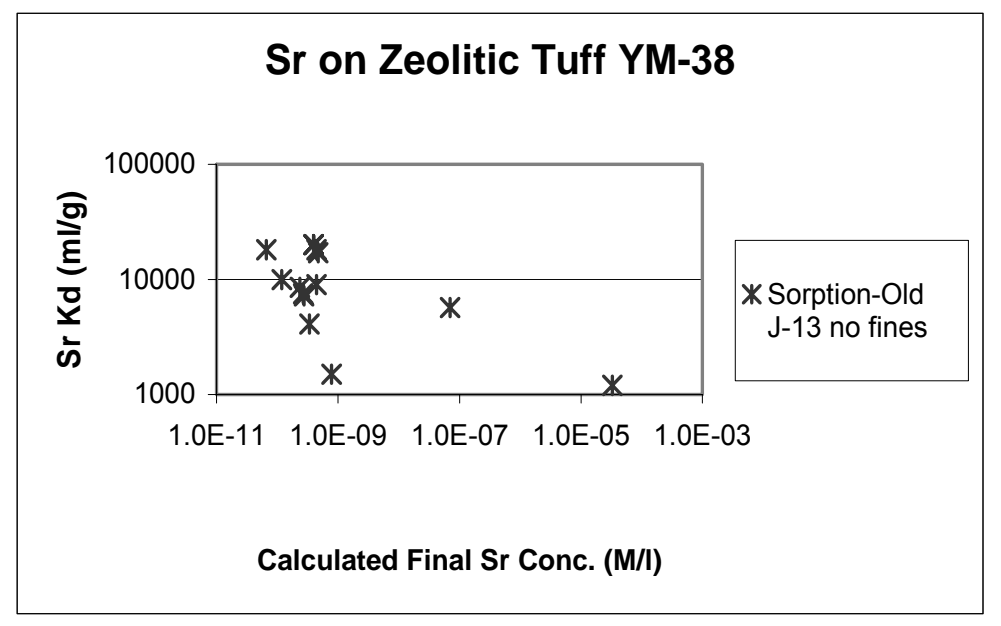

Source DTNs: LA0305AM831341.001 [DIRS 163789]; LA0407AM831341.003 [DIRS 170626].

Figure A-42. Strontium Sorption Coefficients Versus Calculated Final Solution Concentration (M/L) for Sample YM-38 in J-13 Water

The effects of experiment duration on the values of strontium $K_{\mathrm{d}}$ for zeolitic tuff are shown in Figure A-43. The large range in sorption coefficients obtained at a given duration reflects variations in grain size of the crushed-tuff samples used in the experiments, variations in solution strontium concentrations, variations in surface chemistry, and analytical errors and artifacts. Experiments with crushed-tuff samples that include the fines (e.g., particle size $<30 \mu \mathrm{m}$ ) usually have sorption coefficients that are larger than samples from which the fines have been removed (e.g., particle sizes $75-500 \mu \mathrm{m}$ ). This result is partly due to the higher surface area of samples with fines and partly due to mineral fractionation. Mineral fractionation can occur during the sieving process and cause the preferential concentration of very fine-grained minerals (e.g., clays) in the fine fraction. 


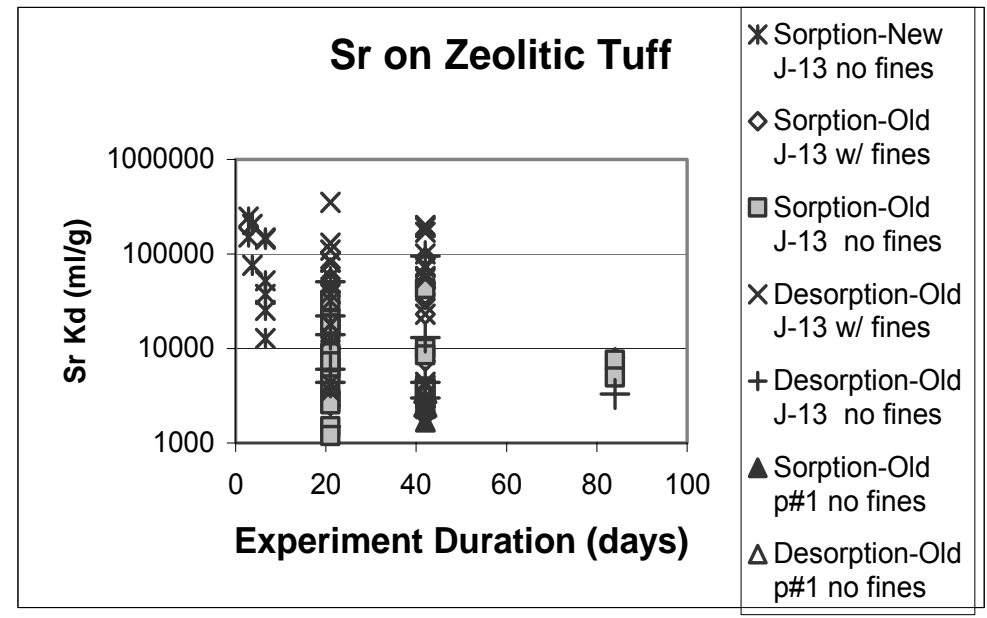

Source DTNs: LA0305AM831341.001 [DIRS 163789]; LA0407AM831341.003 [DIRS 170626].

Figure A-43. Strontium Sorption Coefficients on Zeolitic Tuff Versus Experiment Duration for Sorption (Forward) and Desorption (Backward) Experiments

Figure A-44 shows the effects of experimental duration on sorption coefficients for crushed-tuff samples with the fines removed. The total range of sorption-coefficient values is reduced slightly compared to Figure A-43. The rather limited range of values at 84 days is likely due to the limited number of experiments carried out at this duration. There is no clear trend of sorption-coefficient value with duration. This result likely reflects fast sorption kinetics.

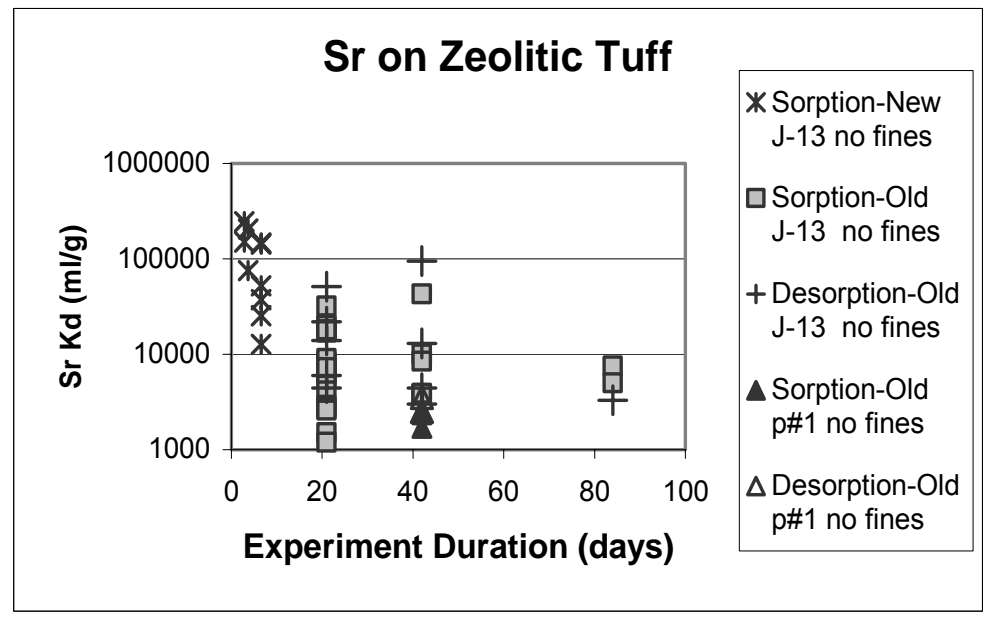

Source DTNs: LA0305AM831341.001 [DIRS 163789]; LA0407AM831341.003 [DIRS 170626].

Figure A-44. Strontium Sorption Coefficients on Zeolitic Tuff With Fine Fraction Removed Versus Experiment Duration for Sorption (Forward) and Desorption (Backward) Experiments 
The strontium sorption-coefficient probability distribution derived for zeolitic tuff in the SZ is a cumulative distribution starting at $100 \mathrm{~mL} / \mathrm{g}$, with a value of $5,000 \mathrm{~mL} / \mathrm{g}$ at 0.5 and a value of $90,000 \mathrm{~mL} / \mathrm{g}$ at 1.0 . The low end of the chosen range was selected based on the minimum value observed in long-term experiments ( $>40$ days) and potential impacts of variations in water chemistry and ion-exchange capacities among zeolitic tuffs at Yucca Mountain. Because there are experiments within the data set that have solution concentrations close to saturation with a strontium carbonate, the effect of nonlinear isotherms is included in the distribution. The upper end of the distribution was chosen as a minimum upper limit given the potential impacts of strontium solution concentrations and ion-exchange capacities.

\section{A7.7.3 Alluvium}

The probability distribution for devitrified tuff will be used as a default for the strontium sorption-coefficient probability distribution in alluvium. Alluvium along the flow path is composed largely of disaggregated tuffaceous materials. Because devitrified tuff makes up a major portion of the volcanic units exposed at the surface, it should be a major component in alluvium. In addition, clays and other secondary minerals are enriched in alluvial materials. The presence of these minerals should result in higher sorption coefficients in alluvial materials compared to intact devitrified tuff.

\section{A7.8 THORIUM}

The solubility of thorium dioxide in waters such as the SZ waters is estimated at $3.2 \times 10^{-9} \mathrm{M} / \mathrm{L}$ at values of $\mathrm{pH}>6.0$ (Hummel et al. 2002 [DIRS 161904], p. 377).

\section{A7.8.1 Devitrified Tuff}

Experiments with Yucca Mountain tuffs were carried out with initial concentrations in the $1.0 \times 10^{-7}$ to $6 \times 10^{-8} \mathrm{M} / \mathrm{L}$ range. Thus, the experiments were initially oversaturated with thorium dioxide. The calculated final thorium concentrations shown in Figure A-45 indicate thorium sorption onto the rock sample brought the final solution concentrations below saturation with thorium dioxide in some cases but not all. The results of experiments oversaturated with thorium dioxide are of questionable value. For the remaining experiments, the sorption coefficients range from 1,213 to 23,800 mL/g. There are no data available for the effect of experimental duration on sorption-coefficient values for Yucca Mountain samples. However, Allard et al. (1983 [DIRS 162982], p. 10) reported that, over experiment durations of 6 hours to 6 weeks, time had little influence on the measured sorption coefficients for thorium on silica in $0.01 \mathrm{M} / \mathrm{L}$ $\mathrm{NaClO}_{4}$. Note that the starting concentrations reported by Allard et al. (1983 [DIRS 162982], p. 6) were below the saturation level for thorium dioxide. 


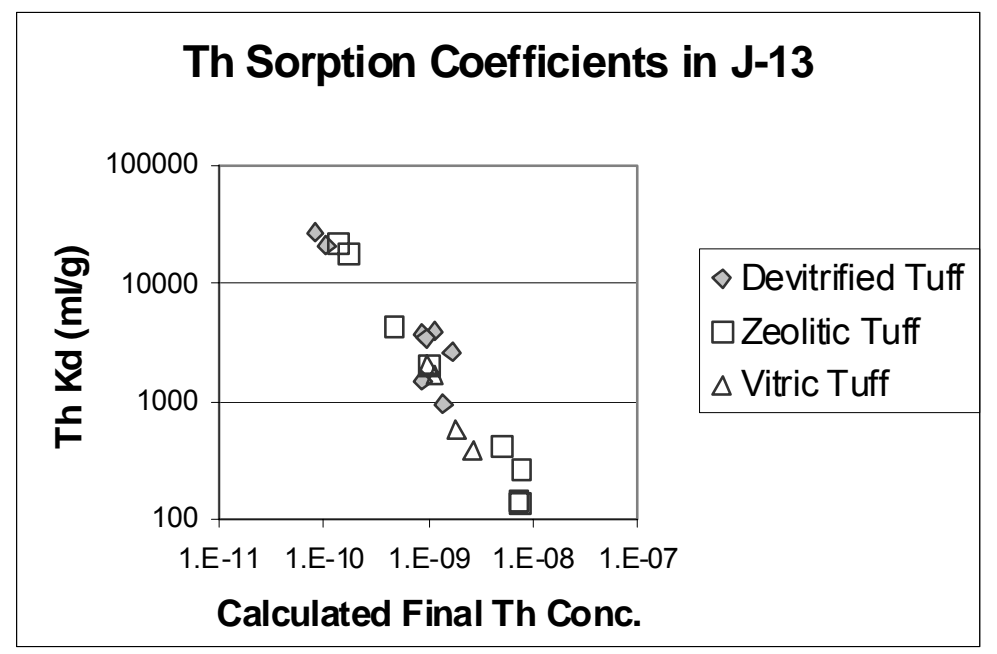

Source DTN: LA0305AM831341.001 [DIRS 163789].

Figure A-45. Thorium Sorption Coefficients on Tuff Versus Calculated Final Thorium Concentration Solution

There are no data available to evaluate the impact of variations in water chemistry on thorium sorption coefficients. However, thorium forms primarily hydroxide complexes at near neutral pH in dilute solutions (Langmuir and Herman 1980 [DIRS 147527], p. 1753). Therefore, water chemistry is expected to have very little influence on thorium sorption-coefficient values in Yucca Mountain groundwaters. However, water chemistry (i.e., $\mathrm{pH}$ ) does impact the solubility of thorium dioxide. This effect is the reason some of the experiments in Figure A-45 were oversaturated with thorium dioxide. As shown in Figure A-46, the lowest sorption coefficients were obtained at near-neutral $\mathrm{pH}$ values where $\mathrm{J}-13$ water was oversaturated with thorium dioxide.

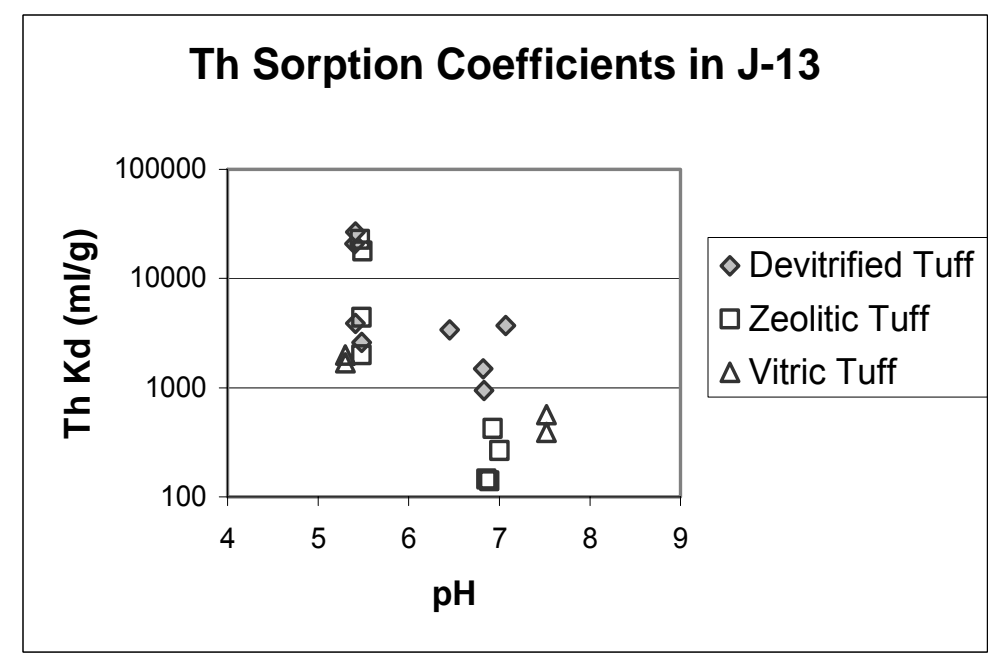

Source DTN: LA0305AM831341.001 [DIRS 163789].

Figure A-46. Thorium Sorption Coefficients on Tuff Versus pH 
On the basis of the experimental data in Figures A-45 and A-46, the range of thorium sorption coefficients expected for devitrified tuffs in the saturated volcanic section at Yucca Mountain is 1,000 to $10,000 \mathrm{~mL} / \mathrm{g}$. This range is intended to reflect the range in surface areas found in devitrified tuffs in the SZ and the range in thorium concentrations expected during SZ transport. The lower end of the range reflects sorption coefficients at thorium concentrations near the solubility limit. The probability distribution type selected is a truncated normal distribution with a mean of 5,500 mL/g and a standard deviation of $1,500 \mathrm{~mL} / \mathrm{g}$.

\section{A7.8.2 Zeolitic Tuff}

Sorption coefficient data for zeolitic tuff are also plotted in Figures A-45 and A-46. Based on the available data, zeolitic tuffs have sorption coefficients for thorium that are similar to those obtained for devitrified tuffs.

On the basis of the data plotted in Figures A-45 and A-46, the range of thorium sorption coefficients selected for zeolitic tuffs in the saturated volcanic section at Yucca Mountain is 1,000 to $10,000 \mathrm{~mL} / \mathrm{g}$. The upper end of this range was selected to reflect the higher surface areas of zeolitic tuffs relative to devitrified tuffs. The probability distribution selected is a truncated normal distribution with a mean of $5,500 \mathrm{~mL} / \mathrm{g}$ and a standard deviation of $1,500 \mathrm{~mL} / \mathrm{g}$.

\section{A7.8.3 Alluvium}

The probability distribution for devitrified tuff will be used as a default for the thorium sorption coefficient distribution in alluvium. Alluvium along the flow path is composed largely of disaggregated tuffaceous materials. Because it has been disaggregated, the surface area of alluvial material should be higher than that of devitrified tuff. In addition, clays and other secondary minerals are enriched in alluvial materials. These characteristics should result in higher sorption coefficients in alluvial materials compared to intact devitrified tuff.

\section{A7.9 URANIUM}

The solubility of uranium obtained from PHREEQC modeling with the file YMP_R3.DAT (DTN: MO0403SPAYMPR3.000 [DIRS 170937]) in J-13 water under oxidizing conditions ranges from $1.7 \times 10^{-4} \mathrm{M} / \mathrm{L}$ at a $\mathrm{pH}$ of 7.1 to $1.9 \times 10^{-4} \mathrm{M} / \mathrm{L}$ at a $\mathrm{pH}$ of 8.5 (output DTN: LA0306AM831343.001 file output/Usatj13.pun.). The solubility of uranium in synthetic $\mathrm{p \# 1}$ water under oxidizing conditions ranges from $1.8 \times 10^{-3} \mathrm{M} / \mathrm{L}$ at a $\mathrm{pH}$ of 6.7 to $2.1 \times 10^{-3}$ $\mathrm{M} / \mathrm{L}$ at a $\mathrm{pH}$ of 8.66 (output DTN: LA0306AM831343.001 file output/Usatp1c.pun.). The solubility-controlling solid in both waters is schoepite.

\section{A7.9.1 Devitrified Tuff}

As shown in Figure A-47, the calculated final uranium concentrations in the sorption experiments were generally below saturation with schoepite. The sorption coefficients obtained in experiments with devitrified tuffs do not show a correlation with the calculated final uranium solution concentrations. 


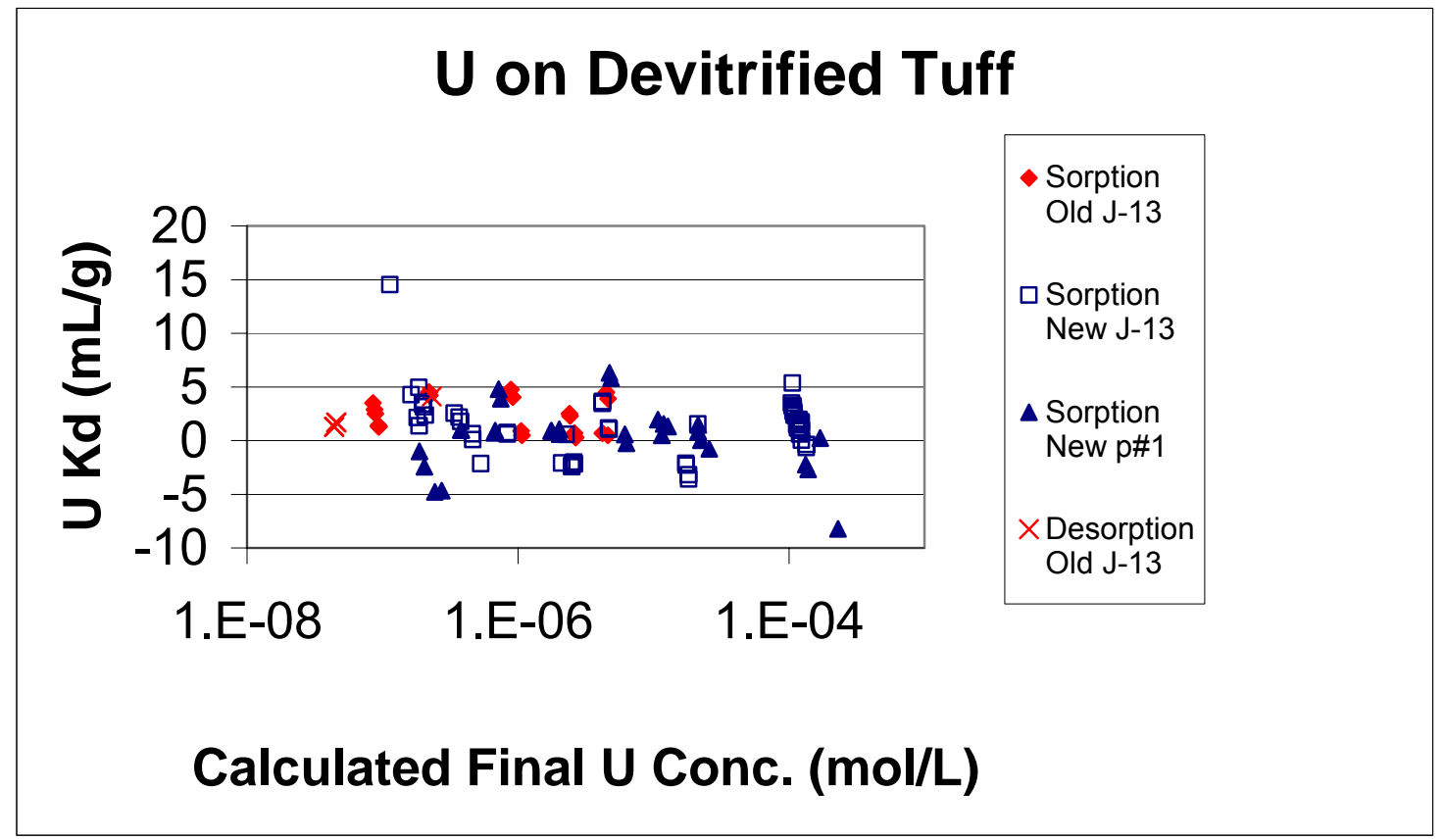

Source DTNs: LA0305AM831341.001 [DIRS 163789]; LA0407AM831341.006 [DIRS 170628].

Figure A-47. Uranium Sorption Coefficients on Devitrified Tuff Versus Calculated Final Uranium Concentration in Solution

Sorption experiments carried out as a function of time are shown in Figure A-48. Beyond approximately 3 days, there is no clear correlation between the sorption coefficients obtained and the duration of the experiments. The data imply that uranium sorption reactions on devitrified tuffs must be relatively fast (i.e., they reach steady state in a few days).

The dependence of the uranium sorption coefficient on water chemistry was tested with experiments using two water compositions (J-13 and synthetic p\#1). The J-13 experimental data are shown as a function of $\mathrm{pH}$ in Figure A-49. The "old data" were obtained in the 1980s and the "new data" in the 1990s. The difference between the two data sets is not statistically significant. The range of values obtained at a given $\mathrm{pH}$ (e.g., 8.4) reflects experimental errors and natural variations in rock properties (e.g., surface area and mineral chemistry). It is not possible to discriminate between these possible causes with the available data. On the basis of the experimental data points, there does not appear to be a correlation between $K_{\mathrm{d}}$ values and $\mathrm{pH}$. However, surface-complexation modeling with PHREEQC, using binding constants derived by Pabalan et al. (1998 [DIRS 162987], p. 124) for uranium on silica points to a clear $\mathrm{pH}$ dependence. Pabalan was the source for uranium binding constants on silica. The binding constants were used in surface complexation modeling calculations to evaluate the impact of variations in water chemistry (e.g., $\mathrm{pH}, \mathrm{J}-13$ versus $\mathrm{p \# 1}$ ) on uranium sorption coefficients). As shown in Figure A-49, the two model curves reflect two different surface areas (2.8 and 5.6 $\mathrm{m}^{2} / \mathrm{g}$ ). The $2.8 \mathrm{~m}^{2} / \mathrm{g}$ surface area is approximately an average value for devitrified tuffs at Yucca Mountain. 


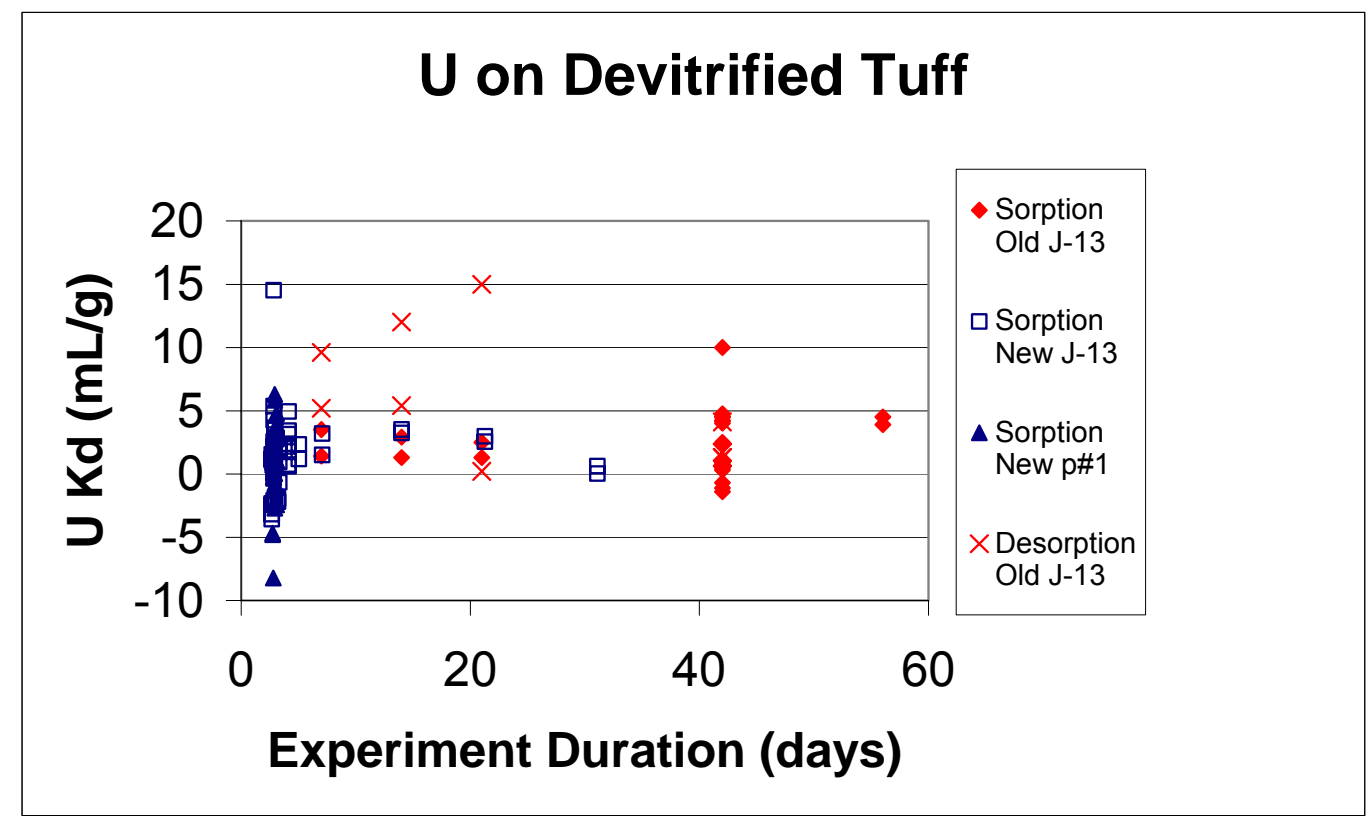

Source DTNs: LA0305AM831341.001 [DIRS 163789]; LA0407AM831341.006 [DIRS 170628].

Figure A-48. Uranium Sorption Coefficients on Devitrified Tuff Versus Experiment Duration for Sorption (Forward) and Desorption (Backward) Experiments

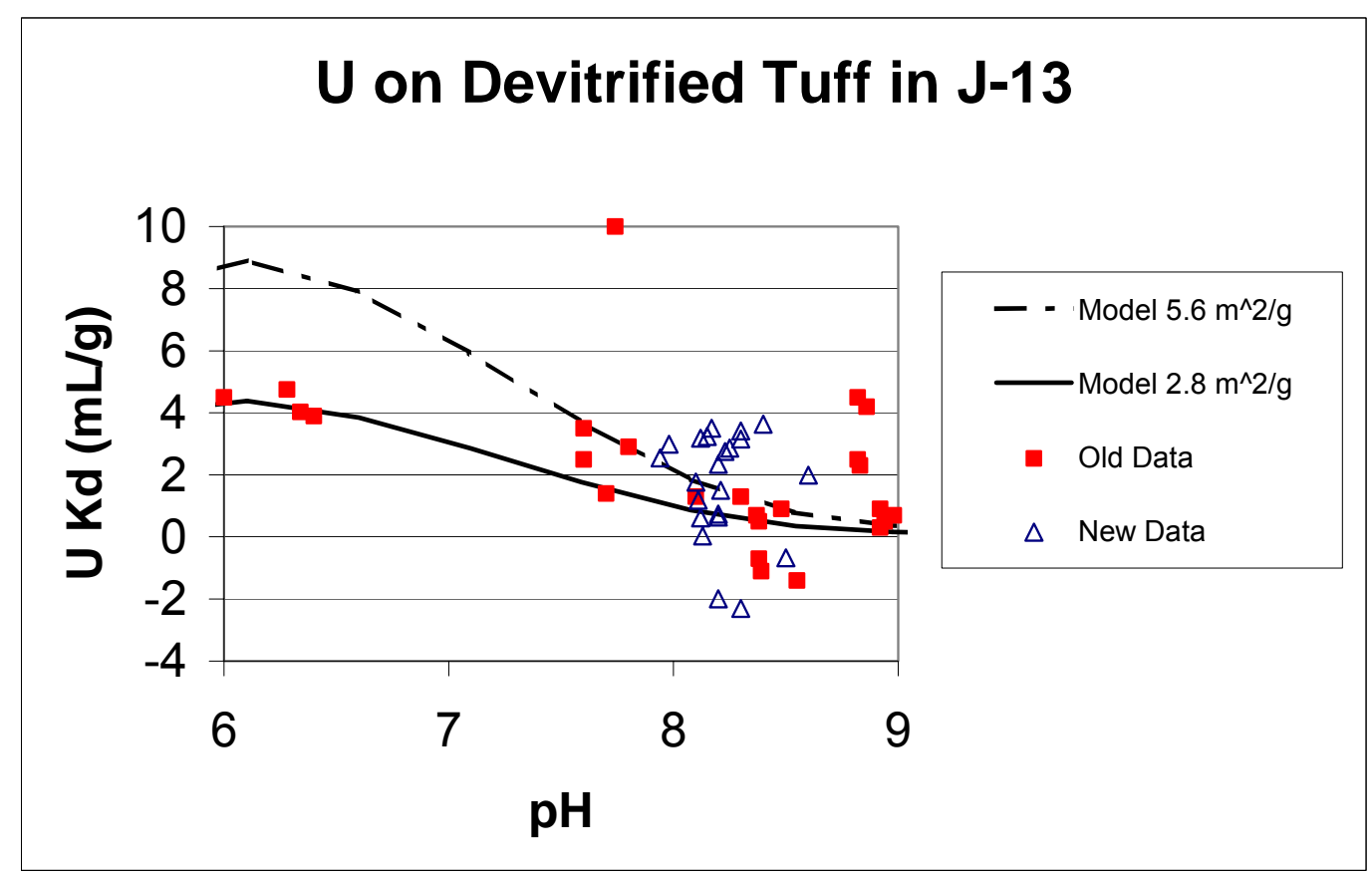

Source DTNs: LA0305AM831341.001 [DIRS 163789]; LA0407AM831341.006 [DIRS 170628].

NOTE: Model curves are from the PHREEQC surface-complexation model (output DTN: LA0306AM831343.001 file output/Udt2j13.pun).

Figure A-49. Uranium Sorption Coefficients on Devitrified Tuff Versus pH 
The sorption coefficients obtained in experiments with synthetic p\#1 water are shown in Figure A-50. The data plotted have substantial experimental errors associated with them as indicated by the magnitude of some of the negative $K_{\mathrm{d}}$ values. These experimental errors result from counting statistics, the stability of counters over time, corrections made for adsorption to container walls, and the $\mathrm{pH}$ of the tracer solution added to the experiment. Taken at face value, the experimental data suggest a trend of increasing values of $K_{\mathrm{d}}$ with increasing $\mathrm{pH}$. However, the surface complexation modeling predicts a decrease in the value of $K_{\mathrm{d}}$ with increasing $\mathrm{pH}$, although the absolute $K_{\mathrm{d}}$ values are rather small.

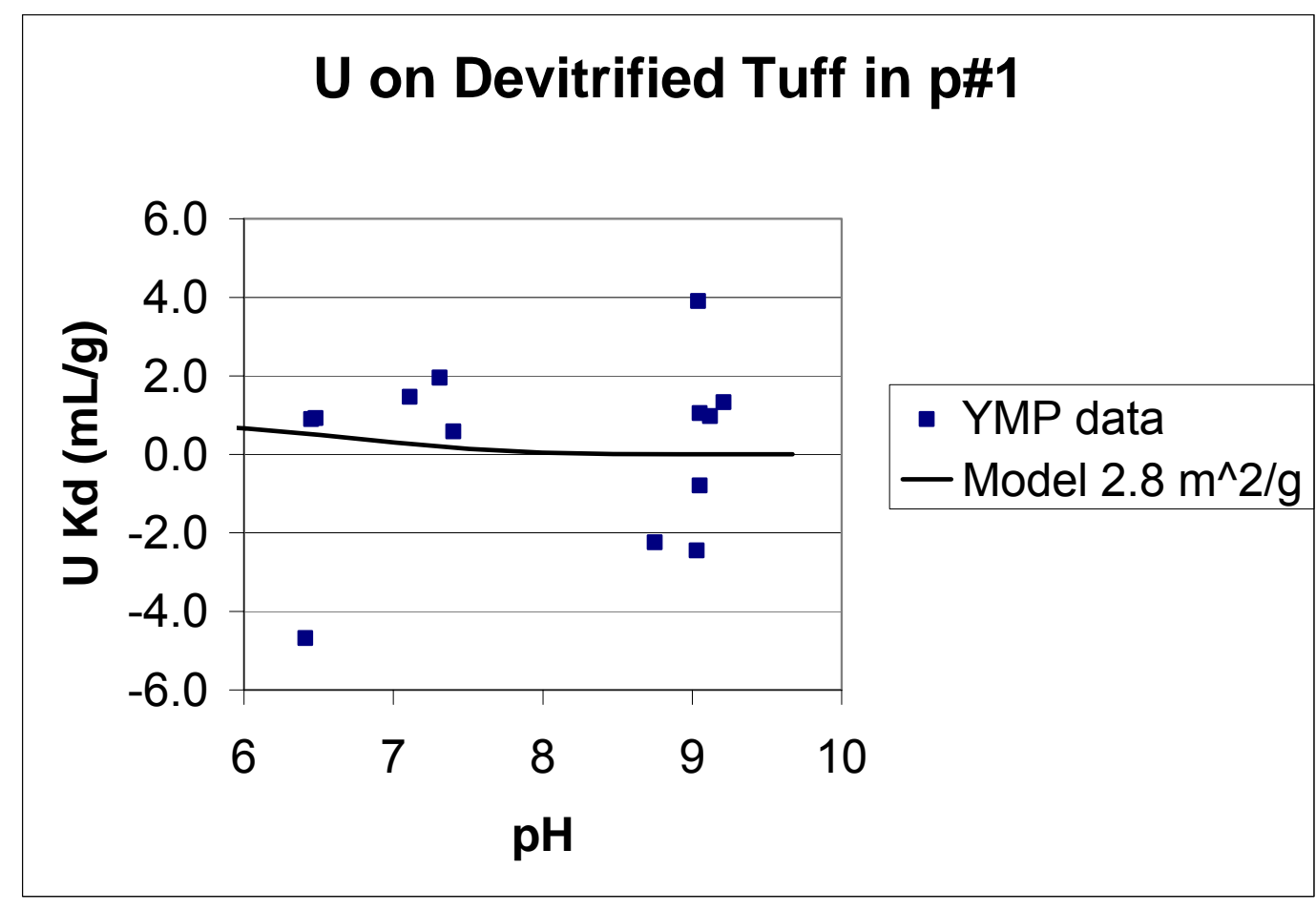

Source DTN: LA0305AM831341.001 [DIRS 163789]; LA0407AM831341.006 [DIRS 170628].

NOTE: Model curve is from the PHREEQC surface-complexation model (output DTN: LA0306AM831343.001 file output/Udtp1.pun).

Figure A-50. Uranium Sorption Coefficients on Devitrified Tuff in $\mathrm{p \# 1} \mathrm{(Volcanics)} \mathrm{Water} \mathrm{Versus} \mathrm{pH}$

On the basis of the experimental data and model curves plotted in Figures A-49 and A-50, a truncated normal distribution was selected for the uranium sorption-coefficient probability distribution for devitrified tuff in the saturated volcanic section with a range of 0 to $4 \mathrm{~mL} / \mathrm{g}$, a median of $2 \mathrm{~mL} / \mathrm{g}$, and a standard deviation of $0.6 \mathrm{~mL} / \mathrm{g}$.

\section{A7.9.2 Zeolitic Tuff}

As shown in Figure A-51, the sorption coefficients obtained in experiments with zeolitic tuffs do not show a clear correlation with the calculated final uranium solution concentrations. The high end of the concentrations plotted is below saturation with a solid uranium phase. 


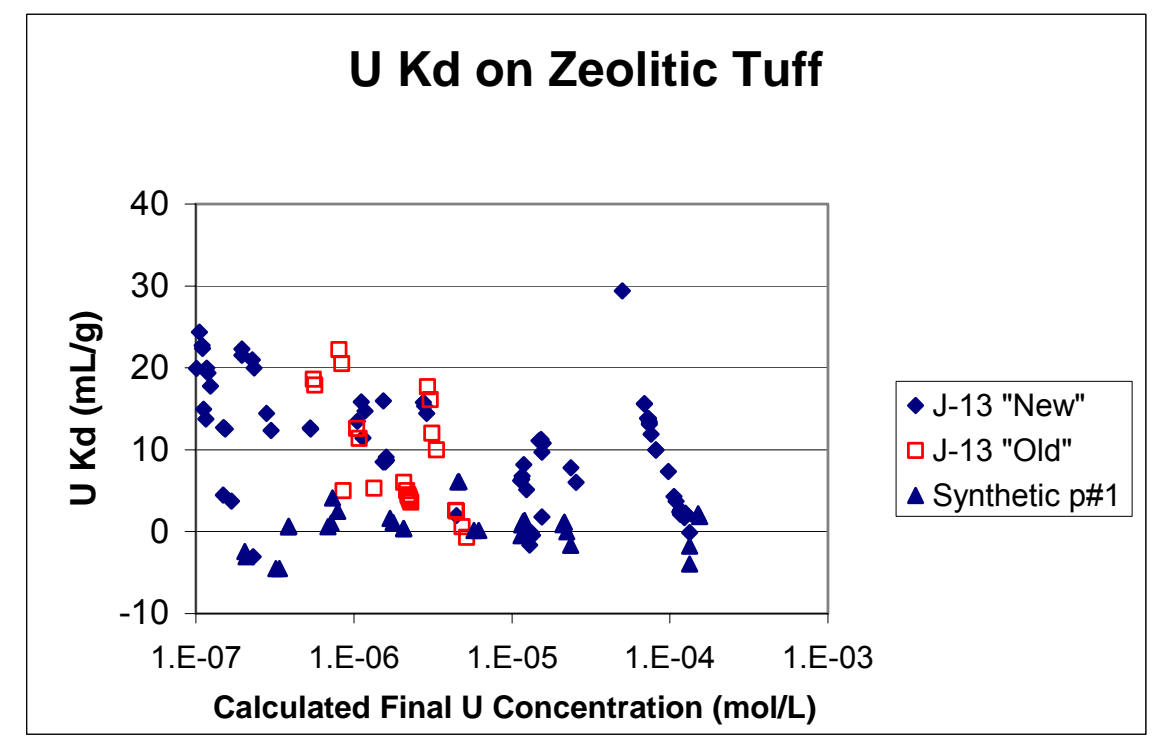

Source DTNs: LA0305AM831341.001 [DIRS 163789]; LA0407AM831341.006 [DIRS 170628].

Figure A-51. Uranium Sorption Coefficients on Zeolitic Tuff Versus Calculated Final Uranium Concentration in Solution

Uranium sorption experiments on zeolitic tuffs carried out as a function of time are shown in Figure A-52. Beyond a period of approximately 3 days, there is no clear correlation between the sorption coefficients obtained and the duration of the experiments. These data imply that uranium sorption reactions on zeolitic tuffs are relatively fast (i.e., they reach steady state in a few days).

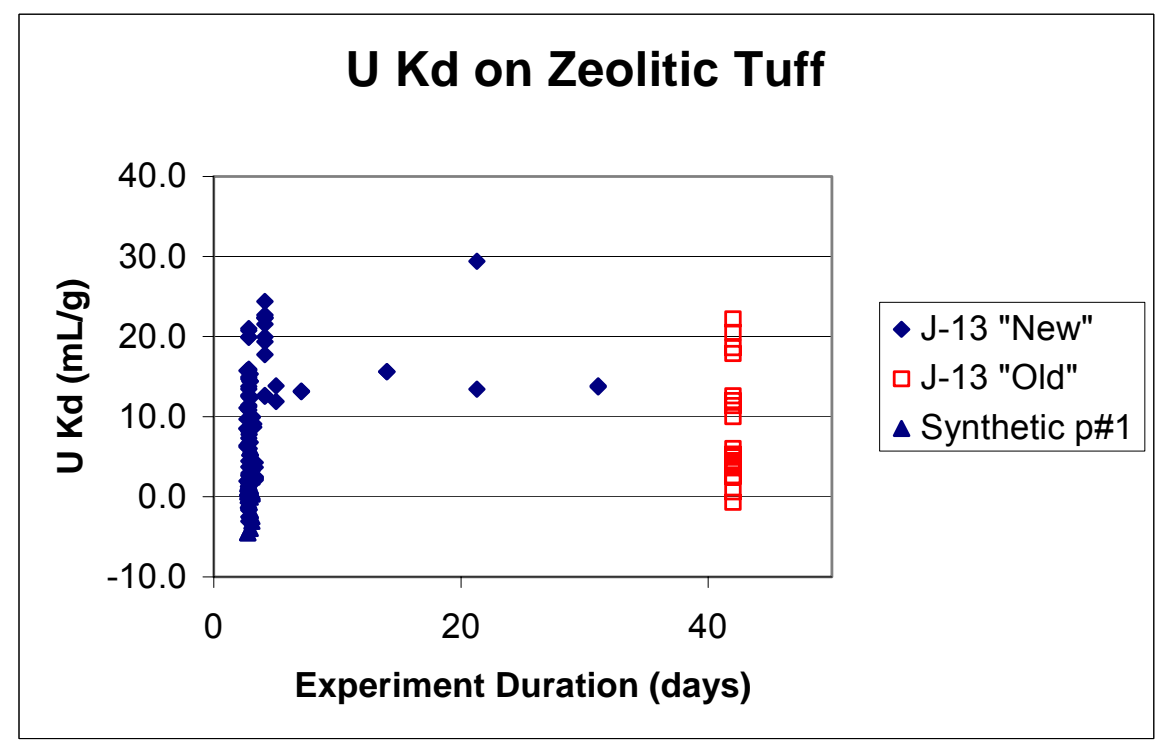

Source DTNs: LA0305AM831341.001 [DIRS 163789]; LA0407AM831341.006 [DIRS 170628].

Figure A-52. Uranium Sorption Coefficients on Zeolitic Tuff as a Function of Experiment Duration 
The dependence of the uranium sorption coefficient on water chemistry was tested with experiments using two water compositions (J-13 and synthetic p\#1). The J-13 data are shown as a function of $\mathrm{pH}$ in Figure A-53. The "old data" were obtained in the 1980s and the "new data" in the 1990s. The difference between the two data sets is not statistically significant. The range of values observed at a given $\mathrm{pH}$ (e.g., 8.4) reflects variations in rock properties and experimental errors. The experimental errors result from such things as counting statistics, the stability of counters over time, the accuracy of corrections for adsorption to container walls, and other experimental artifacts. In some cases, the $\mathrm{pH}$ of the tracer solution added to the experiment seems to have an effect. Some of these errors are random (e.g., counting errors) and others (e.g., adsorption to container walls) may have a nonrandom bias. It is not possible to evaluate these errors separately with the available information. Note that the distribution of data points in Figure A-53 does not indicate a correlation between the values of $K_{\mathrm{d}}$ and $\mathrm{pH}$.

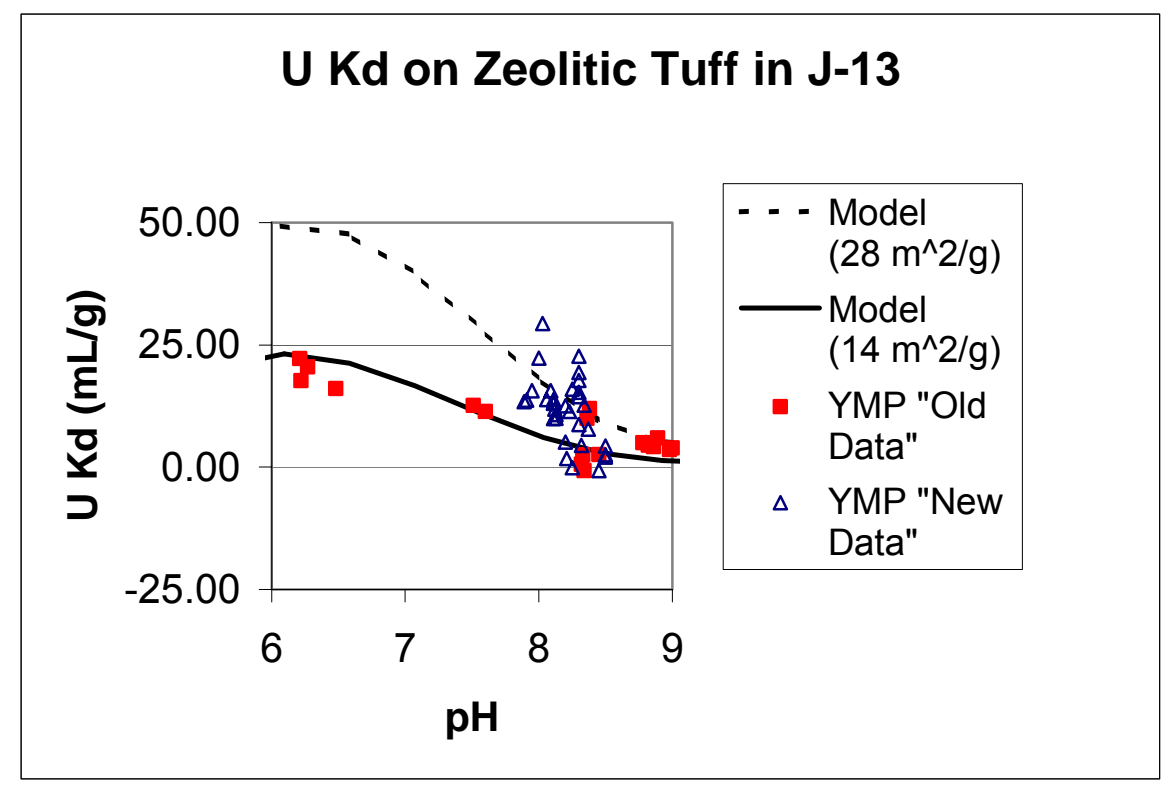

Source DTNs: LA0305AM831341.001 [DIRS 163789]; LA0407AM831341.006 [DIRS 170628].

NOTE: Model curves derived with PHREEQC surface-complexation modeling are also shown (output DTN: LA0306AM831343.001 file output/ Uzeoj13.pun).

Figure A-53. Uranium Sorption Coefficients for Zeolitic Tuff in $\mathrm{J}-13$ Water Plotted as a Function of $\mathrm{pH}$

Surface-complexation modeling was carried out with PHREEQC with the thermodynamic input data file YMP_R3.DAT (DTN: MO0403SPAYMPR3.000 [DIRS 170937]) to provide a framework in which to interpret the experimental data. Binding constants for uranium on silica derived by Pabalan et al. (1998 [DIRS 162987], p. 124) were used in the modeling. Pabalan was the source for uranium binding constants on silica. The binding constants were used in surface complexation modeling calculations to evaluate the impact of variations in water chemistry (e.g., $\mathrm{pH}, \mathrm{J}-13$ versus $\mathrm{p} \# 1$ on uranium sorption coefficients). The modeling results show a clear $\mathrm{pH}$ dependence (Figure A-53). The two model curves reflect two different surface areas. A surface area of $28 \mathrm{~m}^{2} / \mathrm{g}$ was used because it is approximately an average value for zeolitic tuffs and because it is an order of magnitude larger than the average value used for modeling 
devitrified tuffs. A surface area of $14 \mathrm{~m}^{2} / \mathrm{g}$ was also used to show the impact of factor of 2 change in surface area.

The sorption coefficients obtained in experiments with synthetic p\#1 water are shown in Figure A-54. The magnitudes of the negative $K_{\mathrm{d}}$ values plotted are similar to the magnitudes of the positive values plotted. Thus, the net values may be very close to zero. Taken at face value, the experimental data suggest a trend of increasing $K_{\mathrm{d}}$ values with increasing $\mathrm{pH}$. However, the surface complexation modeling predicts an increase in $K_{\mathrm{d}}$ with decreasing $\mathrm{pH}$, although the absolute $K_{\mathrm{d}}$ values are rather small in agreement with the net values obtained from the experimental data.

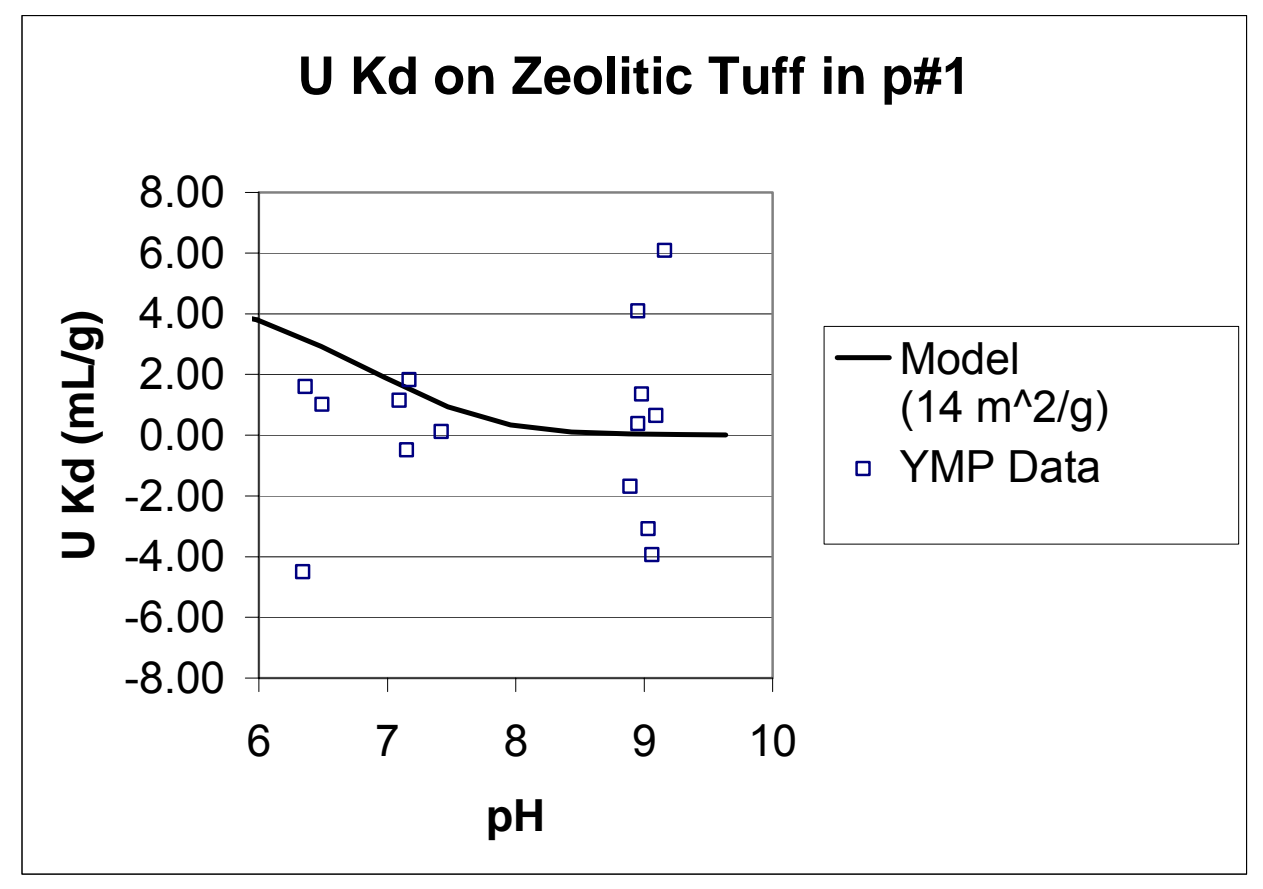

Source DTNs: LA0305AM831341.001 [DIRS 163789]; LA0407AM831341.006 [DIRS 170628].

NOTE: Model curves derived with PHREEQC surface-complexation modeling are also shown (output DTN: LA0306AM831343.001 file output/ Uzeop1.pun).

Figure A-54. Uranium Sorption Coefficients for Zeolitic Tuff in Synthetic $p \# 1$ Water as a Function of $p H$

On the basis of the experimental data and model curves plotted in Figures A-53 and A-54, a truncated normal distribution was selected for the uranium sorption-coefficient probability distribution for zeolitic tuff in the saturated volcanic section with a range of 5 to $20 \mathrm{~mL} / \mathrm{g}$, a median of $12 \mathrm{~mL} / \mathrm{g}$, and a standard deviation of $3.6 \mathrm{~mL} / \mathrm{g}$ (output DTN: LA0310AM831341.002). This distribution emphasizes the J-13 water chemistry over the p\#1 water chemistry. 


\section{A7.9.3 Alluvium}

Sorption coefficients for uranium were measured in batch experiments on cores obtained from three wells (10SA, 22SA, and 19IM1A) drilled into the alluvium (DTN: LA0302MD831341.004 [DIRS 163785]). Following a procedure identical to that used for neptunium, described in Section A7.3.3 of this report, the $K_{\mathrm{d}}$ values for uranium were taken to be described by a $t$ distribution with the following statistics: $\mu_{\mathrm{U}}=4.6 \mathrm{~mL} / \mathrm{g}, \sigma_{\mathrm{U}}=0.58 \mathrm{~mL} / \mathrm{g}$.

Although the $t$ distribution was thought to capture correctly the spatial uncertainty, the question about the representativeness of the experiment does introduce a small amount of additional uncertainty, as discussed for the case of neptunium in Section A7.3.3 of this report. Hence, a cumulative distribution was chosen for uranium. The minimum experimentally observed value was $1.7 \mathrm{~mL} / \mathrm{g}$. Five percent probability was uniformly distributed between $1.7 \mathrm{~mL} / \mathrm{g}$ and the lower bound of the $t$ distribution at $2.9 \mathrm{~mL} / \mathrm{g}$. Since the $t$ distribution had such little variation, it was reasonably represented by a uniform distribution. Hence, 90 percent probability was uniformly distributed between $2.9 \mathrm{~mL} / \mathrm{g}$ and the upper bound of the $t$ distribution at $6.3 \mathrm{~mL} / \mathrm{g}$. The remaining 5 percent probability was uniformly distributed between $6.3 \mathrm{~mL} / \mathrm{g}$ and the maximum experimentally observed value of $8.9 \mathrm{~mL} / \mathrm{g}$.

\section{A8. SUMMARY}

Sorption-coefficient probability distribution functions were derived for the radionuclides of $U$, $\mathrm{Cs}, \mathrm{Np}, \mathrm{Pu}, \mathrm{Am}, \mathrm{Th}, \mathrm{Sr}, \mathrm{Pa}$, and Ra. These are summarized in Table A-4. Experimental and modeling results were used to constrain the distributions. In general, the approach used in the derivation of the distributions tended to underestimate the range and median or mean. This approach was used to provide some conservatism in the derivation given potential scaling uncertainties in the application of these distributions to transport calculations at the Yucca Mountain site.

Table A-4. Small-Scale Probability Distribution Functions for $K_{d} \mathrm{~S}$ in the Saturated Zone Developed on the Basis of Laboratory Data from Core Samples

\begin{tabular}{|c|c|c|l|}
\hline Species & Unit/Analysis & Distribution & \multicolumn{1}{|c|}{$\begin{array}{c}\text { Coefficients Describing Distribution } \\
(\mathbf{m L} / \mathbf{g})\end{array}$} \\
\hline$U$ & Zeolitic & Normal & range $=5-20 ; \mu=12, \sigma=3.6$ \\
\hline & Devitrified & Normal & range $=0-4 ; \mu=2, \sigma=0.6$ \\
\hline & Alluvium & Cumulative & $\left(K_{d}\right.$, prob) $(1.7,0).(2.9,0.05)(6.3,0.95)(8.9,1.0)$ \\
\hline $\mathrm{Np}$ & Zeolitic & Normal & range $=0-6 ; \mu=2.88, \sigma=1.47$ \\
\hline & Devitrified & Exponential & range $=0-2 ; \mu=0.69, \sigma=0.707$ \\
\hline $\mathrm{Pu}$ & Alluvium & Cumulative & $\left(K_{d}\right.$, prob $)(1.8,0).(4.0,0.05)(8.7,0.95)(13,1.0)$ \\
\hline & Zeolitic & Beta & range $=50-300 ; \mu=100, \sigma=15$ \\
\hline & Devitrified & Beta & range $=50-300 ; \mu=100, \sigma=15$ \\
\hline Cs & Alluvium & Beta & range $=50-300 ; \mu=100, \sigma=15$ \\
\hline & Zeolitic & Exponential & range $=4,000-42,000 ; \mu=16,942: \sigma=14,930$. \\
\hline
\end{tabular}


Table A-4. Small-Scale Probability Distribution Functions for $K_{d} \mathrm{~s}$ in the Saturated Zone Developed on the Basis of Laboratory Data from Core Samples (Continued)

\begin{tabular}{|c|c|c|c|}
\hline Species & Unit/Analysis & Distribution & $\begin{array}{c}\text { Coefficients Describing Distribution } \\
(\mathrm{mL} / \mathrm{g})\end{array}$ \\
\hline & Alluvium & Cumulative & range $=100-1,000 ; \mu=728, \sigma=464$ \\
\hline \multirow[t]{3}{*}{ Am } & Zeolitic & Truncated Norm & range $=1,000-10,000 ; \mu=5,500, \sigma=1500$ \\
\hline & Devitrified & Truncated Norm & range $=1,000-10,000 ; \mu=5,500, \sigma=1,500$ \\
\hline & Alluvium & Truncated Norm & range $=1,000-10,000 ; \mu=5,500, \sigma=1,500$ \\
\hline \multirow[t]{3}{*}{$\mathrm{Pa}$} & Zeolitic & Truncated Norm & range $=1,000-10,000 ; \mu=5,500, \sigma=1500$ \\
\hline & Devitrified & Truncated Norm & range $=1,000-10,000 ; \mu=5,500 \sigma=1,500$ \\
\hline & Alluvium & Truncated Norm & range $=1,000-10,000 ; \mu=5,500, \sigma=1,500$ \\
\hline \multirow[t]{3}{*}{$\mathrm{Sr}$} & Zeolitic & Cumulative & $\left(K_{d}\right.$, prob $)(100,0)(5,000,0.5)(90,000,1.0)$ \\
\hline & Devitrified & Uniform & range $=20-400$ \\
\hline & Alluvium & Uniform & range $=20-400$ \\
\hline \multirow[t]{3}{*}{ Th } & Zeolitic & Truncated Norm & $\begin{array}{l}\text { range }=1,000-10,000 ; \mu=5,500, \sigma=1,500 \text { (for a } \\
\text { symmetric distribution and a min and max at }+/-3 \sigma\end{array}$ \\
\hline & Devitrified & Truncated Norm & $\begin{array}{l}\text { range }=1,000-10,000 ; \mu=5,500, \sigma=1,500 \text { (for a } \\
\text { symmetric distribution and a min and max at }+/-3 \sigma\end{array}$ \\
\hline & $\begin{array}{c}\text { Alluvium (same as } \\
\text { devitrified) }\end{array}$ & Truncated Norm & $\begin{array}{l}\text { range }=1,000-10,000 ; \mu=5,500, \sigma=1,500 \text { (for a } \\
\text { symmetric distribution and a min and max at }+/-3 \sigma\end{array}$ \\
\hline \multirow[t]{3}{*}{$\mathrm{Ra}$} & Zeolitic & $\begin{array}{l}\text { Truncated Log- } \\
\text { normal }\end{array}$ & range $=1,000-250,000 ; \mu=100,000, \sigma=31,420$ \\
\hline & Devitrified & Uniform & range $=100-1,000$ \\
\hline & Alluvium & Uniform & range $=100-1,000$ \\
\hline $\mathrm{C} / \mathrm{Tc} / \mathrm{l}$ & Volcanics/Alluvium & Constant & Zero \\
\hline
\end{tabular}

$\mu=$ mean

$\sigma=$ standard deviation

Output DTN: LA0310AM831341.002.

\section{A9. CORRELATIONS FOR SAMPLING OF SORPTION-COEFFICIENT PROBABILITY DISTRIBUTIONS}

In the TSPA, SZ transport calculations are carried out separately for each radionuclide. The sorption-coefficient probability distribution for each radionuclide could be sampled independently in each transport calculation. However, such independent sampling could potentially lead to dose dilution. That is, independent sampling of the distributions could cause radionuclides to travel at independent rates such that the calculated dose at the accessible environment is not representative of maximum possible doses. Similarities in the chemical dependencies of sorption coefficients for the various radionuclides suggest transport rates in the SZ are likely to be correlated for some radionuclides. These correlations are summarized in Table A-5. 
Table A-5. Recommended SZ $K_{d}$ Correlations

\begin{tabular}{|l|l|l|}
\hline \multicolumn{1}{|c|}{ Species } & Unit/Analysis & \multicolumn{1}{c|}{ Correlation } \\
\hline $\mathrm{U}$ & Correlation & $75 \%$ correlation composite (volcanics)/alluvium and $50 \%$ correlation $\mathrm{Np}$ \\
\hline $\mathrm{Np}$ & Correlation & $75 \%$ correlation composite (volcanics)/alluvium and $50 \%$ correlation $\mathrm{U}$ \\
\hline $\mathrm{Pu}$ & Correlation & $50 \%$ correlation composite (volcanics)/alluvium \\
\hline $\mathrm{Cs}$ & Correlation & None \\
\hline $\mathrm{Am} / \mathrm{Th} / \mathrm{Pa}$ & Correlation & $100 \%$ \\
\hline $\mathrm{Sr}$ & Correlation & None \\
\hline $\mathrm{Ra}$ & Correlation & None \\
\hline $\mathrm{C} / \mathrm{Tc} / \mathrm{l}$ & Correlation & $100 \%$ \\
\hline
\end{tabular}

Output DTN: LA0309AM831341.001.

Correlations for sampling sorption-coefficient probability distributions have been derived for the elements americium, neptunium, protactinium, plutonium, thorium, and uranium. The elements americium, protactinium, and thorium sorb primarily by surface-complexation mechanisms and generally have a high affinity for silicate surfaces. In Section A7, the same sorption-coefficient probability distribution $\left(K_{d}=1,000-10,000 \mathrm{~mL} / \mathrm{g}\right)$ has been chosen for all three of these elements. Thus, they are 100 percent correlated by definition. The elements carbon, iodine, and technetium are also 100 percent correlated in that the sorption coefficient is always zero for all three of these elements.

Separate sorption-coefficient probability distributions were derived for neptunium in volcanics and alluvium in Section A7. However, controls on the sorption behavior of neptunium are likely to be similar in volcanics and alluvium, although not identical. A 75 percent correlation has been chosen for sampling of the neptunium sorption coefficients in volcanics and alluvium. The same arguments apply to uranium. Thus, a 75 percent correlation has also been chosen for sampling of the uranium sorption coefficients in volcanics and alluvium. The controls on the sorption behavior of neptunium and uranium are similar but not identical as discussed in Section A7. To account for the similarities, a correlation of 50 percent was chosen for sampling sorption-coefficient distributions for neptunium and uranium. Finally, a correlation of 50 percent was chosen for sampling sorption-coefficient distributions for plutonium in volcanics and alluvium. In the volcanics, the applicable sorption-coefficient distribution is the composite distribution (Appendix C), whereas in the alluvium the sorption-coefficient probability distribution for devitrified tuff was chosen as a default in Section A7. 


\section{APPENDIX B \\ THE COLLOID-FACILITATED TRANSPORT OF REVERSIBLY -ATTACHED RADIONUCLIDES}


The analysis presented in this appendix supports the model component of the SZ Transport model that deals with colloid-facilitated transport of radionuclides that are attached reversibly to the colloids (Section 6.4.2.6). The results of this analysis do not form a direct input to the site-scale SZ transport model.

In general, the majority of radionuclides that sorb to colloids are irreversibly sorbed, with a typical percentage being 90 percent irreversible and 10 percent reversible (BSC 2004 [DIRS 170025]). Radionuclides that are irreversibly sorbed are typically embedded in the colloid or are so strongly sorbed onto the colloid that there is no possibility of detachment for typical transport time scales (thousands of years) through the SZ. On the other hand, reversibly sorbed colloids have measurable desorption rates.

The SZ transport simulations of radionuclides that are irreversibly attached to colloids are conducted for radioisotopes of $\mathrm{Pu}$ and $\mathrm{Am}$. Most radionuclides are taken to sorb onto colloids reversibly (that is, they have measurable desorption rates and can be entirely desorbed from colloids) as shown in Saturated Zone Colloid Transport (BSC 2004 [DIRS 170006], Section 6.3). However, Pu and Am can sorb either reversibly or irreversibly onto colloids with the relative percentages of these obtained from an analysis of waste form degradation and waste package corrosion processes occurring in the repository near-field environment as shown in Saturated Zone Colloid Transport (BSC 2004 [DIRS 170006], Section 6.3.). In general, the majority of the $\mathrm{Pu}$ and Am sorbed to colloids is irreversibly sorbed, with a typical percentage being 90 to 99 percent irreversible and 1 to 10 percent reversible as shown in Waste Form and In-Drift Colloids-Associated Radionuclide Concentrations: Abstraction and Summary (BSC 2004 [DIRS 170025], Section 6.3.3.2). The irreversibly sorbed radionuclides are taken to transport in a manner identical to the colloids onto which they are sorbed. The colloid retardation factor distributions for irreversible colloids are developed in Saturated Zone Colloid Transport (BSC 2004 [DIRS 170006]), so only a brief description is provided here. The transport of the colloids is simulated using the advection-dispersion equation, and colloids are taken to not diffuse. Several field observations have suggested that a small percentage of colloids transport with essentially no retardation in ground water (Kersting et al. 1999 [DIRS 103282], p. 56, 58; Penrose et al. 1990 [DIRS 100811], p. 228), whereas the majority undergoes either reversible or irreversible filtration, which can be described by a retardation factor, $R_{\mathrm{col}}$. In this analysis, filtration is defined as the net effect of chemical sorption of the colloid onto the rock surface and the physical removal of colloids from the advective flow due to sieving and settling. The value of $R_{\text {col }}$ is dependent on several factors such as colloid size, colloid type, and geochemical conditions (e.g., $\mathrm{pH}$, Eh, and ionic strength). These factors are folded into the distribution of $R_{\text {col }}$ that has been developed from field and experimental data collected under varying geochemical conditions with different colloid types and sizes as shown in Saturated Zone Colloid Transport (BSC 2004 [DIRS 170006] Section 6.4.2). Attachment rate constants, $k_{\text {att }}$, detachment rate constants, $k_{\text {det }}$, of colloids to the rock matrix and $R_{\text {col }}$ distributions are related by:

$$
R_{\text {col }}=1+\frac{k_{\mathrm{att}}}{k_{\mathrm{det}}}
$$


The attachment rate constant is also used to determine the fraction of the colloids that transport with no retardation. Specifically, colloids for which one over the attachment rate constant is smaller than the travel time through the system will transport with no retardation. The development of $R_{\text {col }}$ distributions for the volcanics and the alluvium as well as the fraction of colloids that transport unretarded is documented in Saturated Zone Colloid Transport (BSC 2004 [DIRS 170006]).

The SZ transport simulations of radionuclides that are reversibly attached to colloids are conducted for radioisotopes of $\mathrm{Pu}, \mathrm{Am}$, Th, $\mathrm{Pa}$, and $\mathrm{Cs}$ (the rationale for selection of these radionuclides is given in Waste Form and In-Drift Colloids-Associated Radionuclide Concentrations: Abstraction and Summary (BSC 2004 [DIRS 170025], Section 6.3.3.1). Note that the Pu and Am inventories are split into reversibly and irreversibly sorbed fractions. For the reversibly sorbed transport simulations, radioisotopes of $\mathrm{Pu}$ are transported as one group, radioisotopes of Am, Th, and $\mathrm{Pa}$ are transported as a second group, and Cs is transported as a third group. Am, Th, and Pa are being treated as a single group based on the similarity in their absorption characteristics-each of these three radionuclides are strongly sorbed to surfaces generally involving the $\mathrm{OH}$ group, and each of them displays a single valance state in solution and sorption (Allard et al. 1983 [DIRS 162982] pp. 9, 10, and 12). The radionuclides that are reversibly absorbed onto colloids are modeled using the $K_{\mathrm{c}}$ model, which represents the equilibrium partitioning of radionuclides between the aqueous phase and the colloidal phase with the distribution coefficient $K_{\mathrm{c}}$ (Robinson et al. 1997 [DIRS 100416], Equation 8-10, pp. 8-35). The $K_{\mathrm{c}}$ model is a simplified colloid transport model that applies under the following conditions:

1. The contaminant sorbs reversibly to the colloids in addition to interacting with the rock matrix and fractures.

2. The colloids are isolated to the fracture and possess dispersive properties equivalent to that of an aqueous solute.

3. The colloids interact reversibly with the rock surface.

4. Colloids exist throughout the flow system.

Under these conditions, a transport equation for contaminant attached to colloids can be written as

$$
R_{\mathrm{col}} \frac{\partial C_{c}}{\partial t}=D_{z} \frac{\partial^{2} C_{c}}{\partial z^{2}}-v \frac{\partial C_{c}}{\partial z}
$$

where

$C_{c}$ is the concentration of contaminants attached to colloids

$R_{\text {col }}$ is a retardation factor that captures the details of colloid attachment/detachment and reversible filtration processes

$v \quad$ is the pore-water velocity 
$t$ is time

$z \quad$ is the spatial coordinate

$\mathrm{Dz}$ is the coefficient of longitudinal dispersion.

Note that Eq. B-2 represents a mass balance of solute sorbed to colloids, not to colloids alone.

The concentration of contaminants attached to the colloids is related to the aqueous contaminant concentration $C$ using a linear relationship:

$$
C_{c}=K_{c} C
$$

where $K_{\mathrm{c}}$ is the distribution parameter relating the concentrations. Since both $C$ and $C_{\mathrm{c}}$ are expressed as moles contaminant per unit fluid volume, $K_{\mathrm{c}}$ is a dimensionless parameter expressing the ratio of contaminant mass residing on colloids to the mass present in aqueous form. In terms of more commonly defined and measured quantities, $K_{\mathrm{c}}$ is the product of the distribution coefficient for contaminant sorbing onto colloids $\left(K_{\mathrm{d}(\mathrm{colloids})}\right)$ and the concentration of colloidal material available for sorption-mass of colloids per unit fluid volume $\left(C_{\mathrm{d} \text { (colloids) }}\right)$ :

$$
K_{c}=K_{\text {d(colloids) }} C_{\text {colloids }}
$$

Note that the $C_{\text {colloids }}$ term includes the concentration of colloids in the aqueous phase and sorbed to the rock. The transport equation for the contaminant transporting in the aqueous phase is given by:

$$
R_{f} \frac{\partial C}{\partial t}=D_{z} \frac{\partial^{2} C}{\partial z^{2}}-v \frac{\partial C}{\partial z}
$$

where $R_{\mathrm{f}}$ is a retardation factor that captures details of radionuclide attachment/detachment. By combining Equations B-2 and B-3 and making use of Equation B-5, the concentration of the contaminant in the aqueous phase for the fracture can be obtained:

$$
\left(\frac{R_{f}+K_{c} R_{c o l}}{1+K_{c}}\right) \frac{\partial C}{\partial t}=D_{z} \frac{\partial^{2} C}{\partial z}-v \frac{\partial C}{\partial z}
$$

The coupling between the fracture and matrix is identical to the case without colloids if there is no mobility of colloids into the rock matrix. Inspection of Equations B-5 and B-6 reveals that the forms of the transport equations are identical, with different constants in the accumulation loss term. Therefore, the solutions developed thus far need only be modified slightly to include colloid contaminant transport. To do this, a revised constant $\hat{R}_{f}$ is defined as follows:

$$
\hat{R}_{f}=\frac{R_{f}+K_{c} R_{c o l}}{1+K_{c}}
$$

Using $\hat{R}_{f}$ allows us to consider colloidal transport using the advection-dispersion equation. 


\section{INTENTIONALLY LEFT BLANK}




\section{APPENDIX C \\ DERIVATION OF COMPOSITE UPSCALED $K_{d}$ DISTRIBUTIONS}


This appendix provides details of the study performed to derive composite distributions applicable on the scale of the computational grid used in the site-scale SZ transport model for the radionuclides $\mathrm{Cs}, \mathrm{Np}, \mathrm{Pu}, \mathrm{U}, \mathrm{Am}, \mathrm{Th}, \mathrm{Pa}, \mathrm{Sr}$, and $\mathrm{Ra}$. The small scale distributions developed in Appendix A (Tables A-4 and A-5) in conjunction with the analysis presented in this appendix led to the recommended composite distributions for $K_{d}$ s (Tables C-14 and A-5) that form a technical product output of this report (output DTN: LA0310AM831341.002). The data needed as inputs to the analysis presented in this appendix are treated as being "direct input" to the sitescale SZ transport model. These distributions will be used to simulate transport of radionuclides in the SZ site-scale model during the TSPA calculations. The distributions in alluvium for these radionuclides were taken to be the same as the small-scale distributions derived in Appendix A (Table A-4). Given the scope of the work presented here, simulations could not be carried out for all radionuclides, and a choice had to be made. The four radionuclides $\mathrm{Cs}, \mathrm{Np}, \mathrm{Pu}$, and $\mathrm{U}$ were chosen so as to approximately bracket the expected range of values. The distributions in volcanics for Am, Th, Pa, Sr, and Ra were taken to be the same as those in Table A-4. The distributions in volcanics for $\mathrm{Cs}, \mathrm{Np}, \mathrm{Pu}$, and $\mathrm{U}$ were generalized using stochastic modeling (Section C1 of this appendix) to a scale of $500 \mathrm{~m}$, and then modified to include additional uncertainties leading to composite distributions (Section C2 of this appendix) for use with the TSPA calculations.

In the TSPA calculations, radionuclide transport is modeled using a single value of $K_{\mathrm{d}}$ for grid blocks with dimensions 500 meters $(\mathrm{m}) \times 500 \mathrm{~m}$ in the $x$ and $y$ directions. In the field, values of $K_{\mathrm{d}}$ are variable at a scale much smaller than $500 \mathrm{~m}$. Thus, if a uniform single value of $K_{\mathrm{d}}$ is used to model sorption, it is important to use a value that effectively captures variability at a smaller scale and results in the same sorption behavior as if all the small-scale processes were represented explicitly. The factors that affect the sorption behavior of the rock matrix include mineral composition, groundwater chemistry, and the type of radionuclide. Mineral composition and groundwater chemistry are spatially variable at a scale smaller than $500 \mathrm{~m}$. The small-scale $K_{\mathrm{d}}$ distributions for these radionuclides were developed on the basis of laboratory data. This development is given in detail in Appendix A, and the small-scale $K_{\mathrm{d}}$ distributions are presented in Table A-4. The mineral compositions of the volcanic rocks show systematic variability, represented by the mineralogical data summarized in Tables C-1 through Table C-4. Hence, in the volcanics, the small-scale distributions were scaled to the 500-m grid size using stochastic modeling techniques. This development is presented in Section C1 of this appendix. The results of this scaling analysis are presented in Table C-11.

Although the scaled distributions are thought to correctly capture the spatial uncertainty, other sources of uncertainty such as potential fast pathways, reducing conditions along potential transport pathways, higher concentrations of radionuclides during transport in the SZ than the ranges included in the analysis, and competition among radionuclides for sorption sites may not be completely represented in these distributions. To incorporate these other sources of uncertainty, expert judgment was used to develop subjective estimates on the appropriate range of the $K_{d} S$ and percentile points for the distribution. This process is described in Section C2 of this appendix and the results are presented in Table C-14 and Figures C-7 through C-16. The $K_{d}$ distributions presented in this table are the ones recommended for use in TSPA analysis. 


\section{C1. STOCHASTIC MODELING FOR UPSCALING OF $K_{d}$ DISTRIBUTIONS IN THE VOLCANICS}

The stochastic approach used to calculate effective values of $K_{\mathrm{d}}$ for a 500 -m grid block is discussed here. The effect of spatial heterogeneity in $K_{\mathrm{d}}$ values, the effect of upscaling, and the effect of mineralogy were incorporated. The approach included generating spatially heterogeneous distributions of $K_{\mathrm{d}}$ at a scale much smaller than $500 \mathrm{~m}$ and using the heterogeneous distributions to calculate effective $K_{\mathrm{d}}$ values. The heterogeneous distributions were generated by incorporating the effect of spatial variability in rock mineralogy. A stochastic approach was used to generate distributions of effective $K_{\mathrm{d}}$ values, and multiple $K_{\mathrm{d}}$ realizations were used to calculate effective $K_{\mathrm{d}}$ values. The input data used to generate the heterogeneous $K_{\mathrm{d}}$ distributions were derived from experimental data described in Appendix A.

\section{C1.1 DEFINITION OF EFFECTIVE $K_{d}$}

Effective $K_{d}$ was defined as the value of $K_{d}$ that would result in a radionuclide sorption behavior that is similar to the sorption behavior resulting from a heterogeneous distribution of small-scale $K_{d}$ values. This concept is shown schematically in Figure C-1, in which a two-dimensional (2-D) grid block with a uniform effective $K_{d}$ produces radionuclide breakthrough behavior that is similar to that shown by the same grid block with four subgrid blocks with different $K_{d}$ properties.
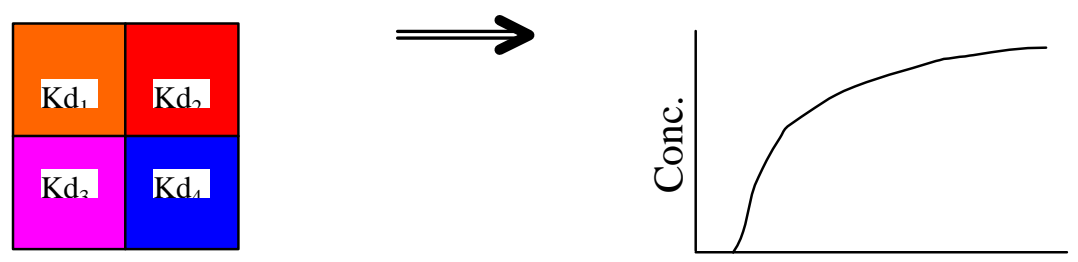

Time
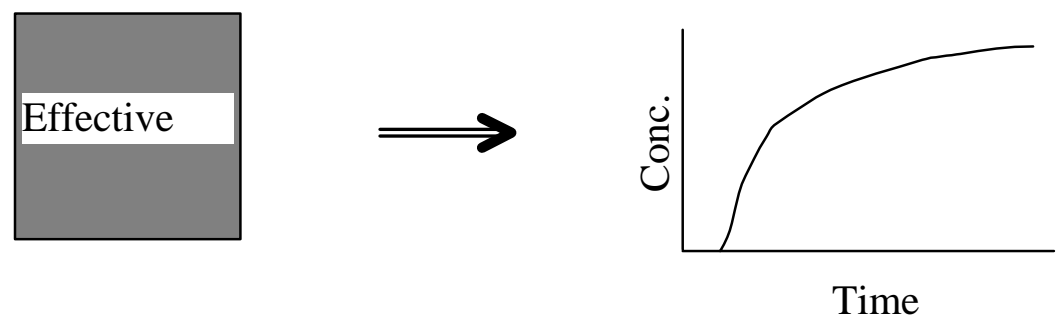

For illustration purposes only.

DTN: N/A

Figure C-1. A Schematic Representation of the Definition of Effective $K_{d}$

With the above definition, the following approach was used to compute an effective $K_{d}$. The retardation coefficient and $K_{d}$ are related to each other by the following equation:

$$
K_{\mathrm{d}}=(\text { retardation coeff. }-1) \frac{\text { Porosity }}{\text { Bulk Density }}
$$


Thus, if the retardation behavior of a system is appropriately calculated, it can be used to calculate the effective $K_{d}$. Effective retardation behavior of a grid block for a particular radionuclide was determined by comparing two breakthrough curves for the same grid block under identical flow conditions. One breakthrough curve was calculated using a dual-porosity transport in which the radionuclide can diffuse from fracture to matrix and get retarded in the matrix (Figure C-2a). The second curve was calculated with identical diffusion behavior but using no retardation in the matrix (Figure $\mathrm{C}-2 \mathrm{~b}$ ). In both calculations, retardation on fracture surface was not included. Using these two curves, effective matrix retardation was calculated by comparing the breakthrough times for 50 percent relative concentration. This concept is further explained schematically in Figure C-3 where relative behavior of two breakthrough curves for a pulse input, with and without matrix sorption, is shown.

a

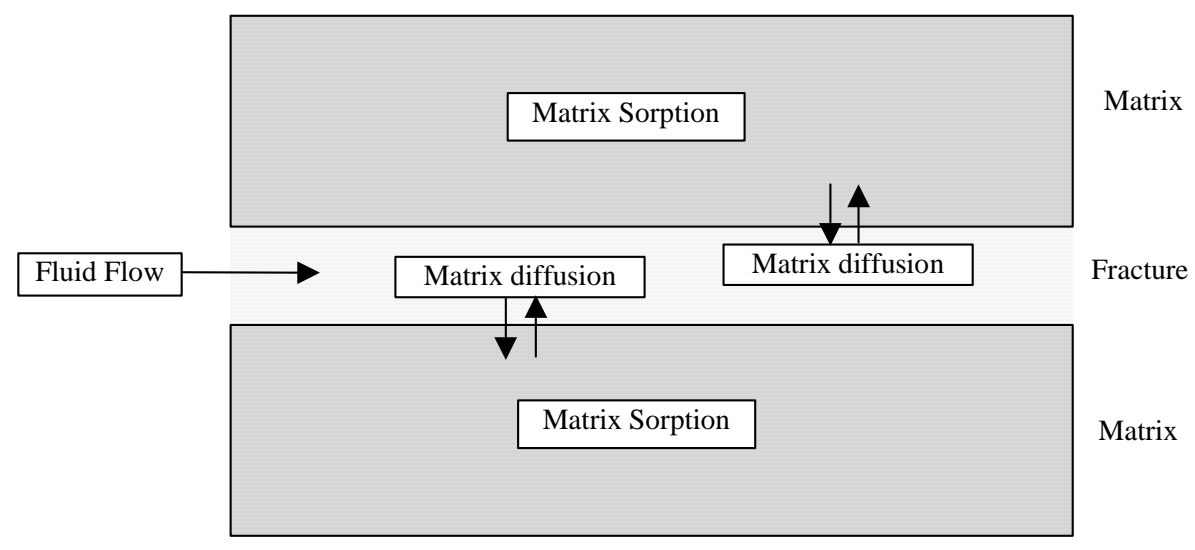

b

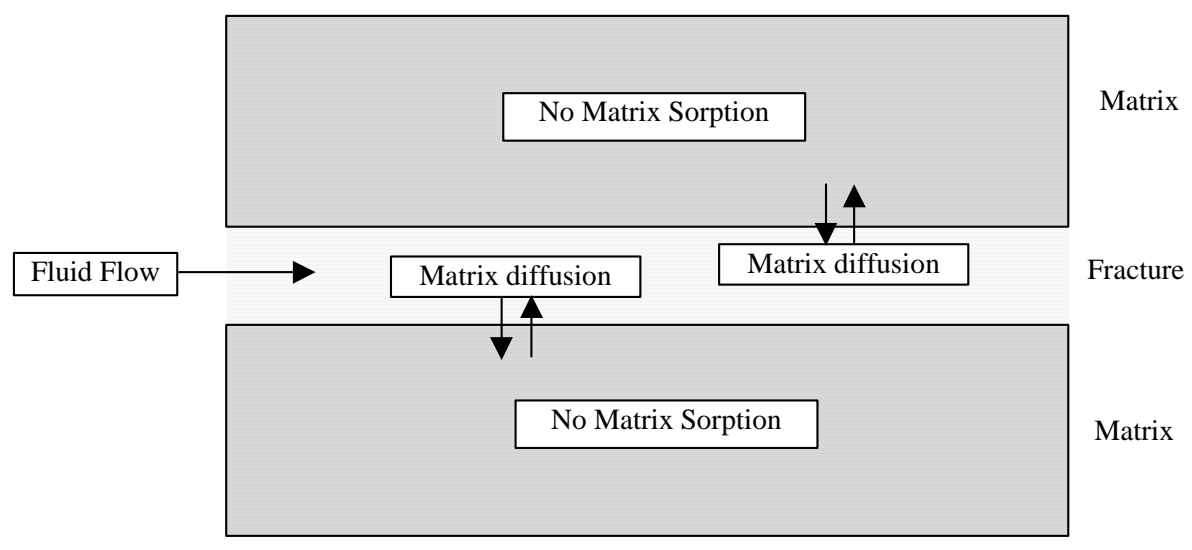

For illustration purposes only

${ }^{\mathrm{a}}$ Transport with diffusion followed by matrix sorption.

${ }^{\mathrm{b}}$ Transport with diffusion followed by no matrix sorption.

Figure C-2. The Processes During Transport of a Radionuclide in a Fractured Media 


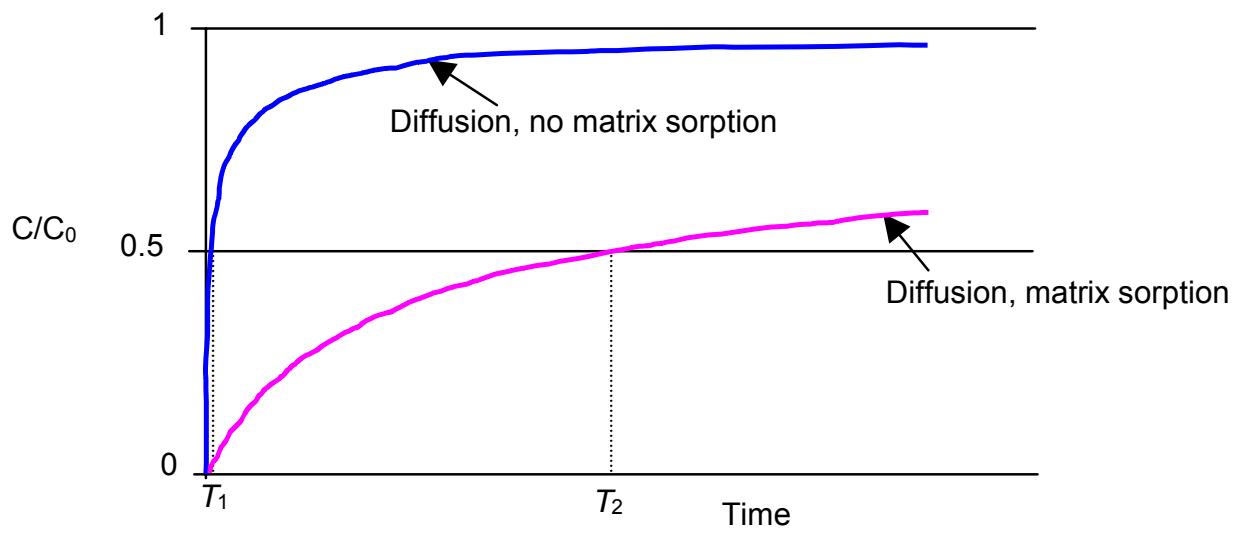

For illustration purposes only.

Figure C-3. Representation of the Breakthrough Curves Used to Calculate Effective Matrix Retardation Behavior

The breakthrough curve for the case with no matrix sorption is much steeper than that for the case with matrix sorption. The times at which 50 percent breakthrough takes place are marked as $T_{1}$ and $T_{2}$ for the cases without matrix sorption and with matrix sorption, respectively. The effective retardation coefficient was calculated as the ratio of these two times:

$$
\text { Effective Retardation }\left(r_{\text {eff }}\right)=\frac{T_{2}}{T_{1}}
$$

This definition of effective retardation was used to calculate effective $K_{d}$ values using Equation C-1. Multiple values of effective $K_{d}$ were calculated using multiple spatially heterogeneous realizations of $K_{d}$ and subsequently were used to generate statistical distribution of effective $K_{d}$. The heterogeneous $K_{d}$ distributions were generated using a geostatistical approach. Before describing the approach, a brief discussion on the method used to perform transport calculations follows.

\section{C1.2 TRANSPORT CALCULATIONS}

As mentioned earlier, a dual-porosity transport model was used to calculate the breakthrough curves. The calculations were performed using the streamline particle-tracking macro 'sptr' in FEHM. The dual-porosity transport model in the sptr macro is based on the analytical solution developed by Sudicky and Frind (1982 [DIRS 105043]) for contaminant transport in a system of parallel fractures. This solution takes into account advective transport in the fractures, molecular diffusion from the fracture into the porous matrix, and adsorption on the fracture surface as well as within the matrix. In this model, all of the above-mentioned processes except adsorption on the fracture surface are represented. The model includes the assumption that there is no sorption on fracture surfaces: see Table 5-1. Input files for the sptr macro were created using cr8sptr.c V2.0 (cr8sptr.c V2.0, STN: 10927-2.0-00) (SNL 2002 [DIRS 163836]). 


\section{C1.2.1 Stochastic Realizations of $K_{d}$}

As mentioned before, the value of $K_{d}$ is dependent on multiple factors, including rock mineralogy and water chemistry, as well as spatial location. This dependence was taken into account when developing $K_{d}$ realizations. As mentioned in Section 6.3 of this model report, due to available data, it was not possible to take into account spatial variability of groundwater chemistry. However, groundwater chemistry was treated as a spatially random variable, and its effect on $K_{d}$ values was incorporated in the $K_{d}$ distribution used as input for generating stochastic realizations (Appendix A). A two-step process was used to capture dependence on rock mineralogy. First, spatial distributions of mineralogically dependent rock types were generated. Data on mineral abundance in rock were available from X-ray diffraction analysis of samples from multiple wells (Table C-1). The mineral abundance data provided the content of the following minerals: smectites, zeolites, tridymite, cristobalite, quartz, feldspar, volcanic glass, analcime, mica, and calcite.

Table C-1. List of Wells for Which Mineral Abundance Data Were Available

\begin{tabular}{|l|l|}
\hline \multicolumn{1}{|c|}{ Data Description } & \multicolumn{1}{c|}{ DTN } \\
\hline Mineralogy, Borehole UE-25 a\#1 & MO0101XRDDRILC.002 (DIRS 163795) \\
\hline Mineralogy, Borehole UE-25 UZ\#16 & $\begin{array}{l}\text { LA000000000086.002 (DIRS 107144); LAJC831321AQ98.005 (DIRS } \\
\text { 109004) }\end{array}$ \\
\hline Mineralogy, Borehole USW G-1 & MO0101XRDMINAB.001 (DIRS 163796) \\
\hline Mineralogy, Borehole USW G-2 & MO0101XRDDRILC.002 (DIRS 163795) \\
\hline Mineralogy, Borehole USW G-3/GU-3 & MO0101XRDMINAB.001 (DIRS 163796) \\
\hline Mineralogy, Borehole USW G-4 & MO0101XRDMINAB.001 (DIRS 163796) \\
\hline Mineralogy, Borehole USW SD-7 & $\begin{array}{l}\text { MO0408K8313211.000 (DIRS 171437); LAJC831321AQ98.005 } \\
\text { (DIRS 109004) }\end{array}$ \\
\hline Mineralogy, Borehole USW SD-9 & $\begin{array}{l}\text { MO0408K8313211.000 (DIRS 171437); LAJC831321AQ98.005 } \\
\text { (DIRS 109004) }\end{array}$ \\
\hline Mineralogy, Borehole USW SD-12 & $\begin{array}{l}\text { MO0408K8313211.000 (DIRS 171437); LAJC831321AQ98.005 } \\
\text { (DIRS 109004) }\end{array}$ \\
\hline Mineralogy, Borehole USW WT-24 & $\begin{array}{l}\text { LASC831321AQ98.001 (DIRS 109047); LADV831321AQ99.001 } \\
\text { (DIRS 109044) }\end{array}$ \\
\hline Mineralogy, Borehole H-6 & MO0106XRDDRILC.003 (DIRS 163797) \\
\hline
\end{tabular}

DTN=data tracking number

These mineral abundance data were used to determine prevalent mineralogic rock types. The rock type was labeled as zeolitic if the zeolitic abundance was greater than 20 percent, as vitric if glass abundance was greater than 80 percent, and as devitrified otherwise. Only the data that were part of the SZ extending $200 \mathrm{~m}$ below the water table were used in the analysis. When mineralogic abundance data were converted to rock-type data with the above definition, it was observed that only zeolitic and devitrified rocks were present for the top $200 \mathrm{~m}$ of the SZ. The observed proportions of the rocks were 60 percent zeolitic and 40 percent devitrified. The data set also included information on the spatial location of rock samples. These data were used to calculate spatial correlation information through indicator semivariograms. Two directional semivariograms were calculated: one in the horizontal direction and another in the vertical direction using GSLIB (GSLIB V1.0GAMV3V1.201, STN: 10398-1.0GAMV3V1.201-00) (LBNL 2000 [DIRS 153099]). The semivariograms were used to calculate the spatial correlation 
parameters. Next, the spatial correlation parameters were used to generate multiple realizations of spatial distribution of rock types. The geostatistical approach of sequential indicator simulations (GSLIB V2.0 MSISIM, STN: 10098-2.0MSISIMV2.0-00) (SNL 2000 [DIRS 149114] was used to generate the spatial distributions. Sequential indicator simulation is a powerful tool that can be used to generate stochastic realizations of parameters. It uses cumulative distribution functions (CDFs) of observed data as input and tries to estimate a discrete, nonparametric true CDF of a simulated parameter. An indicator is a variable used to show the presence or absence of any parameter qualitatively or quantitatively. For example, an indicator can be used to define the presence of a particular rock type at any spatial location. It can also be used to define whether the value of a parameter falls within a certain range of parameter values defined as cutoffs.

After the spatial distributions of rock types were generated, experimental data on $K_{d}$ values (Appendix A) were used to generate spatial distributions of $K_{d}$ values. The experimental data were analyzed to derive rock-type specific statistical distributions for $K_{d}$. The statistical distributions were used to derive the CDF for each radionuclide. Next, indicators were defined at four CDF cutoffs of 0.2, 0.4, 0.6, and 0.8. These cutoffs, along with the spatial correlation information, were then used to generate spatial distributions. Unlike mineral abundance data, spatial information on $K_{d}$ observations was not available. As a result, no spatial correlation functions were available for $K_{d}$ data. In the absence of any spatial correlation functions, the approach used was to generate spatial $K_{d}$ distributions by varying the correlation length and to understand the impact of varying the correlation length on effective $K_{d}$ calculations. Four different values were used for correlation length. This range covers the entire spectrum from completely uncorrelated to fully correlated. Four values were used to represent the entire spectrum while not making the number of simulations excessively large:

- Correlation length equal to a single grid block dimension (4 m) that represents spatially random realizations

- Correlation length equal to the correlation length used to generate permeability realizations $(60 \mathrm{~m})$

- Correlation length equal to the large grid block length (500 m)

- Correlation length equal to the correlation length used to generate rock-type data $(1,000 \mathrm{~m})$.

All of the above values represent the possible range of correlation lengths that $K_{d}$ values can be expected to have. The spatial distributions of $K_{d}$ realizations were generated using the sequential indicator simulation approach. These spatial distributions of $K_{d}$ values were generated for individual rock types. Distributions for each rock type were generated independent of other rock-type distributions. Finally, the rock-type specific $K_{d}$ distributions and rock-type distributions were used to generate integrated $K_{d}$ distributions. The approach used is explained schematically below:

- $K_{d}$ distribution for rock-type ' 1 ': $K_{\mathrm{d} 1}^{1}, K_{\mathrm{d} 2}^{1}, K_{\mathrm{d} 3}^{1}, K_{\mathrm{d} 4}^{1}, K_{\mathrm{d} 5}^{1}, K_{\mathrm{d} 6}^{1}, K_{\mathrm{d} 7}^{1}, \mathrm{~K}, K_{\mathrm{d} n}^{1}$ 
- $K_{d}$ distribution for rock-type ' 0 ': $K_{\mathrm{d} 1}^{0}, K_{\mathrm{d} 2}^{0}, K_{\mathrm{d} 3}^{0}, K_{\mathrm{d} 4}^{0}, K_{\mathrm{d} 5}^{0}, K_{\mathrm{d} 6}^{0}, K_{\mathrm{d} 7}^{0}, \mathrm{~K}, K_{\mathrm{d} n}^{0}$

- Rock-type distribution: $1,0,1,1,1,0,0, \ldots, 1$

- Combined $K_{d}$ distribution: $K_{\mathrm{d} 1}^{1}, K_{\mathrm{d} 2}^{0}, K_{\mathrm{d} 3}^{1}, K_{\mathrm{d} 4}^{1}, K_{\mathrm{d} 5}^{1}, K_{\mathrm{d} 6}^{0}, K_{\mathrm{d} 7}^{0}, \mathrm{~K}, K_{\mathrm{d} n}$, where $\mathrm{n}$ is the number of grid points.

The approach explained above incorporates the effect of spatial heterogeneity and rock mineralogy on the spatial distribution of $K_{d}$. Multiple realizations for the spatial distribution of $K_{d}$ values were generated with this approach.

\section{C1.2.2 Stochastic Realizations of Permeability}

Similar to the $K_{d}$ distributions, spatial distributions of permeability were generated using the stochastic approach. The approach and data used were similar to that in Modeling Sub Gridblock Scale Dispersion in Three-Dimensional Heterogeneous Fractured Media (S0015) (CRWMS M\&O 2000 [DIRS 152259], Sections 5.2 and 6.1). The computer code GS2FEHM.C (GS2FEHM.C V1.0, STN: 10923-1.0-00) (SNL 2002 [DIRS 163837]) was used to create files for the "perm" macro needed as input to FEHM. These permeability realizations represented continuum distributions of permeability for fractured rocks.

\section{C1.3 RESULTS}

\section{C1.3.1 Stochastic Realizations of $\boldsymbol{K}_{\boldsymbol{d}}$}

Figures C-4 and C-5 show the semivariograms calculated from the rock-type data (converted from available mineral abundance data). The figures also show the correlation functions fit to the semivariograms. There is scatter in the data, but the fits are considered reasonable. The parameters for the model fit are shown in Table C-2.

These correlation parameters were used to generate spatial distributions of rock types. The sequential indicator simulation algorithm SISIM (GSLIB V2.0MSISIM;), which is part of GSLIB, was used to generate these distributions. Five different rock-type realizations were generated using this approach. The proportions of zeolitic and devitrified rocks in the five output realizations are shown in Table C-3.

Spatial realizations for $K_{d}$ were generated for four different radionuclides: uranium, neptunium, cesium, and plutonium. Neptunium is shown to be an important contributor to dose CRWMS M\&O 2000 [DIRS 153246], Section 4.1.1) and neptunium to cesium covers the low to high $K_{d}$ spectrum. The small-scale uncertainty distributions based on experimentally available data for these radionuclides (discussed in Appendix A) are given in Table C-4 (reproduced from Table A-4 for convenience). 


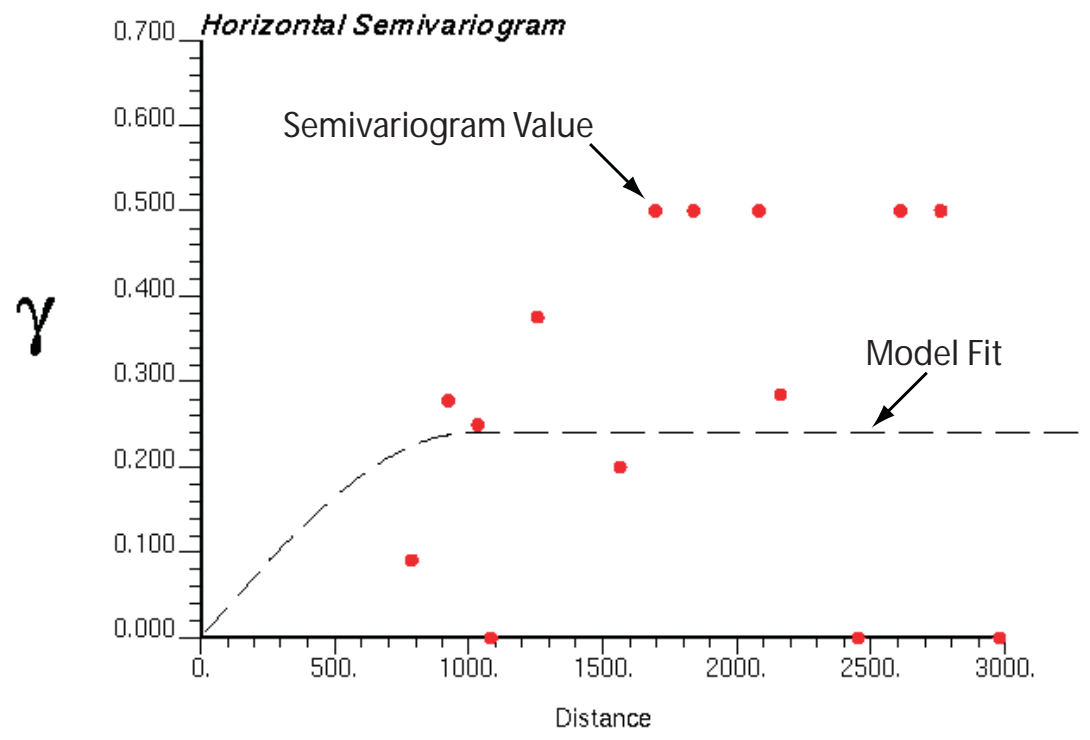

Output DTN: LA0309RP831321.001.

NOTE: The distance on the $x$-axis is in meters.

Figure C-4. Calculated Semivariogram and Model Fit in the Horizontal Direction

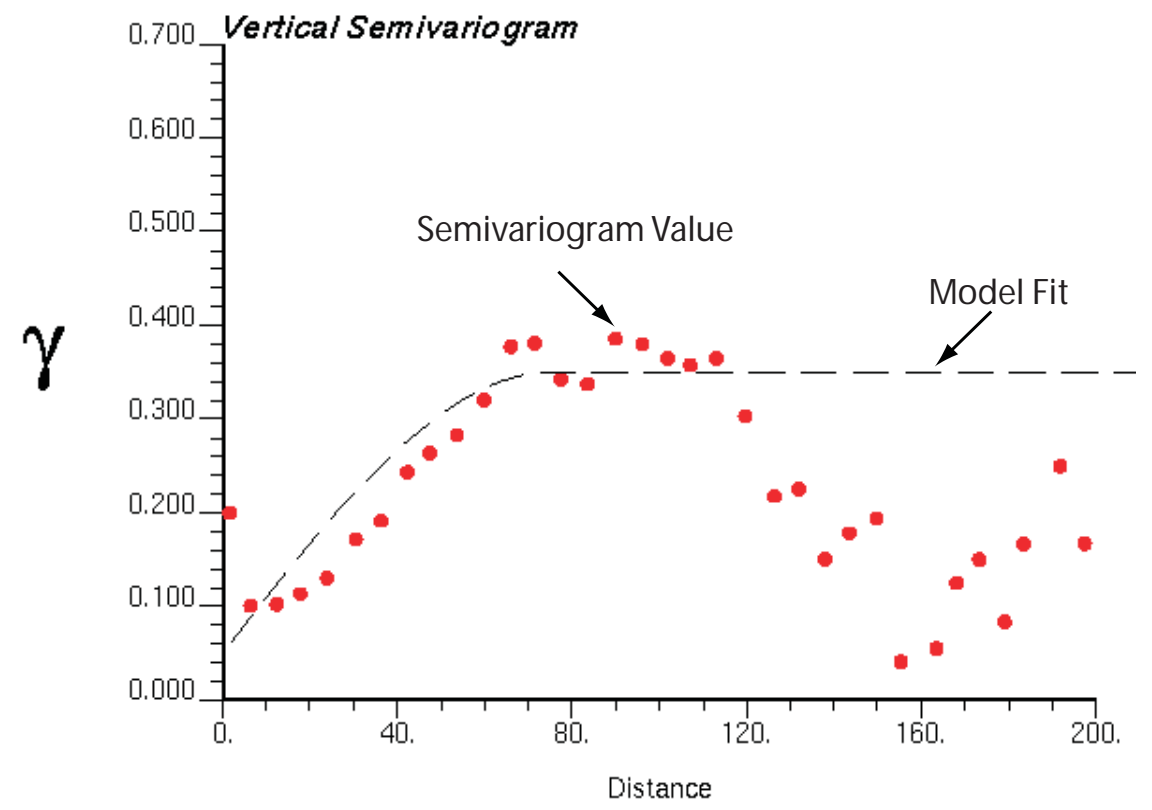

Output DTN: LA0309RP831321.001.

NOTE: The distance on the $x$-axis is in meters.

Figure C-5. Calculated Semivariogram and Model Fit in the Vertical Direction 
Table C-2. Spatial Correlation Parameters for Mineralogic Rock Type Data

\begin{tabular}{|l|c|l|}
\hline \multicolumn{1}{|c|}{ Direction } & Range $(\mathbf{m})$ & \multicolumn{1}{c|}{ Sill } \\
\hline Horizontal & 1,000 & 0.25 \\
\hline Vertical & 75 & 0.35 \\
\hline
\end{tabular}

Output DTN: LA0309RP831321.001.

Table C-3. Proportions of Zeolitic and Devitrified Rocks in Output Realizations

\begin{tabular}{|c|c|c|}
\hline Realization & Zeolitic & Devitrified \\
\hline 1 & 0.6106 & 0.3894 \\
\hline 2 & 0.5746 & 0.4254 \\
\hline 3 & 0.6034 & 0.3966 \\
\hline 4 & 0.5890 & 0.4110 \\
\hline 5 & 0.6270 & 0.3730 \\
\hline
\end{tabular}

Output DTN: LA0309RP831321.001.

Table C-4. Statistical Distributions of Experimentally Observed $K_{d}$ Values

\begin{tabular}{|l|l|l|c|c|c|c|}
\hline \multirow{2}{*}{ Radionuclide } & $\begin{array}{c}\text { Rock- } \\
\text { type }\end{array}$ & Distribution & $\begin{array}{c}\text { Mean } \\
(\mathbf{m L / g})\end{array}$ & $\begin{array}{c}\text { Standard } \\
\text { Deviation } \\
(\mathbf{m L} / \mathbf{g})\end{array}$ & $\begin{array}{c}\text { Minimum } \\
(\mathbf{m L} / \mathbf{g})\end{array}$ & $\begin{array}{c}\text { Maximum } \\
(\mathbf{m L} / \mathbf{g})\end{array}$ \\
\hline \multirow{2}{*}{ Uranium } & Zeolitic & Normal & 12.0 & 3.6 & 5.0 & 20.0 \\
\cline { 2 - 7 } & Devitrified & Normal & 2.0 & 0.6 & 0.0 & 4.0 \\
\hline \multirow{2}{*}{ Cesium } & Zeolitic & Exponential & $16,942.0$ & $14,930.0$ & $4,000.0$ & $42,000.0$ \\
\cline { 2 - 7 } & Devitrified & Normal & 728.0 & 464.0 & 100.0 & 1000.0 \\
\hline \multirow{2}{*}{ Neptunium } & Zeolitic & Normal & 2.88 & 1.47 & 0.0 & 6.0 \\
\cline { 2 - 7 } & Devitrified & Exponential & 0.69 & 0.707 & 0.0 & 2.0 \\
\hline \multirow{2}{*}{ Plutonium } & Zeolitic & Beta & 100.0 & 15.0 & 50.0 & 300.0 \\
\cline { 2 - 7 } & Devitrified & Beta & 100.0 & 15.0 & 50.0 & 300.0 \\
\hline
\end{tabular}

Source: Table A-4.

These distributions were used to derive the CDFs for each radionuclide for each rock type using software routine calc_cdf.c (calc_cdf.c V1.0, STN: 10924-1.0-00) (SNL 2000 [DIRS 149117]). For each CDF, indicators were defined at four CDF cutoffs: 0.2, 0.4, 0.6, and 0.8. As mentioned earlier, in the absence of spatial data, correlation length was parameterized, and four different correlation lengths were used to generate stochastic realizations. This effect of correlation length was studied only for uranium. For other radionuclides, a correlation length of $500 \mathrm{~m}$ was used. Fifty different realizations were generated for each radionuclide and each rock type. Statistics of the output realizations were calculated and compared against the input data. The software routine calc_cdf.c was used to calculate the CDF from the statistical realizations. Tables C-5 through C-8 compare the mean CDFs (calculated from 50 realizations) with the input CDFs for the four cutoffs.

Finally, these rock-type specific $K_{d}$ distributions were combined to generate distributions that were conditioned to the realizations of rock types. The procedure for doing this was outlined in Section C2.3. 
Table C-5. Comparison of Input and Mean Output CDFs for Uranium

\begin{tabular}{|c|c|c|c|c|c|}
\hline \multicolumn{3}{|c|}{ Zeolitic } & \multicolumn{3}{c|}{ Devitrified } \\
\hline $\begin{array}{c}K_{\boldsymbol{d}}(\mathbf{m L} / \mathbf{g m}) \\
\text { Cutoff }\end{array}$ & Input CDF & Output CDF & $\begin{array}{c}K_{d}(\mathbf{m L} / \mathbf{g m}) \\
\text { Cutoff }\end{array}$ & Input CDF & Output CDF \\
\hline 8.97 & 0.2 & 0.2047 & 1.49 & 0.2 & 0.2128 \\
\hline 11.09 & 0.4 & 0.4014 & 1.84 & 0.4 & 0.4050 \\
\hline 12.91 & 0.6 & 0.5964 & 2.15 & 0.6 & 0.5939 \\
\hline 15.03 & 0.8 & 0.7918 & 2.50 & 0.8 & 0.7909 \\
\hline
\end{tabular}

Output DTN: LA0309RP831321.001.

$\mathrm{CDF}=$ cumulative distribution function

Table C-6. Comparison of Input and Mean Output CDFs for Cesium

\begin{tabular}{|c|c|c|c|c|c|}
\hline \multicolumn{3}{|c|}{ Zeolitic } & \multicolumn{3}{c|}{ Devitrified } \\
\hline $\begin{array}{c}\boldsymbol{K}_{\boldsymbol{d}}(\mathbf{m L} / \mathbf{g m}) \\
\text { Cutoff }\end{array}$ & Input CDF & Output CDF & $\begin{array}{c}\boldsymbol{K}_{\boldsymbol{d}}(\mathbf{m L} / \mathbf{g m}) \\
\text { Cutoff }\end{array}$ & $\begin{array}{c}\text { Input CDF } \\
\text { Output CDF }\end{array}$ \\
\hline 4929.3 & 0.2 & 0.2018 & 337.5 & 0.2 & 0.2026 \\
\hline 9478.5 & 0.4 & 0.4058 & 610.5 & 0.4 & 0.4079 \\
\hline 14613.3 & 0.6 & 0.5975 & 845.5 & 0.6 & 0.6012 \\
\hline 24896.8 & 0.8 & 0.8058 & 1118.5 & 0.8 & 1.0 \\
\hline
\end{tabular}

Output DTN: LA0309RP831321.001.

$\mathrm{CDF}=$ cumulative distribution function

Table C-7. Comparison of Input and Mean Output CDFs for Neptunium

\begin{tabular}{|c|c|c|c|c|c|}
\hline \multicolumn{3}{|c|}{ Zeolitic } & \multicolumn{3}{c|}{ Devitrified } \\
\hline $\begin{array}{c}K_{\boldsymbol{d}}(\mathbf{m L} / \mathbf{g m}) \\
\text { Cutoff }\end{array}$ & Input CDF & Output CDF & $\begin{array}{c}K_{d}(\mathbf{m L} / \mathbf{g m}) \\
\text { Cutoff }\end{array}$ & Input CDF & Output CDF \\
\hline 1.65 & 0.2 & 0.2018 & 0.12 & 0.2 & 0.2018 \\
\hline 2.51 & 0.4 & 0.4059 & 0.30 & 0.4 & 0.4059 \\
\hline 3.26 & 0.6 & 0.5976 & 0.51 & 0.6 & 0.5976 \\
\hline 4.12 & 0.8 & 0.8058 & 0.93 & 0.8 & 0.8058 \\
\hline
\end{tabular}

Output DTN: LA0309RP831321.001.

$\mathrm{CDF}=$ cumulative distribution function

Table C-8. Comparison of Input and Mean Output CDFs for Plutonium

\begin{tabular}{|c|c|c|c|c|c|}
\hline \multicolumn{3}{|c|}{ Zeolitic } & \multicolumn{3}{c|}{ Devitrified } \\
\hline $\begin{array}{c}\boldsymbol{K}_{\boldsymbol{d}} \text { (mL/gm) } \\
\text { Cutoff }\end{array}$ & Input CDF & Output CDF & $\begin{array}{c}\boldsymbol{K}_{d}(\mathbf{m L} / \mathbf{g m}) \\
\text { Cutoff }\end{array}$ & Input CDF & Output CDF \\
\hline 87.005 & 0.2 & 0.2054 & 87.005 & 0.2 & 0.2018 \\
\hline 95.094 & 0.4 & 0.4229 & 95.094 & 0.4 & 0.4059 \\
\hline 102.743 & 0.6 & 0.5852 & 102.743 & 0.6 & 0.5976 \\
\hline 112.348 & 0.8 & 0.7855 & 112.348 & 0.8 & 0.8058 \\
\hline
\end{tabular}

Output DTN: LA0309RP831321.001.

$\mathrm{CDF}=$ cumulative distribution function 


\section{C1.3.2 Results of Breakthrough Curve Calculations Using the Particle- Tracking Algorithm}

These multiple $K_{d}$ realizations were used to compute breakthrough curves and model the sorption behavior of each radionuclide. A two-step approach was used. In the first step, steady-state flow fields were computed for 50 different permeability realizations. The properties used for these calculations are shown in Table C-9.

Table C-9. Values of Properties Used in Flow and Transport Calculations

\begin{tabular}{|l|l|}
\hline \multicolumn{1}{|c|}{ Property } & \multicolumn{1}{c|}{ Value } \\
\hline Matrix porosity & $0.22^{\mathrm{a}}$ \\
\hline Rock bulk density & $1,997.5^{\mathrm{a}} \mathrm{kg} / \mathrm{m}^{3}$ \\
\hline Flowing interval porosity & $0.001^{\mathrm{a}}$ \\
\hline Flowing interval spacing & $19.49^{\mathrm{a}} \mathrm{m}$ \\
\hline Hydraulic gradient & $2.9 \times 10^{-4 \mathrm{~b}}$ \\
\hline \\
a Value chosen to fall within the range given in Table 4-2 of this report. \\
b Modeling Sub Gridblock Scale Dispersion in Three-Dimensional Heterogeneous \\
Fractured Media (CRWMS M\&O 2000 [DIRS 152259], pp. 14, Sec. 5.2).
\end{tabular}

The steady-state flow fields were used in the particle-tracking calculations. In these calculations, 4,000 particles were released along one face of the model and were allowed to move under the influence of the steady-state flow field. The locations of the particle releases were determined by a flux-weighted placement scheme. As mentioned in Section C2.1, two sets of particle-tracking calculations were performed for each steady-state flow field. In the first set of calculations, the baseline breakthrough curve was calculated for transport with diffusion from fracture to matrix and no matrix sorption. In the second set of calculations, the breakthrough curve was calculated for transport with diffusion followed by sorption on the matrix. For these calculations, the stochastically generated $K_{d}$ distributions were used. The values of the diffusion coefficient used for these calculations are shown in Table C-10.

Table C-10. Values of Diffusion Coefficients Used for the Particle-Tracking Calculations

\begin{tabular}{|l|l|}
\hline \multicolumn{1}{|c|}{ Radionuclide } & \multicolumn{1}{c|}{ Diffusion Coefficient $\left(\mathrm{m}^{2} / \mathbf{s}\right)$} \\
\hline Anion (Uranium) & $3.2 \times 10^{-11}$ \\
\hline Cation (Plutonium, Cesium, Neptunium) & $1.6 \times 10^{-10}$ \\
\hline
\end{tabular}

Source DTN: LA0003JC831362.001 [DIRS 149557].

These breakthrough curves were used to calculate the effective $K_{d}$ values using the procedure described in Section C2.1. The procedure was repeated for 50 realizations of $K_{d}$. The statistics of the calculated effective $K_{d}$ values are provided in Table C-11. These calculations of stochastic realizations of $K_{d}$ were performed using a correlation length of $500 \mathrm{~m}$. As can be seen from the results, the effective $K_{d}$ distributions are very narrow compared to the distributions of experimentally observed $K_{d}$ values. 
Table C-11. Statistics of Calculated Effective $K_{d}$ Values

\begin{tabular}{|c|c|c|c|c|c|}
\hline Radionuclide & Distribution & Mean & $\begin{array}{c}\text { Standard } \\
\text { Deviation }\end{array}$ & Minimum & Maximum \\
\hline Uranium & Normal & 6.61 & 0.61 & 5.39 & 8.16 \\
\hline Cesium & Normal & $5,188.72$ & 941.55 & $3,000.59$ & $6,782.92$ \\
\hline Plutonium & Normal & 110.17 & 7.45 & 89.90 & 129.87 \\
\hline Neptunium & Normal & 1.48 & 0.23 & 0.99 & 1.83 \\
\hline
\end{tabular}

Output DTNs: LA0309AM831341.001; LA0309RP831341.001; LA0309RP831341.004; LA0309RP831341.002.

A comparison was made as to how well the calculated effective $K_{d}$ values predicted the particle breakthrough behavior with respect to the breakthrough behavior predicted by the heterogeneous $K_{d}$ field (from which the effective value was calculated). In these calculations, a uniform value of $K_{d}$ equal to the effective $K_{d}$ value was used. Figure C-6 shows the two breakthrough curves for one of the $K_{d}$ realizations. The effective value of $K_{d}$ calculated for this realization was $7.32(\mathrm{~mL} / \mathrm{g})$. As can be seen from the figure, the calculated effective $K_{d}$ value captures the breakthrough behavior of the heterogeneous $K_{d}$ field very well.

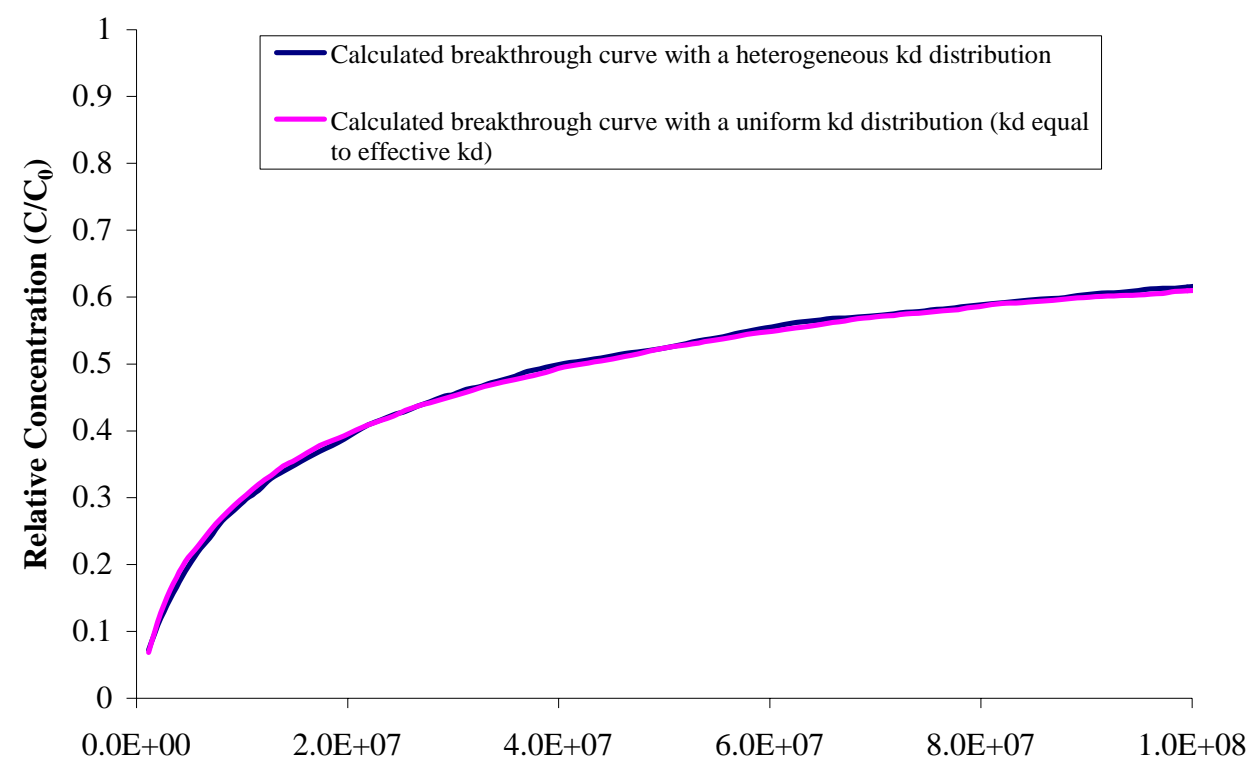

Time (Days)

Output DTNs: LA0309RP831341.003; LA0309RP831341.004.

Figure C-6. Comparison of Breakthrough Behavior Predicted by the Calculated Effective $K_{d}$ 


\section{C1.3.3 Effect of Correlation Length on Effective $K_{d}$ Distributions of Uranium}

As mentioned earlier, the correlation length used in generating the stochastic $K_{d}$ realizations was parameterized because of the lack of the spatial information. The intent was to examine the effect of changes in correlation length on the distribution of effective $K_{d}$ values. Once again, a similar procedure was followed to calculate effective $K_{d}$ values. The heterogeneous $K_{d}$ distributions generated by using different correlation lengths were used for these calculations. Table C-12 details the statistics of the calculated effective $K_{d}$ values along with the correlation length used to generate the heterogeneous $K_{d}$ distributions. As can be seen from the results, variation in the correlation length does not significantly affect the calculated statistics of effective $K_{d}$ values.

Table C-12. Effect of Changes in Correlation Length on Effective $K_{d}$ Distributions for Uranium

\begin{tabular}{|c|c|c|c|c|}
\hline $\begin{array}{c}\text { Correlation Length } \\
(\mathbf{m})\end{array}$ & $\begin{array}{c}\text { Mean } \boldsymbol{K}_{\boldsymbol{d}} \\
(\mathbf{m L / g m})\end{array}$ & Standard Deviation & Minimum & Maximum \\
\hline 4 & 6.71 & 0.49 & 5.70 & 8.13 \\
\hline 60 & 6.79 & 0.47 & 5.42 & 8.14 \\
\hline 500 & 6.61 & 0.61 & 5.39 & 8.16 \\
\hline 1000 & 6.58 & 0.62 & 4.46 & 7.85 \\
\hline
\end{tabular}

Output DTN: LA0309RP831341.004.

\section{C1.3.4 Effect of Variability in the Hydraulic Gradient}

The effect of variability in the hydraulic gradient on calculated effective $K_{d}$ values was studied. These calculations were performed only for uranium and used $K_{d}$ realizations generated with a correlation length of $500 \mathrm{~m}$. Two different values of hydraulic gradient were used: $8.7 \times 10^{-4}$ (3 times mean hydraulic gradient) and $0.967 \times 10^{-4}$ (one-third of mean hydraulic gradient). Steady-state flow fields were calculated with these hydraulic gradients and were subsequently used to calculate particle breakthrough curves. The statistics of the resulting effective $K_{d}$ values are compared with the ones for a mean hydraulic gradient of 0.14 in Table C-13. As can be seen from the results, a variability of three orders of magnitude in hydraulic gradient has not significantly affected the effective $K_{d}$ distributions.

Table C-13. Statistics of Calculated Effective $K_{d}$ Values for Uranium for Different Hydraulic Gradients

\begin{tabular}{|c|c|c|c|c|}
\hline Hydraulic Gradient & $\begin{array}{c}\text { Mean } \boldsymbol{K}_{\boldsymbol{d}} \\
(\mathbf{m L} / \mathbf{g m})\end{array}$ & $\begin{array}{c}\text { Standard Deviation } \\
(\mathbf{m L} / \mathbf{g m})\end{array}$ & $\begin{array}{c}\text { Minimum } \\
(\mathbf{m L} / \mathbf{g m})\end{array}$ & $\begin{array}{c}\text { Maximum } \\
(\mathbf{m L / g m})\end{array}$ \\
\hline $0.967 \times 10^{-4}$ & 6.55 & 0.59 & 5.13 & 7.53 \\
\hline $2.9 \times 10^{-4}$ & 6.61 & 0.61 & 5.39 & 8.16 \\
\hline $8.7 \times 10^{-4}$ & 6.27 & 0.56 & 4.97 & 7.65 \\
\hline
\end{tabular}

Output DTN: LA0309RP831341.004. 


\section{C1.4 SUMMARY OF STOCHASTIC MODELING}

This study was performed to calculate distributions of effective $K_{d}$ for uranium, neptunium, cesium, and plutonium. The effective $K_{d}$ distributions were calculated through a stochastic approach in which multiple values of effective $K_{d}$ were calculated. The value of effective $K_{d}$ was determined by calculating effective retardation resulting from a spatially heterogeneous $K_{d}$ field. The spatially heterogeneous $K_{d}$ fields were calculated using a geostatistical approach. The factors affecting the spatial distribution of $K_{d}$, such as rock mineralogy and spatial heterogeneity, were taken into account while generating the heterogeneous $K_{d}$ fields. As spatial data on $K_{d}$ were not available, the correlation length used to generate the fields was parameterized. The observations of the study were as follows:

- The calculated effective $K_{d}$ values reproduced the sorption behavior of the heterogeneous $K_{d}$ field very well, validating the approach used to determine the effective $K_{d}$ values.

- The distributions of calculated effective $K_{d}$ fields were much narrower than the distributions used as the input. This is to be expected because, in any upscaling study, as the scale gets larger, the variability in effective parameter values gets smaller.

- Variability in correlation length did not significantly affect the effective $K_{d}$ distributions for uranium.

- Variability in hydraulic gradient did not significantly change the effective $K_{d}$ distributions.

\section{C2. DEVELOPMENT OF COMPOSITE $K_{d}$ DISTRIBUTIONS}

In the TSPA calculations, radionuclide transport is modeled using a single value of $K_{d}$ for grid blocks with dimensions $500 \mathrm{~m} \times 500 \mathrm{~m}$ in the $x$ and $y$ directions. In the field, values of $K_{d}$ are variable at a scale much smaller than $500 \mathrm{~m}$. Thus, if a uniform single value of $K_{d}$ is used to model sorption, it is important to use a value that effectively captures variability at smaller scale and results in the same sorption behavior as if all the small-scale processes were represented explicitly. Given the scope of the work presented here, simulations could not be carried out for all radionuclides in both the alluvium and the volcanics, and a choice had to be made. The distributions in alluvium were taken to be the same as the small-scale distributions derived in Appendix A (Table A-4) for all radionuclides. The distributions in volcanics for Am, Th, Pa, Sr, and Ra were also taken to be the same as those in Table A-4. The distribution in volcanics and alluvium for the radionuclides Am, Th, and $\mathrm{Pa}$ are plotted in Figure C-7. The distribution in volcanics and alluvium for the radionuclide $\mathrm{Sr}$ is plotted in Figure C-8. However, in volcanics, the distributions for $\mathrm{Cs}, \mathrm{Np}, \mathrm{Pu}$, and $\mathrm{U}$ were generalized using stochastic modeling (Section $\mathrm{C} 1$ ) to a scale of $500 \mathrm{~m}$, and then modified to include additional uncertainties leading to composite distributions for use with the TSPA calculations. 


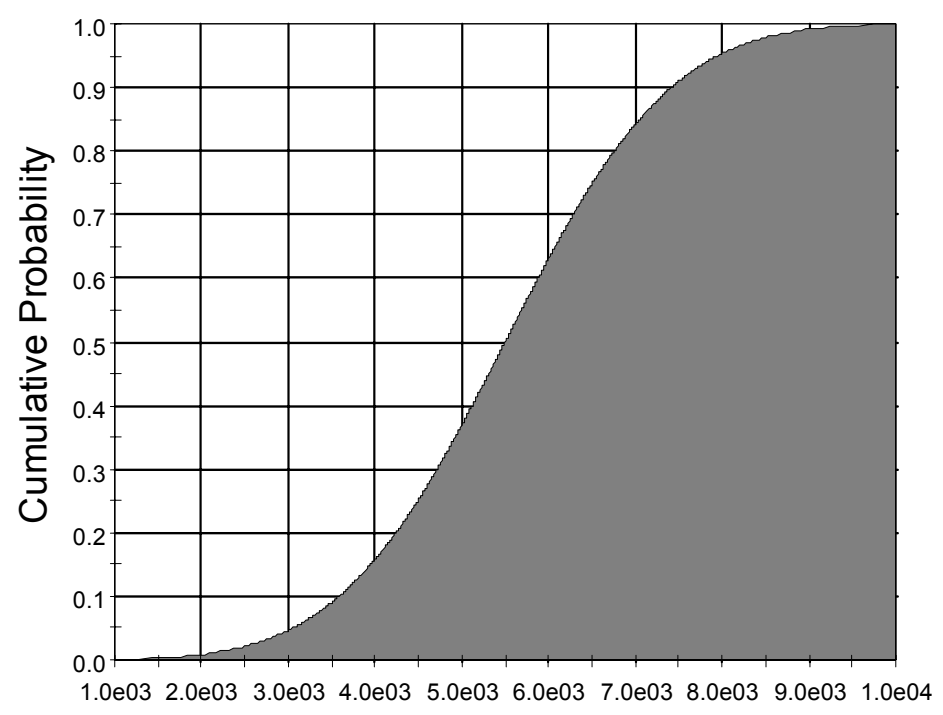

Americum Sorption Coefficient Alluv/Nol Units (ml/g)

Output DTN: LA0309AM831341.001.

Figure C-7. Plot of Composite Cumulative Probability Distribution for Americium, Thorium, and Protactinium in Volcanics and Alluvium

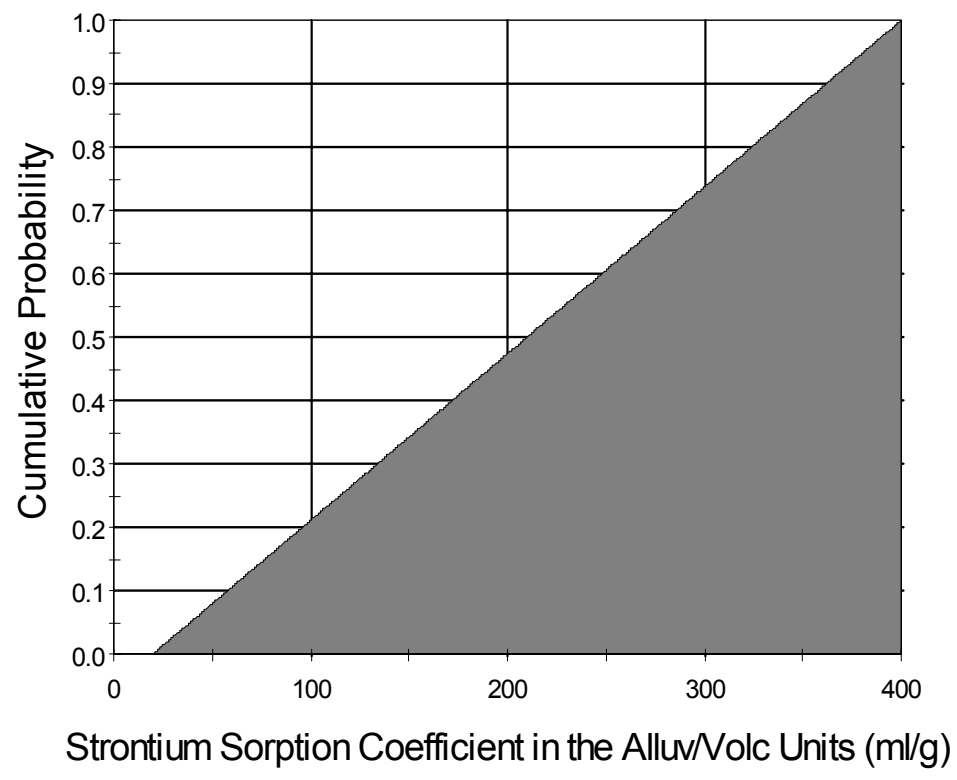

Output DTN: LA0309AM831341.001.

Figure C-8. Plot of Composite Cumulative Probability Distribution for Strontium in Volcanics and Alluvium 
As can be seen by comparing the Tables C-11 and A-4, the stochastically derived upscaled $K_{d}$ distributions in the volcanics are narrow compared to the small-scale distributions. Although the scaled distributions are thought to correctly capture the spatial uncertainty, other sources of uncertainty may not be completely represented in these distributions. Other sources of uncertainty in the volcanics may include potential fast pathways (e.g., along faults) through devitrified tuff, the existence of reducing conditions along potential transport pathways, higher concentrations of radionuclides during transport in the SZ than the ranges included in the analysis, and competition among radionuclides for sorption sites.

To incorporate these other sources of uncertainty in the volcanics, the methods and approach for the development of parameter distributions documented in Guidelines for Developing and Documenting Alternative Conceptual Models, Model Abstractions, and Parameter Uncertainty in the Total System Performance Assessment for the License Application (BSC 2002 [DIRS 158794], Section 4.2) was implemented. In implementing this process, the subject matter expert (SME) who developed the small-scale $K_{d}$ distributions worked with the parameter team lead to assess the impact of these other sources of uncertainty on the recommended distributions. Because the observational data was limited, the SME worked with the parameter team lead and several experts in uncertainty analysis to develop subjective estimates on the appropriate range of the $K_{d} \mathrm{~S}$ and percentile points for the distribution.

The outcome of this process was that the SME judged that the scaled distributions did not completely capture the full-range of $K_{d}$ uncertainty. In the SME's judgment, the full range of the scaled distribution was extended to the minimum and maximum values of the small-scale distribution to create the range for the composite distribution. In this composite distribution 5 percent to 30 percent of the full distribution was assigned to the tails of the composite distribution. The resulting recommended composite distributions are piece-wise uniform distributions that incorporate both the scaled distributions and the small-scale distributions. These composite distributions are given in Table C-14 and their plots are presented in Figures C-9 through C-16. The following paragraphs detail the development of these distributions for $\mathrm{U}, \mathrm{Np}, \mathrm{Pu}$, and Cs.

For uranium in volcanics, the minimum small-scale value was $0 \mathrm{~mL} / \mathrm{g}$. Five percent probability was uniformly distributed between $0 \mathrm{~mL} / \mathrm{g}$ and the lower bound of the scaled distribution at $5.39 \mathrm{~mL} / \mathrm{g}$. Since the scaled distribution had such little variation, it is reasonably represented by a uniform distribution; hence, 90 percent probability was uniformly distributed between $5.39 \mathrm{~mL} / \mathrm{g}$ and the upper bound of the upscaled distribution at $8.16 \mathrm{~mL} / \mathrm{g}$. The remaining 5 percent probability was uniformly distributed between $8.16 \mathrm{~mL} / \mathrm{g}$ and the maximum small-scale experimentally observed value of $20 \mathrm{~mL} / \mathrm{g}$. This distribution is shown schematically in Figure C-9. The distribution for uranium in alluvium is shown in Figure C-10. 
Table C-14. Recommended Composite Distribution for $K_{d} \mathrm{~S}$ In Volcanics and Alluvium

\begin{tabular}{|l|l|l|l|}
\hline \multicolumn{1}{|c|}{ Species } & \multicolumn{1}{|c|}{ Unit/Analysis } & \multicolumn{1}{c|}{ Distribution } & \multicolumn{1}{c|}{$\begin{array}{c}\text { Coefficients describing distribution } \\
(\mathrm{mL} / \mathbf{g})\end{array}$} \\
\hline $\mathrm{U}$ & Composite $($ Volcanics) & Cumulative & $\left(K_{d}\right.$, prob) $(0 ., 0).(5.39,0.05)(8.16,0.95)(20,1.0)$ \\
\hline & Alluvium & Cumulative & $\left(K_{d}\right.$, prob $)(1.7,0).(2.9,0.05)(6.3,0.95)(8.9,1.0)$ \\
\hline $\mathrm{Np}$ & Composite (Volcanics) & Cumulative & $\left(K_{d}\right.$, prob $)(0,0)(0.99,0.05)(1.83,0.90)(6,1.0)$ \\
\hline & Alluvium & Cumulative & $\left(K_{d}\right.$, prob $)(1.8,0).(4.0,0.05)(8.7,0.95)(13,1.0)$ \\
\hline $\mathrm{Pu}$ & Composite (Volcanics) & Cumulative & $\left(K_{d}\right.$, prob) $(10 ., 0).(89.9,0.25)(129.87,0.95)(300,1.0)$ \\
\hline & Alluvium (Devitrified) & Beta & $\mu=100$, range $=50-300, \sigma=15$ \\
\hline $\mathrm{Cs}$ & Composite (Volcanics) & Cumulative & $\left(K_{d}\right.$, prob) $(100 ., 0).(3000.59,0.05)(6782.92,1.0)$ \\
\hline $\mathrm{Am} / \mathrm{Th} / \mathrm{Pa}$ & Alluvium (Devitrified) & Truncated Norm & $\begin{array}{l}\text { range }=100-1,000 \\
\mu=728, \sigma=464\end{array}$ \\
\hline $\mathrm{Sr}$ & Volcanics and Alluvium & Truncated Norm & $\begin{array}{l}\text { range }=1,000-10,000 \\
\mu=5,500, \sigma=1,500\end{array}$ \\
\hline $\mathrm{Ra}$ & Volcanics and Alluvium & Uniform & range $=20-400$ \\
\hline $\mathrm{C} / \mathrm{Tc} / \mathrm{l}$ & Volcanics and Alluvium & Constant & Zero $=100-1,000$ \\
\hline
\end{tabular}

Output DTN: LA0310AM831341.002.

NOTE: Development of the distributions given this table is based on Tables A-4 and C-11.

$\mu=$ mean

$\sigma=$ standard deviation

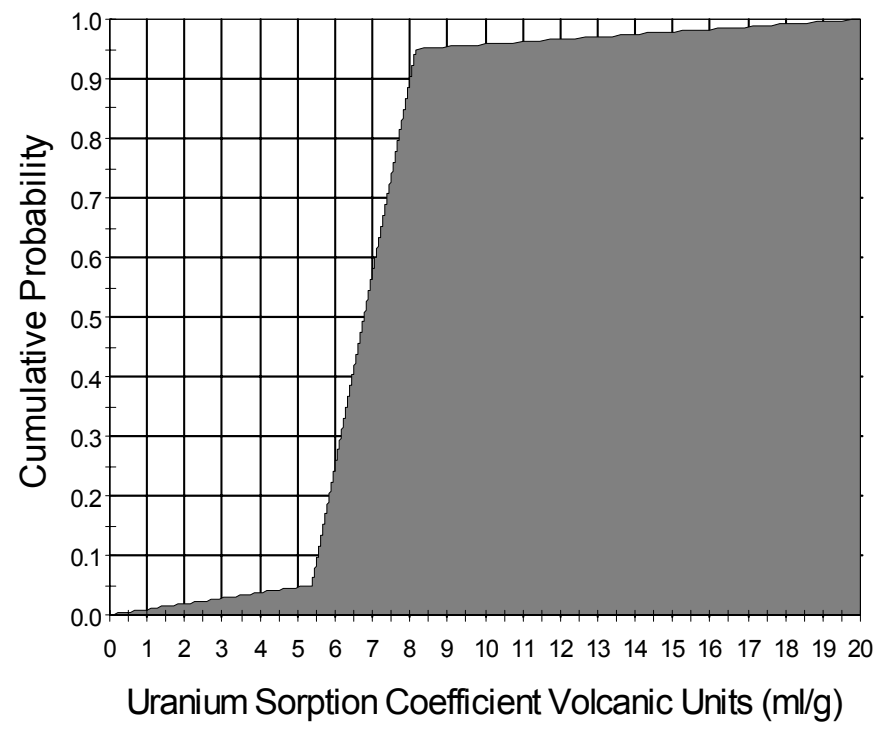

Output DTN: LA0309AM831341.001.

Figure C-9. Plot of Composite Cumulative Probability Distribution for Uranium in Volcanics 


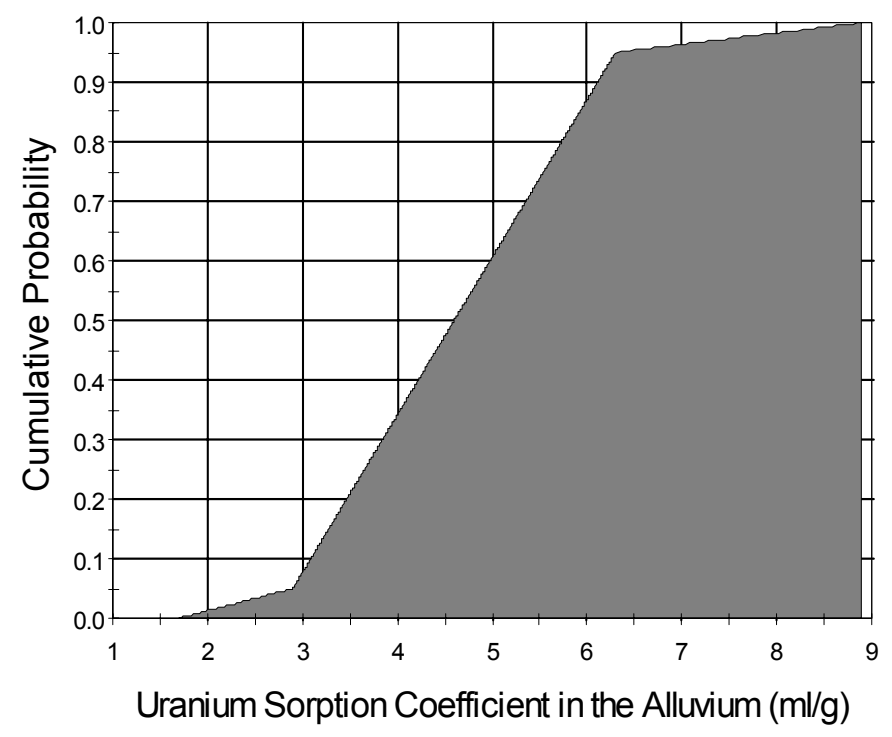

Output DTN: LA0309AM831341.001.

Figure C-10. Plot of Composite Cumulative Probability Distribution for Uranium in Alluvium

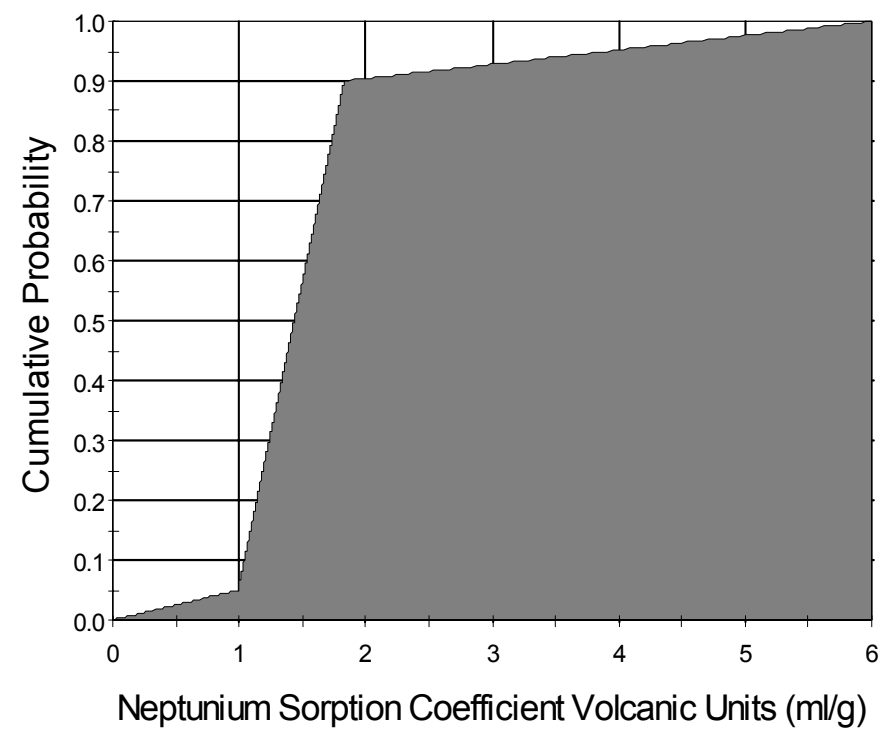

Output DTN: LA0309AM831341.001.

Figure C-11. Plot of Composite Cumulative Probability Distribution for Neptunium in Volcanics 


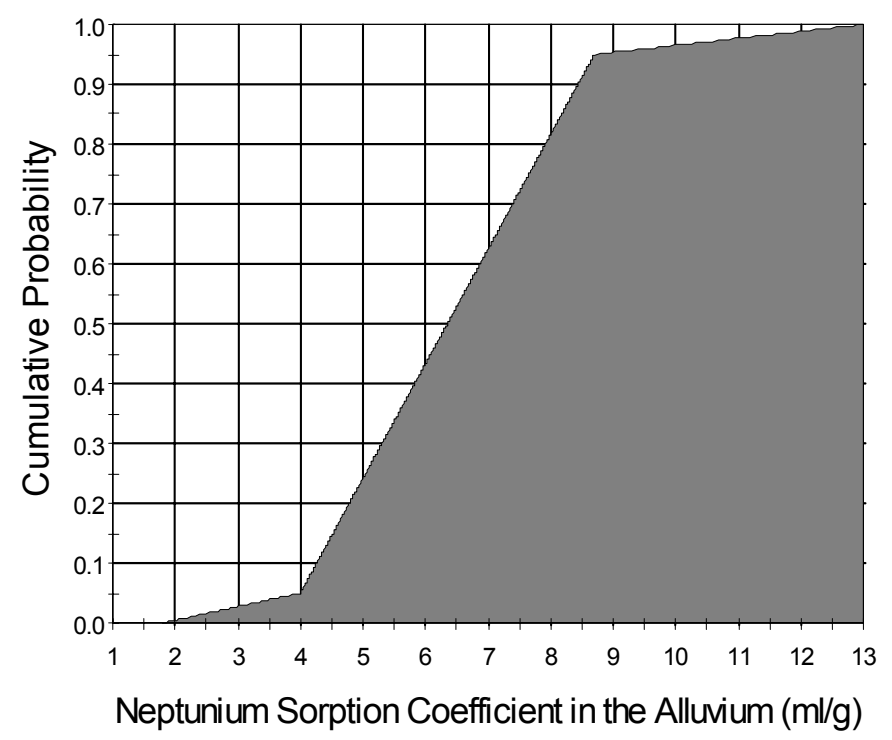

Output DTN: LA0309AM831341.001.

Figure C-12. Plot of Composite Cumulative Probability Distribution for Neptunium in Alluvium

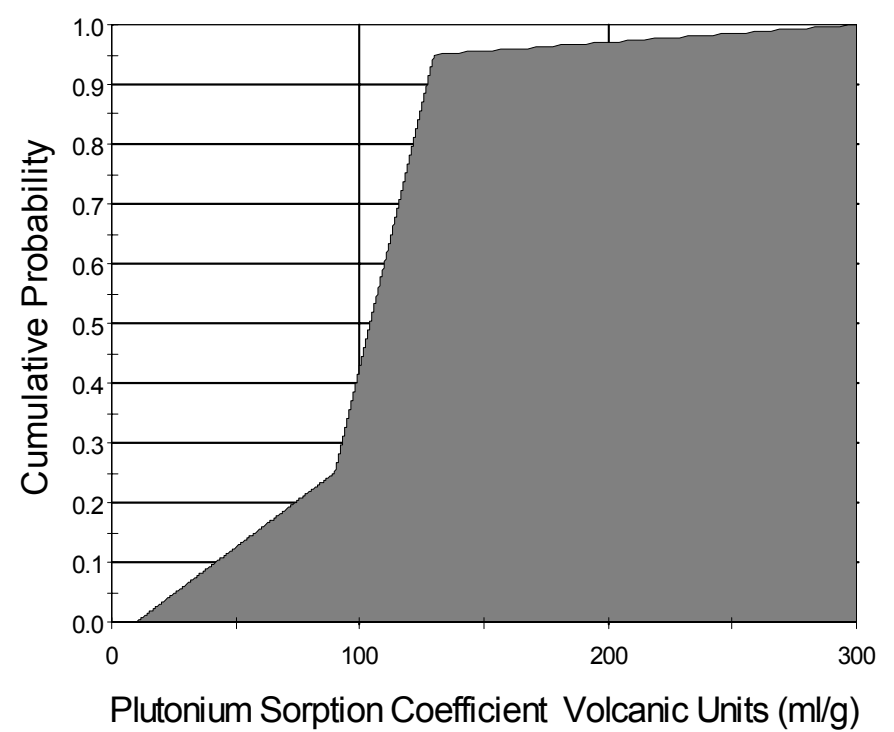

Output DTN: LA0309AM831341.001.

Figure C-13. Plot of Composite Cumulative Probability Distribution for Plutonium in Volcanics 


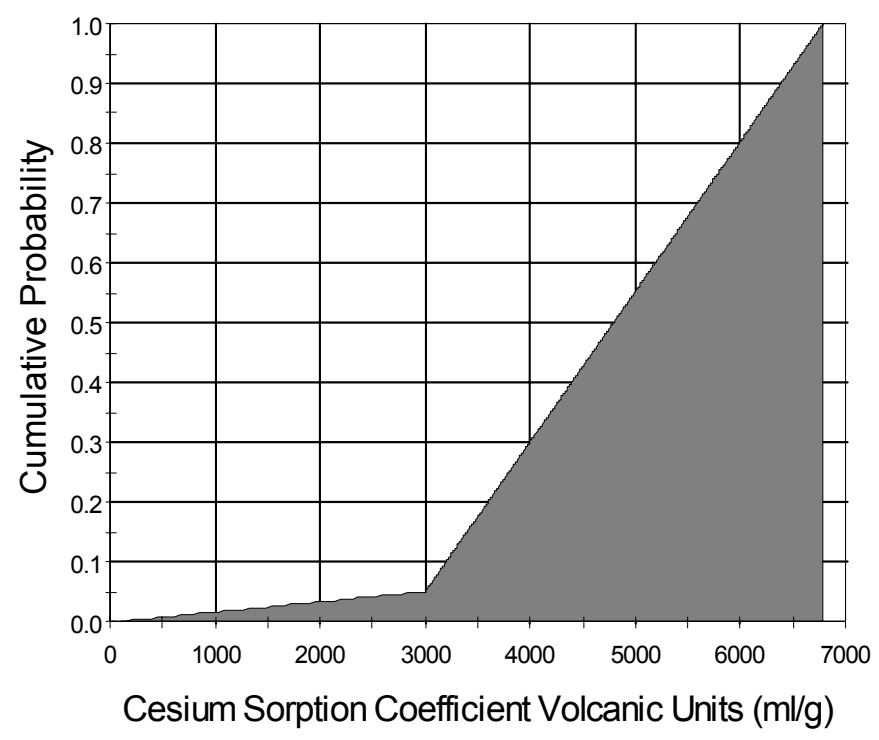

Output DTN: LA0309AM831341.001.

Figure C-14. Plot of Composite Cumulative Probability Distribution for Plutonium in Alluvium

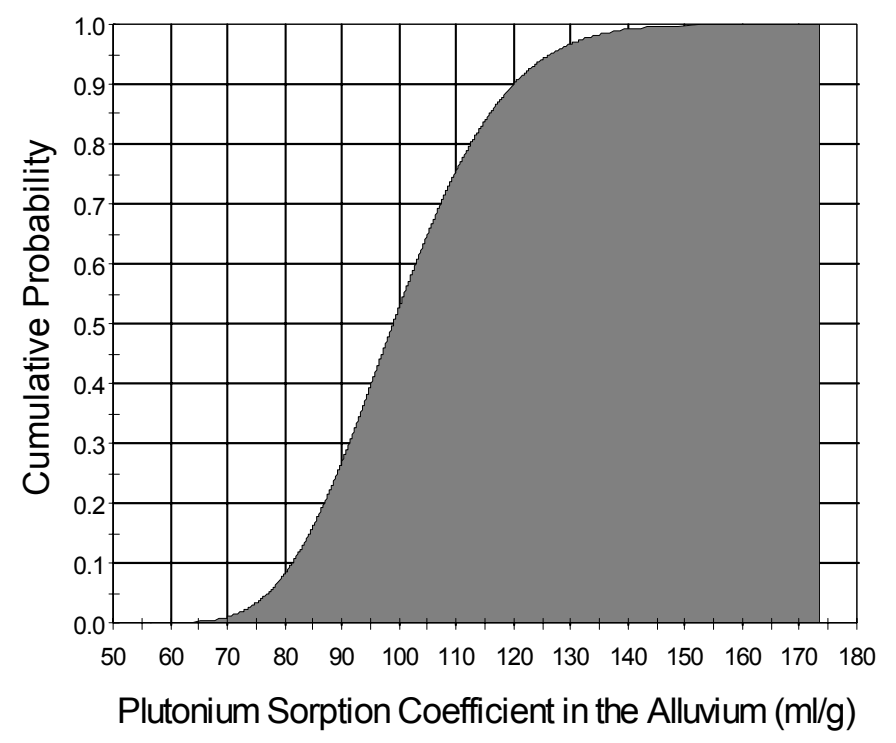

Output DTN: LA0309AM831341.001.

Figure C-15. Plot of Composite Cumulative Probability Distribution for Cesium in Volcanics 


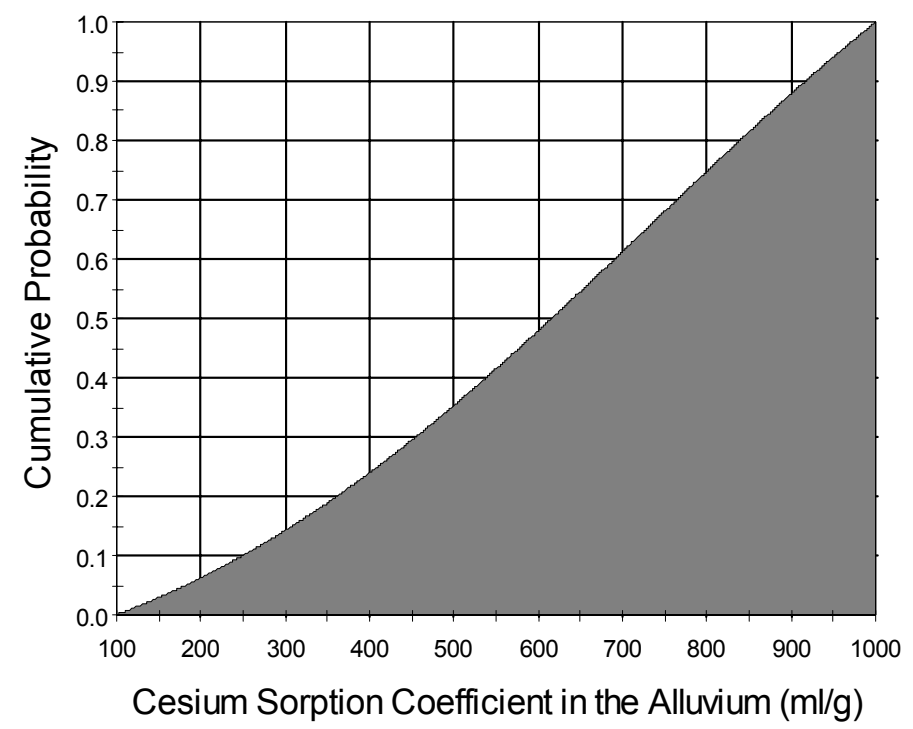

Output DTN: LA0309AM831341.001.

Figure C-16. Plot of Composite Cumulative Probability Distribution for Cesium in Alluvium

For neptunium in volcanics, the minimum small-scale value was $0 \mathrm{~mL} / \mathrm{g}$. Five percent probability was uniformly distributed between $0 \mathrm{~mL} / \mathrm{g}$ and the lower bound of the scaled distribution at $0.99 \mathrm{~mL} / \mathrm{g}$. Since the scaled distribution had such little variation, it is reasonably represented by a uniform distribution; hence, 85 percent probability was uniformly distributed between $1 \mathrm{~mL} / \mathrm{g}$ and the upper bound of the upscaled distribution at $1.83 \mathrm{~mL} / \mathrm{g}$. The remaining 10 percent probability was uniformly distributed between $1.83 \mathrm{~mL} / \mathrm{g}$ and the maximum small-scale experimentally observed value of $6 \mathrm{~mL} / \mathrm{g}$. This distribution is shown schematically in Figure C-11. The distribution for neptunium in alluvium is shown in Figure C-12.

For plutonium in volcanics, the minimum small-scale value was $50 \mathrm{~mL} / \mathrm{g}$. Twenty-five percent probability was uniformly distributed between $10 \mathrm{~mL} / \mathrm{g}$ and the lower bound of the scaled distribution at $89.9 \mathrm{~mL} / \mathrm{g}$. Since the scaled distribution had such little variation, it is reasonably represented by a uniform distribution; hence, 70 percent probability was uniformly distributed between $89.9 \mathrm{~mL} / \mathrm{g}$ and the upper bound of the upscaled distribution at $129.87 \mathrm{~mL} / \mathrm{g}$. The remaining 5 percent probability was uniformly distributed between $129.87 \mathrm{~mL} / \mathrm{g}$ and the maximum small-scale experimentally observed value of $300 \mathrm{~mL} / \mathrm{g}$. This distribution is shown schematically in Figure C-13. The distribution for plutonium in alluvium is shown in Figure C-14.

For cesium in volcanics, the minimum small-scale value was $100 \mathrm{~mL} / \mathrm{g}$. Five percent probability was uniformly distributed between $100 \mathrm{~mL} / \mathrm{g}$ and the lower bound of the scaled distribution at $3,000.59 \mathrm{~mL} / \mathrm{g}$. Since the scaled distribution had such little variation, it is reasonably represented by a uniform distribution; hence, the remaining 95 percent probability was uniformly distributed between $3,000.59 \mathrm{~mL} / \mathrm{g}$ and the upper limit of the scaled distribution at $6,782.92 \mathrm{~mL} / \mathrm{g}$. This distribution is shown schematically in Figure C-15. The distribution for cesium in alluvium is shown in Figure C-16. 


\section{INTENTIONALLY LEFT BLANK}


APPENDIX D

JUSTIFICATION OF LOCAL EQUILIBRIUM APPROACH FOR MODELING RADIONUCLIDE TRANSPORT IN THE SZ 
The analysis presented in this appendix supports screening assessment of an alternative conceptual model that deals with the treatment of sorption reactions as being instantaneous (Table 6.6-1b, Item \#1). The results of this analysis do not form a direct input to the site-scale SZ transport model.

To assess whether the sorption kinetics process needs to be included in the transport model, column test data, under flow rates pertinent to the Yucca Mountain flow system, are used to calculate Damköhler numbers to determine if kinetic effects are important (Triay et al. 1997 [DIRS 100422]). The Damköhler number can be used to determine whether the local equilibrium approach is valid. If valid, kinetic effects can be neglected, and equilibrium models that are computationally much more efficient can be used. Plutonium kinetics is examined in this analysis since plutonium sorption kinetics has been shown to be slower than the other radionuclides in the inventory (see Appendix A). Therefore, if the local equilibrium approach is valid for plutonium, it should be valid for the other radionuclides in the inventory.

The Damköhler number is defined as the rate constant, $k$ (1/time), multiplied by a representative residence time, $T$,

$$
D a=k^{*} T
$$

where $k$ is a first order reaction rate constant.

In Equation D-1, the rate constant quantifies the reaction timescale of the system whereas the residence time quantifies the transport timescale. By multiplying these parameters together, $D a$ provides a basis for evaluating which timescale dominates a system. For cases in which the reaction timescale is much faster than the transport timescale, $D a$ is large and the local equilibrium approach is valid.

Bahr and Rubin (1987 [DIRS 144539], p. 440, Equation 12) demonstrate that the mass balance equation describing solute transport can be separated into an equilibrium and a kinetic component. The smaller the kinetic component, the more accurate are the retardation factors based on the local equilibrium approach.

Bahr and Rubin (1987 [DIRS 144539], p. 450) found that equilibrium was well approximated when the sum of the two Damköhler numbers is greater than 100 and reasonably well estimated when the sum is greater than 10. For evaluation of sorption behavior, separate Damköhler numbers, $D a_{a t t}$ and $D a_{d e t}$, can be computed for attachment and detachment of the sorbing contaminant using $k_{\text {att }}$ and $k_{\text {det }}$, which are the attachment and detachment rate constants for plutonium sorbing onto the mineral surfaces. The magnitude of the kinetic component is inversely proportional to the total Damköhler number. As the sum of the two Damköhler numbers becomes larger, the equilibrium approximation becomes more appropriate.

Valocchi (1985 [DIRS 144579], p. 813, Figure 2) had a similar result, although he used only the reverse rate $\mathrm{k}_{\mathrm{det}}$ to compute a Damköhler number. Valocchi's approach is utilized in this study since a single first order rate best fit the column experiments. Because the Valocchi's approach utilizes one Damköhler number, which results in lower Da numbers than the Bahr and Rubin method, the Bahr and Rubin criteria of 10 and 100 can also be used with the Valocchi approach. Valocchi's approach is used in this study. 
As seen from Equation D-1, to estimate the Damköhler number for the SZ transport model, the reaction rate constants for plutonium sorption must be determined. This determination is done by using laboratory data from column experiments. The general idea behind the calculation is to fit a first-order reaction rate constant to ${ }^{239} \mathrm{Pu}$ column data (DTN: LA0302HV831361.001 [DIRS 163783]). This rate constant, along with a conservative travel time through the fractured volcanics, can be used to estimate a Damköhler number. The Damköhler number indicates whether kinetics are important in a system. A sample calculation is presented below.

1. Determine the pore volume $P V$ of the column:

The pore volume of the column is necessary to calculate the residence time of each column experiment. The pore volume can be calculated by determining the mean breakthrough time for tritium, which is a conservative tracer:

$$
P V=0.5 \text { concentration breakthrough of tritium }
$$

$P V$ for sample G4-268 devitrified tuff with $\mathrm{J}-13$ well water and radionuclides ${ }^{3} \mathrm{H}$ and ${ }^{239} \mathrm{Pu}=7 \mathrm{~mL}$ (DTN: LA0401PR831361.001 [DIRS 166726]).

With the pore volume and flow rate, the residence time $R T$ for each column experiment can be calculated:

$R T$ of Each Column $=P V /$ Flow rate:

Column 1: $\mathrm{RT}=(7 \mathrm{~mL}) /(2.89 \mathrm{~mL} / \mathrm{hr})=2.42 \mathrm{hr}$

Column 2: $R T=(7 \mathrm{~mL}) /(1.12 \mathrm{~mL} / \mathrm{hr})=6.25 \mathrm{hr}$

Column 3: $R T=(7 \mathrm{~mL}) /(0.4 \mathrm{~mL} / \mathrm{hr})=17.5 \mathrm{hr}$

2. Determine the steady-state fractional recoveries concentrations of $\mathrm{Pu}$ in the column for the different flow rates:

For each column experiment, the plutonium fractional recovery plateaus at significantly less than 1.0. The value at which the recovery plateaus is used to determine the kinetic sorption rate of plutonium.

Steady-state concentrations of $\mathrm{Pu}$ at different flow rates:

Column 1: $2.89 \mathrm{~mL} / \mathrm{hr}:$ Fractional Recovery $\mathrm{C} / \mathrm{C}_{0}=0.67$

Column 2: $1.12 \mathrm{~mL} / \mathrm{hr}:$ Fractional Recovery $C / C_{0}=0.31$

Column 3: $0.8 \mathrm{~mL} / \mathrm{hr}$ : Fractional Recovery $\mathrm{C} / \mathrm{C}_{0}=0.06$

3. Determine the kinetic rate constants that fit the Pu column data:

The steady-state fractional recovery versus residence time data was used to obtain the first-order kinetic reaction rate constant. The first-order kinetic rate law (e-kt) yields a result of $k_{\text {for }}=\sim 0.2 \mathrm{hr}^{-1}$. This result is obtained directly from rearrangement of the first-order rate law expression: $k_{\text {for }}=-\ln ($ frac. rec. $) / R T$. 
To calculate the $D a$ number, a reverse kinetic rate is needed. Using a representative value of $K_{d}$ for Pu in devitrified tuff of $100 \mathrm{~mL} / \mathrm{g}$ (Section A7.4.1 of this report), $k_{\text {rev }}=k_{\text {for }} / 100=0.002 \mathrm{hr}^{-1}$ is obtained.

4. Estimate a travel time through the system:

Time to first breakthrough for Np reported in Saturated Zone Flow and Transport Model Abstraction (BSC 2004 [DIRS 170042]), Figure 6-32) is less than 100 years. Hence, a conservative value of 10 years is used for travel time through the fractured volcanics in the SZ.

5. Calculate the Damköhler number:

$$
D a=k_{\mathrm{rev}} R T=175
$$

Valocchi found that equilibrium is well estimated for $D a>100$ (1985 [DIRS 144579], p. 813, Fig. 2).

Based on this analysis, the local equilibrium approach is valid for plutonium transport in the SZ of Yucca Mountain.

It should be noted that colloid-facilitated transport could not be ruled out as an explanation for the early breakthrough of ${ }^{239} \mathrm{Pu}$ in the column experiments used in this analysis. The kinetic interpretation of these column studies is also consistent with a colloid transport interpretation where the sorption and desorption rate constants are equivalent to colloid filtration and detachment rate constants. Another possible explanation for the early ${ }^{239} \mathrm{Pu}$ breakthrough in the column experiments is that microbial growth or other processes in the columns may have resulted in localized reducing conditions that caused some of the injected $\mathrm{Pu}(\mathrm{V})$ to be reduced, sorb strongly to microbial colloidal material, and be transported faster than the portion of $\mathrm{Pu}$ sorbed onto the rock matrix. However, if the early plutonium breakthroughs in the column experiments were a result of colloid-facilitated transport of a portion of the plutonium, then the sorption of rate constants for the soluble plutonium fraction would have to be greater than those obtained assuming that all the plutonium was soluble. This scenario would only strengthen the conclusion that the equilibrium approximation is valid for soluble plutonium over large time scales. 


\section{INTENTIONALLY LEFT BLANK}




\section{APPENDIX E}

TRANSPORT SIMULATIONS WITH ALTERNATE FLOW SCENARIOS 


\section{E1. TRANSPORT SIMULATIONS WITH THE HIGHER WATER TABLE}

The analysis presented in this appendix supports screening assessment of the alternative conceptual model that deals with the effect of water table rise due to future climate conditions (Table 6.6-1a, Item \#4) and of the alternative conceptual model that deals with the effect of alternate flow paths resulting from alternate flow models (Table 6.6-1a, Item \#6). The results of this analysis do not form a direct input to the site-scale SZ transport model.

The SZ site-scale flow model DTN: LA0304TM831231.002 [DIRS 163788] was adapted with a higher water table grid to conduct particle-tracking simulations for a nonsorbing species and for neptunium. These simulations are performed for the estimated wetter, glacial-transition climatic conditions in which the groundwater flux through the SZ is greater than present conditions by a factor of 3.9 as given in Saturated Zone Site-Scale Flow Model (BSC 2004 [DIRS 170037], Section 6.4.5). Multiplying the recharge and the values of permeability for all hydrogeologic units by a factor of 3.9 approximates the higher groundwater flux rates. The expected values for parameters associated with radionuclide transport are applied in these simulations.

The solid line in Figure E-1 shows the results of the transport simulation with the higher water table and estimated groundwater flow under future glacial-transition climatic conditions for a nonsorbing species. All of the simulated breakthrough curves presented in Figures E-1 and E-2 are for transport from beneath the proposed repository to the 18-km regulatory limit of the accessible environment. For comparison, the dashed line in Figure E-1 shows the simulated breakthrough curve using the simplified approach utilized in the TSPA abstraction of radionuclide transport in the SZ as shown in Saturated Zone Flow and Transport Model Abstraction (BSC 2004 [DIRS 170042], Section 6.5). This simplified approach uses the simulated breakthrough curve from the SZ site-scale flow model with the present water table configuration and scales that breakthrough curve to shorter transport times using the groundwater flux multiplier of 3.9 for future glacial-transition climatic conditions. Figure E-2 shows similar results for the transport of neptunium using the two alternative approaches. Neptunium exhibits a small amount of sorption in the matrix of volcanic units and a moderate amount of sorption in the alluvium.

Differences in the simulated transport times for these alternative approaches to climate change in the SZ are primarily attributable to differences in the hydrogeologic units encountered along flow paths from beneath the repository. Flow paths in the SZ pass through a considerably longer length of the upper volcanic confining unit in the adapted site-scale SZ model with the higher water table. The upper volcanic confining unit has significantly lower permeability than the underlying units of the Crater Flat Tuff, leading to longer transport times along these flow paths. In addition, the relatively slower groundwater flow rates in the upper volcanic confining unit allow greater matrix diffusion, leading, in turn, to even longer transport times. There is also greater flow path length through the porous alluvium near the water table to the south and east of the repository in the adapted model with the higher water table. The porous nature of the alluvium also leads to longer simulated transport times through this medium. 
Comparison of the two approaches shown in Figures E-1 and E-2 indicates that the model with the higher water table results in longer simulated transport times for both the nonsorbing species and for neptunium. The simplified approach of scaling the breakthrough curves from the SZ site-scale flow model with the present water table elevations is thus a pessimistic representation of transport in the SZ under wetter glacial-transition climatic conditions relative to the adapted model that incorporates water table rise associated with future conditions. Consequently, the results of these simulations tend to verify the assumption that the flux scaling approach to simulation of climate change is conservative with regard to radionuclide transport in the SZ, relative to the more realistic situation in which water table rise is included in the modeling.

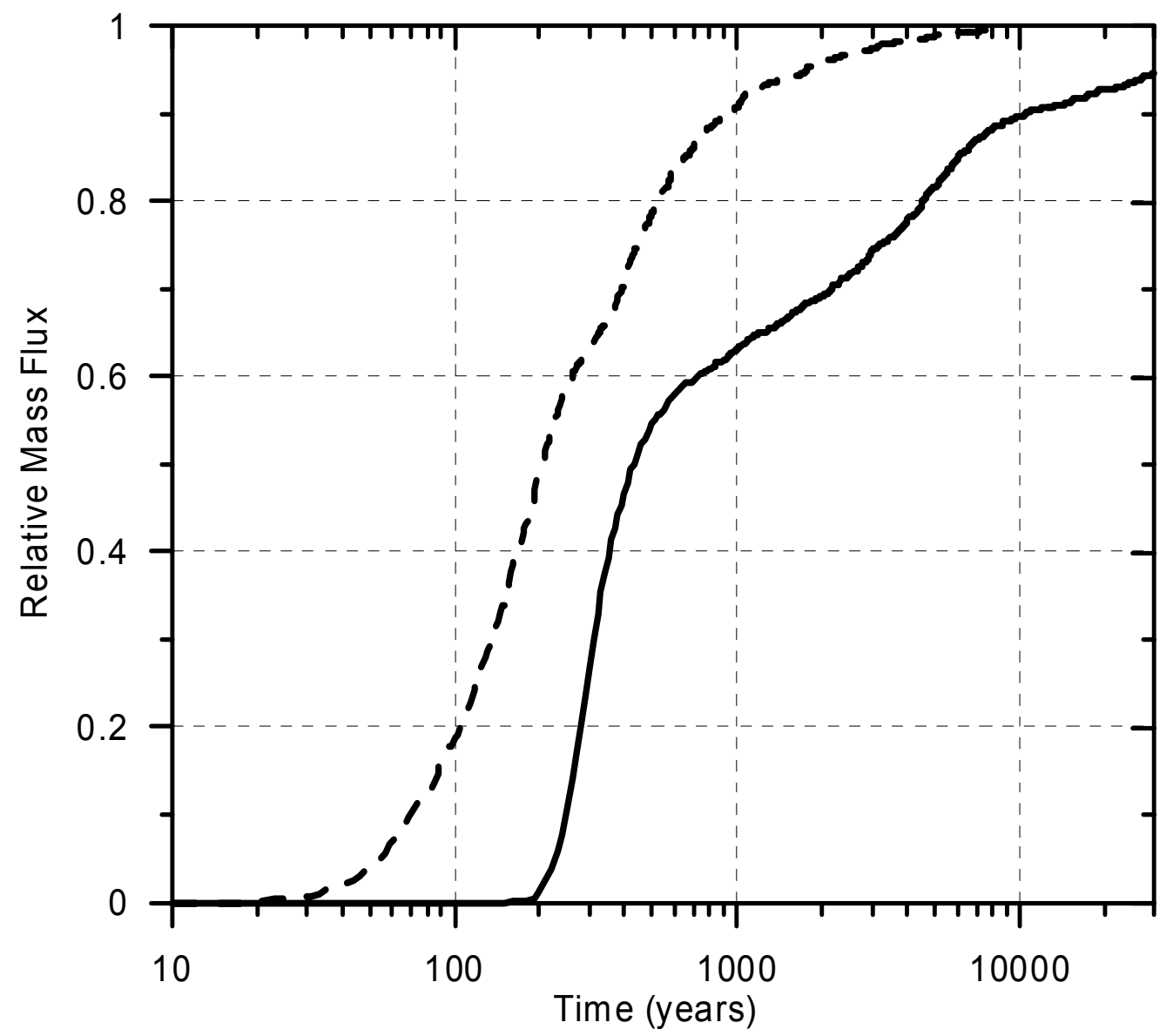

For illustration purposes only.

NOTE: Dashed line is the SZ breakthrough curve at $18 \mathrm{~km}$ for future glacial-transition climatic conditions that has been scaled (by a factor of 3.9) from transport simulation for present-day climatic conditions. The solid line is the SZ breakthrough curve at $18 \mathrm{~km}$ for future glacial-transition climatic conditions that has been simulated with the higher water table model. Breakthrough curves are for a constant source initiated at the water table beneath the repository at time equal to zero and do not include radioactive decay.

Figure E-1. Breakthrough Curves for Nonsorbing Radionuclides for Future Glacial-transition Climatic Conditions Using Two Alternative Approaches 


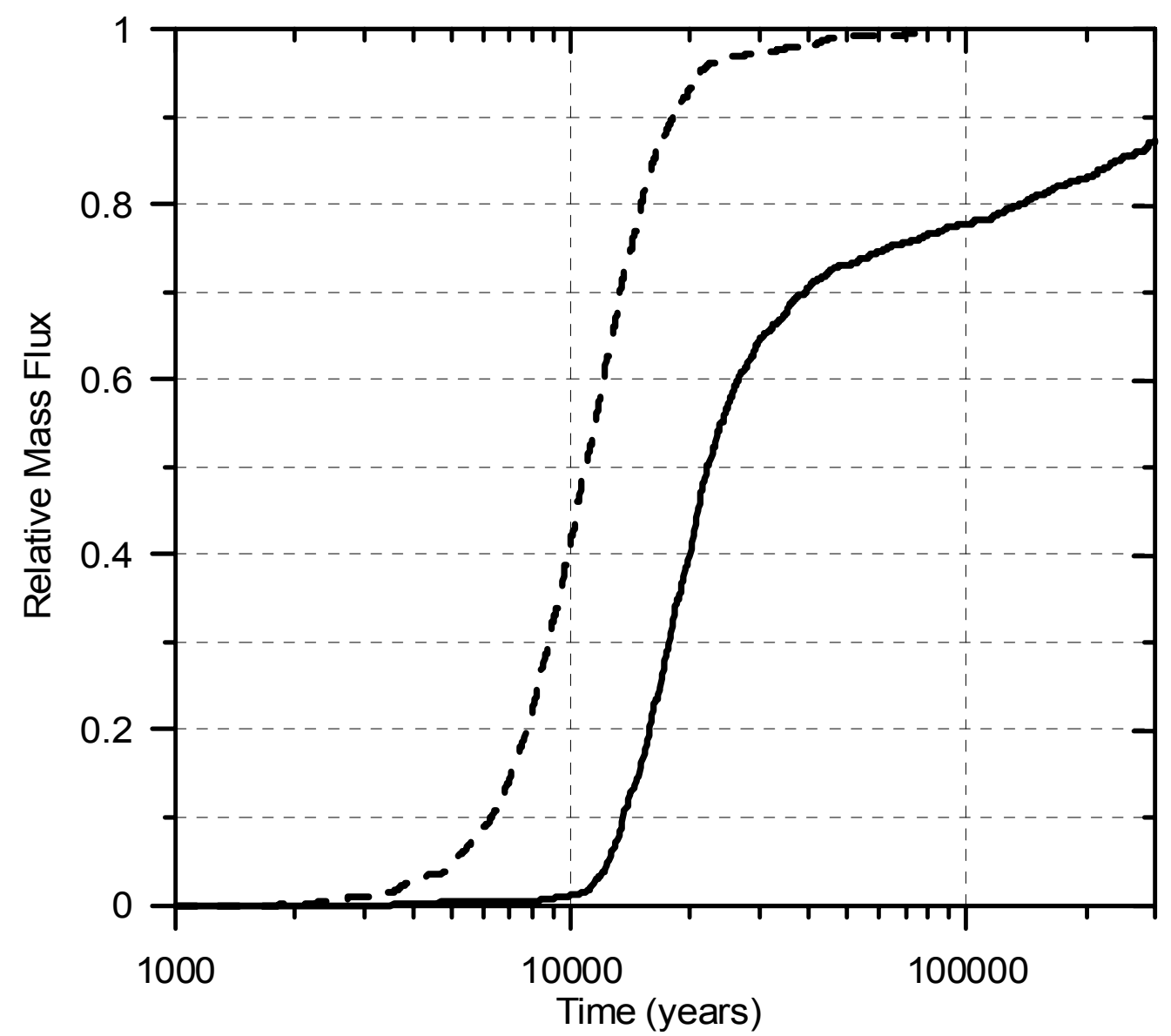

For illustration purposes only.

NOTE: Dashed line is the SZ breakthrough curve at $18 \mathrm{~km}$ for future glacial-transition climatic conditions that has been scaled (by a factor of 3.9) from transport simulation for present day climatic conditions. The solid line is the SZ breakthrough curve at $18 \mathrm{~km}$ for future glacial-transition climatic conditions that has been simulated with the higher water table model. Breakthrough curves are for a constant source initiated at the water table beneath the repository at time equal to zero and do not include radioactive decay.

Figure E-2. Breakthrough Curves for Neptunium for Future Glacial-transition Climatic Conditions Using Two Alternative Approaches

\section{E2. TRANSPORT SIMULATIONS WITH ALTERNATE FLOW MODELS BASED ON REVISED HYDROLOGIC FRAMEWORK MODEL}

Alternate flow models have been developed resulting from a revised hydrologic framework model, additional water level data from the Nye County Early Warning Drilling Program, revised recharge distribution, updated boundary fluxes from the 2001 Death Valley Regional Flow Model, additional permeability data, and further evaluation of the flow model, Saturated Zone Site-Scale Flow Model (BSC 2004 [DIRS 170037] Section 6.4.6).

The hydrologic framework model used in the alternate flow models is more refined with 27 geologic units, as compared to the base-case flow model with 19 geological units. To the north of Yucca Mountain, the east-west barrier that was used in the base-case model has been removed in the alternate flow model and in place of it the geologic units in the region have been 
divided into northern and southern zones at the Claims Canyon Caldera boundary. The region below Yucca Mountain remains comprised of the Crater Flat Group: Prow Pass, Bullfrog, and Tram units. In the alternate flow model, the Crater Flat units are more continuous to the north and west of Yucca Mountain as compared to the base-case flow model. Separate permeability groups were created for Tram Bullfrog and Prow Pass units near Yucca Mountain to accommodate spatial differences between this area and that to the east of Yucca Mountain. Additional zones were included in the alternate flow model to represent the northwest-southeast trending fault zone just to the north of Yucca Mountain, as well as the Ghost Dance Fault and the Dune Wash Fault. In the Fortymile Wash area, the Alluvial Uncertainty Zone that was used in the base-case flow model has been removed from the alternate flow model and replaced with the more detailed geohydrologic information now available.

As in the base-case site-scale SZ flow model, constant head boundary conditions are used around the periphery of the model, but updated 2001 potentiometric surface has been used in alternate flow models (BSC 2004 [DIRS 170037] Section 6.4.6.2). Recharge is applied to the top surface of the alternate flow model in the same manner as the base-case flow model; however, an updated recharge distribution has been used. This results in 40 percent more recharge to the alternate flow model.

Model permeabilities are optimized, as in the base-case flow model, to best match the available water level data and boundary fluxes. Target fluxes at the north, east, and west boundaries of the alternate flow model are considerably different from those used in the base-case model due to improvements in the regional scale model, (BSC 2004 [[DIRS 170037] Section 6.4.6.3). There is considerable uncertainty in the flux values obtained from the regional scale model. To address this uncertainty, five different alternate flow models have been developed and calibrated. These models are identical in all respects except for the target fluxes used on the model boundaries.

It was found in Saturated Zone Site-Scale Flow Model (BSC 2004 [DIRS 170037] Section 6.4.6.3) that the alternate flow models yielded water levels and flux values that compared more favorably with the target data better than the base-case flow model. However, the optimized values of permeabilities for the Crater Flat Group (Tram, Bullfrog, and Prow Pass) were found to be lower than the base-case values for the base-case flow model. Further, permeabilities of these units near Yucca Mountain are an order of magnitude lower than the corresponding values to the east in the Fortymile Wash area. The resulting specific discharge from the alternate flow models was found to be in the range of 0.51 to $0.653 \mathrm{~m} /$ year, significantly lower than the value of $1.3 \mathrm{~m} /$ year for the base-case flow model.

Figures 6-19 through 6-21 of Saturated Zone Site-Scale Flow Model (BSC 2004 [DIRS 170037]) show the flow paths of particles starting at the repository footprint for the base-case flow model and the various alternate flow models. It is seen that the flow paths resulting from the alternate flow models are generally much more southerly than those from the base-case. The alternate flow model flow paths are also deeper. Figures 6-23 through 6-26 of Saturated Zone Site-Scale Flow Model (BSC 2004 [DIRS 170037]) show the various hydrologic units encountered along the flow paths. It is seen that the alternate flow model flow paths encounter volcanic units along a significantly greater portion of the path, and alluvial units along a correspondingly smaller portion of the path, as compared to the base-case flow model. This difference is noteworthy due to the differences in the transport phenomenon of importance in the volcanics as compared to 
the alluvium (Section 6.3). The volcanics are represented by a dual-porosity model with low effective porosities (and resultant high fluid flow velocities), significant matrix diffusion, and retardation in the matrix, while the mechanisms important in the alluvium are advection with much higher effective porosities (with resultant lower fluid flow velocities) and retardation. Colloid-facilitated transport is important in both the media.

Figure E-3 shows the breakthrough curves for the five alternate flow models, as well as the basecase flow model, using base-case transport properties listed in Table 6.4-2. The input files were modified to update the assignment of porosities, diffusivities, and other transport properties to appropriate zones since the unit numbering is different compared to the base-case flow model, as given in Table 6-3 of Saturated Zone Site-Scale Flow Model (BSC 2004 [DIRS 170037]). The median transport time (time for the breakthrough of 50 percent of the input mass at the boundary of the accessible environment at $18 \mathrm{~km}$ ) for the alternate flow models is in excess of 6500 years for nonsorbing radionuclides, as compared to the value of 705 years for the base-case flow model. Thus, the alternate flow models lead to significantly slower transport of the radionuclides as compared to the base-case flow model. It is seen in Figure E-3, that out of the five alternate flow models, model designated AM2 leads to shortest travel times. Hence, as a conservative approach, this model was selected for further studies.

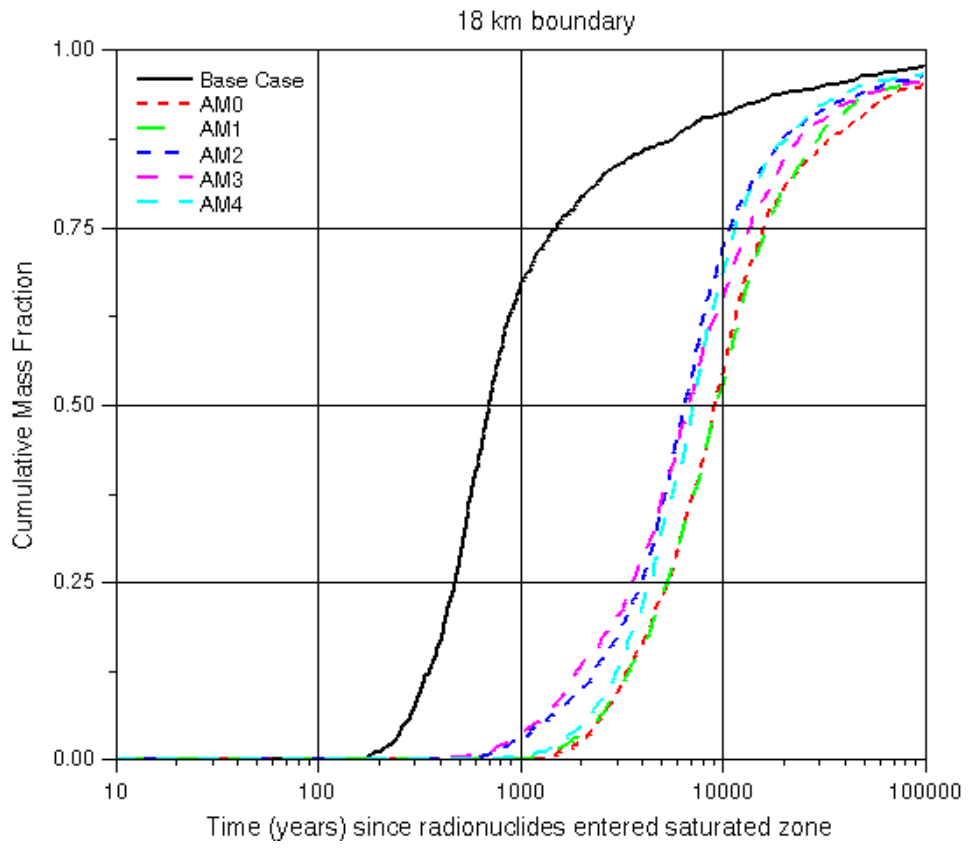

For illustration purposes only.

Figure E-3 Breakthrough Curves at the Boundary to the Accessible Environment at $18 \mathrm{~km}$ for the alternate flow models and the Base-Case Flow Model (Using Base-Case Transport Properties) 
In order to investigate this further, Figure E-4 shows a comparison of the breakthrough curves for AM2 with and without matrix diffusion in the volcanics, along with similar curves for the base-case flow model. It is seen that matrix diffusion leads to significant retardation of the radionuclides, and the effect of this mechanism is more pronounced at the lower velocities encountered in AM2.

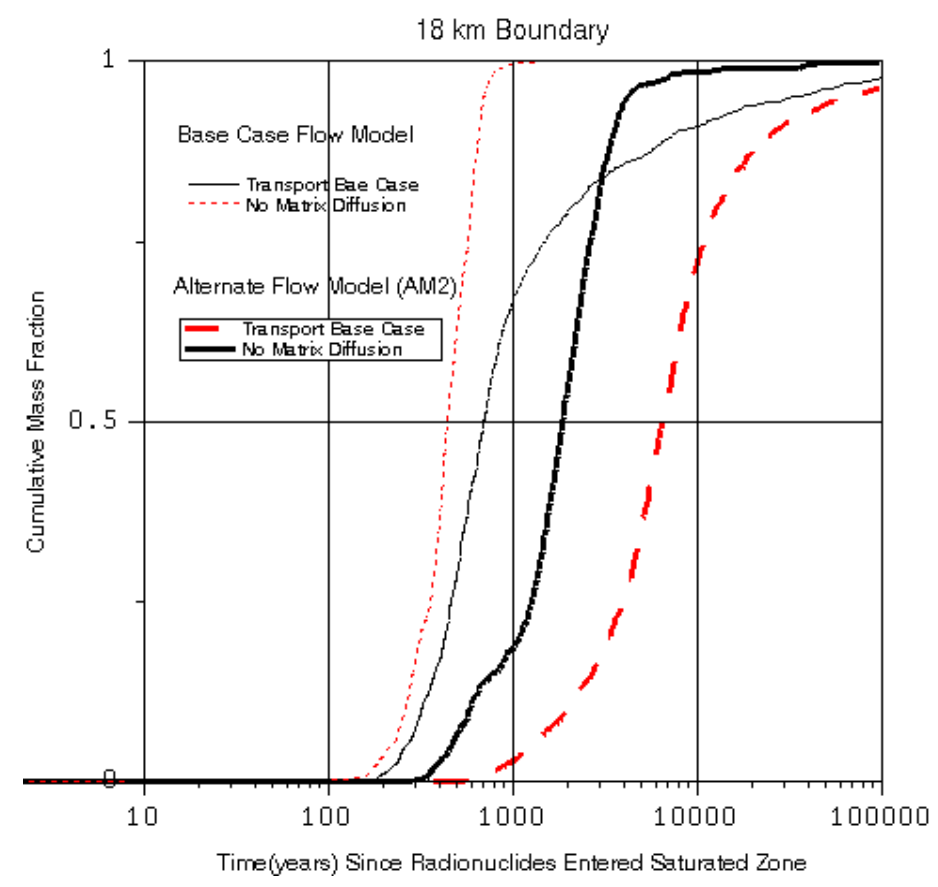

For illustration purposes only.

Figure E-4. Comparison of the Breakthrough Curves for the Alternate Flow Model AM2 With and Without Matrix Diffusion 
The breakthrough curve using the AM2 flow model for the case of a moderately sorbing radionuclide such as $\mathrm{Np}$ is shown in Figure E-5. $K_{d}$ values of $1.3 \mathrm{~mL} / \mathrm{g}$ in the volcanic matrix and $6.3 \mathrm{~mL} / \mathrm{g}$ in the alluvium were used in these calculations. The breakthrough for AM2 without any sorption is shown for comparison. Also shown is the breakthrough curve with the same sorption coefficients using the base-case flow model. It is seen that the transit time for this case is in excess of 75,000 years, much greater than the value that is less than 22,000 years using the base-case flow model.

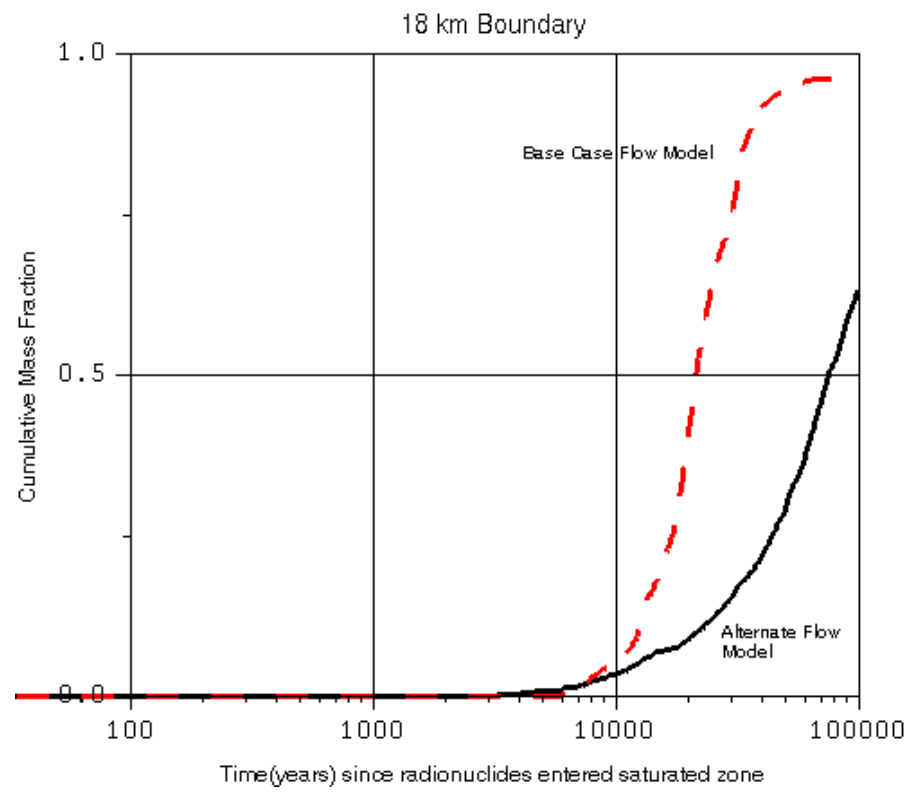

For illustration purposes only.

Figure E-5. Breakthrough Curves for the Case of a Moderately Sorbing Radionuclide Such As Np Using the AM2 Flow Model and Base-Case Flow Model

In conclusion, the alternate flow models AM0 through AM4 lead to transport times significantly longer than that for the base-case flow model. 
INTENTIONALLY LEFT BLANK 


\section{APPENDIX F \\ REDOX MEASUREMENTS IN SATURATED ZONE WATERS}


The data and analysis presented in this appendix supports the rationale for the assumption that for the development of sorption coefficient distributions, groundwater conditions are oxidizing in the site-scale SZ transport model (Table 5-1, Item\# 2). The results of this analysis do not form a direct input to the site-scale SZ transport model.

Variations in the oxidation/reduction (i.e., redox) potential in the SZ along potential transport pathways can have significant impacts on the rates at which redox-sensitive radionuclides are transported from the potential repository. Technetium and the actinide elements neptunium, plutonium, and uranium can each assume several oxidation states depending on redox conditions. The redox conditions along potential transport pathways determine the oxidation states that these radioelements assume in groundwater and in/on solid phases. The lower oxidation states of these elements (e.g., +4) have higher retardation coefficient values (Lieser and Mohlenweg, 1988; Figure 4 [DIRS 106684]) and are, therefore, transported at slower rates than these elements in higher oxidation states (e.g., +5 and +6 ).

Redox potential is basically the voltage of groundwater. The sources for this voltage are oxidation/reduction reactions (i.e., reactions in which electrons are transferred) that occur naturally in aquifers. A redox potential can be measured in groundwater with an inert probe (e.g., platinum electrode). The potentials measured in the field with a probe are referred to as oxidation/reduction potential (ORP) measurements. They are usually referenced to a silver/silver chloride reference electrode. By convention, the standard hydrogen electrode (SHE) has been assigned a voltage of zero at standard conditions. When field ORP measurements referenced to the Ag/AgCl reference electrode are normalized to the standard hydrogen electrode scale, the measurements are called Eh values.

Unfortunately, the measurement of redox potential with a platinum electrode often produces ambiguous results, particularly in oxidizing waters. This has been attributed to an absence of equilibrium among the redox reactions that occur in groundwaters (Lindberg and Runnels 1984 [DIRS 106685]). Fortunately, for the purposes of predicting radionuclide transport, it is generally sufficient to determine whether groundwaters along potential flow paths have redox potentials greater or less than certain critical values. These critical values are those potentials at which neptunium, plutonium, technetium, and uranium, are predominantly in their lower (e.g., +4 ) or higher (i.e., $+5,+6$, and or +7 ) oxidation states. The purpose of this appendix is to discuss analytical data obtained on redox conditions in groundwaters along potential flow paths from the repository to the accessible environment and to evaluate their significance for radionuclide transport.

\section{F1. DISCUSSION OF DATA}

Redox conditions in groundwaters can be characterized by the measurement of various parameters including oxidation/reduction potential (ORP/Eh), (DO, and the concentrations of redox-sensitive constituents such as ferrous iron $\left(\mathrm{Fe}^{2+}\right)$, total iron $\left(\mathrm{Fe}_{\text {total }}\right)$, total manganese $\left(\mathrm{Mn}_{\text {total }}\right)$, nitrate $\left(\mathrm{NO}_{3}{ }^{-}\right)$, nitrite $\left(\mathrm{NO}_{2}{ }^{-}\right)$, ammonium $\left(\mathrm{NH}_{4}{ }^{+}\right)$, sulfide $\left(\mathrm{HS}^{-}\right)$, sulfate $\left(\mathrm{SO}_{4}{ }^{2-}\right)$, as well as other less well known constituents such as different oxidation states of antimony, arsenic, and selenium. The YMP has made measurements of many of these parameters in groundwaters pumped from boreholes along potential flow paths from the repository (Figure F-1). The results are discussed in this appendix. 


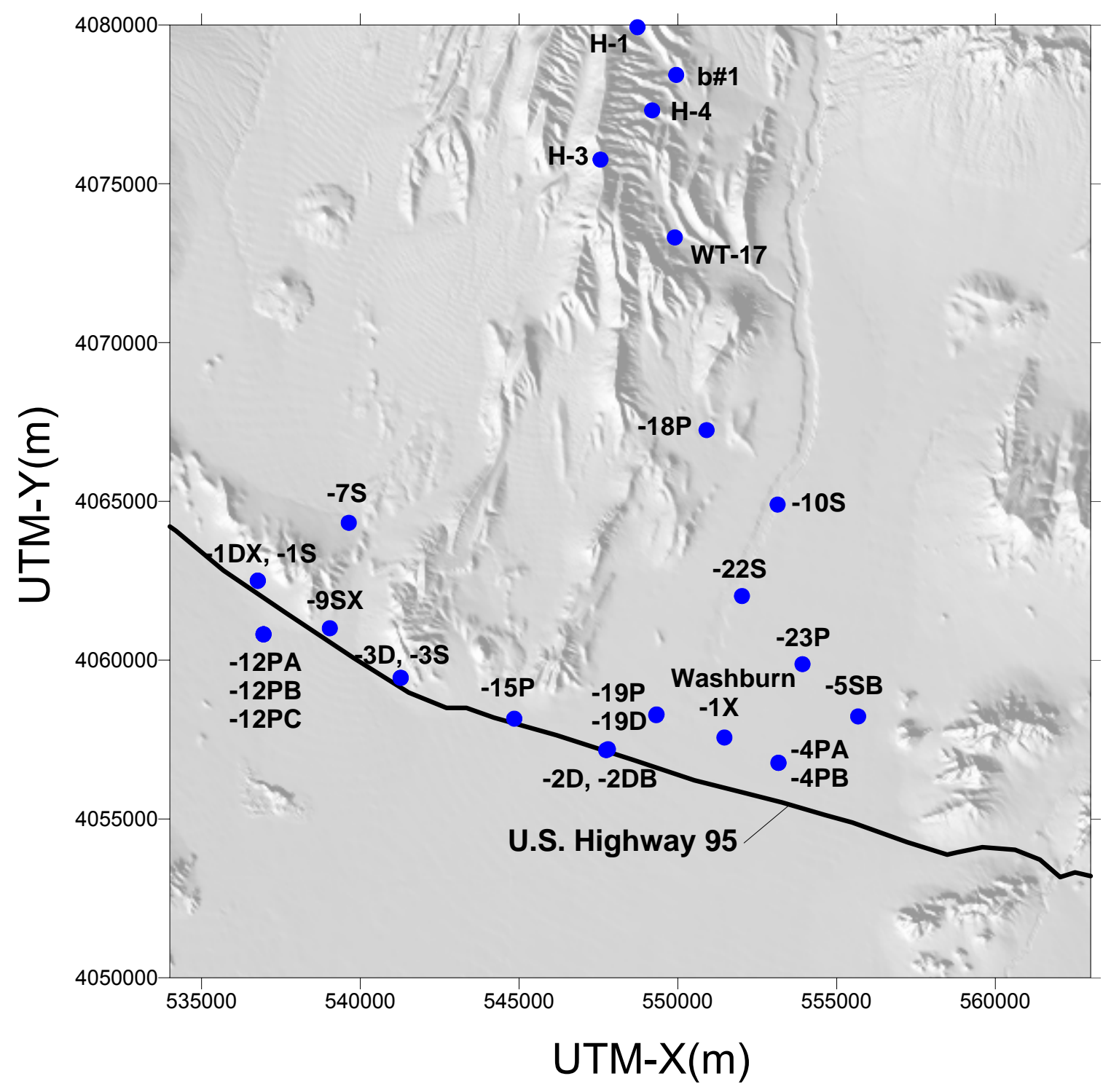

For illustration purposes only.

Figure F-1. Locations of the Wells Sampled for Redox Analysis

In addition to the problem of disequilibrium conditions among different redox reactions in the natural system, there are other factors that can complicate the analysis of redox conditions in aquifers. These factors include borehole construction effects, pumping effects, and analytical problems and errors. The data on redox parameters must be evaluated in light of these factors before the potential impacts on radionuclide transport can be discussed.

\section{F1.1 BOREHOLE CONSTRUCTION EFFECTS}

Borehole construction effects include the use of chemicals and materials during the drilling of a borehole, the use of steel casings and screens below the water table, improper sealing of casing against a borehole wall, and contaminants added to the boreholes during or after construction. After borehole construction has been completed, a borehole is generally "developed" to clean out 
the bulk of any chemicals and materials that may remain in the borehole from construction activities. In addition, when groundwater from a given borehole is to be sampled, at least three borehole volumes of groundwater are purged from the borehole prior to sampling the water. These activities are designed to remove chemicals and materials that were used in borehole construction, remove contaminants that may have entered the borehole after construction, and minimize any potential impacts from the corrosion of steel casing materials.

As a check on whether or not the borehole development and purging activities have produced the desired results, groundwater pumped during purging is routed through a flow-through cell at the surface that allows continuous measurement of water quality parameters $(\mathrm{T}, \mathrm{pH}$, conductivity, DO, and oxidation/reduction potential). Water sampling is begun once three borehole volumes have been purged from the borehole and the water quality parameters have stabilized (i.e., the values have reached a plateau). This procedure helps to avoid most of the potential impacts of borehole construction and contamination. However, the results of sampling of the same boreholes over a period of years suggest that some borehole construction effects take a long time to clean out. This issue is discussed in greater detail in a following section.

Improper sealing of the casing against the borehole wall can impact redox measurements by allowing mixing of waters from different flow zones. These may include waters that have been in contact with the atmosphere. Hydraulic measurements (i.e., heads) are generally used as a guide to detecting borehole sealing problems.

\section{F1.2 PUMPING EFFECTS}

Pumping effects primarily involve the impact of the removal of groundwater from an aquifer. When an aquifer is pumped, water may be produced from more than one horizon even if the borehole is properly sealed. These different horizons may contain waters with different redox properties. Depending on how many producing horizons there are in the packed-off interval and how much each horizon produces, water pumped to the surface could represent a mixture. For example, if some horizons contain water with DO while other horizons contain water with dissolved metals but no DO, the mixture could contain DO and dissolved metals. These mixtures are generally unstable. If the mixture were allowed to stand, the redox-sensitive metals would eventually be oxidized and could precipitate out of solution. If the residence time of water in the pump circuit is sufficiently long, it is possible that the metals could precipitate from the mixed water as it is pumped to the surface. Filtration of these waters before analysis could result in lower metal concentrations than were present at the source. Problems such as these make it difficult to unambiguously interpret redox parameter measurements on groundwaters from some boreholes.

Pumping groundwater from depth to the surface usually involves a decrease of pressure on the water. The pressure decreases generally result in some degassing during which bubbles are formed in the water discharge line. Such degassing can reduce the concentrations of dissolved gases such as carbon dioxide and oxygen in the groundwater. A decrease in the concentration of carbon dioxide in a given groundwater generally leads to a higher $\mathrm{pH}$. Thus, $\mathrm{pH}$ values measured at the surface in a flow-through cell may not reflect the $\mathrm{pH}$ values of in situ groundwater. 
Another potential pumping effect involves air leakage from gas-actuated pumps. These pumps operate on gas pressure delivered to the pump from the surface. If the air hoses or the pump assembly leak air into the water column, measurements of $\mathrm{DO}, \mathrm{pH}$, and other parameters could be impacted. The project has addressed this problem either by using an electric pump or by using a gas other than air (e.g., argon or nitrogen) to pump water from boreholes prior to sampling.

\section{F1.3 ANALYTICAL PROBLEMS AND ERRORS}

Analytical problems include inadequate analytical equipment, analytical equipment failure, improper standards, poor analytical technique, sample contamination, and recording or transcribing errors. Inadequate analytical equipment is a serious problem for redox potential measurements (ORP/Eh) in oxidizing waters. This problem is well known in the analytical community (Langmuir 1997; p. 414 [DIRS 100051]) and is primarily the result of poorly poised solutions. The term "poised" refers to the concentration of redox-sensitive species in solution. When these concentrations are very low, the corresponding exchange currents on the platinum electrode are very low. Under these conditions, the platinum electrode may respond to impurities in the platinum metal or other impurities (e.g., organic species) from either the natural groundwater system or introduced by borehole construction activities.

To further add to the measurement problems, some redox-sensitive species in solution are not very electroactive. That is, they (e.g., DO, nitrite, and nitrate; Stumm and Morgan 1981; p. 493 [DIRS 100829]) do not result in exchange currents on a platinum electrode that properly reflect their concentration in solution. In effect, the measured platinum electrode potentials primarily reflect those redox-sensitive species in solution that are electroactive on platinum. Further, disequilibrium conditions among electroactive redox couples in solution can result in mixed potentials. These are potentials that are some combination of the potentials that would be measured if each electroactive redox couple were present in the absence of others.

Other problems with inadequate analytical equipment include higher detection limits than required for the analysis of redox-sensitive species. For example, in oxidizing groundwaters the concentrations of $\mathrm{Fe}^{2+}$ are commonly below the detection limits of the available field analytical equipment. This limits the accuracy with which the redox state of groundwaters can be defined.

Problems with analytical equipment failure, improper standards, poor analytical technique, sample contamination, and recording or transcribing errors can generally be resolved by fixing the problem once it is recognized. The use of detailed technical procedures has been one approach used to minimize the potential impacts of these problems and thus minimize potential errors.

\section{F2. INTERPRETATION OF REDOX DATA}

\section{F2.1 OXIDIZING GROUNDWATERS}

Platinum electrode (i.e., Eh) measurements on oxidizing groundwaters from the Yucca Mountain area are plotted against DO measurements in Figure F-2. Oxidizing groundwaters are here defined as those having more than $1.0 \mathrm{mg} / \mathrm{L}$ DO. According to Stumm and Morgan (1981; p. 492 [DIRS 100829]), DO is not electroactive on a platinum electrode. That is, the exchange 
current for the $\mathrm{DO} / \mathrm{H}_{2} \mathrm{O}$ couple on platinum does not properly reflect the concentration of DO in solution. To test this idea, Eh values were calculated for the $\mathrm{DO} / \mathrm{H}_{2} \mathrm{O}$ couple based on the following reaction (Langmuir 1997; p. 409 [DIRS 100051]):

$$
\mathrm{O}_{2}(\mathrm{~g})+4 \mathrm{H}^{+}+4 \mathrm{e}^{-}=2 \mathrm{H}_{2} \mathrm{O}
$$

This reaction corresponds to the following equation for Eh (in volts) at $25^{\circ} \mathrm{C}$ (Langmuir 1997; p. 409 [DIRS 100051]):

$$
\mathrm{Eh}=1.23+(0.0592 / 4) *\left(\log \mathrm{pO}^{2}\left[\mathrm{H}^{+}\right] 4\right)
$$

Using a Henry's Law constant of $1.26 \times 10^{-3} \mathrm{M} /$ bar (Langmuir 1997 [DIRS 100051]; p. 409) in water and an atomic weight of oxygen of $31.999 \mathrm{~g} / \mathrm{M}$, this equation yields the calculated Eh values shown in Figure F-2 for the various measured DO values. Clearly, the calculated Eh values do not correspond well to the measured Eh values.

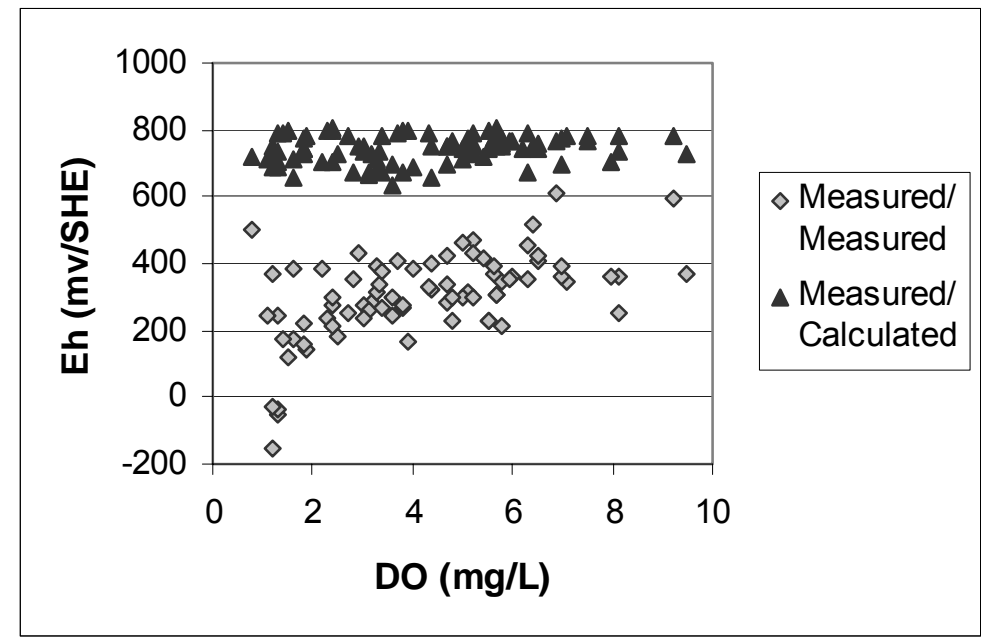

Source DTN: LA0206AM831234.001 [DIRS 160051].

NOTE: Calculated Eh values obtained from measured dissolved oxygen concentrations are also plotted against measured dissolved oxygen concentrations.

Figure F-2. Measured Eh Values Relative to the Standard Hydrogen Electrode Versus Measured Values of Dissolved Oxygen

According to Stumm and Morgan (1981 [DIRS 100829]), the cause for the low exchange currents on platinum is likely the fact that four electron transfers must occur in sequence to produce the equilibrium potential and one of these transfer reactions is very slow. It has been suggested (Stumm and Morgan 1981 [DIRS 100829], p. 462) that the Eh values measured with a platinum electrode in waters containing DO may actually reflect the concentration of one of the reaction intermediates (i.e., hydrogen peroxide). However, this explanation is difficult to test without analyses of the concentration of the reaction intermediates in groundwater. To our knowledge, these analyses have not been performed on Yucca Mountain groundwaters. 
The differences between measured and calculated Eh values in Figure F-2 likely reflect a combination of phenomena. First, the Eh measurements reported in Figure F-2 do not all represent the same equilibration time. During the field measurements, the platinum electrode was in contact with groundwater for as little as 25 minutes or as long as 345 minutes depending on the site (i.e., borehole). At the shorter measurement times, Eh values were generally increasing with time when the measurements were stopped. Thus, if the shorter time measurements had been allowed to go as long as 345 minutes, a larger value would have been obtained for Eh in most cases.

A plot of measurement time and measured Eh in oxidizing groundwaters (i.e., DO $>1.0 \mathrm{mg} / \mathrm{L}$ ) is shown in Figure F-3. Although there is a lot of scatter in the data, repeat measurements on water from the same borehole generally show an increase in measured Eh value with increased measurement time. The example shown with an arrow is from Borehole NC-EWDP-19IM1, zone \#5, which was first measured in November 2001 (low Eh value) and measured again in October 2003 (high Eh value).

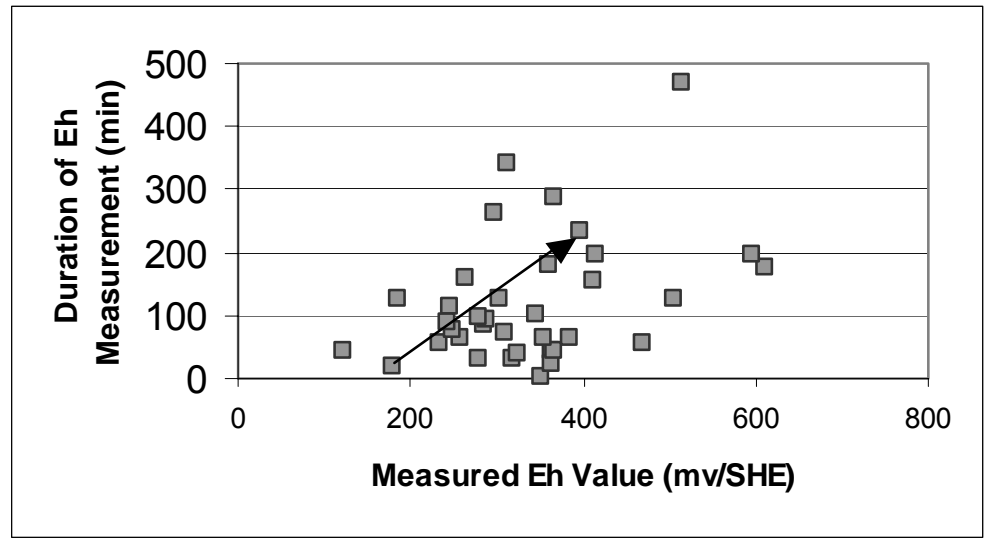

Source DTN: LA0206AM831234.001 [DIRS 160051].

NOTE: Arrow shows example of repeat analyses.

Figure F-3. Plot of Duration of Field Measurement (in minutes) Versus Measured Eh Value in mv Relative to the Standard Hydrogen Electrode for Oxidizing Waters

Second, the measured Eh values likely reflect the presence of other redox-sensitive constituents in the groundwaters that are more electroactive on platinum than the $\mathrm{DO} / \mathrm{H}_{2} \mathrm{O}$ couple. To test this concept, Eh values were calculated for groundwaters on the basis of various redox couples. The couples considered here include $\mathrm{Mn}^{2+} / \mathrm{MnO}_{2}, \mathrm{Fe}^{2+} / \mathrm{Fe}(\mathrm{OH})_{3}$, and $\mathrm{NO}_{2}{ }^{-} / \mathrm{NO}_{3}{ }^{-}$. For the $\mathrm{Mn}^{2+} / \mathrm{MnO}_{2}$ couple, the following reaction can be written assuming pyrolusite is the solubility-controlling solid under oxidizing conditions at $25^{\circ} \mathrm{C}$ (Langmuir 1997 [DIRS 100051]; p. 417):

$$
\mathrm{MnO}_{2} \text { (pyrolusite) }+4 \mathrm{H}^{+}+2 \mathrm{e}^{-}=\mathrm{Mn}^{2+}+2 \mathrm{H}_{2} \mathrm{O}
$$

This reaction corresponds to the following equation for Eh (in volts) at $25^{\circ} \mathrm{C}$ (Langmuir 1997 [DIRS 100051], p. 417):

$$
\text { Eh }=1.23+(0.0296) * \log \left(\left[\mathrm{H}^{+}\right]^{4} /\left[\mathrm{Mn}^{2+}\right]\right)
$$


The Eh values calculated with this equation are plotted against measured $\mathrm{pH}$ values in Figure F-4. The calculated values are based on the assumption that all the Mn measured in the water samples is $\mathrm{Mn}^{2+}$. This assumption is reasonable in that $\mathrm{Mn}^{4+}$ is highly insoluble and would tend to precipitate out of solution. However, this assumption would bias the calculated values to somewhat lower values compared to values that would be obtained if only $\mathrm{Mn}^{2}+$ concentrations were used. As shown in the figure, the calculated Eh values are generally equal to or larger than the Eh values measured with the platinum electrode although they are consistently smaller in value than those calculated on the basis of the $\mathrm{DO} / \mathrm{H}_{2} \mathrm{O}$ couple. The measured Eh values that plot close to the Eh values calculated on the basis of the $\mathrm{Mn}^{2+} / \mathrm{MnO}_{2}$ couple are consistent with the idea that the platinum electrode is reacting to this couple in some of the groundwaters. In Figure F-4, measured Eh values less than $0.0 \mathrm{mv} / \mathrm{SHE}$ either represent mixed waters (see below) or they represent reducing groundwaters that were contaminated with DO during the pumping activities.

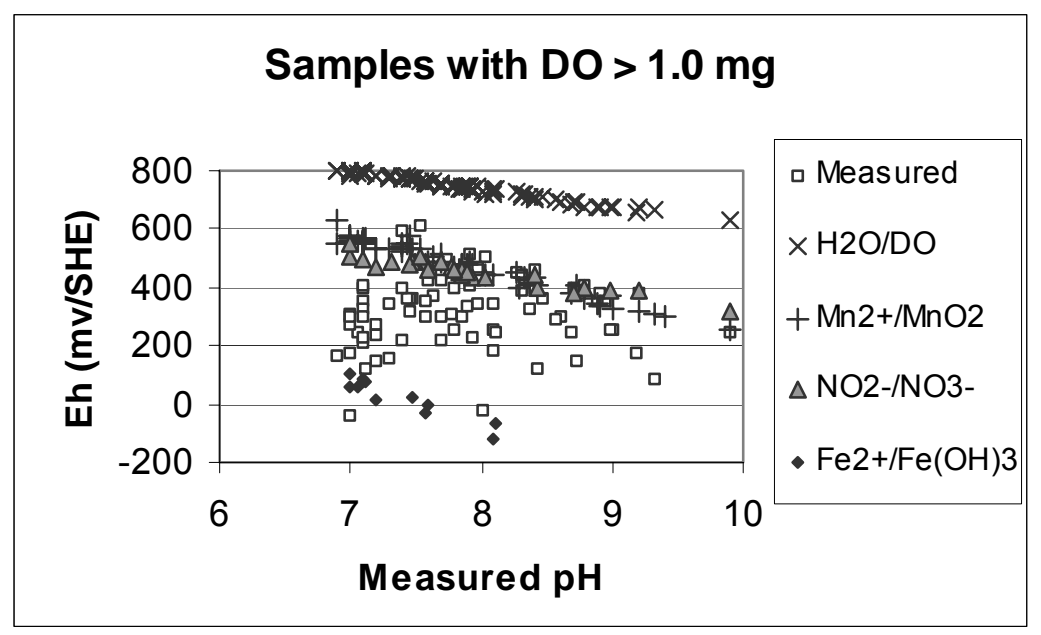

Source DTNs: LA0206AM831234.001 (DIRS 160051); LA0206AM831234.002 (DIRS 163852); LA9907AM831234.009 (DIRS 149209); LA9907AM831234.011 (DIRS 149212); LAAM831311AQ98.007 (DIRS 149520); LAAM831311AQ98.010 (DIRS 149522).

Figure F-4. Measured $\mathrm{pH}$ Values Versus Measured Eh Values and Eh Values Calculated From the $\mathrm{DO} / \mathrm{H}_{2} \mathrm{O}, \mathrm{Mn}^{2+} / \mathrm{MnO}_{2}, \mathrm{NO}_{2}^{-} / \mathrm{NO}_{3}^{-}$, and $\mathrm{Fe}^{2+} / \mathrm{Fe}(\mathrm{OH})_{3}$ Couples in Oxidizing Groundwaters $(\mathrm{DO}>1.0 \mathrm{mg} / \mathrm{L})$

There are a number of reasons why the calculated Eh values might be larger than the measured values plotted in Figure F-4. First, as noted above, measured Eh values often do not reflect steady-state values. That is, with increased measurement times, the measured values tend to increase toward higher values. Second, Equation F-3 may not be the appropriate equation for calculating the control of $\mathrm{Mn}^{2+}$ concentrations in all the groundwaters. That is, the $\mathrm{Mn}^{2+}$ concentration may be controlled by a phase other than $\mathrm{MnO}_{2}$. This is very likely the case for measured Eh values that are $<100 \mathrm{mv}$ but it may also be true for some of the waters with higher measured Eh values. In these cases, the $\mathrm{Mn}^{2+}$ concentration in groundwater may be controlled by equilibria involving phases such as $\mathrm{MnO}_{\mathrm{x}}, \mathrm{Mn}_{2} \mathrm{O}_{3}, \mathrm{MnCO}_{3}$, solid solutions containing these components, and/or by Mn adsorption reactions on other solid phases (e.g., clays, zeolites, feldspars, silica phases). Measurements with negative Eh values in combination with DO values 
greater than $1 \mathrm{mg} / \mathrm{L}$ may reflect mixing of waters with different redox properties in the wellbore. Third, the thermodynamic data on which the calculated Eh values are based may not be accurate.

The lack of consistency between Eh values calculated on the basis of the $\mathrm{DO} / \mathrm{H}_{2} \mathrm{O}$ and $\mathrm{Mn}^{2+} / \mathrm{MnO}_{2}$ couples, as shown in Figure F-4, is not without precedent. According to Stumm and Morgan 1981 [DIRS 100829]; Figure 7.12), a similar situation exists in seawater where the Eh value calculated on the basis of DO is higher ( $740 \mathrm{mv} / \mathrm{SHE})$ than the values calculated from the $\mathrm{Mn}^{2+} / \mathrm{MnO}_{2}$ couple (598 mv/SHE).

The Eh values calculated from the $\mathrm{NO}_{2}{ }^{-} / \mathrm{NO}_{3}{ }^{-}$couple are not subject to uncertainty regarding the identity of the equilibrium solid phase. However, the calculated values (Figure F-4) are sensitive to experimental artifacts and errors and measured $\mathrm{pH}$ values. The concentrations of nitrite measured in most groundwaters in the Yucca Mountain area are in the range of 0.01 to $0.02 \mathrm{mg} / \mathrm{L}$. This is close to the detection limit of the techniques used in the analysis of nitrite. Further, nitrite is present in the environment as preservatives in meat products and other foods. Thus, contamination is always a potential problem.

These factors could bias the Eh values calculated on the basis of the $\mathrm{NO}_{2}^{-} / \mathrm{NO}_{3}{ }^{-}$couple to lower values. This is reflected in the fact that the Eh values calculated on the basis of the $\mathrm{NO}_{2}{ }^{-} / \mathrm{NO}_{3}{ }^{-}$ couple are consistently less than the values calculated based on the DO concentrations in the oxidizing groundwaters (Figure F-4). The $\mathrm{NO}_{2}^{-} / \mathrm{NO}_{3}{ }^{-}$couple is apparently not electroactive on platinum electrodes (Stumm and Morgan 1981 [DIRS 100829]; p. 493). Thus, the measured Eh values plotted in Figure F-4 most likely do not reflect the $\mathrm{NO}_{2}{ }^{-} / \mathrm{NO}_{3}{ }^{-}$couple.

For the $\mathrm{Fe}^{2+} / \mathrm{Fe}(\mathrm{OH})_{3}$ couple, the following reaction can be written assuming amorphous $\mathrm{Fe}(\mathrm{OH})_{3}$ is the solubility controlling (Langmuir 1997 [DIRS 100051]; p. 417):

$$
\mathrm{Fe}(\mathrm{OH})_{3}(\mathrm{am})+3 \mathrm{H}^{+}+\mathrm{e}^{-}=\mathrm{Fe}^{2+}+3 \mathrm{H}_{2} \mathrm{O}
$$

This reaction corresponds to the following equation for Eh (in volts) at $25^{\circ} \mathrm{C}$ (Langmuir 1997 [DIRS 100051]; p. 417):

$$
\text { Eh }=0.975+(0.0592) * \log \left(\left[\mathrm{H}^{+}\right]^{3} /\left[\mathrm{Fe}^{2+}\right]\right)
$$

Most of the groundwaters analyzed for this study did not contain measurable $\mathrm{Fe}^{2+}$. Those that did plot at the low end of the range of measured Eh values (Figure F-4). This suggests the platinum electrode may be responding to ferrous iron in at least some of the water samples. Many of the water samples analyzed had concentrations of $\mathrm{Fe}^{2+}$ and total iron below the detection limit (i.e., $<0.01 \mathrm{mg} / \mathrm{L}$ ). Theoretical Eh values were not calculated for these samples. However, in Figure F-4, these samples would plot somewhere between the highest values calculated on the basis of the $\mathrm{Fe}^{2+} / \mathrm{Fe}(\mathrm{OH})_{3}$ couple and the Eh values calculated on the basis of the $\mathrm{DO} / \mathrm{H}_{2} \mathrm{O}$ couple.

Many of the water samples analyzed contained measurable concentrations of total iron. These total iron concentrations most likely reflect colloidal ferric iron particles less than 0.45 microns in diameter (i.e., less than pore size of filters used in analysis). The origin of these particles is not certain. They could be naturally occurring colloids that were pumped from the producing horizon(s) or they could be artifacts from well construction and/or pumping activities. The fact 
that the platinum electrode Eh values range between the calculated Eh values for the $\mathrm{Fe}^{2+} / \mathrm{Fe}(\mathrm{OH})_{3}$ couple and those calculated for the more oxidizing $\mathrm{Mn}^{2+} / \mathrm{MnO}_{2}$ and $\mathrm{NO}_{2}{ }^{-} / \mathrm{NO}_{3}{ }^{-}$ couples suggests the ferric iron particles may have been formed through the oxidation of dissolved ferrous iron present in the pumped water. In this scheme, the large range in measured Eh values reflects the extent to which ferrous iron in water pumped from depth was oxidized prior to coming in contact with the platinum electrode at the wellhead.

At the DO concentrations found in most of these waters and under equilibrium conditions, the amount of ferrous iron in the waters should be well below the detection limit of the techniques used in the analysis. This suggests the waters containing measurable total iron result from a mixing process. Because the oxidation rate of ferrous iron is relatively fast in neutral to alkaline solutions (Stumm and Morgan 1981 [DIRS 100829]; p. 465), such a mixing process would have to occur during the pumping event. The components of the mixture could be naturally occurring or they could be artifacts of borehole construction and/or pumping activities.

It is considered unlikely that waters containing DO could be kept isolated from waters containing dissolved metals over aquifer thicknesses of 10 to 30 feet (i.e., packed-off interval lengths) in porous media over rather long (i.e., kilometer scale) flow paths. Recharge waters generally contain 8 to $9 \mathrm{mg} / \mathrm{L}$ of DO. As these waters flow downgradient, some of this DO may be consumed in oxidation reactions. However, some groundwaters appear to have retained nearly all of their DO as they moved downgradient (Figure F-2). These waters would have to remain separated from the waters containing dissolved metals from the time they were recharged. How waters with such distinctive redox characteristics could have remained separated from each other in alluvium is very difficult to understand.

This leads to consideration of the possibility that either the metals or the DO concentrations in the waters reflect borehole construction or pumping artifacts. In the case of the metals, obvious sources of reduced (i.e., ferrous) iron are steel casings and screens. Where the borehole casing material is polyvinyl chloride or where there is no casing below the water table, metallic iron could have been introduced into the aquifers during drilling (i.e., from steel drilling equipment). Pumps and their fittings are another potential source of reduced iron.

If the iron present in groundwaters containing DO is an artifact of borehole construction activities, these groundwaters should show progressively lower iron concentrations when sampled over time as the "contaminants" are cleared from the well. The data shown in Table F-1 offer a possible example of this scenario. These are water quality data collected in 2002 and 2003 from Borehole NC-EWDP-22S, which was constructed in 2002 with PVC casing. The data collected in 2003 show lower ferrous and total iron concentrations along with higher DO and nitrate concentrations when compared with data collected in 2002. Manganese and nitrite concentrations show less consistent trends for the two sampling rounds with one unusually high manganese value $(0.168 \mathrm{mg} / \mathrm{L})$ obtained in 2002. Because Eh values were not measured in 2002, no comparison can be made for this parameter. 
Table F-1. Water Quality Data for Samples from Borehole NC-EWDP-22S

\begin{tabular}{|l|l|c|c|c|c|c|c|c|}
\hline \multicolumn{1}{|c|}{ Well } & \multicolumn{1}{|c|}{ Date } & Casing & $\mathrm{Fe}^{+2}$ & $\mathrm{Fe}$ (total) & Mn (total) & Nitrate & Nitrite & DO \\
\hline Number & Measured & Type & $(\mathrm{mg} / \mathrm{L})$ & $(\mathrm{mg} / \mathrm{L})$ & $(\mathrm{mg} / \mathrm{L})$ & $(\mathrm{mg} / \mathrm{L})$ & $(\mathrm{mg} / \mathrm{L})$ & $(\mathrm{mg} / \mathrm{L})$ \\
\hline NC-EWDP-22S-1 & $9 / 11 / 2002$ & $\mathrm{P}$ & 0.02 & 0.10 & 0.017 & 7.5 & 0.008 & 1.3 \\
\hline NC-EWDP-22S-1 & $8 / 5 / 2003$ & $\mathrm{P}$ & $\mathrm{N} / \mathrm{M}$ & $\mathrm{N} / \mathrm{M}$ & $\mathrm{N} / \mathrm{M}$ & $\mathrm{N} / \mathrm{M}$ & $\mathrm{N} / \mathrm{M}$ & 7.1 \\
\hline NC-EWDP-22S-2 & $9 / 10 / 2002$ & $\mathrm{P}$ & 0.01 & 0.15 & 0.023 & 4.2 & 0.008 & $\mathrm{~N} / \mathrm{M}$ \\
\hline NC-EWDP-22S-2 & $8 / 12 / 2003$ & $\mathrm{P}$ & 0.00 & 0.06 & 0.027 & 8.9 & 0.014 & 5.4 \\
\hline NC-EWDP-22S-3 & $9 / 10 / 2002$ & $\mathrm{P}$ & 0.00 & 0.20 & 0.168 & 6.6 & 0.013 & 1.7 \\
\hline NC-EWDP-22S-3 & $9 / 9 / 2003$ & $\mathrm{P}$ & 0.00 & 0.01 & 0.035 & 10.3 & 0.009 & 4.8 \\
\hline NC-EWDP-22S-4 & $9 / 9 / 2002$ & $\mathrm{P}$ & 0.00 & 0.19 & 0.029 & 3.4 & 0.017 & 1.3 \\
\hline NC-EWDP-22S-4 & $9 / 23 / 2003$ & $\mathrm{P}$ & 0.00 & 0.05 & 0.017 & 7.1 & $\mathrm{~N} / \mathrm{M}$ & 5.3 \\
\hline
\end{tabular}

For illustration purposes only

$\mathrm{N} / \mathrm{M}=$ not measured.

The possibility that high DO concentrations are artifacts is considered a less likely general explanation for the disequilibrium conditions observed. Boreholes with multiple completions (i.e., packed-off intervals at different depths) such as NC-EWDP-22S (Table F-1) produce waters from the deepest zones that have a similar DO concentration $(5.5 \mathrm{mg} / \mathrm{L})$ as those produced from the shallower zones (5.0 to $7.1 \mathrm{mg} / \mathrm{L}$ ). It is difficult to understand how waters in the deepest zone could be in contact with the atmosphere unless the cone of depression around the pump reached the pump entry port. Pumping procedures ensured that this situation was avoided in nearly all cases. On the other hand, DO could have been introduced into the pumping circuit in boreholes that do not penetrate very far below the water table (e.g., NC-EWDP-18P). When these boreholes are pumped, water that has been at or near the water table may be drawn into the pumping circuit and mixed with waters containing metals that originate from flow zones deeper in the borehole.

To summarize, the Eh values measured with a platinum electrode in waters containing DO generally do not correspond to Eh values calculated on the basis of the measured DO concentrations. In many cases, the platinum electrode appears to have responded to iron and possibly manganese concentrations in the groundwaters. Further, it appears likely that these metal concentrations are artifacts of borehole construction and/or pumping activities.

The important question to be answered with all these data is "What redox conditions will radionuclides be subject to during transport in oxidizing groundwaters in the SZ?" The data plotted in Figure F-4 indicate a rather broad range of measured Eh values for oxidizing SZ waters. The broadness in the range of measured Eh values may be due in part to natural phenomena such as the mixing of oxidizing and reducing groundwaters, in part to a lack of equilibrium between the platinum electrode and the redox-sensitive species in groundwater, and in part to borehole construction and pumping effects.

The ubiquitous presence of DO in these waters suggests these waters will be oxidizing overall. However, if the radionuclides-of-interest respond to the presence of DO in the same way as the $\mathrm{Mn}^{2+} / \mathrm{MnO}_{2}$ and $\mathrm{NO}_{2}^{-} / \mathrm{NO}_{3}{ }^{-}$couples, the redox potentials will be less than those calculated on the basis of the $\mathrm{DO} / \mathrm{H}_{2} \mathrm{O}$ couple. The range of Eh values calculated on the basis of the $\mathrm{Mn}^{2+} / \mathrm{MnO}_{2}$ and $\mathrm{NO}_{2}^{-} / \mathrm{NO}_{3}{ }^{-}$couples could be used to estimate the redox potential that would be experienced by the radionuclides-of-interest. 
The range of Eh values that these radionuclides would experience during transport can be further narrowed by noting that the range of $\mathrm{pH}$ values measured in $\mathrm{SZ}$ waters is in part an artifact of borehole construction and pumping activities. For example, cement was used in the construction of some boreholes. Cement used below the water table can leach alkaline solutions into the groundwater causing increased $\mathrm{pH}$. More generally, waters brought to the surface from the SZ often outgas carbon dioxide and probably other gases during pumping. Outgassing is evidenced by bubbles that appear in water flowing through the discharge line. The loss of carbon dioxide causes an increase in the measured $\mathrm{pH}$ of the pumped waters (i.e., the waters that pass through the flow-through cell). These effects imply the range of $\mathrm{pH}$ values of groundwaters in the aquifer is smaller than the range indicated by the values measured in the flow-through cell at the wellhead.

In the case of $\mathrm{J}-13$, the in situ $\mathrm{pH}$ value is near 7.0 while the value obtained under normal atmosphere is 8.4. If we assume other groundwaters show similar changes in $\mathrm{pH}$ values between the aquifer depth and the surface, it is likely in situ $\mathrm{pH}$ values would be in the range of 7.0 to 8.0. Further, if we assume the redox conditions to which radionuclides would be exposed in oxidizing SZ groundwaters are represented by the ranges of Eh values calculated from the $\mathrm{Mn}^{2+} / \mathrm{MnO}_{2}$ and $\mathrm{NO}_{2}{ }^{-} / \mathrm{NO}_{3}{ }^{-}$couples, the redox potentials would range from approximately 400 to $500 \mathrm{mv} / \mathrm{SHE}$ at $\mathrm{pH}=8.0$ to 500 to $600 \mathrm{mv} / \mathrm{SHE}$ at $\mathrm{pH}=7.0$. If for some reason the Eh values calculated from the $\mathrm{Mn}^{2+} / \mathrm{MnO}_{2}$ and $\mathrm{NO}_{2}{ }^{-} / \mathrm{NO}_{3}{ }^{-}$couples are not representative of SZ conditions, the range in redox potentials could be as high as 750 to $800 \mathrm{mv} / \mathrm{SHE}$ (i.e., the range calculated for the $\mathrm{DO} / \mathrm{H}_{2} \mathrm{O}$ couple).

\section{F2.2 REDUCING GROUNDWATERS}

The situation is different for reducing waters. In these waters, DO concentrations are low (less than $1.0 \mathrm{mg} / \mathrm{L}$ ) and dissolved metal concentrations are higher than in oxidizing waters. Under these conditions, the platinum electrode does provide useful information on the redox potential of groundwaters largely because exchange currents are larger. The exchange currents are larger because the concentrations of electroactive species in solution are larger. Conversely, Eh values calculated on the basis of dissolved metal concentrations are less definitive in these waters because the identities of the phases that control these concentrations are less obvious.

The boreholes in which reducing conditions have been found in groundwaters in the Yucca Mountain area include boreholes (Figure F-1) on or near Yucca Mountain (H-1, H-3, H-4, 25b\#1, WT-17), a borehole in alluvium to the east of Fortymile Wash (NC-EWDP-5SB), and a group of boreholes to the west of Fortymile Wash (NC-EWDP-1D, 3S, 7SC). The boreholes on Yucca Mountain that contain reducing waters are relatively deep holes that penetrate the Tram Member of the Crater Flat Tuff. This member includes a volcanic unit that contains pyrite $\left(\mathrm{FeS}_{2}\right)$. The pyrite is apparently a primary (i.e., volcanic) constituent of this unit (Castor et al. 1994 [DIRS 102495]). It is thought to have been eroded from the volcanic conduit during the eruption that produced the ash flow. The presence of pyrite in this unit is a likely source of the reducing conditions observed in the groundwaters pumped from these boreholes.

The equilibrium reaction between $\mathrm{Fe}^{2+}$ and $\mathrm{FeS}_{2}$ (pyrite) can be represented with the following equation (Langmuir 1997 [DIRS 100051]; p. 417): 


$$
2 \mathrm{SO}_{4}{ }^{2-}+\mathrm{Fe}^{2+}+16 \mathrm{H}^{+}+14 \mathrm{e}^{-}=\mathrm{FeS}_{2} \text { (pyrite) }+8 \mathrm{H}_{2} \mathrm{O} \text {. }
$$

The following equation is used to calculate Eh values for the $\mathrm{Fe}^{2+} / \mathrm{FeS}_{2}$ couple at $25{ }^{\circ} \mathrm{C}$ (Langmuir 1997 [DIRS 100051]; p. 417):

$$
\mathrm{Eh}=0.362+0.00423 \log \left[\mathrm{Fe}^{2+}\right]\left[\mathrm{SO}_{4}^{-}\right]^{2}\left[\mathrm{H}^{+}\right]^{16} \text {. }
$$

The Eh values measured in these boreholes and the Eh values calculated on the basis of the $\mathrm{Fe}^{2+} / \mathrm{FeS}_{2}$ couple are plotted against measured $\mathrm{pH}$ in Figure F-5. Although the measurements show a similar trend to that shown by the calculated values, the calculated trend is displaced to somewhat lower Eh values. The trend in calculated Eh values based on the $\mathrm{Fe}^{2+} / \mathrm{Fe}(\mathrm{OH})_{3}$ couple seems to correspond more closely to the trend in the measured values. In either case, the measured and calculated values indicate reducing conditions in these wells.

The sample that lies above the main trend in Figure F-5 (more than $400 \mathrm{mv}$ ) represents a water with an oxidizing Eh value and a low DO concentration. This sample likely represents a mixture of oxidizing and reducing waters. Such mixtures could originate in open boreholes or boreholes with multiple screens that are not packed off. For example, if a water with $2.0 \mathrm{mg} / \mathrm{L}$ ferrous iron and no DO from one horizon were mixed with a water with $6.0 \mathrm{mg} / \mathrm{L} \mathrm{DO}$ and no ferrous iron from another horizon in a ratio of 10:1, the DO would eventually oxidize the ferrous iron so that the mixture would contain little or no DO or ferrous iron. However, the total iron concentration could remain elevated if insufficient time had passed for the oxidized iron to precipitate out of solution prior to analysis.

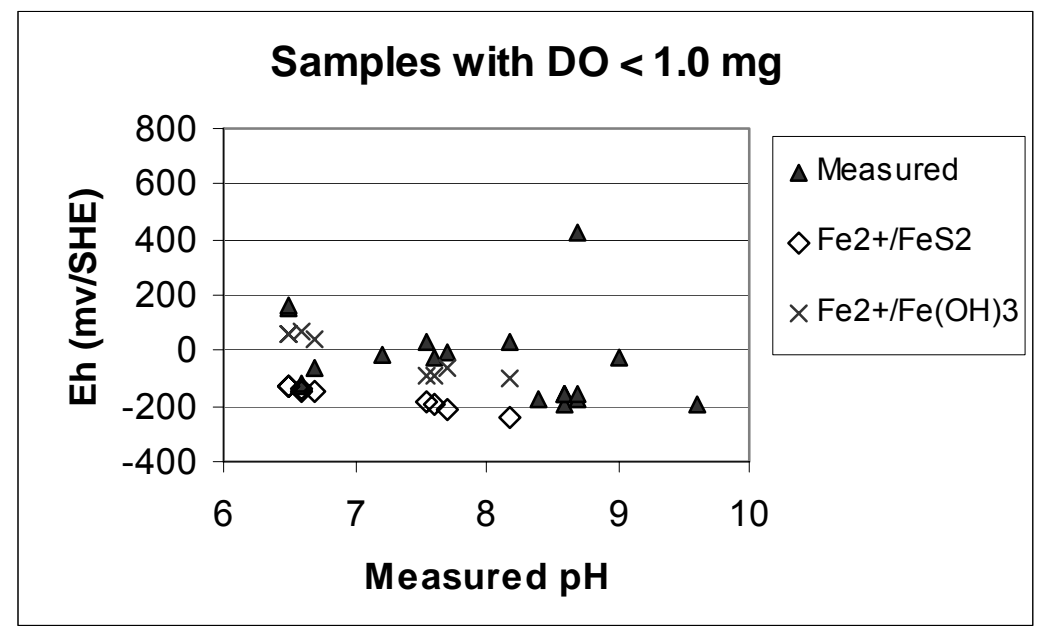

Source DTNs: LA0206AM831234.001 (DIRS 160051); LA0206AM831234.002 (DIRS 163852); LA9907AM831234.009 (DIRS 149209); LA9907AM831234.011 (DIRS 149212); LAAM831311AQ98.007 (DIRS 149520); LAAM831311AQ98.010 (DIRS 149522).

Figure F-5. Measured Eh Values Versus $\mathrm{pH}$ Values and Measured $\mathrm{pH}$ Values Versus Eh Values Calculated on the Basis of the $\mathrm{Fe}^{2+} / \mathrm{FeS}_{2}$ (pyrite) Couple

It is very important to recognize that the presence of reducing conditions in more than one borehole located directly to the east of the repository footprint (e.g., WT-17 and b\#1) suggests there may be a volume of rock east of the repository footprint that contains reducing 
groundwaters. This volume could be a substantial barrier to the transport of redox-sensitive radionuclides in the SZ as discussed in the following section.

The groundwaters showing reducing conditions in boreholes to the east and west of Fortymile Wash near the southern boundary of the site include Boreholes NC-EWDP-1D, 3S, 5SB, 7SC, 12PA and 12 PB. Borehole NC-EWDP-1D is known to contain pyrite based on core descriptions provided by Nye County. The other boreholes may also contain pyrite although core descriptions are not available to test this possibility.

\section{F3. IMPLICATIONS FOR RADIONUCLIDE TRANSPORT}

The discussion in this section is intended to provide a general consideration of the impact of redox conditions on radionuclide transport based on available laboratory and field data than has been acquired to date.

The TSPA calculations for SZ transport use a stochastic approach in which many separate realizations are obtained to provide statistically meaningful results. In each realization for SZ transport, a single value is used for the sorption coefficient of each radionuclide for the volcanic section and another single value is used for the alluvium section (independent values are used for colloid-facilitated transport). It is assumed in the transport model (Section 5, Table 5-1) that redox conditions are oxidizing along all the potential transport pathways from the repository to the accessible environment. Thus, variations in redox conditions in the SZ are not explicitly addressed in the TSPA calculations. This is consistent with the fact that the bulk of the redox measurements obtained on groundwaters in volcanic and alluvial aquifers indicate the presence of oxidizing conditions (i.e., conditions in which the radioelements technetium, neptunium, plutonium, and uranium are in their highest oxidation states). However, there are some volumes of rock in which reducing conditions exist in critical locations important to radionuclide transport. One of these volumes is located directly east of the repository footprint.

Data presented by Lieser and Mühlenweg (1988 [DIRS 106684]) indicate that retardation coefficients for neptunium under reducing conditions are three orders of magnitude higher than they are under oxidizing conditions. Similar differences in retardation coefficient values under reducing versus oxidizing conditions are likely for plutonium, technetium, and uranium. The conditions in most of the boreholes containing reducing groundwaters are sufficiently reducing so that neptunium, plutonium, technetium, and uranium will be predominantly in the +4 oxidation state in these waters. Thus, the site-scale SZ transport model assumes conditions that could be significantly less retarding than those that actually occur in the field for these radionuclides, making the model significantly conservative in regards to the predicted travel times of these radionuclides. Explicit consideration in the transport calculations of the reducing conditions present in the SZ directly east of the repository could result in much longer travel times for redox-sensitive radionuclides. Similarly, the reducing conditions observed in boreholes to the east and west of Fortymile Wash suggest redox-sensitive radionuclides will have much longer transport times through these areas than those that may be predicted by the current TSPA calculations. 


\section{INTENTIONALLY LEFT BLANK}




\begin{abstract}
APPENDIX G
RADIONUCLIDE SORPTION PARAMETERS FROM BATCH AND COLUMN TRANSPORT EXPERIMENTS IN SATURATED ALLUVIUM AND DISCUSSION OF EARLY BREAKTHROUGH BEHAVIOR IN COLUMN EXPERIMENTS
\end{abstract}




\section{G1. INTRODUCTION}

The data and analysis presented in this appendix supports the screening assessment of the alternative conceptual model that deals with the treatment of sorption reactions as being instantaneous (Table 6.6-1b, Item\# 1) in the site-scale SZ transport model. The results of this analysis do not form a direct input to the site-scale SZ transport model.

The saturated alluvium south of Yucca Mountain is expected to serve as a natural barrier to the migration of radionuclides from the repository to the accessible environment. The alluvium consists primarily of materials of volcanic origin, with some enrichment of clays and zeolites relative to the volcanic tuffs at Yucca Mountain.

This appendix describes a series of experiments conducted to better characterize the radionuclide retardation potential of the saturated alluvium. The objectives of the experiments were:

- To evaluate the retardation potential of alluvium for ${ }^{129} \mathrm{I},{ }^{99} \mathrm{Tc},{ }^{237} \mathrm{~Np}$, and ${ }^{233} \mathrm{U}$ by determining distribution coefficients $\left(K_{d} ; \mathrm{mL} / \mathrm{g}\right)$ in batch sorption and desorption experiments using alluvium samples and water collected from boreholes in saturated alluvium along potential flow pathways to the accessible environment.

- To determine the retardation behavior of these radionuclides in column transport experiments using the same water and alluvium as in some of the batch experiments, and to determine whether the sorption parameters measured in batch experiments can be used to predict column transport behavior.

In addition to presenting the experimental results and interpretations, this appendix provides an explanation of apparent inconsistencies between results from batch sorption and column transport experiments. Specifically, the early breakthroughs of a portion of the radionuclide mass in some column transport experiments is discussed in light of results from batch sorption experiments that suggest that much later breakthrough times should have been observed in the column experiments.

\section{G2. EXPERIMENTAL APPROACH}

All batch sorption, desorption, and column transport experiments were conducted under ambient conditions (room temperature, and in contact with atmosphere). Two separate sets of experiments were conducted. The first set was carried out using alluvium samples from boreholes drilled in Phase 1 of the Nye County early-warning drilling program (NC-EWDP-1X, 2D, 3S, 9SX, 19D). Groundwater from Borehole 3S was used in experiments with samples from Boreholes 1D, 2D, 3S, and 9SX while groundwater from Borehole 19D was used in experiments with alluvium samples from 19D. ${ }^{129} \mathrm{I},{ }^{99} \mathrm{Tc}$, and ${ }^{237} \mathrm{~Np}$ were used in these experiments; ${ }^{233} \mathrm{U}$ was not used until the second set of experiments. Column transport experiments were conducted using ${ }^{237} \mathrm{~Np}$ along with tritiated water, which served as a nonsorbing tracer. Groundwater from Borehole 3S and alluvium material from Borehole 2D were used in these column experiments. Because groundwater from Borehole 3S may not be representative of in situ conditions (the water obtained from 3S may have contained organic materials used in well construction), the results obtained in experiments with samples from Boreholes 1X, 2D, 3S, and 9S, were not used 
in the derivation of sorption coefficient probability distributions. However, the results from the batch sorption and column transport experiments using these materials are discussed in this appendix because they exhibit similar qualitative sorption behavior to other sets of alluvium batch and column experiments.

A second set of experiments was carried out using alluvium samples from Phase II Nye County Boreholes NC-EWDP-10SA, 19M1A, and 22SA and groundwater from different zones in NC-EWDP-19D (Zones 1 and 4) and NC-EWDP-10SA. ${ }^{129} \mathrm{I},{ }^{99} \mathrm{Tc},{ }^{237} \mathrm{~Np}$, and ${ }^{233} \mathrm{U}$ were used in these experiments. In addition to batch sorption experiments conducted for all four radionuclides, desorption experiments were carried out for ${ }^{233} \mathrm{U}$. Also, four column transport experiments were conducted using ${ }^{237} \mathrm{~Np}$, and three were conducted using ${ }^{233} \mathrm{U}$. The ${ }^{237} \mathrm{~Np}$ column experiments were carried out using water and alluvium from Borehole 19D. The flow rates in these experiments were varied to span a range of over one order of magnitude. The ${ }^{233} \mathrm{U}$ column experiments were conducted using different combinations of alluvium and water from the three Phase II wells, and all experiments were conducted at the same flow rate. Four scoping column transport experiments (not reported to the Technical Data Management System) were also conducted using ${ }^{129} \mathrm{I}$ and ${ }^{99} \mathrm{Tc}$, but they are not presented in this appendix because they were conducted only to confirm the nonsorbing nature of these radionuclides that was observed in the batch experiments.

As a group, the samples selected for sorption experiments are taken to be representative of alluvium in potential flow paths to the accessible environment. Boreholes NC-EWDP-10SA, 19D, 19M1A, 1X, and 22SA are located within or close to what is believed to be a primary flow pathway that lies under or slightly west of the Fortymile Wash surface channel. The groundwater in these boreholes is, on average, more oxidizing than groundwater from boreholes west (NC-EWDP-1D, 3S, 7S, 9S, 12PA, 12PB, 12PC, 15D, and 15P) and east (NC-EWDP-4P, 5S) of the Fortymile Wash channel (DTN: LA0206AM831234.002 [DIRS 163852]). Field measurements of the redox conditions in alluvium groundwater samples are listed in Table G-1. Oxidizing groundwater should result in smaller $K_{d}$ values for neptunium and uranium compared to reducing groundwater. For uranium, the less sorbing species $\mathrm{U}^{+6}$ is stable at high values (i.e., under more oxidizing conditions) of the Eh and the more sorbing species $\mathrm{U}^{+4}$ is stable at lower values of Eh. This change in sorption behavior of uranium occurs at Eh values in the range 0.0 to $100 \mathrm{mV}$ versus SHE at near-neutral pH (Langmuir 1997 [DIRS 100051], p. 506), and for technetium, the change occurs at $-100-0 \mathrm{mV}$ versus SHE at near-neutral $\mathrm{pH}$ (Langmuir 1997 [DIRS 100051], p. 522). For neptunium, the change in sorption behavior occurs at approximately $230 \pm 30 \mathrm{mV}$ (versus standard hydrogen electrode, SHE) at near-neutral $\mathrm{pH}$ (Langmuir 1997 [DIRS 100051], p. 538). The values of Eh at which these transitions occur are sensitive to $\mathrm{pH}$. However, the site-scale SZ transport model assumes oxidizing conditions for calculating sorption coefficient distributions (Table 5-1). Thus, the derivation of sorption coefficient probability distributions using data obtained on samples from Boreholes NC-EWDP-10SA, 19D, and $19 \mathrm{M} 1 \mathrm{~A}$, leads to conservatism in the prediction of transport rates in alluvium. 
Table G-1. Redox Measurements in Groundwater in Nye County Boreholes

\begin{tabular}{|c|c|c|c|c|c|c|}
\hline $\begin{array}{l}\text { NC-EWDP } \\
\text { Well No. }\end{array}$ & $\begin{array}{c}\text { Sand-Pack Depth } \\
\text { (feet bgs) }\end{array}$ & Sampling Date & $\mathrm{pH}$ & $\begin{array}{c}\text { Dissolved Oxygen } \\
(\mathrm{mg} / \mathrm{L})\end{array}$ & $\begin{array}{c}\text { Eh } \\
\text { mv-SHE }\end{array}$ & $\begin{array}{c}\mathrm{T} \\
\text { (degrees C) }\end{array}$ \\
\hline \multirow[t]{3}{*}{$015 X$} & $152-189$ & $5 / 17 / 99$ & 7.1 & 4.3 & 327 & 27.5 \\
\hline & & $11 / 8 / 99$ & 7.0 & $0.9-3.8$ & $128-272$ & $25.9-26.7$ \\
\hline & & $5 / 18 / 00$ & 7.1 & $3.5-3.9$ & $347-407$ & $27.5-27.9$ \\
\hline \multirow[t]{3}{*}{$01 S x$} & 204-340 & $5 / 17 / 99$ & 7.2 & 2.7 & 249 & 28.4 \\
\hline & & $11 / 8 / 99$ & 7.0 & 1.4 & 172 & 26.9 \\
\hline & & $5 / 18 / 00$ & $7.1-7.2$ & $1.6-1.9$ & $133-146$ & $28.2-28.8$ \\
\hline 01D & 2180.0-2294.7 & $5 / 24 / 00$ & 6.6 & 0.02 & $(-51$ to -131$)$ & 25.9-26.14 \\
\hline \multirow[t]{2}{*}{$03 S$} & $245-275$ & $5 / 20 / 99$ & 8.6 & 1.2 & 370 & 32.9 \\
\hline & & $11 / 15 / 99$ & $8.3-8.5$ & $1.7-2.2$ & $366-386$ & $30.3-31.9$ \\
\hline \multirow[t]{3}{*}{$03 S$} & $295-524.3$ & $5 / 20 / 99$ & 8.7 & 0 & 154 & 32.5 \\
\hline & Open Hole & $11 / 15 / 99$ & $8.5-8.9$ & $0.1-0.4$ & $204-299$ & $27.8-32.0$ \\
\hline & & $5 / 17 / 00$ & 8.8-9.1 & $0.08-0.12$ & (-29 to 41$)$ & $32.4-33.9$ \\
\hline \multirow[t]{2}{*}{ 04PA } & 394.7-496 & $5 / 16 / 00$ & 8.5-9.8 & $3.0-6.3$ & $340-456$ & 23.8-26.3 \\
\hline & & $10 / 26 / 00$ & 7.8-7.9 & $3.8-4.7$ & $309-339$ & $23.5-24.0$ \\
\hline \multirow[t]{2}{*}{ 04PB } & $718-849.5$ & $5 / 26 / 00$ & $6.4-8.1$ & $6.4-8.1$ & 244-249 & 26.6-26.9 \\
\hline & & $10 / 26 / 00$ & $9.7-9.9$ & 2.8-3.6 & $217-242$ & 21.6-23.2 \\
\hline \multirow[t]{2}{*}{ 05SB } & $366.0-499.4$ & $5 / 17 / 00$ & 7.5-7.7 & $0.04-1.5$ & $(-10$ to 37$)$ & $24.2-27.0$ \\
\hline & & $10 / 23 / 00$ & 7.6 & $0.01-0.09$ & (-26 to 49$)$ & $22.8-24.0$ \\
\hline \multirow[t]{2}{*}{$07 S$} & $26-53.2$ & $10 / 23 / 00$ & 7.0-7.1 & $0.7-2.4$ & $144-211$ & $19.7-20.6$ \\
\hline & & $3 / 28 / 01$ & $6.9-7.0$ & 2.3-2.4 & 283-301 & 21.1-21.2 \\
\hline \multirow[t]{2}{*}{$095 x$} & $85.0-126.1$ & $5 / 19 / 99$ & 8.3 & 1.6 & 388 & 28.6 \\
\hline & & $11 / 10 / 99$ & $7.6-8.6$ & 2.1-6.4 & $317-369$ & $25.8-26.6$ \\
\hline \multirow[t]{2}{*}{ 09SX } & 134.8-167.1 & $5 / 19 / 99$ & 7.7 & 5.2 & 432 & 28.1 \\
\hline & & $11 / 10 / 99$ & 7.5-7.7 & $3.6-6.0$ & $354-452$ & 26.1-27.4 \\
\hline \multirow[t]{2}{*}{ 09SX } & 245.4-295.6 & $5 / 18 / 99$ & 7.7 & 2.9 & 430 & 28.5 \\
\hline & & $11 / 9 / 99$ & $7.7-8.1$ & 1.3-3.3 & $196-251$ & 27.1-27.4 \\
\hline \multirow[t]{2}{*}{ 09SX } & 325-397 & $5 / 18 / 99$ & 7.7 & 4.8 & 232 & 29.0 \\
\hline & & $11 / 9 / 99$ & 7.6-7.7 & $3.2-4.9$ & 223-303 & $27.0-27.9$ \\
\hline 12PA & $317.5-389.5$ & $10 / 25 / 00$ & $6.4-6.5$ & $0.3-0.5$ & $122-153$ & $27.2-28.9$ \\
\hline \multirow[t]{2}{*}{$12 \mathrm{~PB}$} & 316.2-399.75 & $5 / 25 / 00$ & 6.6-6.9 & $0.6-3.9$ & $33-167$ & $30.5-30.8$ \\
\hline & & $10 / 25 / 00$ & 6.5 & $0.5-0.8$ & $147-160$ & $27.2-27.6$ \\
\hline \multirow[t]{2}{*}{$12 \mathrm{PC}$} & $160.4-249.6$ & $5 / 25 / 00$ & 7.1 & 4.3-5.2 & $282-302$ & $27.8-27.9$ \\
\hline & & $10 / 26 / 00$ & 7.1 & $5.2-5.5$ & 209-230 & $24.2-24.4$ \\
\hline \multirow[t]{2}{*}{$15 \mathrm{P}$} & 192.6-274.5 & $5 / 23 / 00$ & 7.7 & 4.0-4.7 & $413-424$ & $32.1-32.7$ \\
\hline & & $10 / 26 / 00$ & 7.8 & $4.2-4.4$ & $374-400$ & $26.6-27.5$ \\
\hline
\end{tabular}


Table G-1. Redox Measurements in Groundwater in Nye County Boreholes (Continued)

\begin{tabular}{|c|c|c|c|c|c|c|}
\hline $\begin{array}{l}\text { NC-EWDP } \\
\text { Well No. }\end{array}$ & $\begin{array}{c}\text { Sand-Pack Depth } \\
\text { (feet bgs) }\end{array}$ & Sampling Date & $\mathrm{pH}$ & $\begin{array}{c}\text { Dissolved Oxygen } \\
(\mathrm{mg} / \mathrm{L})\end{array}$ & $\underset{\text { mv-SHE }}{\text { Eh }}$ & $\begin{array}{c}\mathrm{T} \\
\left({ }^{\circ} \mathrm{C}\right)\end{array}$ \\
\hline 19D & \begin{tabular}{|l} 
Zone \# 1 \\
$408.5-437.0$ \\
\end{tabular} & $10 / 17 / 00$ & $8.5-8.7$ & 0.8 & $358-423$ & $31.0-31.3$ \\
\hline $19 D$ & \begin{tabular}{|l} 
Zone \# 3 \\
$568.0-691.0$ \\
\end{tabular} & $9 / 13 / 00$ & 8.4 & $4.8-5.0$ & $388-463$ & $30.3-30.6$ \\
\hline $19 D$ & \begin{tabular}{|l} 
Zone \# 4 \\
717.0-795.0
\end{tabular} & $8 / 27 / 00$ & $8.8-8.9$ & $3.2-3.4$ & $291-376$ & $31.5-31.6$ \\
\hline $19 D$ & Zone \# 5 & $1 / 5 / 02$ & 9.0 & 2.7-3.1 & $\mathrm{N} / \mathrm{A}$ & $31.0-32.6$ \\
\hline $19 \mathrm{P}$ & $351.5-474.5$ & $5 / 23 / 00$ & 8.6-8.7 & $6.7-7.0$ & $324-396$ & 28.9-29.5 \\
\hline \multicolumn{2}{|l|}{ Airport Well } & $6 / 10 / 99$ & 8.3 & 9.5 & 370 & 28.4 \\
\hline \multicolumn{7}{|l|}{ YMP WELLS } \\
\hline WT-17 & 1312-1359 & $7 / 1 / 98$ & $6.1-6.7$ & 0.0 & $(-23$ to -65$)$ & 27.3-29.7 \\
\hline WT-3 & $1053-1093$ & $6 / 22 / 98$ & $7.1-7.6$ & $3.7-6.5$ & $273-422$ & $31.6-33.0$ \\
\hline
\end{tabular}

Source DTN: LA0206AM831234.002 [DIRS 163852].

NOTE: All measurements in this table were conducted in the field using water freshly pumped from the wells.

\section{G3. EXPERIMENTAL RESULTS}

\section{G3.1 NEPTUNIUM BATCH SORPTION RESULTS FROM FIRST SET OF EXPERIMENTS}

The alluvium samples used and their preparation methods for the first set of ${ }^{237} \mathrm{~Np}$ batch sorption experiments are presented in Table G-2. The water compositions used in the experiments are shown in Table G-3. The results of ${ }^{129} \mathrm{I}$ and ${ }^{99} \mathrm{Tc}$ batch sorption experiments are not presented here because they indicated no significant sorption of either radionuclide. The compositions of Boreholes NC-EWDP-03S and NC-EWDP-19D (1 and 2) water were similar. Groundwater from Borehole NC-EWDP-03S had a lower DO concentration, a lower Eh, and a higher organic carbon concentration than the Borehole 19D groundwater. Thus, groundwater from Borehole 3S is more reducing than groundwater from Borehole 19D.

Alluvium samples were characterized primarily by quantitative X-ray diffraction and $\mathrm{N}_{2}$-Brunauer-Emmett-Teller surface area measurements (Table G-4). Samples selected for use in the batch sorption tests represented different boreholes, intervals, sieving methods, and particle sizes.

The mineralogy of the alluvium used in the experiments is summarized in Table G-4. The amount of organic carbon in the samples was negligible. Trace amounts of calcite and hematite were detected in some samples. Alluvium from Borehole NC-EWDP-03S contained a considerable amount of calcite. Dry sieved samples were used for all the experiments except for a column experiment with material from Borehole NC-EWDP-19D. Note that the sieving technique (wet versus dry) had only a minor effect on the mineral composition of the 75 to $500 \mu \mathrm{m}$ fraction of the 19D sample. 
Table G-2. Boreholes and Sample Preparation Methods

\begin{tabular}{|c|c|c|c|c|c|}
\hline \multirow{2}{*}{$\begin{array}{l}\text { Borehole } \\
\text { Location }\end{array}$} & \multirow[b]{2}{*}{ Depth (ft BLS) } & \multirow{2}{*}{$\begin{array}{c}\text { Sample Preparation } \\
\text { Method }^{\mathrm{a}}\end{array}$} & \multicolumn{3}{|c|}{ Particle Size Fraction (wt \%) } \\
\hline & & & $75-2000 \mu \mathrm{m}$ & $75-500 \mu \mathrm{m}$ & $<75 \mu \mathrm{m}$ \\
\hline $2 \mathrm{D}$ & $395-400$ & A & & 59 & 41 \\
\hline $2 \mathrm{D}$ & $400-405$ & $\mathrm{~A}$ & & 60 & 40 \\
\hline $2 \mathrm{D}$ & $405-410$ & A & & 56 & 44 \\
\hline $2 \mathrm{D}$ & $410-415$ & A & & 56 & 44 \\
\hline $9 S$ & $145-150$ & A & & 66 & 34 \\
\hline $9 S$ & $150-155$ & A & & 62 & 38 \\
\hline $9 \mathrm{~S}$ & $155-160$ & A & & 61 & 39 \\
\hline $9 \mathrm{~S}$ & $160-165$ & A & & 61 & 39 \\
\hline $3 S$ & $60-65$ & A & & 54 & 46 \\
\hline $3 S$ & $65-70$ & A & & 64 & 36 \\
\hline $3 S$ & $70-75$ & A & & 59 & 41 \\
\hline $3 S$ & $75-80$ & A & & 66 & 44 \\
\hline $1 \mathrm{X}$ & $390-395$ & $B+A$ & 40 & 39 & 21 \\
\hline $1 X$ & $395-400$ & $B+A$ & 71 & 19 & 10 \\
\hline $1 \mathrm{X}$ & $400-405$ & $B+A$ & 33 & 45 & 22 \\
\hline $1 X$ & $405-410$ & $B+A$ & 51 & 33 & 16 \\
\hline $19 \mathrm{D}$ & $405-425$ & A & ND & ND & ND \\
\hline $19 D$ & $405-425$ & C & 100 & 0 & 0 \\
\hline $19 D$ & $405-425$ & C & 0 & 0 & 100 \\
\hline
\end{tabular}

Source: Ding et al. 2003 (DIRS 164737).

${ }^{a}$ Sample Preparation Method: A) Grind, crush, and dry sieve; B) collect 75 to 2,000 $\mu \mathrm{m}$ size particle materials by dry sieving without grinding or crushing, followed by process $\mathrm{A} ; \mathrm{C}$ ) collect 75 to $2,000 \mu \mathrm{m}$ size particle materials by dry sieving without grinding or crushing processes, follow with washing out the fine particles and collecting particle size range 75 to $2,000 \mu \mathrm{m}$ materials by wet sieving.

$\mathrm{BLS}=$ below land surface; ND = not determined. 
Table G-3. Borehole NC-EWDP-03S and NC-EWDP-19D Water Composition

\begin{tabular}{|c|c|c|c|}
\hline \multirow[b]{2}{*}{ Species } & \multicolumn{3}{|c|}{ Concentration (mg/L) } \\
\hline & $3 S$ (449 ft BLS) & $\begin{array}{c}\text { 19D1 (412-439 ft + 490- } \\
519 \mathrm{ft} B L S)\end{array}$ & 19D2 (412-437 ft BLS) \\
\hline $\mathrm{Na}^{+}$ & 141 & 69.4 & 73.2 \\
\hline $\mathrm{K}^{+}$ & 2.99 & 3.61 & 3.92 \\
\hline $\mathrm{Li}^{+}$ & 0.26 & 0.087 & 0.081 \\
\hline $\mathrm{Ca}^{2+}$ & $0.94 \pm 0.01$ & 7.59 & 7.70 \\
\hline $\mathrm{Mg}^{2+}$ & 0.14 & 0.65 & 0.69 \\
\hline $\mathrm{Mn}^{2+}$ & $<0.002$ & 0.0088 & $<0.0001$ \\
\hline $\mathrm{Fe}^{2+/ 3+}$ & 0.02 & 0.09 & $<0.01$ \\
\hline $\mathrm{Al}^{3+}$ & 0.34 & 0.05 & 0.002 \\
\hline $\mathrm{SiO}_{2}$ & 48.4 & 58.0 & 58.4 \\
\hline $\mathrm{F}^{-}$ & 3.24 & 1.78 & 1.96 \\
\hline $\mathrm{Cl}^{-}$ & 8.68 & 5.61 & 6.52 \\
\hline $\mathrm{NO}_{3}{ }^{-}$ & 0.28 & 4.18 & 4.84 \\
\hline $\mathrm{SO}_{4}{ }^{2-}$ & 50.0 & 23.0 & 23.8 \\
\hline $\mathrm{HCO}_{3}^{-}$ & 261 & 168 & 146 \\
\hline $\mathrm{CO}_{3}{ }^{2-}$ & ND & 0 & 17.9 \\
\hline Alkalinity $\left(\mathrm{CaCO}_{3}\right)$ & 193 & ND & ND \\
\hline $\mathrm{PH}$ & 8.67 & 8.11 & 9.02 \\
\hline Eh $(\mathrm{mv} / \mathrm{SHE})^{\mathrm{a}}$ & 190 & ND & ND \\
\hline DO & 0.02 & ND & ND \\
\hline TOC & 1.5 & $<0.6$ & 0.67 \\
\hline Ionic strength $(\mathrm{mol} / \mathrm{kg})$ & 0.007 & 0.004 & 0.005 \\
\hline
\end{tabular}

For illustration purposes only.

${ }^{\text {a }}$ SHE is the reference electrode for reporting Eh data. Eh(SHE) $)_{\text {sample }}=\mathrm{Eh}(\mathrm{SHE})_{\text {measured for sample }}+$

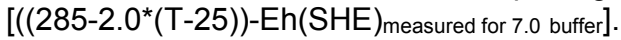

$\mathrm{BLS}=$ below land surface; $\mathrm{DO}=$ dissolved oxygen; $\mathrm{ND}=$ not determined; $\mathrm{SHE}=$ standard hydrogen electrode; TOC=total organic carbon. 
Table G-4. Mineral Abundance and Surface Areas for Selected Alluvium Samples used in Np Sorption Tests

\begin{tabular}{|c|c|c|c|c|c|c|c|c|c|c|c|c|c|c|c|c|}
\hline \multicolumn{4}{|c|}{ Alluvium } & \multicolumn{11}{|c|}{ Quantitative Mineral Abundance for Alluvium Samples (wt \%) } & \multirow{2}{*}{$\begin{array}{c}\text { Surface } \\
\text { Area } \\
\left(\mathrm{m}^{2} / \mathrm{g}\right)\end{array}$} & \multirow{2}{*}{$\begin{array}{c}\text { Organic } \\
\text { Carbon } \\
\text { (wt \%) }\end{array}$} \\
\hline $\begin{array}{l}\text { Sieve } \\
\text { Method }\end{array}$ & $\begin{array}{l}\text { Bore- } \\
\text { hole }\end{array}$ & $\begin{array}{c}\text { Depth } \\
\text { (ft BLS) }\end{array}$ & PS $(\mu \mathrm{m})$ & \begin{tabular}{|c|} 
Smec- \\
tite
\end{tabular} & $\begin{array}{c}\text { Clino- } \\
\text { ptilolite }\end{array}$ & $\begin{array}{c}\text { Kaol- } \\
\text { nite }\end{array}$ & Mica & $\begin{array}{l}\text { Tridy - } \\
\text { mite }\end{array}$ & $\begin{array}{c}\text { Cristo- } \\
\text { batite }\end{array}$ & Quartz & $\begin{array}{l}\text { Feld- } \\
\text { spar }\end{array}$ & Calcite & $\begin{array}{l}\text { Hema- } \\
\text { tite }\end{array}$ & Total & & \\
\hline \multirow[t]{8}{*}{ Dry } & $2 \mathrm{D}$ & $410-415$ & $75-500$ & $2 \pm 1$ & $4 \pm 1$ & $1 \pm 1$ & trace & $3 \pm 1$ & $16 \pm 1$ & $18 \pm 1$ & $54 \pm 8$ & --- & $1 \pm 1$ & $99 \pm 8$ & 1.97 & --- \\
\hline & $9 S x$ & $160-165$ & $75-500$ & $6 \pm 2$ & $3 \pm 1$ & --- & trace & $1 \pm 1$ & $18 \pm 1$ & $14 \pm 1$ & $58 \pm 8$ & --- & trace & $100 \pm 8$ & 2.80 & --- \\
\hline & $3 S$ & $75-80$ & $75-500$ & $1 \pm 1$ & $13 \pm 1$ & $1 \pm 1$ & $1 \pm 1$ & --- & $10 \pm 1$ & $17 \pm 1$ & $53 \pm 8$ & $4 \pm 1$ & --- & $100 \pm 8$ & 3.67 & -- \\
\hline & $19 \mathrm{D}$ & $405-425$ & $75-500$ & $5 \pm 2$ & $7 \pm 1$ & $1 \pm 1$ & trace & $3 \pm 1$ & $13 \pm 1$ & $20 \pm 2$ & $52 \pm 8$ & trace & trace & $101 \pm 9$ & 7.60 & 0.22 \\
\hline & $2 \mathrm{D}$ & $410-415$ & $<75$ & $4 \pm 1$ & $7 \pm 1$ & $1 \pm 1$ & $1 \pm 1$ & $5 \pm 1$ & $14 \pm 1$ & $17 \pm 1$ & $53 \pm 8$ & --- & $1 \pm 1$ & $103 \pm 8$ & 4.27 & --- \\
\hline & $2 \mathrm{D}$ & $395-400$ & $<75$ & $10 \pm 3$ & $13 \pm 1$ & $1 \pm 1$ & trace & $4 \pm 1$ & $13 \pm 1$ & $11 \pm 1$ & $47 \pm 7$ & $1 \pm 1$ & trace & $100 \pm 8$ & 5.55 & --- \\
\hline & 9S & $155-160$ & $<75$ & $19 \pm 6$ & $3 \pm 1$ & trace & $1 \pm 1$ & $4 \pm 1$ & $14 \pm 1$ & $12 \pm 1$ & $50 \pm 7$ & --- & trace & $103 \pm 9$ & 5.69 & --- \\
\hline & $3 S$ & $60-65$ & $<75$ & $7 \pm 2$ & $13 \pm 1$ & $2 \pm 1$ & $1 \pm 1$ & $1 \pm 1$ & $11 \pm 1$ & $12 \pm 1$ & $44 \pm 7$ & $9 \pm 1$ & trace & $100 \pm 8$ & 11.94 & --- \\
\hline \multirow[t]{3}{*}{ Wet } & $19 D$ & $405-425$ & $75-2000$ & $4 \pm 1$ & $5 \pm 1$ & $1 \pm 1$ & $1 \pm 1$ & $3 \pm 1$ & $16 \pm 1$ & $16 \pm 1$ & $49 \pm 7$ & -- & $1 \pm 1$ & $96 \pm 8$ & 5.42 & 0.15 \\
\hline & $19 D$ & \begin{tabular}{|l|}
$405-425$ \\
\end{tabular} & $<75$ & $48 \pm 14$ & $6 \pm 1$ & $1 \pm 1$ & $1 \pm 1$ & $2 \pm 1$ & $5 \pm 1$ & $8 \pm 1$ & $29 \pm 4$ & $1 \pm 1$ & --- & $101 \pm 15$ & 73.65 & 0.76 \\
\hline & 35 & $65-70$ & $<75$ & $30 \pm 9$ & $21 \pm 2$ & $1 \pm 1$ & $2 \pm 1$ & trace & $2 \pm 1$ & $5 \pm 1$ & $25 \pm 4$ & $12 \pm 1$ & --- & $98 \pm 10$ & 37.49 & -- \\
\hline
\end{tabular}

For illustration purposes only.

$\stackrel{1}{1}$ NOTE: Surface area measurements were conducted using MONOSORB N2-Brunauer-Emmett-Teller Single Point Surface Area Analyzer.

BLS=below land surface; PS=particle size; "--." =not detected; trace=amount at less than $0.5 \mathrm{wt} \%$. 


\section{G3.1.1 Kinetics of Neptunium(V) Sorption onto Alluvium}

Kinetic experiments, using the experimental conditions described in Table G-5, were conducted to examine the interaction of $\mathrm{Np}(\mathrm{V})$ and alluvium. The initial sorption kinetics were fast (Figure G-1). After one day of contact between solid and solution, the amount of $\mathrm{Np}(\mathrm{V})$ adsorbed onto alluvium changed little with time in all four tests. The effects of different waters and concentrations of $\mathrm{Np}(\mathrm{V})$ on the kinetic processes of reaction between $\mathrm{Np}(\mathrm{V})$ and alluvium were not systematically evaluated but they appeared to be of less importance than the alluvium characteristics.

Table G-5. Experimental Conditions for Testing Kinetics of $\mathrm{Np}(\mathrm{V})$ Sorption to Alluvium

\begin{tabular}{|c|c|c|c|c|c|}
\hline \multirow[b]{2}{*}{ Test } & \multicolumn{3}{|c|}{ Alluvium } & \multirow{2}{*}{$\begin{array}{c}\text { Neptunium(V) } \\
\text { Initial } \\
\text { Concentration }\end{array}$} & \multirow[b]{2}{*}{ Water Used } \\
\hline & Borehole & Depth (ft BLS) & Particle Size $(\mu \mathrm{m})$ & & \\
\hline Test 1 & $2 \mathrm{D}$ & $410-415$ & $75-500$ & $1 \times 10^{-7} \mathrm{~mol} / \mathrm{L}$ & NC-EWDP-3S \\
\hline Test 2 & $9 S$ & $160-165$ & $75-500$ & $1 \times 10^{-7} \mathrm{~mol} / \mathrm{L}$ & NC-EWDP-3S \\
\hline Test 3 & $3 S$ & $75-80$ & $75-500$ & $1 \times 10^{-7} \mathrm{~mol} / \mathrm{L}$ & NC-EWDP-3S \\
\hline Test 4 & 19D & $405-425$ & $75-2,000$ & $1 \times 10^{-6} \mathrm{~mol} / \mathrm{L}$ & NC-EWDP-19D1 \\
\hline
\end{tabular}

$\mathrm{BLS}=$ below land surface.

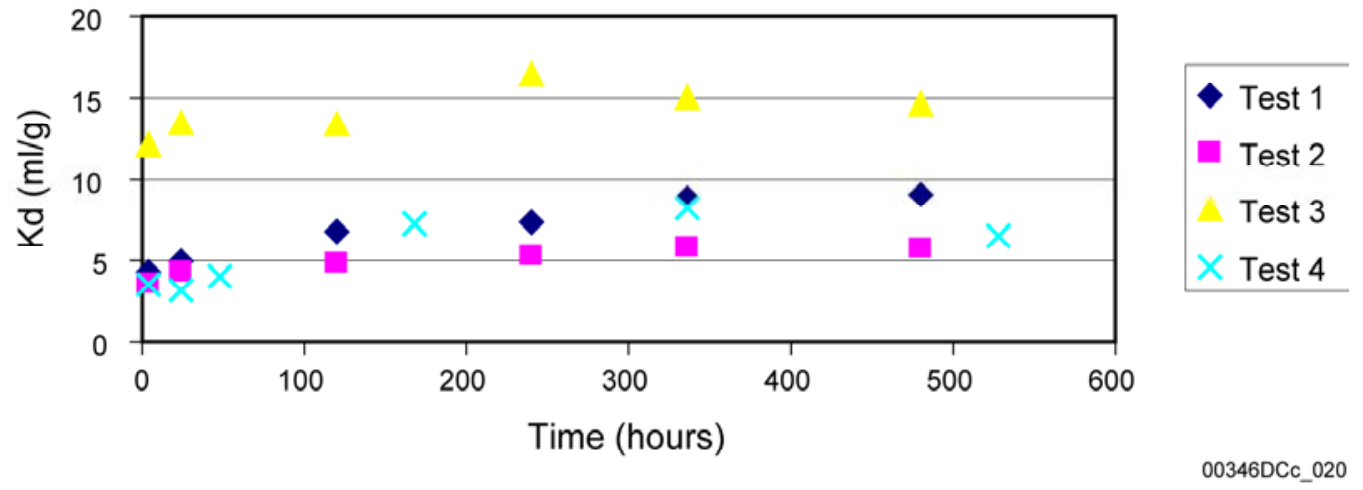

Source DTN: LA0109MD831341.001 (DIRS 156871).

Figure G-1. Sorption Kinetics of ${ }^{237} \mathrm{~Np}$ to Yucca Mountain Alluvium

\section{G3.1.2 Range of $K_{d}$ Values for Neptunium(V)}

The experimentally determined $K_{d}$ values for all the alluvium samples listed in Table G-2 are presented in Figure G-2. The solid-solution contact period for these tests was two weeks. The water used for Borehole NC-EWDP-19D alluvium test was Borehole NC-EWDP-19D1 groundwater. The water used for all other experiments was Borehole NC-EWDP-3S groundwater. The results suggest that the $K_{d}$ of neptunium(V) in alluvium differs from sample to sample and ranges from about 4 to $500 \mathrm{~mL} / \mathrm{g}$. The particle size of the sample appears to play an important role with respect to $K_{d}$ value. In general, the smaller the particle size, the larger the $K_{d}$ value. Alluvium samples from near the surface of Boreholes NC-EWDP-2D and NC-EWDP-3S boreholes have a large adsorption capacity for $\mathrm{Np}(\mathrm{V})$. 


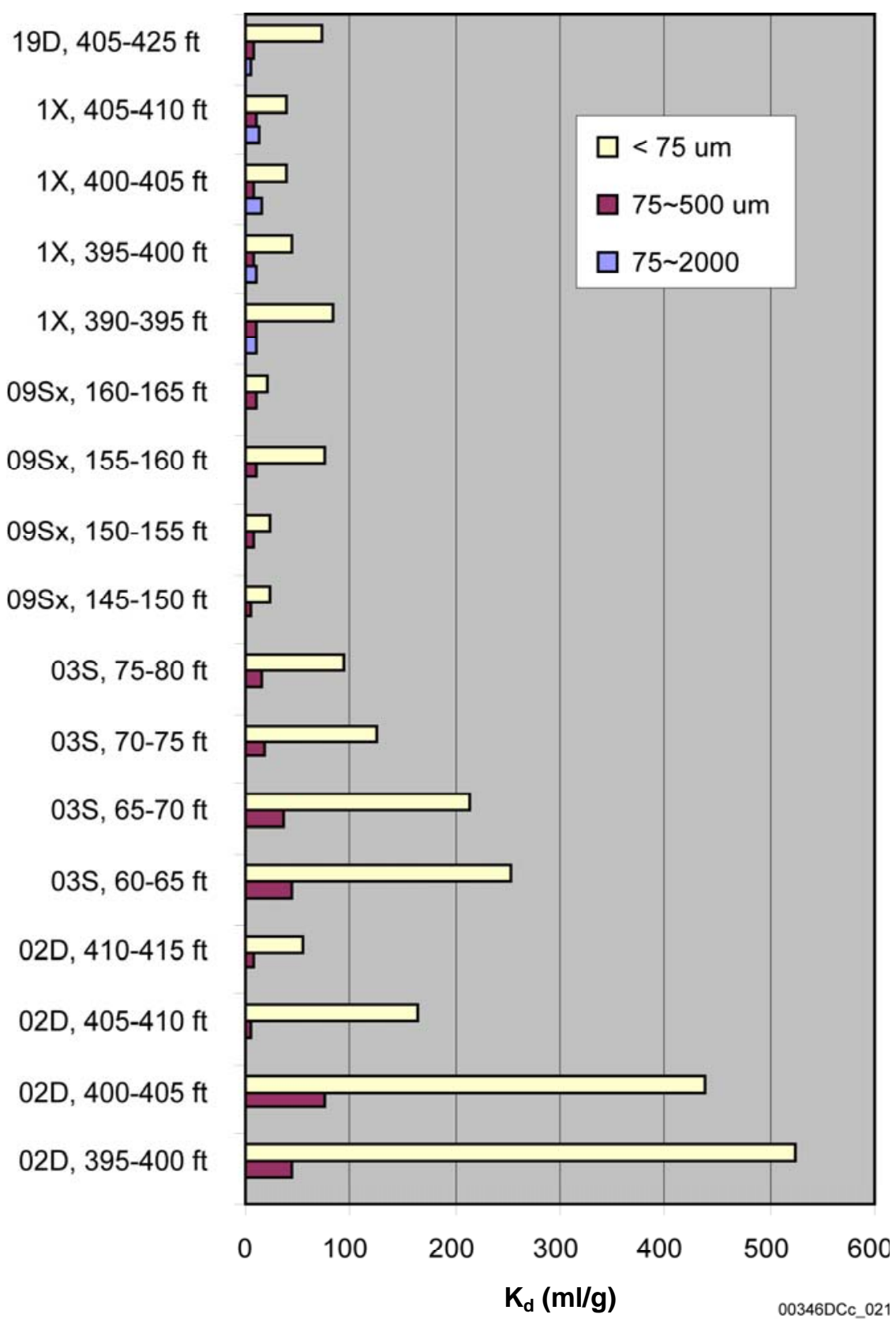

For illustration purposes only

Figure G-2. Batch $K_{d}$ Values for $\mathrm{Np}(\mathrm{V})$ in Different Intervals and Size Fractions 


\section{G3.1.3 Effect of Groundwater Chemistry on Neptunium(V) $K_{d}$ Values}

Adsorption experiments were conducted using ${ }^{237} \mathrm{~Np}$ with alluvium and groundwater from the same boreholes (NC-EWDP-03S and NC-EWDP-19D). The $K_{d}$ values obtained for a given sample with the two different waters were similar (Figure G-3).

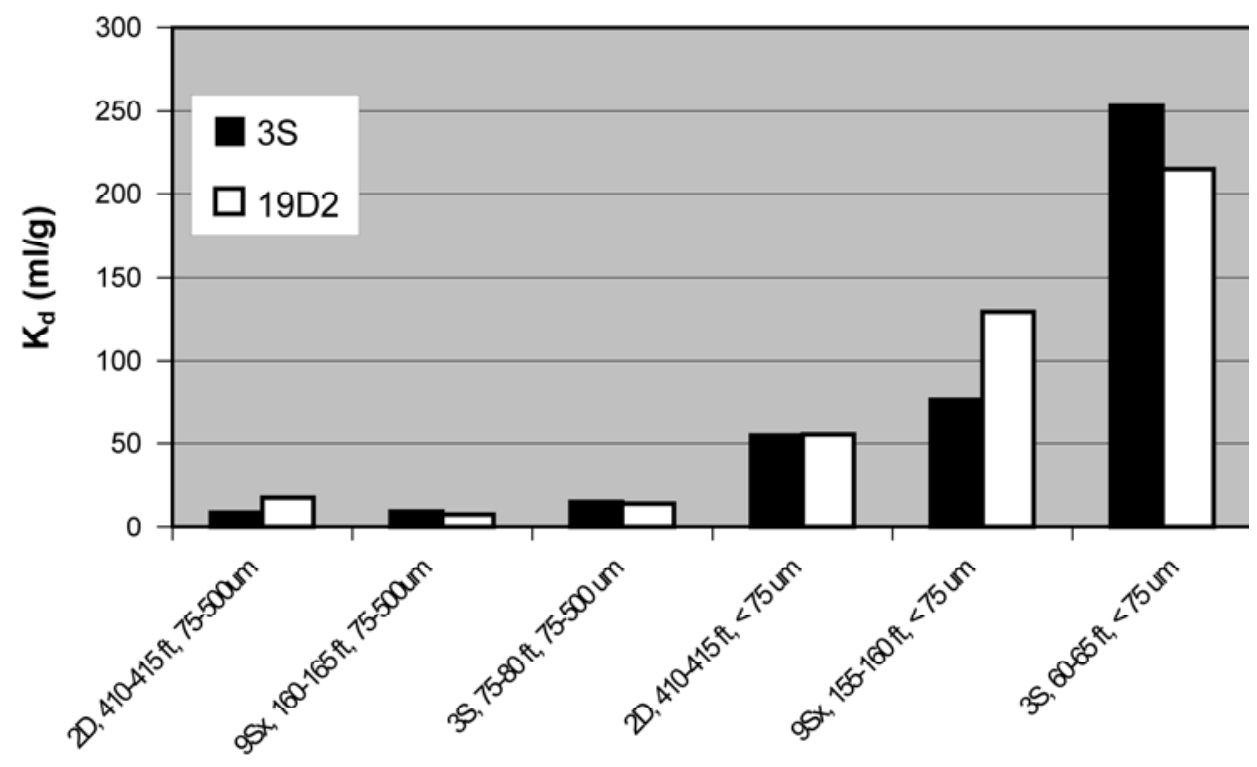

NOTE: The initial concentration of neptunium $(\mathrm{V})$ was $1 \times 10^{-6} \mathrm{M}$. The liquid to solid ratio for all experiments was $20 \mathrm{ml} / \mathrm{g}$. The testing period was 2 weeks.

00346DCc_022

For illustration purposes only

NOTE: The initial concentration of neptunium $(\mathrm{V})$ was $1 \times 10^{-6} \mathrm{M}$. The liquid to solid ratio for all experiments was $20 \mathrm{~mL} / \mathrm{g}$. The testing period was 2 weeks.

Figure G-3. Batch $K_{d}$ Values for Np(V) in Waters from Boreholes NC-EWDP-3S and NC-EWDP-19D

This result suggests that the different redox states of the two waters did not have a significant impact on neptunium sorption behavior. It is likely that the waters used in the experiments had equilibrated with the atmosphere before they were used.

\section{G3.1.4 Effects of Ionic Strength on ${ }^{237} \mathrm{~Np} K_{d}$ Values}

The adsorption of ${ }^{237} \mathrm{~Np}$ in alluvium from Borehole NC-EWDP-19D was examined under various ionic strengths. The original ionic strength of Borehole NC-EWDP-19D water was 0.004 , but this was modified by adding $\mathrm{NaCl}$. The $K_{d}$ of ${ }^{237} \mathrm{~Np}$ did not change significantly with increasing ionic strength (Figure G-4). These results suggest that the reaction mechanism is dominated by surface complexation rather than ion exchange. An additional experiment (not shown) indicated a much larger $K_{d}$ value in deionized water than in the Borehole 19D water, suggesting a possible role of carbonate in suppressing Np sorption in the Borehole 19D water (carbonate was not present in significant quantities in the deionized water). 


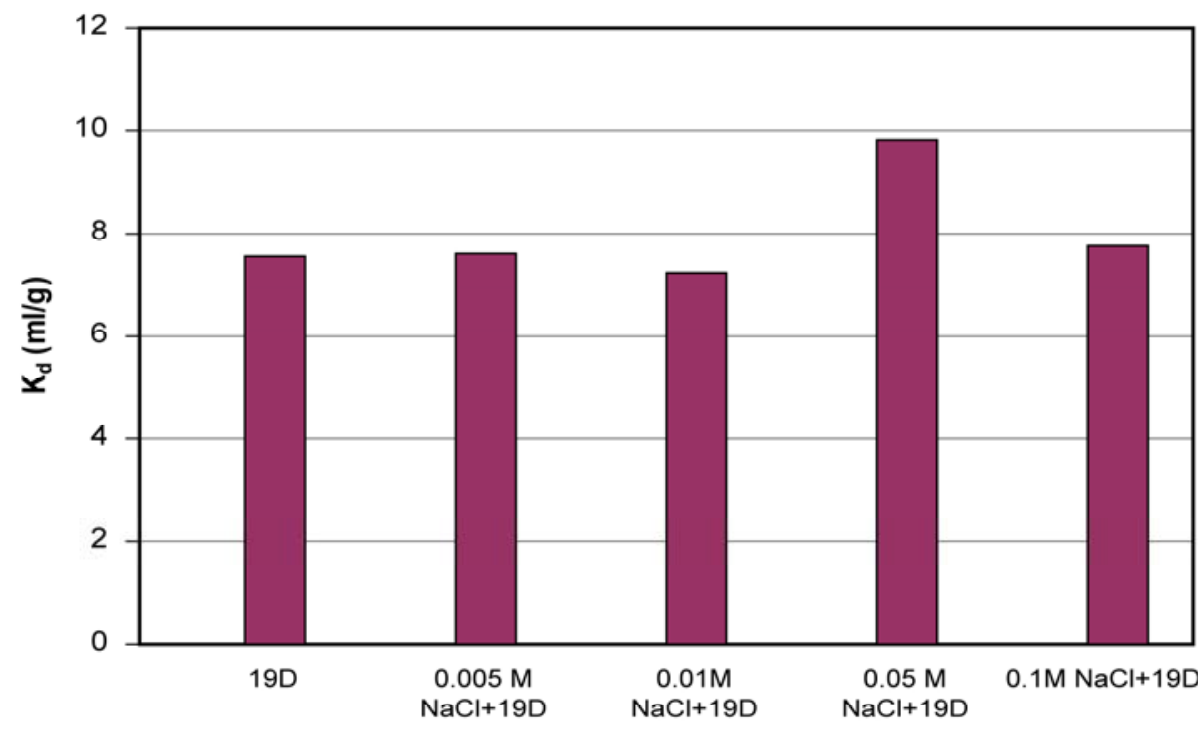

NOTE: Equilibration period is two weeks

00346DCc_023

For illustration purposes only

NOTE: Equilibration period is two weeks.

Figure G-4. Batch $K_{d}$ Values for ${ }^{237} \mathrm{~Np}$ in Solutions with Different lonic Strengths

\section{G3.1.5 ${ }^{237} \mathrm{~Np} K_{d}$ Values in Relation to Surface Area and Secondary Mineral Content}

Surface reactions (e.g., sorption) depend on the surface properties of the sorbent material (e.g., surface area). In general, the larger the surface area of the sample, the larger will be the $K_{d}$ value obtained under the same experimental conditions. Clays and zeolites have larger surface areas than do minerals such as quartz and feldspar. Thus, alluvium containing more clay and zeolites would be expected to have larger $K_{d}$ values.

Experiments were conducted to examine the relationships between surface area, the amount of secondary minerals (combined amounts of smectite and clinoptilolite), and the $K_{d}$ values of ${ }^{237} \mathrm{~Np}$ in alluvium. The results of these experiments are presented in Figure G-5.

Figure G-5 shows that the ${ }^{237} \mathrm{~Np} K_{d}$ values were positively correlated with the amount of smectite and clinoptilolite in the samples and also with the surface area of the samples. However, two samples with extremely high $K_{d}$ values did not have extremely high smectite and clinoptilolite contents, and they also did not have the highest surface areas. Thus, while Np sorption is positively correlated with surface area and mineralogy, trace amounts of minerals such as amorphous Fe and Mn oxides, which were not identified by quantitative XRF, may ultimately exert significant influence on $\mathrm{Np} K_{d}$ values in the alluvium. Studies of sorption of neptunium, plutonium, and americium onto the vitric tuffs of Busted Butte indicated that sorption increases with increasing levels of smectite, $\mathrm{Fe}$, and $\mathrm{Mn}$ oxides in the rock (Turin et al. 2002 [DIRS 164633]). 


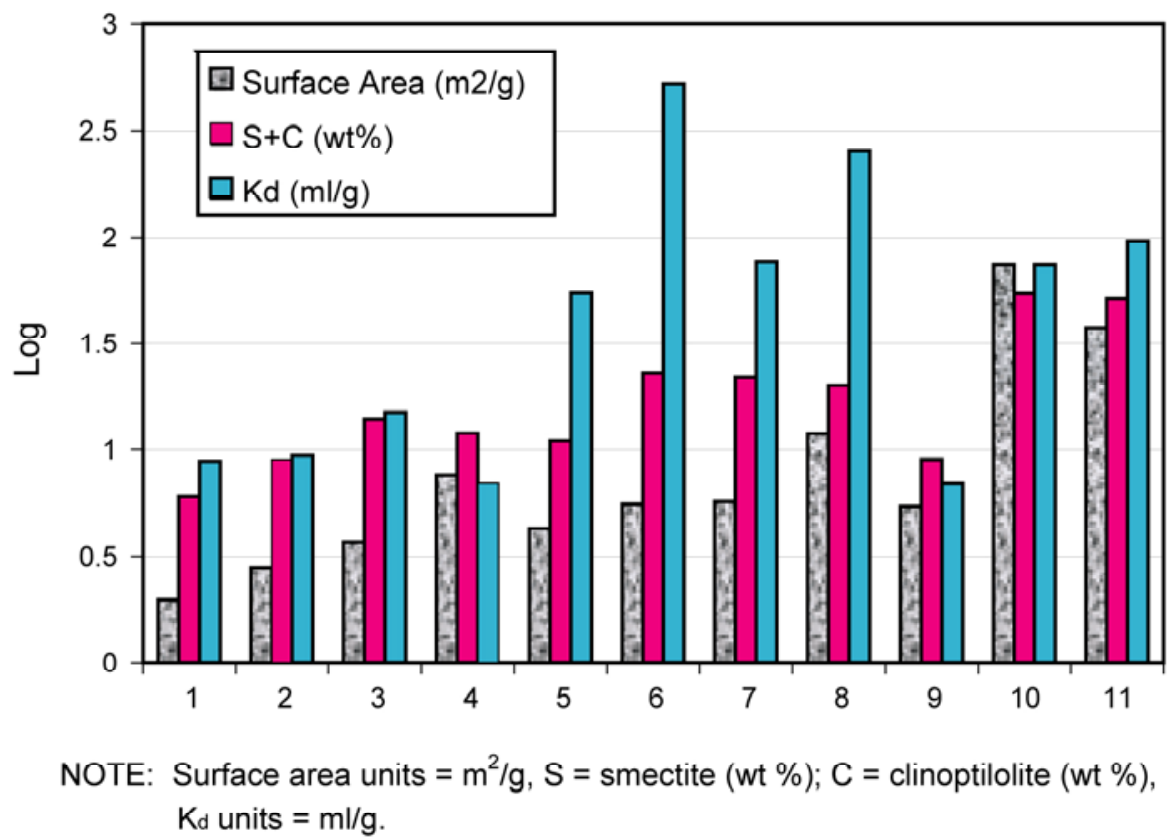

00346DCc 024

For illustration purposes only

NOTE: Surface area units $=\mathrm{m}^{2} / \mathrm{g}, \mathrm{S}=$ smectite (wt \%); $\mathrm{C}=$ clinoptilolite (wt \%), $K_{d}$ units $=\mathrm{mL} / \mathrm{g}$.

Figure G-5. Surface Area, Combined Smectite and Clinoptilolite, and $K_{d}$ Values for $\mathrm{Np}(\mathrm{V})$

\section{G3.2 NEPTUNIUM AND URANIUM RESULTS FROM SECOND SET OF SORPTION AND DESORPTION EXPERIMENTS}

The alluvium samples used in the second set of batch sorption experiments were dry sieved for most experiments. Groundwater used in the experiments was obtained from Boreholes NC-EWDP-19D (Zones 1 and 4) and NC-EWDP-10SA. The chemical composition of Borehole NC-EWDP-19D waters is summarized in Table G-6.

The mineralogy of the alluvium used in the experiments was determined by quantitative X-RF (Table G-7). The major phases in the alluvium samples are silica (i.e., quartz, tridymite, cristobalite), K-feldspar, and plagioclase. The amount of smectite and clinoptilolite in the alluvium differs for the different samples. Among these samples, the sum of the smectite and clinoptilolite in Borehole NC-EWDP-22SA is larger than in NC-EWDP-19IM1A or NC-EWDP-10SA.

\section{G.3.2.1 Batch $K_{d}$ Values for ${ }^{129} \mathrm{I},{ }^{99} \mathrm{Tc},{ }^{233} \mathrm{U}$, and ${ }^{237} \mathrm{~Np}$ in Alluvium}

Under ambient conditions, measured $K_{d}$ values were not statistically distinguishable from zero for ${ }^{129} \mathrm{I}$ and ${ }^{99}$ Tc sorption onto the alluvium, and the reproducibility of the $K_{d}$ measurements was poor (Figure G-6). These results are consistent with earlier results for these two radionuclides in experiments involving alluvium and groundwater from the Phase I Nye County wells. 
Table G-6. Chemical Composition of NC-EWDP-19D Waters

\begin{tabular}{|c|c|c|}
\hline Species & Concentration (mg/L) in Zone 1 & Concentration (mg/L) in Zone 4 \\
\hline Temperature $\left({ }^{\circ} \mathrm{C}\right)$ & 32 & 31 \\
\hline $\mathrm{pH}$ & 7.66 & 9.02 \\
\hline Eh $(\mathrm{mV}-\mathrm{SHE})$ & 342.1 & 493.9 \\
\hline $\mathrm{Na}^{+}$ & 91.50 & 107.30 \\
\hline $\mathrm{K}^{+}$ & 3.70 & 3.40 \\
\hline $\mathrm{Ca}^{2+}$ & 3.70 & 0.92 \\
\hline $\mathrm{Mg}^{2+}$ & 0.31 & 0.03 \\
\hline $\mathrm{SiO}_{2}$ & 22.0 & 18.7 \\
\hline $\mathrm{F}^{-}$ & 2.0 & 2.7 \\
\hline $\mathrm{Cl}^{-}$ & 6.10 & 5.60 \\
\hline $\mathrm{SO}_{4}{ }^{-}$ & 22.0 & 18.7 \\
\hline $\mathrm{HCO}_{3}{ }^{-}$ & 189 & 212 \\
\hline
\end{tabular}

NOTE: $\mathrm{pH}$ and Eh were measured in the laboratory under the conditions of the batch sorption and column transport experiments (DTN: LA0302MD831341.004 [DIRS 163785]).

Table G-7. Quantitative X-ray Diffraction Results of Alluvium Used in Second Set of Experiments

\begin{tabular}{|c|c|c|c|c|c|c|}
\hline \multirow[b]{3}{*}{ Minerals } & \multicolumn{6}{|c|}{ Samples (75 to $500 \mu \mathrm{m}$ fraction, dry sieve) } \\
\hline & \multicolumn{2}{|c|}{ NC-EWDP-19IM1A } & \multicolumn{2}{|c|}{ NC-EWDP-10SA } & \multicolumn{2}{|c|}{ NC-EWDP-22SA } \\
\hline & $725-730^{a, b}$ & $785-790$ & $665-670^{b}$ & $695-700$ & $522-525^{b}$ & $660-665$ \\
\hline Smectite & $6.9^{b}$ & 6.2 & $5.7^{b}$ & 2.6 & $8.3^{b}$ & 4.7 \\
\hline Kaolinite & 1.2 & 1.3 & 0.8 & 0.5 & 2.0 & 1.1 \\
\hline Clinoptilolite & $7.7^{\mathrm{b}}$ & 8.5 & $7.0^{\mathrm{b}}$ & 4.1 & $14.3^{b}$ & 7.9 \\
\hline Tridymite & 7.6 & 7.9 & 3.5 & 2.3 & 8.5 & 10.2 \\
\hline Cristobalite & 5.8 & 6.4 & 7.0 & 5.9 & 5.6 & 7.2 \\
\hline Quartz & 19.2 & 16.1 & 14.0 & 6.0 & 12.8 & 17.3 \\
\hline K-Feldspar & 23.7 & 25.8 & 29.7 & 32.5 & 22.7 & 25.0 \\
\hline Plagioclase & 25.0 & 26.5 & 30.5 & 40.7 & 19.1 & 21.2 \\
\hline Biotite & 1.0 & 3.0 & 3.1 & 2.5 & 2.4 & 2.1 \\
\hline Hematite & 0.7 & 0.7 & 0.8 & 2.4 & 1.0 & 2.5 \\
\hline Total & 98.8 & 102.3 & 102.3 & 99.6 & 96.7 & 99.2 \\
\hline
\end{tabular}

For illustration purposes only.

anterval below land surface (feet).

${ }^{\mathrm{b}}$ Samples selected to conduct kinetic adsorption of ${ }^{233} \mathrm{U}$. 


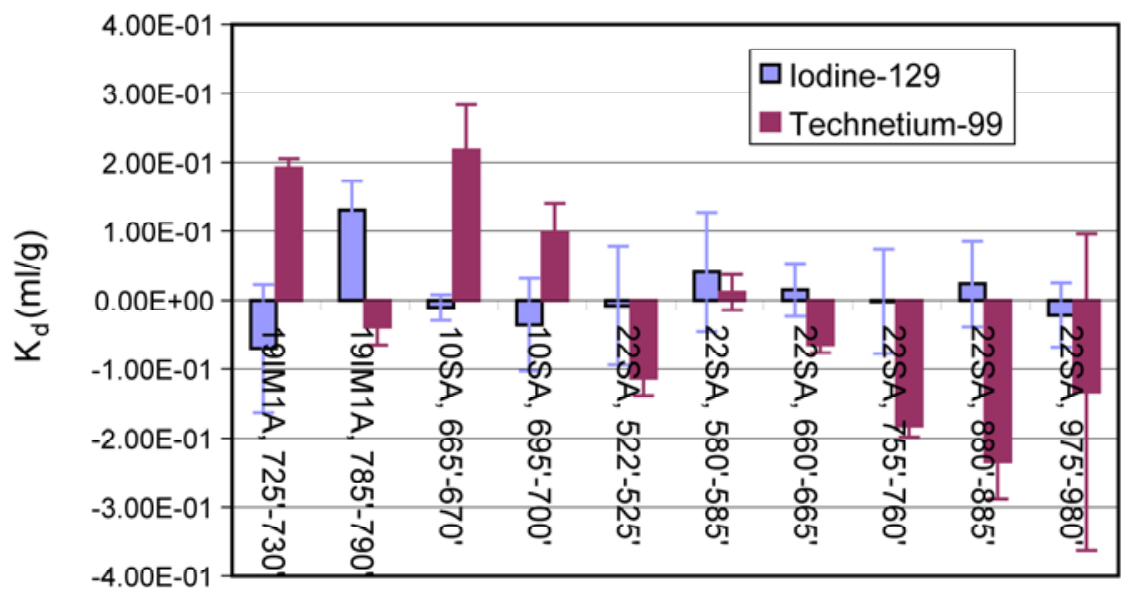

NOTE: Experiments terminated after two weeks. Liquid to solid ratio (L/S) of the experiments is $20 \mathrm{ml} / \mathrm{g}$. NC-EWDP-19D Zone 1 water was used for the experiments with alluvium from NC-EWDP-19IM1A and NC -EWDP-22SA, and NC -EWDP-10 S water was used for the experiments with alluvium from NC-EWDP-10SA.

00346DCc_011

Source DTNs: LA0302MD831341.001 [DIRS 164956]; LA0302MD831341.002 [DIRS 164957].

NOTE: Experiments terminated after two weeks. Liquid to solid ratio (L/S) of the experiments is $20 \mathrm{~mL} / \mathrm{g}$. Borehole NC-EWDP-19D Zone 1 water was used for the experiments with alluvium from Boreholes NC-EWDP-19IM1A and NC-EWDP-22SA, and Borehole NC-EWDP-10 S water was used for the experiments with alluvium from Borehole NC-EWDP-10SA.

Figure G-6. Batch $K_{d}$ Values for ${ }^{129} \mathrm{I}$ and ${ }^{99} \mathrm{Tc}$ in Alluvium

The $K_{d}$ values of ${ }^{237} \mathrm{~Np}$ and ${ }^{233} \mathrm{U}$ in the alluvium were found to differ from sample to sample depending on the depths and types of the alluvium tested (Figure G-7). The $K_{d}$ values range between 3 and $13 \mathrm{~mL} / \mathrm{g}$ for ${ }^{237} \mathrm{~Np}$ and about 3 to $9 \mathrm{~mL} / \mathrm{g}$ for ${ }^{233} \mathrm{U}$. The sorption capacity of alluvium for ${ }^{237} \mathrm{~Np}$ is larger than that for ${ }^{233} \mathrm{U}$. Unlike $K_{d}$ measurements for ${ }^{129} \mathrm{I}$ and ${ }^{99} \mathrm{Tc}$, the reproducibility of ${ }^{237} \mathrm{~Np}$ and ${ }^{233} \mathrm{U}$ sorption measurements was good.

To test if ${ }^{233} \mathrm{U}$ sorption is a function of water composition, adsorption experiments were performed with ${ }^{233} \mathrm{U}$ using water from Borehole NC-EWDP-19D Zones 1 and 4 . The $K_{d}$ values of ${ }^{233} \mathrm{U}$ measured in Zone 4 water were lower than those for Zone 1 (Figure G-8). Major differences in these two waters under the conditions of the laboratory experiments are the $\mathrm{pH}$ of Zone 4 water is considerably higher than that of Zone 1 water (Table G-6), and the DO content of Zone 4 water is higher than water from Zone 1 . Note that the $\mathrm{pH}$ of the waters in the laboratory experiments were significantly different than those measured in the field; the two waters had similar $\mathrm{pH}$ in the field, but the Zone 1 water decreased to $\mathrm{pH}=7.7$ in the lab and the Zone 1 water increased to $\mathrm{pH}=9$. The reasons for these differences in the change in $\mathrm{pH}$ are not fully understood. The lower $\mathrm{pH}$ of the water from Zone 1 is likely a major cause for the higher sorption coefficients obtained in experiments using this water 


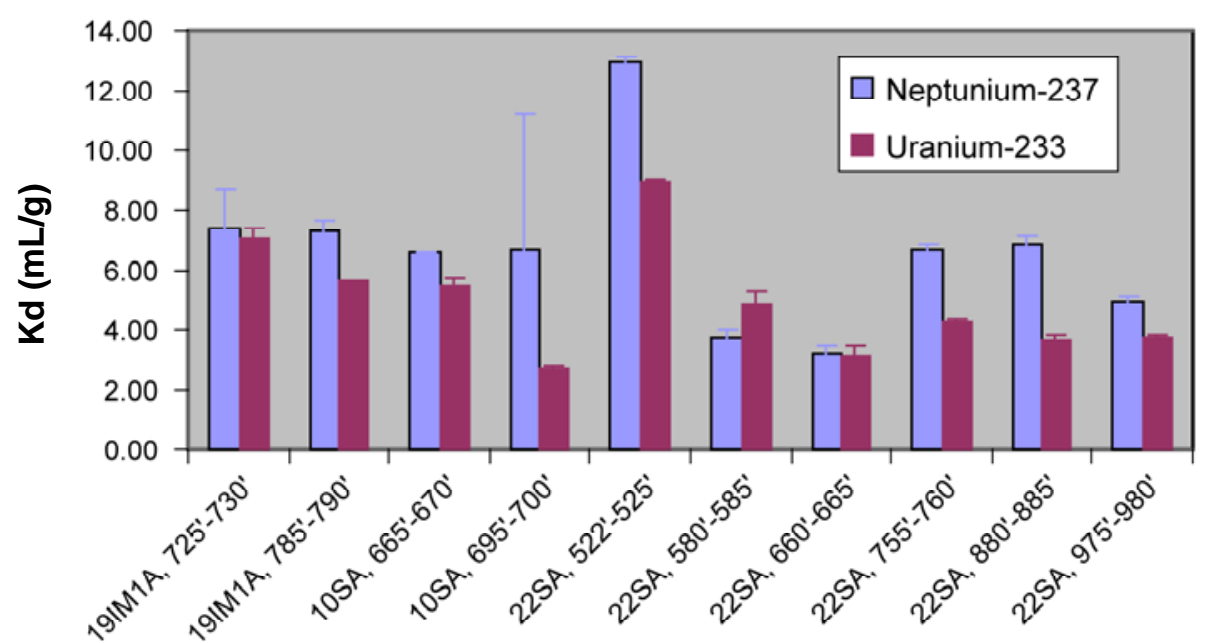

NOTE: Experiments terminated after two weeks. Liquid to solid ratio (L/S) of the experiments is $20 \mathrm{ml} / \mathrm{g}$. NC-EWDP-19D Zone 1 water was used for the experiments with alluvium from NC-EWDP-19IM1A and NC-EWDP-22SA, and NC -EWDP-10S water was used for the experiments with alluvium from NC-EWDP-10SA.

00346DCc_013

NOTE: $\quad$ L/S $=20 \mathrm{~mL} / \mathrm{g}$. NC-EWDP-19D Zone 1 water was used for the experiments with alluvium from NC-EWDP-19IM1A and NC-EWDP-22SA, and NC-EWDP-10S water was used for the experiments with alluvium from NC-EWDP-10SA.

Figure G-7. $\quad K_{d}$ Values for ${ }^{237} \mathrm{~Np}$ and ${ }^{233} \mathrm{U}$ in Alluvium

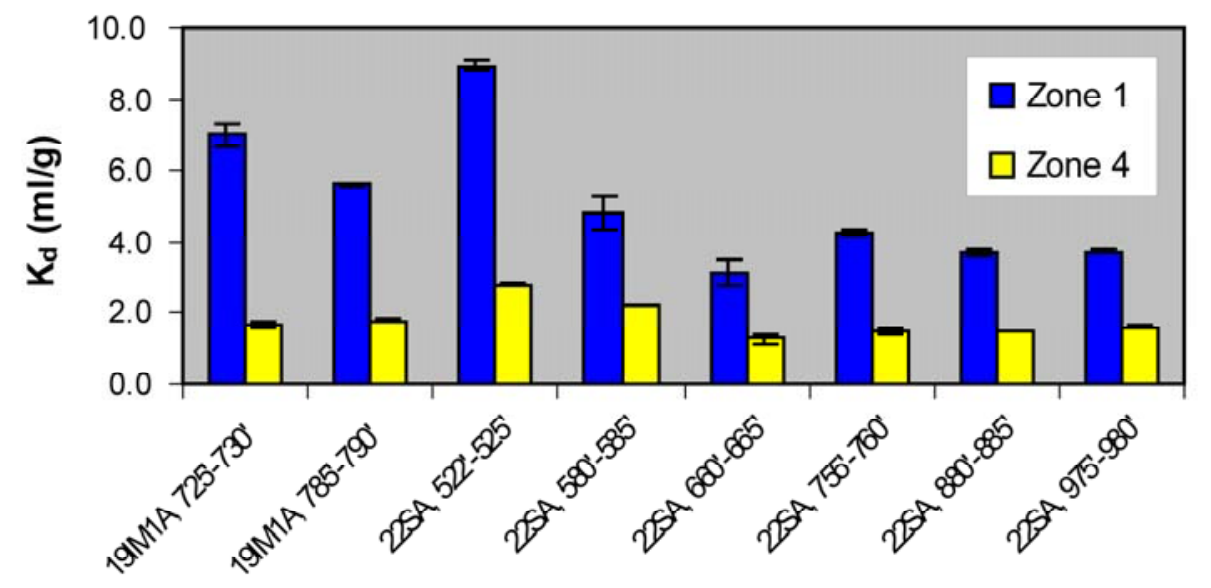

NOTE: Experiments terminated after two weeks. Liquid to solid ratio (L/S) of the experiments was $20 \mathrm{ml} / \mathrm{g}$.

00346DCc_014

Source DTN: LA0302MD831341.004 [DIRS 163785].

NOTE: Experiments terminated after two weeks. Liquid to solid ratio (L/S) of the experiments was $20 \mathrm{~mL} / \mathrm{g}$.

Figure G-8. Batch $K_{d}$ Values for ${ }^{233} \mathrm{U}$ in NC-EWDP-19D Zone 1 and Zone 4 Waters 
The high $\mathrm{pH}$ of the Zone 4 water would have resulted in a significant amount of carbonate ion $\left(\mathrm{CO}_{3}{ }^{\circ}\right)$ present in this water, whereas the Zone 1 water would have had very little carbonate ion present at a $\mathrm{pH}$ of 7.7. Uranium is known to form stable complexes with carbonate ion in solution (Langmuir 1997 [DIRS 100051]), so it would have been more likely to remain in solution at the higher $\mathrm{pH}$ of the Zone 4 water.

\section{G3.2.2 Uranium Sorption and Desorption Kinetics in Alluvium}

Sorption kinetics of ${ }^{233} \mathrm{U}$ was measured in three alluvium samples (Table G-7). After 1 day of exposure, the amount of ${ }^{233} \mathrm{U}$ adsorbed onto alluvium changed little during the remainder of the tests. Thus, the equilibration rate for the uranium sorption reaction is relatively fast (Figure G-9). Higher $K_{d}$ values from the NC-EWDP-22SA sample may be the result of higher smectite and clinoptilolite content (Table G-7).

Multi-step batch desorption experiments of sorbed ${ }^{233} \mathrm{U}$ were conducted (Figure G-10). Most of the uranium desorbed from the alluvium samples was desorbed during the first step. Less uranium was desorbed in subsequent desorption steps. A large fraction (30 to 50 percent) of the sorbed ${ }^{233} \mathrm{U}$ remained sorbed on the solid phase even after three desorption steps. These results suggest that the ${ }^{233} \mathrm{U}$ desorption kinetics were relatively slow and that they were slowing down as the experiments progressed.

Continuous-flow ${ }^{233} \mathrm{U}$ desorption experiments were conducted after the end of some of the sorption experiments. The alluvium material containing sorbed ${ }^{233} \mathrm{U}$ was removed from the test tubes used in the batch experiments and placed in a small "column," where it was then subjected to a continuous flow of fresh water. The effluent from the column was analyzed for ${ }^{233} \mathrm{U}$. The results showed that the release of sorbed ${ }^{233} \mathrm{U}$ slowed down after first $100 \mathrm{~mL}$ of groundwater had contacted the alluvium (Figure G-11), but release continued at a finite rate for the remainder of the experiment. The total duration of the experiment was about 5.5 days, including a $\sim 2.5$-day flow interruption just after $100 \mathrm{~mL}$ eluted.

The concentrations of eluted ${ }^{233} \mathrm{U}$ near the end of the experiment were very close to the detection limit. These results suggest that the desorption of the sorbed ${ }^{233} \mathrm{U}$ was quite slow. Simple linear extrapolation of the trends at the end of the experiments suggests that the total desorption after three weeks would be similar to the total desorption measured after three weeks in the multi-step batch desorption experiments shown in Figure G-10. 


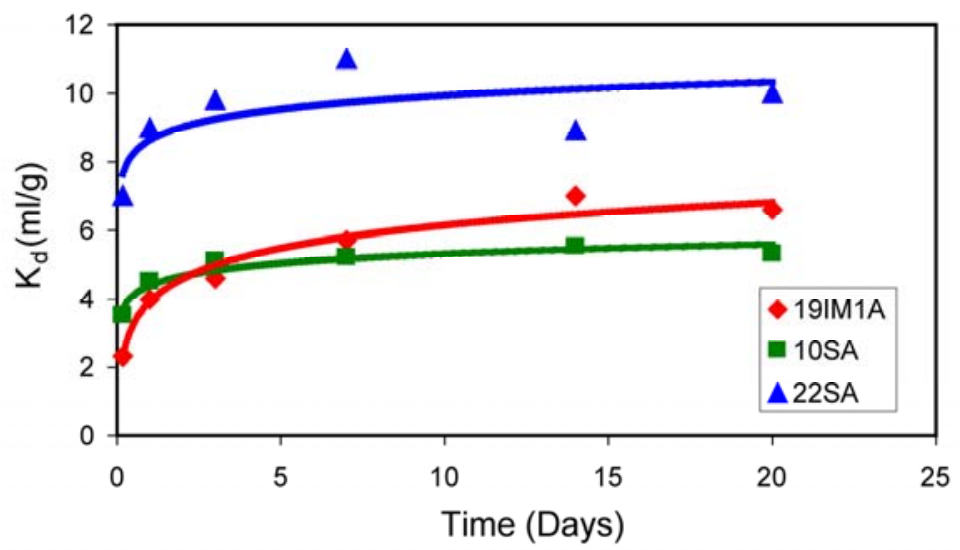

NOTE: L/S $=20 \mathrm{ml} / \mathrm{g}$. NC-EWDP-19D Zone 1 water was used for the experiments with alluvium from NC-EWDP-19IM1A and NC -EWDP-22SA, and NC -EWDP-10S water was used for the experiments with alluvium from NC-EWDP-10SA

00346DCc_012

Source DTNs: LA0302MD831341.003 [DIRS 163784]; LA0302MD831341.004 [DIRS 163785].

NOTE: Experiments terminated after two weeks. Liquid to solid ratio (L/S) of the experiments is $20 \mathrm{~mL} / \mathrm{g}$. NC-EWDP-19D Zone 1 water was used for the experiments with alluvium from NC-EWDP-19IM1A and NC-EWDP-22SA, and NC-EWDP-10S water was used for the experiments with alluvium from NC-EWDP-10SA.

Figure G-9. Batch $K_{d}$ Values for ${ }^{233} \mathrm{U}$ onto Alluvium as a Function of Time

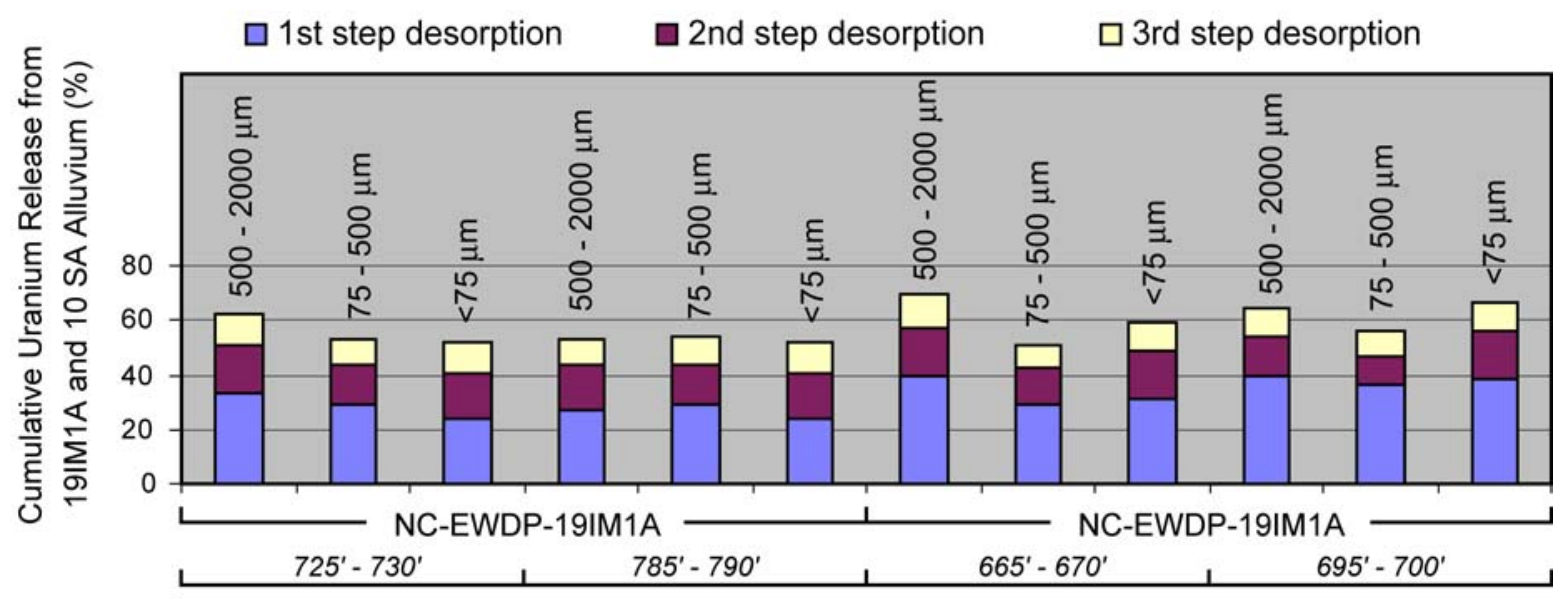

00346DCc_015

For illustration purposes only.

NOTE: The period for each desorption step was one week. L/S ratio for desorption is about $20 \mathrm{~mL} / \mathrm{g}$.

Figure G-10. Cumulative Release of Sorbed ${ }^{233} \mathrm{U}$ from NC-EWDP-19IM1A and NC-EWDP-10SA Alluvium 


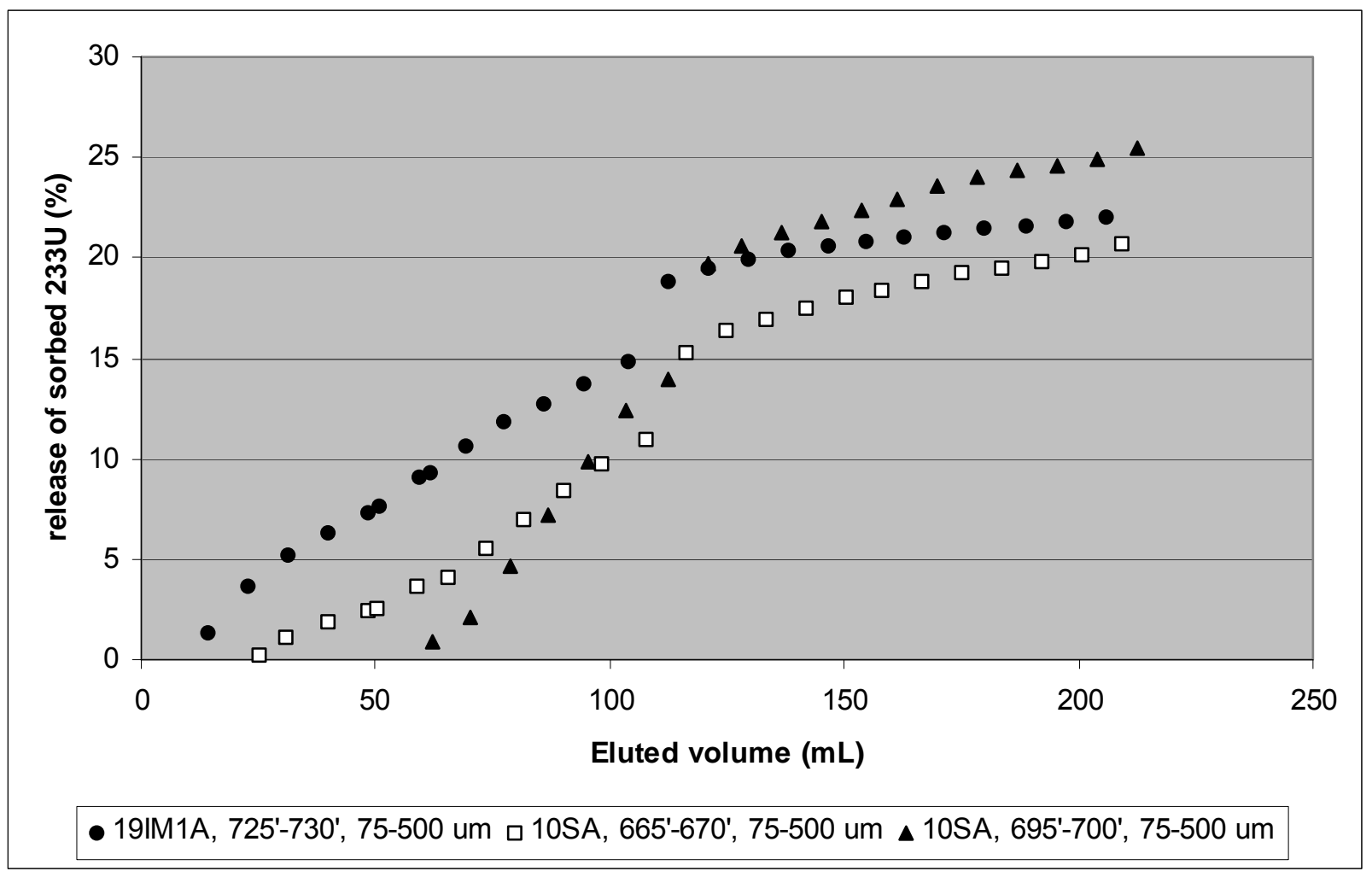

For illustration purposes only.

NOTE: The flow rate is $3 \mathrm{~mL} / \mathrm{h}$. NC-EWDP-19D Zone 1 water was used for NC-EWDP-19IM1A sample, $10 \mathrm{~S}$ water was used for NC-EWDP-10SA samples.

Figure G-11. Release of Sorbed ${ }^{233} \mathrm{U}$ as a Function of Eluted Volume of Groundwater

\section{G4. COLUMN TRANSPORT EXPERIMENTS}

\section{G4.1 NEPTUNIUM COLUMN TRANSPORT EXPERIMENTS}

Two sets of column experiments were performed to investigate $\mathrm{Np}(\mathrm{V})$ transport behavior in saturated alluvium under flowing conditions. Tables G-8 and G-9 list individually the columns used and conditions in the two studies. In all the column experiments, tritium ( $\left.{ }^{3} \mathrm{HHO}\right)$ was used as a nonsorbing tracer. Water from NC-EWDP-03S was used in the experiments in columns 1 and 2, and water from NC-EWDP-19D was used in the experiments in columns 3, 4, and 5. The alluvium used to pack the columns was wet sieved in all cases to remove fine particles that would clog the columns. The latter set of experiments was reported by Ding et al. (2003 [DIRS 164737]). 
Table G-8. Np(V) Column Study (I)

\begin{tabular}{|c|c|}
\hline & Columns 1 and 2 \\
\hline Geologic Medium & $-03 S$ \\
\hline Interval (ft. BLS) & $65-70$ \\
\hline Particle Size $(\mu \mathrm{m})$ & $75-500$ \\
\hline Water Used & $-03 S$ \\
\hline $\mathrm{pH}$ range & $8.5-9.0$ \\
\hline Diameter, cm & 1.0 \\
\hline Length of column $(\mathrm{cm})$ & 60 \\
\hline Porosity in column & 0.44 (Col. 1), 0.45 (Col. 2) \\
\hline Flow rate $(\mathrm{mL} / \mathrm{h})$ & $\begin{array}{c}2 \text { (reduced to } 0.5 \mathrm{~mL} / \mathrm{h} \text { late in the } \\
\text { test) }\end{array}$ \\
\hline
\end{tabular}

For illustration purposes only.

BLS=below land surface.

Table G-9. Np(V) Column Study (II)

\begin{tabular}{|l|c|c|c|}
\hline & Column 3 & Column 4 & Column 5 \\
\hline Geologic Medium & $-19 \mathrm{D}$ & $-19 \mathrm{D}$ & $-19 \mathrm{D}$ \\
\hline Interval (ft. BLS) & $405-425$ & $405-425$ & $405-425$ \\
\hline Particle Size $(\mu \mathrm{m})$ & $75-2,000$ & $75-2,000$ & $75-2,000$ \\
\hline Water Used & $19 \mathrm{D}$ & $19 \mathrm{D}$ & $19 \mathrm{D}$ \\
\hline $\mathrm{pH}$ range & $8.4-8.7$ & $8.4-8.7$ & $8.4-8.7$ \\
\hline Diameter, cm & 2.5 & 2.5 & 2.5 \\
\hline Length of column $(\mathrm{cm})$ & 45 & 45 & 45 \\
\hline Porosity in column & 0.38 & 0.37 & 0.34 \\
\hline Flow rate $(\mathrm{mL} / \mathrm{h})$ & $10,0.6(2$ tests) & 3 & 10 \\
\hline
\end{tabular}

For illustration purposes only.

BLS=below land surface.

Figure G-12 shows the breakthrough curves of neptunium from columns 1 and 2, and Figure G-13 shows the breakthrough curves of neptunium from columns 3, 4, and 5 . The 10$\mathrm{mL} / \mathrm{hr}$ experiment in column 5 was intended to be a duplicate of the $10-\mathrm{mL} / \mathrm{hr}$ experiment in column 3, which was terminated prematurely because of equipment problems. However, this experiment had an abnormally high background alpha activity, which made it impossible to see the tailing behavior that occurred in the other experiments (and suppressed the peak concentration after background subtraction). The earlier breakthrough and peak concentration in this experiment relative to the $10-\mathrm{mL} / \mathrm{hr}$ experiment in column 3 may be related to the fact that column 5 had a significantly greater hydraulic conductivity (by about 35 percent) than column 3 . This higher hydraulic conductivity suggests that there were fewer fine grains and, hence, less surface area and probably less clay and zeolite material in column 5 than in column 3 (despite the fact that the same alluvium material and same sieving method was used). 


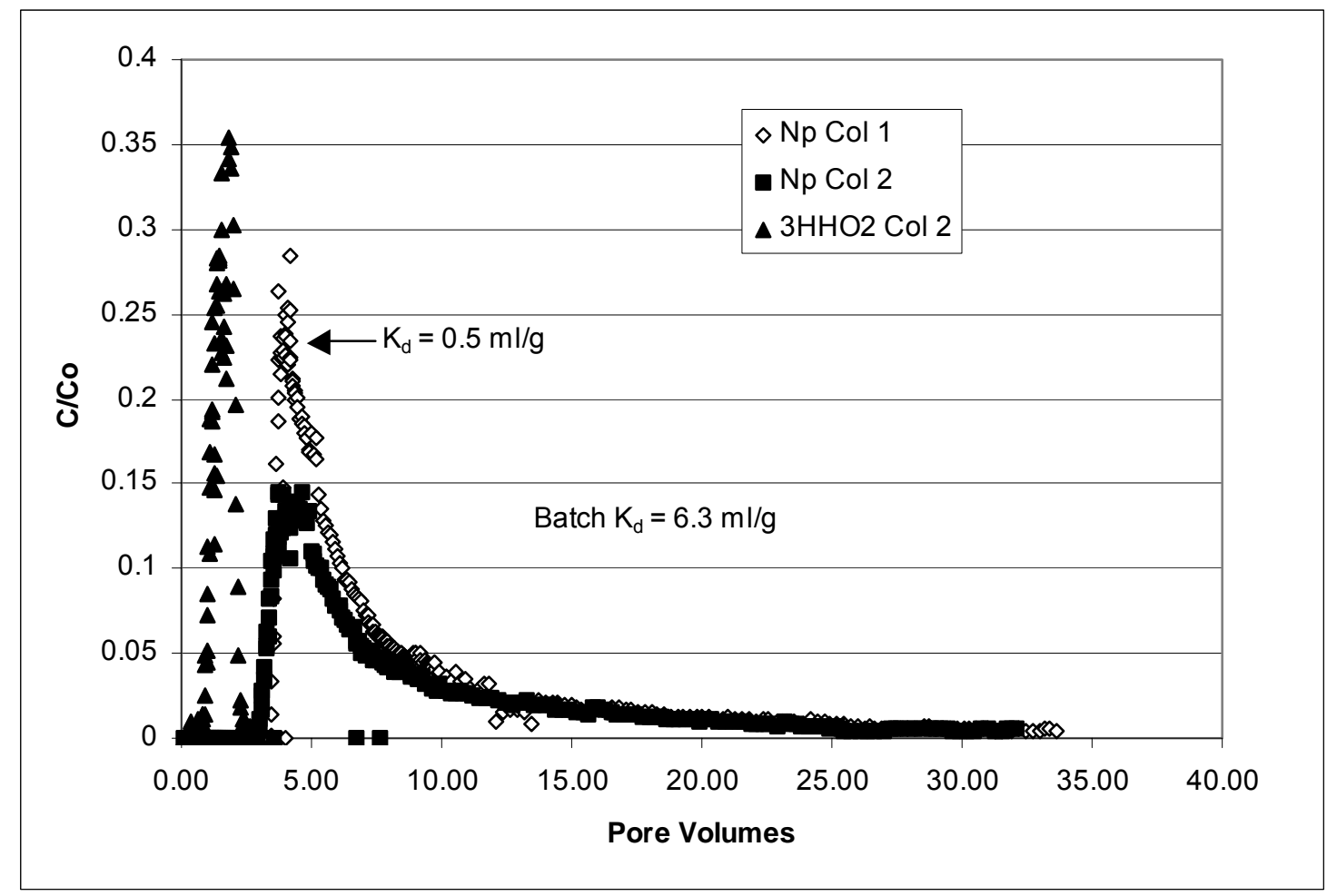

For illustration purposes only.

Figure G-12. Tritiated Water and Neptunium Breakthrough Curves in Columns 1 and 2

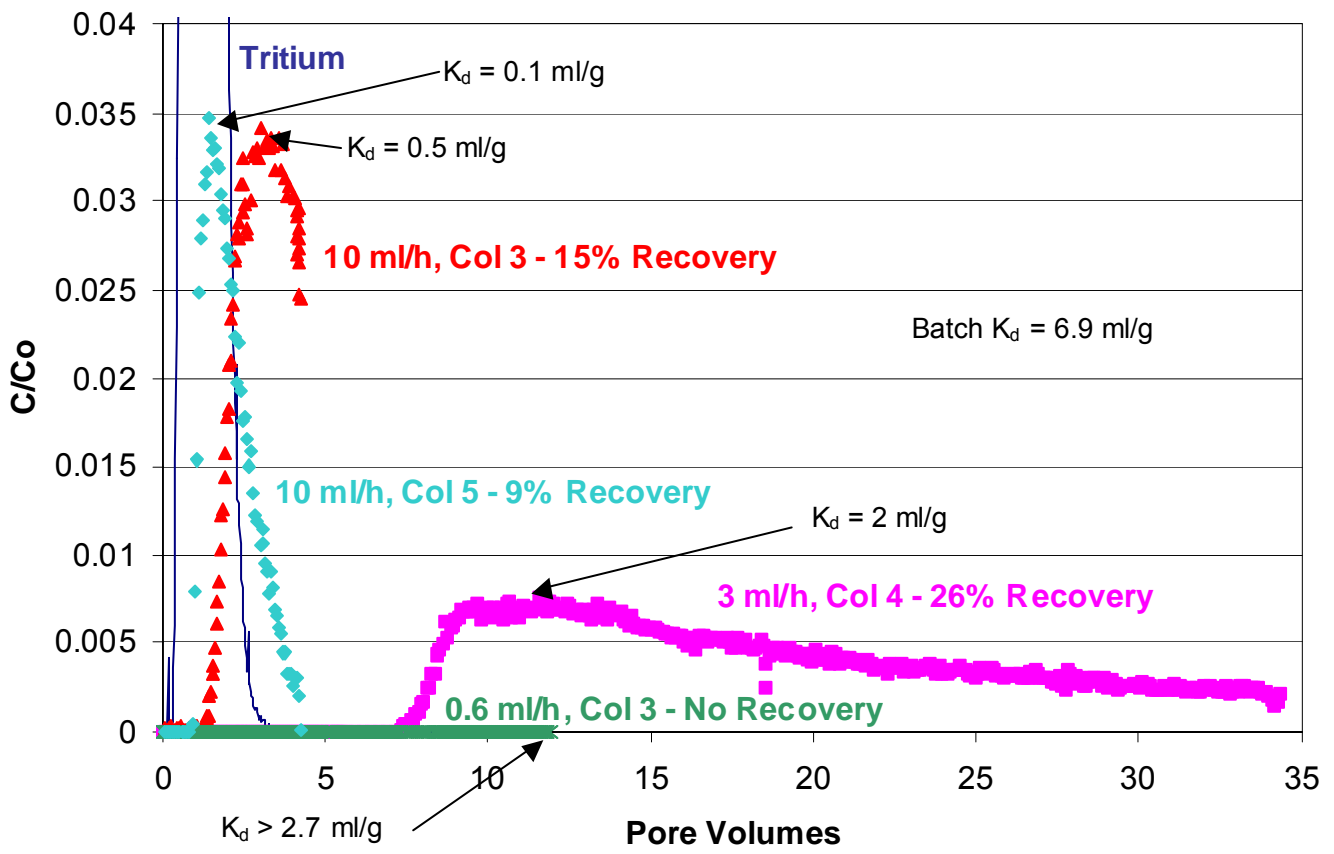

For illustration purposes only. 
NOTE: Column 5 had a significantly higher hydraulic conductivity than column 3 , and the tail of the breakthrough curve was truncated because of an artificially high background concentration (which was subtracted from the measured concentrations).

Figure G-13. Neptunium Breakthrough Curves in Columns 3, 4, and 5

Note that the $0.6-\mathrm{mL} / \mathrm{hr}$ experiment shown in Figure G-13 was conducted in column 3 after the $10-\mathrm{mL} / \mathrm{hr}$ experiment. The column was flushed until no more Np eluted prior to injecting the Np at a rate of $0.6 \mathrm{~mL} / \mathrm{hr}$. As Figure G-13 shows, there was no breakthrough of neptunium after $\sim 12.5$ pore volumes had been eluted in the $0.6-\mathrm{mL} / \mathrm{hr}$ test. The $K_{d}$ value corresponding to a breakthrough at 12.5 pore volumes is approximately $2.7 \mathrm{~mL} / \mathrm{g}$ for the column in which this test was conducted. Thus, all of the neptunium in the $0.6-\mathrm{mL} / \mathrm{hr}$ test had an effective $K_{d}$ value of greater than $2.7 \mathrm{~mL} / \mathrm{g}$. This test is significant because the linear flow velocity in the column was $43 \mathrm{~m} / \mathrm{yr}$, which is consistent with estimates of linear flow velocities in the alluvial aquifer (10 to 80 m/yr) as given in Saturated Zone In-Situ Testing (BSC 2004 [DIRS 170010]).

Figure G-13 indicates that higher flow rates result in a lower effective $K_{d}$ value for at least a portion of the neptunium traveling through the columns. However, despite the early breakthroughs in the experiments at the two higher flow rates, the recoveries of neptunium were still quite low (less than 40 percent), suggesting slow desorption rates for most of the neptunium in the columns. Furthermore, the long tails in these experiments suggest a wide range of desorption rates for neptunium. The minimum possible $K_{d}$ value in the lowest flow rate column test $(2.7 \mathrm{~mL} / \mathrm{g})$ agrees better with the batch studies $\left(K_{d}=6.9 \mathrm{~mL} / \mathrm{g}\right)$ than the $K_{d}$ values for the earliest arriving neptunium in the higher flow rate tests.

The observed differences in neptunium transport as a function of flow rate cannot be explained by a single rate-limited sorption reaction. It is likely that multiple reactions with multiple rates were occurring in the columns because of multiple sorption sites. An approach to modeling these experimental results is discussed later in this appendix. The column experiments reveal that reactive transport processes in heterogeneous alluvium, even at a relatively small scale, are quite complicated and not amenable to simple transport models, at least when flow velocities are high.

\section{G4.2 URANIUM COLUMN TRANSPORT EXPERIMENTS}

Continuous-flow column experiments involving ${ }^{233} \mathrm{U}$ were conducted at room temperature and under ambient conditions at an elution rate of $10 \mathrm{~mL} / \mathrm{hr}$. The elution rate was decreased first to $5 \mathrm{~mL} / \mathrm{hr}$ and then very quickly to $3 \mathrm{~mL} / \mathrm{hr}$ as the experiments progressed. Experimental conditions are presented in Table G-10. The ${ }^{233} \mathrm{U}$ breakthrough curves relative to ${ }^{3} \mathrm{HHO}$ are shown in Figure G-14. In all cases, a small fraction of the uranium broke through at almost the same time as the ${ }^{3} \mathrm{HHO}$, but the vast majority of the uranium mass was significantly retarded. Total uranium recoveries ranged from 26 to 65 percent of the uranium injected. The long tails and incomplete recoveries observed in the column experiments indicate that some of the ${ }^{233} \mathrm{U}$ was slow to desorb from the columns within the time frame of the experiments. These experiments have not yet been quantitatively interpreted to obtain estimates of uranium sorption parameters, but the modeling approach discussed later in this appendix is capable of qualitatively explaining the observed behavior. 
Table G-10. Uranium Column Experiments

\begin{tabular}{|l|c|c|c|}
\hline & Column \#1 & Column \#2 & Column \#3 \\
\hline Geological Medium & $19 I \mathrm{M} 1 \mathrm{~A}$ & $10 \mathrm{SA}$ & $22 \mathrm{SA}$ \\
\hline Interval $(\mathrm{ft} . \mathrm{BLS})$ & $725-730$ & $665-670$ & $522-525$ \\
\hline Particle Size $(\mu \mathrm{m})$ & $75-2,000$ & $75-2,000$ & $75-2,000$ \\
\hline & Column \#1 & Column \#2 & Column \#3 \\
\hline Water Used & $19 \mathrm{D}$ Zone 1 & $10 \mathrm{~S}$ & $19 \mathrm{D}$ Zone 1 \\
\hline pH range & $8.4-8.7$ & $8.2-8.5$ & $8.4-8.7$ \\
\hline Diameter, cm & 2.5 & 2.5 & 2.5 \\
\hline Dry alluvium packed in column $(\mathrm{g})$ & 374.61 & 356.59 & 390.72 \\
\hline Water weight after the saturation $(\mathrm{g})$ & 89.82 & 102.4 & 85.98 \\
\hline Porosity in column & 0.41 & 0.44 & 0.39 \\
\hline
\end{tabular}

For illustration purposes only.

NOTE: The length of columns 1,2 , and 3 are 45,47 , and $45(\mathrm{~cm})$, respectively. The flow rate for all three columns is $10 \mathrm{~mL} / \mathrm{h}$.

$\mathrm{BLS}=$ below land surface.

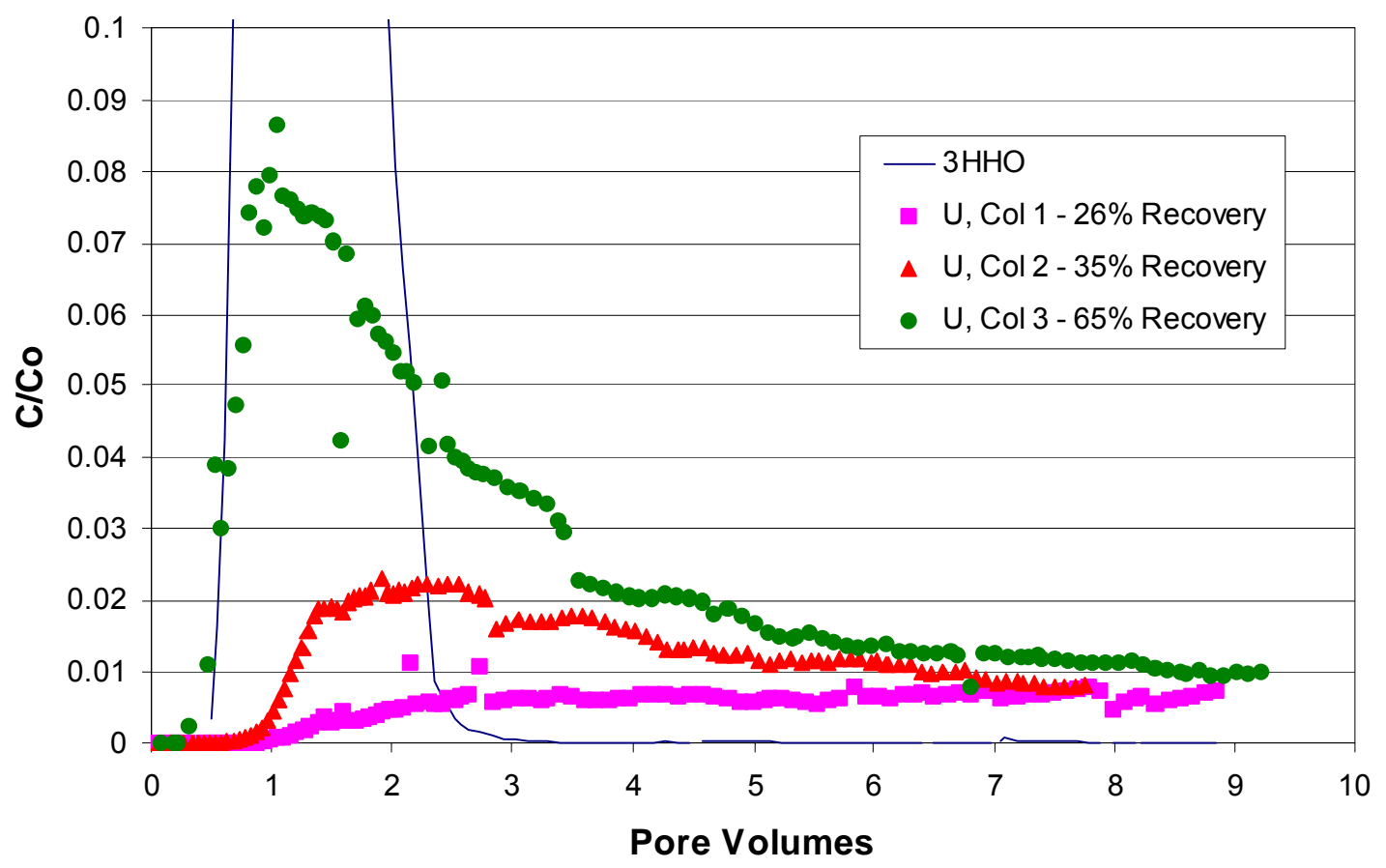

For illustration purposes only.

NOTE: The total recovery of ${ }^{3} \mathrm{HHO}$ was greater than 90 percent in all experiments. The flow rate was $10 \mathrm{~mL} / \mathrm{h}$.

Figure G-14. ${ }^{233} \mathrm{U}$ Breakthrough Curves in the Three Alluvium Column Experiments 


\section{G5. ANALYSIS OF EARLY COLUMN BREAKTHROUGH BEHAVIOR OF ${ }^{237}$ NP AND \\ ${ }^{233}$ U TRANSPORT AND FLOW-RATE DEPENDENCE OF ${ }^{237}$ NP COLUMN TRANSPORT}

The Site-scale SZ transport model described in the body of this report uses the "local equilibrium approach” (Section 6, Table 6.6-1), treating the dissolved and sorbed solutes as being locally in equilibrium. The partitioning is taken to vary linearly with solution concentration and is described by a partition coefficient (the ratio of sorbed solute mass per unit mass of solid to solute concentration in solution at equilibrium), or $K_{d}$ value, which can vary spatially in the flow system depending on the local rock type and groundwater chemistry. The local equilibrium approach results in a linear retardation factor (the ratio of radionuclide travel time to groundwater travel time) that is directly proportional to the $K_{d}$ value. As discussed in Appendix A, the uncertainty and variability in $K_{d}$ values due to uncertainty and variability in rock mineralogy, water chemistry, and other factors is accounted for in the site-scale SZ transport model through the use of $K_{d}$ probability distributions that are based on experimental batch sorption data and equilibrium geochemical modeling.

The combination of the delay in first arrival time relative to a nonsorbing tracer and the long tails and incomplete recoveries of the responses shown in Figure G-14 are inconsistent with linear first-order adsorption and desorption kinetics. The dependencies of both the first and peak arrival times on flow rate or column residence time are also inconsistent with linear first-order kinetics. Figure G-15 shows a representative range of responses that are described by linear first-order adsorption and desorption kinetics. If the adsorption rate is slow enough relative to the flow rate through the column, some of the radionuclide mass will pass through the column with essentially no retardation because the residence time is insufficient for all the mass to sorb. The mass that does sorb gives rise to a long tail in the breakthrough curve. As the reaction rate or the residence time in the column increases, more of the mass sorbs, and eventually the breakthrough curve transitions to the classical retarded response associated with linear equilibrium sorption behavior. Slow kinetics broadens the peak of the response curve relative to fast kinetics, but after some critical residence time in the column is reached, the peak always occurs at the time corresponding to the peak associated with local equilibrium conditions. 


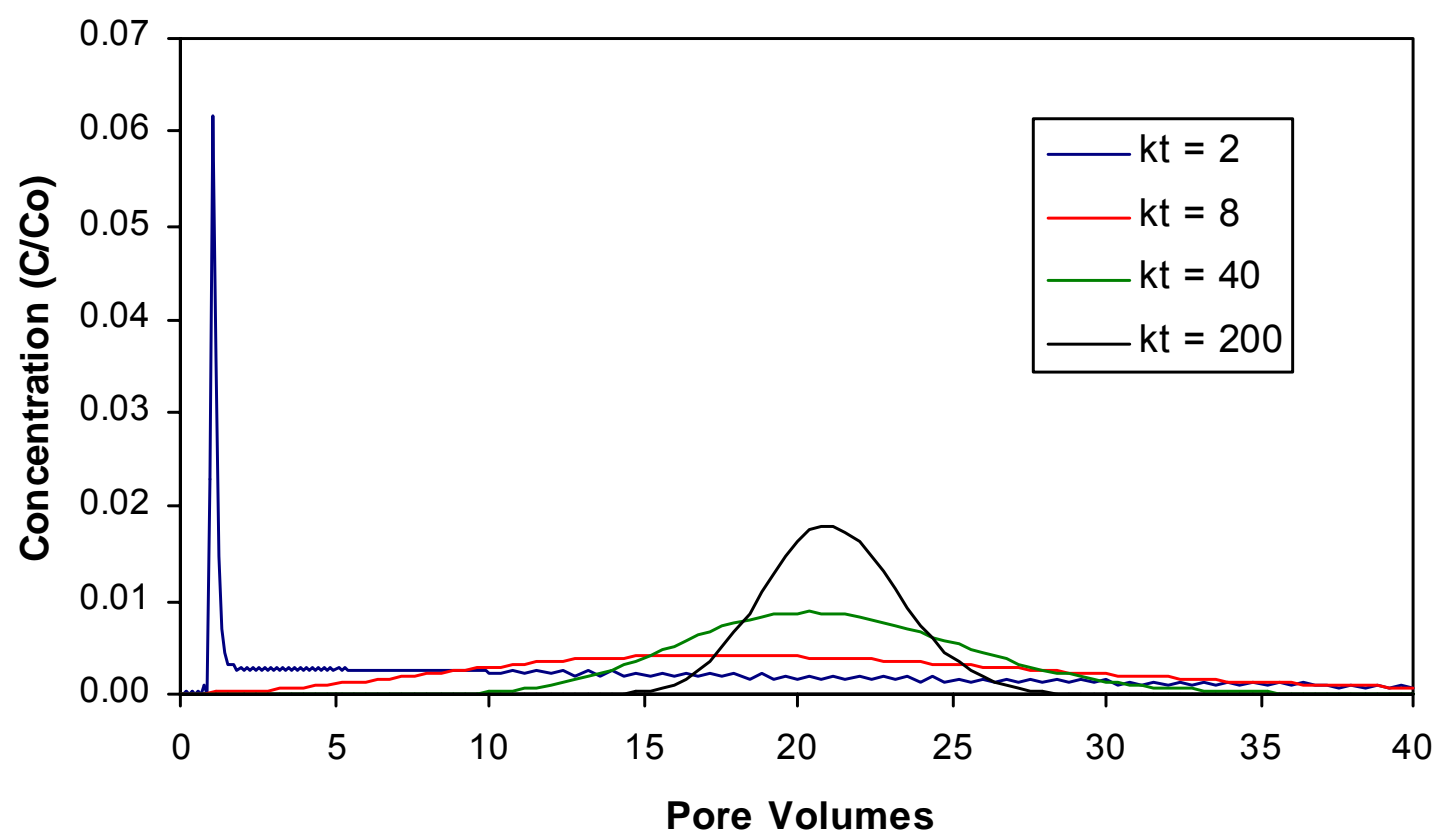

For illustration purposes only.

NOTE: Breakthrough curves generated using RELAP (RELAP V2.0, STN: 10551-2.0-00) (LANL 2002 [DIRS 159065]). The retardation factor for all four cases is 21.

Figure G-15. Predicted Breakthrough Curves as a Function of the Product of Adsorption Rate Constant ( $k$ ) and Nonsorbed Residence Time in the Column ( $t$ )

The initial portions of the responses in Figure G-15 look like classical delays due to equilibrium sorption behavior, but the low mass fractions associated with this early-arriving mass and the long tails are not consistent with equilibrium sorption. Some researchers have suggested using a hybrid equilibrium and slow kinetics approach that implicitly assumes two types of sorption sites: a weaker equilibrium sorption site that affects all the tracer mass (imposing a fixed delay on all the mass) and a stronger, but kinetically controlled sorption site that can significantly delay a substantial fraction of the tracer mass and result in a long tail. This type of analysis could explain reasonably well any of the individual responses shown in Figure G-13, but it cannot explain the different responses as a function of residence time in the columns because a different equilibrium $K_{d}$ value and different sorption and desorption rate constants are needed to explain each response. Multiple first-order linear adsorption and desorption reactions (with each successive reaction having a rate that approximately results in equilibrium sorption behavior at each successive column residence time) would likely improve the ability to match the responses of Figure G-13, but it would probably still be necessary to adjust the rate constants somewhat at each residence time. This adjustment is inherently unsatisfactory because one would expect all of the responses of Figure G-13 to be attributable to a single set of sorption characteristics given that the column material and water chemistry were the same in all the experiments. 


\section{G5.1 ADSORPTION RESIDENCE TIME DISTRIBUTION APPROACH}

One approach to explaining the responses of Figure G-13 is to treat the sorption process as being the result of a distribution of residence times that solute molecules spend adsorbed to the immobile surfaces in the column. This approach is essentially equivalent to assuming that multiple adsorption sites of varying sorption strengths exist on the adsorbent. Even for pure crystalline minerals, one might expect a relatively broad distribution of adsorption residence times caused by edge, corner, or "hole" defect sites, or by element substitutions in crystal lattices. Different adsorption residence times might also be expected for "internal" versus "external" surface area in porous granular materials that are otherwise quite homogeneous. Thus, it seems quite reasonable to expect that the distribution of adsorption residence times (i.e., adsorption site strength) for a complex heterogeneous mixture of materials such as Yucca Mountain alluvium will be very broad.

Assuming a random spatial distribution of different adsorption sites, the sorption process can be simulated by randomly sampling the adsorption residence time distribution either each time a solute molecule collides with an immobile surface or, more practically, for every specified increment of time the solute spends in the solution phase in the column. Adsorption kinetics can be defined by specifying the number of times the distribution is sampled per unit time spent in solution and/or by specifying a distribution with a finite probability of near-zero residence times. For a given distribution, the adsorption kinetics will increase as the distribution is sampled more frequently per unit time spent in the solution phase. Similarly, for a given number of random samples of a distribution, the kinetics will increase as the probability of a near-zero residence time decreases for each sampling event.

These principles can be illustrated by considering a simple binary adsorption residence time distribution, such as that shown in Figure G-16. For this distribution, there is a 50 percent probability that there will be no adsorption (zero adsorption time) and a 50 percent probability of an adsorption time of 1 time unit. Clearly, if this distribution is sampled twice per unit time increment spent in the solution phase in the column (i.e., nonsorbed time increment), the adsorption kinetics will be twice as fast as when the distribution is sampled only once per nonsorbed time increment. That is, only 25 percent of the solute mass will pass through the column without sorption per unit of nonsorbed time in the first case $(0.5 \times 0.5)$, while 50 percent of the mass will pass through without sorbing in the same nonsorbed time increment in the second case. Furthermore, 25 percent of the solute mass in the first case will have an adsorption time of 2 time units and 50 percent will have an adsorption time of 1 time unit, while in the second case there will be no mass with an adsorption time of 2 time units (only 50 percent with an adsorption time of 1 unit). The same results can be obtained by defining two different distributions that are each sampled only once per nonsorbed time increment in the column. For instance, the distribution of Figure G-17 yields the same results for a single random sample as two random samples of the distribution of Figure G-16. Thus, adsorption kinetics can be defined by both the number of random samples per unit nonsorbed time and/or by the shape of the distribution. Furthermore, it follows that for any given number of random samples of an adsorption time distribution, another distribution can be defined that yields the same kinetics after sampling the distribution either a multiple or a divisible factor of the number of times that the first distribution is sampled. 


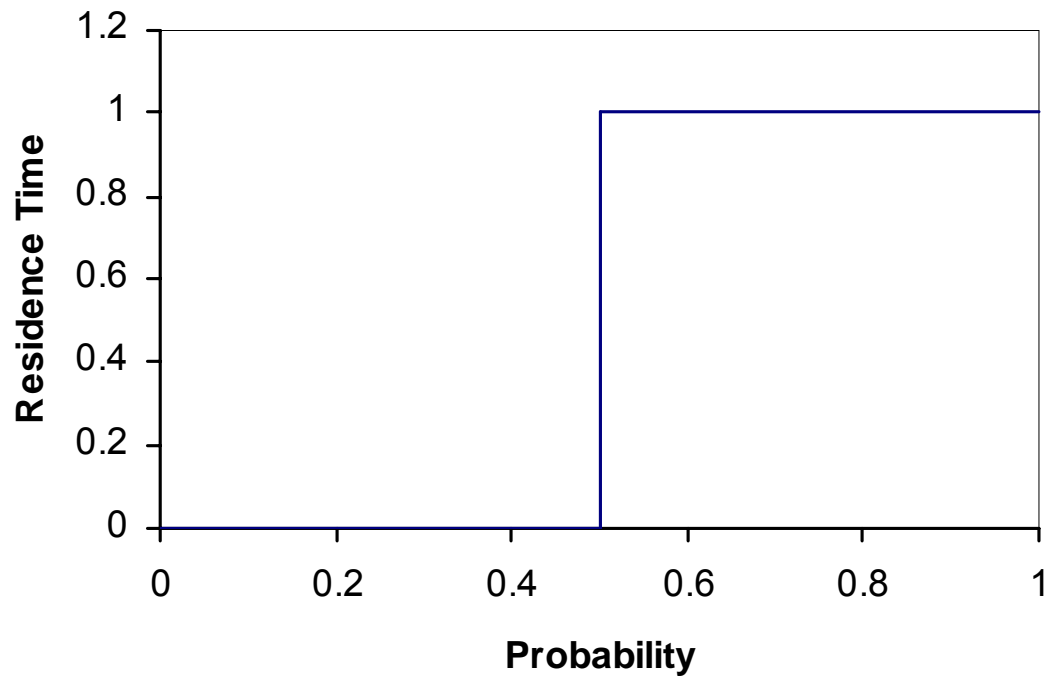

For illustration purposes only.

Figure G-16. A Schematic of Adsorption Residence Time Distribution in which there is a Probability of 0.5 of Having No Sorption, and a Probability of 0.5 of Having an Adsorption Residence Time of 1 (arbitrary time units)

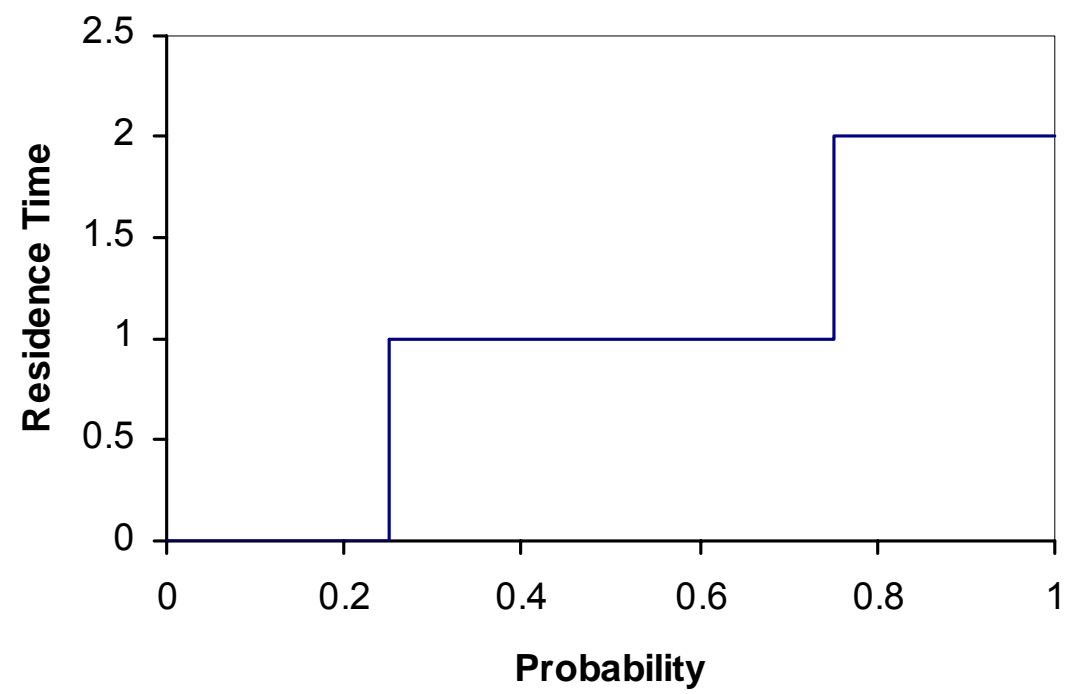

For illustration purposes only.

Figure G-17. A Schematic of Adsorption Residence Time Distribution in which there is a Probability of 0.25 of Having No Sorption, and a Probability of 0.5 of Having an Adsorption Residence Time of 1 , and a Probability of 0.25 of Having an Adsorption Residence Time of 2 (arbitrary time units) 
Another key point is that, for sufficiently long nonsorbed residence times in the column, the effective retardation factor will always be equal to one plus the ratio of the area under the adsorption distribution (in time units) to the nonsorbed residence time increment associated with each random sample of the distribution. For the distribution of Figure G-16, the retardation factor will be two if each random sample of the distribution is associated with one unit of nonsorbed residence time. On the other hand, if the distribution is sampled twice for each unit of nonsorbed residence time, the retardation factor will be three because the distribution will be sampled once for each half unit of nonsorbed residence time (i.e., $1+1 / 0.5$ ). A retardation factor of three will also be obtained if the distribution of Figure G-17 is sampled once for each unit of nonsorbed residence time because the area under the distribution is two time units (i.e., $1+2 / 1$ ). The retardation factor increases linearly with the number of times a distribution is sampled per unit nonsorbed residence time and with the area under the distribution.

Batch sorption experiments, in principle, can also be simulated using this same adsorption residence time distribution approach. Given that the approach is based on associating a randomly sampled adsorption residence time with some number of collision events between solute molecules and adsorbent surfaces, the number of times a distribution is randomly sampled per unit of nonsorbed residence time should scale approximately with the collision frequency. The collision frequency, in turn, should scale with the adsorbent surface area to solution volume ratio in an experiment (assuming that all surface area is equally accessible and the temperature is approximately the same in all experiments). Thus, if a batch sorption experiment is conducted using a surface area to volume ratio of $1 \mathrm{~cm}^{-1}$, and a column experiment is conducted using the same material, but with a surface area to volume ratio of $10 \mathrm{~cm}^{-1}$, the adsorption time distribution corresponding to the material should be sampled 10 times more often per unit of nonsorbed residence time in the column experiment than in the batch experiment.

\section{G5.2 ANALYSIS USING AN EXCEL SPREADSHEET}

Three Excel spreadsheets were set up to simulate batch adsorption, batch desorption, and column transport experiments, respectively, using the adsorption residence time distribution approach. Briefly, these spreadsheets work as follows.

Batch Adsorption Spreadsheet-1,000 “particles” are assumed to represent all the solute mass in a batch sorption experiment. Each particle starts out in the solution phase. The time that each particle spends in the solution phase is given by $t=-t_{a d s} * \ln (P)$, where $t_{a d s}$ is the characteristic mean time spent in the solution phase between adsorption events and $\mathrm{P}$ is a random number generated from a uniform distribution having a minimum of 0 and a maximum of 1 . These randomly sampled times approximate a first-order collision frequency with a collision rate constant of $1 / \mathrm{t}_{\mathrm{ads}}$.

After each particle is "sorbed," another uniformly distributed random number between 0 and 1 is generated and substituted for $\mathrm{P}$ into the following equation to determine the particle adsorption time:

$$
\mathrm{t}=\mathrm{A}\left(10^{\mathrm{BP}^{\mathrm{C}}}+10^{\mathrm{DP}^{\mathrm{E}}}-1\right)
$$

where, A, B, C, D, and E are user-adjustable parameters. This general form of the residence time distribution is used because it can be readily manipulated (by changing the values of $A, B, C, D$, 
and E) to simulate a wide range of possible adsorption/desorption behaviors, including behavior that is consistent with the column transport results of Figure G-13, as will be shown later.

Each particle alternates between the solution phase and the solid phase, with the solution and adsorption residence time distributions being alternately sampled. The fraction of particles sorbed at any given time is taken to be the fraction of solute mass sorbed at that time. The spreadsheet can be used to manually generate an adsorption kinetics curve by plotting the fraction of solute particles that are sorbed as a function of time.

Note that the adsorption process can be specified to have a maximum number of particles that are "strongly" sorbed in any given experiment. This limitation is accomplished by specifying a maximum number of particles that can have an adsorption residence time associated with a probability greater than a specified number. For instance, it can be specified that only 100 particles can be adsorbed at any given time with an adsorption residence time given by $\mathrm{P}>0.95$. When additional particles try to sorb after 100 particles already occupy these "strong" sorption sites, they sorb according to a modified distribution where the parameter $(E$ in Equation G-1) is decreased by a specified amount.

Desorption Spreadsheet-The desorption spreadsheet requires the following information from the adsorption spreadsheet:

- The number of particles that are sorbed at the end of the adsorption experiment,

- The value of P (in Equation G-1) associated with each sorbed particle at the end of the sorption experiment, and

- The parameters A through E of Equation G-1.

This information is used to generate a desorption kinetics curve that gives the fraction of particles originally sorbed that become desorbed as a function of time after stopping the adsorption experiment. It is assumed that there is no "memory" of adsorption residence times between the adsorption and desorption phases; that is, if a particle has already spent $\mathrm{x}$ hours adsorbed during the adsorption experiment, the adsorption residence time in the desorption experiment is not decreased by $\mathrm{x}$ hours when the time associated with the $\mathrm{P}$ value is obtained from Equation G-1. It is also assumed that there is no re-adsorption of desorbed particles - the spreadsheet simply calculates the amount of time each particle spends sorbed to surfaces before desorbing.

Column Transport Spreadsheet-Column transport is simulated as follows:

- 2,500 particles are used to represent the solute mass that transports through a column.

- The residence time for each particle in the solution phase is determined by randomly sampling a normal distribution that has mean given by the mean fluid residence time in the column and a standard deviation given by $\sqrt{2 \tau^{2} / \mathrm{Pe}}$, where $\tau=$ mean fluid residence time in column, and $\mathrm{Pe}=$ Peclet number $=\mathrm{L} / \alpha$, where $\mathrm{L}=$ column length and $\alpha=$ longitudinal dispersivity. 
- The user specifies the number of times the adsorption residence time distribution should be sampled during each particle's movement through the column assuming that the particle spends the mean amount of time in the solution phase. The spreadsheet adjusts the number of times the distribution is sampled (rounded to the nearest integer) based on the ratio of the actual particle residence time in the solution phase to the mean time. When applying the sorption behavior observed in a batch experiment to a column experiment, the "collision frequency" (the number of times the adsorption residence time distribution is sampled per unit time in the solution phase) in the batch experiment should be adjusted for the column experiment based on the ratio of the mass to volume ratios in the two experiments. The spreadsheet contains cells for calculating this conversion.

- Once the number of times to sample the adsorption residence time distribution is determined for each particle, the distribution is sampled that number of times (same process as in the batch adsorption spreadsheet), and the adsorption residence times are added to the solution phase residence time to determine the overall particle residence time in the column.

- The "breakthrough curve" for the solute in the column experiment is determined from the distribution of 2,500 particle residence times in the column.

The use of 1,000 particles for the sorption experiments and 2,500 particles for the column experiments in the Excel spreadsheets is less than optimal, particularly in the case of the column transport simulations, but the spreadsheet implementation of the above algorithms makes it impractical to exceed these numbers by much. Also, the column transport spreadsheet cannot accommodate more than 250 samplings of the adsorption residence time distribution for any given particle. These limitations could be eliminated by implementing the above algorithms in a computer software code (e.g., Fortran or C), but the spreadsheets are adequate for demonstrating and testing the approach and for qualitatively explaining the column transport results of Figure G-13.

\section{G5.3 ANALYSIS OF BATCH EXPERIMENTS}

Before addressing the column results, it is worthwhile to demonstrate the ability of the generalized adsorption residence time distribution approach to mimic first-order adsorption and desorption kinetics. Figure G-18 shows the adsorption residence time distribution that results from first-order desorption kinetics (an "inverse" exponential distribution) with a mean adsorption residence time of 5 hours (i.e., a desorption rate constant of $0.2 \mathrm{hr}-1$ ). Mathematically, this distribution is given by $t=-\ln (1-\mathrm{P}) / \mathrm{k}_{\mathrm{des}}$, where $\mathrm{t}=$ adsorption time, $\mathrm{P}=$ probability ( 0 to 1 , the dependent variable on the $\mathrm{x}$-axis), and $\mathrm{k}_{\mathrm{des}}$ is the desorption rate constant $\left(\mathrm{hr}^{-1}\right)$. Figure G-18 also approximates this residence time distribution using Equation G-1 with $\mathrm{A}=0.001, \mathrm{~B}=4.2, \mathrm{C}=0.265, \mathrm{D}=4.5$, and $\mathrm{E}=10$ (Distribution 1). 


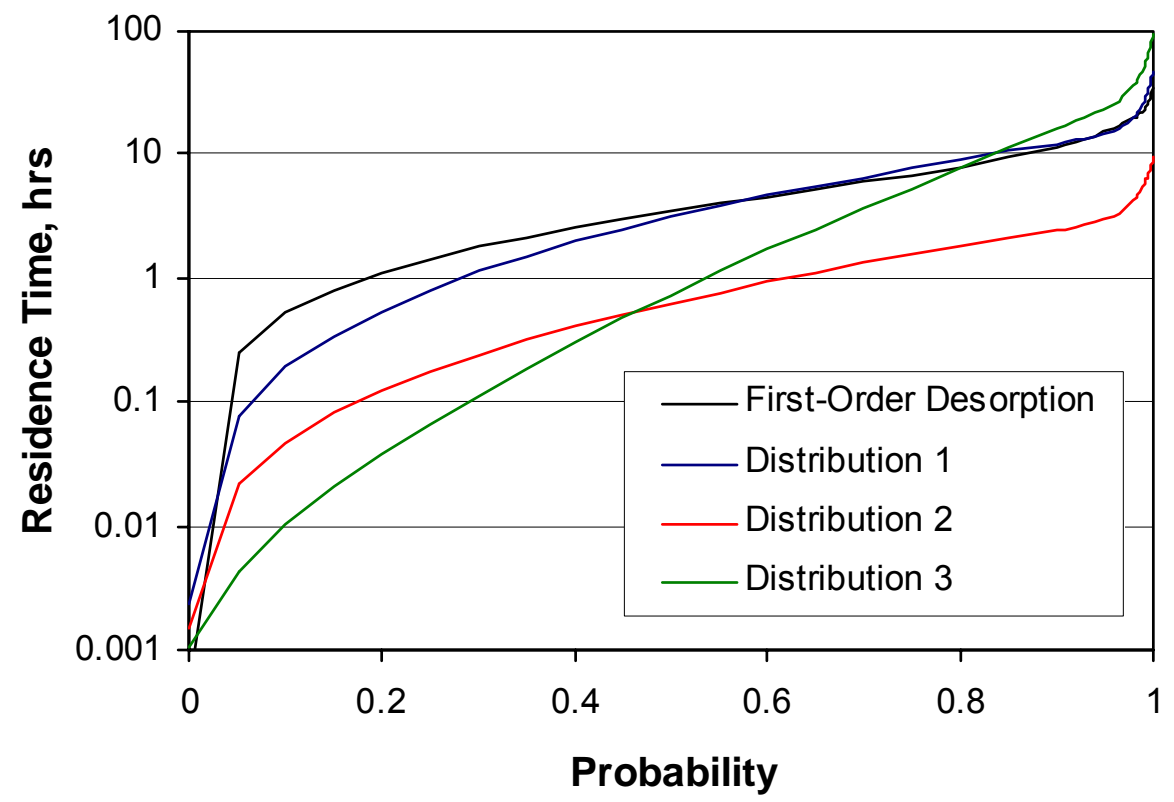

For illustration purposes only.

Figure G-18. Adsorption Residence Time Distribution Corresponding to First-order Desorption with a Rate Constant of $0.2 \mathrm{hr}^{-1}$, and Three Distributions Defined by Equation G-1 that are Discussed in the Text. Distribution 1 is an Approximation of the First-Order Desorption Distribution, Distribution 2 is an Approximation to a First-Order Desorption Distribution with a Rate Constant of $1 \mathrm{hr}^{-1}$, and Distribution 3 Has the Same Area Under the Curve as Distribution 1, but with an Effective Adsorption Rate about 4 Times Lower

Figure G-19 shows the predicted column transport behavior as a function of nonsorbed residence time in the column when Distribution 1 of Figure G-18 is sampled 4 times per hour of nonsorbed time (corresponding to an adsorption rate constant of $4 \mathrm{hr}^{-1}$ ). Breakthrough curves are shown for the same products of rate constant and nonsorbed residence time as in Figure G-15. It is apparent that the spreadsheet breakthrough curves and the curves of Figure G-15 are in reasonably good agreement. The primary difference between the two sets of curves is that the unretarded solute mass associated with the $\mathrm{kt}=2$ curve (the early spike in concentration) is much lower in Figure G-19 than in Figure G-15. Also, the spreadsheet breakthrough curves are much more jagged because they are generated from a relatively small number of particle residence times. 


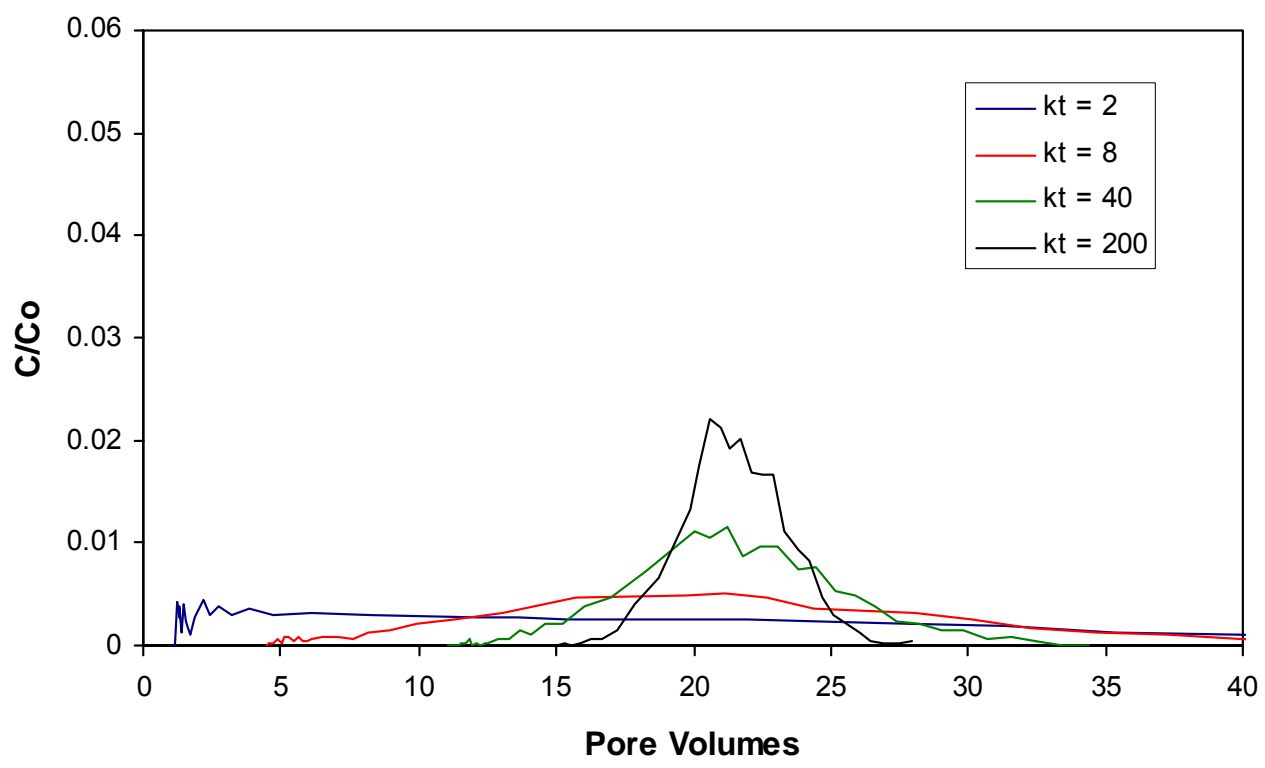

For illustration purposes only

Figure G-19. Breakthrough Curves Computed by Spreadsheet Analysis When Residence Time Distribution 1 From Figure G-18 is Sampled Four Times per Hour of Nonsorbed Residence Time in the Column. The Products of the Adsorption Rate Constant and the Nonsorbed Residence Time Associated with Each Breakthrough Curve are Listed in the Legend. These Products are the Same as Those Associated With the Breakthrough Curves of Figure G-15

Another test of the spreadsheet's ability to simulate first-order adsorption and desorption kinetics is to determine whether it predicts the same breakthrough curves when the nonsorbed residence time is changed but the product of the rate constant and nonsorbed residence time are kept the same (and the ratio of the adsorption and desorption rate constants are also kept the same). A higher adsorption rate was achieved by sampling the adsorption residence time distribution 5 times more frequently than in the slower case (20 times per hour). A higher desorption rate was achieved by changing the values of B, C, and D in Equation G-1 to 3.5, 0.32, and 3.8 (respectively), which lowered the distribution 1 curve in Figure G-18 such that the mean adsorption time was 1 hour instead of 5 hours but preserved the shape of the curve (i.e., distribution 2 in Figure G-18). Thus, both the adsorption and desorption rates were effectively increased by a factor of 5, resulting in no net change in their ratio. Figure G-20 shows that the breakthrough curves for these two cases are in very good agreement when they are plotted as a function of pore volumes. 


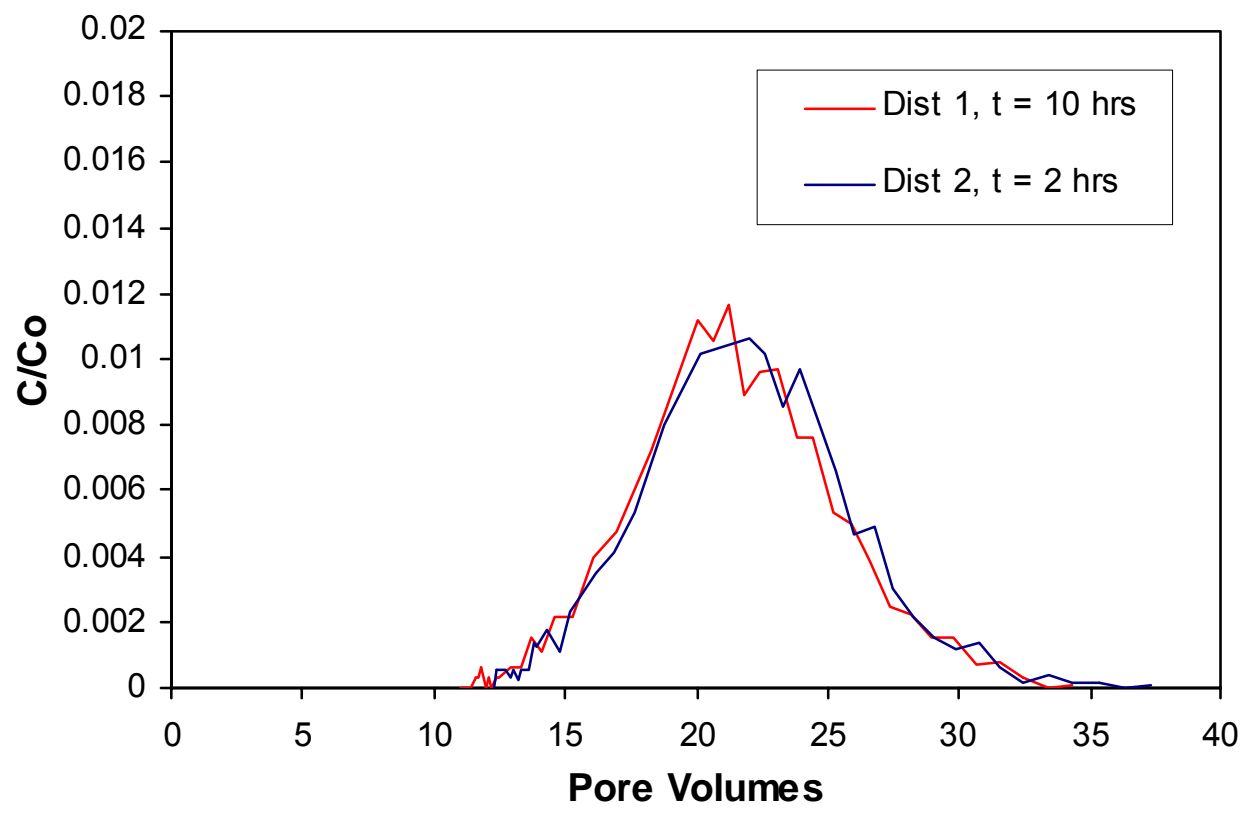

For illustration purposes only.

Figure G-20. Breakthrough Curves Obtained by Sampling Distribution 2 Five Times More Frequently Than Distribution 1 But With the Nonsorbed Residence Time in the Column Being Five Times Longer for Distribution 1

A final test of the column transport spreadsheet is to see if the same breakthrough curve can be obtained by altering the adsorption residence time distribution to have a lower probability of greater residence times while maintaining the same overall area under the curve and decreasing the column flow rate. Distribution 3 in Figure G-18 has the same area under the curve as distribution 1, but the probability associated with larger residence times is smaller than for distribution 1, and the probability associated with shorter residence times is greater than for distribution 1. This greater probability of shorter residence times is offset by having larger residence times at the high end of distribution 3 (to make the areas under the curves equal). From the above discussion, we know that the net effect of a shift from distribution 1 to distribution 3 while maintaining the same distribution sampling frequency should be to decrease both the effective adsorption and desorption rates. Figure G-21 shows that, in fact, very similar column breakthrough curves are obtained using distributions 1 and 3 with the same sampling frequency when the flow rate is about a factor of 4 greater for distribution 1 (indicating that the effective adsorption and desorption kinetics are about 4 times faster for distribution 1). The peaks occur at the same number of pore volumes because the areas under the two distributions are the same, and hence their effective retardation factors are the same. 


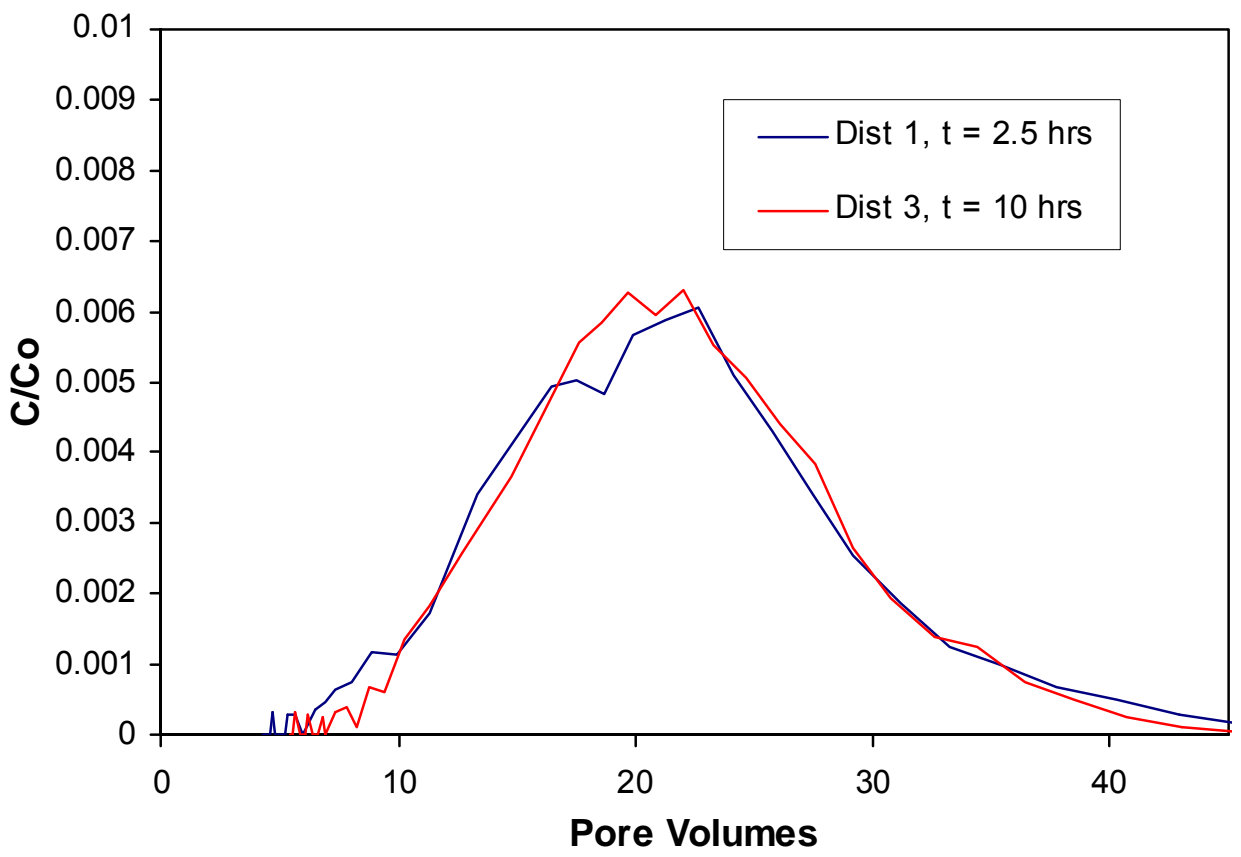

For illustration purposes only.

Figure G-21. Breakthrough Curves Obtained by Sampling Distribution 1 and Distribution 3 at the Same Frequency (four times per hour) With the Nonsorbed Residence Time in the Column Being Four Times Longer for Distribution 3

\section{G5.4 QUALITATIVE SIMULATION OF EARLY COLUMN BREAKTHROUGH BEHAVIOR}

An adsorption residence time distribution such as that shown in Figure G-22 can now be considered. This distribution was selected to yield adsorption and desorption rates that, when the distribution is sampled once every two hours of nonsorbed residence time, are qualitatively similar to those observed for uranium and neptunium in batch experiments. The shape of the distribution is motivated primarily by the uranium desorption data of Figure G-11, which do not exhibit first-order kinetic desorption behavior, but rather indicate that a fraction of the sorbed uranium desorbs very rapidly while the remainder of the uranium desorbs much more slowly. Figure G-23 shows the adsorption rate curve associated with the distribution of Figure G-22, and Figure G-24 shows two desorption rate curves generated after two different time periods of adsorption. Clearly, desorption is predicted to be faster after a shorter adsorption time period, which can be attributed to the fact that there is less opportunity for solute molecules to come in contact with "strong" sorption sites during shorter adsorption times. This aspect of predicted desorption behavior has not been experimentally verified, but the analysis suggests that measuring desorption rates after different periods of adsorption might be a good strategy. Figures G-23 and G-24 are qualitatively similar to Figures G-9 and G-11. 


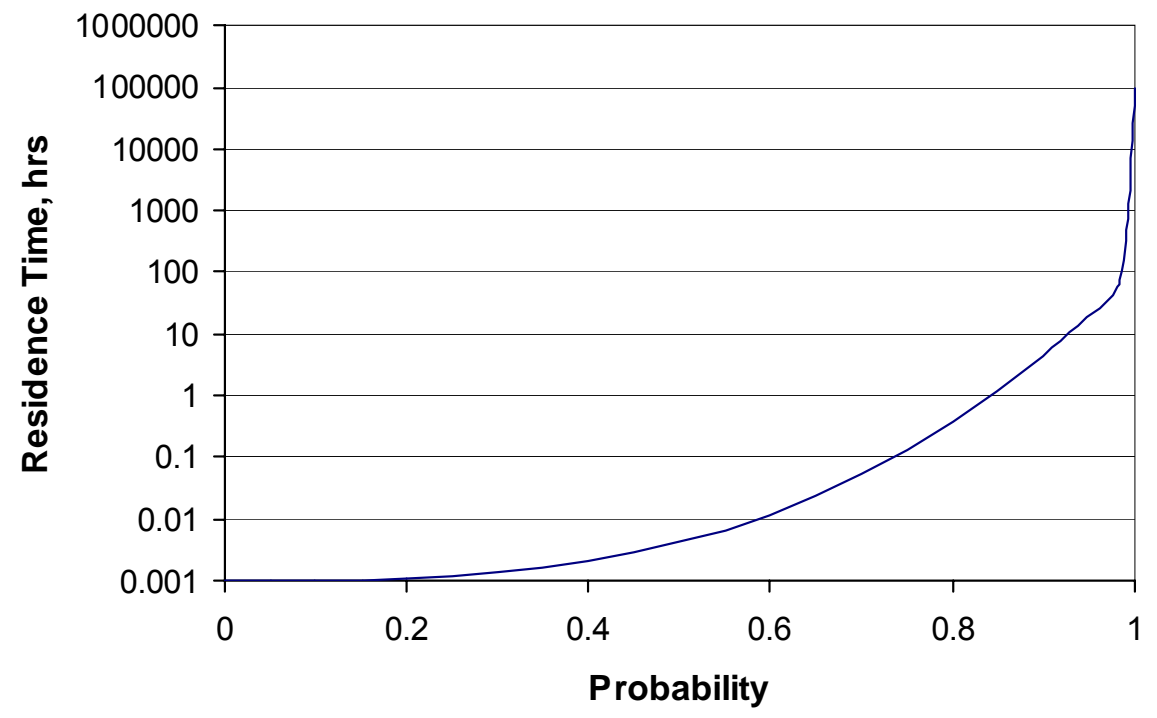

For illustration purposes only.

Figure G-22. Adsorption Residence Time Distribution that has a Low Probability of Very Long Residence Times (i.e., a Small Fraction of Very Strong Adsorption Sites)

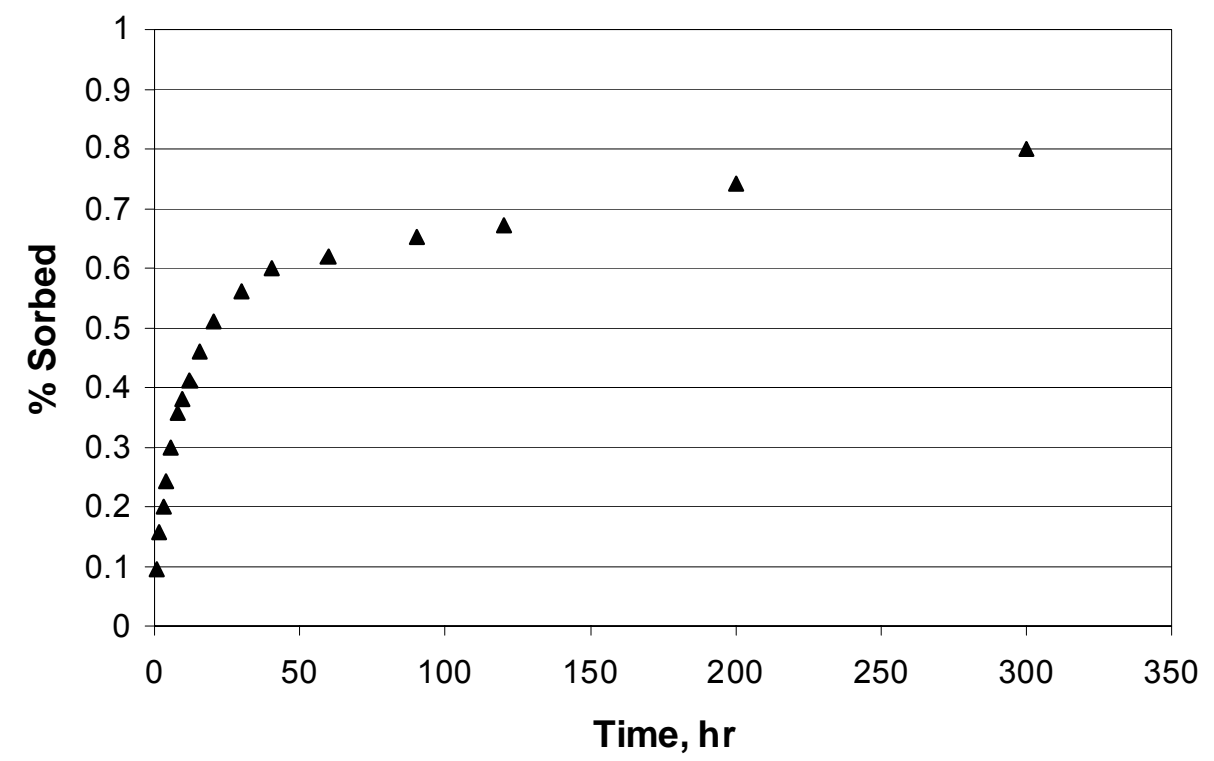

For illustration purposes only.

Figure G-23. Percent of Solute Sorbed as a Function of Time in a Simulated Batch Sorption Experiment Using the Adsorption Residence Time Distribution of Figure G-22 at a Sampling Frequency of Once Every 2 Hours 


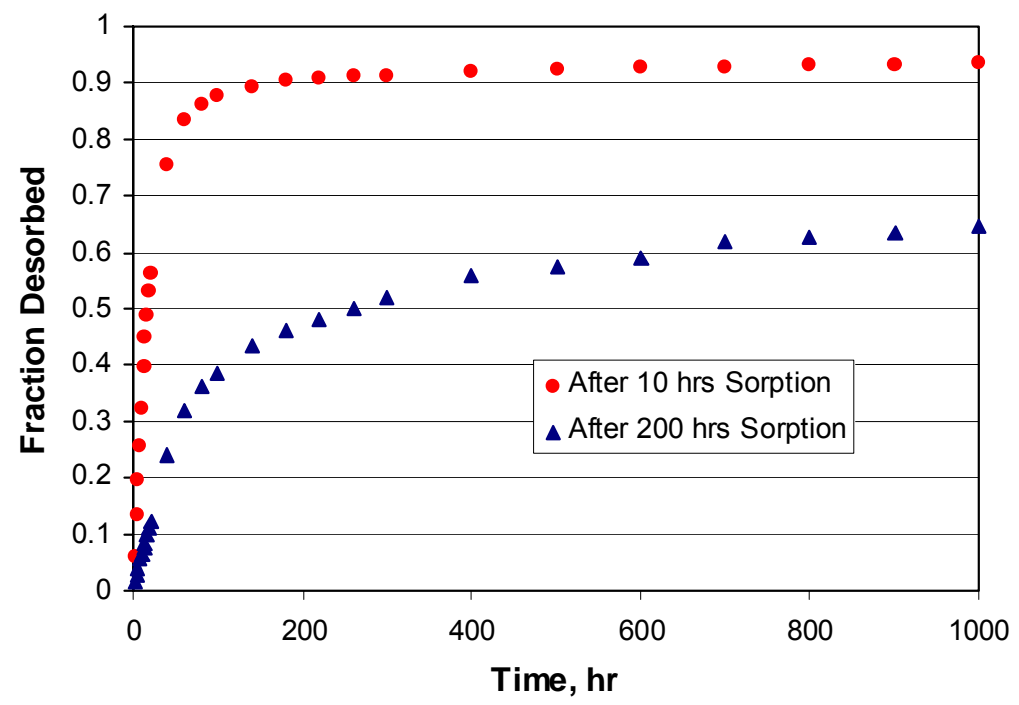

For illustration purposes only.

NOTE: Figure G-23 is the corresponding simulated adsorption experiment.

Figure G-24. Fraction of Solute Desorbed as a Function of Time in Simulated Desorption Experiments Initiated After 10 Hours and 200 Hours of Sorption, Respectively, Using the Adsorption Residence Time Distribution of Figure G-22

When the distribution of Figure G-22 is used in the simulation of column transport experiments, the breakthrough curves of Figure G-25 are obtained at the three different relative flow rates listed in the figure as a function of pore volumes eluted. For these simulations, the adsorption time distribution was sampled 10 times for every hour of nonsorbed residence time in the column. This is an appropriate frequency given the surface area to volume ratios used in the batch and column experiments described earlier in this appendix and the sampling rate of once every two hours used to generate the batch adsorption curve of Figure G-23. The predicted column transport behavior shown in Figure G-25 compares favorably with the Np transport behavior shown in Figure G-13. The increasing delay in the first arrival time of the Np and the decreasing fractional recovery as flow rates decrease can be attributed to the greater probability of encountering "strong” sorption sites (sites with long adsorption residence times) as the nonsorbed residence time in the column increases. Although the experimental breakthrough curves have somewhat sharper leading fronts at the lower flow rates than the curves estimated from the analysis, the shapes of the curves in Figures G-25 and G-13 are qualitatively quite similar. This qualitative agreement between analysis and experiment could probably be improved by employing a more rigorous fitting exercise. 


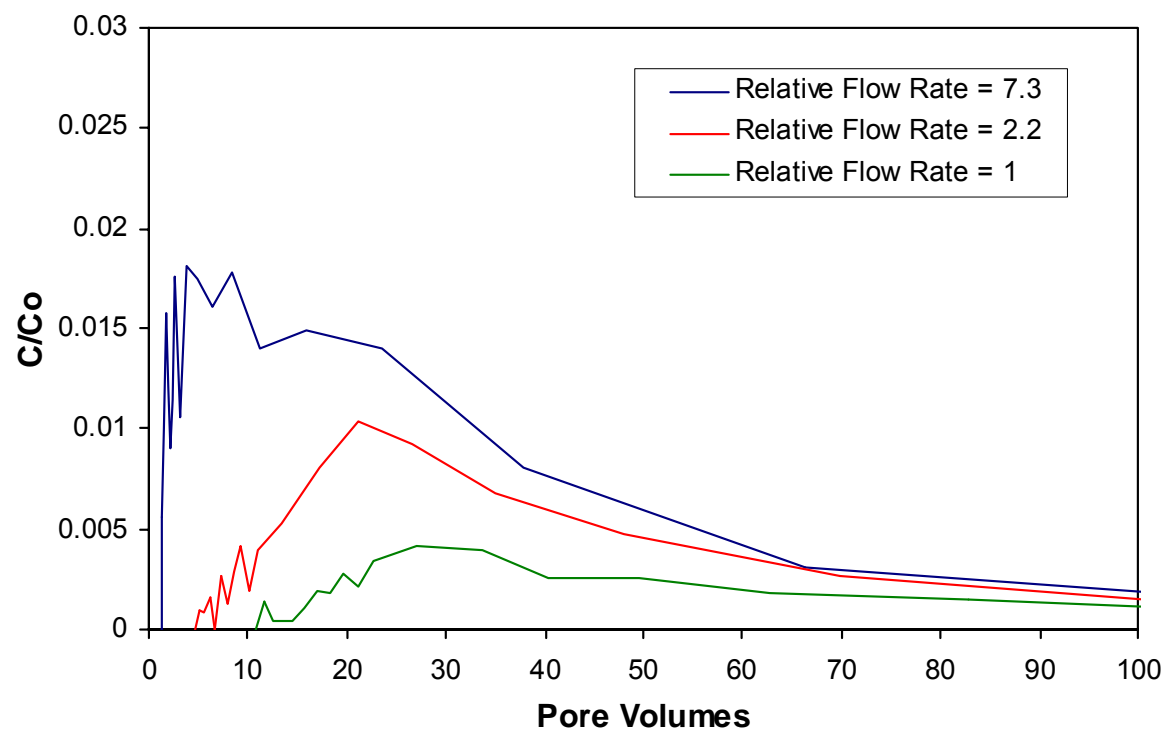

For illustration purposes only.

NOTE: Simulated fractional recoveries are $0.55,0.36$ and 0.17 for the three breakthrough curves at 100 pore volumes.

Figure G-25. Simulated Breakthrough Curves as a Function of Relative Flow Rate for a Solute That Has an Adsorption Residence Time Distribution Given by Figure G-22. These breakthrough curves are qualitatively comparable to those for Neptunium in Figure G-13

In conclusion, the generalized adsorption residence time approach is consistent with the column transport behavior of Figure G-13 (as well as Figures G-12 and G-14) as well as with the batch sorption and, particularly, the desorption experimental results that have been obtained for uranium and neptunium on Yucca Mountain alluvium. A wide range of adsorption residence times, with a small fraction of very large residence times, is necessary to obtain this agreement with experimental results, but such a distribution seems quite reasonable given the heterogeneous nature of the alluvium used in the batch and column transport experiments. The approach is very flexible in that it can be made entirely consistent with a more traditional first-order sorption kinetics, while offering the ability to describe much more complex adsorption and desorption behavior such as that observed experimentally for neptunium and uranium.

\section{G6. IMPLICATIONS FOR SATURATED ZONE RADIONUCLIDE TRANSPORT}

The adsorption residence time distribution analysis approach for radionuclide transport in the SZ near Yucca Mountain has very favorable implications for repository PA. The approach provides a reasonable explanation for why early breakthroughs sometimes occur in laboratory column experiments (i.e., the residence time is insufficient for all the radionuclide mass to come in contact with strong sorption sites). According to this approach, radionuclide solute retardation over long time and distance scales in the field should always be greater than in shorter-duration laboratory column experiments. Furthermore, this analysis suggests that the use of the local equilibrium approach in the SZ radionuclide transport model with $K_{d}$ distributions derived from laboratory batch sorption experiments should, if anything, yield conservative radionuclide transport predictions in the SZ. 


\section{APPENDIX H \\ QUALIFICATION OF SORPTION DATA \\ DTNS: LA0305AM831341.001 AND LA0310AM831341.001}




\section{H1. QUALIFICATION OF SORPTION DATA}

The qualification of DTNs: LA0305AM831341.001 [DIRS 163789] and LA0310AM831341.001 [DIRS 165865] are documented here in accordance with AP-SIII.2Q, Qualification of Unqualified Data. This qualification provides a desired level of confidence that the data are suitable for their intended use, which is limited to the subject of this work product (SZ radionuclide transport) for use in TSPA. The qualification is based on corroboration of data and technical assessment.

\section{H1.1 CORROBORATING DATA}

One important aspect of the data qualification process is corroboration of unqualified data with qualified data. If it can be shown that unqualified sorption coefficient experiments on a given water/rock combination and under a specified set of experimental parameters produced sorption coefficient data that are equivalent to data obtained under a qualified QA program using a similar set of rock/water combinations and experimental parameters, the concordance of the two data sets can be used to support the qualification of the unqualified data set.

Sorption coefficients are subject to a range of factors including some associated with the solid phase, some associated with the liquid phase (groundwater or pore water), and some associated with the element-of-interest. The two main factors associated with the solid phase include mineralogy and surface area. Mineralogy includes mineral type and mineral composition, particularly for minerals that sorb primarily by ion exchange. The factors in the liquid phase that can influence sorption behavior are different for different elements-of-interest. For example, for alkali and alkaline earth elements of interest (i.e., Ba, Cs, Sr, Ra), the concentrations of alkali and alkali earth elements in solution (e.g., $\mathrm{Ca}, \mathrm{K}, \mathrm{Na}, \mathrm{Mg}$ ) are important factors. For an element such as uranium, $\mathrm{pH}$ and the alkalinity are important factors. Factors associated with the element-of-interest include concentration in solution and complexation behavior.

In comparing an unqualified dataset with a qualified dataset for a given element-of-interest, it is important that the factors that can control sorption behavior are at the same or similar values in both datasets unless it can be shown that a given factor does not control sorption behavior for the element-of-interest.

Two examples are cited of comparisons of qualified and unqualified datasets for elements-ofinterest. The first example is for barium sorption coefficients on zeolitic tuff in J-13 water. Qualified and unqualified data points for sorption coefficients and final solution concentrations are shown in Figure H-1. The same water composition was used in both the unqualified and the qualified experiments. Thus, differences in water composition are not an issue in comparisons of the two datasets. The differences in final solution concentrations did not influence the sorption coefficient ranges for the two datasets because the isotherms for barium sorption on zeolitic tuff in J-13 water are essentially linear as shown in Figure A-36. Differences in experimental duration did not influence the sorption coefficient ranges because the barium sorption reactions are fast, as discussed in Section A7.6.2. The fact that the ranges in sorption coefficient values are very similar for the two datasets provides confidence that the experimental procedures used to obtain the unqualified data were appropriate and equivalent to those used to obtain the qualified dataset. 


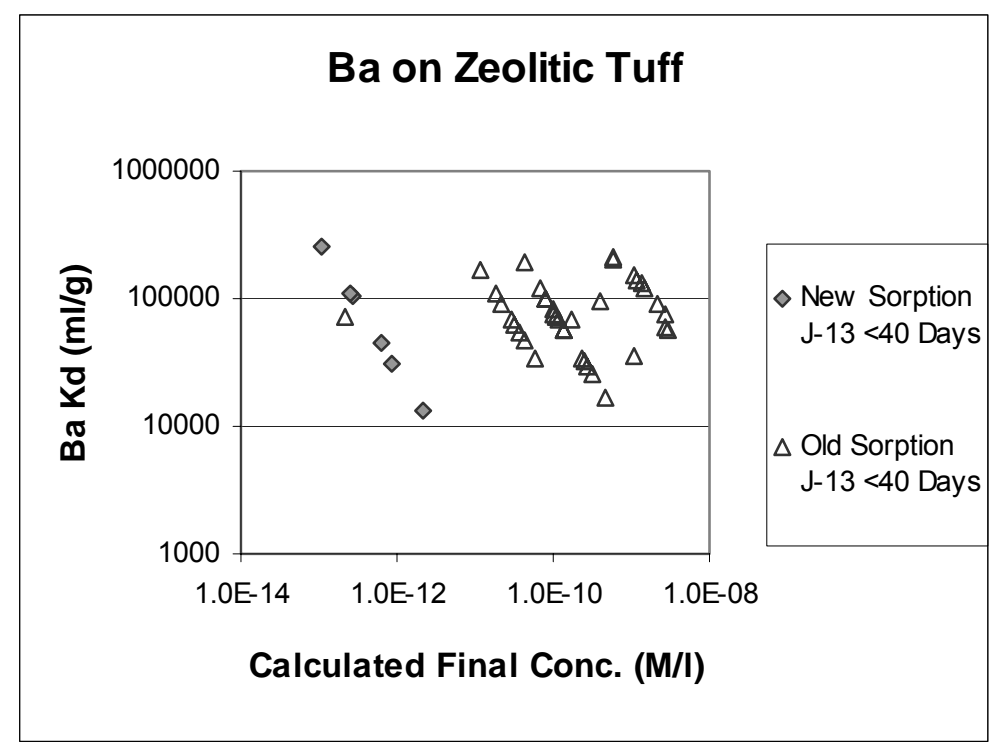

Source DTNs: LA0305AM831341.001 [DIRS 163789]; LA0407AM831341.001 [DIRS 170623].

Figure H-1. Barium Sorption Coefficient on Zeolitic Tuff in J-13 Water Versus Calculated Final Barium-133 Solution Concentration

The second example of a comparison of qualified and unqualified datasets involves uranium sorption on devitrified tuff in J-13 water. Qualified and unqualified data points for sorption coefficients and $\mathrm{pH}$ are shown in Figure $\mathrm{H}-2$. The same water composition was used in both the unqualified and the qualified experiments. Thus, differences in water composition are not an issue in comparisons of the two datasets. The differences in final solution concentrations did not influence the sorption coefficient ranges for the two datasets because the uranium sorption coefficients are not sensitive to final solution concentrations as shown in Figure A-47. Differences in experimental duration did not influence the sorption coefficient ranges because the uranium sorption reactions on devitrified tuff are fast, as discussed in Section A7.9.1. The fact that the ranges in uranium sorption coefficient values are similar for the two datasets, excluding the outlier with a $K_{d}=10 \mathrm{~mL} / \mathrm{g}$, provides confidence that the experimental procedures used to obtain the unqualified data were appropriate and equivalent to those used to obtain the qualified dataset. 


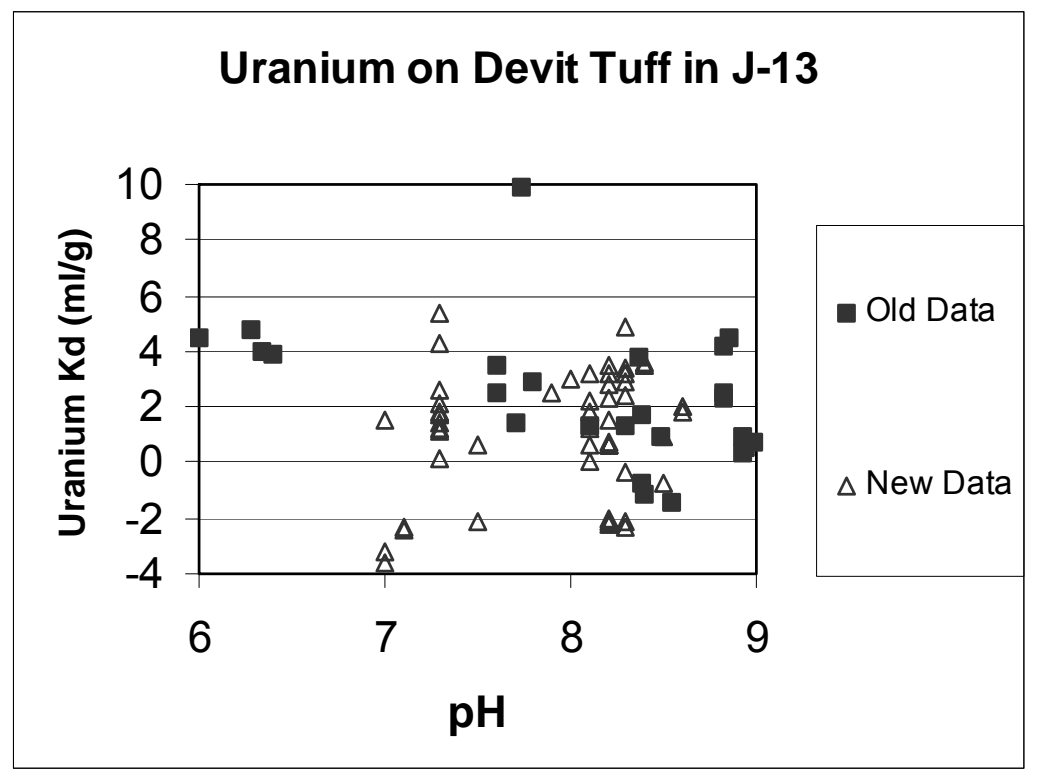

Source DTNs: LA03010AM831341.001 [DIRS 165865]; LA0407AM831341.006 [DIRS 170628].

Figure H-2. Uranium Sorption Coefficient on Devitrified Tuff in J-13 Water Versus pH

\section{H1.2 TECHNICAL ASSESSMENT}

The Los Alamos National Laboratory (LANL) studies of the sorptive behavior of tuff and transport of radionuclides through tuff were performed under the Nevada Nuclear Waste Storage Investigations (NNWSI) project that was managed by the Nevada Operations Office of the DOE. These investigations were performed under the Los Alamos QA program for the NNWSI, with the Materials Science and Technology Division quality assurance organization responsible for the planning and implementation of the Los Alamos QA program.

The Los Alamos National Laboratory Quality Assurance Plan And Procedures For The Nevada Nuclear Waste Storage Investigations (LA-9331-MS) is a collection of the quality assurance documents that provides a detailed account of the Los Alamos NNWSI QA program, including the Quality Assurance Program Plan, the Quality Assurance Program Index and Procedures, work plans, and the detailed procedures developed for the project.

The QA program developed for the NNWSI at Los Alamos was outlined in the Quality Assurance Program Plan and was structured to meet the requirements of NQA-1 and 10 CFR 50, Appendix B, as applied to the evaluation of major geologic formations with regard to their suitability as locations of permanent repositories for high-level radioactive wastes.

The Quality Assurance Program Index (TWS-QI-1 and TWS-CMBQA-QP-02) is a cross-reference between basic requirements standards NQA-1 and 10 CFR 50 showing where these requirements are addressed in the Los Alamos Quality Assurance Manual (QAM) and project implementing procedures. The QAM was the primary compliance document for this work unless otherwise stated in the Quality Assurance Program Plan or in the specific procedure documents written for NNWSI. 
Work plans were the primary planning documents covering the Los Alamos technical activities for the NNWSI. These work plans were written to provide an adequate description of the scope and purpose of the task. They include, directly or by reference, the QA requirements with regard to data validity and documentation. Review boards that consisted of, at a minimum, a management member, a QA member, and an independent technical reviewer who was experienced and competent in the field under review but had no direct program responsibility, approved the work plans as well as the acceptance of final documents. The work plan containing the sorption experiments was TWS-CNC-WP-03, Tuff Experiments-Sorption Ratios and Migration Measurements. This work plan contains the scope of the work, an overview of how the batch sorption studies were to be performed, what procedure would be used to control the experiments, how the measurement of radioactivity would be performed, type of equipment to be used, QA requirements, and documentation of work requirements. The procedure used to control the experiments was TWS-CNC-DP-05, Sorption, Desorption Ratio Determinations of Geologic Materials by a Batch Method. The process used in this procedure is the same process as given in procedure LANL-INC-DP-86, Sorption and Desorption Determinations by a Batch Sample Technique for the Dynamic Transport Task that is the procedure used for the qualified $K_{d}$ data. This latter procedure only provides more detailed guidance.

Training requirements for personnel were delineated in the QAM by procedure QMR 1, Quality Assurance Management and Planning and QMI 1-1, Quality Program Management.

Measuring and testing equipment that require calibration was controlled in accordance with the applicable sections of the QAM (QMR 9 and QMI 9-1). The only equipment used for these experiments that required calibration were the balances and gamma counters. These were controlled in accordance with procedures QMR 9 Control and Calibration of Standards and Measuring and Testing Equipment and QMI 9-1 Acquisition and Calibration Control of Standards and Measuring and Testing Equipment. Quality Assurance Audit of Los Alamos National Laboratories (LANL) Nevada Nuclear Waste Storage Investigations (NNWSI) Project, performed July 16 through 18, 1984, verified that the control of measuring and test equipment was adequate (LANL 1984 [DIRS 171444]. There were no problems found with the measuring and test equipment program. Audit of Los Alamos Scientific Laboratories Quality Assurance Program Plan for Nevada Nuclear Waste Storage Investigations conducted May 1-3, 1979, also found that the measuring and test equipment program was satisfactory (Los Alamos Scientific Laboratories 1979 [DIRS 171445].

Corrective actions for significant conditions adverse to quality were provided in accordance with QMR 12, Corrective Action, of the QAM. Compliance with the QA program was verified by periodic audits that were planned, documented, and carried out in accordance with procedure QMI 15-1, Quality Audits, of the QAM. 


\section{H1.3 SUMMARY}

The programs in place at the time these sorption experiments were performed and the fact that the audits mentioned above did not identify anything that would affect the quality of the data lend support to the conclusion that the data collection methodology and equipment used were appropriate for the type of data under consideration. Corroborating information available for barium and uranium also show the equivalence of the unqualified and qualified data, supporting the adequacy of the unqualified data set. Furthermore, the uncertainty distributions for sorption coefficients generally span a range that is one order of magnitude or more (see Table C-14). The corroborating data comparisons suggest that differences between the unqualified and qualified data are much less than the inherent uncertainty. Therefore, the unqualified data are justified for use in supporting the sorption coefficient distributions to be used in TSPA. 


\section{INTENTIONALLY LEFT BLANK}




\section{APPENDIX I}

QUALIFICATION OF COMPOSITIONS OF WATERS FROM UE-25 J-13 AND UE-25 P\#1(CARBONATE) 


\section{I1.1 PURPOSE}

This report documents the findings of the data qualification team assembled to evaluate selected unqualified hydrology data that were used in the characterization of transport processes in the saturated zone (SZ) at Yucca Mountain, Nevada. These data are qualified for inclusion in the model report Site-Scale Saturated Zone Transport that support the Total System Performance Assessment (TSPA) Model/Analysis for the License Application. This activity has been planned and implemented in accordance with AP-SIII.2Q, Qualification of Unqualified Data.

\section{I1.2 QUALIFICATION TEAM}

Chairperson Sharad Kelkar has 25 years experience in the area of flow and transport through geological media. He has been a member of the Hydrology, Geochemistry, and Geology group at LANL since 1985. In 2002, Mr. Kelkar began his ongoing role as a principal scientist with the Yucca Mountain Project, where he is responsible for documentation in support of the license application for the repository. Mr. Kelkar has contributed to the Yucca Mountain Project in the development of the code FEHM that forms a basis for the flow and transport modeling for the SZ. He has also worked on geothermal energy programs at LANL. Prior to his association with LANL, he worked on laboratory and field testing and engineering analysis in support of petroleum production (Terra Tek Inc., Salt Lake City, Utah, 1982 to 1985) and laboratory and field testing in support of gas recovery programs (Institute of Gas Technology, Chicago, Illinois, 1979 to 1982). Mr. Kelkar received his M.S. degree in Petroleum Engineering from the University of Texas at Austin (1979) for measurements of poro-elastic properties of rocks at elevated pressures and temperatures. Mr. Kelkar received his M.S. degree in Nuclear Physics from the University of Bombay (1974). In addition, Mr. Kelkar has done extensive course-work in hydrology and minerals engineering at the University of California, Berkeley.

Team member: Dr. James Houseworth's professional experience includes groundwater and surface water hydrology and petroleum reservoir engineering. He has provided, over a twelve-year period, technical and management support for performance assessment and site characterization efforts at Yucca Mountain, Nevada, which is being studied by the DOE as a disposal site for high-level radioactive waste. This includes unsaturated zone flow and transport analyses in fractured rock to support performance assessment of the potential repository system and analyses to support construction and testing activities at Yucca Mountain. In addition, he has eight years experience in characterization of petroleum reservoirs including laboratory core analysis methods to determine single and multiphase flow properties and reservoir simulation. Other experience includes numerical modeling of mixing and stratification in surface water reservoirs and the design of outfalls for the discharge of waste heat from nuclear power plants and municipal sewage effluent. Dr. Houseworth received his Ph.D. in 1984 in Environmental Engineering Science from the California Institute of Technology, his M.S. in 1978 in Environmental Engineering Science from California Institute of Technology and his B.S. in 1977 in Environmental Engineering Science from California Institute of Technology

\section{I1.3 DESCRIPTION OF THE DATA TO BE QUALIFIED}

The data that were evaluated and documented in this qualification report are published in the report Groundwater Chemistry Along Flow Paths Between a Proposed Repository Site and the 
Accessible Environment (Ogard and Kerrisk 1984 [DIRS 100783]). These data are shown in Table I-1.

Table I-1 Compositions of Waters from UE-25 J-13 and UE-25 p\#1

\begin{tabular}{|l|c|c|}
\hline \multicolumn{1}{|c|}{ Chemical Constituent } & $\begin{array}{c}\text { UE-25 } \\
\text { J-13 Water }\end{array}$ & $\begin{array}{c}\text { UE-25 p\#1 (Carbonate) } \\
\text { Water (mg/L) }\end{array}$ \\
\hline $\mathrm{Ca}^{2+}$ & 11.5 & 87.8 \\
\hline $\mathrm{Mg}^{2+}$ & 1.76 & 31.9 \\
\hline $\mathrm{Na}^{+}$ & 45 & 171 \\
\hline $\mathrm{K}^{+}$ & 5.3 & 13.4 \\
\hline $\mathrm{SiO}_{2}$ & 64.2 & 64.2 \\
\hline $\mathrm{Cl}^{-}$ & 6.4 & 37 \\
\hline $\mathrm{F}^{-}$ & 2.1 & 3.5 \\
\hline $\mathrm{SO}_{4}{ }^{2-}$ & 18.1 & 129 \\
\hline $\mathrm{HCO}_{3}{ }^{-}$ & 128 & 666 \\
\hline $\mathrm{CO}_{3}{ }^{2-}$ & 0.0 & 0 \\
\hline $\mathrm{pH}$ & 6.9 & 6.7 \\
\hline
\end{tabular}

Ogard and Kerrisk (1984 [DIRS 100783]) Data

\section{I2. QUALIFICATION METHOD AND EVALUATION CRITERIA}

The method used to qualify the data in this report is corroborating data (AP-SIII.2Q, Attachment 3 , Item \#1). The confidence in the data is in question because data collection procedures are unavailable for review.

A comparison of the unqualified data and available qualified corroborating data will be conducted to determine that the unqualified data is acceptable and confidence in the data is warranted.

The team will conduct the comparison by considering the importance of the data to showing that the proposed U.S. Department of Energy repository design meets the performance objectives.

The following data evaluation criteria will be used:

1. Extent and quality of corroborating data

2. Agreement between the data being qualified and the corroborating data.

3. The importance of the data to showing that the proposed U.S. Department of Energy repository design meets the performance objectives of 10 CFR 60.

\section{I3. EVALUATION RESULTS}

The compositions reported by Ogard and Kerrisk (1984 [DIRS 100783]) for J-13 and p\#1 waters were used in the analysis presented in Appendix A because many of the sorption coefficient experiments used in this analysis were performed in the early 1980s. Thus, to make the comparisons of experimental data and model results more direct, the Ogard and Kerrisk data were used in the analysis. The Ogard and Kerrisk data were used as limiting cases to set 
bounding ranges for concentrations of various constituents, which were then used as inputs for the PHREEQC modeling for extrapolating sorption coefficient data (described in Appendix A) to $\mathrm{pH}$ and geochemical compositions for which sufficient laboratory measurements were not available. The results of this modeling, in conjunction with the experimental data, inform the process of expert judgment to arrive at the uncertainty distributions for sorption coefficients for Am (Appendix A7.1), Np (Appendix A7.3), Pu (Appendix A7.4), and U (Appendix A7.9). As such, the recommended distributions for sorption coefficient are expected to be weakly to moderately sensitive to the limits of compositional values that were input to the surface complexation modeling.

The composition of water from J-13 as reported by Ogard and Kerrisk (1984 [DIRS 100783]) and the corroborating data from MO0006J13WTRCM.000 [DIRS 151029] are given in Table I2. The corroborating data set is a verified and qualified data set per AP-SIII.2Q. As noted in Saturated Zone Site-Scale Flow Model (BSC 2004 [DIRS 170037], Section A7.3.1), the uncertainty in concentration of major anions and cations is on the order of 10 percent for most species and 15 percent for $\mathrm{F}^{-}$. Thus, the data from the two sets can be expected to agree within 20 percent of each other for all the constituents except for F-, which should agree within 30 percent of each other. As seen in Table I-2, there is remarkably good agreement between the two data sets. It is seen that for number of constituents, the two data sets agree within the experimental uncertainty given in column 3. For those constituents that the data differences fall somewhat outside the data uncertainty $\left(\mathrm{Ca}_{2}{ }^{+}, \mathrm{Mg}_{2}{ }^{+}, \mathrm{K}^{+}, \mathrm{SiO}_{2}\right)$, the differences are still within the 20 percent limit. Thus the Ogard and Kerrisk data for the composition of the water sample from UE-25 J-13 meet the qualification requirements.

Table I-2. Comparison of Composition of Water from UE-25 J-13

\begin{tabular}{|l|c|c|c|}
\hline \multicolumn{1}{|c|}{ Chemical Constituent } & $\begin{array}{c}\text { Corroborating Data } \\
\text { MO0006J13WTRCM.000 } \\
\text { (DIRS 151029) } \\
\text { Concentration +- } \\
\text { Uncertainty (mg/L) } \\
\text { Concentration) (mg/L) }\end{array}$ & $\begin{array}{c}\text { \% Difference } \\
\text { ABS (100*(Column2- } \\
\text { Column3)/Column2) }\end{array}$ \\
\hline $\mathrm{Ca}^{2+}$ & 11.5 & $13 .+-0.99$ & 13 \\
\hline $\mathrm{Mg}^{2+}$ & 1.76 & $2.01+-0.21$ & 14 \\
\hline $\mathrm{Na}^{+}$ & 45 & $45.8+-2.29$ & 2 \\
\hline $\mathrm{K}^{+}$ & 5.3 & $5.04+-0.61$ & 5 \\
\hline $\mathrm{SiO}_{2}$ & 64.2 & $60.97+-1.85$ & 5 \\
\hline $\mathrm{Cl}^{-}$ & 6.4 & $7.14+-0.61$ & 12 \\
\hline $\mathrm{F}^{-}$ & 2.1 & $2.18+\ldots 0.29$ & 4 \\
\hline $\mathrm{SO}_{4}{ }^{2-}$ & 18.1 & $18.4+-1.03$ & 2 \\
\hline $\mathrm{HCO}_{3}{ }^{-}$ & $128^{(\mathbf{b})}$ & $129.8+-8.6$ & 1 \\
\hline $\mathrm{CO}_{3}{ }^{2-}$ & 0.0 & - & $\mathrm{N} / \mathrm{A}$ \\
\hline $\mathrm{PH}$ & 6.9 & $7.41+-0.44$ & 7 \\
\hline
\end{tabular}

The composition of water from UE-25 p\#1 (carbonate) as reported by Ogard and Kerrisk (1984 [DIRS 100783]) and the corroborating data from MO0006J13WTRCM.000 [DIRS 151029] are given in Table I-3. The corroborating data set is verified and qualified per AP-SIII.2Q. As noted in Saturated Zone Site-Scale Flow Model (BSC 2004 [DIRS 170037], Section A7.3.1), the uncertainty in concentration of major anions and cations is on the order of 10 percent for most 
species and 15 percent for $\mathrm{F}^{-}$. Thus the data from the two sets can be expected to agree within 20 percent of each other. As shown in Column 4 of the Table I-3, this holds for all the constituents except $\mathrm{Cl}^{-}$. The Ogard and Kerrisk value for $\mathrm{Cl}^{-}$is somewhat higher than this range. However, because the data are used for setting limits on the range compositional values, this is considered acceptable. Thus, the Ogard and Kerrisk data for the composition of the water sample from UE25 p\#1(carbonate) meet the qualification requirements.

Table I-3. Comparison of Compositions of Water from UE-25 p\#1(Carbonate)

\begin{tabular}{|l|c|c|c|}
\hline \multicolumn{1}{|c|}{ Chemical Constituent } & $\begin{array}{c}\text { Corroborating Data } \\
\text { MO007MAJIONPH.011 } \\
\text { (DIRS 151524) } \\
\text { mg/L }\end{array}$ & $\begin{array}{c}\text { Ogard and Kerrisk 1984 } \\
\text { (DIRS 100783) Data } \\
\text { (mg/L) }\end{array}$ & $\begin{array}{c}\text { \% Difference } \\
\text { ABS(100*(Column2- } \\
\text { Column3)/Column2) }\end{array}$ \\
\hline $\mathrm{Ca}^{2+}$ & 100. & 87.8 & 12 \\
\hline $\mathrm{Mg}^{2+}$ & 39. & 31.9 & 18 \\
\hline $\mathrm{Na}^{+}$ & 150 & 171 & 14 \\
\hline $\mathrm{K}^{+}$ & 12 & 13.4 & 12 \\
\hline $\mathrm{SiO}_{2}$ & 64.2 & 64.2 & 0 \\
\hline $\mathrm{Cl}^{-}$ & 28 & 37 & 32 \\
\hline $\mathrm{F}^{-}$ & $\mathrm{N} / \mathrm{D}$ & 3.5 & $\mathrm{~N} / \mathrm{A}$ \\
\hline $\mathrm{SO}_{4}{ }^{2-}$ & 160 & 129 & 19 \\
\hline $\mathrm{HCO}_{3}{ }^{-}$ & 694 & 666 & 4 \\
\hline $\mathrm{CO}_{3}{ }^{2-}$ & 0.0 & 0 & $\mathrm{~N} / \mathrm{A}$ \\
\hline $\mathrm{PH}$ & 6.6 & 6.7 & 6 \\
\hline
\end{tabular}

\section{I4. RESULTS}

The qualification team determined, based on the discussions above, that the referenced data are both reasonable and appropriate for the application. This evaluation did not produce any new data.

\section{I5. CONCLUSION}

The data qualification effort documented in this report was performed in accordance with the requirements of the approved Data Qualification Plan included in this appendix. The approved qualification method, corroborating data, was implemented as required. As a result of this qualification evaluation, these data are qualified for inclusion in the model report Site-Scale Saturated Zone Transport. 


\begin{tabular}{|c|c|c|}
\hline BSC & DATA QUALIFICATION PLAN & $\frac{\text { QA: QA }}{\text { Page } 1 \text { of } 1}$ \\
\hline \multicolumn{3}{|c|}{ Section I. Organizational Information } \\
\hline \multicolumn{3}{|l|}{ Qualification Title } \\
\hline \multicolumn{3}{|c|}{ QUALIFICATION OF COMPOSITIONS OF WATERS FROM UE-25 J-13 AND UE-25 P\#1(CARBONATE) } \\
\hline \multicolumn{3}{|l|}{ Requesting Organization } \\
\hline \multicolumn{3}{|c|}{ Regulatory Integration Team - Saturated Zone. } \\
\hline \multicolumn{3}{|c|}{ Section II. Process Planning Requirements } \\
\hline \multicolumn{3}{|c|}{ 1. List of Unqualified Data to be Evaluated } \\
\hline \multicolumn{3}{|c|}{$\begin{array}{l}\text { The data that were evaluated and documented in this qualification report are published in the report Ogard, A.E. and Kerrisk, J.F. } \\
\text { 1984. Groundwater Chemistry Along Flow Paths Between a Proposed Repository Site and the Accessible Environment. LA-10188- } \\
\text { MS. Los Alamos, New Mexico: Los Alamos National Laboratory. ACC: HQS.19880517.2031. [DIRS 100783]. These data are shown } \\
\text { in Table below. }\end{array}$} \\
\hline Chemical Constituent & UE-25 J-13 Water UE-25 p\#1 (Carbonate) Water (mg/ & \\
\hline $\mathrm{Ca} 2+$ & 87.8 & \\
\hline $\mathrm{Mg} 2+$ & 1.76 & \\
\hline $\mathrm{Na}+$ & 171 & \\
\hline $\mathrm{K}+$ & 13.4 & \\
\hline $\mathrm{SiO} 2$ & 64.2 & \\
\hline $\mathrm{Cl}-$ & 6.4 & \\
\hline $\mathrm{F}-$ & 3.5 & \\
\hline SO42- & 18.1 & \\
\hline $\mathrm{HCO}_{3}-$ & 128 & \\
\hline $\mathrm{CO} 32-$ & 0.0 & \\
\hline \multicolumn{3}{|c|}{$\begin{array}{l}\text { A comparison of the unqualified data and available qualified corroborating data will be conducted to determine that the unqualified } \\
\text { data is acceptable and confidence in the data is warranted. } \\
\text { The team will conduct the comparison by considering the importance of the data to showing that the proposed U.S. Department of } \\
\text { Energy repository design meets the performance objectives. } \\
\text { The following data evaluation criteria will be used: } \\
\text { 1. Extent and quality of corroborating data } \\
\text { 2. Agreement between the data being qualified and the corroborating data. } \\
\text { 3. The importance of the data to showing that the proposed U.S. Department of Energy repository design meets the performance } \\
\text { objectives of } 10 \text { CFR } 60 \text {. }\end{array}$} \\
\hline \multicolumn{3}{|c|}{ 3. Data Qualification Team and Additional Support Staff Required } \\
\hline \multicolumn{3}{|c|}{$\begin{array}{l}\text { Qualification Chairperson: Sharad Kelkar, LANL, } 5056674639 \\
\text { Team Member: James Houseworth, LBL, } 7022957632\end{array}$} \\
\hline
\end{tabular}




\begin{tabular}{|l|l|l|}
\hline \multirow{2}{*}{ BSC } & DATA QUALIFICATION PLAN & QA: QA \\
\cline { 3 - 3 } & & Page 2 of 1 \\
\hline
\end{tabular}

1. The following data evaluation criteria will be used:

1. Extent and quality of corroborating data- the corroborating data should be verified and qualified per AP-SIII.2Q

2. Agreement between the data being qualified and the corroborating data should be within the experimental uncertainty.

3. The importance of the data to showing that the proposed U.S. Department of Energy repository design meets the performance objectives of 10 CFR 60 will be considered in evaluating the criteria 1 and 2 above.

5. Identification of Procedures Used

AP-SIII.10Q, Models

Section III. Approval

\begin{tabular}{|l|c|l|l|}
\hline Qualification Chairperson Printed Name & Qualification Chairperson Signature, & Date \\
Sharad Kelkar & Responsible Manager Signature & Date \\
\hline $\begin{array}{l}\text { Responsible Manager Printed Name } \\
\text { Ahmed Monib }\end{array}$ & Chend:/2004 \\
\hline
\end{tabular}

